

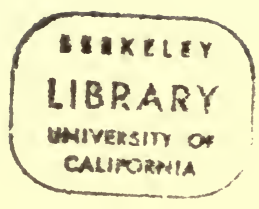








\title{
THEORETICAL MECHANICS
}

AN INTRODUCTORY TREATISE

\author{
ON THE
}

\section{PRINCIPLES OF DYNAMICS.}




\section{Zlonton: C. J. CLAY AND SONS, CAMBRIDGE UNIVERSITY PRESS WAREHOUSE, AVE MARIA LANE,}

Glasgobs: 263, ARGYLE STREET.

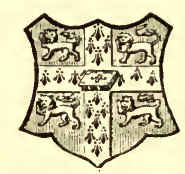

Zleipz̧ig: F. A. BROCKHAUS.

\{elo gork: THE MACMILLAN COMPANY.

Sombay: E. SEYMOUR HALE. 


\title{
THEORETICAL MECHANICS
}

\author{
AN INTRODUCTORY TREATISE
}

ON THE

\section{PRINCIPLES OF DYNAMICS}

WITH APPLICATIONS AND NUMEROUS EXAMPLES.

BY

A. E. H. LOVE, M.A., F.R.S.,

FELLOW AND LECTURER OF ST JOHN'S COLLEGE, CAMBRIDGE.

CAMBRIDGE :

AT THE UNIVERSTTY PRESS.

1897

[All Rights reserved.] 


\section{Cambrioge:}

PRINTED BY J. AND C. F. CLAY, AT THE UNIVERSITY PRESS.

Math. Stat.

Add' 1

GIFT 


\section{PREFACE.}

THE foundations of Mechanical Science were laid by Newton, and his achievements in this department constitute perhaps his most enduring title to fame. Later writers have developed his principles analytically, and have extended the region of their application, but, in regard to the principles themselves, they have acted the part of commentators. Nevertheless we may trace a tendency in modern investigations, which is of the nature of a gradual change in the point of view: there is less search for causes, more inclination to regard the object to be attained as a precise formulation of observed facts. On another side there is an important respect in which modern writers have departed from the form of the Newtonian theory. The philosophical dictum that all motion is relative stands in pronounced contradiction with Newton's dynamical apparatus of absolute time, absolute space, and absolute motion. It has been necessary to reconsider in detail the principles, and the results deduced from them, in order to ascertain what modification would be needed to bring the theory of Rational Mechanics founded by Newton into harmony with the doctrine of the relativity of motion.

The purpose of this book is didactic; it is meant to set before students an account of the principles of Mechanics, which shall be as precise as possible, and which shall be in accordance with modern ideas.

The book is divided into three parts. The first part is preliminary in character, and is intended to accustom the student to the idea of acceleration, and to the fact that a precise description of any motion can be given by a statement of the accelerations involved. In the first Chapter attention has been paid to the determination of position, the essential relativity of position being the key to much that follows. In the second Chapter is introduced the idea of a Vector, and it proves useful to recognise classes 
of Vectors separated by degrees of localisation. In the third Chapter care has been taken to give precise definitions of velocity and acceleration. The fourth Chapter treats of the simpler motions - uniformly accelerated rectilinear motion, parabolic motion, simple harmonic motion, elliptic motion, and central orbits.

The second part is devoted to an exposition of the Principles of Dynamics. Chapter V. contains a statement of the principles. The standpoint adopted is that for which the notion of Mass is the central idea of the subject. In Chapter VI. will be found an analytical formulation of the general theory, so far as to include the equations of motion and the theory of the motion of the centre of inertia. It does not include the equations of Lagrange. Chapter VII. treats of systems of forces, the main point dealt with being the resultant of forces applied to a rigid body. An indication is given here of the application of theoretical mechanics to elastic bodies. Chapter VIII. deals with the theory of work and energy, the equation of energy being regarded primarily as an integral of the equations of motion. A note at the end of Chapter V. indicates very briefly the history of the fundamental principles of Dynamics, and a note at the end of Chapter VIII. describes critically the transformation of the subject from a theory of force to a theory of energy. To avoid interruptions of the argument, the discussion of Units and Dimensions is postponed to an Appendix, and some matters which offer special difficulties, when not treated from the old "absolute" standpoint, are reserved for the last Chapter (XIII.).

The third part of the book is devoted to exemplifying the applications of the general theory. Chapters IX. and X. deal with Dynamics of a Particle; the former referring to free motions of particles, and the latter to constrained and resisted motions. Chapter XI. contains the elements of Rigid Dynamics. Experience shows that students appreciate the theory of momentum most easily in its application to rigid bodies. The subject is treated only in its elementary stages, the geometrical difficulties inherent in the consideration of three dimensional motions making it advisable to postpone their discussion. Chapter XII. contains a 
number of miscellaneous methods and subsidiary theories, relating to impulses, initial motions, small oscillations, and the motion of chains, and it includes also some further applications of the principles of energy and momentum. The order is very different from that adopted in most English text-books; in the ordinary course Mechanics is subordinated to Geometry, the order being that of geometrical difficulty. The order here adopted is meant to be that of the difficulty of the physical notions involved. There can be no doubt that the mechanical meaning of equations of motion in general is easier to grasp than that of equations of initial motion, and the theory of collision involves special hypotheses subsidiary to the general principles of dynamics.

The class of students for whom the book is intended may be described as beginners in Mathematical Analysis. The reader is supposed to have a slight acquaintance with the elements of the Differential and Integral Calculus, and some knowledge of Plane Coordinate Geometry. $\mathrm{He}$ is not assumed to have read Solid Geometry or Differential Equations. The apparatus of Cartesian Coordinates in three dimensions is described, and the solutions of the differential equations that occur are explained. It not infrequently happens that analytical methods are preferred to geometrical ones, as likely to be more helpful to the students whose wants are in view. Chapter XI., and isolated Articles in other chapters, are marked with an asterisk to indicate that in a first reading they may with advantage be omitted. These Articles usually contain matter of such a degree of difficulty that they are likely to be more easily understood after the rest of the book has been read, and further progress in Pure Mathematics has been made. A student attempting to read the book without the guidance of a teacher is recommended to pay the greatest attention in the first place to the unmarked Articles in Chapters IV., IX., X., and XII., and to work out the Examples inserted in the text and attached to such Articles, passing lightly over the more theoretical Chapters, and reverting to them wherever they are referred to. He cannot expect to grasp the whole subject at once in its logical order, and he will find it advisable to read some parts two or three times, connecting them with different special applications. 
In addition to the Examples in the text, some of which are well-known theorems and are referred to in subsequent demonstrations, large collections have been appended to some of the Chapters. It is hoped that these may prove useful to teachers, and to students occupied in revising their work. These Examples are for the most part taken from .University and College Examination papers; others, in very small number, which I have not found in such papers, are taken from the well-known collections of Besant, Routh, and Wolstenholme.

The works which have been most useful to me in connexion with matters of principle are Kirchhoff's Vorlesungen über Mathematische Physik (Mechanik), Pearson's Grammar of Science, and Mach's Science of Mechanics. The last should be in the hands of all students who desire to follow the history of dynamical ideas. In regard to methods for the treatment of particular questions, I am conscious of a deep obligation to the teaching of Mr R. R. Webb.

$\mathrm{I}$ am much indebted to the kindness of friends who have assisted me in the production of this book. Mr J. Larmor has read a large part of the manuscript, and his criticisms and suggestions have been of the greatest value. He has also pointed out a number of errors in the proofs, and has shown a deep and encouraging interest in the progress of the work. Mr J. Greaves has read all the proofs with untiring punctuality and care, and the painstaking industry he has expended upon them leads me to hope that the book may be found free from serious misprints. $\mathrm{He}$ has also helped with many valuable suggestions. Prof. Greenhill also has assisted me with a number of corrections and criticisms. $\mathrm{Mr} \mathrm{R}$. Hargreaves has most kindly performed the distasteful task of verifying a large number of the Examples. His work shows that a sensible proportion of those submitted to him were originally either ambiguous or incorrect, and I fear that many inaccuracies may remain among the others. I shall be grateful for a notice of any correction that ought to be made.

A. E. H. LOVE.

Cambridge, August, 1897. 


\title{
CONTENTS.
}

\section{PRELIMINARY.}

\author{
CHAPTER I.
}

ARTS.

2-6. Position, Time, and Motion. Frame of Reference . . 2-7

\section{CHAPTER II.}

7-13. Definition of a Vector. Composition and Resolution of Vectors . . . . . . . . 8-15

14-27. Localised Vectors, Moments, and Couples. Reduction of a system of Vectors . . . . . . . . . 16-29

\section{CHAPTER III.}

28-33. Definitions of Displacement, Velocity, and Acceleration . $30-34$ 34-38. Notation and Formulæ for Acceleration. Circular Motion 34-38 39, 40. Relative Motion . . . . . . . . . 39-41

\section{CHAPTER IV.}

41-44. Uniformly Accelerated Motion. Gravity . . . . 42-44

45, 46. Parabolic Motion . . . . . . . . . 44-47

47-49. Simple Harmonic Motion . . . . . . . 47-51

50-52. Central Orbits . . . . . . . . . 51-54

53-57. Elliptic Motion. Law of Inverse Square . . . . . 54-59

58-62. Radial and Transversal Accelerations. Differential Equation of Central Orbits . . . . . . . $60-64$

63-66. Apses. Stability of Circular Orbits . . . . . 65-68 Examples . . . . . . . . . . 68-84 


\title{
THE PRINCIPLES OF DYNAMICS.
}

\author{
CHAPTER V. \\ ARTS. \\ PAGES \\ 67-74. Conception of a Body. Mutual Actions. Mass. Density 85-88 \\ 75-77. Force. Motion of a Body under given Forces . . . 88-90 \\ 78-80. Momentum. Kinetic Reaction. Equations of Motion . 90 \\ 81-83. Impulses . • . • • • • • • . 90-92 \\ 84-88. Rational Mechanics and its applications. Postulates of \\ Mechanics . . . . . . . . . . 9 92, 93 \\ 89-94. Field of Force, Weight, Validity of Concept of Mass, \\ Gravitation . . . . . . . . 9 93-98 \\ 95-97. Quantity of Matter. Inertia . . . . . . . 98, 99 \\ Historical Note . . . . . . . . . 100, 101
}

\section{CHAPTER VI.}

98-104. Theory of Parallel Axes and Motion of Centre of Inertia 102-105 105-110. Equations of Motion. D'Alembert's Principle. Independence of Translation and Rotation . . . . . 106-108

111-113. Conservation of Momentum. Impulses . . . . 108-110

\section{CHAPTER VII.}

114-117. Forces acting on Rigid Systems . . . . . 111-114

118-120. Constraints and Resistances. Friction . . . . 114, 115

121-124. Deformable Bodies. Stress and Strain. Surface Tractions 116-118

125-130. Strings and Springs . . . . . . . . 119-122

CHAPTER VIII.

131-138. Work done by Forces. Conservative System. Potential Energy

139-150. Examples of Calculation of Work . . . . . 127-133

151. Equation of Energy . . . . . . . . . 133-135

152-154. Positional and Motional Forces. Conservation of Energy.

Power . . . . . . . . . 135-138

155. Kinetic Energy produced by Impulses _ . • • . 138, 139

156-159. Virtual Work. Variational Equation of Motion . . 139-141

Critical Note . . . . . . . . . 141-144 


\section{METHODS AND APPLICATIONS.}

ARTS.

\section{CHAP'TER IX.}

160-174. Dynamics of a Particle. Given Fields of Force . . 145-154

175-177. Problem of Two Bodies. Planetary Motions . . . 154-159

178-181. Disturbed Elliptic Motion . . . . . . . 159-161

Examples . . . . . . . . . . . 162-171

\section{CHAPTER X.}

182-187. Constrained Motion under Gravity. Atwood's Machine . 172-177

188-190. Simple Circular Pendulum. Small Oscillation . . . 178-180

191-194. Finite Motion of Pendulum . . . . . . 180-182

195-202. Motion on Smooth Curve. Rotating Tube. Rough Curve 182-189

203-207. Motion on a Surface. Conical Pendulum . . . . 189-195

208-213. Resisting Medium . . . . . . . . 195-200

Examples . . . . . . . . . . . . . $201-226$

\section{CHAPTER XI.}

214. Two-dimensional Motion of Rigid Body. Angular Velocity 227, 228 215-217. Moments of Inertia . . . . . . . . 228-231

218-220. Momentum, Kinetic Energy, and Kinetic Reaction of Rigid Body . . . . . . . . . . 232-235

221-224. Equations of Motion. Pendulum . . . . . $\quad$. 235-238

225. Illustrative Problems. Inertia, Friction, Stress . 238-247

Examples . . . . . . . . . . 247-255

\section{CHAPTER XII.}

226. Introductory Remarks

227-236. Impulses. Restitution of Form in collision. Impulsive Changes of Motion . . . . . . . . 256-269

237-244. Impulsive motion of rigid bodies . . . . . 269-278

245-249. Initial motions of systems of particles . . . . 278-282

250-252. Initial motions of systems of rigid bodies . . . . . 282-286

253,254 . Small oscillations of systems of particles . . . . 286-289

255-257. Small oscillations of systems of rigid bodies . . . 289-292

258, 259. Applications of the Principles of Energy and Momentum 293-295

260-264. Stability of steady motions. Principles of Energy and

Momentum applied to systems of rigid bodies . . 295-301

265-268. Motion of a chain, restricted freedom . . . . 301-304

269-274. General motion of a chain in two dimensions. Initial

Motion and Impulsive Motion . . . . . 304-310

Examples . . . . . . . . . . 310-350 


\section{CHAPTER XIII.}

ARTS.

275-284. Rotation of the Earth, Weight, Acceleration due to Gravity, Pendulum

285-289. Relativity of Force. Universal Gravitation

290, 291. Measurement of time. Effect of change of time-measuring process

\section{APPENDIX.}

292-299. Measurement, Units, and Dimensions 


\section{CORRIGENDA.}

p. 3, line 25 from the top, insert positive before number.

p. 68 , Example 2, the path is a rectangular hyperbola.

p. 69, Example 14 for $\cos ^{-1}\left\{\right.$ read $\left\{\cos ^{-1}\right.$.

p. 70, Example 17, the path is a conic.

p. 70, Example 18, for sine read hyperbolic sine.

p. 71, Example 24, the third component should be $\dot{w}-(u w x+v w y) / r^{2}$.

p. 72, Example 36, the range is $2 R$, not $R$.

p. 75, Example 60, for $2 h v^{2}$ a read $2 h v^{2} g$.

p. 76, Example 66, for $\frac{1}{2} \pi+\theta$ read $\frac{1}{2} \pi-\theta$.

p. 79, Example 94, result should be $\frac{\sqrt{ } 2}{3 \pi}\left(1+\frac{2}{n}\right) \sqrt{ }\left(1-\frac{1}{n}\right)$ years.

p. 80, Example 105, result should be $e^{3} h^{2} X P /\left(l S L^{3}\right)$.

p. 82 , Example 121, the acceleration contains an additional term varying inversely as the cube of the distance.

p. 84, Example 138 for $4 \kappa^{2}\left(2 a^{-3}-\ldots\right)$ read $4 \kappa^{2}\left(2 r^{-3}-\ldots\right)$.

p. 164, Example 17, result should read

$$
h_{0}^{2}\left(\sin \theta \frac{d u}{d \theta}-u \cos \theta\right)-\frac{1}{2} \mu\left\{(\sin 3 \theta-\sin \theta) \frac{d u}{d \theta}-2 \cos \theta\right\}=C .
$$

p. 164, Example 18, result should read

$$
\frac{d^{2} u}{d \theta^{2}}+u=\left\{P-f(t) \frac{d u}{d \theta}\right\} / u^{2}\{F(t)\}^{2} .
$$

p. 165 , line 3 from the bottom, for $u / a$, read $\frac{1}{3} a u^{3}$.

p. 224, Example 155,

$$
\text { for } \frac{g x}{a^{2}}=\int_{p}^{a} P^{-\frac{2}{p}} d p \quad \text { read } \frac{g x}{a^{2}}=\int_{p}^{a} P^{-\frac{2}{n}} d p .
$$

p. 263 , line 9 from the top,

$$
\begin{aligned}
\text { for } & \left\{(U-u)^{2}-\left(U^{\prime}-u^{\prime}\right)^{2}\right\} \\
\text { read } & \left\{\left(U-U^{\prime}\right)^{2}-\left(u-u^{\prime}\right)^{2}\right\} .
\end{aligned}
$$

p. 289, line 11 from the bottom, for kinectic read kinetic. 



\section{PRELIMINARY.}

\section{CHAPTER I.}

\section{INTRODUCTORY.}

1. Mechanics is a Natural Science; its data are facts of experience, its principles are generalisations from experience. The possibility of Natural Science depends on a principle which is itself derived from multitudes of particular experiences-the "Principle of the Uniformity of Nature." This principle may be stated as follows-Natural events take place in invariable sequences. The object of Natural Science is the description of the facts of nature in terms of the rules of invariable sequence which natural events are observed to obey. These rules of sequence, discovered by observation, suggest to our minds certain general notions in terms of which it is possible to state the rules in abstract forms. Such abstract formulas for the rules of sequence which natural events obey we call the "Laws of Nature." When any rule has been established by observation, and the corresponding Law formulated, it becomes possible to predict a certain kind of future events.

The Science of Mechanics is occupied with a particular kind of natural events, viz. with the motions of material bodies. Its object is the description of these motions in terms of the rules of invariable sequence which they obey. For this purpose it is necessary to introduce and define a number of abstract notions suggested by observations of the motions of actual bodies. It is then possible to formulate laws according to which such motions take place, and these laws are such that the

L. 
future motions and positions of bodies can be deduced from them, and predictions so made are verified in experience. But in the process of formulation the Science assumes a highly abstract character. The fundamental notions involved have, for the most part, names in common use, such as "force" for example, but the common use of such names is never precise, and for scientific purposes the meaning to be attached to them must be made definite. This is done, as in Geometry, by means of definitions and postulates. Except in the statement of the postulates nothing ought to be taken from experience, all the results ought to be logically deduced. There is thus an abstract logical theory of Mechanics, of the same nature as Geometry, in which all that is assumed is suggested by experience, all that is found is proved by reasoning. The test of the validity of a theory of this kind is its consistency with itself, the test of its value is its ability to furnish rules under which natural events actually fall. In what follows we shall be mainly occupied with the exposition of the theory, we shall not detail the observations and experiments by which the fundamental notions were suggested, nor shall we do more than indicate in particular cases the kinds of natural events to which applications of our theory can be made.

2. Motion of a point. We have said that our object is the description of the motions of bodies. The necessity for a simplification arises from the fact that, in general, all parts of a body have not the same motion, and the simplification we make is to consider the motion of so small a portion of a body that the differences between the motions of its parts are unimportant. How small the portion must be in order that this may be the case we cannot say beforehand, but we avoid the difficulty thus arising by regarding it as a geometrical point. We think then in the first place of the motion of a point.

Motion may be defined as change of position taking place in time.

In regard to this definition it is necessary to make clear two things: one is the measurement of time, and the other is the meaning of the phrase "change of position."

3. Measurement of time. Time may be measured by any process which goes on continually. The amount of the 
process that is effected in any interval of time is supposed to be measurable, and the measure of this amount can be taken to measure the interval, so that equal intervals of time are those in which equal amounts of the process selected as time-measurer take place, and different intervals are in the ratio of the measures of the amounts of the process that take place in them. In any interval of time many processes may be going on. Of these one is selected as a time-measurer; we shall call it the standard process. "Uniform processes" are such that equal amounts of them are effected in equal intervals of time, that is, in intervals in which equal amounts of the standard process are effected. Processes which are not uniform are said to be "variable." It is clear that processes which are uniform when measured by one standard may be variable when measured by another standard. The choice of a standard being in our power, it is clearly desirable that it should be so made that a number of processes uncontrollable by us should be uniform or approximately uniform; it is also clearly desirable that it should have some relation to our daily life. The process actually adopted for measuring time is the average rotation of the Earth relative to the Sun*, and the unit in terms of which this process is measured is called the "mean solar second." In the course of this book we shall assume that time is measured in this way, and we shall denote the measure of the time which elapses between two particular instants by the letter $t$, then $t$ is a real number (in the most general sense of the word "number") and the interval it denotes is $t$ seconds.

4. Determination of Position. Position of a point relative to a set of points $\uparrow$ is not definite until the set includes four points which do not all lie in one plane. Suppose $O, A, B, C$ to be four such points; one of them, $O$, is chosen and called the origin, and the three planes $O B C, O C A, O A B$ are the faces of a trihedral angle having its vertex at $O$. The position of a point $P$ with reference to this trihedral angle is determined as follows:-we draw $P N$ parallel to $O C$ to meet the plane $A O B$ in $N$, and we draw $N M$ parallel to $O B$ to meet $O A$ in $M$; then the lengths $O M, M N, N P$ determine the position of $P$. Any particular length, e.g. one centimetre, being taken as the unit of length, each of these

* See Chapter XIII.

+ The phrase "position of a point" means its position relative to other points. 
lengths is represented by a number (in the general sense) viz. by the number of centimetres contained in it. It is clear that $O P$

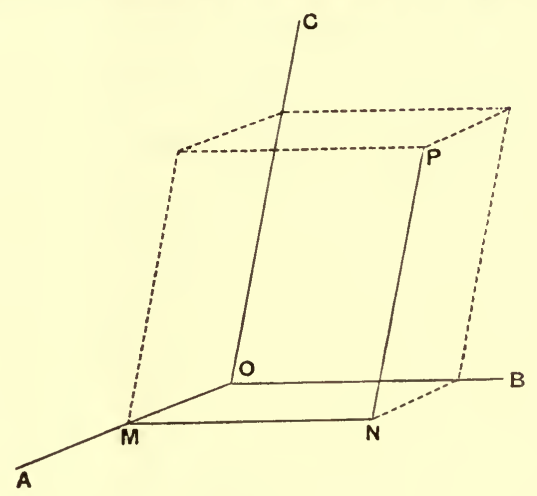

Fig. 1.

is a diagonal of a parallelepiped and that $O M, M N, N P$ are three edges no two of which are parallel. The position of a point is therefore determined by means of a parallelepiped whose edges are parallel to the lines of reference, and one of whose diagonals is the line joining the origin to the point.

It is generally preferable to take the set of lines of reference to be three lines mutually at right angles, then the faces of the trihedral angle are also at right angles; sets of lines so chosen are called systems of rectangular axes, and the planes that contain two of them are coordinate planes*. It is clear from the figure

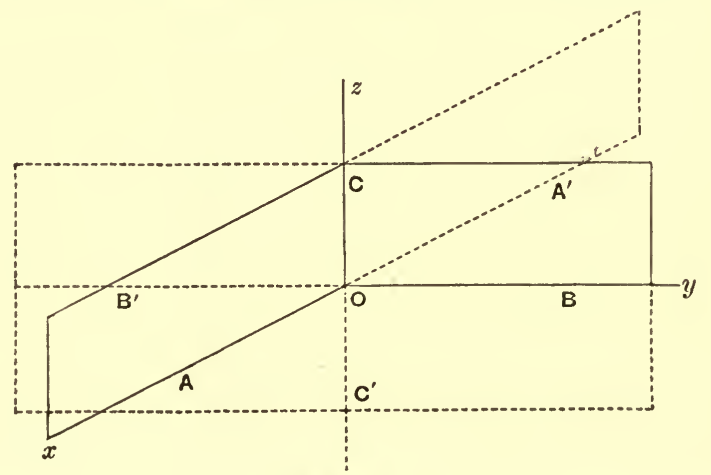

Fig. 2. only.

* We shall, in the course of this book, make use of rectangular coordinates 
that a set of rectangular coordinate planes divide the space about a point into eight compartments, the particular trihedral angle $O A B C$ being one compartment. The lengths $O M, M N, N P$ of Fig. 1, taken with certain signs, are called the coordinates of the point $P$, and are denoted by the letters $x, y, z$. The rule of signs is that $x$ is equal to the number of units of length in the length $O M$ when $P$ and $A$ are on the same side of the plane $B O C$, and is equal to this number with a minus sign when $P$ and $A$ are on opposite sides of the plane $B O C$, and similarly for $y$ and $z$. We can express the conventions as regards sign by means of the following table, in which $A^{\prime}, B^{\prime}, C^{\prime}$ denote points in $A O, B O, C O$ produced:-

\begin{tabular}{|c|c|c|c|c|c|c|}
\hline & & & & sign of $x$ & sign of $y$ & sign of $z$ \\
\hline & he & ingle & $O A B C$ & + & + & + \\
\hline$"$ & $"$ & $"$ & $O A^{\prime} B C$ & - & + & + \\
\hline$"$ & $"$ & $"$ & $O A B^{\prime} C$ & + & - & + \\
\hline$"$ & $"$ & $"$ & $O A^{\prime} B^{\prime} C$ & - & - & + \\
\hline$"$ & $"$ & $"$ & $O A B C^{\prime}$ & + & + & - \\
\hline$"$ & $"$ & $"$ & $O A^{\prime} B C^{\prime}$ & - & + & - \\
\hline " & & $"$ & $O A B^{\prime} C^{\prime}$ & + & - & - \\
\hline$"$ & & $"$ & $O A^{\prime} B^{\prime} C^{\prime}$ & - & - & - \\
\hline
\end{tabular}

It is clear that the coordinates $x, y, z$ determine the position of the point $P$ with reference to the lines $O A, O B, O C$.

A set of lines of reference such as $O A, O B, O C$ with respect to which the position of a point $P$ can be determined will be called a frame of reference. 
5. Change of position. Suppose that a point which, at any particular instant, had a position $P$ with reference to any frame, has at some later instant a position $Q$ relative to the same frame. The point is said to have undergone a "change of position" or a displacement. Let the line $P Q$ be drawn. It is clear that the displacement is precisely determined by this line; we say that it is represented by this line. Suppose the line $P Q$ drawn through $P$ to be produced indefinitely both ways, a parallel line may be drawn through any other point,

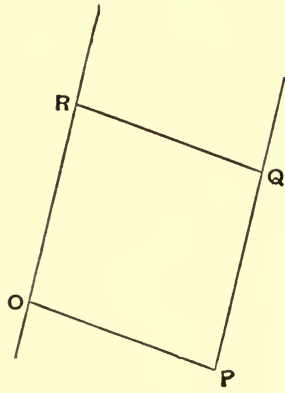

Fig. 3. for instance through $O$, and then this line determines a particular direction; this is the direction of the displacement. Of the two senses in which this line may be described one, $O R$, is the sense from $O$ towards the point $R$ which is the fourth corner of a parallelogram having $O P, P Q$ as adjacent sides; this is the sense of the displacement. The measure of the length of $P Q$ is the number of units of length it contains; this number is the magnitude of the displacement. The subsequent position, $Q$, is entirely determined by (1) the previous position, $P,(2)$ the direction of the displacement, (3) the sense of the displacement, (4) the magnitude of the displacement.

Further it is clear that exactly the same change of position is effected in moving a point from $P$ to $K$ by

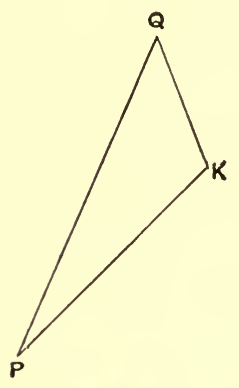

Fig. 4. the straight line $P K$, and from $K$ to $Q$ by the straight line $K Q$, as in moving, the point from $P$ to $Q$ directly by the straight line $P Q$. That is to say, displacements represented by lines $P K, K Q$ are equivalent to the displacement represented by the line $P Q$.

Displacement is a quantity, for one displacement can be greater, equal to, or less than another; but two displacements in different directions, or in different senses, are clearly not equivalent to each other; and thus displacement belongs to the class of mathematical quantities known as vectors or directed 
quantities. We shall proceed in the next Chapter to discuss the geometry of vectors, and shall take up the proper development of our subject in the following Chapter.

6. Note on the determination of frames of reference. To determine a frame of reference we require to be able to mark a point, a line through that point, and a plane through that line. Suppose $O$ to be the point, $O A$ a line through the point, $A O B$ a plane through the line. We can draw on the plane a line at right angles to $O A$ meeting it in $O$, and we can erect at $O$ a perpendicular to the plane. The three lines so determined can be a frame of reference.

In practice we cannot mark a point but only a small part of a body, for example we may take as origin a place on the Earth's surface; then at the place we can always determine a particular line, the vertical at the place, and, at right angles to it, we have a particular plane, the horizontal plane at the place; on this plane we may mark the line which points to the North, or in any other direction determined with reference to the points of the compass, we have then a frame of reference. Again we might draw from the place lines in the directions of any three visible stars, these would form a frame of reference. Or again we might take as origin the centre of the Sun, and as lines of reference three lines going out from thence to three stars. The choice of a suitable frame of reference, like the choice of the time-measuring process, is in our power, and it is manifest that some motions which we wish to describe will be more simply describable when the choice is made in one way than when it is made in another. We shall return to this matter in Chapter XIII. 


\section{CHAPTER II.}

\section{GEOMETRY OF VECTORS.}

7. Definition of a Vector. A vector is an object of mathematical reasoning which requires for its determination (1) a number called the magnitude of the vector, (2) the direction of a line called the direction of the vector, (3) the sense in which the line is supposed drawn from one of its points, called the sense of the vector, and which obeys a certain rule of mathematical operation to be presently stated.

Let any particular length be taken as unit of length. Then from any point a straight line can be drawn to represent the vector* in magnitude, direction, and sense. The sense of the line is indicated when two of its points are named in the order in which they are arrived at by a point describing the line.

The rule of mathematical operation to which vectors are subject is a rule for replacing one vector by other vectors to which it is (by definition) equivalent.

This rule may be divided into two parts and stated as follows :-

(1) Vectors represented by equal and parallel lines drawn from different points in like senses are equivalent.

(2) The vector represented by a line $A C$ is equivalent to the vectors represented by the lines $A B, B C$, the points $A, B, C$ being any points whatever.

* The line is not the vector. The line possesses a quality, described as extension in space, which the vector may not have. From our complete idea of the line this quality must be abstracted before the vector is arrived at. On the other hand the vector is subject to a rule of operation to which a line can only be subjected by means of an arbitrary convention. 
8. Examples of Equivalent Vectors. If $A C, A^{\prime} C^{\prime}$ are equal and parallel lines, their ends can be joined by two lines $A A^{\prime}, C C^{\prime}$ which are equal and parallel; then the vectors represented by $A C, A^{\prime} C^{\prime}$ are equivalent; vectors represented by $A C$, $C^{\prime} A^{\prime}$ are not equivalent.

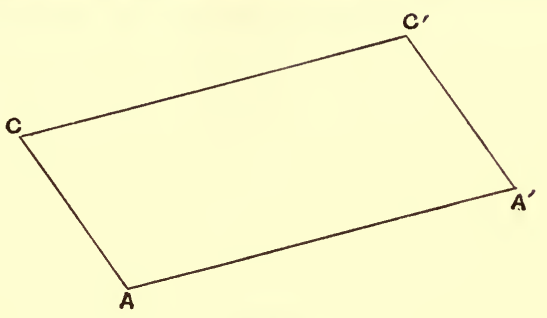

Fig. 5.

Again if $A, B, C$ are any three points, and a parallelogram $A, B, C, D$ is constructed having $A B, B C$ as adjacent sides, $A D$ and $B C$ are equivalent vectors. Also the vector $A C$ is equivalent to the vectors $A B, B C$, or $A D, D C$, or $A B, A D$.

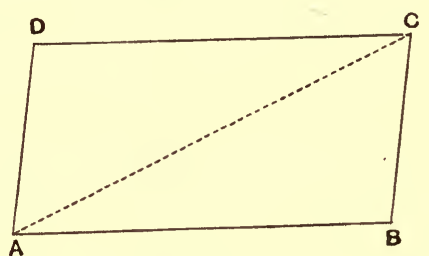

Fig. 6 .

Further if a polygon (plane or gauche) is constructed, having $A C$ as one side, and having any points $P, Q, \ldots T$ as corners, the vector represented by $A C$ is equivalent to the vectors represented by $A P, P Q, \ldots T C$. This is clear because by definition the vectors $A P, P Q$ can be replaced by $A Q$, and so on. The statement is independent of the number of sides of the polygon, and of the order in which its corners are taken, no corner being taken more than once, provided the points $A, C$ are regarded as the first and last corners. [The restriction that no corner is to be taken

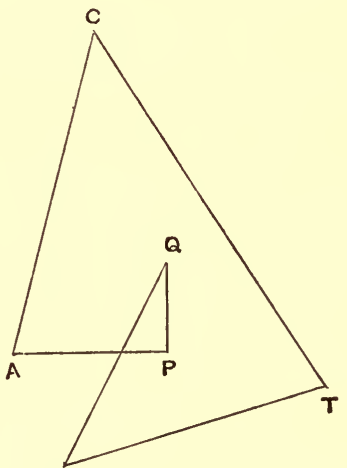

Fig. 7. more than once will be presently removed.]

In particular if the polygon is a gauche quadrilateral $A B D C$ a parallelepiped can be constructed having its edges parallel to $A B$, $B D, D C$, and having $A C$ as one diagonal. Then the vector $A C$ is equivalent to the vectors represented by the edges $A B, A P, A Q$ which meet in $A$. (See Fig. 8.)

The case of this which is generally most useful is the case 
where the edges of the parallelepiped are the axes of reference relative to which the positions of points are determined.
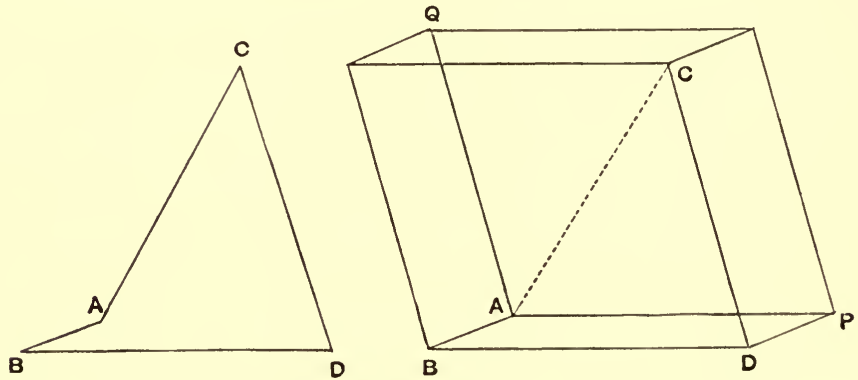

Fig. 8.

9. Components and Resultant. A set of vectors equivalent to a single vector are called components, and the single vector to which they are equivalent is called their resultant.

The operation of deriving a resultant vector from given component vectors is called composition, we compound the components to obtain the resultant; the operation of deriving components in particular directions from a given vector is called resolution, we resolve the vector in the given directions to obtain the components in those directions.

It is clear from the constructions in the preceding article that we can resolve a vector in one way into components parallel to any two given lines which are in a plane to which the vector is parallel, and again we can resolve the vector in one way into components parallel to any three given lines not in the same plane.

When the directions of the component vectors are at right angles to each other the components are called resolved parts of the resultant vector in the corresponding directions.

Thus, if we take a system of rectangular coordinate axes, any vector parallel to a coordinate plane, e.g. the plane of $(x, y)$, can be resolved into components parallel to the axes of $x$ and $y$, these are the resolved parts of the vector in the directions of the axes of $x$ and $y$.

Again, taking a three dimensional system of rectangular axes, any vector can be resolved into components parallel to the 
axes of $x, y$, and $z$, and these are the resolved parts of the vector in the directions of these axes.

In the former case taking $O P$ to represent the vector, and drawing $P M$ at right angles to $O x, O M$ and $M P$ represent the

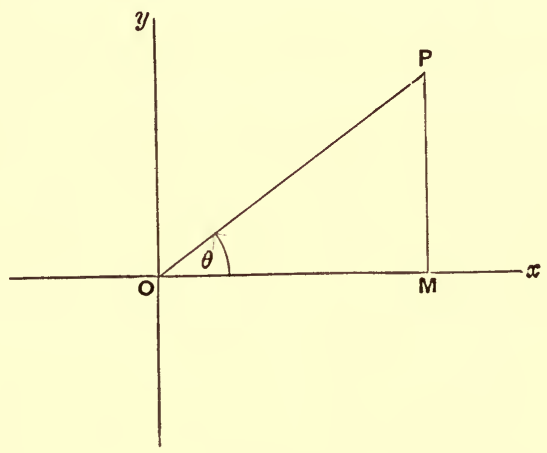

Fig. 9.

resolved parts of the vector parallel to the axes. If $R$ is the magnitude of the vector represented by $O P$, and $\theta, \phi$ the angles* between the lines $O P$ and $O x, O y$, then $R \cos \theta$ and $R \cos \phi$ are the magnitudes of the resolved parts respectively, and these are the projections of $O P$ on the axes.

More generally, taking $O P$ to represent the vector, and drawing a parallelepiped with $O$ and $P$ as opposite corners and with its faces parallel to the coordinate planes, the resolved parts of the vector in the directions of the axes are numerically equal to the projections of $O P$ on the axes. If $R$ is the magnitude of the vector represented by $O P$, and if $l, m, n$ are the cosines of the

* In Fig. $9 \cos \phi$ is $\sin \theta$, but it is easy to draw a figure, e.g. Fig. 10, which makes it appear that $\cos \phi$ is $-\sin \theta$. With the usual conventions in regard to the signs of trigonometrical functions we shall always have

$$
\cos \phi=\sin \theta
$$

provided $\theta$ is the angle traced out by a line $O P$ starting from $O x$ and turning round $O$ in the direction $O x$ to $O y$.

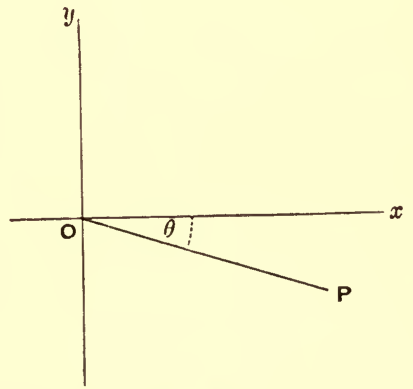

Fig. 10. 
angles which $O P$ makes with $O x, O y, O z$ respectively the resolved parts in these directions are $R l, R m$, and $R n$ respectively.

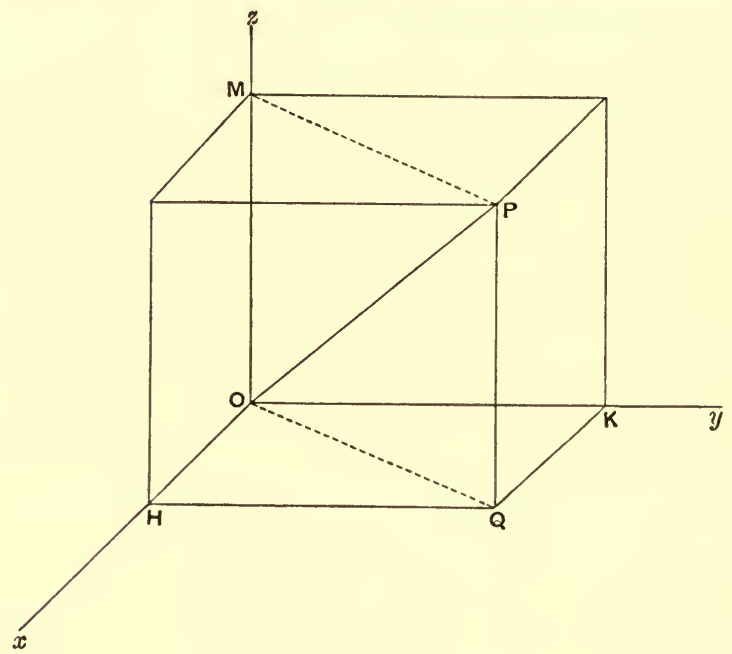

Fig. 11.

This rule determines the senses as well as the magnitudes of the resolved parts ; thus, when $\cos \theta$, in the first case, and $l$, in the second case, are negative, the component parallel to the $x$ axis is in the negative direction of that axis, i.e. in the direction $x O$ produced.

It is clear from this rule that when the magnitudes and signs of the resolved parts of a vector in the directions of three mutually rectangular lines are given the vector is uniquely determinate, that is to say there is one and only one vector which has given resolved parts parallel to three such lines.

The construction in the former of these cases is a construction for the resolved parts of a

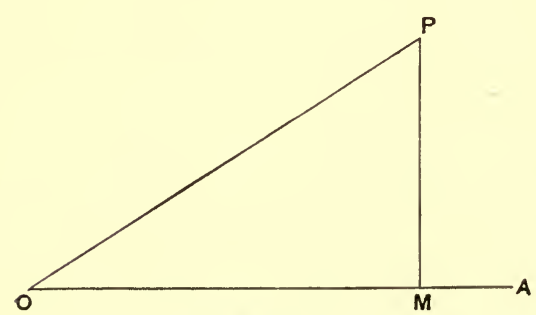

Fig. $9 a$. vector parallel and perpendicular to a line. As before, let $O P$ be a line representing the vector, and $O A$ a line parallel and perpendicular to which the vector is to be resolved. Draw $P M$ at right angles to $O A$. Then the 
vector is equivalent to vectors represented by $O M, M P$, and the magnitudes of these are respectively $R \cos \theta$ and $R \sin \theta$, where $R$ is the magnitude of the vector to be resolved, and $\theta$ is the angle between its direction and $O A$.

The vector represented by $M P$ is the resolved part of the vector represented by $O P$ at right angles to the line $O A$.

10. Composition of any number of vectors. I. Consider first the case where all the vectors are parallel to a plane, and take it to be the plane of $x, y$. Let $O P_{1}, O P_{2}$, $\ldots O P_{n}$ be lines representing the vectors, (supposed to be $n$ in number,) in magnitude, direction, and sense, and let $\theta_{1}, \theta_{2}, \ldots \theta_{n}$ be the angles the lines $O P_{1}, O P_{2}, \ldots O P_{n}$ make with $O x$, i.e. the angles traced

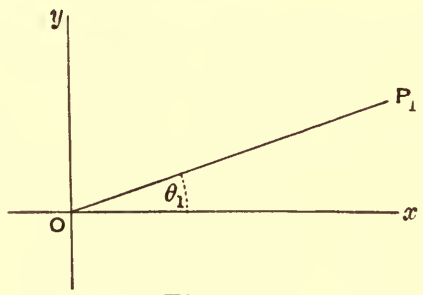

Fig. 12. out by a revolving line turning about $O$ from $O x$ towards $O y$. Let $r_{1}, r_{2}, \ldots r_{n}$ denote the magnitudes of the vectors.

Then the vector represented by $O P_{1}$ may be replaced by vectors $r_{1} \cos \theta_{1}$ parallel to $O x$, and $r_{1} \sin \theta_{1}$ parallel to $O y$, and similarly for the others.

All the resolved parts parallel to $O x$ are equivalent to a single vector $X$ parallel to $O x$ given by

$$
X=r_{1} \cos \theta_{1}+r_{2} \cos \theta_{2}+\ldots+r_{n} \cos \theta_{n}=\Sigma(r \cos \theta) .
$$

All the resolved parts parallel to $O y$ are equivalent to a single vector $Y$ parallel to $O y$ given by

$$
Y=r_{1} \sin \theta_{1}+r_{2} \sin \theta_{2}+\ldots+r_{n} \sin \theta_{n}=\Sigma(r \sin \theta) .
$$

The vector whose resolved parts parallel to $O x$ and $O y$ are $X$ and $Y$ is the resultant of all the vectors. Let the magnitude of this vector be $R$, and let its direction and sense be those of a line going out from $O$ and making an angle $\psi$ with $O x$.

Then we have $R \cos \psi=X$, and $R \sin \psi=Y$.

These two equations determine the magnitude $R$ and the angle $\psi$, viz. : $R$ is the numerical value of $\sqrt{ }\left(X^{2}+Y^{2}\right)$, and $\psi$ is that one among the angles whose tangents are $Y / X$ for which the sine has the same sign as $Y$ and the cosine has the same sign as $X$. 
II. Consider the more general case where the vectors are not parallel to a plane. Let $r_{1}, r_{2}, \ldots r_{n}$ be the magnitudes of the vectors, and call any one of these numbers $r$. Let $l, m, n$ be the cosines of the angles which the line representing this vector in direction and sense makes with the axes of $O x, O y, O z$. Then this vector may be resolved into $r l, r m, r n$ parallel to the lines $O x, O y$, $\mathrm{O} z$, and the whole set of vectors is equivalent to a vector whose resolved parts parallel to the axes are $X, Y, Z$, where $X=\Sigma r l$, $Y=\Sigma r m, Z=\Sigma r n$, the summations extending to all the vectors of the set. The resultant is therefore a vector whose magnitude, $R$, is the numerical value of $\sqrt{ }\left(X^{2}+Y^{2}+Z^{2}\right)$, and such that the line representing it in direction and sense makes with the axes $O x, O y$, $O z$ angles whose cosines are $X / R, Y / R, Z / R$.

11. Vectors equivalent to zero. When the magnitude of the resultant of any set of vectors is zero the set of vectors is said to be equivalent to zero. Thus two equal vectors parallel to the same line, and in opposite senses, are equivalent to zero.

It is clear that the sum of the resolved parts, in any direction, of a set of vectors equivalent to zero is equal to zero.

Again vectors parallel and proportional to the sides of a polygon, and with senses determined by the order of the corners when a point travels round the polygon, are equivalent to zero.

This last statement enables us to do away with the restriction (Art. 8) that in the resolution of a vector into components parallel to the sides of a polygon not more than two sides of the polygon may meet in a point.

12. Centroids. Although not immediately connected with the subject of this Chapter, it is convenient here to introduce the definitions of the centroids of figures.

Consider in the first place a figure consisting of isolated points $A_{1}, A_{2}, \ldots A_{n}$. Let any plane be drawn, and let $x_{1}, x_{2}, \ldots x_{n}$ be the distances of the points from the plane, a positive sign being given to the $x$ 's of the points on one side of the plane, and a negative sign to the $x$ 's of the points on the other side of the plane. Then there will be a parallel plane whose distance from this plane is $\frac{1}{n}\left(x_{1}+x_{2}+\ldots+x_{n}\right)$; the sign of this quantity determines the relative situation of the planes. We may say that the distance of this plane from the plane first drawn is the average distance of the points from the plane first drawn. 
The centroid of the system of points is the point whose distance from any plane is the average distance of the points from the plane.

If $x_{r}, y_{r}, z_{r}$ are the coordinates of any point $A_{r}$ of the set, referred to any axes, the coordinates of the centroid, referred to the same axes, are

$$
\frac{1}{n} \sum_{1}^{n} x_{r}, \quad \frac{1}{n} \sum_{1}^{n} y_{r}, \quad \frac{1}{n} \sum_{1}^{n !} z_{r} .
$$

If the origin is at the centroid it is clear that each of these sums is zero.

In the same way we may define the centroid of a line, an area, or a volume. Let $d \boldsymbol{\tau}$ denote a differential element of the line, area, or volume, infinitesimal in all its dimensions, and let $x, y, z$ be the coordinates of a point within the element. Then the centroid is the point whose coordinates are

$$
\frac{\int x d \tau}{\int d \tau}, \quad \frac{\int y d \tau}{\int d \tau}, \frac{\int z d \tau}{\int d \tau},
$$

the integrations extending through the line, area, or volume.

We may also define the centroid of a set of points for different multiples. Let $x_{r}, y_{r}, z_{r}$ be the coordinates of one of the points, $m_{r}$ the corresponding multiple. Then the centroid of the points for multiples $m_{1}, m_{2}, \ldots m_{n}$ is the point whose coordinates are

$$
\frac{\sum_{1}^{n} m_{r} x_{r}}{\sum_{1}^{n} m_{r}}, \quad \frac{\sum_{1}^{n} m_{r} y_{r}}{\sum_{1}^{n} m_{r}}, \quad \frac{\sum_{1}^{n} m_{r} z_{r}}{\sum_{1}^{n} m_{r}} .
$$

This definition holds for all real values of the multiples $m$ provided their sum is not zero.

13. Theorem of the Composition of Vectors. The rule for the composition of vectors may be stated in terms of the centroid of a set of points.

Let the vectors be represented in direction by lines $O A_{1}, O A_{2}, \ldots O A_{n}$ going out from an origin, $O$, to points $A_{1}, A_{2}, \ldots A_{n}$, and let the magnitudes of the vectors be $m_{1} . O A_{1}, m_{2} . O A_{2}, \ldots m_{n} . O A_{n}$, where $m_{1}, m_{2}, \ldots m_{n}$ are any real numbers. When any number $m_{r}$ is negative, the sense of the corresponding vector is to be from $A_{r}$ towards $O$. Let $G$ be the centroid of the points $A_{1}, A_{2}, \ldots A_{n}$ for multiples $m_{1}, m_{2}, \ldots m_{n}$.

Then the resultant of all the vectors is represented in direction by the line $O G$, its magnitude is $O G \cdot \sum_{1}^{n} m_{r}$, and its sense is from $O$ to $G$ or from $G$ to $O$ according as the sum $\sum_{1}^{n} m_{r}$ is positive or negative.

By taking rectangular axes having $O$ as origin, and resolving the vectors along them, it is clear from the definition of $G$ that the resolved part, parallel to an axis, of the vector described is the sum of the resolved parts, parallel to the same axis, of the given vectors.

This proves the theorem. 
14. Localised Vectors. The vectors we have so far considered have no relation to any particular point, they are equally well represented by lines drawn from any point, and they have no relation to any particular line, they are equally well represented by segments of all lines parallel to their direction. They may be called unlocalised vectors. But it is often important to consider objects of mathematical reasoning which, in other respects, have the properties of vectors, but which have relations with particular points or particular lines.

$A$ vector localised at a point is an object of mathematical reasoning which, like an unlocalised vector, is defined by its magnitude, direction, and sense, and also by a point and by a rule of equivalence, viz.:-two sets of vectors localised at the same point are equivalent if two sets of unlocalised vectors with the same magnitudes, directions, and senses are equivalent.

There is in general no rule of equivalence for vectors localised at different points.

$A$ vector localised in a line is a vector localised at any point in a particular line, which is in the direction of the vector, with the additional rules of equivalence that two vectors localised in the same line are equivalent if they have the same magnitude and the same sense, and that two vectors localised in lines which meet are equivalent to a single vector localised in a line.

All the constructions in the previous Articles apply to vectors localised at points and to vectors localised in lines, provided all components and resultants are localised at the proper points or in the proper lines. In particular a vector localised at a point is equivalent to components (or resolved parts) of the same magnitudes, directions, and senses as if it were unlocalised, provided these components and resolved parts are localised at the same point; also a vector localised in a line is equivalent to components (or resolved parts) of the same magnitudes, directions, and senses as if it were unlocalised, provided these components and resolved parts are localised in lines which meet in a point on the line of the resultant.

Thus a vector localised at $O$ may be represented (as in Fig. 11) by a line $O P$, and is equivalent to vectors localised at $O$ and represented by lines $O H, O K, O M$; and a vector localised in the 
line $O P$, having the same magnitude and sense, is equivalent to vectors localised in any three lines parallel to $O x, O y, O z$, meeting in a point on $O P$, and having the magnitudes and senses of $O H$, $O K, O M$.

The differences between the three classes of vectors may be expressed thus :-

A vector (unlocalised) is equivalent to any parallel vector of equal magnitude and like sense. Thus the line representing the vector may be drawn from any point.

A vector localised in a line is equivalent to any vector of equal magnitude and like sense localised in the same line. The line representing it may be drawn from any point in a particular line, and is a segment of that line.

A vector localised at a point is not equivalent to any other single vector. The line representing it must be drawn from the point.

A vector localised in a line is clearly determined by its components parallel to three given lines and by one point of the line, in particular the line in which it is localised is thereby determined.

\section{Equivalent systems of vectors localised in lines.} Let two vectors be localised in lines which meet in a point $A$. They may be localised at the point $A$. They are then equivalent to a determinate resultant localised at $A$, and since, by definition, they are equivalent to a vector localised in a line, this line is the line of the resultant through $A$.

Let $A B, A D$ be the lines in which the vectors are localised, the order in which the points are named indicating the senses. Let $P$ and $Q$ be their magnitudes; and take the segments $A B$ and $A D$ to be proportional to $P$ and $Q$. Construct a parallelogram $A B C D$ having $A B, A D$ as adjacent sides. Then a vector localised in the line

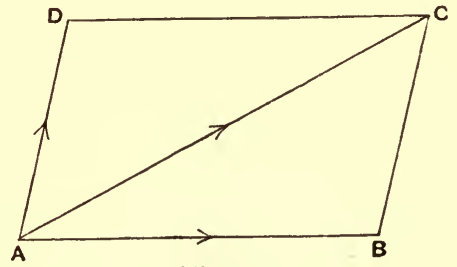

Fig. 13. 
$A C$, of sense indicated by the order $A, C$, and of magnitude $R$, such that

$$
P: Q: R=A B: A D: A C,
$$

is equivalent to the two given vectors localised in $A B, A D$.

Except in the case of parallel vectors this construction enables us to replace a system of vectors localised in lines lying in a plane by a single vector localised in a line in the plane. We can replace two of the vectors by their resultant, then this resultant and a third by their resultant, and so on.

But the difficulty encountered when we seek to replace pairs of parallel vectors can generally be removed, and the method by which this is accomplished will at the same time furnish a method for replacing a given set of localised vectors by simpler equivalent sets of localised vectors which is of much more direct application than the method just described.

The principle on which this method rests is that as two equal vectors of opposite senses localised in the same line are equivalent to zero, any set of vectors localised in lines is equivalent to any other set which differs from it only by containing, in addition to the original vectors, pairs of equal and opposite vectors, the vectors of any pair being localised in the same line.

16. Moments. The moment about a line $L$ of a vector localised in another line $L^{\prime}$ is the product, with a certain sign, of

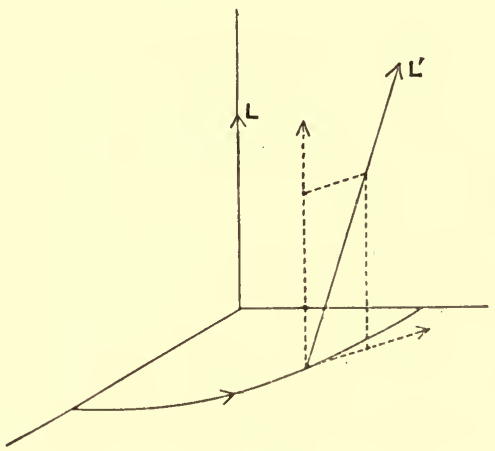

Fig. 14.

the magnitude of the resolved part of the vector at right angles to the line $L$ and the length of the common perpendicular to the two lines. The rule of signs is as follows:-One of the two senses 
in which the line $L$ may be described is chosen, and called the positive sense. Describe a circle with its centre at the point where the common perpendicular meets $L$, with its plane at right angles to $L$, and with the common perpendicular for radius; the resolved part of the vector at right angles to $L$ is localised in a tangent to this circle. We suppose the circle described in the sense of this resolved part. Now if the sense of description of the circle and the positive direction of the line $L$ are related like the rotation and translation of a right-handed screw the sign of the moment is plus, if not it is minus. The rule of signs may be stated in a simpler equivalent form. Imagine a watch placed in the plane of the circle above described with the positive direction of the line $L$ running from the back towards the face, then if the sense of the tangent to the circle is that in which the hands go round the sign is -, if opposite to this the sign is + .

The student must accustom himself to the above rule of signs. It is known as "the right-handed screw rule." He will be assisted by observing that in the figure of a set of rectangular axes the positive senses of the axes may be taken in the same way as in Articles 4 and 9 , and then the positive senses of rotation about them are those of a line turning in the planes $(y, z),(z, x),(x, y)$ from $O y$ towards $O z$, from $O z$ towards $O x$, and from $O x$ towards $O y$.

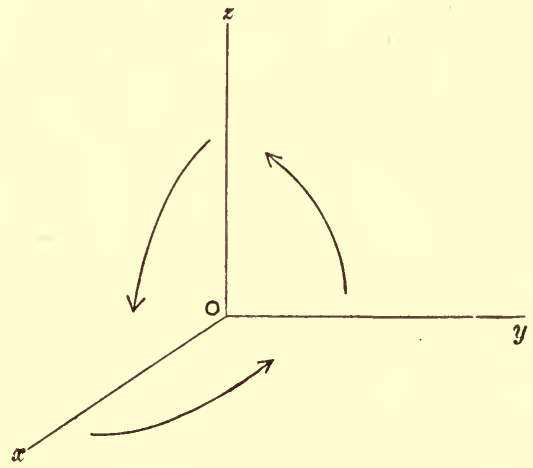

Fig. 15.

In a corner of a room, for example, let the student look towards one wall ; its plane may be taken for the plane $(y, z)$, the wall on his left will be the plane $(z, x)$ the floor will be the plane $(x, y)$. If he hangs his watch on either wall, or places it on the floor, so that he can see the face, the hands will go round in a direction opposite to one of those shown in the diagram. An ordinary screw turned so as to move in the positive direction of either axis will turn round in the way shown in the diagram. 
The moment about a line of a vector localised at a point is defined to be the moment about the line of a vector with the same magnitude, direction, and sense, but localised in a line through the point.

17. Moments about a point. When we are concerned with vectors localised in lines that lie in a plane, or with vectors localised at points in a plane and having their directions parallel to that plane, the moments of these vectors about a line perpendicular to the plane are defined to be the moments of the vectors about the point where this line meets the plane. For the sake of simplicity we may also give the following definition:-

The moment about a point $O$ of a vector in a plane through $O$ and localised in a line $L$ is the product, with a certain sign, of the magnitude of the vector and the perpendicular upon $L$ from the point $O$. The rule of signs is that when the sense of the vector is opposite to that of the motion of the hands of a watch, placed with its face in the plane, and the centre of its face at 0 , the sign is + , otherwise the sign is - . The watch must be supposed to have its face always turned the same way.

18. Lemma. The moment about a point $O$ of a vector localised at a point $A$ is identical with the moment about $O$ of the resolved part of the vector at right angles to $O A$.

This follows at once by using the first form of the definition, and it can be deduced

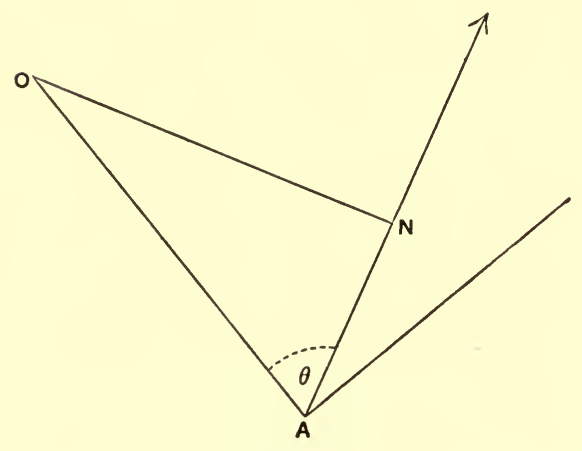

Fig. 16. from the second form as follows :-

Let $\theta$ be the angle the direction of the vector makes with the line $A O$, and draw $O N$ at right angles to the line of the vector. The magnitude of the resolved part of the vector at right angles to $A O$ is $R \sin \theta$, where $R$ is the magnitude of the vector. The perpendicular from $O$ on the line of the vector is the line $O N$, and it is equal to $O A \cdot \sin \theta$. 
Now moment of $R$ about $O=R . O N$

$$
\begin{aligned}
& =R . O A \sin \theta \\
& =R \cdot \sin \theta \cdot O A \\
& =\text { moment about } O \text { of resolved part } \\
& \quad \text { at right angles to } O A .
\end{aligned}
$$

19. Theorem of Moments. The sum (with proper signs) of the moments about a point $O$ of two vectors localised at a point $A$ is equal to the moment of their resultant about 0 .

Let $P_{1}$ and $P_{2}$ be the magnitudes of the vectors, $\theta_{1}$ and $\theta_{2}$ the angles the lines representing them drawn from $A$ make with $A O, R$ the magnitude of the resultant, $\phi$ the angle the line representing it makes with $A O$. Then the magnitudes of the resolved parts at right angles to $A O$ are $P_{1} \sin \theta_{1}$, $P_{2} \sin \theta_{2}$, and $R \sin \phi$, and we know (Article 10) that $R \sin \phi=P_{1} \sin \theta_{1}+P_{2} \sin \theta_{2}$.

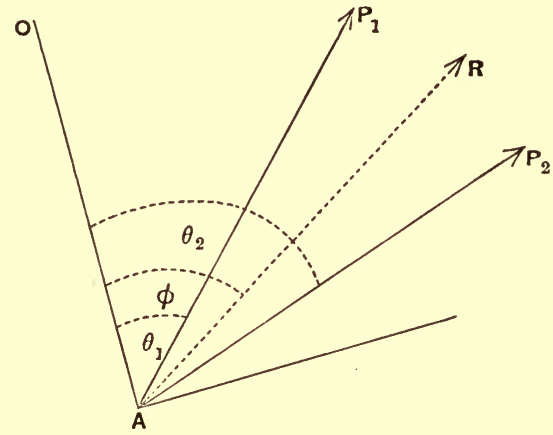

Fig. 17.

Now sum of moments of $P_{1}$ and $P_{2}$ about $O$

$$
\begin{aligned}
& =O A\left(P_{1} \sin \theta_{1}+P_{2} \sin \theta_{2}\right) \\
& =O A \cdot R \sin \phi \\
& =\text { moment of } R \text { about } 0 .
\end{aligned}
$$

This result can be immediately extended to any number of vectors localised at a point.

20. Couples. A pair of vectors of equal magnitudes and opposite senses localised in parallel lines is called a couple.

The plane of the two parallel lines is the plane of the couple.

The moment of one of the vectors about any point on the line of the other is the moment of the couple. The magnitude of the moment is, of course, a number, which is the product of the numbers expressing the magnitude of the vector and the distance between the lines.

A line drawn from any point, in a certain sense, at right angles 
to the plane of the couple, and of length containing a number of units of length equal to the number which expresses the moment of the couple, is called the axis of the couple. The sense in which the line is drawn is that of a parallel line meeting the line of one of the vectors, and such that the moment of the other about it is positive.

The series of three theorems we are now going to prove lead to the result that a couple is equivalent to an unlocalised vector represented by its axis.

21. Theorem I. Two couples in the same plane are equivalent if they have the same moment.

We shall prove that two couples in the same plane, of equal

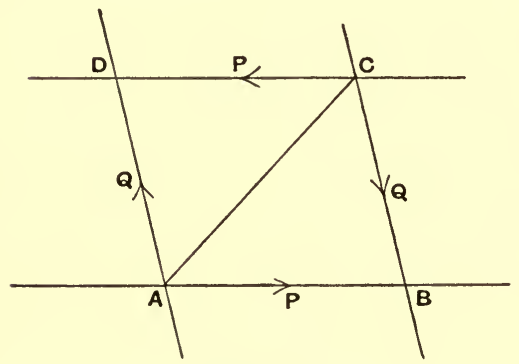

Fig. 18. moments, in opposite senses, are equivalent to zero.

The lines in which the vectors are localised, being two pairs of parallel lines, form a parallelogram. Let this be $A B C D$.

Let the vectors of one couple be of magnitude $P$, and be localised in the lines $A B, C D$; and let the vectors of the other couple be of magnitude $Q$, and be localised in the lines $A D, C B$.

Let the unit of length be so chosen that $A B$ represents $P$ in magnitude.

Then the area of the parallelogram is of magnitude equal to the moment of the couple.

Hence $A D$ represents $Q$ in magnitude.

Now the vectors $P$ and $Q$ localised in the lines $A B, A D$, and proportional to those lines, are equivalent to a vector localised in the line $A C$, and proportional to that line. The sense of this vector is $A C$.

Also the vectors $P$ and $Q$ localised in the lines $C D, C B$, and proportional to those lines, are equivalent to a vector localised in the line $C A$, and proportional to that line. The sense of this vector is $C A$. 
It follows that the set of four vectors $P, P$, and $Q, Q$ are equivalent to zero.

This theorem shows that a couple may be replaced by any other couple of the same moment and sense and in the same plane.

Before going on to Theorem II. we must prove a general theorem concerning the resultant of two vectors localised in parallel lines but not forming a couple. This theorem might be proved independently of Theorem I., but the proof of it becomes very much simplified if Theorem $I$. is assumed.

22. Vectors localised in parallel lines. Let $P, Q$ be the magnitudes of two vectors localised in parallel lines, $A$ and $B$ any points on these lines, $d$ the distance between the lines.
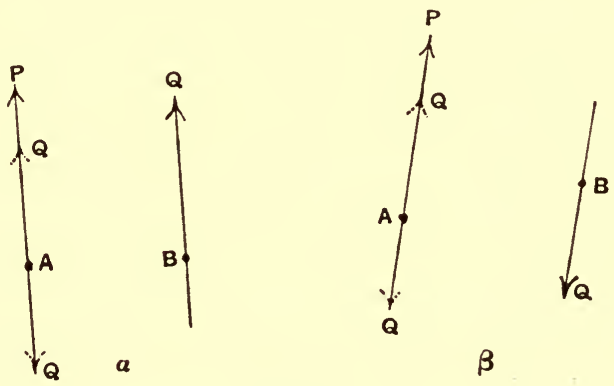

$\beta$

Fig. 19.

In the line in which $P$ is localised introduce two vectors each of magnitude $Q$ and in opposite senses. Then the pair of vectors is equivalent to a couple of moment $Q d$ and a single vector localised in the same line as $P$. The magnitude of the single vector is $P+Q$ (Fig. 19, $\alpha$ ) if $P$ and $Q$ have like senses, and is $P \sim Q$ (Fig. 19, $\beta$ ) if $P$ and $Q$ have opposite senses, and its sense is that of $P$ or $Q$ when these have like senses, but when $P$ and $Q$ have opposite senses it is that of the vector $P$ if $P>Q$, that of the vector $Q$ if $Q>P$. In either case let the magnitude of the single vector be $R$, and in the second case, to fix ideas, take $Q>P$.

Replace the couple of moment $Q d$ by a couple in the same plane consisting of two vectors each of magnitude $R$, one of them being localised in the same line as $P$ and in the opposite direction to $R$, and the other in a parallel line at a distance $Q d / R$ on that 
side of the first line which makes the moment have the right sign. The figures $\alpha$ and $\beta$ show this for the two cases.

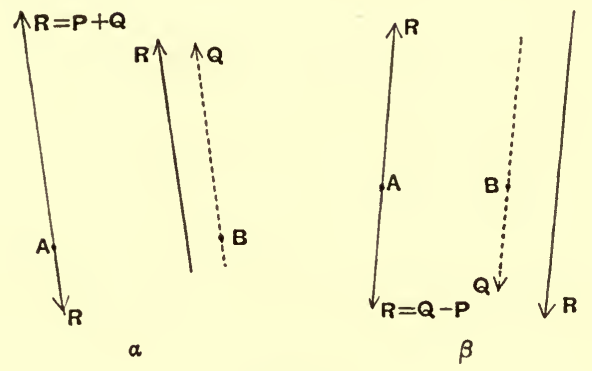

Fig. 20.

When $P$ and $Q$ have like senses the system is equivalent to a vector of magnitude $P+Q$ localised in a line parallel to the lines of $P$ and $Q$, between these lines, and at a distance $d^{\prime}$ from the line of $P$ such that $(P+Q) d^{\prime}=Q d$. The sense of this vector is that of $P$ and $Q$.

When $P$ and $Q$ have opposite senses, and $Q>P$, the system is equivalent to a vector of magnitude $Q-P$ localised in a line parallel to the line of $P$ or $Q$, on the side of the line of $Q$ remote from the line of $P$, and at a distance $d^{\prime}$ from the line of $P$ such that $(Q-P) d^{\prime}=Q d$. The sense of this vector is that of $Q$.

It is clear that in both cases the parallel vectors have a single resultant, localised in a parallel line, and of such magnitude and sense that its moment about any point in the plane of the two parallel vectors is equal to the sum of the moments of the two parallel vectors about the same point.

The particular case of this theorem required in our proof of Theorem II. is that the resultant of two vectors of equal magnitude and like sense, localised in parallel lines, is a vector of twice the magnitude and of the same sense, localised in a line midway between the two parallels.

23. Theorem II. Two couples in parallel planes are equivalent if they have the same moment.

We shall prove that two couples in parallel planes of equal moments in opposite senses are equivalent to zero.

Let the vectors of one couple be of magnitude $P$, and be 
localised in the lines $A B, C D$; and let the vectors of the other couple be of magnitude $Q$, and be localised in the lines $A^{\prime} D^{\prime}, C^{\prime} B^{\prime}$.

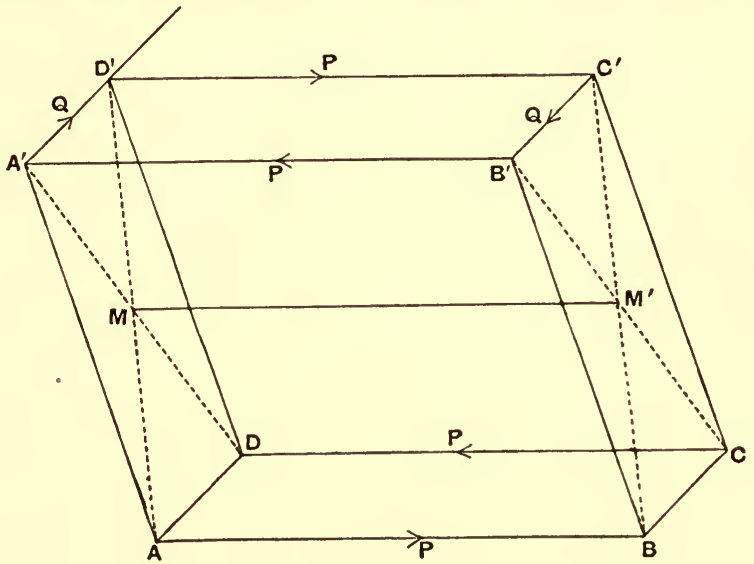

Fig. 21.

Through $A^{\prime} D^{\prime}$ and $B^{\prime} C^{\prime}$ draw a pair of parallel planes meeting the lines of the couple $P$ in the points $A, D, B, C$.

Through $A B$ and $C D$ draw a pair of parallel planes meeting the lines of the couple $Q$ in the points $A^{\prime}, B^{\prime}, C^{\prime}, D^{\prime}$.

These two pairs of planes with the planes of the two couples form a parallelepiped.

Replace the couple $Q$ in its plane by an equivalent couple consisting of vectors localised in the lines $B^{\prime} A^{\prime}$ and $D^{\prime} C^{\prime}$. These vectors are both of magnitude $P$, and have the senses indicated by the order of the letters.

Now parallel vectors $P$ localised in lines $A B, D^{\prime} C^{\prime}$, and having the senses indicated, are equivalent to a vector of magnitude $2 P$ localised in the line $M M^{\prime}$ joining the middle points of $A D^{\prime}$ and $B C^{\prime}$. The sense of this vector is $M M^{\prime}$.

Also parallel vectors $P$ localised in lines $C D, B^{\prime} A^{\prime}$ are equivalent to a vector of magnitude $2 P$ localised in the same line $M M^{\prime}$. The sense of this vector is $M^{\prime} M$.

It follows that the set of four vectors $P, P$, and $Q, Q$ are equivalent to zero.

This theorem shows that a couple may be replaced by any couple of the same moment in any parallel plane. 
24. Theorem III. Couples obey the vector law of composition.
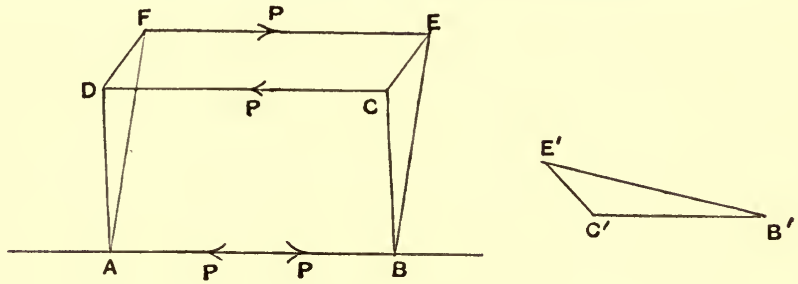

Fig. 22 .

Let the planes of the couples meet in the line $A B$.

Replace the couple in one plane by any couple having one of its vectors localised in $A B$ in the sense $A B$.

Let the two vectors be of magnitude $P$, and let the other be localised in the line $C D$.

Replace the couple in the other plane by a couple having one of its vectors localised in $B A$ in the sense $B A$.

We can take these vectors also to be of magnitude $P$, and then the other will be localised in a certain line $F E$ in the plane of the second couple.

Let $A B$ represent $P$ in magnitude, and through the points $A$, $B$ draw planes at right angles to $A B$ cutting the lines $C D$ and $E F$ in the points named $C, D, E, F$.

Then the two couples are seen to be equivalent to a single couple, whose vectors are of magnitude $P$, and are localised in the lines $C D, F E$.

The figures $A B C D, A B E F, C D F E$ are rectangles, and their areas are proportional to the moments of the couples. These areas are in the ratios of the lengths of $B C, B E, C E$.

Hence if we turn the triangle $B C E$ through a right angle in its plane its sides will be parallel and proportional to the axes of the couples. Let $B^{\prime} C^{\prime} E^{\prime}$ be the new triangle; then it is clear that if $E^{\prime} B^{\prime}$ represents the axis of the second couple in sense, the sense of the first is $B^{\prime} C^{\prime}$, and the sense of the resultant is $E^{\prime} C^{\prime}$.

Thus the axis of a couple which has the magnitude, direction, and sense of a line $E^{\prime} C^{\prime}$ is the axis of the resultant of two component couples, the axes of the components having the magnitudes, 
directions, and senses of two lines $E^{\prime} B^{\prime}$ and $B^{\prime} C^{\prime}$. This is the vector law.

25. System of localised vectors in a plane. Let a vector of any magnitude $P$ be localised in a line $A B$, and let $O$ be any point not in the line $A B$. Through $O$ draw a line parallel to $A B$, and let there be two vectors each of magnitude $P$ and of opposite senses localised in this line. Then the system of vectors is equivalent to a vector localised in the line through $O$ parallel to $A B$, of magnitude $P$, and having the sense of the original vector in $A B$, and a couple of moment $P p$, where $p$ is the distance of $A B$ from $O$, and of a definite sense, with its axis perpendicular to the plane $A O B$.

Any given system of vectors in a plane can in this way be replaced by a vector localised at a point

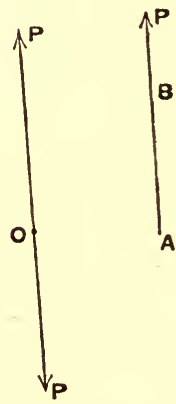

Fig. 23. $O$ in the plane, which is the resultant of equal parallel vectors in the senses of the given vectors, but localised in lines through 0 , and a couple whose axis is perpendicular to the plane and whose moment is $\Sigma( \pm P p)$, where $P$ is the magnitude of any one of the original vectors, $p$ the perpendicular on its line from 0 , and the sign of each term is determinate.

Let $R$ be the resultant of the vectors at $O$, and $G$ the moment of the couple. If $R$ is not zero replace $G$ by two localised vectors, each of magnitude $R$, one localised in the line of $R$ through $O$ and in the sense opposite to $R$, and the other in a parallel line at a distance $G / R$ from 0 . The whole system is then equivalent to this last vector.

If $R$ is zero the whole system is equivalent to the couple $G$.

If $R$ and $G$ are both zero the system is equivalent to zero.

Thus any system of vectors localised in lines lying in a plane is equivalent to a single vector localised in a line lying in the

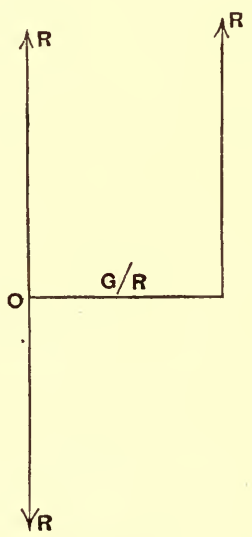

Fig. 24. 
plane, or to a couple whose axis is perpendicular to the plane, or to zero.

The single vector, and the couple, in the cases where the system is equivalent to a single vector or a couple, are determinate and unique.

The conditions of equivalence of two systems of vectors localised in lines lying in a plane are that when one system is equivalent to a single vector the other is equivalent to a single vector of the same magnitude and sense localised in the same line, when one system is equivalent to a couple the other is equivalent to a couple of the same magnitude and sense, when one system is equivalent to zero the other is equivalent to zero.

\section{Analysis of vectors localised in lines in a plane.} Take any origin $O$, and any rectangular axes of $x, y$ in the plane.

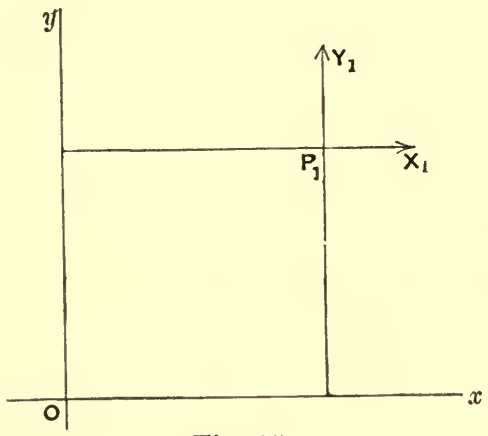

Fig. 25. Let $X_{1}, Y_{1}$ be the resolved parts parallel to the axes of one of the vectors, and let $P_{1}\left(x_{1}, y_{1}\right)$ be any point on the line in which it is localised. Then the moment of the couple, when this vector is replaced by an equal vector localised in a line through $O$ and a couple, is the moment of this vector about $O$, i.e. it is the sum of the moments of the resolved parts $X_{1}, Y_{1}$ about $O$, and this sum is $x_{1} Y_{1}-y_{1} X_{1}$. Hence the whole system is equivalent to a vector localised in a line through $O$, whose resolved parts parallel to the axes are $\Sigma X$ and $\Sigma Y$, and a couple in the plane, whose moment is $\Sigma(x Y-y X)$.

\section{General analysis of vectors localised in lines.} Take any origin $O$, and any rectangular axes of $x, y, z$. Let $X, Y, Z$ be the resolved parts parallel to the axes of one of the vectors, and $x, y, z$ the coordinates of a point in the line in which it is localised. Introduce a pair of equal and opposite vectors localised in a line through $O$ parallel to the line of this vector, and resolve them into components localised in the axes. The 
magnitudes of these components are $X, Y, Z$. The original vector is thus replaced by vectors $X, Y, Z$ localised in the axes, and by three couples about the axes, whose moments (by last Article) clearly are

$$
y Z-z Y, z X-x Z, x Y-y X
$$

respectively.

Hence any system of vectors localised in lines can be replaced by a single vector localised in a line through the origin, whose

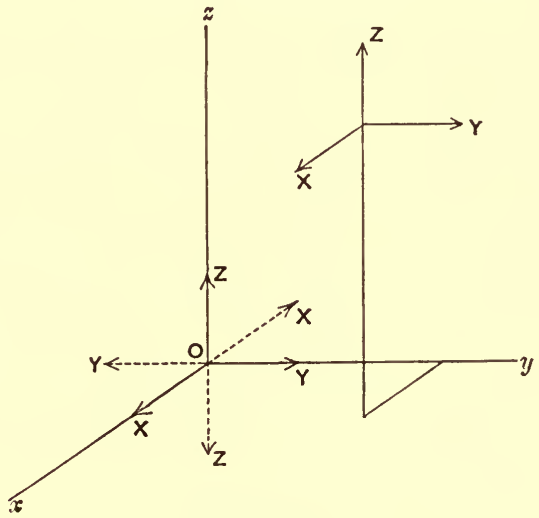

Fig. 26.

resolved parts parallel to the axes are $\Sigma X, \Sigma Y, \Sigma Z$, and by a couple, equivalent to component couples about the axes, whose moments are $\Sigma(y Z-z Y), \Sigma(z X-x Z), \Sigma(x Y-y X)$, where $X, Y, Z$ are the resolved parts of any one of the original vectors parallel to the axes, and $x, y, z$ are the coordinates of any point in the line in which that vector is localised. 


\section{CHAP'TER III.}

\section{VELOCITY AND ACCELERATION.}

28. Displacement. Let $x, y, z$ be the coordinates of a moving point at any particular instant with reference to any particular frame, $x^{\prime}, y^{\prime}, z^{\prime}$ the coordinates of the point at a subsequent instant, with reference to the same frame, then $x^{\prime}-x$, $y^{\prime}-y, z^{\prime}-z$ are the components, parallel to the axes, of a vector quantity called the displacement of the point. The vector is not localised.

29. Velocity in a straight line. Consider in the first place a point moving in a straight line, e.g. one of the lines of reference, and let $s$ be the number of units of length it passes over in $t$ units of time. Then it may happen that the two numbers $s$ and $t$ have a constant ratio whatever number we take for $t$. The point is then said to move uniformly in the line, and the fraction $\frac{s}{t}$ is defined to be the measure of its velocity. A point moving uniformly describes equal lengths in equal times.

Again consider the case where the point moves in a straight line, but the number of units of length passed over in any interval of time does not bear a constant ratio to the number of units of time in the interval. In this case there will be equal intervals of time in which the point describes unequal lengths; in the one of two equal intervals in which it describes the greater length we should say it was moving faster, in the other, in which it describes the shorter length, we should say it was moving more slowly. We have thus an idea of velocity of a point not moving uniformly, let us try to make it precise. 
For a point moving in a straight line we may define the average velocity in any interval of time to be the fraction

\section{number of units of length described in an interval number of units of time in the interval}

When the point is not moving uniformly this fraction is a variable number, which has a definite value when the measure of the interval is given and the first instant of the interval is given. Taking the first instant of the interval always the same, and taking for the measure of the interval a series of diminishing numbers, we obtain a series of fractions, which we assume* approach a limiting value as the measure of the interval is indefinitely diminished. This limiting value is defined to be the velocity of the point at the first instant of the interval. We might in the same way define the velocity of a point at the last instant of an interval.

We can now define the velocity of a point moving in a straight line at any instant. It is the limit of the average velocity in an indefinitely small interval of time beginning or ending at the instant.

The two limits are in general the same; when they are different we call them the velocity just after the instant and the velocity just before the instant respectively.

Let $t$ be the measure of the interval of time which has elapsed since some particular instant, chosen as the origin of time, and suppose that at the end of this interval the point has described a length $s$ measured from some particular point in the line of its motion. We say that the point is at $s$ at time $t$. In the same way suppose that it is at $s^{\prime}$ at time $t^{\prime}$. Then in the interval $t^{\prime}-t$ it describes a length $s^{\prime}-s$, and its average velocity in the interval is $\frac{s^{\prime}-s}{t^{\prime}-t}$. The number $s$ is a function of the number $t$, and the limit of the fraction just written is the number known as the differential coefficient of $s$ with respect to $t$. The velocity of the moving point is accordingly measured by $\frac{d s}{d t}$.

The number $s^{\prime}-s$ is the measure of the displacement of the point during the interval $t^{\prime}-t$. When the velocity is uniform it is measured by the

* We do not require to consider any other case. 
displacement in a unit of time. If the unit of time were replaced by a smaller unit the displacement in it would be replaced by a shorter length, and this length would measure the velocity in terms of the new unit of time. However short an interval is taken for the unit of time the length described in it measures the velocity in terms of it. When we wish to recall this fact, and to bring it into connection with the definition of variable velocity we say that the latter is measured by "the rate of displacement per unit of time," but we must not attach to this phrase any other meaning than that which has just been explained, i.e. the phrase means nothing but the limit of the fraction number of units of length described in an interval number of units of time in the interval

when the interval is indefinitely diminished.

30. Velocity in general. When the point is not moving in a straight line it will have a displacement in any interval $t^{\prime}-t$ parallel to each of the three axes of reference; suppose these displacements to be $x^{\prime}-x, y^{\prime}-y, z^{\prime}-z$. Then we assume that each of the fractions

$$
\frac{x^{\prime}-x}{t^{\prime}-t}, \frac{y^{\prime}-y}{t^{\prime}-t}, \frac{z^{\prime}-z}{t^{\prime}-t},
$$

has a limit, and these limits are, as above, the rates of displacement per unit time parallel to the axes. They are defined to be the component velocities parallel to the axes. We can thus define the velocity of a moving point in general to be a vector, localised in a line through the position of the point, whose resolved part in any direction is the rate of displacement of the point in that direction per unit time.

As before, $x, y, z$ are functions of $t$, and the component velocities in the directions of the axes are

$$
\frac{d x}{d t}, \frac{d y}{d t}, \frac{d z}{d t}
$$

At any instant the point is moving along the tangent to a curve, called its path or trajectory. This tangent is the line drawn through the point in the direction of the velocity, i.e. it is the limiting position of a chord drawn from the point to an indefinitely near point of the curve. Let $s$ be the arc of the curve measured from some particular point of the curve up to the position of the moving point at time $t$, and let $s^{\prime}$ be the corresponding arc for time $t^{\prime}$. Then the length of the chord joining the two positions is the magnitude of the vector whose components parallel to the axes are $x^{\prime}-x, y^{\prime}-y, z^{\prime}-z$, and this chord becomes ultimately equal to the element of arc $(d s)$ of the curve as the two positions approach to coincidence. Thus the magnitude of the 
velocity of the moving point at time $t$ is $\frac{d s}{d t}$, where $s$ is the length of the arc of the path measured, in the sense of description of the path, from some particular point of it to the position of the moving point at time $t$. The magnitude of the velocity of a point is often called its speed, and, when it is independent of the time, the point is said to move with uniform speed whether its path is straight or curved.

31. Measurement of velocity. The measure of any particular velocity is a number expressing the ratio of the velocity to the unit of velocity.

The unit velocity is that with which a point describes one unit of length uniformly in each unit of time.

The number expressing a velocity is the ratio of a number expressing a length to a number expressing an interval of time. It therefore varies inversely as the unit of length and directly as the unit of time.

Velocity is accordingly said to be a quantity of one dimension in length and of minus one dimension in time, or its dimension symbol is $L T^{-1}$, where $L$ ștands for length, and $T$ for time.

32. Acceleration. A point moving with a variable velocity, relative to any frame, is said to have an acceleration relative to that frame.

When the point is moving in such a way that its velocity increases by equal amounts in equal intervals of time, however short the intervals may be, it is said to have a uniform acceleration, provided the velocity acquired in every interval has the same direction and sense.

Uniform acceleration is determined as regards magnitude, direction, and sense by the velocity added in a unit of time.

When the acceleration is not uniform, the moving point is said to have a variable acceleration.

The acceleration of a point moving in a straight line is the rate of increase of its velocity per unit of time. This is a short way of expressing the following definition :-

Let $v$ be the velocity of the point at time $t$, and $v^{\prime}$ its velocity at time $t^{\prime}$, then its acceleration is the limit of the fraction $\frac{v^{\prime}-v}{t^{\prime}-t}$ when the interval $t^{\prime}-t$ is indefinitely diminished, or in words it is 
the limit of the fraction

number of units of velocity added in an interval of time number of units of time in the interval

when the interval is indefinitely diminished. The number $v$ is a function of the number $t$, and its differential coefficient with respect to $t$ is the acceleration, i.e. the acceleration is measured by $\frac{d v}{d t}$.

When the point is not moving in a straight line it will in general have a variable velocity parallel to each of the lines of reference (coordinate axes). Let $u, v, w$ be component velocities parallel to these axes at time $t$, and $u^{\prime}, v^{\prime}, w^{\prime}$ corresponding components at time $t^{\prime}$, then the fractions $\frac{u^{\prime}-u}{t^{\prime}-t}, \frac{v^{\prime}-v}{t^{\prime}-t}, \frac{w^{\prime}-w}{t^{\prime}-t}$ are assumed to have limits when the interval $t^{\prime}-t$ is indefinitely diminished, and these limits are the differential coefficients $\frac{d u}{d t}, \frac{d v}{d t}, \frac{d w}{d t}$. The vector which has these components parallel to the axes is defined to be the acceleration of the point, or in other words we define the acceleration of a moving point to be the vector, localised in a line through the point, whose resolved part in any direction is the rate of increase of the velocity in that direction per unit of time.

33. Measurement of Acceleration. The measure of any particular acceleration is the number expressing the ratio of the acceleration to the unit acceleration.

The unit acceleration is that uniform acceleration with which a moving point gains a unit of velocity in a unit of time.

The number expressing an acceleration is the ratio of a number expressing a velocity to a number expressing an interval of time. It therefore varies inversely as the unit of length and directly as the square of the unit of time.

Acceleration is accordingly said to be a quantity of one dimension in length and of minus two dimensions in time, or its dimension symbol is $L T^{-2}$.

34. Notation for velocities and accelerations. We have so frequently to deal with differential coefficients of quantities with regard to the time that it is convenient to use 
for them an abbreviated notation. We shall therefore denote the differential coefficient of any quantity $q$ with regard to the time $t$ by placing a dot over the $q$, thus $\dot{q}$ stands for $\frac{d q}{d t}$.

Now suppose $x, y, z$ are the coordinates of a moving point at time $t$, then its component velocities parallel to the axes are denoted by $\dot{x}, \dot{y}, \dot{z}$.

Again suppose $u, v, w$ are the component velocities of a point parallel to the axes, then its component accelerations are denoted by $\dot{u}, \dot{v}, \dot{w}$.

Since $\dot{u}=\frac{d \dot{x}}{d t}, \dot{v}=\frac{d \dot{y}}{d t}, \dot{w}=\frac{d \dot{z}}{d t}$ it is convenient to write for them $\ddot{x}, \ddot{y}, \ddot{z}$ respectively. This recalls the fact that the component accelerations parallel to the axes are the second differential coefficients of the coordinates.

In the same way when we have to deal with any function of the time, say $q$, we may write $\ddot{q}$ for $\frac{d^{2} q}{d t^{2}}$, as we write $\dot{q}$ for $\frac{d q}{d t}$, where as usual $\frac{d^{2} q}{d t^{2}}$ is written for $\frac{d}{d t}\left(\frac{d q}{d t}\right)$. Also, following the analogy of the case where $q$ is $x, y$, or $z$, we may call $\dot{q}$ the velocity with which $q$ increases, and $\ddot{q}$ the acceleration with which $q$ increases.

\section{Acceleration of a point describing a plane curve.} Suppose that the moving point describes a plane curve which occupies a fixed position with reference to the axes. To fix ideas we may take it to be in the plane of $(x, y)$.

Let $v$ be the velocity at any point $P$ of the curve, $v^{\prime}$ the velocity at a neighbouring point $Q$, and $\Delta \phi$ the angle $Q T A$

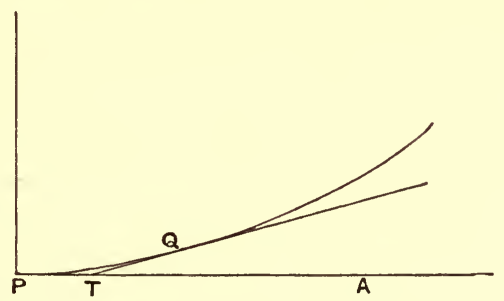

Fig. 27. 
between the tangent at $P$ and the tangent at $Q$. Also let $\Delta t$ be the time taken by the point to move from $P$ to $Q$, and let $\Delta s$ be the length of the arc $P Q$.

The velocity at $Q$ can be resolved into components $v^{\prime} \cos \Delta \phi$ in the direction of the tangent at $P$ and $v^{\prime} \sin \Delta \phi$ in the direction of the normal at $P$.

Hence the acceleration in the direction of the tangent at $P$ is the limit of $\frac{v^{\prime} \cos \Delta \phi-v}{\Delta t}$ when $\Delta t$ is indefinitely diminished. In passing to the limit $\cos \Delta \phi$ differs from unity by an infinitesimal of the second order, and $v^{\prime}$ differs from $v$ by an infinitesimal of the first order, viz. by the limit of $\Delta v$ the increment of the velocity. Thus the above limit is $\frac{d v}{d t}$ or $\dot{v}$. Since we have

$$
\frac{d v}{d t}=\frac{d v}{d s} \cdot \frac{d s}{d t}=v \frac{d v}{d s}
$$

we may write $v \frac{d v}{d s}$ for the component acceleration parallel to the tangent, and we may also write $\ddot{s}$ for it, since $v$ is $\dot{s}$.

Again the acceleration in the direction of the normal at $P$ is the limit of $\frac{v^{\prime} \sin \Delta \phi}{\Delta t}$, and this is the same as the limit of

$$
\frac{v^{\prime}}{v} v \frac{\sin \Delta \phi}{\Delta \phi} \frac{\Delta \phi}{\Delta s} \frac{\Delta s}{\Delta t}
$$

and the limits of these factors in order are $1, v, 1, \frac{1}{\rho}, v$, where $\rho$ is the radius of curvature of the curve at $P$. Thus the acceleration in the direction of the normal is $\frac{v^{2}}{\rho}$.

*36. Acceleration of a point describing a tortuous curve. We add here an investigation of the acceleration of a point describing a tortuous curve which occupies a fixed position with respect to the axes.

We recall the facts that if $x, y, z$ are the rectangular coordinates of a point of a curve and $s$ the arc measured from some particular point of the curve to the point $(x, y, z)$, the direction cosines of the tangent, in the sense in which $s$ increases, are $\frac{d x}{d s}, \frac{d y}{d s}, \frac{d z}{d s}$, satisfying the relation $\left(\frac{d x}{d s}\right)^{2}+\left(\frac{d y}{d s}\right)^{2}+\left(\frac{d z}{d s}\right)^{2}=1$; 
the direction cosines of the principal normal directed towards the centre of curvature are $\rho \frac{d^{2} x}{d s^{2}}, \rho \frac{d^{2} y}{d s^{2}}, \rho \frac{d^{2} z}{d s^{2}}$, satisfying the relation

$$
\frac{1}{\rho^{2}}=\left(\frac{d^{2} x}{d s^{2}}\right)^{2}+\left(\frac{d^{2} y}{d s^{2}}\right)^{2}+\left(\frac{d^{2} z}{d s^{2}}\right)^{2}
$$

where $\rho$ is the radius of circular curvature; and the direction cosines of the binormal are $\rho\left(\frac{d^{2} y}{d s^{2}} \frac{d z}{d s}-\frac{d^{2} z}{d s^{2}} \frac{d y}{d s}\right), \rho\left(\frac{d^{2} z}{d s^{2}} \frac{d x}{d s}-\frac{d^{2} x}{d s^{2}} \frac{d z}{d s}\right), \rho\left(\frac{d^{2} x}{d s^{2}} \frac{d y}{d s}-\frac{d^{2} y}{d s^{2}} \frac{d x}{d s}\right)$. We recall also the relation $\frac{d x}{d s} \frac{d^{2} x}{d s^{2}}+\frac{d y}{d s} \frac{d^{2} y}{d s^{2}}+\frac{d z}{d s} \frac{d^{2} z}{d s^{2}}=0$.

In the expressions $\ddot{x}, \ddot{y}, \ddot{z}$ for the component accelerations parallel to the axes we change the independent variable from $t$ to $s$.

We have, writing $v$ for the speed, so that $v$ stands for $\dot{s}$,

$$
\ddot{x}=\frac{d^{2} x}{d t^{2}}=\frac{d}{d t}\left(\frac{d x}{d t}\right)=\frac{d s}{d t} \frac{d}{d s}\left(\frac{d s}{d t} \frac{d x}{d s}\right)=v \frac{d}{d s}\left(v \frac{d x}{d s}\right),
$$

so that

$$
\ddot{x}=v \frac{d v}{d s} \frac{d x}{d s}+v^{2} \frac{d^{2} x}{d s^{2}},
$$

so

$$
\ddot{y}=v \frac{d v}{d s} \frac{d y}{d s}+v^{2} \frac{d^{2} y}{d s^{2}}
$$

and

$$
\ddot{z}=v \frac{d v}{d s} \frac{d z}{d s}+v^{2} \frac{d^{2} z}{d s^{2}} \text {. }
$$

If we multiply these component accelerations in order by the direction cosines of the tangent and add, we obtain the component acceleration parallel to the tangent to the curve in the sense in which $s$ increases; we thus find for this component the expression

$$
v \frac{d v}{d s}\left[\left(\frac{d x}{d s}\right)^{2}+\left(\frac{d y}{d s}\right)^{2}+\left(\frac{d z}{d s}\right)^{2}\right]+v^{2}\left(\frac{d x}{d s} \frac{d^{2} x}{d s^{2}}+\frac{d y}{d s} \frac{d^{2} y}{d s^{2}}+\frac{d z}{d s} \frac{d^{2} z}{d s^{2}}\right) \text {, or } v \frac{d v}{d s} \text {. }
$$

Again, if we multiply by the direction cosines of the principal normal and add, we obtain the component acceleration parallel to the principal normal directed towards the centre of curvature; we thus find for this component the expression

$$
v \frac{d v}{d s} \rho\left[\frac{d x}{d s} \frac{d^{2} x}{d s^{2}}+\frac{d y}{d s} \frac{d^{2} y}{d s^{2}}+\frac{d z}{d s} \frac{d^{2} z}{d s^{2}}\right]+v^{2} \rho\left[\left(\frac{d^{2} x}{d s^{2}}\right)^{2}+\left(\frac{d^{2} y}{d s^{2}}\right)^{2}+\left(\frac{d^{2} z}{d s^{2}}\right)^{2}\right] \text {, or } \frac{v^{2}}{\rho} \text {. }
$$

Finally, if we multiply by the direction cosines of the binormal and add, we find no component acceleration parallel to the binormal.

Thus the acceleration of a point describing a tortuous curve is in the osculating plane of the curve, and its resolved parts parallel to the tangent and principal normal are $v \frac{d v}{d s}$ and $\frac{v^{2}}{\rho}$, exactly as in the case of a point describing a plane curve. As in that case, the expression for the former component may be replaced by $\dot{v}$, or by $\ddot{s}$. 
37. Angular velocity and acceleration. Suppose a line, for example the line joining the positions at any time of two moving points, moves so as always to be in the same plane with reference to any frame. To fix ideas we shall take the plane to be the coordinate plane of $(x, y)$. Suppose the line makes an angle $\theta^{*}$ with the axis $x$ at time $t$, and an angle $\theta+\Delta \theta$ with the same axis at time $t+\Delta t$. Then $\Delta \theta$ is the measure of the angle turned through by the line in the interval measured by $\Delta t$, and the limit of the ratio of these two numbers is $\dot{\theta}$, the differential coefficient of $\theta$ with respect to $t$. This number, $\dot{\theta}$, is called the angular velocity of the line. In the same way $\ddot{\theta}$ is called the angular acceleration of the line.

The definition of the angular velocity of a line which does not remain in one plane is deferred.

38. Uniform circular motion. Suppose that, relative to any frame, the path of a moving point is a circle which occupies a fixed position relative to the frame, and suppose that the speed is uniform.

Let time be measured from the instant when the point was at $A$ on the circle, and let $\operatorname{arc} A P,=s$, and

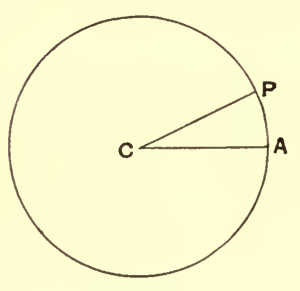

Fig. 28. $\angle A C P$, $=\theta$, be the arc described by the point, and the angle described by the radius vector, in the interval, $t$, from the instant when the point was at $A$.

Let $a$ be the radius of the circle. Then $s=a \theta$, and therefore $\dot{s}=a \dot{\theta}$.

Hence, the speed $\dot{s}$ being assumed constant, the angular velocity $\dot{\theta}$ is also constant.

Now the acceleration parallel to the tangent, being the rate of increase of $\dot{s}$ per unit of time, is zero.

The acceleration parallel to the normal is $\frac{\dot{s}^{2}}{\rho}$ where $\rho$ is the radius of curvature. For a circle of radius $a, \rho=a$, and we have seen that $\dot{s}=a \dot{\theta}$. Hence the acceleration of a point describing a circle with uniform speed is directed to the centre, and its magnitude is $a \dot{\theta}^{2}$, where $a$ is the radius, and $\dot{\theta}$ is the angular velocity of the radius joining the centre to the point.

* We shall generally take the angle to be measured in circular measure. 
39. Relative coordinates and relative motions. Let $x_{1}, y_{1}, z_{1}$ be the coordinates of a point $A$ at time $t$ referred to axes with origin at $O, x_{2}, y_{2}, z_{2}$ the coordinates of a second point $B$ at the same time referred to the same axes, and $\xi, \eta, \zeta$ the coordinates of $B$ at the same time referred to parallel axes through $A$. Then $\xi, \eta, \zeta$ are called the coordinates of $B$ relative to $A$.

We have

$$
\left.\begin{array}{l}
x_{2}=x_{1}+\xi, \\
y_{2}=y_{1}+\eta \\
z_{2}=z_{1}+\zeta .
\end{array}\right\}
$$

Let accented letters denote at time $t^{\prime}$ the quantities that correspond to unaccented letters at time $t$, thus let $x_{1}{ }^{\prime}, y_{1}{ }^{\prime}, z_{1}{ }^{\prime}$ be the coordinates of $A^{\prime}$, the position of $A$ at time $t^{\prime}$. Then as before

$$
\left.\begin{array}{l}
x_{2}^{\prime}=x_{1}{ }^{\prime}+\xi^{\prime}, \\
y_{2}^{\prime}=y_{1}^{\prime}+\eta^{\prime}, \\
z_{2}^{\prime}=z_{1}^{\prime}+\zeta^{\prime} .
\end{array}\right\}
$$

By subtraction we deduce

$$
\left.\begin{array}{l}
x_{2}^{\prime}-x_{2}=\left(x_{1}^{\prime}-x_{1}\right)+\left(\xi^{\prime}-\xi\right), \\
y_{2}^{\prime}-y_{2}=\left(y_{1}^{\prime}-y_{1}\right)+\left(\eta^{\prime}-\eta\right), \\
z_{2}^{\prime}-z_{2}=\left(z_{1}^{\prime}-z_{1}\right)+\left(\zeta^{\prime}-\zeta\right) .
\end{array}\right\}
$$

The terms on the left are the components parallel to the axes of the displacement of $B$.

The terms in the first brackets on the right are the components parallel to the axes of the displacement of $A$.

The terms in the second brackets on the right are the components of the displacement of $B$ relative to parallel axes with origin at $A$.

Thus we have the result:- The displacement of a point $B$ relative to axes at $O$ is compounded of the displacement of a point $A$ relative to the same axes and the displacement of $B$ relative to parallel axes through $A$.

By dividing equations (2) by $t^{\prime}-t$ and passing to the limit when $t^{\prime}-t$ is indefinitely diminished, or, what is the same thing, by differentiating equations (1) with respect to $t$, we find

$$
\dot{x}_{2}=\dot{x}_{1}+\dot{\xi}, \quad \dot{y}_{2}=\dot{y}_{1}+\dot{\eta}, \quad \dot{z}_{2}=\dot{z}_{1}+\dot{\zeta},
$$

and by differentiating again we find

$$
\ddot{x}_{2}=\ddot{x}_{1}+\ddot{\xi}, \quad \ddot{y}_{2}=\ddot{y}_{1}+\ddot{\eta}, \quad \ddot{z}_{2}=\ddot{z}_{1}+\ddot{\zeta} .
$$


These equations may be expressed in words as follows:-

The $\left\{\begin{array}{c}\text { velocity } \\ \text { acceleration }\end{array}\right\}$ of $B$ relative to axes at $O$ is compounded of the $\left\{\begin{array}{c}\text { velocity } \\ \text { acceleration }\end{array}\right\}$ of $A$ relative to the same axes and the $\left\{\begin{array}{c}\text { velocity } \\ \text { acceleration }\end{array}\right\}$ of $B$ relative to parallel axes through $A$.

The discussion of the motion of $B$ relative to axes whose origin is $A$ but which move so as not to be always parallel to the axes whose origin is at $O$ is deferred for the present.

40. Geometry of Relative motion. The geometrical view of relative motion is instructive, and leads easily to results of some importance. For shortness we shall speak of displacement, velocity, and acceleration of a point relative to a second point, meaning thereby displacement, velocity, and acceleration of the point relative to axes through the second point parallel to the axes of reference.

Let $A$ be the position at any time $t$ of a point which moves relatively to a frame having its origin at $O$, and let $A^{\prime}$ be its position at time $t^{\prime}$. From $O$ draw $O H$ equal and parallel to $A A^{\prime}$, and in the same sense; the vector represented by $O H$ is the displacement of $A$.

Similarly let $B$ be the position at time $t$ of a second point referred to the same frame, and $B^{\prime}$ its position at time $t^{\prime}$. From $O$ draw $O K$ equal and parallel to $B B^{\prime}$, and in the same sense; the vector represented by $O K$ is the displacement of $B$.
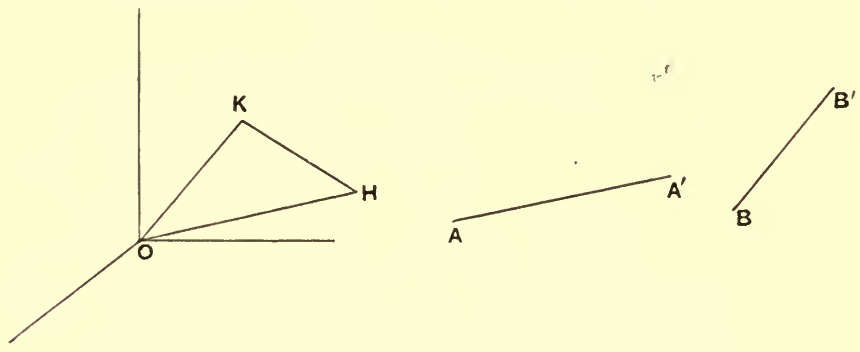

Fig. 29.

Then the displacement of $B$ relative to $A$ is the vector that must be compounded with the displacement of $A$ in order that the resultant may be the displacement of $B$. 
Join $H K$. Then the vector $O K$ is compounded of $O H, H K$.

Hence $H K$ represents the displacement of $B$ relative to $A$ in magnitude, direction, and sense.

Now the vector $H K$ is the resultant of $H O, O K$.

Hence to obtain the displacement of $B$ relative to $A$ we must compound the displacement of $B$ with the reversed displacement of $A$. The resultant is the required relative displacement.

In the same way the $\left\{\begin{array}{c}\text { velocity } \\ \text { acceleration }\end{array}\right\}$ of $B$ relative to $A$ is the $\left\{\begin{array}{c}\text { velocity } \\ \text { acceleration }\end{array}\right\}$ which must be compounded with the $\left\{\begin{array}{c}\text { velocity } \\ \text { acceleration }\end{array}\right\}$ of $A$ in order that the resultant may be the $\left\{\begin{array}{c}\text { velocity } \\ \text { acceleration }\end{array}\right\}$ of $B$.

Since the velocity of a point in any direction is the rate of increase of its displacement in that direction per unit time, and since its acceleration in any direction is the rate of increase of its velocity in that direction per unit time, we have the rules :-

The $\left\{\begin{array}{c}\text { velocity } \\ \text { acceleration }\end{array}\right\}$ of $B$ relative to $A$ is the resultant of the $\left\{\begin{array}{c}\text { velocity } \\ \text { acceleration }\end{array}\right\}$ of $B$ and the $\left\{\begin{array}{c}\text { velocity } \\ \text { acceleration }\end{array}\right\}$ of $A$ reversed.

The compositions and resolutions described in this Article are to be effected as if the vectors involved were not localised, but the velocity and acceleration of $B$ relative to $A$ are to be regarded as localised in lines through $B$. 


\section{CHAPTER IV.}

\section{DESCRIPTION OF MOTION IN TERMS OF ACCELERATION.}

41. In this chapter we propose to discuss with some detail a number of important particular cases in which the motion of a point is deduced from the value of its acceleration relative to a frame: in all these cases the path is a straight line or a plane curve.

The moving point will be considered as defining the position from time to time of a very small part of a body, and will frequently be described as a particle.

\section{Rectilinear motion with uniform acceleration.} Let the point move in a straight line, say the axis of $x$ with uniform acceleration $f$ in the positive direction of the axis; let $x_{0}$ be the coordinate of its position at the instant from which $t$ is measured, and let $u$ be the velocity of the moving point in the positive direction of the axis when $t=0$.

Then we are given

$$
\ddot{x}=f \text {, }
$$

with the conditions $x=x_{0}$ when $t=0$, and $\dot{x}=u$ when $t=0$.

Writing $v$ for $\dot{x}$, so that $v$ is the velocity at time $t$, we are given

$$
\dot{v}=f,
$$

with the condition $v=u$ when $t=0$.

Now one function of $t$ having the constant $f$ for its differential coefficient is the function $f t$, and the most general expression for a function having this differential coefficient is $f t+C$, where $C$ is an arbitrary constant. Hence $v$ must be of the form $f t+C$.

Putting $t=0$, we find $u=C$, so that the constant is determined.

Hence

$$
v=u+f t \text {, or } \dot{x}=u+f t \text {. }
$$


Again one function of $t$ having the function $u+f t$ for its differential coefficient is $u t+\frac{1}{2} f t^{2}$, hence $x$ must be of the form $C^{\prime}+u t+\frac{1}{2} f t^{2}$, where $C^{\prime}$ is an arbitrary constant.

Putting $t=0$, we find $x_{0}=C^{\prime}$, so that the constant is determined.

Hence

$$
x=x_{0}+u t+\frac{1}{2} f t^{2} .
$$

If $s$ is the distance described in the interval $t, s$ is $x-x_{0}$, so that

$$
s=u t+\frac{1}{2} f t^{2}
$$

By elimination of $t$ between this equation and the equation $v=u+f t$, we find

$$
v^{2}-u^{2}=2 f s
$$

In particular the velocity acquired in moving from rest over a distance $s$ is $\sqrt{2 f s}$. This is described as the "velocity due to falling through $s$ with an acceleration $f$."

\section{Examples.}

1. Prove that, when the acceleration is uniform, the average velocity in any interval of time is the velocity at the middle of the interval.

2. Obtain the formula $v^{2}-u^{2}=2 f s$ by multiplying both sides of the equation $\ddot{x}=f$ by $\dot{x} d t$ and integrating.

3. Suppose the distance $s$ divided into a great number of equal segments, and the sum of the velocities after describing those segments divided by their number, a velocity will be obtained which will have a limit when the number of segments is increased indefinitely, and this limit may be called the average velocity in the distance. Prove that, when the initial velocity is zero, this average velocity is equal to $\frac{2}{3}$ of the final velocity.

44. Acceleration due to gravity. The importance of the case just discussed arises from the fact that it is very nearly realised in nature. Suppose the frame of reference consists of lines fixed with reference to the Earth's surface at a place, one of them being the vertical at the place, i.e. the line in which a loaded flexible string or chain at the place would hang so as to be at rest relative to the Earth. Then it is very nearly true that, if a body were let fall near this place, it would drop with a constant acceleration in the vertical direction, or all its points would move downwards with this acceleration. When the body is let fall in the exhausted receiver of an air-pump, then it is found that, the more perfect the vacuum, the more nearly is the acceleration independent of the shape, size and material of the 
body. The acceleration of a free falling body at a place is known as the acceleration due to gravity at the place. Its magnitude depends upon the latitude of the place, in London it is about 981.8 when the centimetre is the unit of length, and 32.2 when the foot is the unit of length, the unit of time in both cases being the mean solar second. The letter $g$ is commonly used to denote this acceleration.

45. Parabolic motion. Let the velocity of a particle at time $t=0$ be parallel to the plane of $(x, y)$, and let its acceleration be uniform and parallel to the negative direction of the axis of $y$. Take $g$ to be the measure of the acceleration.

Since the acceleration parallel to the axis $z$ is always zero, the particle does not acquire velocity parallel to this axis; and, since at time $t=0$ it has no velocity parallel to this axis, it undergoes no displacement parallel to this axis; thus the point moves in a plane parallel to the plane $(x, y)$.

At time $t=0$ suppose the velocity of the particle is $V$ in a direction making an angle $\alpha$ with the axis $x$.

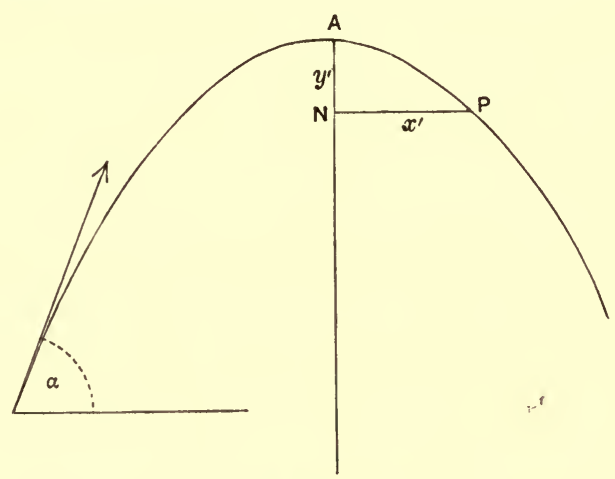

Fig. 30 .

We have the equations $\quad \ddot{x}=0$,

$$
\ddot{y}=-g \text {, }
$$

with the conditions that when $t=0$,

$$
\dot{x}=V \cos \alpha, \quad \dot{y}=V \sin \alpha .
$$

Since $\ddot{x}=0$, we have $\dot{x}=V \cos \alpha$ always.

Since $\ddot{y}=-g$, we have $\dot{y}=V \sin \alpha-g t$ after an interval $t$. 
Thus, after an interval measured by $V \sin \alpha / g, \dot{y}$ vanishes, and the particle has no velocity parallel to the axis $y$, it is therefore moving parallel to the axis $x$. Previously to this it had a velocity in the positive direction of the axis $y$, and after this it has a velocity in the negative direction of the same axis. Its path therefore has a vertex, which is reached after an interval

$$
V \sin \alpha / g,=t_{0} \text { say, }
$$

from the beginning of the motion.

If we refer the motion to parallel axes of $x^{\prime}, y^{\prime}\left(y^{\prime}\right.$ being positive in the opposite sense to $y$ ) through the vertex $A$, and take $t^{\prime}$ to measure the time of moving from the vertex $A$ to any point $P$ we shall have

$$
\frac{d^{2} x^{\prime}}{d t^{\prime 2}}=0, \text { with } \frac{d x^{\prime}}{d t^{\prime}}=V \cos \alpha \text {, and } x^{\prime}=0 \text {, at time } t^{\prime}=0,
$$

and $\quad \frac{d^{2} y^{\prime}}{d t^{\prime 2}}=g$, with $\frac{d y^{\prime}}{d t^{\prime}}=0$, and $y^{\prime}=0$, at time $t^{\prime}=0$.

Hence $x^{\prime}=V \cos \alpha t^{\prime}, y^{\prime}=\frac{1}{2} g t^{\prime 2}$. Eliminating $t^{\prime}$, we have

$$
x^{\prime 2}=\frac{2 V^{2} \cos ^{2} \alpha}{g} y^{\prime}
$$

so that the path of the particle is a parabola with vertex at $A$.

We might have deduced this result analytically from the equations $\ddot{x}=0$, $\ddot{y}=-g$. Integrating and determining the constants so that when $t=0, x=x_{0}$, $\dot{x}=V \cos a$, and $y=y_{0}, \dot{y}=V \sin a$, we find

$$
\begin{aligned}
& x=x_{0}+V \cos a \cdot t, \\
& y=y_{0}+V \sin a \cdot t-\frac{1}{2} g t^{2} .
\end{aligned}
$$

Eliminating $t$ we have

$$
y-y_{0}-\frac{V^{2} \sin ^{2} a}{2 g}+\frac{1}{2 g}\left[V \sin a-g \frac{x-x_{0}}{V \cos a}\right]^{2}=0,
$$

the equation of a parabola whose axis is parallel to the axis $y$, and whose vertex is at the point

$$
x=x_{0}+\frac{V^{2} \sin a \cos a}{g}, \quad y=y_{0}+\frac{V^{2} \sin ^{2} a}{2 g} .
$$

The theorem of this Article was discovered by Galilei.

46. Examples. [In these examples the axis $y$ is supposed to be the vertical at a place.]

1. Write down the length of the latus rectum of the above parabola.

2. Show that the height of the directrix above the starting point is $V^{2} / 2 g$.

3. If $v$ is the velocity at any point of the path show that the point is at a distance $v^{2} / 2 g$ below the directrix. 
4. Prove that the time until the particle is again in the horizontal plane through the point of projection is $2 V \sin a / g$. [This is called the time of flight on the horizontal plane through the point of projection.]

5. Prove that the distance from the starting point of the point where the particle strikes the horizontal through the starting point is $V^{2} \sin 2 a / g$. [This is called the range on the horizontal plane through the point of projection.]

6. To find the range and time of flight on an inclined plane through the point of projection. Let $\theta$ be the inclination of the plane to the horizon.

Resolve up the plane, and at right angles to it. The resolved accelerations are

$$
-g \sin \theta, \quad-g \cos \theta
$$

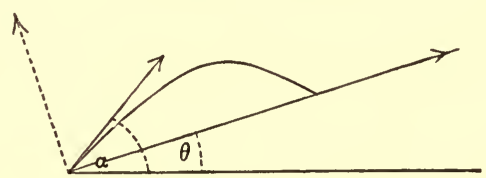

Fig. 31.

the resolved initial velocities are

$$
V \cos (a-\theta), \quad V \sin (a-\theta) ;
$$

the resolved velocities at time $t$ are

$$
V \cos (a-\theta)-g t \sin \theta, \quad V \sin (a-\theta)-g t \cos \theta ;
$$

the distances described in time $t$ parallel and perpendicular to the inclined plane are

$$
V t \cos (a-\theta)-\frac{1}{2} g t^{2} \sin \theta, \quad V t \sin (a-\theta)-\frac{1}{2} g t^{2} \cos \theta .
$$

The time of flight is obtained by making the second of these equal to zero, it is

$$
\frac{2 V \sin (a-\theta)}{g \cos \theta} \text {. }
$$

The range is found by substituting this value for $t$ in $V t \cos (a-\theta)-\frac{1}{2} g t^{2} \sin \theta$.

Prove that the range in question is

$$
\frac{2 V^{2} \cos ^{2} a}{g \cos \theta}(\tan a-\tan \theta)
$$

and that this is the same as

$$
\frac{V^{2}}{g \cos ^{2} \theta}[\sin (2 a-\theta)-\sin \theta]
$$

7. Prove that, when the velocity of projection is given, the range on an inclined plane is greatest when the direction of projection bisects the angle between the plane and the vertical.

8. Show that if a parabola is constructed having its focus at the point of projection $S$, its axis vertical, and its vertex at a height $V^{2} / 2 g$ above the point 
of projection, then the parabolic path for which the range on a line through $S$ is greatest touches this parabola at the point where the line cuts it.

[From this it follows that all possible paths of particles moving with uniform acceleration $g$ downwards, and starting from a point $S$ with given velocity $V$, touch a paraboloid of revolution about the vertical through $S$ having its focus at $S$. This paraboloid is the envelope of the trajectories of such particles.]

9. If $v$ is the velocity at any point of the parabolic path, and $\rho$ the radius of curvature at the point, verify that $v^{2} / \rho$ is equal to the component of $g$ along the normal.

10. Prove that, in the different trajectories possible under gravity between two points $A, B$, the times of flight are inversely proportional to the velocities when vertically over the middle point of $A B$.

11. Two particles describe the same parabola under gravity. Prove that the intersection of the tangents at their positions at any instant describes a coaxial parabola as if under gravity. Prove also that, if $\tau$ is the interval between the instants when they pass through the vertex, the distance between the vertices of the two parabolas is $\frac{1}{8} g \tau^{2}$.

12. A particle moves under gravity from the highest point of a sphere of radius $c$. Prove that it cannot clear the sphere unless its initial velocity exceeds $\sqrt{ }\left(\frac{1}{2} g c\right)$.

13. Prove that there are in general two directions in which a particle may be projected with a given velocity so as to strike a given object, and find how far off in a given direction the object may be.

14. Prove that the greatest range on an inclined plane through the point of projection is equal to the distance through which the particle would fall during the time of flight.

\section{Simple Harmonic Motion. A point moving in a} straight line with an acceleration directed to a point in the line and proportional to the distance is said to have a simple harmonic motion.

Let the line be the axis $x$, and the point towards which the acceleration is directed the origin; then the acceleration is in the negative direction of the axis when $x$ is positive, and in the positive direction when $x$ is negative, so that the formula for it is

$$
\ddot{x}=-\mu x,
$$

where $\mu$ is a positive constant.

Let time be measured from an instant at which $x=\alpha$ and $\dot{x}=0$. With the origin as centre and with radius $a$ describe a 
circle, and when the moving point is at $N$ on one diameter of this circle, so that $O N=x$, draw $N P$ at right angles to this diameter, and consider the motion of the point $P$.

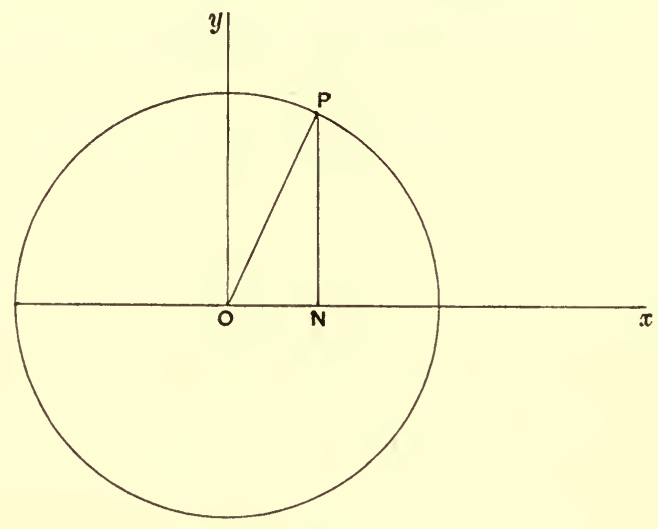

Fig. 32.

Let the angle $N O P=\theta$.

Then $x,=a \cos \theta$, and $y,=a \sin \theta$, are coordinates of $P$.

By differentiating we have

$$
\dot{x}=-a \sin \theta \dot{\theta}, \quad \ddot{x}=-a \sin \theta \ddot{\theta}-a \cos \theta \dot{\theta}^{2}
$$

hence

$$
\ddot{x}=-\left(y \ddot{\theta}+x \dot{\theta}^{2}\right) \text {; }
$$

since

$\ddot{x}=-\mu x$,

we must have

$$
\ddot{\theta}=0 \text {, and } \dot{\theta}^{2}=\mu \text {. }
$$

Hence the point $P$ describes the circle uniformly; the angular velocity of the radius vector is uniform and equal to $\sqrt{ } \mu$, and the angle $\theta=t \sqrt{ } \mu$.

The distance of the point $N$ from $O$ at time $t$ is given by

$$
x=a \cos (t \sqrt{ } \mu) \text {. }
$$

The velocity of the point is directed along $x O$, and its magnitude is $a \sqrt{ } \mu \sin (t \sqrt{ } \mu)$.

The above process shows that the solution of the equation

$$
\ddot{x}=-\mu x \text {, }
$$

with the conditions that when $t=0, x=a, \dot{x}=0$, is $x=a \cos (t \sqrt{ } \mu)$. 
It follows, by changing the epoch from which time is measured, that the complete solution must be of the form

$$
a \cos \left\{\sqrt{ } \mu\left(t-t_{0}\right)\right\},
$$

and this can be expressed in the form*

$$
A \cos (t \sqrt{ } \mu)+B \sin (t \sqrt{ } \mu) .
$$

Let the moving point have at time $t=0$ a position denoted by $x_{0}$ and a velocity denoted by $\dot{x}_{0}$; we know, that at any time $t$, $x$ must be given by an equation of the form

$$
x=A \cos (t \sqrt{ } \mu)+B \sin (t \sqrt{ } \mu) .
$$

To determine the constant $A$ put $t=0$, we have $x_{0}=A$.

To determine the constant $B$, differentiate with respect to $t$, we have

$$
\dot{x}=-A \sqrt{ } \mu \sin (t \sqrt{ } \mu)+B \sqrt{ } \mu \cos (t \sqrt{ } \mu) .
$$

Now put $t=0$ and we find

$$
\dot{x}_{0}=B \sqrt{ } \mu .
$$

Hence the solution of the equation $\ddot{x}=-\mu x$, with the conditions that $x=x_{0}$ and $\dot{x}=\dot{x}_{0}$ when $t=0$, is

$$
x=x_{0} \cos (t \sqrt{ } \mu)+\frac{\dot{x}_{0}}{\sqrt{ } \mu} \sin (t \sqrt{ } \mu) .
$$

It is to be observed that the whole motion is periodic, that is repeats itself after equal intervals of time; the period is $\frac{2 \pi}{\sqrt{ } \mu}$.

The equation $x=a \cos (t \sqrt{ } \mu-\epsilon)$ represents simple harmonic motion with period $2 \pi / \sqrt{ } \mu$, in this form $a$ is called the amplitude of the motion, it is the greatest value of $x$, and $\epsilon$ determines the phase of the motion.

48. Composition of simple harmonic motions. We consider the case where the moving point has a simple harmonic motion of period $\frac{2 \pi}{\sqrt{\mu}}$ parallel to each of the axes $x$ and $y$, the acceleration in each case being directed towards the origin.

* The student who is acquainted with the methods of solving linear differential equations will recognize that this is the known form of the complete primitive of the differential equation. 
We have the equations $\ddot{x}=-\mu x$,

$$
\ddot{y}=-\mu y,
$$

and we deduce that $x$ and $y$ must be given by equations of the form

$$
\begin{aligned}
& x=A \cos (t \sqrt{ } \mu)+B \sin (t \sqrt{ } \mu), \\
& y=C \cos (t \sqrt{ } \mu)+D \sin (t \sqrt{ } \mu),
\end{aligned}
$$

where $A, B, C, D$ are arbitrary constants depending on the initial conditions, viz. $A$ and $C$ are the coordinates, and $B \sqrt{ } \mu, D \sqrt{ } \mu$ the resolved velocities at the instant $t=0$.

Solving the above equations for $\cos (t \sqrt{ } \mu)$ and $\sin (t \sqrt{ } \mu)$, we have $(A D-B C) \cos (t \sqrt{ } \mu)=D x-B y,(A D-B C) \sin (t \sqrt{ } \mu)=A y-C x$, eliminating $t$, we find

$$
(D x-B y)^{2}+(A y-C x)^{2}=(A D-B C)^{2},
$$

so that the path of the moving point is an ellipse whose centre is the origin, and whose position with reference to the origin and axes is fixed. The whole motion is clearly periodic with period $2 \pi$ $\sqrt{\cdot \mu} \cdot$

Let us change the axes to the principal axes of the ellipse, and suppose the moving point is at one extremity $(x=a)$ of the major axis at the instant $t=0$, then at this instant $x=a, y=0$, and, since the point is moving at right angles to the major axis, $\dot{x}=0$. Suppose $\dot{y}=b \sqrt{ } \mu$. Then we must have at time $t$

$$
x=a \cos (t \sqrt{ } \mu), \quad y=b \sin (t \sqrt{ } \mu) .
$$

Thus $b$ is the semi-axis minor, and $t \sqrt{ } \mu$ is the eccentric angle at time $t$.

The point therefore moves so that its eccentric angle increases uniformly with angular velocity $\sqrt{ } \mu$.

\section{Examples.}

1. Prove that when the equation is $\ddot{x}=\mu x$, where $\mu$ is positive, and the initial conditions are that $x=x_{0}$ and $\dot{x}=\dot{x}_{0}$ when $t=0$, then at any time $t$

$$
x=x_{0} \cosh (t \sqrt{ } \mu)+\frac{\dot{x}_{0}}{\sqrt{ } \mu} \sinh (t \sqrt{ } \mu) .
$$

2. Prove that when the acceleration is directed from the origin and is proportional to the distance the path is an hyperbola. 
3. In simple harmonic motion given by $\ddot{x}=-\mu x$ starting from $x=\alpha$, prove, by multiplying both sides of the equation by $\dot{x} d t$ and integrating, that $\dot{x}^{2}=\mu\left(a^{2}-x^{2}\right)$ for all positions of $x$.

4. In the elliptic motion of Article 49 prove that the velocity $v$ at distance $r$ from the centre is given by

and evaluate the constant.

$$
v^{2}+\mu r^{2}=\text { const., }
$$

5. In the hyperbolic motion of Example 2 prove that the velocity $v$ at distance $r$ from the centre of the hyperbola is given by

and evaluate the constant.

$$
v^{2}=\mu r^{2}+\text { const. }
$$

50. Central Acceleration. In the motion just described (Article 48) the resultant acceleration is of magnitude $\mu r$ and is directed along the radius vector towards the origin, $r$ being the length of this radius vector. An acceleration always directed towards or from a point which occupies a fixed position relative to a frame is called a central acceleration. The point through which the acceleration always passes is called the centre. We shall now prove a general theorem* with reference to central accelerations :-

The path of a point moving with a central acceleration is in a plane through the centre, and the radius vector drawn from the centre to the point describes equal areas in equal times.

Let the line of the velocity at any instant be drawn, and a plane drawn through the centre and this line. All the circumstances of the motion being symmetrical with regard to this plane, there is no more reason why the point should move out of it on one side than on the other. The point therefore moves in the plane.

Let the plane of motion be the plane of $(x, y)$, and let the centre towards or from which the acceleration is directed be the origin. Then, since the acceleration is localised in a line through the origin, its moment about the origin is zero, or we have

$$
x \ddot{y}-y \ddot{x}=0 .
$$

The left-hand member of this equation is the differential coefficient of $x \dot{y}-y \dot{x}$, so that we have

$$
\frac{d}{d t}(x \dot{y}-y \dot{x})=0 \text {. }
$$

* Due to Newton, Principia, Lib. I. Sect. II. Prop. 1. 
Hence

$$
x \dot{y}-y \dot{x}=\text { const., }=h \text { say. }
$$

The left-hand member of this equation is the moment of the velocity about the origin, and is therefore equal to the product $p v$, where $v$ is the velocity of the moving point in any position, and $p$ is the perpendicular from the origin on the tangent to the path at this position. We therefore have

$$
p v=h \text {. }
$$

Now let $s$ be the arc of the curve measured in the direction in which the curve is described from

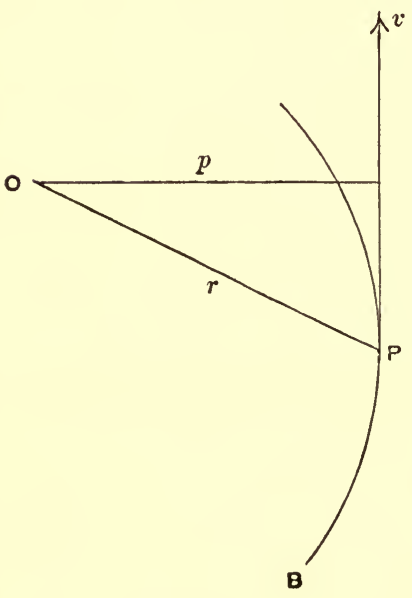

Fig. 33. some point $B$ of the curve up to the position $P$ of the moving point at time $t, A$ the area described by the radius vector while the moving point describes the arc $s$. Then $p \Delta s$ is ultimately twice the area of the infinitesimal triangle described by the radius vector in the interval $\Delta t$, taken to describe the small arc $\Delta s$, so that $p \dot{s}=2 \dot{A}$. Also $\dot{s}=v$.

Hence $h,=p \dot{s}$, is twice the rate at which area is described by the radius vector, and, $h$ being constant, the radius vector describes areas uniformly, i.e. it describes

equal areas in equal times.

The quantity $h$ is twice the area described in a unit of time.

The path described by a point $P$ which moves with an acceleration in the line joining $P$ to a point $O$ is called a central orbit, and is said to be described about the point $O$.

51. Formula for the central acceleration. Let $f$ be the magnitude of the central acceleration at $P$, supposed directed towards $O, r$ the radius vector $O P, \rho$ the radius of curvature of the path at $P$.

The resolved part of the acceleration parallel to the normal at $P$ is $f \frac{p}{r}$. 
But this resolved part of the acceleration is $\frac{v^{2}}{\rho}$.

Hence $\quad \frac{v^{2}}{\rho}=f \frac{p}{r}$.

From this equation and the equation $v p=h$ we may eliminate $v$, and obtain the equation

$$
f=\frac{h^{2} r}{p^{3} \rho} .
$$

Since $\rho=r \frac{d r}{d p}$, we may also write this equation

$$
f=\frac{h^{2}}{p^{3}} \frac{d p}{d r}
$$

When the curve is given we can hence deduce the acceleration to a given point in its plane required for the description of the curve. When $f$ is given in terms of $r$ we can find by integration the $(p, r)$ equation of the curve.

\section{Examples.}

1. Show that, when the orbit is an ellipse described about the centre, the acceleration is proportional to the radius vector.

2. In the same case show that the velocity at any point is proportional to the length of the diameter conjugate to the diameter through the point.

3. Points move from a position $P$ with a velocity $V$ in different directions with an acceleration to a point $C$ proportional to the distance. Prove that all the elliptic trajectories described have the same director circle.

Let the tangent at $P$ to one of the trajectories meet the director circle in $T$, and let $Q$ be the point of contact of the other tangent to this trajectory drawn from $T$. Prove that the trajectory in question touches at $Q$ an ellipse having $C$ as centre, $P$ as one focus, and $C T$ as the length of the semi-axis major.

[This ellipse is the envelope of the trajectories of points starting from $P$ with the given velocity and moving about $C$ with the given central acceleration.]

4. Show that the central acceleration when a circle is described as a central orbit about a point on the circumference is $8 h^{2} a^{2} / r^{5}, a$ being the radius of the circle.

5. Show that the central acceleration when an equiangular spiral is described as a central orbit about its pole is proportional to $r^{-3}$.

6. Show that for an ellipse described as a central orbit about any point $O$ in its plane the central acceleration at any point $P$ is proportional to $r / q^{3}$ where $r$ is the radius vector $O P$, and $q$ is the perpendicular from $P$ on the polar of 0 . 
7. Interpret the formula $v^{2}=f \rho p / r$ so as to show that the velocity at any point $P$, when a curve is described as a central orbit about a point $O$, is that due to falling with constant acceleration, equal to that at $P$, through one quarter of the chord of curvature in the direction $P O$.

53. Elliptic motion about a focus. Let an ellipse of semi-axes $a, b$ be described as a central orbit about a focus $S$. Let $S^{\prime}$ be the second focus, $e$ the eccentricity, $l$ the semi-latus rectum.

Let $P$ be any point on the ellipse; let $r$ and $r^{\prime}$ be the radii vectores drawn from $S$ and $S^{\prime}$ to $P$; let $p$ and $p^{\prime}$ be the perpen-

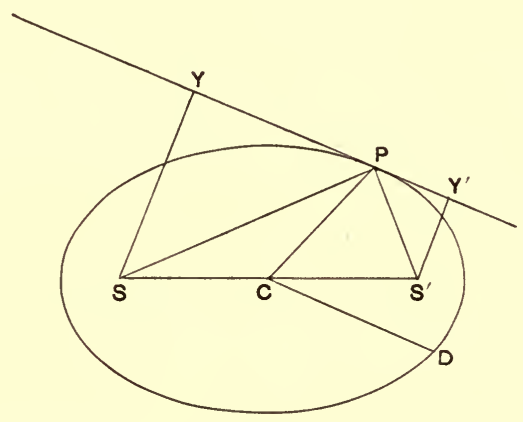

Fig. 34 .

diculars from $S$ and $S^{\prime}$ on the tangent at $P$; let $C$ be the centre and $C D$ the semi-diameter conjugate to $C P$.

Then

$$
\rho=C D^{3} / a b, \quad r r^{\prime}=C D^{2}, \quad p p^{\prime}=b^{2}, \quad r+r^{\prime}=2 a, \quad b^{2}=a l .
$$

Also since $\angle S P Y=\angle S^{\prime} P Y^{\prime}$, we have

$$
\frac{p}{r}=\frac{p^{\prime}}{r^{\prime}}, \text { and therefore each of these }=\frac{\sqrt{p p^{\prime}}}{\sqrt{r r^{\prime}}}=\frac{b}{C D} .
$$

Now the acceleration, $f$, is given by

$$
\begin{aligned}
f & =\frac{h^{2} r}{p^{3} \rho} \\
& =\frac{h^{2} r a b}{C D^{3}}\left(\frac{C D}{b r}\right)^{3}=\frac{h^{2}}{r^{2}} \frac{a}{b^{2}}=\frac{h^{2}}{r^{2} l} .
\end{aligned}
$$

Thus the acceleration varies inversely as the square of the distance $r$, and, if we write $\mu / r^{2}$ for it, we have $h^{2}=\mu l$. 
The periodic time in which the ellipse is described is

$$
\frac{2 \pi a b}{h}=\frac{2 \pi a^{\frac{3}{2}}}{\sqrt{ } \mu} \text {. }
$$

\section{Examples.}

1. Prove that the velocity $v$ at any point of the ellipse is given by the equation

$$
v^{2}=\mu\left(\frac{2}{r}-\frac{1}{a}\right) \text {. }
$$

2. Prove that if any conic is described as a central orbit about a focus the acceleration is $\mu / r^{2}$ towards the focus, and $\mu=h^{2} / l$.

Prove also that when the conic is a parabola $v^{2}=2 \mu / r$, and when it is a hyperbola $v^{2}=\mu(2 / r+1 / a)$.

3. Prove that in elliptic motion about a focus $S$ the velocity at any point $P$ is perpendicular and proportional to the radius vector from the other focus to the point $W$, where $S P$ produced meets a circle centre $S$ and radius $2 a$.

[From the formula in Example 1, this circle is called the "circle of no velocity."]

4. Prove that the velocity at $P$ can be resolved into two constant components, one at right angles to the radius vector $S P$, and the other at right angles to the major axis.

5. Points move from a position $P$ with a velocity $V$ in different directions with an acceleration to a point $S$ varying inversely as the square of the distance. Prove that all the trajectories have equal transverse axes.

Let the line from $P$ to the second focus of one of the trajectories (supposed elliptic) meet that trajectory in $P^{\prime}$. Prove that this trajectory touches at $P^{\prime}$ an ellipse with $S$ and $P$ as foci and a definite major axis.

[This ellipse is the envelope of the trajectories of points starting from $P$ with the given velocity and describing ellipses as central orbits about $S$.]

6. To find the time of describing any are of the ellipse described as a central orbit about a focus.

Draw the auxiliary circle $A Q A^{\prime}$.

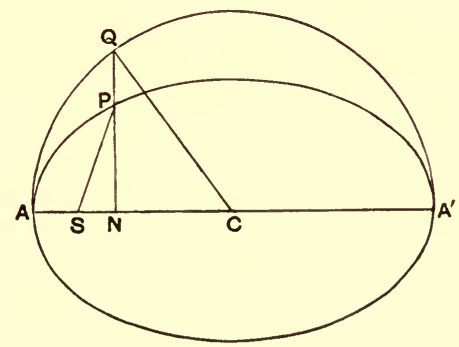

Fig. 3ว. 
Let $\phi,=\angle Q C A$ in the figure, be the eccentric angle of $P$, and $\theta,=\angle A S P$, the vectorial angle.

Then curvilinear area $A S P=$ curvilinear area $A N P$-triangle $S P N$

$$
=\frac{b}{a}(\text { curvilinear area } A N Q)-\text { triangle } S P N \text {. }
$$

Now curvilinear area $A N Q=$ sector $A C Q$ - triangle $C Q N$

$$
=\frac{1}{2}\left(a^{2} \phi-a^{2} \sin \phi \cos \phi\right) \text {, }
$$

and

$$
\text { triangle } S P N=\frac{1}{2} b \sin \phi(a e-a \cos \phi) \text {. }
$$

Hence curvilinear area $A S P=\frac{1}{2} a b(\phi-e \sin \phi)$.

Let $t$ be the time from $A$ to $P$ then, since $h$ is twice the area described per unit of time,

Thus

$$
\begin{aligned}
& h t=a b(\phi-e \sin \phi) . \\
& t=\frac{a^{\frac{3}{2}}}{\sqrt{ } \mu}(\phi-e \sin \phi) .
\end{aligned}
$$

The quantity $\sqrt{ } \mu / a^{\frac{3}{2}}$ is known as the "mean motion" and is denoted by $n$, so that the time in question is given by

$$
n t=\phi-e \sin \phi \text {. }
$$

Prove that $\theta$ is connected with $\phi$ by the equation

$$
\begin{aligned}
\cos \phi & =\frac{e+\cos \theta}{1+e \cos \theta}, \text { and that, if } e \text { is small, } \\
\theta & =n t+2 e \sin n t \text { approximately. }
\end{aligned}
$$

7. Two points describe the same ellipse in the same periodic time, starting together from one end of the major axis; one of them has an acceleration to a focus $S$, and the other an acceleration to the centre $C$. Prove that, if $\phi_{1}$ and $\phi_{2}$ are their eccentric angles at any instant, then $\phi_{1}-\phi_{2}=e \sin \phi_{1}$.

8. Two points describe ellipses of latera recta $l$ and $l^{\prime}$ in different planes about a common focus, and the accelerations to the focus are equal when the distances are equal. Show that, when the relative velocity of the points is along the line joining them, the tangents to the ellipses at the positions of the points meet the line of intersection of the planes in the same point, and that the focal distances, $r$ and $r^{\prime}$, make with this line angles $\theta$ and $\theta^{\prime}$ such that

$$
\frac{r \sin \theta}{\sqrt{ } \iota}=\frac{r^{\prime} \sin \theta^{\prime}}{\sqrt{ } l^{\prime}}
$$

55. Motion with a central acceleration varying inversely as the square of the distance. We give here a version of Newton's investigation* of the orbit described by a point which moves from a given position $P$, with a given velocity $V$, in a given direction $P T$, and has an acceleration to a point $S$ varying inversely as the square of the distance from $S$.

\footnotetext{
* Principia, Lib. I. Sect. III. Prop. 17.
} 


\section{Lemma.}

Given a point $P$, a tangent $P T$, a focus $S$, and the focal chord of curvature $P Q$, one conic, and only one, can be described, and this conic is an ellipse, parabola, or hyperbola according as $P Q<$, $=$, or $>4 S P$.

Let $U$ be the middle point of $P Q$. Draw $P G$ at right angles to $P T$, and $U G$ parallel to $P T$; draw $U O$ and $G K$ at right angles to $S P$ meeting $P G$ and $S P$ in $O$ and $K$ respectively.

Then by similar triangles $O P U$, $U P G, G P K$ we have

$O P: P U=P U: P G=P G: P K$.

Whence $O P=\frac{P G^{3}}{P K^{2}}$.

Now describe a conic with focus $S$

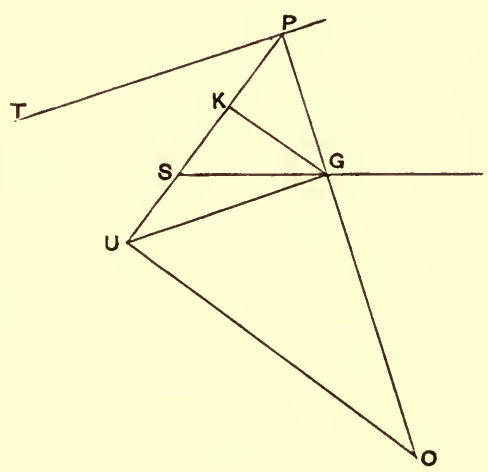

Fig. 36. and axis $S G$ to touch $P T$ at $P, G$ is the foot of the normal, and $P K$ is the semi-latus rectim. Hence $O$ is the centre of curvature.

Since $S G: S P=$ eccentricity, the conic is determinate and unique.

Since a semicircle on $P U$ as diameter passes through $G$, we have when $S P>\frac{1}{2} P U, S G<S P$; when $S P<\frac{1}{2} P U, S G>S P$; when $S P=\frac{1}{2} P U, S G=S P$.

Thus the conic is an ellipse, parabola, or hyperbola according as

$$
S P>,=\text {, or }<\frac{1}{2} P U \text {. }
$$

Now let a point move from $P$ with velocity $V$ in direction $P T$ and have an acceleration $\mu /(\text { distance })^{2}$ towards $S$.

Find $Q$ in $P S$ produced so that

$$
V^{2}=2 \frac{\mu}{S P^{2}} \frac{P Q}{4} .
$$

Then by Example 7 on p. 54, $P Q$ is the chord of curvature of the path in direction $P S$.

With $S$ as focus describe a conic touching $P T$ at $P$, and having $P Q$ for focal chord of curvature at $P$.

Let a point describe this conic as a central orbit about $S$ starting with velocity $V$ at $P$, the two moving points have at starting the same position, velocity, and acceleration, and their accelerations are always the same when their distances from $S$ are the same, they therefore describe the same orbit. 
The orbit in question

$$
\text { is an ellipse if } \frac{1}{4} P Q<S P \text { i.e. if } V^{2}<\frac{2 \mu}{S P},
$$

it is a parabola if $\frac{1}{4} P Q=S P$ i.e. if $V^{2}=\frac{2 \mu}{S P}$,

it is a hyperbola if $\frac{1}{4} P Q>S P$ i.e. if $V^{2}>\frac{2 \mu}{S P}$.

\section{Motion in a straight line with an acceleration to}

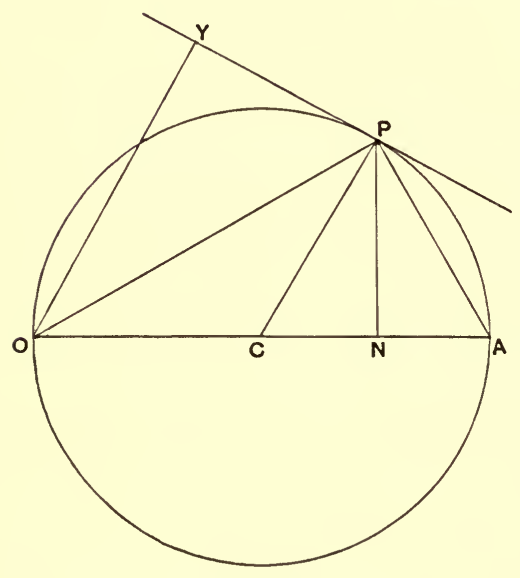

Fig. 37.

a point in the line varying inversely as the square of the distance. Let a point $N$ move in a straight line $O A$, starting from $A$, so that, when $O N=x, \ddot{x}=-\mu / x^{2}$.

On $O A$ as diameter describe a circle, and let $C$ be its centre, and $a$ its radius; draw $N P$ at right angles to $O A$, and consider the motion of the point $P$ on the circle.

We shall show that, if $P$ describes the circle with an acceleration towards $O$, the point $N$ will have the acceleration named.

By Example 4 of p. 53 we have

acceleration of $P=\begin{gathered}8 h^{2} a^{2} \\ O P^{5}\end{gathered}$, where $h$ is twice the rate at which $O P$ describes areas about $O$.

To resolve in direction $A O$ multiply by $O N / O P$ and observe that $O N: O P=O P: O A$. Thus

$$
\text { acceleration of } N=\frac{8 h^{2} a^{2} O N}{O P^{6}}=\frac{8 h^{2} a^{2} O N}{(2 a \cdot O N)^{3}}=\frac{h^{2}}{a} \frac{1}{O N^{2}} \text {. }
$$

Hence if we take the point $N$ to start at a distance $2 a$ from $O$ and put $h^{2}=\mu a$, then when $O N=x, N$ will have an acceleration 
$\mu / x^{2}$ towards $O$, i.e. we shall have

$$
\ddot{x}=-\mu / x^{2} \text {. }
$$

Since the radius vector $O P$ describes areas uniformly we can utilise the figure to express the position in terms of the time.

Let angle $A O P=\theta$, and let $t$ be the time of going from $A$ to $N$. Then

$$
x=O N=\frac{O P^{2}}{2 a}=\frac{(2 a \cos \theta)^{2}}{2 a}=2 a \cos ^{2} \theta,
$$

and $h t=$ twice the curvilinear area $A O P$

$=$ twice the sector $A C P+$ twice the triangle $O C P$

$=2 a^{2} \theta+a^{2} \sin 2 \theta$

thus $t=\frac{a^{\frac{3}{3}}}{\sqrt{ } \mu}(2 \theta+\sin 2 \theta)$.

Thus the coordinate $x$ and the time $t$ are both expressed in terms of a parameter $\theta$.

\section{Examples.}

1. The same results may, of course, be arrived at by integrating the equation $\ddot{x}=-\frac{\mu}{x^{2}}$ with the conditions that when $t=0, x=2 \alpha, \dot{x}=0$.

Multiplying both sides by $\dot{x} d t$ and integrating, we find

$$
\frac{1}{2} \dot{x}^{2}=\frac{\mu}{x}+C, \text { where } C \text { is an arbitrary constant; }
$$

putting $t=0$, we have

$$
C=-\frac{\mu}{2 \alpha} \text {. }
$$

Thus

$$
\dot{x}^{2}=\mu\left(\frac{2}{x}-\frac{1}{a}\right) \text {. }
$$

Hence

$$
t=\int_{2 a}^{x} \frac{-d x}{\sqrt{\mu \frac{2 a-x}{a x}}} .
$$

By putting $x=2 \alpha \cos ^{2} \theta$ in this deduce the result in the text.

2. Find the time of falling to $O$.

3. Prove that as $N$ approaches $O$ the velocity increases without limit.

[We shall see hereafter that when a natural system is devised in such a way that a point of a body moves as here described, either the body cannot pass through the point corresponding to $O$, or before it reaches $O$ the formula for the acceleration changes and the velocity at $O$ is finite.] 
58. Radial and transversal velocities and accelerations. Suppose a point is describing a plane curve which occupies a fixed position with reference to a frame. To fix ideas we may suppose it to be in the plane of $(x, y)$. Let $r, \theta$ be the polar coordinates of the point at time $t$, referred to the origin as pole and the axis $x$ as initial line. It is required to express in terms of $r, \theta$ and their differential coefficients the resolved parts of the velocity and acceleration parallel and perpendicular to the radius vector.

Let $u, v$ be the resolved parts of the velocity parallel and perpendicular to the direction of $r$. The resolved parts of the

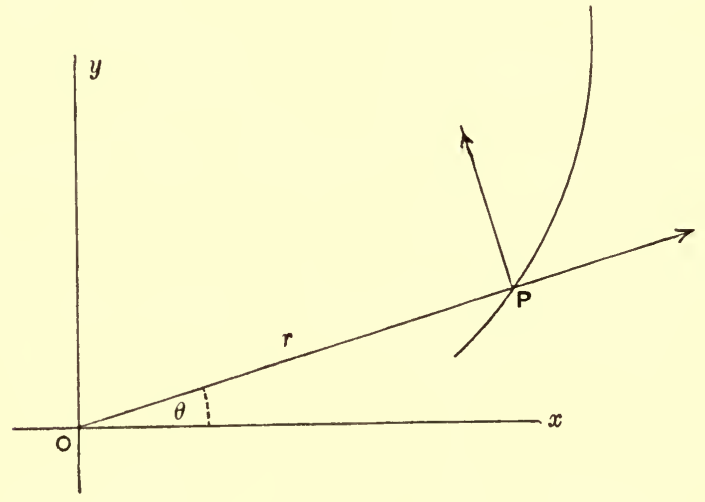

Fig. 38.

same velocity parallel to the axes are $\dot{x}, \dot{y}$, and we have therefore, by resolving parallel to the axes,

$$
\begin{aligned}
& u \cos \theta-v \sin \theta=\dot{x}=\frac{d}{d t}(r \cos \theta)=\dot{r} \cos \theta-r \dot{\theta} \sin \theta \\
& u \sin \theta+v \cos \theta=\dot{y}=\frac{d}{d t}(r \sin \theta)=\dot{r} \sin \theta+r \dot{\theta} \cos \theta .
\end{aligned}
$$

Solving these equations we find $u=\dot{r}, v=r \dot{\theta}$.

Again let $\alpha, \beta$ be the resolved parts of the acceleration parallel and perpendicular to the direction $r$. The resolved parts of the same acceleration parallel to the axes of $x$ and $y$ are $\ddot{x}$ and $\ddot{y}$. 
We have therefore, by resolving parallel to the axes

$$
\begin{aligned}
\alpha \cos \theta-\beta \sin \theta=\ddot{x} & =\frac{d}{d t}(\dot{r} \cos \theta-r \dot{\theta} \sin \theta) \\
& =\left(\ddot{r}-r \dot{\theta}^{2}\right) \cos \theta-(r \ddot{\theta}+2 \dot{r} \dot{\theta}) \sin \theta \\
\alpha \sin \theta+\beta \cos \theta=\ddot{y} & =\frac{d}{d t}(\dot{r} \sin \theta+r \dot{\theta} \cos \theta) \\
& =\left(\ddot{r}-r \dot{\theta}^{2}\right) \sin \theta+(r \ddot{\theta}+2 \dot{r} \dot{\theta}) \cos \theta .
\end{aligned}
$$

Solving these equations we find $\alpha=\ddot{r}-r \dot{\theta}^{2}, \beta=r \ddot{\theta}+2 \dot{r} \dot{\theta}$.

Here it is important to observe that the acceleration along the radius vector is the resolved part parallel to that line of the acceleration relative to the frame $O x, O y$; it is not the acceleration with which the radius vector increases.

Further it is to be observed that the acceleration, $\beta$, at right angles to the radius vector can be expressed in the form $\frac{1}{r} \frac{d}{d t}\left(r^{2} \dot{\theta}\right)$, where the expression $r^{2} \dot{\theta}$ is equal to the moment of the velocity about the origin.

By expressing that, in central orbits, the resolved acceleration at right angles to the radius vector vanishes, we should obtain a new proof of the formula $p v=h$, and we should find $h=r^{2} \dot{\theta}$. Comparing this Article with Article 50 we verify the well-known formula of Differential Calculus

$$
x d y-y d x=r^{2} d \theta \text {. }
$$

\section{Examples.}

1. A point $P$ describes a curve relative to axes through $O$. Prove that, relative to parallel axes through $P, O$ describes a curve equal in all respects to that described by $P$. Prove also that any point dividing $O P$ in a constant ratio describes a similar curve relative to the axes through $O$ or $P$.

2. The motion of a point is referred to polar coordinates $r, \theta, \phi$ with origin at the origin of a set of rectangular axes of $x, y$, $z$, with the axis $z$ for polar axis, and the plane $(x, z)$ for initial meridian; prove that the resolved parts of the velocity along the radius vector, the tangent to the meridian, and the tangent to the parallel through the position at time $t$ are $\dot{r}, r \dot{\theta}$, and $r \sin \theta \dot{\phi}$, and that the resolved parts of the accelerations along the same lines are

$$
\ddot{r}-r \dot{\theta}^{2}-r \sin ^{2} \theta \dot{\phi}^{2}, \quad \frac{1}{r} \frac{d}{d t}\left(r^{2} \dot{\theta}\right)-r \sin \theta \cos \theta \dot{\phi}^{2}, \quad \text { and } \quad \frac{1}{r \sin \theta} \frac{d}{d t}\left(r^{2} \sin ^{2} \theta \dot{\phi}\right) \text {. }
$$


3. Apply the results of Example 2 to obtain a formal proof of the statement that, when the acceleration of a moving point is always directed towards or from a point, fixed relatively to a frame, the path of the point, relative to that frame, is a plane curve.

4. The motion of each of two points relative to a certain frame is uniform rectilinear motion, and the straight paths intersect. Prove that the acceleration with which the distance between the points increases is inversely proportional to the cube of that distance, and find the path of either point relative to the other.

5. Relatively to a certain frame a point $O$ describes a straight line uniformly with velocity $V$, and a second point $P$ describes a curve in such a way that the line $O P$ describes areas uniformly; prove that the resolved part perpendicular to $O P$ of the acceleration of $P$ is $2 V v \sin \phi / O P$, where $v$ is the velocity of $P$, and $\phi$ the angle the tangent to its path makes with $O P$.

6. Relatively to a certain frame, a point $A$ describes a circle (centre $O$ ) uniformly, and a point $B$ moves with an acceleration always directed to $A$. If the area covered by the line $A B$ is described uniformly, prove that the resolved part parallel to $O A$ of the velocity of $B$ is proportional to the perpendicular from $B$ on $O A$ produced.

60. Differential equation of central orbit. The equations that hold for a point describing a central orbit about the origin with acceleration $f$ towards the origin are

$$
\begin{aligned}
\ddot{r}-r \dot{\theta}^{2} & =-f, \\
r^{2} \dot{\theta} & =h,
\end{aligned}
$$

where $h$ is a constant determined by the initial conditions.

By using the second of these equations we can change the independent variable in the first from $t$ to $\theta$. We write $u$ for $r^{-1}$, then we have

$$
\dot{\theta}=h u^{2}, \frac{d}{d t}=h u^{2} \frac{d}{d \theta},
$$

so that the first equation becomes

$$
h u^{2} \frac{d}{d \theta}\left(h u^{2} \frac{d r}{d \theta}\right)-h^{2} u^{3}=-f
$$

and, since $u^{2} \frac{d r}{d \theta}=-\frac{d u}{d \theta}$, and $h$ is constant, this is

$$
\frac{d^{2} u}{d \theta^{2}}+u=\frac{f}{h^{2} u^{2}},
$$

This is the differential equation of the path. The integral of the equation will be a relation between $u$ and $\theta$ containing two arbitrary constants, and this is the polar equation of the path. 


\section{Integration of the equation when $f$ is a function}

of r. Multiply both sides of the equation by $\frac{d u}{d \theta}$, and let $\phi(u)$ be the indefinite integral of $\iota^{-2} f$, we have

$$
\frac{1}{2}\left[\left(\frac{d u}{d \theta}\right)^{2}+u^{2}\right]=\frac{1}{h^{2}} \phi(u)+A,
$$

where $A$ is constant.

Suppose the initial condition is that the starting point is at a distance $c$ from the origin and the initial velocity is $V$ in a direction making an angle $\alpha$ with the radius vector. We have $h=V c \sin \alpha$. Also, by a well-known formula, if $p$ is the perpendicular from the origin on the tangent at the point $(u, \theta)$,

$$
\frac{1}{p^{2}}=u^{2}+\left(\frac{d u}{d \theta}\right)^{2} \text {. }
$$

To determine the constant $A$, express that equation (1) holds in the initial condition, we have

$$
\frac{1}{2 c^{2} \sin ^{2} \alpha}=\frac{1}{V^{2} c^{2} \sin ^{2} \alpha} \phi\left(\frac{1}{c}\right)+A .
$$

$A$ is now determined, and equation (1) can be written

$$
\left(\frac{d u}{d \theta}\right)^{2}=\psi(u)
$$

where $\psi$ is a well-defined functional expression.

Integrating this equation we have

$$
\theta-\alpha=\int \frac{d u}{\sqrt{\psi(u)}}
$$

where $\alpha$ is an arbitrary constant depending on the choice of the initial line.

\section{Examples of Integration.}

1. Let the acceleration $f$ be inversely proportional to the square of the distance.

We have $f=\mu u^{2}$ so that the equation is

$$
\frac{d^{2} u}{d \theta^{2}}+u=\frac{\mu}{h^{2}} .
$$

Write $l$ for $h^{2} / \mu$ and $v$ for $u-\frac{1}{l}$, then the equation is

$$
\frac{d^{2} v}{d \theta^{2}}+v=0
$$


and the complete primitive is (by Article 47)

$$
v=A \cos (\theta-a),
$$

where $A$ and $a$ are arbitrary constants. If we put now $e$ for $A l$ we have

$$
\frac{l}{r}=1+e \cos (\theta-a),
$$

so that the orbit is a conic of latus rectum $l,=h^{2} / \mu$, and of arbitrary eccentricity $e$ having the origin as one focus.

This investigation of the possible central orbits with acceleration $\mu / r^{2}$ may be taken to replace Newton's investigation, of which a version was given in Article 55.

2. To find all the orbits which can be described with a central acceleration varying inversely as the cube of the distance.

We have $f=\mu u^{3}$ so that

$$
\begin{gathered}
\frac{d^{2} u}{d \theta^{2}}+u=\frac{\mu}{h^{2}} u . \\
\frac{d^{2} u}{d \theta^{2}}+u\left(1-\frac{\mu}{h^{2}}\right)=0 .
\end{gathered}
$$

or

There are three cases according as $h^{2}>=$ or $<\mu$.

(1) When $h^{2}>\mu, 1-\frac{\mu}{h^{2}}$ is positive, put it equal to $n^{2}$.

Then all the possible orbits are of the form $u=A \cos (n \theta+a)$, they include a straight line for the case $n=1$.

(2) When $h^{2}=\mu$ we have $\frac{d^{2} u}{d \theta^{2}}=0$ so that $u=A \theta+B$ where $A$ and $B$ are arbitrary constants. If $A=0$ the orbit is a circle, otherwise it is a hyperbolic spiral, as we see by choosing the constant $B$ so as to write the above

$$
u=A(\theta-a) \text {. }
$$

(3) When $h^{2}<\mu, 1-\frac{\mu}{h^{2}}$ is negative, put it equal to $-n^{2}$.

Then all the possible orbits are of the form

$$
u=A \cosh (n \theta+a) \text { or } u=a e^{n \theta}+b e^{-n \theta} .
$$

Putting $a$ or $b$ equal to zero we have an equiangular spiral.

3. By integration of the equation

$$
\frac{d^{2} u}{d \theta^{2}}+u=\frac{\mu}{h^{2} u^{3}},
$$

prove that all the orbits that can be described with a central acceleration proportional to the distance are ellipses having the origin as centre.

4. If $f$ is any function of $r$ show that one of the possible orbits is a circle described about its centre. 
63. Apses. An apse is a point of a central orbit at which the tangent is at right angles to the radius vector.

There is a theory concerning the distribution of the apses when the central acceleration is a single-valued function of the distance, i.e. for the case where the acceleration depends only on the distance and is always the same at the same distance.

Let $A$ be an apse on a central orbit described about a point $O$, $f$ the central acceleration, supposed a single-valued function of distance, $T A T^{\prime}$ a line through $A$ at right angles to $A O$. Then a point starting from $A$ at right angles to $A O$ with a certain velocity would describe the orbit, suppose $V$ is this velocity.

If a point starts from $A$ with velocity $V$ in direction $A T$ or $A T^{\prime}$, and has the acceleration $f$ towards $O$, it describes the orbit; so that two points starting from $A$

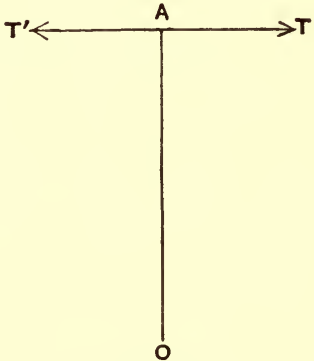

Fig. 39. in these two directions with the same velocity $V$ and the same acceleration $f$ describe the same orbit. Since the two points have the same acceleration at the same distance, the curves they describe are clearly equal and similar, and are symmetrically placed with respect to the line $A O$. Thus the orbit is symmetrical with respect to $A O$ in such a way that chords drawn across it at right angles to $A O$ are bisected by $A O$. The parts of the orbit on either side of $A O$ are therefore optical images in the line $A O$.

Now let the point start from $A$ in direction $A T$, and let $B$ be the next apse of the orbit that it passes through, also let $A^{\prime}$ be the next apse after $B$ that it passes through. Then the parts $A O B$, $B O A^{\prime}$ of the orbit are optical images in the line $O B$, and the angle $A O B$ is equal to the angle $A^{\prime} O B$, and the line $A O$ is equal to the line $A^{\prime} O$. In the same way the next apse the point passes through will be at a distance from $O$ equal to $O B$, and thus all the apses are at distances from $O$ equal

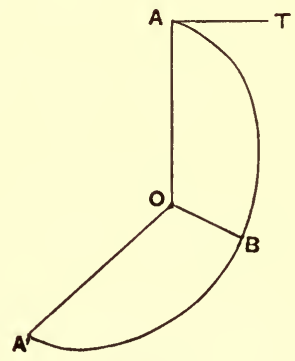

Fig. 40. 
[CHAP. IV.

to either $O A$ or $O B$, these are called the apsidal distances, and the angle between consecutive apses in the order in which the moving point passes through them is always equal to $A O B$, this is called the apsidal angle.

The theory just explained is usually stated in the form:There are two apsidal distances and one apsidal angle.

It is clear that the radius vector is a periodic function of the vectorial angle with period twice the apsidal angle.

\section{Examples.}

1. If the apsidal distances are equal the orbit is a circle described about its centre.

2. Write down the lengths of the apsidal distances and the apsidal angle for (1) elliptic motion about the centre, (2) elliptic motion about the focus, (3) all the orbits that can be described with a central acceleration varying inversely as the cube of the distance.

3. Explain the following paradox:-Four real normals can be drawn to an ellipse from a point within its evolute, and in Example 6 on p. 53 we found the central acceleration to any point requisite for the description of an ellipse; there are apparently in this case four apsidal distances and fotir apsidal angles.

65. Apsidal angle in nearly circular orbit. Suppose the central acceleration is $f(r)$ at distance $r$, then a circle of radius $c$ described about its centre is a possible orbit with $\frac{1}{2} h$ for rate of describing area provided

$$
\begin{aligned}
\frac{1}{c}\left(\frac{h}{c}\right)^{2} & =f(c), \\
h^{2} & =c^{3} f(c) .
\end{aligned}
$$

or

Let us suppose the point is at any instant near to the circle, and that it is describing an orbit about the origin with this $h$.

The equation of its path is

$$
\frac{d^{2} u}{d \theta^{2}}+u=\frac{f(r)}{h^{2} u^{2}} .
$$

At the instant in question $u$ is nearly equal to $\frac{1}{c}$; if it was precisely $\frac{1}{c}$, and if the point was moving at right angles to the radius vector, the point would describe the circle of radius $c$. We 
assume that it is always so near to the circle that the difference $u-\frac{1}{c}$ is so small that we may neglect its square, the investigation we give will determine under what condition this assumption is justifiable.

Put $u=\frac{1}{c}+x$ and write $\phi(u)$ for $f(r)$, and $a$ for $\frac{1}{c}$, so that

Then

$$
h^{2}=\phi(a) / a^{3} \text {. }
$$

$$
\begin{aligned}
\frac{d^{2} x}{d \theta^{2}}+x+a= & \frac{a^{3} \phi(a+x)}{\phi(a)} \frac{1}{(a+x)^{2}} \\
= & \frac{a}{\phi(a)}\left[\phi(a)+x \phi^{\prime}(a)+\ldots\right]\left[1-\frac{2 x}{a}+\frac{3 x^{2}}{a^{2}}-\ldots\right], \\
& \frac{d^{2} x}{d \theta^{2}}+x\left\{3-\frac{a \phi^{\prime}(a)}{\phi(a)}\right\}=0,
\end{aligned}
$$

or

neglecting $x^{2}$.

Now if $3-\frac{a \phi^{\prime}(a)}{\phi(a)}$ is positive we may put it equal to $\kappa^{2}$, and then the solution of the above equation is of the form

$$
x=A \cos (\kappa \theta+\alpha),
$$

so that the greatest value of $x$ is $A$, and by taking $A$ small enough $x$ will be as small as we please and the neglect of $x^{2}$ will be justified.

In this case $u$, and therefore $r$, will be a periodic function of $\theta$ with period $2 \pi / \sqrt{ }\left\{3-\frac{a \phi^{\prime}(a)}{\phi(a)}\right\}$, the orbit is nearly circular and its apsidal angle is $\pi / \sqrt{ } /\left\{3-\frac{a \phi^{\prime}(a)}{\phi a}\right\}$.

Again, if $3-\frac{a \phi^{\prime}(a)}{\phi(a)}$ is negative we may put it equal to $-\kappa^{2}$, and then the solution of the above equation is of the form

$$
x=A e^{\kappa \theta}+B \dot{e}^{-\kappa \theta},
$$

and it is clear that one of the terms increases in geometrical progression whether $\theta$ increases or diminishes, so that $x$ will very soon be so great that its square can no longer be neglected, 
whatever the number we agree to neglect may be. In this case the orbit tends to depart widely from the circular form.

In the former of these cases the circular motion is said to be stable, in the latter unstable.

\section{Examples.}

1. If $f(r)=r^{-n}$ or $\phi(u)=u^{n}$, prove that the possible circular orbits are stable when $n<3$ and unstable when $n>3$.

2. For $n=3$ prove that the circular orbit is unstable, and find the orbit described by a point moving with the $h$ for circular motion in a circle of radius $c$ through a point near the circle.

3. If $f(r)=r^{-4}$ prove that the curve described with the $h$ for circular motion in a circle of radius $c$ is either the circle $r=c$ or one of the curves

$$
\frac{r}{c}=\frac{\cosh \theta+1}{\cosh \theta-2}, \quad \frac{r}{c}=\frac{\cosh \theta-1}{\cosh \theta+2} .
$$

\section{EXAMPLES.}

1. Prove that the time in which it is possible to cross a road of breadth $c$, in a straight line, with the least uniform velocity, between a stream of omnibuses of breadth $b$, following at intervals $a$, moving with velocity $V$, is

$$
\frac{c}{V}\left(\frac{a}{b}+\frac{b}{a}\right)
$$

2. A particle moves in the plane of two rectangular axes so that the resolved parts of its velocity parallel to the axes are proportional to its distances from two other rectangular lines in the plane. Prove that its path is an equiangular spiral or a hyperbolic spiral.

3. Three horses in a field are at a certain moment at the angular points of an equilateral triangle. Their motion relatively to a person driving along a road is in direction round the sides of the triangle (in the same sense) and in magnitude equal to the velocity of the carriage. Show that the three horses are moving along concurrent lines.

4. A straight line $A B$ turns with uniform angular velocity about a point $A$, retaining a constant length, and a second straight line $B C$ of constant length moves so that $C$ is always in a certain straight line through $A$. Prove that the velocity of $C$ is proportional to the intercept which $B C$ makes on the line through $A$ at right angles to $A C$.

5. A point $P$ moves uniformly in a circle; $Q$ is a point in the same radius at double the distance from the centre; $P R$ is a tangent at $P$ equal to the arc described by $P$ from the beginning of the motion; show that the acceleration of $R$ is represented in direction and magnitude by $R Q$. 
6. A point $C$ describes a circle of radius $r$ with angular velocity $\omega^{\prime}$ about the centre $O$, and a point $P$ moves so that $C P$ is always equal to $a$ and turns with angular velocity $\omega$ in the plane of the circle described by $C$. Prove that the angular velocity of $O P$ is

$$
\frac{1}{2}\left\{\omega\left(R^{2}+a^{2}-r^{2}\right)+\omega^{\prime}\left(R^{2}-a^{2}+r^{2}\right)\right\} ! R^{2},
$$

where $R$ is the length of $O P$.

7. Two points move uniformly in straight lines. At any time the distance between them is $a, V$ is their relative velocity, $u$ and $v$ are the resolved parts of $V$ parallel and perpendicular to the direction of $a$. Show that, when they are nearest together, their distance is $a v / V$, and that the time until they arrive at this position is $a u / V^{2}$.

8. Two points $A$ and $B$ move with uniform velocities $u, v$ in two straight lines containing an angle $a$; prove that the time from the position in which $A B$ is least to that in which it is double its least value is

$$
\sqrt{ } 3 c v \sin \alpha /\left(u^{2}+v^{2}-2 u v \cos a\right),
$$

where $c$ is the distance $A B$ when $A$ crosses the path of $B$.

9. Prove that when a particle moves along a plane curve the velocity of the foot of the perpendicular from the origin on the direction of motion is $r v / \rho, v$ being the velocity of the particle, $r$ its distance from the origin, and $\rho$ the radius of curvature of its path.

10. Two particles start simultaneously from the same point and move along two straight lines, one with uniform velocity, the other with uniform acceleration. Prove that the line joining the particles at any time touches a fixed parabola.

11. A particle moves with uniform acceleration along the tangent to its path and describes $\operatorname{arcs} s_{1}, s_{2}, s_{3}$ in the $n_{1}, n_{2}, n_{3}$ th seconds after any particular instant; prove that

$$
s_{1}\left(n_{2}-n_{3}\right)+s_{2}\left(n_{3}-n_{1}\right)+s_{3}\left(n_{1}-n_{2}\right)=0 .
$$

12. Two boats start off to race with velocities $v, v^{\prime}$, and move with accelerations $f, f^{\prime}$, the result being a dead heat. Prove that the length of the course is

$$
2\left(v-v^{\prime}\right)\left(v f^{\prime}-v^{\prime} f\right) /\left(f-f^{\prime}\right)^{2} .
$$

13. A body is projected vertically upwards with velocity $v$; after a time $t$ a second body is projected vertically with velocity $v^{\prime}(<v)$. If they meet as soon as possible

$$
t=\left\{v-v^{\prime}+\sqrt{ }\left(v^{2}-v^{2}\right)\right\} / g .
$$

14. A particle moves in the axis $x$ with acceleration $\mu / x^{2}$ towards the origin, starting from rest at $x=a$. Show that the time of arriving at a distance $x$ is

$$
\sqrt{ }\left(\frac{a^{3}}{2 \mu}\right) \cos ^{-1}\left\{\sqrt{ } \frac{x}{a}+\sqrt{ }\left(\frac{x}{a}-\frac{x^{2}}{a^{2}}\right)\right\} .
$$


15. A particle moves in a straight line under a force tending to a fixed point in the line which, at distance $r$, is equal to $\mu / r^{2}-b^{2} \mu /\left(r^{3} a\right)$, and starts from rest at distance $a+\sqrt{ }\left(a^{2}-b^{2}\right)$. Prove that it will come to rest at distance $a-\sqrt{ }\left(a^{2}-b^{2}\right)$ in time $\pi a^{\frac{3}{2}} / \sqrt{ } \mu$, and will oscillate between these distances.

16. A particle moves along the axis $x$, starting from rest at $x=\alpha$; for an interval $t_{1}$ from the beginning of the motion the acceleration is $-\mu x$, for a subsequent interval $t_{2}$ the acceleration is $\mu x$, and at the end of this interval the particle is at the origin; prove that

$$
\tan \left(\sqrt{ } \mu t_{1}\right) \tanh \left(\sqrt{ } \mu t_{2}\right)=1 \text {. }
$$

17. A particle moves with an acceleration always directed to a point moving uniformly in a straight line, and the line joining the point to the position of the particle at any time is normal to the path of the particle; prove that the path is an ellipse.

18. A particle moves so that the angular velocity of the radius vector from a fixed point and the acceleration along it are both constant, prove that the acceleration at right angles to it varies as the sine of the angle between it and a fixed straight line.

19. A particle is moving in a parabola and at distance $r$ from the focus its velocity is $v$; show that its acceleration is compounded of $\frac{1}{4 r} \frac{d}{d r}\left(v^{2} r\right)$ parallel to the axis and $\frac{r}{4} \frac{d}{d r}\left(\frac{v^{2}}{r}\right)$ along the radius vector outwards.

20. A particle is describing an involute of a given curve; prove that its accelerations along the tangent and normal to its path are $\frac{d}{d t}(s \dot{\psi})$ and $s \dot{\psi}^{2}$ respectively, where $s$ is the arc of the given curve, $\psi$ the angle which the tangent makes with a fixed straight line.

21. Prove that, if the acceleration of a point describing a tortuous curve makes an angle $\psi$ with the principal normal, then $\tan \psi^{\prime}=\frac{\rho}{v} d v$.

In the case of a plane curve the condition that the acceleration is always directed to the same point is that the equation $\sin \psi+\frac{d}{d s} \frac{\rho \cos \psi}{1-\rho \frac{d \psi}{d s}}=0$ must be satisfied at every point.

22. The position of a point is given by the perpendiculars $\xi, \eta$ on two fixed lines containing an angle $a$ with each other, prove that the component velocities in the directions $\xi, \eta$ are

$$
(\dot{\xi}+\dot{\eta} \cos a) / \sin ^{2} a \text { and }(\dot{\eta}+\dot{\xi} \cos a) / \sin ^{2} a \text {. }
$$


23. Prove that the component accelerations of a moving particle are $X$ parallel to the axis of $x$, and $R$ perpendicular to the radius vector, where

and

$$
\begin{gathered}
X=\frac{\dot{r} \ddot{r}\left(r^{2}-x^{2}\right)-(\dot{r} x-\dot{x} r)^{2}}{x\left(r^{2}-x^{2}\right)}, \\
R=\frac{r}{x} \frac{(\ddot{r} \dot{r}-x \ddot{x})\left(r^{2}-x^{2}\right)-(\dot{r} x-\dot{x} r)^{2}}{\left(r^{2}-x^{2}\right)^{\frac{3}{2}}} .
\end{gathered}
$$

24. The position of a point is given by $x, y, r$, where $x, y, z, r$ have their usual signification relative to rectangular axes; show that the component accelerations are

$$
\dot{u}+\frac{u w}{r}, \dot{v}+\frac{v w}{r},(\dot{v}-u w x+v w y) / r^{2},
$$

$u, v, w$ being component velocities in the directions of $x, y, r$.

25. If $x, y$ are the coordinates of a point referred to rectangular axes turning with angular velocity $\omega$, prove that the accelerations in the directions of the axes are

$$
\ddot{x}-y \dot{\omega}-2 \dot{y} \omega-\omega^{2} x \text { and } \ddot{y}+x \dot{\omega}+2 \dot{x} \omega-\omega^{2} y \text {. }
$$

26. Prove that, if rectangular axes $O x, O y$ revolve with uniform angular velocity $\omega$, and the component velocities of a point $(x, y)$ parallel to the axes are $A / x$ and $B / y$, then the square of the distance of the point from the origin increases uniformly with the time.

27. The sides $C A, C B$ of a triangle are fixed in position and the side $A B$ is of constant length. The velocities of $A$ and $B$ along $C A$ and $C B$ are $u$ and $v$, the corresponding accelerations are $U, V$, and $\omega$ is the angular velocity of $A B$; prove that

$$
\begin{array}{rlrl}
u \cos A+v \cos B & =0, & & u \sin A \sim v \sin B=c \omega, \\
U \cos A+V \cos B=-c \omega^{2}, & U \sin A \sim V \sin B=c \dot{ }
\end{array}
$$

28. Two axes $O x, O y$ are inclined at an angle $a$ and rotate with angular velocity $\omega$ about $O$. Show that the component velocities are

$$
\dot{x}-\omega x \cot \alpha-\omega y \operatorname{cosec} a, \quad \dot{y}+\omega y \cot a+\omega x \operatorname{cosec} a \text {. }
$$

If the position of a point is defined by the perpendiculars $\xi, \eta$ drawn to the instantaneous positions of $O x, O y$, prove that the component velocities $u, v$ in these directions are given by

$$
\left.\begin{array}{l}
u=(\dot{\xi}+\dot{\eta} \cos a) / \sin ^{2} a+\omega \eta / \sin a \\
v=(\dot{\eta}+\dot{\xi} \cos a) / \sin ^{2} a-\omega \xi / \sin a
\end{array}\right\},
$$

and the component accelerations are

$$
\begin{aligned}
& \dot{u}-\omega u \cot a+\omega v \operatorname{cosec} a, \\
& \dot{v}+\omega v \cot a-\omega u \operatorname{cosec} a .
\end{aligned}
$$

29. Two fixed points are taken on a circle and any point on the circle is at distances $r_{1}, r_{2}$ from thern, the radii vectores $r_{1}, r_{2}$ containing an angle $a$; prove that the component velocities in the directions of $r_{1}$ and $r_{2}$ are $u_{1}, u_{2}$ where

$$
u_{1}+u_{2} \cos a=\dot{r}_{1}, \quad u_{2}+u_{1} \cos a=\dot{r}_{2},
$$

and that the component accelerations in the same directions are

$$
\dot{u}_{1}-u_{2} \dot{r}_{2} / r_{1} \text { and } \dot{u}_{2}-u_{1} \dot{r}_{1} / r_{2} \text {. }
$$


30. The radii vectores from two fixed points distant $c$ apart to the position of a particle are $r_{1}, r_{2}$, and the velocities in these directions are $u_{1}, u_{2}$; prove that the accelerations in the same directions are

$$
\dot{u}_{1}+\frac{1}{2} \frac{u_{1} u_{2}}{r_{1}^{2} r_{2}}\left(r_{1}^{2}-r_{2}^{2}+c^{2}\right) \text {, and } \dot{u}_{2}+\frac{1}{2} \frac{u_{1} u_{2}}{r_{1} r_{2}^{2}}\left(r_{2}^{2}-r_{1}^{2}+c^{2}\right) \text {. }
$$

31. The radii vectores from three fixed points to the position of a particle are $r_{1}, r_{2}, r_{3}$, and the velocities in these directions are $u_{1}, u_{2}, u_{3}$; prove that the accelerations in these directions are

$$
\dot{u}_{1}+u_{1}\left(\frac{u_{2}}{r_{2}}+\frac{u_{3}}{r_{3}}\right)-\frac{u_{1}}{r_{1}}\left(u_{2} \cos \theta_{12}+u_{3} \cos \theta_{13}\right),
$$

and the two similar expressions, in which $\theta_{23}, \theta_{31}, \theta_{12}$ are the angles contained by the directions of $\left(r_{2}, r_{3}\right),\left(r_{3}, r_{1}\right)$ and $\left(r_{1}, r_{2}\right)$.

32. Three tangents to the path of a particle whose acceleration is constant and always in the same direction form a triangle $A B C$; the velocities are $u$ along $B C, v$ along $C A, w$ along $A B$. Prove that

$$
\frac{B C}{u}+\frac{C A}{v}+\frac{A B}{w}=0 .
$$

33. Prove that the angular velocity of a projectile about the focus of its path varies inversely as its distance from the focus.

34. Prove that when a shot is projected from a gun at any angle of elevation, the shot as seen from the point of projection will appear to descend past a vertical target with uniform velocity.

35. A particle is projected from a platform with velocity $V$ and elevation $\beta$. On the platform is a telescope fixed at elevation $a$. The platform moves horizontally in the plane of the particle's motion, so as to keep the particle always in the centre of the field of view of the telescope. Show that the original velocity of the telescope must be $V \sin (a-\beta) \operatorname{cosec} a$, and its acceleration $g \cot \boldsymbol{a}$.

36. A cricketer in the long field has to judge a catch which he can secure with equal ease at any height from the ground between $k_{1}$ and $k_{2}$; show that he must estimate his position within a length

$$
R\left\{\sqrt{ }\left(1-\frac{k_{2}}{h}\right)-\sqrt{ }\left(1-\frac{k_{1}}{h}\right)\right\},
$$

where $R$ is the range on the horizontal and $h$ the greatest height the ball attains.

37. If $a$ is the requisite elevation of a cannon for a mark on a target at a horizontal range $R$, and if the axis of the trunnions of the cannon is inclined to the horizontal at an angle $\beta$, the shot will strike the target at a distance $R \tan a \sin \beta$ on one side, and $R \tan a(1-\cos \beta)$ below the mark aimed at.

38. A heavy particle is projected from a point $A$ with the least velocity of projection $V$ so as to pass through a point $B$; show that the velocity at $B$ is $V \tan \beta$, where $2 \beta$ is the angle which $A B$ makes with the vertical. 
39. A heavy particle is projected from a point $A$ so as to pass through another point $B$; show that the least velocity with which this is possible is $\sqrt{ }(2 g l) \cos \frac{1}{2} a$, and that the highest point of the path is at a height $l \cos ^{4} \frac{1}{2} a$ above $A$, where $A B=l$ and makes an angle $a$ with the vertical.

40. From a fort a buoy was observed at a depression $i$ below the horizon, and a gun was fired at an elevation $a$, but the shot was observed to strike the water at a depression $i^{\prime}$. Show that to strike the buoy the elevation should be $\theta$, where

$$
\frac{\cos \theta \sin (\theta+i)}{\cos a \sin \left(a+i^{\prime}\right)}=\frac{\cos ^{2} i \sin i^{\prime}}{\cos ^{2} i^{\prime} \sin i}
$$

41. A particle is to be projected so as just to pass through three equal rings, of diameter $d$, placed in parallel vertical planes at distances $a$ apart, with their highest points in a horizontal straight line at a height $h$ above the point of projection. Prove that the elevation must be $\tan ^{-1} \frac{2 \sqrt{ }(h d)}{a}$.

42. A particle is projected from a point on a horizontal table so as to pass through the four upper corners of a regular polygon of an even number of sides which stands in a vertical plane with one side on the table. If $R$ and $r$ are the radii of the circumscribed and inscribed circles of the polygon, prove that the range on the plane is $2 \sqrt{ }\left(R^{4}-5 R^{2} r^{2}+8 r^{4}\right) / R$ and that the greatest height of the particle above the polygon is $\frac{1}{2} R^{2}\left(R^{2}-r^{2}\right) /\left\{r\left(2 r^{2}-R^{2}\right)\right\}$.

43. A man standing at a distance $a$ from a net of height $h$ wishes to strike a ball over the net so that it may fall to the ground within a distance $b(<a)$ on the other side of the net. Prove that the square of the maximum horizontal velocity which should be imparted to the ball increases in harmonic progression as the height at which the ball is struck increases in arithmetic progression, so long as the height does not exceed $h(1+a / b)$; and that for heights $h$ and $2 h$ these maximum horizontal velocities are in the ratio $\sqrt{ }(a-b): \sqrt{ } a$.

44. A man travelling round a circle of radius $a$ with speed $v$ throws a ball from his hand at a height $h$ above the ground, with a relative velocity $V$, so that it alights at the centre of the circle. Show that the least possible value of $V$ is given by $V^{2}=v^{2}+g\left\{\sqrt{ }\left(a^{2}+h^{2}\right)-h\right\}$.

45. If $A$ and $B$ are two given points, and $C$ a given point on the line joining them, prove that, in the different trajectories possible under gravity between $A$ and $B$, the time of flight varies as $\sqrt{C D}$, where $D$ is the point in which the trajectory meets the vertical through $C$.

46. In any trajectory between two points $A, B$, the intercept on a vertical line through a point $C$ on $A B$ between $C$ and the trajectory is $\frac{1}{2} g t_{1} t_{2}$, where $t_{1}$ is the time from $A$ to the vertical through $C$, and $t_{2}$ the time from that vertical to $B$.

47. A particle is projected with elevation $a$ from a point on a plane of inclination $\beta$ in a vertical plane containing a line of greatest slope. Prove that, if the elevation of the point of the path most distant from the inclined plane is $\gamma$, then $\tan a+\tan \beta=2 \tan \gamma$. 
48. A particle is projected with velocity $V$ at any elevation, $\alpha$, greater than the least positive value of $\cos ^{-1} \frac{1}{3}$; show that its path will cut two planes through the point of projection at right angles; that, if their inclinations to the horizontal are $\beta$ and $\gamma$, then $\beta+\gamma=\alpha$; and that the time of passing from one to the other is

$$
\sin (\beta \sim \gamma) V / g \text {. }
$$

49. A heavy particle starts, with a velocity $u$ at an inclination $\gamma$ to the horizontal, from a point in a plane of inclination $a$, such that $2 \sqrt{ } 2 \tan a=\sqrt{ } 3 \tan \gamma$. Show that, for different positions of the vertical plane of motion, the greatest projection of the range on a horizontal line perpendicular to the line of greatest slope is

$$
\begin{aligned}
& 5 \sqrt{ } 5 \\
& 4 \sqrt{ } 6 \frac{u^{2}}{g} \sin 2 \gamma
\end{aligned}
$$

50. Two inclined planes intersect in a horizontal line and are inclined to the horizontal at angles $a$ and $\beta$. A particle is projected from a point in the former, distant $\alpha$ from the intersection, so as to strike the latter at right angles; show that the velocity of projection is

$$
\sqrt{ }(2 g a) \sin \beta / \sqrt{ }\{\sin a-\sin \beta \cos (a+\beta)\} .
$$

51. If the velocity $v$ at any point of the path of a projectile under gravity is suddenly diminished by one-half, prove that the focus of the new trajectory is nearer to the projectile by the distance $\frac{3}{8} v^{2} / g$, and that the curvature of the path is quadrupled.

52. Two heavy particles are projected from a point with equal velocities, their directions of projection being in the same vertical plane; $t, t^{\prime}$ are the times taken by the particles to reach the other point where their paths intersect, and $T, T^{\prime \prime}$ are the times taken to reach the highest points of the paths : show that $t T^{\prime}+t^{\prime} T^{\prime \prime}$ is independent of the directions of projection.

53. Three particles are projected from the same point in the same vertical plane with velocities $v_{1}, v_{2}, v_{3}$ at elevations $\beta_{1}, \beta_{2}, \beta_{3}$. Prove that the foci of their paths lie in a straight line if

$$
\frac{\sin 2\left(\beta_{2}-\beta_{3}\right)}{v_{1}^{2}}+\frac{\sin 2\left(\beta_{3}-\beta_{1}\right)}{v_{2}^{2}}+\frac{\sin 2\left(\beta_{1}-\beta_{2}\right)}{v_{3}^{2}}=0 .
$$

54. Three particles are projected from a given point in given directions. Prove that after an interval of time $t$ they form a triangle of area proportional to $t^{2}$. If the directions of projection of two of them are in the same vertical plane, show that the plane of the triangle will pass through the point of projection after a time $\frac{2}{g} \frac{u v \sin (\beta-\alpha)}{u \cos a-v \cos \beta}$, where $u, v$ are the initial velocities and $a, \beta$ the initial elevations of these two particles.

55. A number of particles are projected simultaneously from a point, and move under gravity; prove that, if tangents are drawn to their paths from any point in the vertical line through the point of projection, the points of contact will be simultaneous positions of the particles.

56. Particles are projected from the same point with equal velocities under gravity; prove that the vertices of their paths are on an ellipse. If 
they are all equally elastic and impinge on a vertical wall the vertices of their subsequent paths also lie on an ellipse.

57. A shot is fired with velocity $\sqrt{ }(2 g h)$ from the top of a mountain which is in the form of a hemisphere of radius $r$. Show that the furthest points of the mountain which can be reached by the shot are at a distance (measured in a straight line) $r-\sqrt{ }\left(r^{2}-4 r h\right)$ from the point of projection.

58. A gun is placed on a fort situated on a hill side of inclination $a$ to the horizon. Show that the area commanded by it is $4 \pi h(h+d \cos a) \sec ^{3} a$, where $\sqrt{ }(2 g h)$ is the muzzle-velocity of the shot, and $d$ the perpendicular distance of the gun from the hill side.

59. A gun is mounted at a given spot so as to command the horizontal plane on which it stands. Its mounting is such that the direction, in which it is pointed, must lie in a given plane inclined to the horizontal at an angle $a$. Prove that the part of the plane commanded is an ellipse of eccentricity $\sin a$, the muzzle-velocity being constant.

60. At a horizontal distance $a$ from a gun there is a wall of height $h\left(>a-g a^{2} / v^{2}\right)$, and a shot is fired with velocity $v$ in the vertical plane at right angles to that of the wall. Prove that the distance commanded by the gun on the other side of the wall is

$$
\frac{2 h a}{g\left(a^{2}+h^{2}\right)} \sqrt{ }\left(v^{4}-a^{2} g^{2}-2 h v^{2} a\right)
$$

provided this expression is real.

61. It is required to throw a ball from a given point with a given velocity $V$ so as to strike a vertical wall above a horizontal line on the wall. It is found that when projected in the vertical plane at right angles to the wall the elevation must lie between $\theta_{1}$ and $\theta_{2}$. Prove that the points on the wall towards which the ball may be directly projected lie within a circle of radius

$$
V^{2} \sin \left(\theta_{1}-\theta_{2}\right) /\left\{g \sin \left(\theta_{1}+\theta_{2}\right)\right\} \text {. }
$$

62. Water issues from a fountain jet in such a manner that the velocity of emission in a direction making an angle $\theta$ with the vertical is $\sqrt{ }(g a \operatorname{cosec} \theta)$, the jet being at a height $h$ above the centre of a circular basin. Prove that, if all the water is to fall into the basin, its radius must not be less than

$$
\left[2 a\left\{a+\sqrt{ }\left(a^{2}+h^{2}\right)\right\}\right]^{\frac{1}{2}} \text {. }
$$

63. Prove that if the sole effect of a wind on the motion of a projectile is to produce an acceleration $f$ in a horizontal direction, the locus of points in a horizontal plane which can just be reached with a given velocity $v$ of projection is an ellipse of eccentricity $f / \sqrt{ }\left(f^{2}+g^{2}\right)$ and area $\pi v^{4} \sqrt{ }\left(f^{2}+g^{2}\right) / g^{3}$.

64. A particle is projected so as to enter in the direction of its length a smooth straight tube of small bore fixed at an angle of $45^{\circ}$ to the horizon, and to pass out again at the other end of the tube. Show that the latera recta of its paths before entering and after leaving the tube differ by $\sqrt{ } 2$ times the length of the tube. 
65. Prove that if two heavy particles projected in the same vertical plane at the same instant from two given points with the same velocity meet, the sum of the inclinations of the directions of projection must be constant, and that for a constant velocity of projection and different directions of projection the locus of the point of meeting is a parabola.

66. A man standing on the edge of a cliff throws a stone with given velocity $u$ at a given inclination to the horizon, in a plane perpendicular to the edge of the cliff; after an interval $\tau$ he throws another stone from the same spot with given velocity $v$ at an angle $\frac{1}{2} \pi+\theta$ with the line of discharge of the first stone and in the same plane. Find $\tau$ so that the stones may strike each other, and show that the maximum value of $\tau$ for different values of $\theta$ is $2 v^{2} / w g$, and occurs when $\sin \theta=v / u, w$ being the vertical component of $v$.

67. Two particles describe the same ellipse in the same time as a central orbit about the centre. Prove that the point of intersection of their directions of motion describes a concentric ellipse as a central orbit about the centre.

68. Two particles are projected in parallel directions from two points in a straight line passing through a point $O$, with velocities proportional to their distances from $O$, and each particle has an acceleration to $O$ equal to $\mu$ (distance). Prove that all the tangents to the path of the inner cut off, from that of the outer, arcs described in equal times.

69. Two particles describe concentric and coaxial ellipses about the common centre with accelerations which are equal at equal distances, the sum of the axes of one ellipse being equal to the difference of the axes of the other; and the particles start in opposite directions from corresponding extremities of the transverse axes. Prove that the line joining them is of constant length, and turns with uniform angular velocity.

70. From all points on the circumference of a circle, to the centre of which tends a force varying as the distance, particles are projected towards a point on the circumference with velocities varying as their distances from the point. Prove that at any instant the particles lie on a circle.

71. Particles are projected from points on a sphere of radius $a$ with velocity $\sqrt{ }(g b)$ and move with an acceleration to the centre equal to $g r / a$ at distance $r$. Prove that the part of the surface on which they fall is the smaller of the two segments into which the sphere is divided by a small circle of radius $b$.

72. A body is describing an ellipse of eccentricity $\frac{1}{2}$ under a force to the centre, and when it is at one end of the latus rectum the centre of force is suddenly transferred to the foot of the corresponding directrix. Prove that the times which elapse in the two possible cases before the body reaches the major axis are to one another as $2: 1$. 
73. A particle $P$ describes a rectangular hyperbola with an acceleration ${ }_{\mu} C P$ from the centre $C$; a point $Y$ is taken in $C P$ so that $C P . C Y=a^{2}$; prove that the rate at which $P$ and $Y$ separate is

$$
\sqrt{ } \mu C P\left(1-\frac{a^{2}}{C P^{2}}\right)^{\frac{1}{2}}\left(1+\frac{a^{2}}{C P^{2}}\right)^{\frac{8}{2}}
$$

where $2 \alpha$ is the transverse axis.

74. If the acceleration of a particle is directed to a point $S$ and varies inversely as the square of the distance, prove that there are two directions in which it can be projected from a point $P$ so as to pass through a point $Q$, and that the velocity of arrival at $Q$ is the same for both. Prove also that the angle between one of the directions of projection and $P Q$ is the same as the angle between the other and $P S$.

75. A particle describes an elliptic orbit about a focus; prove that the angular velocity at any point about the other focus varies inversely as the square of the normal at the point.

76. A particle describes any conic about a focus; prove that the total velocity acquired in moving from one point to another is in the direction of the line joining the focus to the pole of the chord joining the points.

77. Prove that the periodic time of a particle projected with velocity $V$ from a point distant $r$ from the origin, and having an acceleration $\mu / r^{2}$ to the origin, is

$$
\frac{2 \pi}{\sqrt{ } \mu}\left(\frac{2}{r}-\frac{V^{2}}{\mu}\right)^{-\frac{3}{2}}
$$

78. Prove that the greatest radial velocity of a particle describing an ellipse about a focus is

$$
2 \pi a e\left(1-e^{2}\right)^{-\frac{1}{2}} / T
$$

where $2 a$ is the major axis, $e$ the eccentricity, and $T$ the periodic time.

79. A particle describes an ellipse as a central orbit about a focus, and a second particle describes the same ellipse in the same time with uniform angular velocity about the same focus. The particles start together from the farther apse. Prove that the angle the line joining the particles subtends at the focus is greatest when the angle described by the first particle is $\cos ^{-1}\left\{1-\left(1-e^{2}\right)^{\frac{3}{4}}\right\} / e, e$ being the eccentricity.

80. A particle describes an ellipse of axes $2 a, 2 b$ about a focus. Prove that the average distance of the particle from the focus for an indefinitely great number of instants corresponding to equal differences of vectorial angle is $b$, and that the average distance of the particle from the focus for an indefinitely great number of equidistant instants of time is $a\left(1+\frac{1}{2} e^{2}\right)$, where $e$ is the eccentricity.

81. When a parabola is described as a central orbit about a focus, prove that the direction of motion at any point, $P$, meets the directrix in a point, $Q$, whose velocity is inversely proportional to the abscissa of $P$. 
82. When an hyperbola is described as a central orbit about a focus prove that the rate at which areas are described about the centre is inversely proportional to the distance from the focus.

83. Prove that the central orbit described with acceleration $\mu /{\text { (distance })^{2}}^{2}$ by a particle projected with velocity $V$ from a point where the distance is $R$ is a rectangular hyperbola if the angle of projection is

$$
\operatorname{cosec}^{-1}\left\{V \sqrt{ }\left(V^{2} R^{2}-2 \mu R\right) / \mu\right\} \text {. }
$$

84. A particle describes an ellipse about a focus, and at any point of the orbit the acceleration begins to be directed to the centre and to vary as the distance, its magnitude being unaltered. Prove that the new orbit is an ellipse having double contact with the old orbit and entirely within it.

85. A particle describing an ellipse about a focus has its velocity suddenly doubled and turned through a right angle, and proceeds to describe a parabola, the law of the acceleration being unaltered; the axis of the parabola is at right angles to the axis of the ellipse. Prove that the eccentricity of the ellipse is $\frac{1}{2} \sqrt{ } 2$.

86. A particle describes an ellipse about a focus $S$ starting from one end of the major axis, and arrives at the end of the minor axis in time $T$. At the end of this time the centre of force is transferred without altering its intensity to the other focus $H$, and the particle moves for a second interval $T$ under the action of the force to $H$. Find the position of the particle, and show that if the centre of force were transferred back to $S$ after the second interval $T$ the particle would begin to describe an ellipse of eccentricity $\left(3 e-e^{2}\right) /(1+e)$, where $e$ is the eccentricity of the first ellipse.

87. A body is revolving in an ellipse of eccentricity $\frac{1}{2}$, under the action of a force to the focus $S$, and when it is at a distance $S P$ from $S$ equal to the latus rectum, a blow is given to it perpendicular to $S P$ such that its new direction is perpendicular to the major axis. Show that the dimensions of the orbit are unaltered, but the major axis is turned through an angle $S P H$, where $H$ is the second focus.

88. A body is moving in a given hyperbola under the action of a force tending to a focus $S$; when it arrives at any point $P$, the force suddenly becomes repulsive: find the position and magnitude of the axes of the new orbit, and show that the difference of the squares of the eccentricities of the new and old orbits is proportional to $S P$.

89. Find, when possible, the point in an elliptic orbit about a focus at which if the centre of force were transferred to the empty focus the orbit would be a parabola. Prove that there is no such point unless the eccentricity is greater than $\sqrt{ } 5-2$.

90. A particle is describing a circle under a force to a point $S$ on the circumference. At a point $P$ on the circle the force changes to the inverse square, its magnitude being unaltered, and the particle proceeds to describe an ellipse. On $P S$ produced a point $Q$ is taken so that $S Q={ }_{3}^{4} S P, Q T$ is drawn perpendicular to the tangent at $P$, and $S Q T R$ is a parallelogram. Show that the middle point of $T R$ is the centre of the ellipse. 
91. A particle is describing a circle of radius $c$ as a central orbit about a point distant $c / \sqrt{ } 3$ from the centre. When the line joining this point to the particle subtends a right angle at the centre of the circle the law of the acceleration suddenly changes, and thereafter it varies inversely as the square of the distance, but the magnitude of the acceleration does not change discontinuously. Prove that the major axis of the new elliptic orbit is $16 c / 5 \sqrt{ } 3$ and that its eccentricity is $\sqrt{ } 19 / 8$.

92. Prove that the focal radius and vectorial angle of a particle describing an ellipse of small eccentricity $e$ at time $t$ after passing the nearer apse are approximately given by the equations

$$
\begin{aligned}
& r=a\left(1-e \cos n t+\frac{1}{2} e^{2}-\frac{1}{2} e^{2} \cos 2 n t\right), \\
& \theta=n t+2 e \sin n t+{ }_{4}^{5} e^{2} \sin 2 n t,
\end{aligned}
$$

where $2 \alpha$ is the major axis and $2 \pi / n$ is the periodic time.

Prove also that if $e^{2}$ is neglected the angular velocity about the other focus is constant.

93. Prove that the time of describing the smaller part of an elliptic orbit about a focus cut off by a focal chord is $\sqrt{ }\left(\alpha^{3} / \mu\right)(2 \phi-\sin 2 \phi)$, where $2 \alpha \sin \phi$ is the chord of the auxiliary circle that corresponds to the focal chord, and $2 a$ is the major axis of the orbit.

94. If the perihelion distance of a comet is $\frac{1}{n}$ th of the radius of the earth's orbit, supposed circular, show that the comet will remain within the earth's orbit for

$$
\frac{2}{3 \pi}(1+1 / n) \sqrt{ }\left(\frac{1}{2}-1 / n\right) \text { years, }
$$

the comet's orbit being parabolic.

95. If the parabolic orbits of two comets intersect the orbit of the earth, supposed circular, in the same two points, and if $t_{1}, t_{2}$ are the times in which the comets move from one of these points to the other, prove that

$$
\left(t_{1}+t_{2}\right)^{\frac{3}{2}}+\left(t_{1}-t_{2}\right)^{\frac{3}{2}}=\left(\frac{4}{3 \pi} T\right)^{\frac{3}{2}} \text {, where } T \text { is a year. }
$$

96. The times of passage of a particle between two points distant $d$ apart in the two parabolic orbits that can be described about the same focus with the same law of acceleration are $T_{1}, T_{2}$, and the distances of the points from the focus are $r_{1}, r_{2}$. Prove that

$$
\left(T_{1}-T_{2}\right)^{2}:\left(T_{1}+T_{2}\right)^{2}=\left(r_{1}+r_{2}-d\right)^{3}:\left(r_{1}+r_{2}+d\right)^{3} .
$$

97. Three focal radii $S P, S Q, S R$ of an elliptic orbit about a focus $S$ are determined, and the angles between them. Show that the ellipticity may be found from the equation $b \Delta=a \Delta^{\prime}$, where $\Delta$ is the area of the triangle $P Q R$, and $\Delta^{\prime}$ is the area of a triangle whose sides are

$$
2 \sqrt{ }(S Q . S R) \sin \frac{1}{2} Q S R,
$$

and two similar expressions. 
98. A particle describes a circle as a central orbit about a point $O$. Prove that the sum of the velocities at any two points collinear with $O$ is constant.

99. A circle is described as a central orbit about a point $O$ on the circumference; if the tangent to the circle meets the diameter through $O$ produced in $R$, prove that the velocity of $R$ is proportional to

$$
\sqrt{ }\left(4 a^{2}-r^{2}\right) / r\left(2 a^{2}-r^{2}\right)^{2}
$$

where $a$ is the radius of the circle.

100. A particle is projected from $A$ with velocity $\sqrt{ }\left(\frac{1}{2} \mu\right) / O A^{2}$ and moves with an acceleration $\mu /(\text { distance })^{5}$ directed to $O$, the direction of projection making an angle $a$ with $O A$. Prove that the particle will arrive at $O$ after a time

$$
\frac{O A^{3}}{\sqrt{ }(2 \mu)} \frac{a-\sin a \cos a}{\sin ^{3} a} .
$$

101. A particle describes a circle as a central orbit about an excentric point. In any diameter $A B$ of the circle points $S, S^{\prime}$ are taken such that $S A: S^{\prime} A=S B: S^{\prime} B=e$. Prove that, if $V$ and $V^{\prime}$ are the velocities of the particle at any point on the portion of the circle concave to $S^{\prime}$, when the circle is described about $S$ and $S^{\prime}$ respectively, and if $V=V^{\prime}$ at $A$, then $1 / V-e / V^{\prime}$ is constant.

102. Prove that the acceleration with which a particle $P$ can describe a circle as a central orbit about a point $S$ is inversely proportional to $S P^{2} \cdot P P^{\prime 3}$, where $P P^{\prime}$ is the chord through $S$.

If points are taken on the orbit such that the squares of their distances from $S$ are in arithmetic progression, the corresponding velocities are in harmonic progression.

103. Prove that the accelerations with which the same circle can be described as a central orbit about two points $R, S$ in its plane in the same periodic time are in the ratio $S G^{3}: R P^{2} . S P, P$ being any point on the circle and $S G$ being a straight line drawn from $S$ parallel to $R P$ to meet the tangent at $P$ in $G$.

104. A particle is moving with uniform velocity $\sqrt{ }\left(\frac{1}{2} \mu\right) / c^{2}$ in a given straight line, and when it is at a certain point it begins to have an acceleration $\mu r /\left(r^{2}+b^{2}\right)^{3}$ towards a point $S$ distant $a$ from the line. Prove that, if $c^{2}>\left(a^{2}+b^{2}\right)$, there are two positions of the point for which the subsequent orbit is a circle, and that the two circles cut at an angle $\omega$ given by

$$
c^{2} \sin \frac{1}{2} \omega=2 a \sqrt{ }\left(c^{2}-a^{2}-b^{2}\right) .
$$

105. A particle describes an ellipse of latus rectum $2 l$ about the point $X$ where the axis meets a directrix. Prove that the acceleration is $\ell^{2} X P /\left(l S M^{3}\right)$, where $S$ is the focus corresponding to $X$, and $M$ is the foot of the perpendicular from $P$ on the major axis. 
106. An ellipse is described as a central orbit about a point $O$ on the major axis ; prove that the acceleration at $P$ varies as $P L^{3} / O P^{2}$ where $L$ is the point of intersection of $O P$ and the diameter conjugate to that passing through $P$.

107. When a particle describes an ellipse as a central orbit about any point in its plane the sum of the reciprocals of the velocities at the extremities of any diameter is independent of the position of the point and varies as the periodic time.

108. Any conic whose centre is $C$ is described as a central orbit about any point $R$. Prove that the acceleration at $P$ is proportional to $C G^{3} / R P^{2}$, $C G$ being drawn parallel to $R P$ to meet the tangent at $P$ in $G$.

109. A particle $P$ describes a parabola as a central orbit about a point $O$ on the axis ; prove that the acceleration is $\mu\{1 / O P+1 / O p\}^{-3} . O P^{-2}, p$ being the other point of intersection of $O P$ with the curve; prove also that the time of passing from one extremity of the ordinate through $O$ to the other is $\frac{8}{3} \sqrt{ }(2 / \mu)$.

110. A particle describes a parabola, latus rectum $4 a$, with an acceleration tending to a point on the axis distant $c$ from the vertex. Prove that the time of moving from the vertex to a point distant $y$ from the axis is proportional to $y+\frac{1}{12} y^{3} / a c$.

111. Prove that any conic can be described by a particle with an acceleration always at right angles to the transverse axis and varying inversely as the cube of the distance from it.

If a particle is describing an ellipse in this manner, and at one end of one of the equiconjugate diameters the acceleration is suddenly changed in sense without being altered in magnitude, prove that the particle will proceed to describe an hyperbola having the axes of the ellipse as asymptotes.

112. A particle describes an ellipse with acceleration parallel to a diameter. Show that the acceleration must vary inversely as the cube of the ordinate of the conjugate diameter.

113. A particle moves with an acceleration $\mu y^{-3}$ towards the axis $x$, starting from the point $(0, k)$ with velocities $U, V$ parallel to the axes of $x, y$. Prove that it will not strike the axis $x$ unless $\mu>V^{2} k^{2}$, and that, in this case, it strikes it at a distance $U k^{2} /(\sqrt{ } \mu-V k)$ from the origin.

114. A particle describes a cycloid with an acceleration always perpendicular to the base, prove that its magnitude is proportional to the inverse fourth power of the radius of curvature at each point of the curve.

115. Show that a particle can describe an equiangular spiral of angle $a$ and pole $S$ with an acceleration $\mu / S P^{n}$ whose direction makes a constant angle $\beta$ with the tangent to the spiral provided

$$
\tan a=\frac{1}{2}(n-1) \tan \beta \text {. }
$$


116. Prove that the velocity and acceleration of a particle describing an equiangular spiral as a central orbit about the pole are at any instant the same as those of a particle describing a certain ellipse with centre at the pole as a central orbit about the centre, the axes of the ellipse being proportional to the distance from the pole.

117. If an equiangular spiral whose pole is $O$ is described as a central orbit about any point $S$, prove that the acceleration at $P$ is inversely proportional to $O P . S P^{2} \cdot \sin ^{3} \phi$, where $\phi$ is the angle the radius vector $S P$ makes with the tangent at $P$.

118. Prove that the acceleration towards the centre of the fixed circle with which a particle can describe an epicycloid is proportional to $r / p^{4}$, where $r$ is the radius vector and $p$ the perpendicular from the centre to the tangent.

119. The curve $r=a+b \theta$ is described as a central orbit about the origin with initial distance $a$ and initial velocity $V$ in a direction making an angle $\frac{1}{4} \pi$ with the initial radius vector. Find the formula for the acceleration.

120. Prove that the acceleration with which the curve $r=\alpha \sin n \theta$ can be described as a central orbit about the origin is proportional to

$$
2 n^{2} a^{2} r^{-5}-\left(n^{2}-1\right) r^{-3} \text {. }
$$

121. Prove that the curve $r=a\left(1+\frac{1}{2} \sqrt{ } 6 \cos \theta\right)$ is a central orbit about the origin for acceleration inversely proportional to the fourth power of the distance.

122. If the curve $r^{2 n}+b^{2 n}+2 \alpha^{n} r^{n} \cos n \theta=0$ is described as a central orbit about the origin with areal velocity $\frac{1}{2} h$, prove that the central acceleration is

$$
2 h^{2}\left(b^{2 n}-a^{2 n}\right) \frac{d}{d r}\left\{r^{2 n-2} /\left(r^{2 n}-b^{2 n}\right)^{2}\right\} .
$$

123. If any curve is described as a central orbit about a point $O$ the velocity of the foot of the perpendicular from $O$ on the tangent varies inversely as the chord of curvature through $O$.

124. A particle is describing a central orbit about a point $S$, and $h$ is twice the rate at which the radius vector describes areas. Another particle moves so that at any instant its distance $(r)$ from $S$ is equal to that of the first particle, and the angular velocity of its radius vector is less than that of the first particle in the ratio $\sin a: 1$. Show that the second particle has an acceleration to $S$ less than that of the first particle by $h^{2} \cos ^{2} a / r^{3}$.

125. A series of particles are describing the same curve as a central orbit about a point $O$ with an acceleration whose tangential component is $h^{2} / p^{2} \phi^{\prime}(p)$. Prove that if the line density at any time is constant and $=\rho_{0}$, the line density $\rho$ at any subsequent time $t$ is given by

$$
\phi(p)+h t=\phi\left(p \rho_{0} / \rho\right),
$$

$\frac{1}{2} h$ being the rate of description of areas about $O$, and $p$ the perpendicular from $O$ on the tangent. 
126. If inverse curves with respect to $O$ can be described as central orbits about $O$ with accelerations $f, f^{\prime}$, prove that

$$
\frac{r^{3} f}{h^{2}}+\frac{r^{\prime 3} f^{\prime}}{h^{\prime 2}}=\frac{2}{\sin ^{2} \phi},
$$

where $h$ and $h^{\prime}$ are constants, $r$ and $r^{\prime}$ are corresponding radii vectores, and $\phi$ is the angle $r$ or $r^{\prime}$ makes with the tangent.

127. If $f$ is the acceleration and $\frac{1}{2} h$ the areal velocity in a central orbit about a point $O$, prove that the angular acceleration $a$ about $O$ satisfies the equation

$$
\frac{d a^{2}}{d u}-6 \frac{a^{2}}{u}=8 h^{2} u^{4}\left(f-h^{2} u^{3}\right)
$$

where $u$ is the reciprocal of the distance from 0 .

128. If the central acceleration is $\mu\left[2\left(a^{2}+b^{2}\right) u^{5}-3 a^{2} b^{2} u^{7}\right]$, the initial distance $a$, and the initial velocity $\sqrt{ } \mu / \alpha$ at right angles to the radius vector, determine the orbit.

129. A particle describes a central orbit about the origin with acceleration $\mu u^{3}\left(n^{2}+1-2 n^{2} a^{2} u^{2}\right)$, starting from an apse at distance $a$ with the velocity from infinity; prove that it describes the curve

$$
r=\alpha \cosh n \theta \text {. }
$$

130. A particle describes a central orbit with acceleration

$$
\mu\left[4(\alpha / r)^{9}+(a / r)^{3}-32(r / a)^{3}\right]
$$

starting from a point for which $r=a$ with velocity $3 \sqrt{ }(2 \alpha \mu)$ in a direction making an angle $\frac{1}{4} \pi$ with the radius vector. Prove that the path is

$$
r^{3}=\frac{1}{2} a^{3} \operatorname{coth} 2 \theta \text {. }
$$

131. If the central acceleration is $2 \mu\left(u^{3}-\alpha^{2} u^{5}\right)$ and the particle is projected from an apse at distance $\alpha$ with velocity $\sqrt{ } \mu / \alpha$, the time until the distance is $r$ is

$$
\frac{1}{2 \sqrt{ } \mu}\left[a^{2} \log \frac{r+\sqrt{ }\left(r^{2}-a^{2}\right)}{a}+r \sqrt{ }\left(r^{2}-a^{2}\right)\right] .
$$

132. A particle moving with a central acceleration $\mu\left(u^{4}+2 \alpha u^{5}\right)$ starts from a point at distance $\alpha$ from the origin in a direction making an angle $\left(\pi-\cot ^{-1} 2\right)$ with the radius vector and with the velocity from infinity. Show that the equation of the path is $r=\alpha(1-2 \sin \theta)$.

133. A particle describes a central orbit with acceleration $\mu r /\left(r^{2}-a^{2}\right)^{2}$ towards the origin being projected from an apse at distance $3 \alpha$ with velocity $\sqrt{ }(2 \mu) / 4 a$. Show that it describes the cardioid $r^{2}=a^{2}+\frac{8}{9} p^{2}$. Prove also that if the equations of the cardioid are

$$
\begin{aligned}
& x=2 \alpha \cos \phi-\alpha \cos 2 \phi \\
& y=2 \alpha \sin \phi-\alpha \sin 2 \phi
\end{aligned}
$$

the time between two points $\phi_{1}$ and $\phi_{2}$ is the same as in an elliptic orbit about a focus between two points whose eccentric angles are $\phi_{1}$ and $\phi_{2}$, the periodic times being the same. 
134. A particle moves in a nearly circular orbit with an acceleration $\mu+\nu(r-a)$, $a$ being the mean radius; show that the apsidal angle is $\pi \omega / \sqrt{ }\left(3 \omega^{2}+\nu\right)$, where $\omega$ is the mean angular velocity.

135. If the central acceleration is $\mu u^{5}$ the velocities at the two apsidal distances satisfy the relation $v_{1}^{2}+v_{2}^{2}=2 h^{4} / \mu$.

136. A particle describes a central orbit with acceleration $\mu\left(\gamma^{-5}-\frac{1}{8} a^{2} r^{-7}\right)$ starting from a point where $r=a$ with velocity $\frac{5}{4} \sqrt{ }(2 \mu) / \alpha^{2}$ at an inclination $\sin ^{-1} \frac{4}{5}$ to the radius vector. Prove that its path is

$$
1-\theta=a \sqrt{ } 3 / \sqrt{ }\left(4 r^{2}-a^{2}\right) .
$$

137. A particle describes a central orbit with acceleration $\mu /(r-a)^{2}$ towards the origin, starting with the velocity from infinity from a point at any distance $c$ between $a$ and $2 a$ at an angle $2 \cos ^{-1} \sqrt{ }(a / c)$. Prove that the path described is given by the equation

$$
\frac{1}{2} \theta=\tanh ^{-1} \sqrt{ }\{(r-a) / a\}-\tan ^{-1} \sqrt{ }\{(r-a) / a\} .
$$

138. A particle moving with a central acceleration $4 \kappa^{2}\left(2 a^{-3}-3 r a^{-4}-2 r^{3} a^{-6}\right)$ starts from a point distant $\frac{4}{3} a$ from the origin in direction making an angle $\tan ^{-1} 27 / 125$ with the radius vector with such velocity that the rate of description of areas is $k$. Show that the equation of the orbit is

$$
1+\frac{r^{2}}{a^{2}}=\operatorname{coth}^{2}\left(\frac{a}{\sqrt{ }\left(a^{2}+r^{2}\right)}+\theta+\tanh ^{-1} \frac{3}{5}-\frac{3}{5}\right) .
$$




\section{THE PRINCIPLES OF DYNAMICS.}

\section{CHAPTER V.}

\section{MASS AND FORCE.}

67. We have seen in the last Chapter how a description of the motion of a point can be furnished by a statement of its acceleration, we have now to see how this knowledge can be made available for a complete description of the motions of bodies. To this end it is necessary to form a conception of bodies and of the nature of their mutual actions. The elements that enter into this conception have all been suggested by experience, but the conception is much more precise in detail than the observations that suggested it. It will therefore be best in the first place to regard the conception as purely ideal, and afterwards to explain how it is applied to obtain a description of the motions of natural bodies.

The first point to which attention must be directed is the distinction between homogeneity and heterogeneity. If we divide a body into a number of parts of exactly equal size and of like shape, then it may happen that we are unable to distinguish the parts one from another by any difference of quality. The parts may be all of the same weight, as tested by a balance, they may all be equally hard, and so on. When this is the case the body is said to be homogeneous, otherwise it is said to be heterogeneous. Now experience shows that a heterogeneous body can be divided into parts each more nearly homogeneous than the body, and this suggests that a heterogeneous body may be regarded as made up of homogeneous parts, each part being itself a body.

The first step in forming our ideal conception is to acquire the notion of a material figure, suggested as a representation of a homogeneous body or of a homogeneous part of a body. 
68. Material Figure. Mean Acceleration. Imagine that all the points which are within a certain volume at time $t$ define particles (Art. 41) which are moving relatively to a certain frame. The figure constructed with points in the positions of these particles at any subsequent time is called a material figure, ${ }^{*}$ provided the accelerations of the points are subject to certain rules to be expressed in the two following Articles.

Whatever these accelerations may be we can define, as the mean acceleration of the figure at time $t$, a vector, localised in a line through the centroid of the figure, whose resolved part in any direction is equal to the average acceleration per unit volume of the points of the figure in that direction.

Thus if $x, y, z$ are the coordinates of one point of the figure at time $t, d v$ an infinitesimal volume containing the point, and $v$ the volume of the figure at time $t$, the resolved parts of the mean acceleration of the figure parallel to the axes are

$$
\frac{1}{v} \int \ddot{x} d v, \quad \frac{1}{v} \int \ddot{y} d v, \quad \frac{1}{v} \int \ddot{z} d v,
$$

the integrations extending through the volume.

It is clear that the mean acceleration at any instant is the acceleration of the centroid of the figure at that instant.

The rules, above mentioned, to which the accelerations of the points of the figure are subjected, are rules governing the magnitudes, directions, and senses of the mean accelerations of two or more material figures.

69. Mutual Action. Imagine that the positions (or velocities) of two material figures affect their mean accelerations relative to the same frame; in other words that there is some relation between these accelerations and the positions (or velocities) of points in the two figures. Then we say that one of them acts on the other and produces acceleration in it.

Imagine that two material figures act on each other so that each produces in the other a mean acceleration relative to a certain frame. Let $f, f^{\prime}$ be the magnitudes of these accelerations.

Now we subject these mean accelerations to a rule, viz. they are localised in the same line, the line joining the centroids, and have opposite senses.

* Cf. Maggi, Teoria matematica del movimento dei corpi. Milan, 1896. 
70. Mass-ratio. Let $v$ and $v^{\prime}$ be the volumes of two material figures, $f$ and $f^{\prime}$ the magnitudes of the mean accelerations produced by $v^{\prime}$ in $v$ and by $v$ in $v^{\prime}$; then the ratio $f^{\prime}: f$ is defined to be the mass-ratio of the two figures $v$ and $v^{\prime}$, and $f: f^{\prime}$ is the mass-ratio of the two figures $v^{\prime}$ and $v$. We make it a rule that the mass-ratio of two material figures is independent of the time.

Suppose $v^{\prime \prime}$ is the volume of a third material figure. Suppose that by the mutual action of $v^{\prime \prime}$ and $v$ there are produced in $v$ and $v^{\prime \prime}$ mean accelerations of magnitudes $f_{1}$ and $f_{1}^{\prime \prime}$ relative to the same frame as before.

Suppose that by the mutual action of $v^{\prime \prime}$ and $v^{\prime}$ there are produced in $v^{\prime}$ and $v^{\prime \prime}$ mean accelerations of magnitudes $f_{2}^{\prime}$ and $f_{2}^{\prime \prime}$ relative to the same frame as before.

Then we make it a rule that these are not independent but connected by the relation $\frac{f}{f^{\prime}} \cdot \frac{f_{1}^{\prime \prime}}{f_{1}} \cdot \frac{f_{2}^{\prime}}{f_{2}^{\prime \prime}}=1$.

This amounts to saying that

$$
\frac{\text { mass ratio of } v \text { and } v^{\prime}}{\text { mass ratio of } v \text { and } v^{\prime \prime}}=\text { mass ratio of } v^{\prime \prime} \text { and } v^{\prime} \text {. }
$$

71. Mass. If we associate the number 1 with any particular material figure $A$, then we can associate a definite positive number $m$ with any other material figure $B$, this number is the mass-ratio of the two figures $A$ and $B$. We call it the mass of $B$. According to this the mass-ratio of two figures, $A$ and $B$, is the ratio of the mass of $A$ to the mass of $B$.

72. Conception of a body. We imagine a body to be made up of material figures* each of which is a homogeneous part of the body and has a definite mass, and we define the mass of the body to be the sum of the masses of the material figures of which it is made up.

To cover all cases we imagine the material figures of which a body is made up to occupy infinitesimal elements of volume with infinitesimal masses, and we speak of one such figure as a particle of the body.

Since the mass of a portion of a body however small may be taken as the unit of mass there will be no inconsistency in the idea of an isolated particle of finite mass.

* Cf. Critical Note at the end of Chapter VIII. 
We shall be much occupied in what follows with the theory of the motions of systems of such particles; but, keeping at present to the conception of a body as made up of particles, we regard bodies as bounded by surfaces and continuously occupying finite volumes, and we say that at any point within the surface of the body there is a particle of the body.

73. Density. Any part of a body (so conceived) will have a certain mass, and it may be part of our conception that whatever part we take, the mass of the part has a constant ratio to the volume. When this is so we say that the body is homogeneous, and call the ratio in question the density of the body. When the ratio of the mass of a part of the body to its volume is conceived as variable from part to part of the body, then, taking a series of parts of diminishing volumes containing the same point we get a series of numbers for the ratios of their masses to their volumes; the limit of this series when the volume is indefinitely diminished is defined to be the density of the body at the point. It is clear that, if $\rho$ is the density of a body at any point, and $d v$ an infinitesimal volume containing the point, then $\int \rho d v$, the integration being taken throughout the volume occupied by the body, is the mass of the body.

74. Mutual actions of Bodies. We conceive of the particles of bodies as acting one upon another so as to produce accelerations relative to a frame, and we conceive that the acceleration of any particle relative to the frame is the resultant of component accelerations contributed by the actions of other particles, and then the system of component accelerations of the different particles is always a system consisting of pairs of accelerations, the accelerations of a pair being the contribution $f$ of a particle (of mass $m^{\prime}$ ) to the acceleration of another particle (of mass $m$ ), and the contribution $f^{\prime}$ of the particle of mass $m$ to the acceleration of the particle of mass $m^{\prime}$. The accelerations $f$ and $f^{\prime}$ are localised in the line joining the particles $m$ and $m^{\prime}$, have opposite senses, and have magnitudes such that $m f=m^{\prime} f^{\prime}$. The product $m f$ or $m^{\prime} f^{\prime}$ is taken to measure the mutual action of the two particles.

75. Force. We say that the particles of bodies exert forces one on another. 
Let $f$ be the magnitude of the acceleration contributed to a particle of mass $m$ by the action of a particle of mass $m^{\prime}$, this acceleration is a vector localised in the line joining the particles. We define the force exerted by the particle $m^{\prime}$ upon the particle $m$ to be a vector localised at a point (the position of $m$ ), of magnitude $m f$, and having the direction and sense of the acceleration $f$. force.

The line joining the particles is called the line of action of the

The definition includes the statement that the force exerted by $m$ on $m^{\prime}$ is equal and opposite to that exerted by $m^{\prime}$ on $m$.

76. Resultant Force. Since, by the definition, a force is a vector localised at a point, there can be no proper resultant of a system of forces except when they act on a particle. Nevertheless it is convenient to regard a system of forces in general as equivalent to other systems, in the same way as if the forces were vectors localised in their lines of action. We can thus determine for any system of forces a resultant force at any origin and a resultant couple exactly as was done for vectors localised in lines in Article 27. We shall see hereafter that when a force acts on any particle of a rigid body it produces the same acceleration in all points of the body as it would do if acting on any other particle of the body lying in the line of action of the force. The force- and couple-resultants of a system of forces regarded as vectors localised in lines are therefore the resultant force and couple for the same system of forces acting on a rigid body (See Article 115).

77. Motion of a body. We shall show hereafter that for any body there is a certain point, known as the centre of mass or centre of inertia, which moves like a particle of mass equal to the mass of the body acted upon by a force, which is the force that would be the resultant of the system of forces applied to the body if the body were rigid.

The centre of inertia of a homogeneous spherical body is its centre of figure. All the points of a rigid body moving without rotation, have the same acceleration as its centre of inertia. Thus (assuming the system of definitions and rules given to be applicable to natural bodies) it is easy to devise cases in which the motion of the centre of inertia of a natural body can be observed. 
When we speak of the acceleration of a body we must be understood to mean the acceleration of the centre of inertia of the body supposed to be rigid.

78. Momentum. The momentum of a particle is defined to be a vector localised in the line of its velocity, having the sense of the velocity, and of magnitude equal to the product of the mass of the particle and its velocity.

79. Kinetic Reaction. The kinetic reaction of a particle is a vector localised in the line of its acceleration, having the sense of the acceleration, and of magnitude equal to the product of the mass of the particle and its acceleration.

The magnitude of this vector, its sense, and the line in which it is localised are the same as those of the rate of change of momentum of the particle per unit of time.

80. Equations of motion. Since the acceleration of a particle is the resultant of the accelerations contributed to it by the actions of other particles, its kinetic reaction is the resultant of the kinetic reactions produced by these particles; these component kinetic reactions are vectors identical in magnitude and sense with the forces acting on the particle, and are localised in the lines of action of these forces. It follows that the resolved part, parallel to any line, of the kinetic reaction of the particle is the sum of the resolved parts, parallel to the same line, of the forces that act on the particle.

The equations expressing this equivalence are called the equations of motion of the particle.

Thus let $x, y, z$ be the coordinates of the particle at time $t, m$ its mass, and let $X, Y, Z$ be the resolved parts parallel to the axes of the resultant force acting upon it. The equations of motion are

$$
m \ddot{x}=X, m \ddot{y}=Y, m \ddot{z}=Z \text {. }
$$

81. Impulse of a force. Let $\dot{x}_{0}, \dot{y}_{0}, \dot{z}_{0}$ be the resolved parts of the velocity at the instant when $t=t_{0}$. On integrating the above equations we have

$$
m \dot{x}-m \dot{x}_{0}=\int_{t_{0}}^{t} X d t \text { and two similar equations. }
$$


The time-integral of a force between limits corresponding to the beginning and end of any interval is called the impulse of the force during the interval. It is the vector (unlocalised) whose resolved part in any direction is the time-integral between the same limits of the resolved part of the force in that direction.

The above equations can be stated in words as follows:- the change of momentum of a particle in any interval is equal to the impulse of the force acting on it during the interval.

82. Impulsive action. Many changes of motion of natural bodies take place with such rapidity that it is very difficult to observe the gradual transition from one state of motion to another, and it is therefore convenient, in our ideal system, to allow for the possibility of sudden changes of motion. This is done by supposing that the mutual action of two particles, or the resultant action of many particles on one particle, can increase without limit as the particle passes through some position. Let $X_{1}, Y_{1}, Z_{1}$ be the sums of the resolved parts parallel to the axes of those forces exerted on a particle $m$ which do not remain finite throughout an interval of time denoted by $\tau$, and suppose the instant at the middle of this interval denoted by $t$, is the instant at which $X_{1}, Y_{1}, Z_{1}$ cease to be finite. Then we suppose that

$$
L t_{\tau=0} \int_{t-\frac{1}{2} \tau}^{t+\frac{1}{2} \tau} X_{1} d t,=X, L t_{\tau=0} \int_{t-\frac{1}{2} \tau}^{t+\frac{1}{2} \tau} Y_{1} d t,=Y, L t_{\tau=0} \int_{t-\frac{1}{2} \tau}^{t+\frac{1}{2} \tau} Z_{1} d t,=Z,
$$

are finite. We observe that these quantities are the limits of the impulses of the forces during an indefinitely short interval containing the instant $t$. The vector, localised at the position of the particle, whose resolved parts parallel to the axes are $X, Y, Z$ is defined to be the impulse exerted on the particle at the instant $t$.

If the velocity of the particle just before the instant $t$ has resolved parts $u_{0}, v_{0}, w_{0}$ parallel to the axes, and just after has resolved parts $u, v, w$ parallel to the axes, we have the equations

$$
m u-m u_{0}=X, m v-m v_{0}=\underline{Y}, m w-m w_{0}=Z,
$$

and these equations can be stated in words in the form:-the change of momentum of a particle in any direction is equal to the impulse acting on the particle in that direction. These equations are called equations of impulsive motion. 
83. Impulsive motion of a body. The motion of a body whose particles are subject to impulses is determined from the motion of a particle at its centre of inertia in the same way as when it is subject to forces. Thus the changes of momentum produced in two bodies by their impulsive mutual action are equal and opposite, or the changes of velocity produced in them are inversely as their masses.

84. Rational Mechanics. The system of definitions and rules which we have laid down lead to a system of differential equations for determining the motions, relative to a frame, of a system of particles, or of a body or a system of bodies, conceived to be made up of particles. It may be regarded as a purely ideal system, and its validity is unaffected by the question whether it has or has not any relation to the observed motions of natural bodies. The subject, so treated, is known as Rational Mechanics. The objects of which it treats are pure objects of thought. Its development consists in the logical deduction of particular results from the general principles laid down.

85. Mechanics of Natural Bodies. The application of Rational Mechanics to the formulation of the Laws that govern the motions of natural bodies consists in the statement that it is possible to assign masses to the bodies and to choose a frame of reference determined by parts of natural bodies, such that the observed motions of natural bodies, relative to the frame, obey the Laws of Rational Mechanics within certain limits of exactness; that in fact the observed motions coincide with the motions described in the phraseology of Rational Mechanics so closely that no discrepancy can be observed.

There are motions, e.g. the phenomena of diffusion, which cannot easily be brought under the above statement. A mechanical theory of such phenomena, like that of any other phenomenon, is a statement concerning bodies, as conceived in Rational Mechanics, which will move as the natural bodies are observed to move.

86. Mutual Action. Natural bodies act on each other in various ways. The sun warms a stone, rain wets a coat, buffers stop a train. Among these actions many are clearly actions in 
which motion of a body relative to a frame is set up, altered, or stopped. Such actions are mechanical.

We replace in imagination the natural body whose motion is observed to be changing by a body as conceived in Rational Mechanics, and attribute to that body a certain mass, called the mass of the natural body; then, from the observed changes of motion, we can find expressions for the forces that act on the body. The solution of the equations of motion of the body with the attributed mass, under the action of the deduced forces, gives a rule for determining the position of the body at any time. We state that the mass may be so chosen that the observed position coincides with the determined position.

87. Postulates of Mechanics. The method indicated may be summed up in the following postulates :-

(1) Every body, and every individual part of a body, has a constant mass, and the mass of the body is the sum of the masses of its parts.

(2) Every body may be conceived as made up of particles (continuously filling the volume within the surface of the body) which act upon one another so as to produce accelerations.

(3) All changes of motion arise from the mutual actions of the particles of bodies.

(4) The component accelerations produced in two particles by their mutual action are localised in the line joining the particles, have opposite senses, and are of magnitudes inversely proportional to the masses of the particles.

(5) In no circumstances do the surfaces of two bodies intersect so as to contain a finite volume, or two bodies never at the same instant occupy the same space.

88. Frame of reference. The accelerations mentioned in postulates (2) and (4) are, of course, accelerations relative to a frame. It is possible to choose frames for which the postulates apply to natural bodies and also frames for which they do not. This matter will occupy us again in Chapter XIII.

89. Field of Force. We can seldom discover what component accelerations, arising from the mutual actions of particles, 
must be assumed in order that the observed accelerations may be the resultants; all we can generally observe is the resultant acceleration of a body or of a part of a body. Thus, having fixed upon our frame of reference, and attributed a mass to a body, we can find by observation the resultant force acting on the body. This resultant is in fact a vector localised at the position of the centre of inertia of the body, having the direction and sense of the acceleration with which the centre of inertia moves, and equal to the product of the mass and the acceleration. In most cases the acceleration has a value depending, in a simple way, on the relative positions of the body, neighbouring bodies, and the frame of reference. When this is the case we call the region where such acceleration can be observed a field of force, and define the intensity of the field at the point to be a vector localised at the point, and having the magnitude, direction, and sense of the acceleration. We may also say that every particle gives rise to a field of force, and regard any field as a region where such forces combine at any point to produce a resultant.

90. Gravity. We have stated already in Article 44, that in the neighbourhood of the Earth any body, small enough for us to handle or to move by machinery, falls towards the Earth with an acceleration approximately constant, and in a vertical direction. We attribute this acceleration to the action of the particles of which the Earth is conceived to be made up on the particles of which the body is conceived to be made up. The neighbourhood of the Earth is a field of force, and the acceleration at any point in the field, i.e. the intensity of the field, is, at any place, directed vertically downwards and is equal to $g$, where $g$ is the acceleration described in Article 44, and there called the acceleration due to gravity.

91. Weight. In the neighbourhood of the Earth the resultant force on a free falling body is numerically equal to the product of the mass of the body and the acceleration due to gravity. This force is called the weight of the body.

We conceive that this force is always acting on the body whether it is falling freely or not. If the body is supported, or is moving with an acceleration unequal to $g$ or not in the vertical direction, we conceive it to be acted upon by other forces as well. 
At any place on the Earth's surface the mass of a body is proportional to its weight.

92. Determination of the mass of a body. To determine the masses of bodies near the Earth's surface, small enough to be handled, it is only necessary to weigh the bodies in a common balance. Bodies which equilibrate when weighed are of equal mass.

Any particular body is chosen, and its mass is taken to be unity. Other bodies are weighed against it and their sizes adjusted until they separately balance with it. When a body balances with $n$ of these unit bodies its mass is $n$. In the same same way taking a smaller mass than the unit, and adjusting it so that precisely $n$ masses equal to the smaller mass balance the unit, we have $n$ bodies each of which has mass $1 / n$. In this way we can see how it is possible to determine the mass of a body with a degree of accuracy depending only on the sensitiveness of the balance employed. The mass of a body is a positive number, in the general sense, which expresses the mass ratio of the body and a body of unit mass.

93. Objective Validity of the conception of mass. The ratio of the masses of any two small bodies can be determined with great accuracy by weighing them.

Now it is part of the conception of mass that when two bodies act on each other so as to produce changes of motion, the changes of velocity produced in any time by their mutual actions are inversely as their masses.

The objective validity of the conception is confirmed if the ratio of the masses deduced by direct observation of the velocities produced by their mutual action coincides with the ratio of the masses determined by weighing the bodies.

Direct experiments of this nature can be devised of which the principle is as follows:-

Two spherical bodies are suspended by strings from points in the same horizontal line, the strings being of such lengths, and the points at such distances, that, when the strings are vertical, the bodies are in contact and the line of centres is horizontal. 
Suppose $m$ and $m^{\prime}$ are the masses of the bodies (determined by weighing them), $u$ and $u^{\prime}$ the velocities in

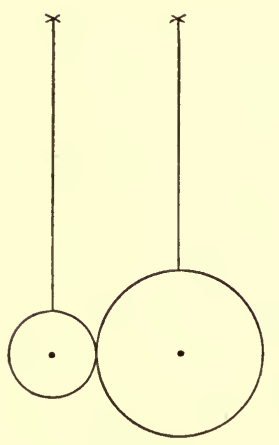

Fig. 41. the line of centres just before they come into contact, $v$ and $v^{\prime}$ the velocities in the line of centres just after they come into contact. Consider a case where the strings are vertical at the instant of collision, and the bodies rebound after collision. With the velocities $u$ and $u^{\prime}$ the bodies approach each other, with the velocities $v$ and $v^{\prime}$ they separate. Hence the changes of velocity in the line of centres are $u+v$ and $u^{\prime}+v^{\prime}$.

Now the velocities $u, v, u^{\prime}, v^{\prime}$ can all be determined by observing the heights from which the bodies were dropped and the heights to which they rise; and it is found that

$$
m(u+v)=m^{\prime}\left(u^{\prime}+v^{\prime}\right) .
$$

An instrument for making this experiment is known as a Ballistic Balance. It is no part of the plan of this book to describe the details of instruments.

94. Gravitation. We have described methods of determining the masses of bodies which can be handled. But these yield no way of assigning masses to the Earth, the Sun, or other celestial bodies. Also we have said that it is not generally possible to assign the component accelerations produced in any one of the particles of which a natural body is regarded as made up by the action of particles of other natural bodies. There is however a class of cases of great generality and importance for which this can be done. We can in fact in the case of particles at a distance apart which can be measured by ordinary means, (by a divided scale,) or at any greater distance, state a rule for the intensity of the field of force due to each particle, and then the masses can be once for all assigned so that the resultant intensity at any point coincides with the result of observation.

The rule in question is known as the Law of Gravitation: it is that the force between two particles of masses $m, m^{\prime}$ at a distance $r$ is of magnitude $\gamma \frac{m m^{\prime}}{r^{2}}$, where $\boldsymbol{\gamma}$ is a constant independent of $m$, $m^{\prime}, r$ and of the time, and the sense of the force exerted by $m^{\prime}$ on 
$m$ is from $m$ to $m^{\prime}$. The last part of the statement is usually expressed by saying that the force is an attraction.

The Theory of Attractions is the theory by means of which the resultants of forces exerted by the particles of gravitating bodies are calculated. For our immediate purpose the most important result of this theory is that, for a spherical body of uniform density, (or having its mass so distributed that the surfaces of equal density are concentric spheres,) the resultant attraction of the body on a particle outside it is the same as that of a particle at the centre, of mass equal to the mass of the body. This result can be briefly (and less precisely) stated in the form that spheres attract each other as if their masses were concentrated at their centres.

The direct application of the law of gravitation to celestial mechanics requires for its complete statement the choice of a frame of reference. We shall say at present that the origin is in the Sun and that the lines of reference go out thence to stars so distant that they have no observable annual parallax. (See Chapter XIII.)

Relatively to this frame the Earth and each of the planets describe paths which are very approximately ellipses having one focus in the Sun, and the radii vectores from the Sun to any planet describe equal areas in equal times, while the square of the periodic time in which any planet describes its ellipse is proportional to the cube of the axis major of the ellipse.

The statement just made is a statement of facts of observation. Remembering the results of Articles 50 to 55, we can interpret it by saying that each planet has an acceleration towards the Sun varying inversely as the square of the distance.

This statement is in accordance with the Law of Gravitation if we suppose the mass of the Sun to be great compared with the masses of the planets.

The statement made in Article 91 concerning the field of force near the Earth is also clearly included in the Law of Gravitation; for distances from the Earth's surface within which we can manipulate bodies, the attraction of the Earth on a body is practically constant.

The constant $\gamma$ can be determined by direct experiment. 
A knowledge of the acceleration $g$ can then be used to deduce the mass of the Earth, and Astronomical observations can then be applied to determine the masses of the Sun and the Planets. It is only in this way that these masses can be assigned.

95. Measurement of Mass. To any particular body we may assign the mass unity, then the mass of any other body has a ratio to the mass of the chosen body which is a real positive number. This number measures the mass of the body.

The unit of mass is not expressible in terms of the units of length and time.

In British measure the unit of mass is the mass of a particular lump of platinum, kept in the Royal Exchequer, and defined by Act of Parliament to be a pound. The mass of any body which when weighed against this standard pound would balance it is a mass of one pound.

In the C.G.S. system of units the unit of mass is the $\frac{1}{1000}$ th part of the mass of a certain lump of platinum known as the "Kilogramme des Archives," made by Borda, and is defined to be a gramme.

The mass of a cubic centimetre of pure water at a temperature of $4^{\circ}$ Centigrade under a barometric pressure represented by 760 millimetres of mercury is very approximately equal to 1 gramme.

96. Quantity of matter in a body. The mass of a body is the quantity of matter in the body.

This is a definition of quantity of matter.

We are accustomed to estimate the quantities of matter in bodies by weighing them, that is we think of one body as containing more or less material than another according as, when weighed, the former weighs more or less than the latter. This method manifestly cannot be applied to determine the quantity of matter in the Earth, the Moon, or any other celestial body.

The acceleration with which a free body falls towards the Earth is variable with latitude, and increases in passing from the Equator to the Poles. Thus the weight of a body, measured by the product of this acceleration and the mass of the body, is variable with latitude, and increases as we pass from the equator to the poles. 
It is inconsistent with the idea of "the quantity of matter in a body" that this quantity should be liable to increase or diminution when the body is transported from place to place. Rather the idea of the quantity of matter in a body is the idea of something which cannot be increased or diminished so long as the body remains the same. This idea with the rule that the quantity of matter in a body is the sum of the quantities of matter in its parts combine to point to mass as the measure of quantity of matter.

The common use of the word "weight" covers two notions which are essentially distinct, the notion of pressure which a heavy body exerts on a support, and the notion of quantity of matter. In scientific speaking or writing, different words must be used to express distinct notions.

97. Inertia. Besides serving as a measure of the quantity of matter in a body, the mass of a body is connected with a property of bodies which is a subject of common observation. The postulate that all changes of motion arise from the mutual actions of particles includes the statement that if any body could be freed entirely from the action of all other bodies it would have no acceleration. To produce any particular. change of motion in a body, a particular set of forces must be applied to it, and these must be exerted on the particles of the body by the particles of some other body. The tendency of the body, apart from the action of other bodies, to persist in its state of motion at any instant is known as its inertia. It is evident that, the greater the mass of the body, the greater must be the force applied to it to produce in a given interval of time any particular change of velocity. The mass of the body can thus be taken to measure the tendency in question, and for this reason the mass of a body is sometimes called its inertia. 


\section{HISTORICAL NOTE.}

Before the time of Galilei there existed no dynamical theory, and the statical results that had been obtained were of a vague character. The older statical researches professed to give an account of the action of force in certain circumstances, but the forces so considered were at first entirely undefined. The idea had however been established that a force prevents motion, which would take place in the absence of the force, and this gave the notion that force was directed, its direction being opposite to that in which motion would take place if the force were not applied. Further the force exerted to prevent any motion was supposed to be measurable by the tension of a chain pulled in the direction of the force so as to produce the same effect, and the tension itself was supposed to be measurable by the weight the chain would support if it passed over a smooth pulley and produced the required effect. The whole of the ideas thus sketched depended on gravity, there was nothing cosmical about the scheme.

Galilei discovered by experiment that the velocity of a falling body is proportional to the time during which it has been falling, and was thus led to the idea of acceleration. He recognised in the motion of a body on a smooth horizontal plane that a body, which the existing statical theory would have regarded as under no force, moves uniformly in a straight line, and he was thus led to connect the presence of force rather with the production of acceleration, than with the prevention of motion.

Newton found that the idea of acceleration, thus introduced by Galilei, availed for the description of the motions of the bodies of the solar system equally with the motion of falling bodies near the earth's surface, and he made the idea of force, as that which produces acceleration, the cardinal notion in his philosophy. Newton also introduced the idea of mass, as distinct from weight, and stated that the mass of a body is the quantity of matter it contains. He formulated his theory in a series of definitions, in the three celebrated Laws of Motion, which he described as Axiomata sive Leges Motus, and in the Scholia attached thereto. We give here a translation of the three Laws of Motion:

"First Law. Every body remains in its state of rest or of uniform motion "in a straight line, except in so far as it is compelled by impressed forces "to change its state."

"Second Law. Change of motion is proportional to the impressed moving "force, and takes place in the direction in which that force is impressed."

"Third Law. Reaction is always equal and opposite to action; or the "actions of two bodies one on the other are always equal and oppositely "directed." 
The definitions preceding the laws introduce the notions of mass, and of impressed moving force as an action on a body by which its state of motion changed, and as proportional to what we now call momentum generated in a given interval. The scholia attached to the laws contain a demonstration of the theorem of the parallelogram of forces, and an account of the determination of masses by direct experiment with the ballistic balance. The latter is given as a verification of the Third Law.

We have already alluded to the application which Newton made of his theory to the problems of celestial mechanics. It was in this connexion that he introduced the Law of Gravitation. The application to ordinary terrestrial mechanics was not considered by him in detail, and thus it happened that when d'Alembert propounded his principle (Article 107) it was accepted as a new principle. It is virtually included in the Third Law.

It has been the task of modern criticism to disentangle in Newton's theory the definitions, and the postulates. That the Laws of Motion are of the nature of postulates is clearly recognised in Newton's word Axiomata, but it is now held that they also partake of the nature of definitions. According to the account we have given, there is an essential physical discovery presented in the Newtonian theory:-the discovery that bodies possess the properties we associate with the word "mass"; these properties are concisely expressed in Postulates I-IV of Article 87; the rest of the theory is of the nature of definition. In particular the theorem of the parallelogram of forces is a consequence of the definition of force as a vector. 


\section{CHAPTER VI.}

\section{GENERAL THEOREMS.}

98. Centre of Inertia. The centre of inertia of a system of particles is the centroid of points, which are the positions of the particles, for multiples, which are the masses of the particles; it is the point whose coordinates $\bar{x}, \bar{y}, \bar{z}$ relative to any frame are given by the equations

$$
\bar{x} \Sigma m=\Sigma(m x), \quad \bar{y} \Sigma m=\Sigma(n y), \quad \bar{z} \Sigma m=\Sigma(m z),
$$

where $m$ is the mass of a particle of the system at the point $(x, y, z)$, and the summation extends to all the particles.

If $\rho$ is the density of a body at a point $(x, y, z)$, the coordinates of the centre of inertia of the body are $\bar{x}, \bar{y}, \bar{z}$, where

$$
\bar{x}=\frac{\iiint x \rho d x d y d z}{\iiint \rho d x d y d z}
$$

with similar expressions for $\bar{y}, \bar{z}$ obtained by interchanging $x$ with $y$ and $z$ respectively, the integrations extending throughout the body.

Since the centre of inertia of a body small enough to be handled coincides with its centre of gravity as defined in Statics, we shall denote it by the letter $G$.

99. Relative coordinates. Let the coordinates of the position of a particle of mass $m$ be $x, y, z$ referred to any system of axes, and let $\bar{x}, \bar{y}, \bar{z}$ be the coordinates of the centre of inertia of the system of particles referred to the same axes, then we may put

$$
x=\bar{x}+x^{\prime}, \quad y=\bar{y}+y^{\prime}, \quad z=\bar{z}+z^{\prime},
$$

and the quantities $x^{\prime}, y^{\prime}, z^{\prime}$ are the coordinates of the position of the particle relative to parallel axes through the centre of inertia.

From the definition of the centre of inertia it follows that

$$
\Sigma\left(m x^{\prime}\right)=0, \quad \Sigma\left(m y^{\prime}\right)=0, \quad \Sigma\left(m z^{\prime}\right)=0,
$$

the summations referring to all the particles. 
100. Linear Momentum. The momentum of a particle has been defined to be a vector localised in the line of the velocity of the particle, with the sense of the velocity, and of magnitude equal to the product of the mass and the velocity.

The resolved parts parallel to the axes of the momentum of a particle of mass $m$ at the point $(x, y, z)$ are $m \dot{x}, m \dot{y}, m \dot{z}$.

The momenta of the particles of a system can be replaced by a resultant momentum localised in a line through the centre of inertia, and a couple. The resultant momentum has as its resolved parts parallel to the axes

$$
\Sigma(m \dot{x}), \quad \Sigma(m \dot{y}), \quad \Sigma(m \dot{z}) .
$$

Differentiating the equations such as $\bar{x} \Sigma m=\Sigma(m x)$, we obtain such equations as $\dot{\bar{x}} \Sigma m=\Sigma(m \dot{x})$, where $\dot{\bar{x}}$ stands for $\frac{d \bar{x}}{d t}$.

We thus see that the resultant momentum is equal to the momentum of a particle of mass equal to the mass of the system, placed at the centre of inertia, and moving with the velocity of the centre of inertia.

The momentum just described is called the linear momentum of the system. The particle at the centre of inertia just described will be referred to as the "particle G."

101. Kinetic Reaction. The kinetic reaction of a particle has been defined as a vector localised in the line of the acceleration of the particle, with the sense of the acceleration, and of magnitude equal to the product of the mass and the acceleration.

The resolved parts, parallel to the axes, of the kinetic reaction of a particle of mass $m$ at the point $(x, y, z)$ are $m \ddot{x}, m \ddot{y}, m \ddot{z}$.

The kinetic reactions of the particles of a system can be replaced by a resultant kinetic reaction localised in a line through the centre of inertia, and a couple. The resultant kinetic reaction has as its resolved parts parallel to the axes

$$
\Sigma(m \ddot{x}), \Sigma(m \ddot{y}), \Sigma(m \ddot{z}) \text {. }
$$

Differentiating the equations such as $\dot{\bar{x}} \Sigma m=\Sigma(m \dot{x})$ we find such equations as $\ddot{x} \Sigma m=\Sigma(m \ddot{x})$.

We thus see that the resultant kinetic reaction is that of the particle $G$, i.e. it is the same as that of a particle of mass equal to the mass of the system placed at the centre of inertia and moving with it. 
102. Moment of Momentum. The moment of momentum of a system of particles about any axis is the sum of the moments of the momenta of the particles about the same axis.

The momenta can be reduced to a linear momentum localised in a line through a point and a couple, and then the moment of momentum is the couple. The magnitude and direction of the couple depend upon the point chosen.

The moment of momentum about a line is the resolved part of this couple about the line, when the point chosen is on the line.

The moments of momentum of a system of particles about the axes of reference are

$$
\Sigma m(y \dot{z}-z \dot{y}), \quad \Sigma m(z \dot{x}-x \dot{z}), \quad \Sigma m(x \dot{y}-y \dot{x}) .
$$

In these expressions put $x=\bar{x}+x^{\prime}, \ldots$, so that $x^{\prime}, y^{\prime}, z^{\prime}$ are the coordinates relative to parallel axes through the centre of inertia, and $\dot{x}^{\prime}, \dot{y}^{\prime}, \dot{z}^{\prime}$ are the resolved parts of the velocity relative to the centre of inertia. Then, observing that $\Sigma m x^{\prime}=0, \Sigma m \dot{x}^{\prime}=0$, and similarly for $y^{\prime}$ and $z^{\prime}$, we find the above expressions become three such as

$$
(\bar{y} \dot{z}-\bar{z} \dot{\bar{y}}) \Sigma m+\Sigma m\left(y^{\prime} \dot{z}^{\prime}-z^{\prime} \dot{y}^{\prime}\right) \text {. }
$$

We may state our result in words:-The moment of momentum of a system of particles about any axis is equal to the moment of the momentum of the particle $G$ about the same axis, together with the moment of momentum in the motion relative to $G$ about a parallel axis through $G$.

It is now clear that, when the momenta of the particles of the system are reduced to a linear momentum, localised in a line through the centre of inertia, and a couple, the couple is the moment of momentum in the motion relative to parallel axes through the centre of inertia.

The phrase angular momentum is frequently used for moment of momentum.

103. Moment of Kinetic Reaction. The moments of the kinetic reactions of a system of particles about the axes of reference are

$$
\Sigma m(y \ddot{z}-z \ddot{y}), \quad \Sigma m(z \ddot{x}-x \ddot{z}), \quad \Sigma m(x \ddot{y}-y \ddot{x}) .
$$


Putting again $x=\bar{x}+x^{\prime}, \ldots$ and observing that

$$
\sum m \ddot{x}^{\prime}=0, \quad \Sigma m \ddot{y}^{\prime}=0, \quad \Sigma m \ddot{z}^{\prime}=0,
$$

we find that these expressions become three such as

$$
(\bar{y} \ddot{z}-\bar{z} \ddot{\bar{y}}) \Sigma m+\Sigma m\left(y^{\prime} \ddot{z}^{\prime}-z^{\prime} \ddot{y}^{\prime}\right) \text {. }
$$

We may state our result in words:-The sum of the moments of the kinetic reactions of a system of particles about any axis is equal to the moment about that axis of the kinetic reaction of the particle $G$, together with the sum of the moments of the kinetic reactions in the motion relative to $G$ about a parallel axis through $G$.

Since, as in Article 50, $y \ddot{z}-z \ddot{y}=\frac{d}{d t}(y \dot{z}-z \dot{y})$,

the moment of any kinetic reaction about any axis is equal to the rate of increase of a corresponding moment of momentum about the same axis per unit of time.

Thus the rate of increase of the moment of momentum of a system about any axis is the same as the sum of the moments of the kinetic reactions about that axis.

It is now clear that, when the kinetic reactions of the particles of a system are reduced to a resultant kinetic reaction localised in a line through the centre of inertia, and a couple, the couple is the rate of increase of the moment of momentum of the motion relative to parallel axes through the centre of inertia.

104. Kinetic energy. The kinetic energy of a particle is half the product of its mass and the square of its velocity.

For a particle of mass $m$ at $(x, y, z)$ it is

$$
\frac{1}{2} m\left(\dot{x}^{2}+\dot{y}^{2}+\dot{z}^{2}\right) \text {. }
$$

The kinetic energy of a system of particles is the sum of the kinetic energies of the particles. It is the quantity

$$
\frac{1}{2} \Sigma m\left(\dot{x}^{2}+\dot{y}^{2}+\dot{z}^{2}\right) \text {. }
$$

Putting $x=\bar{x}+x^{\prime}, \ldots$ and remembering that

$$
\Sigma m \dot{x}^{\prime}=0, \quad \Sigma m \dot{y}^{\prime}=0, \quad \Sigma m \dot{z}^{\prime}=0,
$$

we find that this expression becomes

$$
\frac{1}{2}\left(\dot{x}^{2}+\dot{\bar{y}}^{2}+\dot{\bar{z}}^{2}\right) \Sigma m+\frac{1}{2} \Sigma m\left(\dot{x}^{\prime 2}+\dot{y}^{\prime 2}+\dot{z}^{\prime 2}\right) .
$$

We may state this result in words:-The kinetic energy of a system of particles is the kinetic energy of the particle $G$ together with the kinetic energy in the motion relative to parallel axes through $G$. 


\section{Equations of motion of a system of particles.} Let $m_{1}$ be the mass of one particle of the system, $x_{1}, y_{1}, z_{1}$ its coordinates at time $t, X_{1}, Y_{1}, Z_{1}$ the sums of the resolved parts parallel to the axes of the forces exerted on this particle by particles not forming part of the system, $X_{1}^{\prime}, Y_{1}^{\prime}, Z_{1}^{\prime}$ the sums of the resolved parts parallel to the axes of the forces exerted on the same particle by the remaining particles of the system.

The equations of motion of this particle are

$$
m_{1} \ddot{x}_{1}=X_{1}+X_{1}^{\prime}, m_{1} \ddot{y}_{1}=Y_{1}+Y_{1}^{\prime}, m_{1} \ddot{z}_{1}=Z_{1}+Z_{1}^{\prime} .
$$

Similarly the equations of motion of a second particle of mass $m_{2}$ at $\left(x_{2}, y_{2}, z_{2}\right)$ may be written

$$
m_{2} \ddot{x}_{2}=X_{2}+X_{2}^{\prime}, m_{2} \ddot{y}_{2}=Y_{2}+Y_{2}^{\prime}, m_{2} \ddot{z}_{2}=Z_{2}+Z_{2}^{\prime} \text {. }
$$

We shall write as the type of such equations

$$
m \ddot{x}=X+X^{\prime}, m \ddot{y}=Y+Y^{\prime}, m \ddot{z}=Z+Z^{\prime} .
$$

Then $(X, Y, Z)$ is the type of the external forces, and $\left(X^{\prime}, Y^{\prime}, Z^{\prime}\right)$ is the type of the internal forces.

106. Law of Internal Action. The system of the internal forces between the parts of a system is equivalent to zero.

Since the action between a pair of particles of a system is a pair of equal and opposite forces in the line joining the particles, the resultant of all these forces regarded as vectors localised in these lines is zero, the sum of their resolved parts parallel to any line is zero, and the sum of their moments about any axis is zero.

Thus, in the notation of the last Article, one of the forces contributing to the resultant whose resolved parts are $X_{1}^{\prime}, Y_{1}^{\prime}, Z_{1}^{\prime}$ is the action of $m_{2}$ on $m_{1}$, and one of the forces contributing to the resultant whose resolved parts are $X_{2}^{\prime}, Y_{2}^{\prime}, Z_{2}^{\prime}$ is the action of $m_{1}$ on $m_{2}$. These are equal and opposite forces in the line joining $m_{1}$ and $m_{2}$. The resultants whose resolved parts are the forces typified by $X^{\prime}, Y^{\prime}, Z^{\prime}$ are made up entirely of such components.

Hence we have $\Sigma X^{\prime}=0, \Sigma Y^{\prime}=0, \Sigma Z^{\prime}=0$, and

$$
\Sigma\left(y Z^{\prime}-z Y^{\prime}\right)=0, \Sigma\left(z X^{\prime}-x Z^{\prime}\right)=0, \Sigma\left(x Y^{\prime}-y X^{\prime}\right)=0 \text {. }
$$

\section{Simplified forms of the equations of motion.} Adding the left-hand members of all the $x$-equations of motion, and remembering that $\Sigma X^{\prime}=0$, we obtain $\Sigma m \ddot{x}=\Sigma X$. 
In like manner we have

$$
\Sigma m \ddot{y}=\Sigma Y \text {, and } \Sigma m \ddot{z}=\Sigma Z .
$$

Again multiplying the $z$-equations by the $y$ 's and the $y$-equations by the $z^{\prime}$ s, adding, and remembering that $\Sigma\left(y Z^{\prime}-z Y^{\prime}\right)=0$, we have

$$
\Sigma m(y \ddot{z}-z \ddot{y})=\Sigma(y Z-z Y) .
$$

In like manner we have

$$
\Sigma m(z \ddot{x}-x \ddot{z})=\Sigma(z X-x Z) \text {, and } \Sigma m(x \ddot{y}-y \ddot{x})=\Sigma(x Y-y X) \text {. }
$$

Our equations may be stated in words :-

(1) The sum of the resolved parts in any direction of the kinetic reactions of a system of particles is equal to the sum of the resolved parts of the external forces in the same direction.

(2) The sum of the moments about any axis of the kinetic reactions of a system of particles is equal to the sum of the moments of the external forces about the same axis.

The result may also be briefly stated in the form:-When the external forces are regarded as localised in their lines of action the kinetic reactions and the external forces are two equivalent systems of localised vectors.

This result, in a slightly different form, was first stated by D'Alembert in his Traité de Dynamique, 1743. It is known as D'Alembert's Principle.

108. Motion of the centre of inertia. Since the resultant of the kinetic reactions of the particles of a system is the kinetic reaction of a particle of mass equal to the mass of the system placed at the centre of inertia and moving with it, we see that

$$
\ddot{\bar{x}} \Sigma m=\Sigma X, \ddot{y} \Sigma m=\Sigma Y, \ddot{z} \Sigma m=\Sigma Z,
$$

so that the centre of inertia moves like a particle of mass equal to the mass of the system under the action of the resultant of all the forces applied to the system.

109. Motion about the centre of inertia. In the equations such as $\Sigma m(y \ddot{z}-z \ddot{y})=\Sigma(y Z-z Y)$ put $x=\bar{x}+x^{\prime}, \ldots$ The left-hand member of the equation just written becomes

$$
[(\ddot{y}-\bar{z} \ddot{y}) \Sigma m]+\Sigma m\left(y^{\prime} \ddot{z}^{\prime}-z^{\prime} \ddot{y}^{\prime}\right),
$$


and the right-hand member becomes

$$
[\bar{y} \Sigma Z-\bar{z} \Sigma Y]+\Sigma\left(y^{\prime} Z-z^{\prime} Y\right) \text {. }
$$

The terms in square brackets in the two members are identical, and we thus have such equations as

$$
\Sigma m\left(y^{\prime} \ddot{z}^{\prime}-z^{\prime} \ddot{y}^{\prime}\right)=\Sigma\left(y^{\prime} Z-z^{\prime} Y\right) \text {. }
$$

These can be stated in words:-The rate of increase of the moment of momentum in the motion relative to $G$ about any line through $G$, is equal to the sum of the moments of the external forces about the same line.

110. Independence of translation and rotation. From the results of the last two articles we see that the motion of the centre of inertia is determined by the external forces independently of any motion relative to the centre of inertia, and the motion relative to the centre of inertia is determined independently of the motion of the centre of inertia.

111. Conservation of Linear Momentum. When the resultant external force on a system has no resolved part parallel to a particular line, the sum of the resolved parts of the kinetic reactions of the particles parallel to that line is zero. Hence the rate of increase of the resolved part of the linear momentum of the system parallel to that line is zero, or the resolved part of the linear momentum parallel to the line is constant.

In such a case the resolved part, parallel to the line, of the velocity of the centre of inertia is constant.

A simple example is afforded by the motion of a body near the Earth's surface; the horizontal component of the velocity of the centre of inertia of the body is constant. As in Article 45, the centre of inertia describes a parabola however the body moves about it.

The motion of the centre of inertia of a body which is so remote from every other body that the resultant force acting upon it can be disregarded is uniform motion in a straight line. The same holds for the centre of inertia of any system of bodies or particles supposed removed from all external action. We note that any such statement implies that a definite frame of reference has been chosen, a point to which we shall revert in Chapter XIII. 
112. Conservation of angular momentum. When the sum of the moments of the external forces on a system about a particular axis is zero, the sum of the moments of the kinetic reactions about the same axis is zero, and therefore the moment of momentum of the system about the axis is constant.

A simple example is afforded by the motion of a particle acted on by a force always passing through a point whose position relative to the frame of reference is fixed. For such a case we have, as in Article 50, an equation expressing that the moment of the velocity about the point is constant. Thus the equation $p v=h$ for central accelerations may be regarded as an example of the principle just stated.

When all the external forces acting on a system reduce to a resultant force at the centre of inertia without any couple, the sum of the moments of the external forces about any axis through the centre of inertia vanishes; the rate of increase of the moment of momentum about any such axis therefore vanishes, and the moment of momentum of the system about any such axis is constant. We note that in this statement it is implied that the axis occupies a fixed position relative to the frame of reference.

113. Equations of Impulsive Motion. As in Article 105 , let $X+X^{\prime}$ be the sum of the resolved parts parallel to the axis $x$ of all the forces, external and internal, that act on a particle $m$; and, as in Article 82 suppose $X$ and $X^{\prime}$ do not remain finite at time $t$, but that the impulses of $X$ and $X^{\prime}$ are finite, or that $X$ and $X^{\prime}$, defined by the equations

$$
\mathrm{Lt}_{\tau=0} \int_{t-\frac{1}{2} \tau}^{t+\frac{1}{2} \tau} X d t=\underset{\bullet}{X}, \mathrm{Lt}_{\tau=0} \int_{t-\frac{1}{2} \tau}^{t+\frac{1}{2} \tau} X^{\prime} d t=X^{\prime}
$$

are finite. Let $\dot{x}$ and $\dot{\xi}$ be the resolved parts parallel to the axis $x$ of the velocity of $m$ just after the instant $t$ and just before this instant respectively. Then we have the equation

$$
m(\dot{x}-\dot{\xi})=\underset{X}{ }+\underline{X}^{\prime} \text {. }
$$

In like manner the impulsive changes of velocity parallel to the axes $y$ and $z$ will be determined by equations which may be written

$$
\begin{aligned}
& m(\dot{y}-\dot{\eta})=Y+\underline{Y}^{\prime} \\
& m(\dot{z}-\dot{\zeta})=\underline{Z}+\underline{Z}^{\prime}
\end{aligned}
$$


Observing that it is a consequence of the Law of internal action (Article 106) that the system $X^{\prime}, \underline{Y}^{\prime}, Z^{\prime}$ is equivalent to zero, and operating as in Article 107, we can deduce equations which may be stated in words :-

The change of momentum of the particle $G$ in any direction is equal to the sum of the resolved parts of the external impulses in that direction.

The change of the moment of momentum of the system about any axis is equal to the sum of the moments of the external impulses about that axis. 


\section{CHAPTER VII.}

\section{SYSTEMS OF FORCES.}

114. Conception of a rigid system. We can imagine that a system of particles moves in such a way that the distance between any two particles of the system is constant. Such a system is said to be rigid.

In particular if the particles of a rigid system continuously fill a surface the system is a rigid body, and the surface is the surface of the body.

In a rigid system the motion of the whole system is determined when the motion of three of its particles is determined. For the three particles determine a frame of reference relatively to which all the particles of the system have invariable positions.

To determine the positions of all the particles of a rigid system relative to a frame is therefore the same thing as determining the position of one frame, $F$, relative to another. This requires the determination of the positions of the origin of the frame $F$, of one of its lines of reference, and of a plane through that line. The position of a point depends on three quantities, the coordinates of the point. The position of a line through a point depends on two quantities, since the line may make any angle with one of the axes, and the plane through it parallel to that axis may make any angle with a coordinate plane, but these two angles determine the line. The position of a plane through a line depends on one quantity, which may be taken to be the angle it makes with the plane drawn through the line parallel to one of the axes of reference. Thus the positions of all the particles of a rigid system relative to a frame are determined when six quantities such as those specified are given.

It follows that the velocities and accelerations of all the particles of a rigid system must be expressible in certain definite 
forms in terms of the velocities and accelerations with which six particular quantities increase.

In order that a system may be rigid it is necessary that the component accelerations contributed to any particle by the actions of the other particles should satisfy such conditions that the resultant acceleration of any particle of the system is expressible in the proper form in terms of six quantities such as those above described. It follows that the internal forces between the particles of the system must be so adjusted as to produce such accelerations. The internal forces in question are not subject to any other condition, and we may arbitrarily assume any system of internal forces provided the assumed system obeys this condition.

115. Forces applied to a rigid system. Suppose a rigid system is in motion in any manner, and suppose, accordingly, that the internal forces are adjusted to satisfy the condition just described.

Let two forces equal in magnitude and opposite in sense be applied to two particles, the line of action of the forces being the line joining the particles. To fix ideas, let $m, m^{\prime}$ be the masses of the particles, and let the force applied to $m$ be of magnitude $R$ in the sense from $m$ to $m^{\prime}$.

The internal forces will be altered in some way consistent with the rigidity of the system, and it is clear that, if the internal force assumed to act between the two particles $m$ and $m^{\prime}$ before the application of the forces $R$ is modified by compounding with it a repulsion of magnitude $R$ between these particles, the resultant forces on all the particles will be the same as before the application of the forces $R$ to $m$ and $m^{\prime}$, and the system will still be rigid, and will be moving exactly as before.

It follows that the motion of the rigid system is unaffected by the application to any two particles of the system of a pair of equal and opposite forces in the line joining the particles.

Now let $F$ be any external force applied to a particle $m$ of the system, and let $m^{\prime}$ be a particle in the line of action of $F$. Suppose applied at $m^{\prime}$ a force of the same magnitude, direction and sense as $F$, and suppose applied at $m$ a force of the same magnitude and in the same direction as $F$ but opposite in sense. The motion of the system is unaffected.

Thus the effect of a force applied to any particle of a rigid 
system is the same as that of an equal parallel force of like sense applied to any other particle of the system which lies in the line of action of the force.

We may express this result by saying that forces applied to particles of a rigid system may be regarded as vectors localised in their lines of action instead of vectors localised at the positions of the particles. This is the result referred to in Article 76.

The result is known as the Principle of the Transmissibility of Force.

116. Inviolable conditions. A rigid system is an example of a system which moves in such a way that certain geometrical relations are maintained, and the internal forces between the particles of the system are so adjusted from instant to instant that the conditions are never violated.

The notion thus introduced may be extended to cases where the system is not rigid, or where external forces are adjusted so that certain geometrical conditions are never violated. In such cases generally some external forces are given, and others are in our power, and the latter can be adjusted as stated.

\section{Reaction of bodies in contact. The Postulate 5} of Article 87 frequently involves a geometrical relation which is to be maintained.

This happens when two bodies are in contact. So long as they are not separated their surfaces touch, they never intersect.

The maintenance of this condition requires a particular application of force to both.

When the surfaces touch at isolated points, or at a point, the forces required to maintain the condition may be regarded as applied to the particles which are at the points of contact in the directions of the common normals.

Let $A$ and $B$ be the bodies, $P$ a point of contact. Then the forces applied to $P$, considered as a particle of $A$, are of two sorts, forces exerted by the other particles of $A$, and forces exerted by the particles of $B$, the latter are regarded as having a finite resultant, known as the reaction of $B$ upon $A$ at the point $P$. In like manner the reaction of $A$ upon $B$ at the point $P$ is made up of 
component forces between particles, viz. of the forces exerted by the other particles of $A$ upon the particle at $P$.

The two resultant forces thus defined are equal and opposite, since otherwise the particle at $P$ (of infinitesimal mass) would have infinite acceleration.

We can imagine two cases: (1) where the reaction of $A$ on $B$ is directed along the common normal at $P$, it is then the force required to maintain the condition; (2) where the reaction of $A$ on $B$ is not directed along the common normal, but nevertheless the condition is maintained.

In the first case the reaction is known as the pressure between the bodies at $P$, and the surfaces are said to be smooth at $P$.

In the second case the resolved part of the reaction along the common normal is called the pressure between the bodies at $P$, and the resolved part at right angles to the normal is called the friction at $P$, and the surfaces are said to be rough at $P$.

When the surfaces are in contact over a finite area we are at liberty to assume reactions, such as those described in the case of contact at points, to act upon the bodies at every point of the area of contact, such reactions having finite resultants and finite sums of moments about any axis.

118. Constraints. More generally, when a system moves so that one of its particles always lies on a prescribed geometrical curve or surface, this condition can be maintained by a force applied to the particle along the normal to the curve or surface, and then the force is known as a constraint. We may say that constraints are forces applied to the particles of a system moving in a field of force, in directions at right angles to the paths of the particles, and required for the maintenance of geometrical conditions. Constraints must arise from the actions of particles, but the particles producing them are not necessarily particles of a body on whose surface the constrained particle moves.

119. Friction. We have already defined the friction between two rough bodies at a point of contact to be the resolved part of the reaction at right angles to the common normal.

As before, let $A$ and $B$ be the bodies, $P$ a point of contact, $R$ the pressure, and $F$ the friction at $P$. 
Each of the bodies is regarded as having a particle at $P$.

The particle of $A$ at $P$ will have a certain velocity, and similarly for the particle of $B$ at $P$. The velocity of the particle of $A$ at $P$, relative to axes parallel to the axes of reference drawn through the particle of $B$ at $P$, is the relative velocity of the points in contact considered as the velocity of a point of $A$ relative to $B$. In like manner there is an equal and opposite relative velocity of the points in contact considered as the velocity of a point of $B$ relative to $A$.

The condition of contact is that the relative velocity just described is localised in a line in the tangent plane at $P$, or that the resolved part of this velocity in the direction of the common normal vanishes.

The first law of Friction is that the friction on $\left\{\begin{array}{l}A \\ B\end{array}\right\}$ at $P$ is opposite in sense to the relative velocity of the points in contact considered as the velocity of a point of $\left\{\begin{array}{l}A \\ B\end{array}\right\}$ relative to $\left\{\begin{array}{l}B \\ A\end{array}\right\}$.

The second law of Friction is that the friction $F$ and the pressure $R$ are connected by a relation of inequality $F \leqslant \mu R$, where $\mu$ is a constant depending only on the materials of which the bodies are composed. The constant $\mu$ is called the coefficient of friction.

When the relative velocity above described is zero, the motion is described as rolling. In order that rolling may take place it is generally necessary that the coefficient of friction should exceed a certain number depending on the circumstances of the case. A motion of two bodies in contact which is not one of pure rolling is described as a motion of sliding or slipping. The rule for the direction of friction may be stated in the form that friction tends to prevent slipping. When slipping takes place $F=\mu R \quad$ When the bodies are sufficiently rough to prevent slipping throughout the motion they are sometimes said to be perfectly rough.

120. Resistances. Friction belongs to a class of forces known as resistances. The characteristics of a resistance are that it acts on a particle in the direction of its velocity relative to some frame (not necessarily the frame of reference) in the opposite sense to this velocity. 
121. Deformable bodies, strain. A system of particles which is not rigid can move so that the distance between two particles changes. Whenever this can happen a particular configuration is chosen as a standard, and, in a configuration in which the distance between any two particles is greater or less than that in the standard, the system is said to be strained.

For a continuous system of particles forming a deformable body it can be shown that the motion of the body can be precisely specified by the following method:-Choose one particle of the body, one line of particles going out from the chosen particle, one plane of particles passing through the chosen line. In the deformation of the body the line and the plane respectively become a curve and a curved surface of changing forms, but the curve has a tangent line, and the surface a tangent plane, passing through the chosen particle. The particle, the tangent line, and the tangent plane determine a frame relative to which all the particles of the body have at any time definite positions. The determination of the position of this frame relative to the frame of reference is, as we have seen in Article 114, the same problem as the determination of the position at any time of a rigid body. The determination of the positions of other particles of the body relative to this frame can be shown to depend upon the determination of six quantities continuously variable from point to point, and known as the components of strain. The theory which effects this determination is the Theory of Elasticity.

In the case of a solid body, for which it may be assumed that the deformation is always small, the above method can be applied with success. But it is manifestly less applicable in the case of a fluid, or of a plastic solid undergoing finite deformation.

In all cases, however, the equations of motion of a system of particles, obtained in Article 107, have to be applied to every portion into which the system can be divided. To succeed in this process we must consider more closely the resultants of internal forces between parts of a system.

122. Stress. Conceive that in a continuous (or discontinuous) system of particles, exerting forces on each other, a plane is drawn, and a closed curve marked on the plane. Some of the lines between particles cross the plane within the curve. Let the 
forces acting in these lines on the particles on one side of the plane be reduced to a resultant, at the centroid, $A$, of the area within the curve, and a couple. Now let the curve, remaining in its plane and similar to itself, be indefinitely contracted, so that it tends to the point $A$ at the original centroid. At any stage of the process let $R$ be the resultant, and $\sigma$ the area in question. Then a vector localised at the point $A$, of magnitude equal to the limiting value of the ratio $R / \sigma$, and in the direction and sense which $R$ takes in the limit when $\sigma$ is indefinitely small, is called the stress at $A$ across the plane.

The stress is conceived to be exerted by the part of the system on one side of the plane upon the part on the other side of the plane. Since the stress was compounded from internal actions between particles on the two sides of the plane, there is an equal and opposite stress exerted by the part of the system on the second side of the plane upon the part on the first side.

When the stress is normal to the plane it is called tension or pressure according as it acts on the part of the system on one side of the plane in the sense from that side towards the other side or in the opposite sense.

Now the method by which the general equations of motion, for any part of a body, are applied to deformable bodies consists in regarding the internal forces as always equivalent to stresses across the elements of the surface of any part of the body.

\section{Reaction of bodies in contact realised as resultant}

stress. To define stress at a point across a plane we began by considering the forces exerted by particles on one side of the plane upon particles on the other side, and the resultant of these forces for a finite area of the plane. This resultant may be called the resultant stress across the area.

In the same way when two bodies are in contact over a finite plane area we may define the resultant stress which either exerts on the other across the area.

If the area is indefinitely diminished, so that it is contracted to a point, it is manifest that the resultant stress so defined ought to be regarded as reducing to the reaction between the two bodies described in Article 117. 
The identification of the resultant stress with the reaction requires us to make the supposition that the bodies are rigid. For it is then immaterial whether the action of the particles of $B$ upon the particles of $A$ in lines passing indefinitely close to the point of contact $P$ are regarded as exerted upon the particle of $A$ at $P$ or upon other particles of $A$.

If we regard a rigid body as the limiting case of a deformable body when all the numbers expressing deformations fall below any assigned number, however small, then it is clear that there will be a certain order of approximation to which it will be correct to neglect the distinction between forces exerted by the particles of $B$ upon the particles of $A$ and the forces exerted by the particles of $B$ upon the particle at $P$.

In this way the conception of rigid bodies and the reactions at their points of contact becomes of importance in Mechanics.

\section{Bodily forces and surface-tractions. Consider any} deformable system of particles continuously distributed so as to form a body. In any infinitesimal volume $d v$, where the density is $\rho$, we say there is a particle of mass $\rho d v$.

The field of force due to the actions of the particles of other bodies would produce in this particle a resultant acceleration $f$, where $f$ is the intensity of the field of force at the position of the particle.

The force exerted on the particle by the particles of other bodies is accordingly $\rho f d v$ in the direction and sense of $f$.

Since this force is proportional to the mass of the particle, the resultant of such forces on all the particles in a small volume contains the mass within the volume as a factor. Such forces are known as bodily forces.

On the other hand the resultants, for any part of a surface bounding a portion of a body, of the stresses exerted on that portion by other portions of the body, or by portions of other bodies, in contact with it over the part of the surface, are known as surface tractions. The expressions for the resolved parts of such tractions on small areas contain as factors the areas of the surfaces across which they act. 
125. Tension of a string or chain. In Mechanics a string is regarded as a mere line of particles, but the mass of any segment of the string of finite length is supposed to be finite. When the mass is proportional to the length the string is said to be uniform, but when this is not the case the ratio of the number of units of mass in any segment to the number of units of length in the segment has a finite limit when the length of the segment is indefinitely diminished, and this ratio is called the line-density or mass per unit length of the string.

A plane drawn across a string, at right angles to the tangent to the line of the string at the point where the plane meets it, is called a normal plane. The resultant stress across the normal plane is conceived to be a single force at the point where the line meets the plane, and directed along the tangent. This stress is a tension, (Article 122) and is called the tension of the string at the point.

Any portion of a string between two particles $P, P^{\prime}$ moves under the action of the external forces on its particles and the tensions at its ends. The motion of its centre of inertia depends on these forces only.

In particular if $P^{\prime}$ is very close to $P$, and if $S$ is the sum of the resolved parts parallel to the tangent at $P$ (in the sense $P P^{\prime}$ ) of the external forces applied to the particles in the segment $P P^{\prime}, \rho$ the line density, $\Delta s$ the length of $P P^{\prime}, f$ the acceleration of the centre of inertia of $P P^{\prime}$ in the direction of the tangent at $P, T$ the tension at $P, T^{\prime \prime}$ the tension at $P^{\prime}$, and $\Delta \phi$ the angle between the tangents at $P$ and $P^{\prime}$, we have the equation

$$
\rho \Delta s . f=T^{\prime} \cos \Delta \phi-T+S .
$$

Now when $P^{\prime}$ approaches indefinitely close to $P$ the expression $\frac{T^{\prime} \cos \Delta \phi-T}{\Delta s}$ will have a limit which is $\frac{d T}{d s}$ (cf. Article 35 ), and $\frac{S}{\rho \Delta s}$ will have a finite limit which is the acceleration produced in a particle at $P$ by the external forces. Suppose that the external forces are bodily forces, and suppose that this acceleration is $F$.

Then our equation can be written

$$
\rho f=\frac{d T}{d s}+\rho F
$$


A case of great importance is that in which $\rho$ is regarded as infinitesimal. For small values of $\rho$ there is clearly an order of approximation to which it is correct to write the above equation $\frac{d T}{d s}=0, \rho f$ and $\rho F$ being smaller than a number which we agree to neglect; then, to the same order of approximation, $T$ is constant.

When the mass of a string is neglected the tension is constant throughout any portion which is subject only to bodily forces and terminal tensions.

A string of which the mass is neglected will frequently be called a thread, and one of which the mass is not neglected a chain.

\section{Reaction of string in contact with surface. When} the string is in contact with a body, there will be forces which are not bodily forces arising from the mutual actions of the particles of the string and the particles of the body exerted across the surface of the body. When the surface is smooth the resultant of these actions upon any element of the string will be directed along the normal to the surface, and will therefore have no resolved part in the direction of the tangent line to the string. The equation (A) will therefore still hold, and, as before, it will follow that when the mass of a string is neglected the tension is constant throughout any portion in contact with a smooth surface.

Whether the surface is smooth or not there will be a finite pressure per unit length at any point. Taking as before $P, P^{\prime}$ to be the positions of two particles of the string near together, the reaction of the surface upon the part of the string between $P$ and $P^{\prime}$ can be resolved into a pressure along the normal at $P$, and tangential forces in the tangent plane at $P$ to the surface. If $P P^{\prime}$ is of length $\Delta s$, then these will in general be the products of finite quantities and $\Delta s$. Thus if $R \Delta s$ is the pressure on the element, $R$ is the pressure at $P$ per unit length, and $R$ is always a finite quantity.

127. Extensible string. When the string changes its length there is always a particular length for each portion of it such that the tension is throughout zero. When every portion has this length the string is said to be in its natural state.

Let $\Delta s_{0}$ be the length of a short piece of the string in its natural state, and $\Delta s$ the length of the same piece in any other 
state. We suppose $\Delta s_{0}$, having one and the same particle always at one of its ends, to be indefinitely reduced. Then we define the limit of the ratio $\frac{\Delta s-\Delta s_{0}}{\Delta s_{0}}$, when $\Delta s_{0}$ is indefinitely diminished, to be the extension of the string at the point to which $\Delta s_{0}$ approaches. Thus the extension is measured by the ratio of the increase of length to the natural length.

In an extensible string the tension at any point is a constant multiple of the extension at the same point, or, if $\lambda$ is a certain constant depending on the material of the string, and of the dimensions of force, the tension is $\lambda \epsilon$, where $\epsilon$ is the extension.

The constant $\lambda$ of an extensible string is the modulus of elasticity of the string.

When the mass of the string is neglected the tension is constant throughout, and so is the extension*. In this case the extension is equal to $\left(l-l_{0}\right) / l_{0}$, where $l_{0}$ is the length of the string in its natural state (called the natural length), and $l$ is the length of the string when extended.

128. Inextensible string. A string which exerts tension, but is never sensibly extended, must be thought of as the limit to which an extensible string approximates, when the extension $\epsilon$ remains always less than a number which we agree to neglect, but the modulus $\lambda$ is very great, in such a way that the product $\lambda \epsilon$ is of the order which we agree to retain.

129. Spring. The word spring is used in Rational Mechanics to express an idea suggested by generalising the idea of an extensible string. We imagine a line of particles in which the stress across a plane normal to the line is always either pressure or tension, and a natural state of the system in which it vanishes. When the length of any element exceeds its natural length there is extension, and tension equal to the product of $\lambda$ and the extension. When the length of any element is less than its natural length there is contraction, measured by the ratio of the diminution of length to the natural length, and pressure equal to the product of $\lambda$ and the contraction. The constant $\lambda$ is the modulus of elasticity of the spring.

* There is a reservation for contact with a rough body. 
130. Stress and Strain. The tension of a string is an example of stress, and the extension or contraction of a spring is an example of strain. The six components of strain in a body, referred to in Article 121, are quantities in terms of which the extensions and contractions of all the lines joining particles of a body which are near together can be expressed. The law that the tension is proportional to the extension is the particular case for elastic strings of a general law connecting the stresses and strains in a body. It is of almost universal application when the strains are sufficiently small. 


\section{CHAPTER VIII.}

\section{THEORY OF WORK AND ENERGY.}

131. Work of a Force. Consider a force of magnitude $P$ applied to a particle at a point $A$.

Let the particle be displaced to a point $A^{\prime}$ so that the displacement $A A^{\prime}$ and the line of action of $P$ include an angle $\theta$.

The product $P \cdot A A^{\prime} \cdot \cos \theta$ is defined to be the work of the force in the displacement of the particle. It is the product of the magnitude of the force and the resolved part of the displacement in the direction of the force, it is also the product of the magnitude of the displacement and the resolved part of the force in the direction of the displacement.

Since the sum of the resolved parts in any direction of a set of forces acting on a particle is the resolved part of their resultant in that direction, it is clear that the sum of the works of the components in any displacement is equal to the work of the resultant in that displacement.

132. Work done. When the displacement is infinitesimal, the work is infinitesimal. The work of a force in any infinitesimal displacement of the point at which it is localised is defined to be the work done.by the force in that displacement.

Let $X, Y, Z$ be the resolved parts of the force parallel to the axes of reference, $\delta x, \delta y, \delta z$ the resolved parts of the displacement, then the work done is

$$
X \delta x+Y \delta y+Z \delta z
$$

When the work done by a force is negative it is said to be done "against" the force. 
133. Work done by a force in a finite displacement. Let a particle move from a point $P_{0}$ to a point $P$ by any path. Choose on the path $n-1$ intermediate points $P_{1}, P_{2}, \ldots P_{n-1}$. Let $F_{1}$ be the magnitude of the resultant force acting on the particle at any point between $P_{0}$ and $P_{1}, \theta_{1}$ the angle between the line of action of $F_{1}$ and the displacement $P_{0} P_{1}$; let $F_{2}$ be the magnitude of the resultant force acting on the particle at any point between $P_{1}$ and $P_{2}, \theta_{2}$ the angle between the line of action of $F_{2}$ and the displacement $P_{1} P_{2}$; proceeding in this way we can assign the meanings of all the quantities that enter into the sum

$$
F_{1} \cdot P_{0} P_{1} \cdot \cos \theta_{1}+F_{2} \cdot P_{1} P_{2} \cdot \cos \theta_{2}+\ldots+F_{n} \cdot P_{n-1} P \cdot \cos \theta_{n} .
$$

Now let the number $n$ be indefinitely increased, and let all the chords $P_{r} P_{r+1}$ be indefinitely diminished. The sum just written tends to a limit represented by

$$
\int F \cdot \cos \theta \cdot d s
$$

where $d s$ is any element of the path, $F$ the resultant force on the particle as it passes over the element, and $\theta$ the angle between the line of action of $F^{\prime}$ and the tangent to the path at the element, the integral being a line-integral taken along the path.

The integral just written down is clearly the sum of the elements of work done by the force on the particle in an infinite series of infinitesimal displacements equivalent to a displacement from the point $P_{0}$ to the point $P$. It is defined to be the work done by the force in the actual displacement of the particle.

If $X, Y, Z$ are the resolved parts, parallel to the axes, of the resultant force on the particle at any point of its path, $d x, d y, d z$ the resolved parts of an infinitesimal displacement from that point to a neighbouring point, the expression for the work done may be written

$$
\int X d x+Y d y+Z d z
$$

where the integral is a line-integral taken along the path.

134. Work done by a system of forces. Suppose a system of forces to be applied to a system of particles. If we form a sum of terms, each term being the work done by the forces on one of the particles in the displacement of that particle along its path, 
from the position it occupies at time $t_{0}$ to the position it occupies at time $t$, the sum so formed is the work done by the forces of the system in the displacements of the particles from their positions at time $t_{0}$ to their positions at time $t$.

In the notation of the last Article this work is represented by $\Sigma \int F \cdot \cos \theta \cdot d s$ or by $\Sigma \int X d x+Y d y+Z d z$, the integrals being line-integrals along the paths of the particles, and the summations extending to all the particles.

135. Conservative system. The work done by a system of forces in the displacements of a system of particles from a set of initial positions to a set of final positions is expressed analytically in terms of quantities depending on the actual paths of the particles. If the path of any particle were replaced by a different curve the work done by the force on that particle would be in general different, and the work of the system would also be different.

But there is a most important class of cases in which the work done by the forces as the particles pass from one set of positions to another is independent of the paths, and depends only on the initial and final positions. When this is the case the system is said to be conservative. Otherwise the system is said to be non-conservative.

It is clearly necessary, for a system of forces to be conservative, that, in any position of the particles, the forces acting upon them should be one-valued functions of the quantities defining their positions. This condition though necessary is not sufficient.

136. Work Function. For a conservative system there exists a function of the quantities that define the positions of the particles, which represents the work done by all the forces of the system as its particles pass to those positions from any particular set of positions. This function is known as the Work Function.

The particular set of positions from which the particles pass will be referred to as the standard position.

The work done in passing from the position at any time $t$ to the standard position is numerically equal to the work done in passing from the standard position to the position at time $t$, but 
has the opposite sign. For the forces are the same, at the same points, in magnitude, direction, and sense, but the infinitesimal displacements have their senses changed.

Since the work done in passing from one set of positions, denoted by $A$, to another, denoted by $B$, is independent of the paths, it is clearly equal to the sum of the works done in passing from the position $A$ to the standard position, and from the standard position to the position $B$. It is therefore equal to the difference

(value of work function in position $B$ ) - (value of work function in position $A$ ).

137. Potential Energy. The work function in any position $A$ with its sign changed is the work that would be done by the forces if the system passed from the position $A$ to the standard position. It is defined to be the Potential Energy of the system in the position $A$.

Only systems for which a work function exists, i.e. only conservative systems, can possess potential energy.

For the sake of precision we present our previous statements in the following form:-A system in which the work done by all the forces on all the particles, as they pass from one set of positions to another, is independent of the paths of the particles, is said to be a conservative system, and the work done by the forces of such a system, as its particles pass from any set of positions to a determinate standard set of positions, is called the potential energy of the system in the former set of positions.

*138. Analytical condition for conservative system. Any position of the system is supposed to be defined by assigning particular values to a certain set of geometrical quantities.

Suppose these quantities are $\theta, \phi, \psi, \ldots$

Then the co-ordinates of every particle of the system can be expressed in terms of the values of $\theta, \phi, \psi, \ldots$ at time $t$.

Let $x, y, z$ be the co-ordinates of any particle of the system at time $t$, $X, Y, Z$ the resolved parts, parallel to the axes, of the resultant force on it.

We must have such equations as

$$
x=f(\theta, \phi, \psi, \ldots)
$$

where $f$ denotes a one-valued function.

Differentiating we have

$$
d x=\frac{\partial f}{\partial \theta} d \theta+\frac{\partial f}{\partial \phi} d \phi+\ldots
$$


It follows that any expression which is linear in the differentials such as $d x$ is linear in the differentials such as $d \theta$.

Now $X, Y, Z$ will be one-valued functions of $\theta, \phi, \psi \ldots$ since, as remarked in Article 135, the system cannot otherwise be conservative.

Hence $\Sigma(X d x+Y d y+Z d z)$, the summation referring to all the particles, is capable of being expressed in the form

$$
\Theta d \theta+\Phi d \phi+\Psi d \psi+\ldots
$$

where $\Theta, \Phi, \Psi$ are finite one-valued functions of $\theta, \phi, \psi, \ldots$

Now it follows from the last paragraph of Article 136 that the work done in the small displacement represented by $d \theta, d \phi, d \psi, \ldots$ is the differential of the work function in the position represented by $\theta, \phi, \psi, \ldots$ Let this function be $W$.

Then we have an equation of the form

$$
d W=\Theta d \theta+\Phi d \phi+\Psi d \psi+\ldots,
$$

expressing that the right-hand member is the complete differential of the one-valued function $W$.

This result gives us such equations as

$$
\Theta=\frac{\partial W}{\partial \theta}, \quad \Phi=\frac{\partial W}{\partial \phi}, \ldots
$$

with the necessary corollaries

$$
\frac{\partial \theta}{\partial \phi}=\frac{\partial \Phi}{\partial \theta}, \ldots
$$

If there are $n$ quantities such as $\theta$, there are $\frac{1}{2} n(n-1)$ equations such as that last written. These equations constitute the analytical conditions that the system may be conservative.

\section{Examples of Calculation of Work.}

139. Work done in the rising or falling of a body. Let $M$ be the mass of any body, $g$ the acceleration due to gravity, $z_{0}$ the height of the centre of inertia of the body above a particular horizontal plane, and let the body move to any other position in which $z$ is the height of its centre of inertia above the same horizontal plane.

The work done by the weight of the body is $\operatorname{Mg}\left(z_{0}-z\right)$.

If $z_{0}<z$ the centre of inertia of the body is raised through a height $z-z_{0}$ and work of amount $\operatorname{Mg}\left(z-z_{0}\right)$ is done against the weight.

If the body is guided from the first position to the second under the action of forces whose resultant is always directed vertically upwards, and whose magnitude is always indefinitely little greater than the weight of the body, then the work done by these forces is $M g\left(z-z_{0}\right)$. 
140. Work done by the mutual actions between two particles. Let $A, B$ be the initial positions of the particles, $F$ the force between them. To

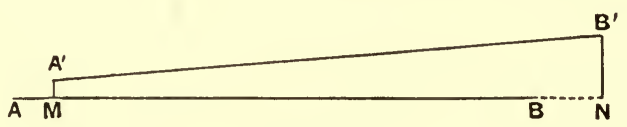

Fig. 42.

fix ideas suppose $F$ to act on the particle at $A$ from $A$ towards $B$. Let the length $A B=l$.

Let very small displacements be made so that $A$ comes to $A^{\prime}$ and $B$ comes to $B^{\prime}$; let the line $A^{\prime} B^{\prime}$ make with $A B$ an angle $\theta$, which must be very small when the displacements are very small. Also let $A^{\prime} B^{\prime}=l^{\prime}$, then $l^{\prime}-l$ also is very small.

Let $M, N$ be the projections of $A^{\prime}, B^{\prime}$ on $A B$; to fix ideas suppose $M$ is in $A B$, and $N$ in $A B$ produced.

The work done by the two forces $F$ is

$$
\begin{aligned}
& F \cdot A M-F \cdot B N \\
= & F(A M-B N) \\
= & F(A B-M N) \\
= & F\left(l-l^{\prime} \cos \theta\right) \\
= & F\left[\left(l-l^{\prime}\right)+l^{\prime}(1-\cos \theta)\right] .
\end{aligned}
$$

Now $1-\cos \theta$ is an infinitesimal of the second order when $\theta$ is of the first order, and the work done is therefore $F\left(l-l^{\prime}\right)$ to the first order of infinitesimals.

141. Case where the distance is invariable. When the particles move in such a way that the distance between them is unaltered, the sum of the works done by the forces exerted by the first on the second and by the second on the first is an infinitesimal of the second order when the displacements are of the first order. Hence in any finite displacement no work is done by such forces.

Examples of this are (1) the tension of an inextensible string does no work; (2) the forces between the particles of a rigid body do no work.

142. Work of gravitating system. Let $m, m^{\prime}$ be the masses of two particles of the system, $r$ the distance between them, and let this distance become $r+\delta r$, the work done is, by Art. 140,

$$
-\frac{\gamma m m^{\prime}}{r^{2}} \delta r \text {. }
$$

Hence the work done by the forces between these particles as their distance changes from $r_{0}$ to $r_{1}$ is

$$
\begin{aligned}
& -\int_{r_{0}}^{r_{1}} \frac{\gamma m m^{\prime}}{r^{2}} d r . \\
= & \gamma m m^{\prime}\left(\frac{1}{r_{1}}-\frac{1}{r_{0}}\right) .
\end{aligned}
$$


The work done in any displacement of the system is therefore

$$
\gamma\left[\Sigma \frac{m m^{\prime}}{r_{1}}-\Sigma \frac{m m^{\prime}}{r_{0}}\right],
$$

where $m, m^{\prime}$ are the masses of two particles, $r_{0}$ their initial distance, and $r_{1}$ their final distance, and the summation extends to all the pairs of particles.

For calculating the work function of such a system the most convenient position to choose as the standard position is one in which all the distances, such as $r_{0}$, are indefinitely great; then the work function is $\gamma \mathbf{\Sigma}\left(\mathrm{mm}^{\prime} / r\right)$ where $r$ is the distance between the particles $m$ and $m^{\prime}$.

143. Potential Function. The potential function of a gravitating system at a point is the work function for the system and a unit mass at the point, when the standard positions of all the particles of the system are the positions they occupy, and that of the unit mass is at an infinite distance.

If $m$ is the mass of any particle of the system, and $r$ the distance of this particle from the point, the potential function of the system at the point is $\gamma \Sigma(m / r)$.

If there is a particle of the system at the point that particle is to be omitted from the sum.

Let $V$ be the potential at $P$, and $V^{\prime}$ the potential at $P^{\prime}$; then $V^{\prime}-V$ is the work done by the forces in a displacement in which the particle of unit mass is displaced from $P$ to $P^{\prime}$, and the particles of the system are not displaced.

Let $F$ he the resultant force on the particle of unit mass at $P$, i.e. the intensity of the field at $P$, and let $X, Y, Z$ be its resolved parts parallel to the axes of reference; then, if $P P^{\prime}$ is infinitesimal, and $\delta x, \delta y, \delta z$ are its projections on the axes of reference, and if $V^{\prime}=V+\delta V$, we must have

$$
\delta V=X \delta x+Y \delta y+Z \delta z \text {. }
$$

If $P P^{\prime}$ is parallel to the axis of $x, \delta V$ is the change made in $V$ by changing $x$ into $x+\delta x$ without altering $y$ or $z$, and thus we have

$$
X=\frac{\partial V}{\partial x} \text {. }
$$

In the same way $Y=\frac{\partial V}{\partial y}$, and $Z=\frac{\partial V}{\partial z}$.

This shows that the resolved part in any direction of the force on the particle is the rate of variation of the potential per unit of length in that direction.

144. Potential energy of gravitating system. Let $m_{1}, m_{2}, \ldots$ be the masses of the particles, $r_{12}$ the distance between the positions of $m_{1}$ and $m_{2}$, and similarly for the others, and let the standard position be that in which all the distances, such as $r_{12}$, are indefinitely great.

L. 
The potential energy is

$$
-\gamma \Sigma \frac{m_{1} m_{2}}{r_{12}}
$$

the summation being extended to all the pairs of particles.

It is clear that this can be written

$$
-\frac{1}{2}\left[m_{1} \gamma\left(\frac{m_{2}}{r_{12}}+\frac{m_{3}}{r_{13}}+\ldots\right)+m_{2} \gamma\left(\frac{m_{1}}{r_{12}}+\frac{m_{3}}{r_{23}}+\ldots\right)+\ldots\right],
$$

and then the factor multiplying $m_{1}$ is the potential of the system at the point occupied by $m_{1}$, the factor multiplying $m_{2}$ is the potential of the system at the point occupied by $m_{2}$, and so on.

Thus the potential energy of the system is $-\frac{1}{2} \Sigma m V$, where $m$ is the mass of any particle, and $V$ the potential of the system at the point occupied by it.

145. Gravity. Consider the particular case of a heavy body near the Earth's surface. We regard the Earth as a spherical body with its mass so arranged that its attraction on a particle at an external point is the same as that of a particle at its centre of mass equal to the mass of the Earth. Let $E$ be this mass.

Then the particles of the Earth exert on a particle of mass $m$ near its surface a force $\gamma \frac{E m}{r^{2}}$ where $r$ is the distance of the particle from the Earth's centre.

This is very nearly equal to the weight of the particle, viz. to $m g$, where $g$ is the acceleration due to gravity.

We therefore have $\gamma \frac{E}{r^{2}}=g$ approximately.

When the particle is displaced so that $r$ becomes $r+\delta r$ the work done by the forces is $-m g \delta$.

Now when the particle remains near the Earth's surface at a place, $\delta r$ is the height above a horizontal plane through which it is raised, and the height through which the centre of inertia of a system of such particles is raised is

$$
\frac{\Sigma m \delta r}{\Sigma m} \text {. }
$$

Hence the work done in the displacement of the body is $M g h$, where $M$ is the mass of the body and $h$ the height through which its centre of inertia falls.

This is the same result as that differently expressed in Article 139.

146. Examples of conservative systems. The gravitating system discussed above is an example of a conservative system. No matter by what paths the particles move from one set of positions to another the work done depends only on the initial and final distances. 
It is clear that this is the case also whenever the force between any two particles is a one-valued function of their distance and does not depend upon any other variable.

There is a more general case possible, viz., when the force between two particles is a one-valued function of their distance and of the other distances between particles of the system; provided such functions satisfy certain conditions.

147. Potential Energy of Stretched String. In the case of gravitation the work done by internal forces in any displacement can be calculated directly, even for the case of a continuous body, but in other cases, where the internal forces themselves are only assigned as regards their resultants across small elements of area, the work done by the forces constituting these resultants is calculated indirectly by the application of the Principle of Virtual Work. (Article 157.)

We shall carry out the calculation for an extensible string.

Suppose an element of the string of natural length $\Delta s_{0}$, which will presently be taken infinitesimal, is in equilibrium under the tensions at its ends and any bodily forces, and suppose $\epsilon$ is the extension of the element, and $\lambda$ the modulus of elasticity. Then, with sufficient approximation, the length is $(1+\epsilon) \Delta s_{0}$, and the tension at either end is $\lambda \epsilon$. The bodily force acting on the element is of the order $\lambda \epsilon . \Delta s_{0}$, viz., of the order of the difference of tensions at the ends.

Now let the ends be slightly displaced so that the length is increased, and let the extension become $\epsilon+\Delta \epsilon$, so that the length is $(1+\epsilon+\Delta \epsilon) \Delta s_{0}$. The work done by the tensions at the ends is $\lambda \epsilon . \Delta \epsilon . \Delta s_{0}$. The work done by the bodily forces is of the same order as the product of this quantity and $\Delta s_{0}$.

Let $\Delta W^{\prime}$ be the work done by the internal forces during the displacement, then, with sufficient approximation, the equation of Virtual Work is

$$
\Delta W^{\prime}+\lambda \epsilon . \Delta \epsilon . \Delta s_{0}=0 .
$$

It thus appears that the work done by the internal forces within the element $d s_{0}$ of the string in an indefinitely small displacement $d \epsilon$ is $d W^{\prime}$, where

$$
d W^{\prime}=-d s_{0} \cdot \lambda \epsilon . d \epsilon
$$

Hence the work done by the internal forces in the string, when it is stretched from its natural length until the extension at any element becomes $\epsilon$, is $W^{\prime}$, where

$$
\begin{aligned}
W^{\prime} & =-\int d s_{0} \int_{0}^{e} \lambda \epsilon d \epsilon \\
& =-\frac{1}{2} \lambda \int \epsilon^{2} d s_{0},
\end{aligned}
$$

the integral being taken along the string.

When the string is uniformly extended we find

$$
W^{\prime}=-\frac{1}{2} \lambda \epsilon^{2} s_{0}=-\frac{1}{2}(\lambda \epsilon)\left(s_{0} \epsilon\right)
$$


where the two factors are the tension in the stretched state and the increase of length.

Referring to the definition in Article 138 we see that the potential energy of the stretched string is $\frac{1}{2} \lambda \epsilon^{2} s_{0}$, when the extension is uniform, and is $\frac{1}{2} \lambda \int \epsilon^{2} d s_{0}$ in any case, the standard state being that in which the string has its natural length. This potential energy is equal to the work that would be done by the internal forces between the parts of the string if the string were allowed to contract freely to its natural length.

148. Spring. In the same way, for a spring whose mass is neglected, whether the spring is extended or contracted, the potential energy when the length is $l$ is $\frac{1}{2} \lambda \frac{\left(l-l_{0}\right)^{2}}{l_{0}}$, where $l_{0}$ is the natural length, and $\lambda$ the modulus of elasticity.

149. Localisation of Potential Energy. The potential energy of a gravitating system and the potential energy of a stretched string are two examples of the potential energy that arises from internal forces between the parts of a system.

But the two cases present a marked difference. In the case of the string we are able definitely to assign a certain amount of the potential energy to every element of the string, that amount depending only on the state of the element. We can do this in such a way that every change in the energy so assigned to the element corresponds to a definite change in the state of that element. We may therefore say that the energy is located in the element. The amount so located in an infinitesimal element is $\frac{1}{2} \lambda \epsilon^{2} d s_{0}$. We can think of it as possessed by the element, just as kinetic energy is possessed by a particle.

In the case of the gravitating system we are not able to assign a certain amount of the potential energy to any part of the system, in such a way that changes of the energy so assigned correspond to changes in the state of that part, independently of changes in the position of the part relative to other parts. We cannot, in any way that shall be completely satisfactory, locate some portion of the energy in one part of the system, another portion in another part of the system, and so on. For instance in the case of the heavy body near the Earth's surface we cannot locate the energy in the body, nor in the Earth, nor in any definite proportion some of it in the body and some in the Earth. We have to think of it as possessed by the system, not by the bodies composing the system.

It is however frequently convenient, when discussing the motion of a body in a conservative field, to attach, in imagination, the potential energy of the system to the body, and when we do this we shall speak of the energy as "potential energy of the body in the field." For example, the potential of a gravitating system at a point, with its sign changed, is the potential energy of a unit mass at the point in the field of the gravitating system. In the case of a heavy body near the Earth's surface the expression Mgh of Article 139 is the potential energy of the body in the field of the Earth's gravitation, when its centre of inertia is at a height $h$ above a particular horizontal plane. 
150. Forces which do no work. Constraints. Among forces which do no work we have already had occasion to notice,

(1) the tension of an inextensible string,

(2) the internal forces between the parts of a rigid body.

Both of these are of the nature of constraints, viz., they are forces required to maintain geometrical conditions.

Other examples of forces which do no work are

(1) the pressure between smooth surfaces,

(2) the reaction between rough surfaces which roll on each other.

These also are forces required to maintain geometrical conditions.

In the case of smooth surfaces there is no relative displacement of the points that come into contact in the direction of the pressure, and thus as much work is done against the pressure on one body as is done by the pressure on the other body at any point of contact.

In the case of rough surfaces which roll on each other there is no relative displacement of the points that come into contact in any direction, and thus as much work is done against the pressure on one body as is done by the pressure on the other body at any point of contact, and as much work is done against the friction on one body as is done by the friction on the other body at any point of contact.

Care is required in the application of these results to the discussion of the motion of one body in contact with another body. When both bodies are in motion, relatively to the frame of reference, usually the pressure, or reaction, on one body at a point of contact does work, and this work must not be omitted from the work function (if there is one) of the forces acting on that body. Thus it may well happen that there is no energy equation (Article 151) for a single body, forming part of a system for which there is an energy equation.

When however a body slides in contact with a smooth, or rolls in contact with a rough, surface which has a fixed position relative to the frame of reference, then the pressure, or reaction, at a point of contact does no work,

The notion of constraint, explained in Article 118, may be generalised. We shall call forces which do no work constraints.

151. Equation of Energy. As in Article 105 let $m_{1}$ be the mass of any particle of a system, $x_{1}, y_{1}, z_{1}$ its coordinates at time $t$, $X_{1}, Y_{1}, Z_{1}$ the resolved parts, parallel to the axes, of the resultant of all the forces exerted upon it by particles outside the system, $X_{1}^{\prime}, Y_{1}^{\prime}, Z_{1}^{\prime}$ the resolved parts parallel to the axes of the resultant of all the forces exerted upon it by the other particles of the system. The equations of motion are such as

$$
m_{1} \ddot{x}_{1}=X_{1}+X_{1}^{\prime} \text {. }
$$


We multiply the $x_{1}$ equation by $\dot{x}_{1}$, the $y_{1}$ equation by $\dot{y}_{1}$, and so on, and add together all the equations thus obtained. This process gives

$$
\Sigma m(\ddot{x} \dot{x}+\ddot{y} \dot{y}+\ddot{z} \dot{z})=\Sigma\left[\left(X+X^{\prime}\right) \dot{x}+\left(Y+Y^{\prime}\right) \dot{y}+\left(Z+Z^{\prime}\right) \dot{z}\right],
$$

the summation extending to all the particles.

The left-hand member of this equation is

$$
\frac{d}{d t}\left[\frac{1}{2} \sum m\left(\dot{x}^{2}+\dot{y}^{2}+\dot{z}^{2}\right)\right] \text {, or } \frac{d T}{d t},
$$

where $T$ is the kinetic energy of the system (Art. 104).

If then we multiply by $d t$, and integrate both sides with respect to $t$ from $t_{0}$ up to $t$, we obtain the equation

$$
T-T_{0}=\Sigma \int\left(X+X^{\prime}\right) d x+\left(Y+Y^{\prime}\right) d y+\left(Z+Z^{\prime}\right) d z,
$$

where $T_{0}$ is the kinetic energy at time $t_{0}$, and the integrals on the right are line-integrals taken along the paths of the particles from their positions at time $t_{0}$ to their positions at time $t$.

The right-hand member of the equation last written is the work done by all the forces (internal and external) acting on all the particles as they move from their positions at time $t_{0}$ to their positions at time $t$, and the equation can be stated in words:-the change of the kinetic energy of the system, as it passes from one set of positions to another set of positions, is equal to the work done by the forces in the same displacements.

When the system is conservative, let $V$ be the potential energy in the position at time $t$, and $V_{0}$ the potential energy in the position at time $t_{0}$, then the right-hand member of the equation is $V_{0}-V$. The equation can therefore be written

$$
T+V=T_{0}+V_{0}
$$

and this result can be expressed in words:-The total energy of the system, or the sum of the kinetic energy and the potential energy, is a constant quantity.

The equations of motion of a conservative system always possess an integral which expresses the constancy of the total energy, and this integral is, as we have seen, equivalent to the statement that the change of kinetic energy between two positions is equal to the work done by the forces on the particles as they pass from the first position to the second position. The latter statement is true 
whether the system is conservative or not, but it is not always an integral of the equations of motion. In order that it may be so, we must be able to calculate the work done without solving the equations of motion, and, except for conservative systems, this is generally impossible.

152. Positional and Motional Forces. We may imagine cases in which the forces acting on a particle have definite magnitudes, directions, and senses determined by the positions of the particles of the system and of other systems, or, in other words, where the forces are one-valued functions of the geometrical quantities defining the position. Such forces are said to be positional.

We can imagine other cases in which the forces in any positions depend on the velocities in those positions or on the paths by which those positions are reached. Such forces are said to be motional.

The characteristic of positional forces is that the work done by them as the system passes, by any set of paths, from a position $A$ to a position $B$ is numerically equal to the work done in passing, by the same set of paths, from the position $B$ to the position $A$, but has the opposite sign.

Now suppose a system moves under the action of positional forces only. Let it pass by one set of paths from the position $A$ to the position $B$, and by another set of paths from the position $B$ to the position $A$. If the system is conservative no work is done, and the kinetic energy at the end of the cycle is the same as that at the beginning. If the system is not conservative the paths can be adjusted so that the work done in some cycle is positive: the kinetic energy at the end of the cycle is greater than at the beginning. By repeatedly performing the cycle the system can continually acquire kinetic energy. Such a system would constitute a "perpetual motion."

In Nature no perpetual motion has ever been found, and it is therefore in accordance with observed facts to subject the forces of Rational Mechanics to the restriction that positional forces are always conservative.

Motional forces on the other hand are generally non-conservative. For example, the friction between two rough bodies is 
reversed in sense when the relative motion of the points of contact is reversed in sense. Since the friction always acts so as to oppose slipping, the work done by the friction in any displacement is negative (or zero), and thus friction in a system never increases the kinetic energy. Systems in which we recognise the action of forces always tending to diminish the kinetic energy without producing an equivalent in potential energy are said to be dissipative.

153. Conservation of Energy. In any system every increase in the kinetic energy can be regarded as produced by forces which do work, and every decrease by forces against which work is done. In a conservative system every increase of kinetic energy is accompanied by a diminution of potential energy, and conversely. In such a system the total energy, as well as the total mass, remains always the same, and all motions are processes in which redistributions of the energy among the parts of the system, or else transformations of the energy, from kinetic to potential or from potential to kinetic, take place.

In a conservative system let $E$ be the total energy. Let $T$ be the kinetic energy, and $W$ the work-function, in any position. The equation of energy can be written

$$
T=E+W
$$

showing that the kinetic energy is a one-valued function of the quantities that define the position of the system.

The principle of the Conservation of Energy is a principle which asserts that the occurrence of dissipative forces in the formulation of the laws which govern any phenomenon is invariably due to an imperfect analysis of the circumstances. The suggestion is that when the circumstances are completely analysed, and the motions of all the parts of the system are allowed for, the total kinetic energy will be found to be in every case a function of position only, as it is in the case of a system moving under conservative positional forces. This is the same thing as saying that for a system isolated from the action of external bodies, or under such external actions only as can be represented by conservative positional forces, there is a constant total energy, some part of which is at any instant kinetic energy, and the remainder potential energy. 
Cases in which the mechanical description of the motion, according to the principles laid down in Chapters V. and VII., requires the assumption of motional forces can only be brought under the principle by supposing that the motions of the centres of inertia of parts of the system, motions of rotation of these parts, and strains effected in them, are not all the motions of which such systems are capable. In the Chapters referred to we assumed that bodies might be treated as continuous, and, on that assumption, there cannot be any motions of bodies other than those mentioned. There are however very many phenomena which suggest that natural bodies are of discontinuous structure, and, on this assumption, the mechanical descriptions, assuming continuity, would be first approximations in which volumes of a certain order of smallness are treated as infinitesimal. This can be expressed in another way by saying that the motion of a particle is motion of translation only, and that in the mechanical description of the motion of a body portions of finite size are treated as particles. Such a treatment affords an adequate description of many motions.

When we wish to retain the method of treatment by assumption of continuity, and at the same time to adopt the Principle of the Conservation of Energy, we make a compromise by imagining that there are forms of energy which are neither kinetic nor potential, that processes can be imagined by which quantities of energy in any form can be transformed into equal quantities of energy in any other form, and that in particular any form of energy can be transformed into mechanical potential energy. Now mechanical potential energy of a system in any position is measured by the amount of work which would be done by the forces of the system if the system passed from that position into a certain standard position.

We are thus able to make the following definition :-

The energy of a system in any state is its capacity for doing work, and is measured by the amount of potential energy the system would possess if all its energy were transformed into mechanical potential energy.

The Principle of the Conservation of Energy is then the statement that the energy of a system is a quantity which can be 
neither increased nor diminished by any internal action, but can exist in any of the forms of which energy is susceptible.

When we do not retain the conception of bodies as continuous, but make a more complete analysis of the circumstances of any motion, we are guided by the same principle in the form first stated, viz.: when all motions are allowed for, the total energy of the system is constant, and exists only in the forms of kinetic and potential energy.

154. Power. When work is done by the action of a system $S$ upon a system $S^{\prime}$ the forces exerted by the particles of $S$ upon the particles of $S^{\prime}$ do work in the displacements of the particles of $S^{\prime}$. In cases where the energy can be localised the energy of the system $S^{\prime}$ is increased, and that of $S$ diminished, by a quantity equal to the amount of work so done. The number of units of work done in any interval bears a definite ratio to the number of units of time in the interval; and, when the interval is indefinitely short, this ratio has a limit, which is the rate at which work is being done per unit of time.

The power of a system acting on another system is the rate per unit of time at which the first system does work upon the second.

Corresponding to each force between particles of the two systems there is a certain power measured by the product of the magnitude of the force and the resolved part, in its direction, of the velocity of the particle on which it acts, or by the product of the magnitude of the velocity of the particle and the resolved part, in its direction, of the force exerted upon it. The sum of all these powers is the power of the first system acting on the second.

The power can be measured equally by the rate at which work is done upon the second system or by the rate at which the first system does work.

Thus in any machine performing mechanical work a certain amount of energy is expended, and an equal amount of work done, per unit of time; and the machine is said to be "working up to a power" measured by the rate at which the work is done.

155. Kinetic Energy produced by Impulses. As in Article 113 let $\dot{x}, \dot{y}, \dot{z}$ be the resolved parts parallel to the axes of the velocity of the particle of mass $m$ just after an impulse, $\dot{\xi}, \dot{\eta}, \dot{\zeta}$ 
the similar resolved parts of the velocity just before the impulse, $X, Y, Z$ the sums of the resolved parts parallel to the axes of the external impulses applied to $m, X^{\prime}, Y^{\prime}, Z^{\prime}$ the sums of the similar resolved parts of the internal impulses, $T$ and $T_{0}$ the kinetic energies of the system just after and just before the impulses.

We have such equations as

$$
m(\dot{x}-\dot{\xi})=X+X^{\prime} .
$$

Also

$$
\begin{aligned}
T-T_{0} & =\frac{1}{2} \Sigma m\left(\dot{x}^{2}+\dot{y}^{2}+\dot{z}^{2}\right)-\frac{1}{2} \Sigma m\left(\dot{\xi}^{2}+\dot{\eta}^{2}+\dot{\zeta}^{2}\right) \\
& =\frac{1}{2} \Sigma m(\dot{x}-\dot{\xi})(\dot{x}+\dot{\xi})+\text { two similar terms } \\
& =\frac{1}{2} \Sigma\left(X+\dot{X}^{\prime}\right)(\dot{x}+\dot{\xi})+\text { two similar terms. }
\end{aligned}
$$

Thus, the change of kinetic energy produced by impulses is the sum of the products of all the impulses and the arithmetic means of the velocities, in their directions, of the particles to which they are applied just before and just after the impulsive actions.

It is very important to notice that the internal impulses may not be omitted from the equation here obtained, just as the internal forces may not be omitted from the equation of energy of Article 151.

156. Virtual Work. We have defined the work done by a force when the particle on which it acts undergoes any finite or infinitesimal displacement.

Suppose the particle is in motion in any manner, then our definition applies not only to the actual displacement of the particle but also to any other possible displacement.

The work done by a force in an infinitesimal displacement which is not necessarily the actual displacement is called the virtual work of the force for that displacement, and the displacement itself is called a virtual displacement.

157. Principle of Virtual Work. The sum of the virtual works of all the forces on a system in equilibrium vanishes in every infinitesimal displacement.

The sum in question vanishes, not by the quantities that express the displacements becoming indefinitely small, but by the factors that multiply these quantities in the sum being indefinitely small.

We have already seen that the work of a system of forces acting on a particle is equal to the work of their resultant, and this applies to infinitesimal displacements. Now when the particle is in equilibrium under the forces the resultant vanishes identically. Hence the virtual work of the forces vanishes.

Again, for a system of particles in equilibrium under the action of a system of forces each particle is in equilibrium, and therefore the principle enunciated holds good for each particle. It follows by addition that the principle holds for the system. 
From the theorem of Article 140 it follows that the virtual work of the forces between the particles of a rigid system is an infinitesimal of a higher order than the virtual displacements. This work may therefore be omitted in the calculation of the virtual work for the system. Hence for a system of rigid bodies in equilibrium we have the result that the sum of the virtual works of all the external forces applied to all the rigid bodies vanishes for every possible infinitesimal displacement.

*158. Virtual moment of localised vector. In the same way in which we defined the virtual work of a force we may define a quantity connected with any vector localised at a point. Such a quantity will be called the virtual moment of the vector. Further, the definition may be made to apply to a vector localised in a line through the point. We shall say that the product of any infinitesimal displacement of a point and the resolved part, in the direction of the displacement, of a vector localised in a line through the point is the virtual moment of the vector for that displacement.

The virtual moment of a force for any displacement is the virtual work of the force in that displacement.

It is clear, as in Article 131, that the virtual moments of two equivalent systems of vectors localised at a set of points, or in lines through the points, are equal.

*159. Variational equation of motion. Since the kinetic reactions of a system of particles and the forces acting upon them are equivalent systems of vectors, it follows that, for every infinitesimal displacement, the virtual moment of the kinetic reactions is equal to the virtual work of the forces.

The virtual work of the forces is the work done in the infinitesimal displacement, and this is equal to the infinitesimal change that would be made in the kinetic energy if that displacement were executed.

It follows that the infinitesimal change in the kinetic energy is equal to the virtual moment of the kinetic reactions.

Analytically, the virtual displacement $\delta x, \delta y, \delta z$, of any particle can be expressed in terms of the quantities $\theta, \phi, \ldots$ that define the position of the system and their differentials $\delta \theta, \delta \phi, \ldots$ The kinetic reactions $m \ddot{x}, m \ddot{y}, m \ddot{z}$, can be expressed in terms of $\theta, \phi, \ldots$ their velocities $\dot{\theta}, \dot{\phi}, \ldots$, and their accelerations $\ddot{\theta}, \ddot{\phi}, \ldots$, and the equation stated above can be written

$$
\Sigma m(\ddot{x} \delta x+\ddot{y} \delta y+\ddot{z} \delta z)=\Sigma\left[\left(X+X^{\prime}\right) \delta x+\left(Y+Y^{\prime}\right) \delta y+\left(Z+Z^{\prime}\right) \delta z\right] .
$$

The left-hand member can be transformed into an expression linear in $\delta \theta$, $\delta \phi, \ldots$ with coefficients depending on $\theta, \phi, \ldots, \dot{\theta}, \dot{\phi}, \ldots, \ddot{\theta}, \ddot{\phi}, \ldots$, and the righthand member can be transformed into an expression of the form $\theta \delta \theta+\Phi \delta \phi+\ldots$. To secure the equality of the two sides for all values of the ratios $\delta \theta: \delta \phi: \ldots$, it is necessary and sufficient that the coefficients of $\delta \theta, \delta \phi, \ldots$ on the two sides should be equal.

The equations thus obtained must be the equations of motion of the system expressed in terms of the quantities $\theta, \phi, \ldots$ which define its position 
at any time. The general equation above written is therefore equivalent to all the equations of motion of the system. It is called the general variational equation of motion.

The expression of the coefficients of $\delta \theta, \delta \phi, \ldots$ in the left-hand member is beyond the scope of this book.

\section{CRITICAL NOTE.}

THE conception of bodies, as continuous and made up of particles, and the conception of the mutual actions of bodies, as made up of forces between particles, are, as a matter of historical fact, the two conceptions upon which the existing science of Mechanics has been based. They possess further the advantages, (1) that it is possible to found upon them a strictly logical deductive theory, in fact the theory sketched in the preceding Chapters and to be exemplified in subsequent Chapters, and (2) that this theory provides an adequate abstract formulation of the rules obeyed by the motions of the bodies of the solar system, and of matter in bulk under ordinary conditions. It has thus historically developed into a scheme which successfully coordinates an immense number of disconnected observations concerning matters of fact. Accordingly this theory constitutes a science, a logically valid and practically valuable method of representing observed facts by abstract formulas.

An objection has been raised against this method of formulation*, that at the outset it admits a possibility which it afterwards excludes-the possibility of non-conservative positional forces. This objection seems to me to have no weight, since we should expect that, in a theory logically deduced from definitions and postulates, the postulates could not all be introduced at the beginning, but rather that, at certain stages of the process of deductive argument, it would happen that a choice among different possibilities would offer itself. At such stages additional postulates might be introduced, and, in fact, the postulating of the Law of Gravitation as a general law of force is an example of the method of making such a choice among postulates. In all such cases that postulate is to be preferred which accords with the simplest expression of observed facts.

There are however other objections of a more serious character. The first of these is that the theory cannot be complete, or that it aims at an unattainable precision. In order to state completely in terms of the theory the rules that govern the motions of bodies we should require to know what law of force between the hypothetical particles ought to be assumed in the cases in

* By Hertz in his Principien der Mechanik, Leipzig, 1894. 
which we cannot take it to be the law of gravitation. No success has attended the efforts of those physicists who have sought after a law of force to account for cohesion, and little success has been attained in seeking similarly to account for the phenomena of elasticity. In the Rational Mechanics of cohesion and elasticity it has been necessary simply to assume that there could be forces between particles in any positions of such magnitudes as would produce agreement with facts of observation; on the other hand no proof has been adduced of the impossibility of such forces. In the absence of such proof, and of more cogent objections, the theory would retain its value.

The most important of the objections against the theory is directed against the fundamental conception of bodies on which it rests. This conception in fact competes with a different one, which has been deduced from physical and chemical observation and experiment. I mean the molecular hypothesis. Referring to Article 67 , it will be observed that we have inferred from the apparent diminution of heterogeneity of a body with the size of the part considered that the smallest parts of bodies are homogeneous, and the whole of our theory really turns on this supposition. Now if we should have any reason to suppose that the approach to homogeneity does not continue indefinitely with diminution of size, but that, after a certain limit of smallness with approximate homogeneity has been reached, further progressive diminution of size would be accompanied by accentuated heterogeneity; then it would appear that our theory could at best be a first approximation. Now the observed facts on which the molecular hypothesis is founded are all of the kind just indicated; they all point to the existence of structure in parts of bodies extremely small compared with any parts that we can actually separate from the rest for purposes of observation and experiment.

Here then we have arrived at an apparent contradiction. Starting* with statical considerations, concerning the mutual actions of approximately rigid bodies, with experiments on falling bodies, and with Astronomical observations, we have been led to a certain hypothesis concerning the structure of bodies. Starting with a different set of experiments and observations we are led to form a quite opposite hypothesis, and there can be no doubt that the second hypothesis is better established than the first. Thus some part of the system of postulates on which we based our Rational Mechanics, though valid in logic, is not a true representation of facts, and it is desirable to endeavour to reconcile the opposing hypotheses by giving up something not really essential, but actually treated as fundamental, when the general problems of Physics are approached from one side or the other.

To seek ground for reconciliation let us look a little more closely at the results of our theory. That bodies behave as if they were made up of particles possessing what we have called definite mass-ratios, i.e. that bodies have the property we call mass, affects the motions of systems of bodies in a perfectly definite way. There is for each body a definite mass, which is always a constant coefficient entering in the same place in the equations governing its motion. There is absolutely no doubt that we must attribute

* See Historical Note at the end of Chapter V. 
to every body of ordinary dimensions the property of mass. Whether any physical meaning can be attached to the phrase "mass of a molecule" is not a question with which we need here concern ourselves.

In the next place we have arrived at certain quantities which we call quantities of energy, and we have admitted the existence of two kinds of energy, one depending on motion, the other on position. In our theory the notion of energy was derived by a long process from the notion of force. Now, if we had developed our theory a little further by purely analytical processes, we should have found, at least for conservative systems, that the equations of motion of such a system could be deduced by a particular process of differentiation from a knowledge of two analytical expressions, one representing the kinetic energy, and the other the potential energy. The method by which this can be done is, in fact, the method of the variational equation indicated in Article 159. The virtual work of the forces, which was the right-hand member of the equation, was shown to be derivable by differentiation from the expression for the potential energy. The virtual moment of the kinetic reactions can be shown to be derivable by certain differentiations from the kinetic energy. This important result is due to Lagrange. It thus appears that from the expressions for the kinetic and potential energies of a conservative system alone, if properly set down, all the equations of motion can be deduced. In particular the equations of motion of the bodies of the solar system can be so deduced. It is absolutely necessary to attribute to every body of ordinary dimensions the possession, in general, of kinetic energy, and to attribute to every system of bodies the possession of energy, only a part of which is kinetic.

Here then we seem to have some grounds for the kind of compromise we are seeking. The suggestion is that we should retain the conceptions of mass and energy, and the separation of forms of energy, one form being kinetic. In accordance with this suggestion mass will be a property of bodies which affects their motions precisely as before; to formulate this property we have merely to make the mass of a body a constant coefficient of a term in the kinetic energy ; further force will be an expression for the amount of mutual action, which will be derivable from the expression for the potential energy whenever the system is conservative; and thus, in many cases, observed facts, stated in the beginnings of the science in terms of resultant forces, can be stated in the same way still; and we have seen that, except for gravitation, nothing about forces other than resultants can be stated both with precision and so as to agree with facts of observation.

Now if we were asked what this mass or energy that we are talking about is, we should have to confess that we did not precisely know, that we could not expect to know until we had explored and formulated the whole domain of physical science. We should have to say that the notions were reached by means of a provisional theory in which they had perfectly definite meanings; that they had been further generalised because the old theory succeeded in representing some facts, and failed in representing others, and in such representations these two were the most important among the really 
representative conceptions; that these conceptions corresponded with facts in special ways, the correspondence being rather with the average and general properties of bodies of ordinary dimensions than with the precise and particular properties of their hypothetical particles.

We have now arrived at the conclusion that, if we are prepared to abandon precise definition and the purely logical deductive method, as unsuited to a science at present incompletely known, we may construct a physical theory, indefinite in parts and incomplete in details, but nevertheless available for co-ordinating the results obtained by physical investigation, and capable of being itself advanced towards perfection thereby. 


\section{METHODS AND APPLICATIONS.}

\section{CHAPTER IX.}

MOTIONS OF FREE PARTICLES IN GIVEN FIELDS OF FORCE.

160. THE application of the principles which have been laid down in previous Chapters to the discussion of the motions of particles in particular circumstances is the part of our subject usually described as "Dynamics of a Particle." We shall devote to it the two following chapters, and shall have occasion to refer to it again in Chapter XII. This part of our subject divides itself into two main branches, referring respectively to free motions under given forces, and to constrained and resisted motions taking place under forces which are not all given. We confine our attention in the present Chapter to free motions.

We have thus to consider the motion of one particle, or of several particles, relative to a given frame, when each particle is under the action of forces relative to the frame which are given at every point. Physically such forces are presumed to arise from the actions upon the particle of other particles; but for our purpose the important thing is that they produce given accelerations, and in many cases the way in which the acceleration arises need not be taken into account. Further when we are considering the motion of a single particle, whose acceleration is given, it is immaterial what mass we attribute to the particle, and we can therefore, if we wish, take its mass to be the unit of mass; but for the sake of comparison with other cases we shall generally take it to have a given mass. When we are considering the motions of several particles we must suppose each particle to have a given mass.

L. 
For a single particle the kind of application we can make of our theory appears as an extension and generalisation of the results of Chapter IV.; the importance of such applications arises from the theorem of Article 108, according to which the centre of inertia of a body moves like a particle under the resultant of the forces applied to the body.

We shall not in what follows, as we have hitherto, continually mention the frame of reference, and repeat that the motion discussed is motion relative to the frame; but this is always to be understood. Thus when we speak of a fixed point or a fixed line we shall mean a point or a line occupying a definite position relative to the frame of reference; when we speak of the path of a particle we shall mean its path relative to the frame of reference.

161. Method of Particle Dynamics. The method of formation of the equations of motion has been described in Article 80. It consists in equating the product of the mass of the particle and its resolved acceleration in any direction to the resolved part of the force acting upon it in that direction. The equations thus arrived at are differential equations. The left-hand member of any equation contains differential coefficients of geometrical quantities with respect to the time. The right-hand member is, in general, a given function of geometrical quantities. Although there are many cases in which equations of this kind can be solved, there exists no general method for solving them.

Diversity can arise, in regard to the formation of the equations, only from the choice of different directions in which to resolve. Thus we may resolve parallel to the axes of reference, or we may resolve along the radius vector from the origin to a particle, and in directions at right angles thereto, or again we may resolve along the tangent to the path of a particle and in directions at right angles thereto. The most suitable directions to choose in particular cases are determined by the circumstances.

In regard to the solution of equations of motion we can only premise that in cases where there is an equation of energy (Article 151), or an equation of conservation of linear momentum, or of angular momentum (Articles 111 and 112), these equations are first integrals of the equations of motion. 
162. Conservative Field. As in Article 80 let $m$ be the mass of the particle, $x, y, z$ its coordinates at time $t, X, Y, Z$ the resolved parts of the forces acting upon it; then, if the field of force is conservative, $X d x+Y d y+Z d z$ is the complete differential of a function $W$, so that

$$
X=\frac{\partial W}{\partial x}, \quad Y=\frac{\partial W}{\partial y}, \quad Z=\frac{\partial W}{\partial z} .
$$

The equations of motion of the particle are

$$
m \ddot{x}=\frac{\partial W}{\partial x}, m \ddot{y}=\frac{\partial W}{\partial y}, m \ddot{z}=\frac{\partial W}{\partial z},
$$

and they possess the integral (Article 151)

$$
\frac{1}{2} m\left(\dot{x}^{2}+\dot{y}^{2}+\dot{z}^{2}\right)=W+\text { const. }
$$

If $v$ is the magnitude of the velocity of the particle at time $t$, this equation can be written

$$
\frac{1}{2} m v^{2}=W+\text { const. }
$$

This equation applies to all the particular cases discussed in Chapter IV.

163. Conservation of Linear Momentum. Suppose the axis $x$ is a direction in which there is no resolved part of the force acting on the particle. The equation of motion by resolution parallel to the axis $x$ is $m \ddot{x}=0$, and it follows that $\dot{x}$ is constant throughout the motion, or the resolved velocity in any direction in which the resolved part of the force vanishes is constant. This is a special case of the general principle considered in Article 111.

164. Conservation of Angular Momentum. Suppose the axis $z$ is a line about which the forces acting on the particle have no moment. Then we have

$$
x Y-y X=0 \text {. }
$$

Hence multiplying the equations

$$
m \ddot{x}=X, m y=Y
$$

respectively by $y$ and $x$, and subtracting, we have

$$
m(x \ddot{y}-y \ddot{x})=0,
$$

and this equation possesses the integral

$$
x \dot{y}-y \dot{x}=\text { const. }
$$

This is a special case of the general principle considered in Article 112. 
165. Motion of a body under gravity. The frame of reference is supposed to be fixed relatively to the Earth and to have its origin at a place on the Earth's surface near which the motion takes place. Then it is approximately true that the field of force is of intensity $g$ directed vertically downwards, and we treat this statement as exact.

The equations of conservation of linear momentum show that the motion takes place in the vertical plane through the direction of projection, and that the horizontal velocity in that plane is constant.

Let the axes of $x$ and $y$ be a fixed horizontal and vertical in this plane, the positive sense of the axis $y$ being upwards, then we have

$$
\dot{x}=\text { const. }=u \text { say. }
$$

The kinetic energy of the body is $\frac{1}{2} m\left(\dot{x}^{2}+\dot{y}^{2}\right)$.

The potential energy of the body in the field (Article 149) is mgy.

Hence the equation of energy is

$$
\frac{1}{2} m\left(\dot{x}^{2}+\dot{y}^{2}\right)+m g y=\text { const. }
$$

This equation may be written

$$
\frac{1}{2} m u^{2}\left\{1+\left(\frac{d y}{d x}\right)^{2}\right\}+m g y=\text { const. }
$$

or, by choice of the constant

giving

$$
\begin{aligned}
& \frac{d y}{\sqrt{y_{0}-y}}=\frac{\sqrt{ }(2 g)}{u} d x, \\
& \left(x-x_{0}\right)^{2}=\frac{2 u^{2}}{g}\left(y_{0}-y\right),
\end{aligned}
$$

where $x_{0}$ is a constant of integration. This equation represents a parabola with axis vertical and vertex upwards, the point $\left(x_{0}, y_{0}\right)$ being the vertex.

166. Motion under a central force. For a particle under the action of a force to a fixed point which is a function of the distance from that point, the principles of the conservation of energy and momentum supply all the first integrals of the equations of motion. Drawing a plane through the fixed point and the tangent line to the path at any instant, the linear momentum perpendicular to this plane is constantly zero, so that the particle moves in the plane. The moment of momentum about an axis through the fixed point perpendicular to the plane remains constant, and this gives us an equation of the form

$$
p v=h,
$$

where the notation is that of Article 50, and we see that $m h$ is the moment of momentum about this axis. The kinetic energy of the particle is $\frac{1}{2} m v^{2}$, and the potential energy of the particle in the field is

$$
\int^{r} m f d r
$$


$m f$ being the central attractive force, and thus the equation of energy is

$$
\frac{1}{2} m v^{2}+\int^{r} m f d r=\text { const. }
$$

This equation can be written

$$
\frac{1}{2} \frac{1}{p^{2}}=\frac{1}{h^{2}} \int_{r} f d r+A,
$$

and can thus be identified with the equation (1) at the top of p. 63.

167. Production of simple harmonic motion. We have explained in Article 127 that the tension in an extensible string is proportional to the extension, so that a body attached to such a string will be subject to a force in the line of the string, of the amount indicated, so long as the string remains stretched. Thus if the string is attached to two fixed points whose distance apart is greater than its natural length, and the body is attached to any point of it, then, when the body is displaced in the line of the string from its position of equilibrium, there will be a force urging it towards its position of equilibrium, and proportional to its displacement. It will therefore have a simple harmonic motion.

Again, as we have explained in Article 129, the stress in a spring is tension proportional to the extension when the spring is extended, and pressure proportional to the contraction when the spring is compressed, so that a body attached to the spring will be acted upon by a force proportional to its displacement from the position in which it would rest. It will therefore have a simple harmonic motion.

We may also state here that in very small vibrations of a system about a position of equilibrium each particle executes a motion compounded of simple harmonic motions in various directions.

Taking the case of the body attached to the spring, moveable in the line of the spring, and under no force except that arising from the action of the spring, let $x$ be the displacement of the end of the spring to which the body is attached, the other end being fixed, the stress in the spring is tension $\mu x$ when the spring is extended through $x$, and pressure $\mu x$ when the spring is compressed through $x$, where $\mu$ is a constant depending only on the spring. This constant is known as the "strength" of the spring. 
Thus, if $m$ is the mass of the body, the equation of motion of the body is

$$
m \ddot{x}=-\mu x \text {. }
$$

The mass of the spring being neglected, the kinetic energy is $\frac{1}{2} m \dot{x}^{2}$, and the potential energy is $\frac{1}{2} \mu x^{2}$, and thus we have the equation of energy $\frac{1}{2} m \dot{x}^{2}+\frac{1}{2} \mu x^{2}=$ const., from which the equation of motion might be deduced by differentiating with respect to the time.

\section{Examples.}

1. A particle of mass $m$ is attached to the middle point of an elastic thread, of natural length $a$ and modulus $\lambda$, which is stretched between two fixed points. Prove that, if no forces act on the particle other than the tensions in the parts of the thread, it can oscillate in the line of the thread with a simple harmonic motion of period $\pi \sqrt{ }(m a / \lambda)$.

2. A particle of mass $m$ is attached to one end of an elastic thread, of natural length $\alpha$ and modulus $\lambda$, the other end of which is fixed. The particle is displaced until the thread is of length $a+b$, and is then let go. Prove that, if no forces act on the particle except the tension of the thread, it will return to the starting point after a time $2\left(\pi+2 \frac{\alpha}{b}\right) \sqrt{ } \frac{m \alpha}{\lambda}$.

3. Prove that, if a body is suddenly attached to an unstretched vertical elastic thread and let fall under gravity, the greatest subsequent extension is twice the statical extension of the thread when supporting the body.

4. Prove that, if a spring is held compressed by a given force and the force is suddenly reversed, the greatest subsequent extension is three times the initial contraction.

5. An elastic thread of natural length $a$ has one end fixed, and a particle is attached to the other end, the modulus of elasticity being $n$ times the weight of the particle. The particle is at first held with the thread hanging vertically and of length $\alpha^{\prime}$, and is then let go from rest. Show that the time until it returns to its initial position is

$$
2\left(\pi-\theta+\theta^{\prime}+\tan \theta-\tan \theta^{\prime}\right) \sqrt{ }(\alpha / n g),
$$

where $\theta, \theta^{\prime}$ are acute angles given by

$$
\sec \theta=n \alpha^{\prime} / \alpha-n-1, \sec ^{2} \theta^{\prime}=\sec ^{2} \theta-4 n,
$$

and $\alpha^{\prime}$ is so great that real values of these angles exist.

169. Gravitation. The case of a central force varying inversely as the square of the distance is the case of gravitation, at least when the mass of one gravitating body is great compared with that of the other. Thus for a body near the Earth's surface the action of the Earth on the body produces an acceleration which is nearly constant and in a vertical direction. A correction to this statement will be made by saying that the force in question is directed to the centre of the Earth, and varies inversely as the 
square of the distance therefrom. Thus for bodies near the Earth's surface there is a correction of gravity for height above the Earth's surface. In fact, if $g$ is the value of the acceleration due to gravity at the surface, and $a$ the Earth's radius, the acceleration due to gravity at a height $h$ above the surface is

$$
g a^{2} /(a+h)^{2} \text {. }
$$

Naturally, the correction only becomes sensible at heights which can be reached with difficulty, as in the ascent of a balloon, or at the top of a high mountain.

The correction for depth below the Earth's surface, as for instance at the bottom of a deep mine, depends on a result in the Theory of Attractions, according to which the force exerted by a uniform gravitating sphere at an internal point is proportional to the distance from the centre. Thus, if $h$ now denotes depth below the Earth's surface, the acceleration due to gravity at depth $h$ may be taken to be

$$
g(a-h) / a .
$$

There are other corrections of gravity at least as important as those here mentioned. One of them arises from the heterogeneity of the material of the Earth, another from the fact that the Earth is not spherical. Another correction depending on the choice of the frame of reference will occupy us later in Chapter XIII.

\section{Examples.}

1. Consider the motion of a particle under the action of a uniform fixed gravitating sphere, of density $\rho$ and radius $a$, and suppose the particle to start from rest at a distance $b(>a)$ from the centre. It will move directly towards the centre with an acceleration $\frac{4}{3} \pi \gamma \rho \alpha^{3} / x^{2}$ at distance $x$, so long as $x>a$, and when $x=a$, it will have a velocity given by

$$
\frac{1}{2} \dot{x}^{2}=\frac{4}{3} \pi \gamma \rho \alpha^{3}\left(\frac{1}{a}-\frac{1}{b}\right) \text {. }
$$

Now suppose a fine tunnel is bored through the centre of the sphere in the direction of motion of the particle. When the particle passes into the tunnel its acceleration becomes $\frac{4}{3} \pi \gamma \rho x$ at distance $x$, and it moves with a simple harmonic motion. The velocity at distance $x$ is given by the equation

$$
\frac{1}{2} \dot{x}^{2}+\frac{2}{3} \pi \gamma \rho x^{2}=\text { const., }
$$

and the constant is determined from the expression given above for the velocity at the instant of entering the tube.

Prove that the velocity at the centre is

$$
\sqrt{ }\left\{\frac{4}{3} \pi \gamma \rho a^{2}(3-2 a / b)\right\} \text {. }
$$

[This is the result referred to by anticipation in Example 3 of Article 57.] 
2. Show that a gun at the sea level can command $1 / n^{2}$ of the Earth's surface if the greatest height to which it can send a shot is $1 / n$ of the Earth's radius, variations of gravity due to height above the surface being taken into account.

3. A particle falls to the Earth from a height $h$. Prove that the time of falling is $\left(1+\frac{5}{6} h / R\right) \sqrt{ }(2 h / g)$ approximately, where $R$ is the Earth's radius, and $g$ is the acceleration due to gravity at its surface, and the square of $h / R$ is neglected.

\section{Motion in one plane, radial and transverse reso-}

lutions. When the force acting on a particle is resolved into a radial force $R$ and a transverse force $T$ always acting in one plane, the equations of motion can be written

$$
\left.\begin{array}{rl}
m\left(\ddot{r}-r \dot{\theta}^{2}\right) & =R, \\
\frac{m}{r} \frac{d}{d t}\left(r^{2} \dot{\theta}\right) & =T
\end{array}\right\} .
$$

By the process of Article 60 we can change the independent variable from $t$ to $\theta$, and thus obtain the differential equation of the path.

Also when there is a work function $W$ we have

$$
R=\frac{\partial W}{\partial r}, \text { and } T=\frac{1}{r} \frac{\partial W}{\partial \theta},
$$

and then there is an energy equation of the form

$$
\frac{1}{2} m\left(\dot{r}^{2}+r^{2} \dot{\theta}^{2}\right)=W+\text { const. }
$$

\section{Examples.}

1. Writing $h$ for $r^{2} \dot{\theta}$, and $u$ for $r^{-1}$, prove that the differential equation of the path will be found by eliminating $h$ between the equations

$$
\frac{d}{d \theta}\left(\frac{1}{2} h^{2}\right)=\frac{T}{m u^{3}}, \quad h^{2}\left(\frac{d^{2} u}{d \theta^{2}}+u\right)=-\frac{1}{m u^{2}}\left(R+\frac{T}{u}, d u\right) .
$$

2. Supposing $R=0, T=\mu r$, prove that the kinetic energy acquired in describing a closed curve is $2 \mu \times$ (area of curve).

[This is an example of non-conservative positional forces referred to in Article 152, it is in accordance with that Article to say that such forces do not occur in natural systems.]

173. Motion under several central forces. When a particle moves under the action of central forces directed to a number of different fixed points, each force being a function of the distance from the point towards which it acts, we cannot usually by help of general principles write down any first integral of the equations of motion except the equation of energy. A number of theorems relating to motions under such forces can however be proved. We give a few examples with some indications of methods. 


\section{Examples.}

1. A particle of mass $m$ moves under the action of forces to two fixed points $A, A^{\prime}$ of magnitudes $m \mu / r^{2}, m \mu^{\prime} / r^{\prime 2}$ respectively, where $r$ and $r^{\prime}$ are the distances of the particle from $A$ and $A^{\prime}$, and $\mu$ and $\mu^{\prime}$ are constants. The equations of motion possess an integral of the form

$$
r^{2} r^{\prime 2} \dot{\theta} \dot{\theta}^{\prime}=\alpha\left(\mu \cos \theta-\mu^{\prime} \cos \theta^{\prime}\right)+\text { const. }
$$

where $a$ is the distance $A A^{\prime}$.

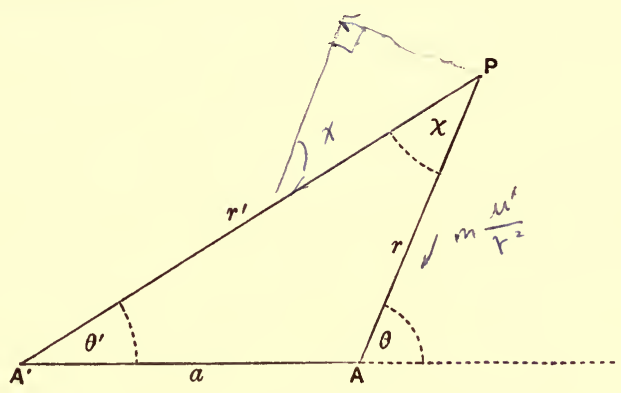

Fig. 43.

Resolving at right angles to the radius vector $r$, we have

$$
m \frac{1}{r} \frac{d}{d t}\left(r^{2} \dot{\theta}\right)=m \frac{\mu^{\prime}}{r^{\prime 2}} \sin \chi, \text { where } \chi \text { is the angle } A P A^{\prime},
$$

so that

$$
r^{\prime 2} \frac{d}{d t}\left(r^{2} \dot{\theta}\right)=\mu^{\prime} r \sin \chi=\mu^{\prime} a \sin \theta^{\prime},
$$

similarly

$$
r^{2} \frac{d}{d t}\left(r^{\prime 2} \dot{\theta}^{\prime}\right)=-\mu r^{\prime} \sin \chi=-\mu a \sin \theta .
$$

Multiplying by $\dot{\theta}^{\prime}$, and $\dot{\theta}$, adding, and integrating, we have an equation of the given form.

2. A particle of mass $m$ moves under the action of forces to two fixed points of magnitudes $m \mu r, m \mu^{\prime} r^{\prime}$. Prove, with the notation of Example 1, that there is an integral equation of the form

$$
\mu r^{2} \dot{\theta}+\mu^{\prime} r^{\prime 2} \dot{\theta}^{\prime}=\text { const. }
$$

3. A given plane curve can be described by a particle under central forces to each of $n$ given points, when the forces act separately. Prove that it can be described under the action of all the forces, provided the particle is properly projected.

Let $f_{\kappa}$ be the acceleration produced in the particle by the force to the $\kappa$ th centre $O_{\kappa}, v_{\kappa}$ the velocity of the particle at any point when the curve is described under this force, $r_{\kappa}$ the distance of the point from $O_{\kappa}$, and $p_{\kappa}$ the perpendicular from $O_{\kappa}$ on the tangent to the curve at the point, $\rho$ the radius of curvature and $d s$ the element of arc of the curve at the point. Then we are given that

$$
v_{\kappa} \frac{d v_{\kappa}}{d s}=-f_{\kappa} \frac{d r_{\kappa}}{d s}, \quad \frac{v_{\kappa}^{2}}{\rho}=f_{\kappa} \frac{p_{\kappa}}{r_{\kappa}} .
$$


Now the curve can be described under all the forces if there exists a velocity $V$ satisfying the two equations

$$
V \frac{d V}{d s}=-\sum_{1}^{n} f_{\kappa} \frac{d r_{\kappa}}{d s}, \quad \frac{V^{2}}{\rho}=\sum_{1}^{n} f_{\kappa} \frac{p_{\kappa}}{r_{\kappa}},
$$

and it is clear that these are satisfied by

$$
V^{2}=\sum_{1}^{n} v_{\kappa}^{2} \text {. }
$$

Thus the condition is that the kinetic energy when all the forces act must be the sum of the kinetic energies when they act separately.

4. Prove that a lemniscate $r r^{\prime}=c^{2}$, where $2 c$ is the distance between the points from which $r$ and $r^{\prime}$ are measured, can be described under the action of forces $m \mu / r$ and $m \mu / r^{\prime}$ directed to those points.

5. A particle describes a plane orbit under the action of two central forces each varying inversely as the square of the distance, directed towards two points symmetrically situated in a line perpendicular to the plane of the orbit. Show that the general $(p, r)$ equation of the orbit, referred to the point where the line joining the centres of force meets the plane as origin, is of the form

$$
\left(1-a^{2} / p^{2}\right)^{2}=b^{2} /\left(c^{2}+r^{2}\right),
$$

where $c$ is the distance of either centre of force from the plane, and $a$ and $b$ are constants.

6. A point describes a semi-ellipse, bounded by the axis minor, and its velocity, at a distance $r$ from the nearer focus, is $a \sqrt{ }\{f(a-r) / r(2 \alpha-r)\}$, $2 a$ being the axis major, and $f$ a constant. Prove that its acceleration is compounded of two, each varying inversely as the square of the distance, one tending to the nearer focus, and the other from the farther focus.

175. The Problem of Two Bodies*. Two particles which attract each other according to the law of gravitation are projected in any manner. It is required to show that the relative motion is parallel to a fixed plane, and that the relative orbits are conics, and to determine the periodic time when the orbits are elliptic.

The principle of the conservation of linear momentum shows that the centre of inertia of the two particles moves uniformly in a straight line. The accelerations of the particles, and the velocity of either relative to the other, are unaltered, if we refer them to a frame whose axes are parallel to those of the original frame of reference, and whose origin is at the centre of inertia. We shall suppose this to be done.

* The Problem of Two Bodies was solved by Newton, Principia, Lib. I. Sect. xI. Props. 57-63. 
Then the acceleration of each particle is in the line joining it to the origin, and the velocities of the particles are localised in lines which lie in a plane containing the origin, the motion of each particle therefore takes place in this plane.

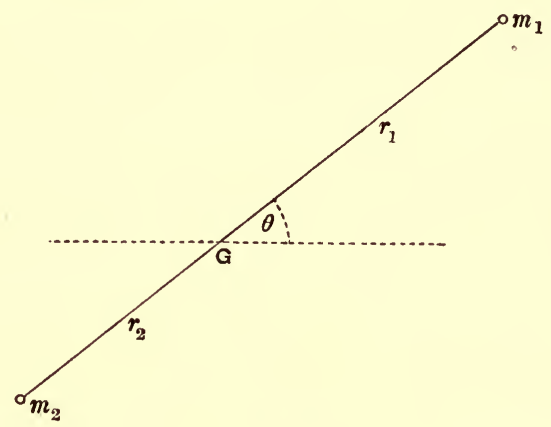

Fig. 44.

Now let $G$ be the centre of inertia, $m_{1}, m_{2}$ the masses of the particles, $r_{1}, r_{2}$ their distances from $G$ at time $t, \theta$ the angle the line joining them makes with any fixed line in the plane of motion, also let $r,=r_{1}+r_{2}$, be the distance between the particles at time $t$, and let the force between them be $\gamma m_{1} m_{2} / r^{2}$.

Then the equations of motion of $m_{1}$ are

$$
\left.\begin{array}{l}
m_{1}\left(\ddot{r}_{1}-r_{1} \dot{\theta}^{2}\right)=-\gamma m_{1} m_{2} / r^{2}, \\
m_{1} \frac{1}{r_{1}} \frac{d}{d t}\left(r_{1}^{2} \dot{\theta}\right)=0
\end{array}\right\} .
$$

Since $r_{1}=m_{2} r /\left(m_{1}+m_{2}\right)$, these equations become

$$
\left.\begin{array}{l}
\ddot{r}-r \dot{\theta}^{2}=-\gamma\left(m_{1}+m_{2}\right) / r^{2}, \\
\frac{d}{d t}\left(r^{2} \dot{\theta}\right)=0
\end{array}\right\},
$$

and it is clear that the equations of motion of $m_{2}$ would lead us to the same two equations.

The equations last written show that the acceleration of $m_{1}$ relative to $m_{2}$, or of $m_{2}$ relative to $m_{1}$, is $\gamma\left(m_{1}+m_{2}\right) / r^{2}$, and that there is no transverse acceleration. Thus either particle describes a central orbit about the other with acceleration varying inversely as the square of the distance, and by Example 1, p. 63, this orbit is a conic described about a focus. 
Further, when the orbit is an ellipse, its major axis, $2 a$, is the sum of the greatest and least distances between the particles, and the periodic time is by Article 53 equal to

$$
2 \pi \frac{a^{\frac{3}{2}}}{\sqrt{ }\left\{\gamma\left(m_{1}+m_{2}\right)\right\}} \text {. }
$$

\section{Examples.}

1. If the particles are projected with velocities $v, v^{\prime}$ in directions containing an angle $a$ from points whose distance apart is $R$, prove that the relative orbit is an ellipse, parabola, or hyperbola according as

$$
v^{2}+v^{2}-2 v v^{\prime} \cos a<=\text { or }>2 \gamma\left(m_{1}+m_{2}\right) / R .
$$

2. $S, P$, and $E$ denote the masses of the Sun, a planet, and the Earth; the major axis of the planet's orbit is $k$ times that of the Earth's orbit, and its periodic time is $n$ years ; prove that

$$
n^{2}=k^{3}(S+E) /(S+P) \text {. }
$$

[Kepler's Third Law of Planetary motion quoted in Article 94 states that $n^{2}=k^{3}$ approximately. This would follow if $S$ were great compared with $P$ or E.]

3. Two gravitating spheres of masses $m, m^{\prime}$, and radii $a, a^{\prime}$, are allowed to fall together from a position in which their centres are at a distance $c$, it is required to find the time until they are in contact.

We may suppose the centre of inertia at rest, and take $x$ for the distance between the centres of the spheres at time $t$. Then their velocities are

$$
\frac{m^{\prime} \dot{x}}{m+m^{\prime}} \text { and } \frac{m \dot{x}}{m+m^{\prime}} \text {. }
$$

Hence the kinetic energy of the system is

$$
\frac{1}{2} m\left(\frac{m^{\prime} \dot{x}}{m+m^{\prime}}\right)^{2}+\frac{1}{2} m^{\prime}\left(\frac{m \dot{x}}{m+m^{\prime}}\right)^{2}=\frac{1}{2} \frac{m m^{\prime}}{m+m^{\prime}} \dot{x}^{2} .
$$

The potential energy, measured from the position in which the distance was $c$ as standard position, is (Article 142),

$$
\gamma m m^{\prime}\left(\frac{1}{c}-\frac{1}{x}\right) \text {. }
$$

Hence we have, by the equation of energy,

$$
\dot{x}^{2}=2 \gamma\left(m+m^{\prime}\right)\left(\frac{1}{x}-\frac{1}{c}\right),
$$

and the time required is

$$
\frac{1}{\sqrt{\left\{2 \gamma\left(m+m^{\prime}\right)\right\}}} \int_{a+a^{\prime}}^{c} \sqrt{\frac{c x}{c-x}} d x .
$$

If then we find an angle $\theta$ such that $a+a^{\prime}=c \cos ^{2} \theta$, we shall have for the required time

$$
\frac{c^{\frac{3}{2}}(\theta+\sin \theta \cos \theta)}{\sqrt{ }\left\{2 \gamma\left(m+m^{\prime}\right)\right\}}
$$


4. A body, of mass $\mathrm{km}$, describes an ellipse of eccentricity $e$ and axis major $2 \alpha$ under the action of a fixed gravitating body of mass $m$. Prove that; if $m$ is let go when the distance between the bodies is $R$, the eccentricity $e^{\prime}$ of the subsequent relative orbit is given by the equation

$$
e^{2}-e^{2}=\frac{k\left(1-e^{2}\right)}{(1+k)^{2}}\left\{k+2\left(1-\frac{a}{R}\right)\right\} .
$$

5. Two gravitating particles of masses $m, m^{\prime}$ are describing relatively to each other elliptic orbits of eccentricity $e$ and axis major $2 \alpha$, their centre of inertia being at rest. Prove that, if $m$ is suddenly fixed when the particles are at a distance $R$, the eccentricity $e^{\prime}$ of the orbit subsequently described by $m^{\prime}$ is given by the equation

$$
\left(m+m^{\prime}\right)\left(\frac{2}{R}-\frac{m+m^{\prime}}{a m} \frac{1-e^{\prime 2}}{1-e^{2}}\right)=m\left(\frac{2}{R}-\frac{1}{a}\right) .
$$

177. General Problem of Planetary Motion. Consider in the general case the motion of a system of particles which act on each other according to the law of gravitation. The acceleration of any particle $P$ is compounded of accelerations to each of the others, and any one of the components thus arising is of the form $\gamma m / r^{2}$, where $m$ is the mass of one of the particles and $r$ is its distance from $P$. For such a system there would exist seven first integrals of the equations of motion. In fact the Principle of the Conservation of Linear Momentum would give three such integrals, representing that the centre of inertia of the system moves uniformly in a straight line; the Principle of the Conservation of Moment of Momentum would give three integrals, representing that the moment of momentum of the system about any one of the axes of reference is a constant quantity; the Principle of the Conservation of Energy would also give an integral equation.

But, even in the case of three particles, these integrals do not suffice for a complete description of the motions of the system, and up to the present time no other integral of the equations has been obtained except for special circumstances of projection.

Now the solar system affords an example of a system such as that here considered, for the Sun and each of the planets are approximately spherical, and it is consonant with results of observation to assume that the component actions between the particles of which, for purposes of Rational Mechanics, these bodies are assumed to be made up, reduce to resultants in the lines joining their centres of inertia. 
Thus we cannot deduce from the law of gravitation an exact account of the motions of the bodies forming the solar system. But there are a number of circumstances which conduce to the possibility of deducing from this law such an approximate account of the motions in question as shall be sufficiently exact to agree with observation over a long period of time. Among these we may mention (1) that the mass of the Sun is great compared with that of the other bodies, even the mass of Jupiter being less than $\frac{1}{1000}$ th part of that of the Sun, (2) that all the orbits are nearly circular and lie nearly in one plane.

Now it follows from the first of these statements that all the forces acting on any planet are small compared with the attraction of the Sun, and thus an approximate description of the motion might be obtained by leaving these forces out of account. The approximate equations can be completely solved, as we have seen in Article 175. If then, starting at any instant, we could conceive that a planet moved under no force except the attraction of the Sun, it would describe an ellipse with the Sun in one focus; and, since at starting it would have the position and velocity which it actually has, this ellipse would touch the actual path.

The ellipse in question is known as the "instantaneous ellipse," and the motion in it is of the kind described in Articles 53-55. The method of Planetary Theory is to determine this ellipse and to determine how it changes from time to time. For the determination of the ellipse we observe that the plane of the ellipse will cut any other plane through the Sun in a line, so that, in particular, the orbit of any planet cuts the Earth's orbit in a line through the Sun, this line is known as the "line of nodes;" the position of the line of nodes and the angle between the two planes determine the plane of the ellipse; the angle in question is known as the "inclination." The position of the ellipse in its plane is determined by means of the angle contained between the line of nodes and the axis major. The shape and size of the ellipse are determined by its eccentricity and its major axis. One further element is necessary in order to determine the position in terms of the time, and this is arranged for by choosing an epoch from which to measure time. 
Thus the elements of a planet's orbit are:

$a$ the semi-axis major,

$e$ the eccentricity,

$i$ the inclination,

$\Omega$ the longitude of the node,

$\varpi$ the longitude of the perihelion,

$\epsilon$ the epoch,

where $\epsilon$ is the longitude of the planet at time $t=0$. It is to be noticed that $\Omega$ is measured in the plane of the Earth's orbit, while $\epsilon$ and $\varpi$ are measured in the plane of the planet's orbit from a definite line in the plane of the orbit.

178. Disturbed Elliptic Motion. It would be beyond the scope of this work to explain the methods by which the variations of the elements are determined, but it appears to be not without interest to examine the rates of variation of some of the elements produced by small forces acting in given directions. We shall consider quite generally the rate at which changes are produced in the elements $a, e, \varpi$ of an elliptic orbit by small tangential and normal forces in the plane of the orbit.

179. Tangential disturbing force. Suppose a particle $P$, describing an elliptic orbit about a focus $S$, receives a small tangential impulse increasing its velocity by $\delta v$. Let $R$ be the distance of the particle from $S$ at the instant, $\mu / r^{2}$ the acceleration to $S$ when the distance is $r, a+\delta a$ the semi-axis major of the orbit immediately after the impulse.

We have, by Example 1 of Article 54,

$$
\begin{gathered}
v^{2}=\mu\left(\frac{2}{R}-\frac{1}{a}\right), \\
(v+\delta v)^{2}=\mu\left(\frac{2}{R}-\frac{1}{a+\delta a}\right),
\end{gathered}
$$

giving

$$
\frac{\delta a}{a^{2}}=\frac{2 v \delta v}{\mu} \text { approximately. }
$$

Again, if $h$ is the moment of the velocity about $S$ before the 
impulse, $h+\delta h$ afterwards, since the tangent to the path is unaltered, we have

$$
\begin{gathered}
\frac{h+\delta h}{v+\delta v}=\frac{h}{v}, \\
\delta h=h \frac{\delta v}{v} .
\end{gathered}
$$

giving

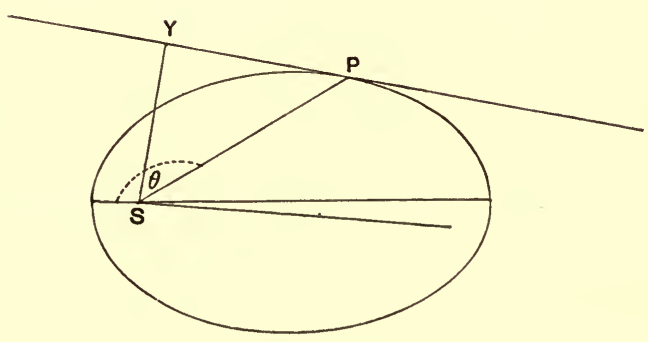

Fig. 45.

Hence if $l$ is the semi-latus rectum before the impulse, $l+\delta l$ afterwards, we have

$$
\mu(l+\delta l)=h^{2}\left(1+\frac{\delta v}{v}\right)^{2}, \text { with } h^{2}=\mu l
$$

giving

$$
\delta l=2 l \frac{\delta v}{v} \text { approximately. }
$$

Now $l=\alpha\left(1-e^{2}\right)$, and if $e$ becomes $e+\delta e$,

$$
\left(1-e^{2}\right) \delta a-2 e a \delta e=2 a\left(1-e^{2}\right) \frac{\delta v}{v}
$$

giving $\delta e=\frac{\left(1-e^{2}\right)}{e}\left[a \frac{v \delta v}{\mu}-\frac{\delta v}{v}\right]=\frac{1-e^{2}}{e} \frac{\delta v}{v} a\left[\frac{v^{2}}{\mu}-\frac{1}{a}\right]$,

or

$$
\delta e=\frac{1-e^{2}}{e} \frac{2 \delta v}{v} a\left(\frac{1}{R}-\frac{1}{a}\right) \text {. }
$$

Further the angle $\theta$ which $S P$ makes with the axis major is given by the equation $l / R=1+e \cos \theta$ and it is clear that

$$
\delta \theta=-\delta \varpi,
$$

hence

$$
\delta l / R=\frac{\delta e}{e}\left(\frac{l}{R}-1\right)+e \sin \theta \delta \varpi
$$


If the particle is subject to a disturbing force producing a small tangential acceleration $f$ we shall have

$$
\begin{gathered}
\dot{a}=\frac{2 a^{2} v f}{\mu}, \quad \dot{e}=\frac{2 f}{v} \frac{l}{e}\left(\frac{1}{R}-\frac{1}{a}\right), \\
e \sin \theta \dot{\varpi}=\frac{2 l}{R} \frac{f}{v}-\frac{\dot{e}}{e}\left(\frac{l}{R}-1\right) .
\end{gathered}
$$

180. Normal disturbing force. Suppose the particle to receive an impulse imparting to it a velocity $\delta v$ in the direction of the normal outwards. Then the resultant velocity is, to the first order, unaltered, and consequently $a$ is unaltered, or $\delta a=0$.

If $p$ is the perpendicular from the focus $S$ on the tangent at $P$, meeting it in $Y$, then the value of $h$ is increased by $P Y \delta v$, or we have

$$
\delta h=\sqrt{ }\left(R^{2}-p^{2}\right) \delta v .
$$

Hence

$$
\mu \delta l=2 h \delta h=2 p v \delta v \sqrt{ }\left(R^{2}-p^{2}\right) ;
$$

also $\delta l=-2 a e \delta e$, so that

$$
\delta e=-\frac{p v \delta v}{\mu a e} \sqrt{ }\left(R^{2}-p^{2}\right) .
$$

Again, $l / R=1+e \cos \theta$, so that

$$
-2 a e \delta e / R=\left(\frac{l}{R}-1\right) \frac{\delta e}{e}+e \sin \theta \delta \varpi .
$$

If the particle is subject to a disturbing force producing a small normal acceleration $f$ we have

$$
\dot{a}=0, \dot{e}=-\frac{p f v}{\mu a e} \sqrt{ }\left(R^{2}-p^{2}\right), e \sin \theta \dot{\varpi}=-\dot{e}\left(\frac{2 a e}{R}+\frac{l-R}{e R}\right) .
$$

\section{Examples.}

1. For a small tangential impulse prove that

$$
\delta e=2 \delta v(e+\cos \theta) / v, \quad \delta \varpi=2 \delta v \sin \theta / e v .
$$

2. For a small normal impulse prove that

$$
\delta e=-r \delta v \sin \theta / a v, \quad \delta \varpi=\delta v(2 a e+r \cos \theta) / \alpha e v .
$$

3. For a small radial impulse prove that

$$
\delta a=2 a^{2} e \delta v \sin \theta / h, \quad \delta e=h \delta v \sin \theta / \mu, \quad \delta \varpi=-h \delta v \cos \theta / e \mu .
$$

4. For a small transversal impulse prove that $\delta \alpha=2 \delta v a^{2}(1+e \cos \theta) / h, \quad \delta e=\delta v\{r(e+\cos \theta)+l \cos \theta\} / h, \quad \delta \varpi=\delta v \sin \theta(l+r) / e h$. 


\section{EXAMPLES.}

1. A particle is suspended from a point by an elastic thread and oscillates in the vertical line through the point of suspension. Prove that the period is the same as that of a simple pendulum of length equal to the excess of the length of the thread in the position of equilibrium above its natural length.

2. A particle is attached to one end of an elastic thread of natural length $l$, the other end of which is fixed to a point on a smooth horizontal table. When the particle is at rest on the table, with the thread straight but unstretched, it receives a blow, which, if directed along the thread would make the particle move to a maximum distance $2 l$ from the fixed end. Prove that, if the direction of the blow makes an angle $a$ with the thread, the maximum length of the thread during the motion is the greatest root of the equation

$$
x^{4}-2 l x^{3}+l^{4} \sin ^{2} a=0 .
$$

3. A particle is attached to a fixed point by means of an elastic thread of natural length $3 a$, whose coefficient of elasticity is six times the weight of the particle. When the thread is at its natural length, and the particle is vertically above the point of attachment, the particle is projected horizontally with a velocity $3 \sqrt{ }\left(\frac{1}{2} \alpha g\right)$; prove that the angular velocity of the thread will be constant, and that the particle will describe the curve

$$
r=a(4-\cos \theta) \text {. }
$$

4. A heavy particle is fastened to the free ends of a number of elastic threads which pass through fixed smooth rings, each ring being at a distance from the fixed end of the thread which passes through it equal to the natural length of the thread. Prove that if the particle is projected in any direction it describes an ellipse about its position of equilibrium as centre.

5. Prove that a body ejected from the Earth with velocity exceeding seven miles per second will not in general return to the Earth, and may leave the solar system.

6. Prove that the least velocity with which a body could be projected from the North Pole so as to meet the Earth's surface at the Equator is nearly $4 \frac{1}{2}$ miles per second, and that the angle of elevation is $22 \frac{1}{2}^{\circ}$.

7. A particle is projected from the Earth's surface so as to describe a portion of an ellipse whose axis major is $\frac{3}{2}$ of the Earth's radius. Prove that, if the direction of projection makes an angle $30^{\circ}$ with the vertical, the time of flight is

$$
\frac{3}{4} \sqrt{ }(3 a / g)\left(\tan ^{-1} \sqrt{ } 6+\mathfrak{N}^{\prime} \frac{2}{3}\right),
$$

where $\alpha$ is the Earth's radius and $g$ is the value of gravity at its surface. 
8. A stream of particles originally moving in a straight line $K$ with velocity $V$ is under the influence of a gravitating sphere of radius $R$, whose centre moves with velocity $v$ in a straight line intersecting the line $K$ and making with it an angle $a$. Prove that, if the distance of the sphere from the line is originally very great, a length

$$
\frac{2 R}{v \sin a} \sqrt{ }\left(V^{2}-2 V v \cos a+v^{2}+2 g R\right)
$$

of the line of particles will fall upon the sphere, $g$ being the force per unit mass at the surface of the sphere.

9. A particle is projected with velocity less than that from infinity under a force tending to a fixed point and varying inversely as the $n$th power of the distance. Prove that if $n$ is not $<3$ the particle will ultimately fall into the centre of force.

10. A particle moves under a central force varying inversely as the $n$th power of the distance $(n>1)$, the velocity of projection is that due to a fall from restat infinity, and the direction of projection makes an angle $\beta$ with the radius vector of length $R$. Prove that the maximum distance is $R \operatorname{cosec}^{\frac{2}{n-3}} \beta$ when $n>3$, and that the particle goes to infinity if $n=$ or $<3$.

11. Prove that the time of describing any part of a central orbit is

$$
\int \frac{r d r}{\sqrt{ }\left\{2 r^{2}(C+V)-h^{2}\right\}},
$$

taken between appropriate limits, where $V$ is the potential, and $C$ and $h$ are constants depending on the initial conditions.

12. Prove that, if a possible orbit under a central force $\phi(r)$ is known, a possible orbit under a central force $\phi(r)+\lambda r^{-3}$ can be found. In particular prove that a particle projected from an apse at distance $a$ with velocity $\sqrt{ }(\lambda+\mu) / a$, under an attraction

$$
\frac{1}{2} \mu(n-1) \alpha^{n-3} r^{-n}+\lambda r^{-3}, \quad(n>3),
$$

will arrive at the centre in time

$$
\frac{a^{2}}{2} \sqrt{\frac{\pi}{\mu}} \Gamma\left(\frac{n+1}{2 n-6}\right) / \Gamma\left(\frac{2}{n-3}\right) .
$$

13. A particle moves under a central force and is projected with velocity $v_{0}$ from a point at distance $r_{0}$ in a direction making an angle $a$ with the radius vector. Prove that the apsidal distances are the real roots of the equation for $r$

$$
W r^{2} /\left(r_{0}^{2} \sin ^{2} a-r^{2}\right)=\frac{1}{2} v_{0}^{2},
$$

where $W$ is the work done by the central attractive force as the particle moves from the point of projection to any point $r, \theta$. 
14. A particle is describing a circular orbit of radius $a$ under a force to the centre producing an acceleration $f(r)$ at distance $r$, and a small increment of velocity $\Delta u$ is given to it in the direction of motion. Prove that the apsidal distances of the disturbed orbit are

$$
a \text { and } a+4 \Delta u \frac{\sqrt{ }\{a f(\alpha)\}}{3 f(a)+a f^{\prime}(\alpha)} .
$$

Prove also that, if the increment of velocity imparted to the particle is directed radially, the apsidal distances are approximately

$$
a \pm \Delta u \frac{\sqrt{ } a}{\sqrt{ }\left\{3 f(\alpha)+a f^{\prime}(a)\right\}} .
$$

15. A particle moves under a central force $\mu(1+8 k \cos 2 \theta) / r^{2}$ being projected from an apse on the initial line at distance $c$ with velocity $\sqrt{ }(\mu / c)$; show that the next apsidal distance is $c /(1+3 \kappa)$.

16. A particle moves under a central force proportional to $u^{2}(c u+\cos \theta)^{-3}$ towards the centre. Show that the orbit is one of the conics given by the equation $(c u+\cos \theta)^{2}=a+b \cos 2(\theta+a)$.

17. A particle moves in a plane under a radial force $P$ and a transverse force $T$, where

$$
P=-\mu u^{3}(3+5 \cos 2 \theta), \quad T=\mu u^{3} \sin 2 \theta ;
$$

prove that a first integral of the differential equation of the path can be expressed in the form

$$
h_{0}^{2}\left(\sin \theta \frac{d u}{d \theta}-u \cos \theta\right)+\frac{2}{\mu}\left[(\sin 3 \theta-\sin \theta) \frac{d u}{d \theta}+u \cos 3 \theta\right]=C,
$$

where $h_{0}^{2}$ and $C$ are constants.

18. A particle moves under the action of a central force $P$ and a transverse disturbing force $\frac{1}{r} f(t)$. Prove that

where $F(t)=\int f(t) d t$.

$$
\frac{d^{2} u}{d \theta^{2}}+u=\frac{P-f(t)}{u^{2}\left\{F^{\prime}(t)\right\}^{2}},
$$

19. Prove that in a plane field of force of which the potential referred to polar coordinates is

$$
\frac{a}{r^{4}}+\frac{\beta}{r^{6}}\left(1+3 \sin ^{2} \theta\right)
$$

a particle, if projected in the proper direction with the velocity from infinity, will describe a curve of the form

$$
\begin{array}{cc} 
& (r-a \sin \theta)(r-b \sin \theta)=a b, \\
\text { provided } & \frac{2}{a b}+\frac{4}{(a+b)^{2}}+\frac{a}{\beta}=0 .
\end{array}
$$

20. A particle of mass $m$ describes a circle (centre $C$ ) in period $T$ under the action of a force to a fixed point $S$. Prove that the force can be resolved into two directed to inverse points $O, O^{\prime}$ in $C S$ and equal respectively to

$$
\frac{16 m \pi^{2}}{T^{2}} \frac{C O^{2}}{C S^{2}} \frac{C P^{6}}{O P^{5}} \text { and } \frac{16 m \pi^{2}}{T^{2}} \frac{C O^{2}}{C S^{2}} \frac{C P^{6}}{O^{\prime} P^{5}} \text {. }
$$


21. A particle describes a circle with acceleration $\mu r^{-5}$ towards a point on the circumference. Prove that it will still describe the circle if acted on by a repulsive force from the centre producing acceleration $\mu^{\prime} a^{-5}$ provided it starts from rest at a point where $r=a\left(\mu / 2 \mu^{\prime}\right)^{\frac{1}{4}}, a$ being the radius of the circle.

22. A particle describes an ellipse under two forces, functions of the distance, one to each focus. If the law of force to one focus is $\mu r$, prove that to the other it must be $\mu r+\mu^{\prime} / r^{2}$.

23. An ellipse is described under the action of two forces, one to each focus. Show that the force per unit of mass along the focal radius vector $r$ is

$$
\frac{a v^{2}}{2 r(2 a-r)}-\frac{1}{4} \frac{d v^{2}}{d r}
$$

where $2 a$ is the major axis and $v$ the velocity.

24. Two centres of force of equal strength, one attractive and the other repulsive, are placed at two points $S$ and $H$, each force varying inversely as the square of the distance. Show that a particle placed anywhere in the plane bisecting $S H$ at right angles will oscillate in a semi-ellipse of which $S$ and $H$ are foci.

25. A body is placed at rest in a plane through two fixed centres of force, each varying inversely as the square of the distance, at a point where the forces are equal. Prove that it will oscillate in an arc of an hyperbola if both forces attract, and in the arc of an ellipse if one force attracts and the other repels.

26. A particle describes a parabola under two forces, one constant and parallel to the axis, and the other passing through the focus; prove that the latter force varies inversely as the square of the focal distance. Prove also that, if the force through the focus is repulsive, and numerically equal, at the vertex, to the constant force, the particle will come to rest at the vertex; and find the time occupied in describing any arc of the curve.

27. A particle describes a circle under the action of forces, tending to the extremities of a fixed chord, which are to each other at any point inversely as the distances $r, r^{\prime}$ from the point to the ends of the chord. Determine the forces, and prove that the product of the component velocities along $r$ and $r^{\prime}$ varies inversely as the length of the perpendicular from the position of the particle to the chord; also show that the time from one end of the chord to the other is

$$
\frac{a}{V} \frac{(\pi-a) \cos a+\sin a}{\cos ^{2} \frac{1}{2} a},
$$

where $V$ is the velocity of the particle when moving parallel to the chord, $a$ the radius of the circle, and $a$ the angle between $r$ and $r^{\prime}$.

28. A particle moves under the action of a repulsive force $\mu\left(u^{2}-a u^{3}\right)$ from a fixed point, and a force $\mu\left(1 / c^{2}-u / a\right)$ parallel to a fixed line, $1 / u$ being the distance from the point. Show that, if it starts from rest at a point where the forces are equal, it describes a parabola of which the point is the focus. 
29. If a curve is described under a force $P$ tending to the origin and a normal force $N$, prove that

$$
p^{2} \frac{d}{d r}\left(N r \frac{d r}{d p}\right)+\frac{d}{d r}\left(P p^{3} \frac{d r}{d p}\right)=0 .
$$

30. A particle is projected from an apse of Bernouilli's Lemniscate along the tangent with velocity $\sqrt{ } \mu / a$ and moves under the action of forces

$$
\mu r^{\prime 2} \frac{r^{\prime}-r}{\left(3 r r^{\prime}-r^{2}\right)^{3}}, \quad \mu r^{2} \frac{r-r^{\prime}}{\left(3 r r^{\prime}-r^{\prime 2}\right)^{3}},
$$

to the nearer and further poles respectively, $r$ being the distance from the nearer pole, and $r^{\prime}$ from the further pole. Show that it describes the lemniscate.

31. A particle $P$ moves under the action of two fixed centres of force $S_{1}, S_{2}$ producing accelerations $\mu_{1} / r_{1}{ }^{2}$ and $\mu_{2} / r_{2}{ }^{2}$ towards $S_{1}$ and $S_{2}$, where $r_{1}, r_{2}$ are the distances $S_{1} P, S_{2} P$. Prove that if the motion does not take place in a fixed plane there is an integral equation of the form

$$
\left(r_{1}^{2} \dot{\theta}_{1}\right)\left(r_{2}^{2} \dot{\theta}_{2}\right)+h^{2} \cot \theta_{1} \cot \theta_{2}=c\left(\mu_{1} \cos \theta_{1}+\mu_{2} \cos \theta_{2}\right)+\text { const. }
$$

where $\theta_{1}, \theta_{2}$ are the angles $S_{2} S_{1} P$ and $S_{1} S_{2} P, c$ is the distance $S_{1} S_{2}$, and $h$ is the moment of the velocity about the line of centres.

32. A thin spherical shell of small radius, moving without rotation, describes a circle of radius $R$ with velocity $V$ about a gravitating centre of force $O$, and when its centre is at a point $A$ bursts with an explosion which generates velocity $v$ in each fragment directly outwards from the centre. Prove that the fragments all pass through the line $A O$ within a length

$$
8 V^{3} v R /\left(V^{4}-6 V^{2} v^{2}+v^{4}\right),
$$

and that if $v$ is small the stream of fragments will form a complete ring after a time approximately equal to $\frac{1}{3} \pi R / v$.

33. Two particles are under the action of forces tending to a fixed point and varying as the distance from that point, the force being the same at the same distance in each case; the particles also attract each other with a different force varying as the distance between them; prove that the orbit of either particle relative to the other is an ellipse and the periodic time is $2 \pi / \sqrt{ }\left(\mu+2 \mu^{\prime}\right), \mu$ and $\mu^{\prime}$ denoting the forces on unit mass respectively at unit distance.

34. A series of particles which attract each other with forces varying directly as the masses and distance are under the attraction of a fixed centre of force which also varies directly as the distance; prove that, if they are projected in parallel directions from points lying on a radius vector passing through the centre of force with velocities inversely proportional to their distances from the centre of force, they will at any subsequent time lie on a hyperbola. 
35. A body of mass $M$ is moving in a straight line with velocity $U$, and is followed, at a distance $r$, by a smaller body of mass $m$ moving in the same line with velocity $u$. The bodies attract each other according to the law of gravitation. Prove that the smaller body will overtake the other after a time

where

$$
\begin{gathered}
\left(\frac{r}{1+w}\right)^{\frac{3}{2}} \frac{\pi+\sqrt{ }\left(1-w^{2}\right)+\cos ^{-1} w}{\sqrt{ }\{\gamma(M+m)\}}, \\
1-w=\frac{r(U-u)^{2}}{\gamma(M+m)} .
\end{gathered}
$$

36. Two bodies, masses $m, m^{\prime}$, are describing relatively to each other circular orbits under their mutual gravitation, $\alpha$ and $\alpha^{\prime}$ being their distances from the centre of inertia. If $V$ is the relative velocity, and $m$ receives an impulse $m V$ towards $m^{\prime}$, prove that the two bodies proceed to describe, relatively to the centre of inertia, parabolas whose latera recta are $2 \alpha$ and $2 \alpha^{\prime}$.

37. Two gravitating spheres of masses $m, m^{\prime}$ moving freely have relative velocity $V$ when at a great distance apart, and in the absence of gravitation one would pass the other at a minimum distance $d$. Prove that the relative orbits are hyperbolic, and that the direction of the relative velocity will be ultimately turned through an angle

$$
2 \tan ^{-1}\left\{\gamma\left(m+m^{\prime}\right) / V^{2} d\right\} .
$$

38. In a system of two gravitating bodies, $M$ and $m$, initially $M$ is at rest, and $m$ is projected with velocity $\sqrt{ }\{\gamma(M+m) / d\}$ at right angles to the line joining the bodies, $d$ being the distance between the bodies. Prove that the path of $M$ is a succession of cycloids and that $M$ comes to rest at a cusp after equal intervals of time.

39. In a system of two gravitating bodies of masses $M$ and $m$ the relative orbit is an ellipse of semi-axes $\alpha$ and $b$. Prove that if the mass of the second body could be suddenly doubled the eccentricity of the new orbit would be

$$
\left.\frac{1}{M+2 m} \sqrt{ }(M+2 m)^{2}-\frac{b^{2}}{a^{2}}(M+m)(M+2 m)-\frac{b^{2}}{a} \frac{m}{\gamma} v^{2}\right],
$$

where $v$ is the relative velocity at the instant of the change.

40. Two gravitating particles whose distance is $r$, are describing circles uniformly about their common centre of gravity with angular velocity $\omega$, and a small general disturbance in the plane of motion is communicated to the system, so that after any time $t$ the distance is $r+u$, and the line joining the particles is in advance of the position it would have occupied if the steady motion had not been disturbed by the angle $\phi$; obtain the equation

$$
2 \dot{u}-r \omega \phi=3 \omega t(r \dot{\phi}+2 \omega u)+\text { const. }
$$

squares of $u$ and $\dot{\phi}$ being neglected.

41. Two equal particles $P, Q$ are projected from points equidistant on opposite sides of a third particle $S$, with a velocity due to their distance under the attraction of $S$ only. All three particles are gravitating, and the directions 
of projection are at right angles to $P Q$. If $b$ is the conjugate axis of the orbit described by either $P$ or $Q, e$ its eccentricity, and $b^{\prime}, e^{\prime}$ those of the relative orbit of $P$ and $S$ (in the absence of $Q$ ), $P$ being projected in the same manner as before, then $b=2 b^{\prime}$, and $(1-e) /(1+e)=\frac{1}{4}\left(1-e^{\prime}\right) /\left(1+e^{\prime}\right)$.

42. If two bodies of masses $E$ and $M$ move under their mutual gravitation and that of a fixed body of mass $S$ so that the three are always in one plane, prove that

$$
(E+M)^{2} H+E M h=\text { const. }
$$

where $h$ is the rate at which $M$ describes area about $E$ and $H$ is the rate at which the centre of inertia of $M$ and $E$ describes area about $S$.

If the three bodies are free, prove that the equation must be altered to

$$
S(E+M)^{2} H+(S+E+M) E M h=\text { const. }
$$

43. If three bodies of masses $m_{1}, m_{2}, m_{3}$, subject only to their mutual attractions $P_{23}, P_{31}, P_{12}$, remain at constant distances from one another, those distances are in the ratios

$$
m_{1} P_{23}: m_{2} P_{31}: m_{3} P_{12} \text {. }
$$

44. Three equal particles $A, B, C$, attracting each other with a force proportional to the distance, and equal to $\mu$ per unit mass at unit distance, are placed at the corners of an equilateral triangle of side $2 \alpha$. The particle $A$ is projected towards the centre of the triangle with velocity $c \sqrt{ } \mu$, the other particles being set free at the instant of projection. Prove that the three particles will first be in a straight line after a time

$$
\frac{1}{\sqrt{(3 \mu)}} \sin ^{-1} \frac{a}{\sqrt{\left(a^{2}+\frac{1}{9} c^{2}\right)}}
$$

45. When a particle is at the nearer apse of an ellipse , of eccentricity $e$ described about the focus, the force on unit mass at unit distance is increased by the small fraction $\frac{1}{n}$ of itself : when the particle is at the further apse, the force becomes less than its original value by the same amount. Prove that the time taken in this revolution is less than the original period by the fraction $\frac{6 e}{n\left(1-e^{2}\right)}$ of itself.

46. A particle describes an elliptic orbit about a focus and, when at the end of the minor axis, it receives a small impulse towards the centre equal to $\frac{1}{n}$ th of its momentum. Show that the eccentricity $e$ is increased or diminished by $\frac{1}{n} \sqrt{ }\left(1-e^{2}\right)$ according to the direction of motion at the instant. 
47. If, at any point of an elliptic orbit about a focus, the force ceases to act for a given very short time, find the angle through which the apse line will have turned and the change of the eccentricity, and show that they are respectively proportional to the resolved parts of the force parallel and perpendicular to the apse line.

48. A particle of mass $m$ describes an ellipse about a focus, $\mu m$ being the force at unit distance; when the particle is at an extremity of the minor axis it receives a small impulse $m V$ in a direction perpendicular to the plane of the orbit; prove that the eccentricity of the orbit will be diminished by $\frac{1}{2} V^{2} a e / \mu$, and that the angle which the axis major of the orbit makes with the distance from the focus will be increased by

$$
\frac{V^{2} a}{2 \mu} \frac{2-e^{2}}{e \sqrt{ }\left(1-e^{2}\right)},
$$

where $2 \alpha$ is the axis major, and $e$ the eccentricity of the orbit.

49. An ellipse of eccentricity $e$ and latus rectum $l$ is described freely about a focus by a particle of mass $m$, the angular momentum being $m h$. A small impulse $m u$ is given to the particle, when at $P$, in the direction of its motion; prove that the apse line is turned through an angle proportional to the intercept made by the auxiliary circle of the ellipse on the tangent at $P$, and which cannot exceed lu/eh.

50. If the velocity of a periodic comet is suddenly increased near its aphelion by a small amount $\delta V$, prove that the changes produced in the eccentricity and axis major are given by the equations

$$
\delta e=-2 \delta V \sqrt{ }(l / \mu), \quad \delta a=2 \delta V \sqrt{ }\left\{a^{3}(1-e) / \mu(1+e)\right\},
$$

where the letters have their usual meanings for elliptic motion.

51. A comet describes about the Sun an ellipse of eccentricity $e$ nearly equal to unity. At a point where the radius vector makes an angle $\theta$ with the apse line, the comet is instantaneously affected by a planet so that its velocity is increased in the ratio $n+1: n$, where $n$ is great, without altering its direction. Show that if the new orbit is a parabola

$$
e=1-\frac{4}{n} \cos ^{2} \frac{1}{2} \theta \text { nearly. }
$$

52. A body is revolving in an elliptic orbit with acceleration $\mu / r^{2}$ to a centre of force in one focus $S$, and when at $P$ it receives impulsively a small velocity $\delta v$ in the direction $P M$ at right angles to the major axis. Prove that the major axis will turn through the angle $\frac{\delta v}{e h} \frac{S M . P M}{S P}$.

53. At a point $P$ of an ellipse, described under a force to a focus $S$, the direction of motion is deflected through a small angle $\beta$ without alteration of 
magnitude. Prove that at a point $Q$ on the original ellipse the deviation of the new path, measured along the normal at $Q$, is

$$
{ }_{\beta}^{P H} \sqrt{Q H \cdot Q S} \sin Q H P,
$$

where $H$ is the second focus, and $C B$ the semi-axis minor.

54. If, when a particle, describing an elliptic orbit about a focus, is at an end of the axis minor, the centre of force is suddenly shifted a small distance $a a$ towards the particle, the eccentricity $e$ of the orbit will be unaltered, but the axis major will be turned through an angle $a \sqrt{ }\left(e^{-2}-1\right)$.

55. If the particle (of the last Example) is at an end of the latus rectum, and the centre of force is suddenly shifted a small distance $a a$ towards the centre, show that, to a first approximation, the eccentricity is diminished by $a$, and the major axis is turned through an angle $\alpha a / l$, where $l$ is the semi-latus rectum, while the periodic time is unaltered. Also prove that, to a second approximation, the periodic time is increased by $3 a^{2} \alpha^{3} / 2 l^{3}$ of its original value.

56. If when the particle (of the last Example) is at any point distant $r^{*}$ from the centre of force, the centre of force is suddenly shifted a small distance $k$ perpendicular to the plane of the orbit; prove that the periodic time is increased in the ratio $1+\frac{3}{2} \frac{a k^{2}}{r^{3}}: 1$. Also, if the change takes place when the particle is at an end of the latus rectum, the angle between the apse line and the radius vector is altered by

$$
\frac{1+2 e^{2}}{\left(1-e^{2}\right)^{2}} \frac{k^{2}}{2 e a^{2}}
$$

57. A particle is describing an ellipse under a force to a focus $S$; and, when the particle is at $P$, the centre of force is suddenly moved a short distance $x$ parallel to the tangent at $P$. Prove that the axis major is turned through the angle $\frac{x}{S G} \sin \phi \sin (\theta-\phi)$, where $G$ is the foot of the normal, $\theta$ the angle the normal makes with $S G$, and $\phi$ the angle the tangent makes with $S P$.

58. Defining the instantaneous orbit under a central force varying as the distance as that which would be described if the resistance ceased to act, show that, if at any point the resistance produces a retardation $f$, the rates of variation of the principal semi-axes are given by the equations

$$
\frac{\dot{a}}{a\left(a^{2}-r^{2}\right)}=\frac{\dot{b}}{b\left(r^{2}-b^{2}\right)}=-\frac{f}{v\left(a^{2}-b^{2}\right)},
$$

where $v$ is the velocity and $r$ the radius vector at the instant. 
59. In the last Example there is no resistance but there is a disturbance which produces a normal acceleration $g$. Show that the maxima of the rates of variation of the principal semi-axes of the instantaneous ellipse are given by the equations

$$
\frac{\dot{a}}{b}=-\frac{\dot{b}}{a}=\frac{ \pm g}{(a+b) \sqrt{ } \mu},
$$

where $\mu$ is the central force on unit mass at unit distance.

60. A particle $P$ describes an ellipse under a central force producing an, acceleration $k^{2}$ (distance) directed to a point $O$. When $P$ is at an end of the axis major, $O$ begins to move with a simple harmonic motion $\mu \sin \lambda t$. Show that the motion of $P$ may be represented at any time by motion in an ellipse whose centre is fixed and axis minor is constant and whose semi-axis major is variable according to the equation

$$
a=a_{0}+\frac{\mu k}{\lambda^{2}-k^{2}}(\lambda \sin k t-k \sin \lambda t) \sec k t .
$$




\section{CHAPTER X.}

\section{MOTION UNDER CONSTRAINTS AND RESISTANCES.}

182. THE second main subdivision of "Dynamics of a Particle" relates to motion of a particle in a given field of force when the force of the field is not the only force acting on the particle, but there are unknown forces also acting upon it and enforcing some conditions. This subdivision includes all cases where the particle is constrained to move on a given curve or surface smooth or rough, all cases where the motion of one particle is partly determined by that of another with which it is connected in an invariable manner, and we shall extend it to include all cases of particles subject to motional forces (Article 152).

\section{Motion on a smooth curve in a vertical plane} under gravity. The motion is entirely determined by the equation of energy

$$
\frac{1}{2} v^{2}+g y=\text { const., }
$$

where $v$ is the velocity of the particle when it is at a height $y$ above a fixed horizontal plane. The velocity is therefore always that due to falling from a definite level which is the same throughout the motion.

Let $\rho$ be the radius of curvature at the point where the velocity is $v$, and $\phi$ the angle which the normal drawn inwards (towards the centre of curvature) makes with the vertical drawn downwards, then, taking $m$ for the mass of the particle and $R$ for the pressure of the curve on the particle, we have by resolving along the normal

$$
m \frac{v^{2}}{\rho}=m g \cos \phi+R,
$$

where $R$ is supposed to act inwards. 
When the constraint is applied by means of a smooth tube within which the particle moves, or when the particle is the centre of inertia of a small ring sliding on a smooth wire in the form of the curve, $R$ can act either inwards or outwards. When the constraint is applied by means of a smooth surface the particle is either on the concave side or on the convex side of its path on the surface ; in the former case $R$ acts inwards, in the latter outwards.

When the pressure, if there is any, must act inwards or must act outwards the particle leaves the curve at the instant when the pressure changes sign. Putting $R=0$, we find as the condition for this

$$
v^{2}=g \rho \cos \phi,
$$

showing that when the particle leaves the curve the velocity is that due to falling through one quarter of the vertical chord of curvature.

If we wish to find the acceleration along the tangent to the curve we have only to resolve along the tangent, and the required acceleration is the resolved part of $g$ along the tangent.

184. Motion down an inclined tube. The case just discussed includes in particular the case of a particle sliding down a line of greatest slope on a smooth inclined plane, or sliding down a smooth straight tube held in an inclined position.

If $a$ is the inclination to the horizon, the acceleration down the tube (or down the plane) is $g \sin a$, and the pressure on the tube (or on the plane) is $m g \cos a$, where $m$ is the mass of the particle.

The motion of the particle is uniformly accelerated motion, and the discussion of Article 42 applies to it. The first experimental determination of the value of $g$ was made by observing the velocity acquired in sliding down an inclined plane.

\section{Examples.}

1. Prove that the time of descent down all chords of a vertical circle starting from the highest (or ending at the lowest point) is the same.

2. Prove that the line of quickest descent from a point $A$ to a curve, which is in a vertical plane containing $A$, is the line from $A$ to the point of contact with the curve of a circle described to have $A$ as its highest point and to touch the curve. Prove also that the line of quickest descent from a curve to a point $A$ is the line from $A$ to the point of contact with the curve of a circle described to have $A$ as its lowest point and to touch the curve. 
3. Prove that each of the lines of quickest descent in Example 2 bisects the angle between the normal to the curve and the vertical at the point where it meets the curve, and hence show that the line of quickest descent from one given curve to another in the same vertical plane bisects the angle between the normal and the vertical at both ends.

4. When the tube (or the plane) of Article 184 is rough, and the coefficient of friction is $\mu$, prove that the acceleration down the line of greatest slope is $g(\sin a \pm \mu \cos a)$, where the upper or lower sign is to be taken according as the particle is moving up or down. Also find the acceleration with which a particle moves on a rough horizontal plane.

5. Example 4 is generally assumed to apply to the motion of a railway train. Consider the motion on straight horizontal rails, and let $m$ be the mass of the train, $P$ the resultant force acting upon it apart from the resistance, $\mu \mathrm{n} g$ the resistance, then $f$ the acceleration with which the train moves is given by the equation

$$
m f=P-\mu m g,
$$

and, if $P=\mu m g$, the train moves with uniform velocity. The force $P$ is known as the "pull of the engine," but it is not the tension in a coupling between the engine and the rest of the train.
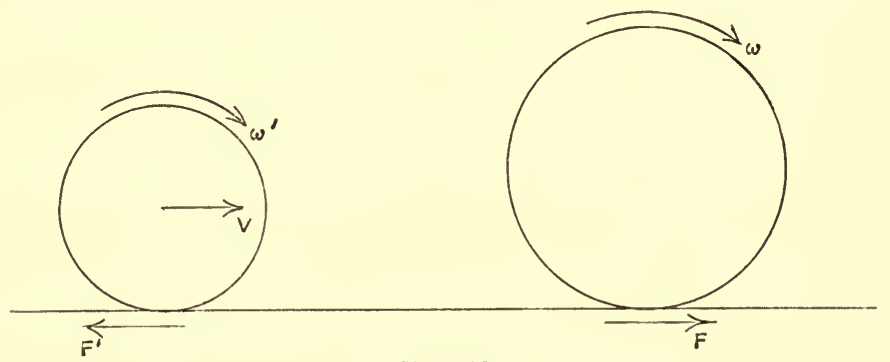

Fig. 46.

To see how the force $P$ arises we consider the motion of the driving wheel and the motion of a wheel of one of the coaches of the train. The left-hand circle represents the coach-wheel, and the right-hand circle the driving wheel. At starting the centre of the driving wheel is at rest, and the machinery is so contrived that a couple is exerted upon it tending to turn it rapidly in the direction shown by the arrow-head marked $\omega$. The wheel slips (or "skids") on the rail, and, at the point of contact, friction is exerted in the direction opposite to that in which the point of contact slips, this is shown by $F$ in the figure. So long as the steam is "on" the couple is exerted on the wheel, and there is sliding friction $F$ as shown. The resultant of such frictions for all parts on which they act is the force $P$. Again, consider the coach-wheel rolling on the rail with an angular velocity in the direction shown by the arrow marked $\omega^{\prime}$. The motion of the coach on the rails is in the direction of the arrow marked $V$. In the absence of friction the coach would slip over the rails in this direction. Thus the friction of the rails on the coach-wheels is in the 
direction of the arrow marked $F^{\prime \prime}$, and this friction is rolling friction. The resultant of all such frictions as $F^{\prime \prime}$ is the resistance to the motion of the train.

It appears that the resistance of the rails arises from rolling friction, and the pull of the engine from sliding friction, and, since the ratio of rolling friction to pressure at a point of contact is always less (for the same materials) than that of sliding friction to pressure, there is no difficulty in seeing how a train can be set in motion by a locomotive of smaller mass than the train.

The resistance of the air is not sufficiently great to affect the argument.

6. A carriage is slipped from an express train, going at full speed, at a distance $l$ from a station, and comes to rest at the station. Prove that the rest of the train will then be at a distance $M l /(M-m)$ beyond the station, $M$ and $m$ being the masses of the whole train and of the carriage slipped, and the pull of the engine being constant.

7. Prove that the extra work required to take a train from one station to stop at the next at a distance $l$ in an interval $t$ is

$$
\frac{2 l}{g t^{2}} \frac{1}{k} /\left\{\left(\frac{1}{m}+\frac{1}{n}\right)\left(\frac{1}{m}+\frac{1}{n}+\frac{1}{k}\right)\right\}
$$

times the work required to run through without stopping, where the incline of the road is 1 in $m$, and the resistance of the road and the brake power per unit mass are equal to the components of gravity down uniform inclines of 1 in $n$ and 1 in $k$ respectively.

8. A cylinder whose section is a parabola is placed with its generators horizontal, the axis of a normal section vertical, and the vertex upwards, and a particle is projected along it in a vertical plane. Prove that if it leaves the parabola anywhere it does so at the point of projection.

9. A particle is projected from the lowest point of a vertical section of a smooth hollow circular cylinder whose axis is horizontal so as to move round inside the cylinder. Prove that, if the velocity is that due to falling from the highest point, the particle leaves the circle when the radius through it makes with the vertical an angle $\cos ^{-1} \frac{2}{3}$.

Find the least velocity of projection in order that the particle may describe the complete circle.

10. A particle is constrained to describe a circle by means of an inextensible thread, and leaves the circle when the thread makes an angle $\beta$ with the vertical drawn upwards. Prove that when it strikes the circle again the thread makes an angle $3 \beta$ with the same vertical.

11. Prove that a particle projected in any manner on a smooth plane of inclination $a$ to the horizon describes a parabola as if under gravity diminished in the ratio $\sin a: 1$. 


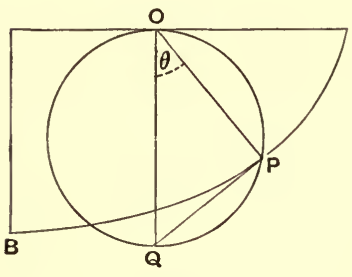

Fig. 47.

12. When a particle moves on a smooth cycloid under gravity, the vertex of the cycloid being at the lowest point, the equation of motion, by resolution along the tangent in direction $Q P$, may be written

$$
\ddot{s}=-g \sin \theta \text {, }
$$

$s$ being the arc measured from the vertex to $P$, and $\theta$ the angle the normal $O P$ makes with the vertical. Now, by a known property of the cycloid, $s=4 a \sin \theta$, where $a$ is the radius of the generating circle, and thus the above equation becomes

$$
\ddot{s}=-\frac{g}{4 a} s,
$$

showing that the motion in $s$ is simple harmonic with period $2 \pi \sqrt{ }(4 a / g)$. Thus the time taken to fall to the vertex from any point on the curve is independent of the starting point, and in fact is $\pi \sqrt{ }(a / g)$.

[This property is known as the "Isochronism of the cycloid."]

13. Show that the time a train, if unresisted, takes to pass through a tunnel under a river in the form of an arc of an inverted cycloid of length $2 s$ and height $h$ cut off by a horizontal line is

$$
\frac{s}{\sqrt{2 g h}} \cos ^{-1}\left(\frac{v^{2}-2 g h}{v^{2}+2 g h}\right),
$$

where $v$ is the velocity with which the train enters and leaves the tunnel.

\section{Motion of two bodies connected by an inexten-} sible thread. We shall consider the case of two bodies, of masses $m$ and $m^{\prime}$, attached

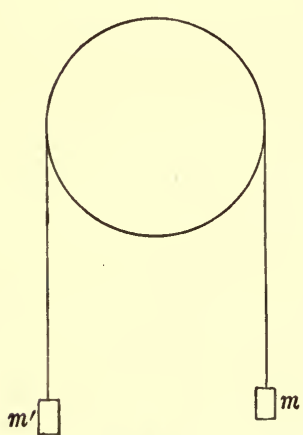

Fig. 48. to the ends of an inextensible thread which passes over a smooth pulley. We neglect the mass of the thread.

The bodies will move like particles at their centres of inertia.

The particles in question move in the line of the thread.

Suppose that the particle of mass $m$ has descended through a distance $x$ in the interval $t$. Then $m^{\prime}$ has risen through a distance $x$. Hence at any instant the velocities of the particles are equal in magnitude.

The kinetic energy is therefore $\frac{1}{2}\left(m+m^{\prime}\right) \dot{x}^{2}$.

Also the potential energy is Const. $+m^{\prime} g x-m g x$. 
Hence the equation of energy can be written

$$
\frac{1}{2}\left(m+m^{\prime}\right) \dot{x}^{2}=\left(m-m^{\prime}\right) g x+\text { const. }
$$

By differentiating this equation we obtain

$$
\left(m+m^{\prime}\right) \ddot{x}=\left(m-m^{\prime}\right) g,
$$

showing that the acceleration of either particle is in magnitude $\frac{m-m^{\prime}}{m+m^{\prime}} g$.

The tension $T$ of the thread can be obtained from the equation of motion of either particle. Thus we have for $m$

giving

$$
\begin{aligned}
& m \ddot{x}=m g-T, \\
& T=\frac{2 m m^{\prime}}{m+m^{\prime}} g .
\end{aligned}
$$

An instrument of which the above is the principle is known as Atwood's machine. It is manifest that the masses can be so adjusted as to make the acceleration much smaller, and therefore much more accurately measurable, than the acceleration $g$ of a free falling body. When the ratio of the masses of the two bodies is known, experiments with this instrument yield a determination of the value of $g$.

\section{Examples.}

1. In Atwood's machine the mass $m^{\prime}$ is rigid, the mass $m$ consists of a rigid portion of mass $m^{\prime}$ and a small additional piece lightly resting upon it. As $m$ descends it passes through a ring, by which the additional piece is lifted off. Prove that, if $m$ starts from a height $h$ above the ring, and if the time it takes to fall a distance $k$ after passing through the ring is $t$, then

$$
g=\frac{2\left(m-m^{\prime}\right)}{m+m^{\prime}} \frac{k^{2}}{h t^{2}} \text {. }
$$

2. Two particles of masses $M, m$ are connected by an inextensible thread of negligible mass which passes through a small smooth ring on a smooth fixed horizontal table. When the thread is just stretched, so that $M$ is at a distance $c$ from the ring, and the particles are at rest, $M$ is projected on the table at right angles to the thread. Prove that until $m$ reaches the ring $M$ describes a curve whose polar equation is of the form

$$
r=c \cos \{\theta \sqrt{ }(1+m / M)\} .
$$

3. Two particles of masses $M, m$ are connected by an inextensible thread of negligible mass; $M$ describes on a smooth table a curve which is nearly a circle with centre at a point $O$, and the thread passes through a small smooth hole at $O$ and supports $m$. Prove that the apsidal angle of $M$ 's orbit is *

$$
\pi \sqrt{ }\left\{\frac{1}{3}(1+m / M)\right\} \text {. }
$$


188. Simple Circular Pendulum. A particle constrained to describe a circle in a vertical plane is called a "simple circular pendulum." When the constraint is applied by means of an inextensible thread of negligible mass with one extremity fixed, or when the particle is within a hollow cylinder with a horizontal axis, the particle can leave the circle. For the present we shall suppose the particle to be within a circular tube, fixed in a vertical plane.

Let $l$ be the radius of the circle. This is called the length of the pendulum.

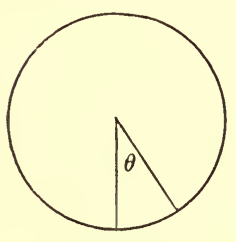

Fig. 49.

Let $\theta$ be the angle the radius through the particle makes with the vertical at time $t$.

The kinetic energy is $\frac{1}{2} m l^{2} \dot{\theta}^{2}$, where $m$ is the mass of the particle.

The potential energy of the particle in the field is $m g l(1-\cos \theta)$, the standard position of the particle being at the lowest point.

Hence the equation of energy may be written, after division by $m l$,

$$
\frac{1}{2} l \dot{\theta}^{2}=g \cos \theta+\text { const. }
$$

where the constant depends on the velocity at the lowest point.

189. Small Oscillation. Differentiating the equation of energy last written with respect to the time, we have

$$
l \ddot{\theta}=-g \sin \theta \text {. }
$$

This equation might have been obtained by resolving along the tangent to the circle.

If $\theta$ is very small throughout the motion we may put $\theta$ for $\sin \theta$, and thus

$$
\ddot{i \theta}=-g \theta .
$$

This equation shows (Article 47) that the motion in $\theta$ is simple harmonic with a period $2 \pi \sqrt{ }(l / g)$.

The pendulum swings from side to side of the vertical. Suppose it to start from rest in a position near the equilibrium position but slightly displaced to the right. It falls to the equilibrium position in time $\frac{1}{2} \pi \sqrt{ }(l / g)$, passes through it, and proceeds to the left until its displacement is numerically equal to that at 
starting, and comes to rest after an interval $\frac{1}{2} \pi \sqrt{ }(l / g)$ from the equilibrium position. The motion is then reversed. The time from rest to rest is $\pi \sqrt{ }(l / g)$. This is known as the time of a "beat," the period $2 \pi \sqrt{ }(l / g)$ is the time of a "complete oscillation."

A pendulum which beats seconds is known as a "seconds pendulum;" the time of a complete oscillation of such a pendulum is two seconds. The length of the seconds pendulum at a place is given by the equation

$$
\pi \sqrt{ }(l / g)=1
$$

Pendulum experiments afford the most exact method of determining the value of $g$.

\section{Examples.}

1. Prove that, in the case of one-sided constraint, the particle cannot leave the circle unless it has risen above the centre.

2. Prove that, if in London $g=981 \cdot 17$, the units being the centimetre and the second, then the length of the seconds pendulum there is $99 \cdot 413$ centimetres.

3. A balloon ascends with constant acceleration and reaches a height of $900 \mathrm{ft}$. in one minute. Show that a pendulum clock carried with it will gain at the rate of $27 \cdot 8$ seconds per hour, approximately.

4. If $l_{1}$ is the length of a pendulum which gains $n$ seconds in an hour, and $l_{2}$ the length of a pendulum which loses $n$ seconds in an hour, $n$ being small, prove that the square root of the true length of the seconds pendulum is the harmonic mean between $\sqrt{ } l_{1}$ and $\sqrt{ } l_{2}$.

5. The bob of a pendulum which is hung close to the face of a cliff is attracted to the cliff with a horizontal force of intensity $f$. Show that the time of a beat is

$$
\pi l^{\frac{1}{2}} /\left(g^{2}+f^{2}\right)^{\frac{1}{4}}
$$

where $l$ is the length of the pendulum.

6. A bead slides on a smooth circular wire of radius $\alpha$, whose plane is inclined at an angle $a$ to the vertical. Find the period of its small oscillations about the lowest point.

7. Show that, if the suspending fibre of a simple pendulum is slightly extensible, its vibrations of small amplitude take place in an arc having its centre of curvature at a distance below the point of suspension equal to three times the statical increase of length due to the weight of the bob, while the period is simply that due to the stretched length of the fibre.

8. Prove that on taking a pendulum down a mine, the time of vibration is increased or diminished according as the mean density of the Earth at its surface is greater or less than two-thirds of the Earth's mean density. 
9. Find the greatest angle through which a person can oscillate on a swing, the ropes of which can support a tension equal to twice the person's weight.

*191. Finite Oscillation. More generally, suppose the pendulum to start from rest in a position in which the radius makes an angle $\alpha$ with the vertical. Then, in the notation of Article $188, \dot{\theta}=0$ when $\theta=\alpha$, and the equation of energy of that Article can be written

or

$$
\begin{gathered}
\frac{1}{2} l \dot{\theta}^{2}=g(\cos \theta-\cos \alpha), \\
\frac{1}{4} \theta^{2}=\frac{g}{l}\left(\sin ^{2} \frac{\alpha}{2}-\sin ^{2} \frac{\theta}{2}\right),
\end{gathered}
$$

showing that the pendulum oscillates between two positions in which it is inclined to the vertical at an angle $\alpha$ on the right and left sides of the vertical.

To express the position of the pendulum in terms of the time $t$, since it was in the equilibrium position, we introduce a new variable $\psi$ defined by the equation

$$
\sin \frac{\alpha}{2} \sin \psi=\sin \frac{\theta}{2}
$$

with the further conditions that as $\theta$ increases from 0 to $\alpha, \psi$ increases from 0 to $\frac{1}{2} \pi$; as $\theta$ diminishes from $\alpha$ to $0, \psi$ increases from $\frac{1}{2} \pi$ to $\pi$; as $\theta$ diminishes from 0 to $-\alpha, \psi$ increases from $\pi$ to $\frac{3}{2} \pi$; and as $\theta$ increases from $-\alpha$ to $0, \psi$ increases from $\frac{3}{2} \pi$ to $2 \pi$. With these conventions there is one value of $\psi$ corresponding to every instant in a complete period.

Now we have

$$
\begin{gathered}
\frac{1}{2} \dot{\theta} \cos \frac{\theta}{2}=\dot{\psi} \sin \frac{\alpha}{2} \cos \psi, \\
\sin ^{2} \frac{\alpha}{2}-\sin ^{2} \frac{\theta}{2}=\sin ^{2} \frac{\alpha}{2} \cos ^{2} \psi, \\
\dot{\psi}^{2}=\frac{g}{l}\left(1-\sin ^{2} \frac{\alpha}{2} \sin ^{2} \psi\right) .
\end{gathered}
$$

Hence the time $t$ from the instant when the particle was passing through the lowest point in the direction in which $\theta$ increases is given by the equation

$$
t=\sqrt{\frac{l}{g}} \int_{0}^{\psi} \frac{d \psi}{\sqrt{\left(1-\sin ^{2} \frac{\alpha}{2} \sin ^{2} \psi\right)}},
$$


where the square root is always to be taken positive. The complete period is

$$
4 \sqrt{\frac{l}{g}} \int_{0}^{\frac{\pi}{2}} \frac{d \psi}{\sqrt{\left(1-\sin ^{2} \frac{\alpha}{2} \sin ^{2} \psi\right)}}
$$

With the above relation between $t$ and $\psi, \sin \psi$ is said to be an Elliptic Function of $t \sqrt{ } \frac{g}{l}$, and the relation is written

$$
\sin \psi=\operatorname{sn}\left(t \sqrt{\frac{g}{l}}\right) \quad\left(\bmod \sin \frac{\alpha}{2}\right) .
$$

The function has a real period, and the integral

$$
\int_{0}^{\frac{\pi}{2}} \frac{d \psi}{\sqrt{\left(1-\sin ^{2} \frac{\alpha}{2} \sin ^{2} \psi\right)}}
$$

is one quarter of this period.

The position of the pendulum at any time $t$ is determined by the equation

$$
\sin \frac{\theta}{2}=\sin \frac{\alpha}{2} \operatorname{sn}\left(t \sqrt{\frac{g}{l}}\right) \quad\left(\bmod \sin \frac{\alpha}{2}\right) .
$$

*192. Complete Revolution. If the constant in the energy equation of Article 188 is such that $\dot{\theta}$ never vanishes it must be greater than $g$, and the velocity at the lowest point is greater than that due to falling from the highest point. Hence there will be some velocity at the highest point. Let us suppose the velocity at the highest point is that due to falling through a height $h$; then, when $\theta=\pi$

$$
l^{2} \dot{\theta}^{2}=2 g h,
$$

and for any other value of $\theta$

or

$$
\begin{gathered}
\frac{1}{2} l \dot{\theta}^{2}=g\left(\cos \theta+1+\frac{h}{l}\right), \\
\frac{1}{4} \dot{\theta}^{2}=\frac{g(h+2 l)}{2 l^{2}}\left(1-\frac{2 l}{h+2 l} \sin ^{2} \frac{\theta}{2}\right),
\end{gathered}
$$

giving $\sin \frac{\theta}{2}=\operatorname{sn}\left(\frac{t}{k} \sqrt{ } \frac{g}{l}\right) \quad(\bmod k)$, where $k^{2}=2 l /(h+2 l)$. 
The period of a complete revolution is

$$
2 k \sqrt{\frac{l}{g}} \int_{0}^{\frac{\pi}{2}} \frac{d \phi}{\sqrt{ }\left(1-k^{2} \sin ^{2} \phi\right)} \text {. }
$$

*193. Limiting case. In the case where the pendulum is projected from the position of equilibrium with velocity equal to that due to falling from the highest point the equation can be integrated by logarithms.

The constant in the energy equation of Article 188 must then be chosen so that $\dot{\theta}$ vanishes when $\theta=\pi$, and the equation therefore is

$$
\frac{1}{2} l \dot{\theta}^{2}=g(1+\cos \theta)
$$

which may be written

$$
\frac{1}{4} \dot{\theta}^{2}=\frac{g}{l} \cos ^{2} \frac{\theta}{2}
$$

The time of describing an angle $\theta$ is therefore $t$, where

$$
t=\sqrt{\frac{l}{g}} \int_{0}^{\frac{\theta}{2}} \frac{d x}{\cos x}=\sqrt{\frac{l}{g}} \log \left(\sec \frac{\theta}{2}+\tan \frac{\theta}{2}\right) .
$$

It is to be noted that the particle approaches the highest point indefinitely, but does not reach it in any finite time.

The same equations may be used to describe the motion of the particle from a position indefinitely close to the unstable position of equilibrium at the highest point of the circle.

\section{*194. Examples.}

1. Prove that the time of a finite oscillation when the fourth power of $a$, the angle of oscillation, is neglected, is $2 \pi\left(1+\frac{1}{16} a^{2}\right) \sqrt{ }(l / g)$.

2. Prove that, in the limiting case of Article 193,

$$
\theta=2 \tan ^{-1} \sinh \{t \sqrt{ }(g / l)\} \text {. }
$$

3. Prove that if a seconds pendulum makes a complete finite oscillation in four seconds the angle $a$ is about $160^{\circ}$.

195. Motion on a smooth plane curve under any positional forces. Suppose that a particle of mass $m$ is constrained to move on a given smooth plane curve under the action of given forces in the plane. Let $s$ be the arc of the curve measured from some point of the curve up to the position of 
the particle at time $t$. Let $S$ be the tangential component of the forces in the direction in which $s$ increases, and $N$ the component along the normal inwards. Let $v$ be the velocity of the particle in the direction in which $s$ increases, and $R$ the pressure of the curve on the particle. We shall write down the equations for the case where the particle is on the inside of the curve, and $R$ accordingly acts inwards. The equations for the case in which $R$ acts outwards can be obtained by changing the sign of $R$.

By resolving along the tangent and normal we obtain

$$
\left.\begin{array}{rl}
m v \frac{d v}{d s} & =S, \\
m \frac{v^{2}}{\rho} & =N+R
\end{array}\right\} .
$$

These are the equations of motion. The former can be integrated so as to express the velocity in terms of the position. In fact, since the forces $S$ and $N$ depend only on position, and since the coordinates of any point of the curve can be regarded as functions of a single parameter, it is clear that, on the curve, $S$ is a function of the parameter, and $d s$ is the product of a function of the parameter and its differential. Hence we have

$$
\frac{1}{2} m v^{2}=\int S d s+\text { const. }
$$

- where the integral is of the form $\int^{\theta} f(\theta) \phi(\theta) d \theta$ in which $\theta$ is the parameter, $\dot{S}$ is $f(\theta)$, and $d s$ is $\phi(\theta) d \theta$.

This is a case in which the work done by the forces between two positions can be calculated whether the system of forces is conservative or not, and the equation can be interpreted in the form

$$
\text { change of kinetic energy = work done, }
$$

where the "change of kinetic energy" on the left means the excess of the kinetic energy in the position at time $t$ above that in some definite standard position, and the "work done" on the right means the work done by the forces in the displacement of the particle along the curve from the definite standard position to the position at time $t$.

In the case of conservative positional forces $\int S d s$ is the excess 
of the value of the work function in the position at time $t$ above its value in the standard position.

The integral equation gives the velocity $v$ in each position, and the second of the equations of motion gives the pressure $R$. In the case of one-sided constraint the particle leaves the curve when $R$ changes sign.

\section{Examples.}

1. Prove that, when the particle leaves the curve, the velocity is that due to falling under the force kept constant through one quarter of the chord of curvature in the direction of the force.

2. Prove that, when the curve is a free path under the given forces for proper velocity of projection, then for any other velocity of projection, the pressure varies as the curvature.

*197. Smooth plane tube rotating in its plane. Suppose that a particle of mass $m$ moves

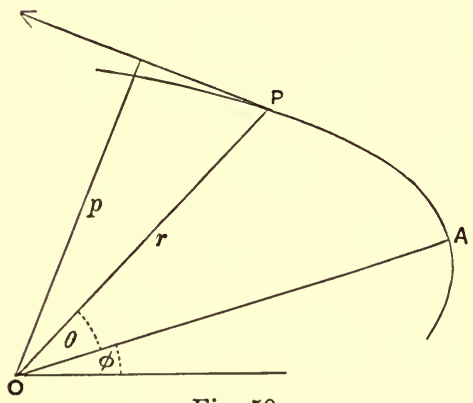

Fig. 50. in a smooth plane tube, and that the tube rotates in its plane about a point $O$ rigidly connected with it. Let $O A$ be any particular radius vector of the tube, and $\phi$ the angle $O A$ makes with a fixed line in the plane of the tube. Then $\dot{\phi}$ is the angular velocity of the tube. We shall write $\omega$ for $\dot{\phi}$.

Let $P$ be the position of the particle in the tube at time $t$. Let $O P=r$, and $\angle A O P=\theta$. Then $r$ and $\theta$ are polar coordinates of $P$ referred to $O A$ as initial line, and $r$ and $\theta+\phi$ are polar coordinates of $P$ referred to a fixed initial line. Let $\rho$ be the radius of curvature of the tube at $P$.

Let $v$ be the velocity of the particle relative to the tube. Then, if $\operatorname{arc} A P=s, v$ is $\dot{s}$, the direction of $v$ is that of the tangent to the tube, and the resolved parts of $v$ along $O P$ and at right angles to $O P$ are $\dot{\boldsymbol{r}}$ and $r \dot{\boldsymbol{\theta}}$.

Now the resolved accelerations of the particle along $O P$ and at right angles to $O P$ are

$$
\ddot{r}-r(\dot{\theta}+\dot{\phi})^{2},
$$


and

$$
\frac{1}{r} \frac{d}{d t}\left\{r^{2}(\dot{\theta}+\dot{\phi})\right\}
$$

These may be written

$$
\left.\begin{array}{l}
\ddot{r}-r \dot{\theta}^{2}-2 r \dot{\theta} \omega-r \omega^{2}, \\
\frac{1}{r} \frac{d}{d t}\left(r^{2} \dot{\theta}\right)+2 \dot{r} \omega+r \dot{\omega}
\end{array}\right\} .
$$

Of these the terms independent of $\omega$ are equivalent to $v \frac{d v}{d s}$ along the tangent to the tube at $P$ and $v^{2} / \rho$ inwards along the normal to the tube.

The terms containing $2 \omega$ as a factor are equivalent to $2 \omega v$ inwards along the normal to the tube. This can be seen by considering that $\dot{r}$ along $O P$ and $r \dot{\theta}$ transverse to $O P$ are equivalent to $v$ along the tangent in the direction in which $s$ increases, and that we have, as multipliers of $2 \omega$, the components of this resultant turned through a right angle.

Now we can resolve a vector in the direction $O P$ into components along the tangent at $P$ to the tube and inwards along the normal by multiplying by $\frac{d r}{d s}$ and $\frac{p}{r}$, where $p$ is the perpendicular from $O$ on the tangent; similarly for a vector transverse to $O P$.

Hence finally the accelerations resolved along the tangent and normal to the tube are

$$
\left.\begin{array}{c}
v \frac{d v}{d s}-\omega^{2} r \frac{d r}{d s}+\dot{\omega} p, \\
\frac{v^{2}}{\rho}+2 \omega v+\omega^{2} p+\dot{\omega} r \frac{d r}{d s}
\end{array}\right\} .
$$

Now let the particle move in the tube under the action of forces in the plane of the tube whose resolved parts along the tangent and normal to the tube are $S$ and $N$, and let $R$ be the pressure of the tube on the particle. Then the equations of motion are

$$
\left.\begin{array}{c}
m\left[v \frac{d v}{d s}-\omega^{2} r \frac{d r}{d s}+\dot{\omega} p\right]=S, \\
{\left[\frac{v^{2}}{\rho}+2 \omega v+\omega^{2} p+\dot{\omega} r \frac{d r}{d s}\right]=N+R}
\end{array}\right\}
$$


*198. Newton's Revolving Orbit. Suppose that the form of the tube in Article 197 is a free path under a central force to 0 . Let the tube turn about $O$ with an angular velocity $\dot{\phi}$ which is always equal to $n \dot{\theta}$, where $n$ is constant, and $\dot{\theta}$ is the angular velocity of the radius vector in the free path when the particle is at $(r, \theta)$. Then the path traced out by the particle is a free path under the original central force and an additional central force which varies inversely as the cube of the distance.

Let $f$ be the central acceleration in the free path, and $\frac{1}{2} h$ the rate of description of areas. Then we are given

$$
\left.\begin{array}{rl}
\ddot{r}-r \dot{\theta}^{2} & =-f, \\
r^{2} \dot{\theta} & =h
\end{array}\right\} .
$$

Now, in the tube $\dot{\phi}=n \dot{\theta}$, so that

and

$$
\begin{aligned}
r^{2}(\dot{\theta}+\dot{\phi}) & =h(1+n), \\
\ddot{r}-r(\dot{\theta}+\dot{\phi})^{2} & =-f-r \dot{\theta}^{2}\left(2 n+n^{2}\right), \\
& =-f-\frac{h^{2}}{r^{3}}\left(2 n+n^{2}\right) .
\end{aligned}
$$

Hence the path traced out by the particle in the revolving tube is a free path with a central acceleration to 0 made up of two terms, one of them being $f$, and the other being inversely proportional to $r^{3}$.

This result may be stated in another form as follows:-Relatively to a certain frame a particle describes a central orbit about the origin with central acceleration $f$; if a second frame with the same origin rotates about the origin relatively to the first frame, with an angular velocity always the same multiple of that of the radius vector in the said central orbit, the path of the particle relatively to the second frame is again a central orbit with the central acceleration increased by an amount inversely proportional to the cube of the distance.

\section{*199. Examples.}

1. A particle moves in a tube in the form of an equiangular spiral which rotates uniformly about the pole, and is under the action of a central force to the pole of the spiral. Prove that if there is no pressure on the tube the central force at distance $r$ nust be of the form $A r+B r^{-3}$, where $A$ and $B$ are constants.

2. Prove that motion which, relatively to any frame, can be described as motion in a central orbit with acceleration $\mu /(\text { distance })^{3}$ towards the origin 
and moment of velocity $h$ may be described, relatively to a different frame with the same origin, as uniform motion in a straight line, provided $h^{2}>\mu$.

3. A particle moves in a smooth plane tube, and is under a central force to a fixed point about which the tube rotates uniformly. Prove that, if the pressure is always zero, the central force is

$$
m\left[r \omega^{2}+2 r \omega\left(h-r^{2} \omega\right) / p^{2}+\left(h-r^{2} \omega\right)^{2} p^{-3} d p / d r\right]
$$

where $m$ is the mass of the particle, $m h$ is its moment of momentum about the fixed point, $\omega$ is the angular velocity of the tube, $r$ is the radius vector, and $p$ the perpendicular from the fixed point on the tangent to the tube at the position of the particle.

\section{*200. Motion on a rough plane curve under gravity.} When a particle is constrained to describe a plane curve in a vertical plane under gravity but there is frictional resistance to the motion as well as pressure on the curve we assume that the friction is $\mu$ times the pressure, where $\mu$ is the coefficient of friction. The friction acts in the tangent to the curve in the sense opposite to that of the velocity.

The equations of motion take different forms in different circumstances. We shall choose for investigation the case where the particle is on the outside of the curve, and

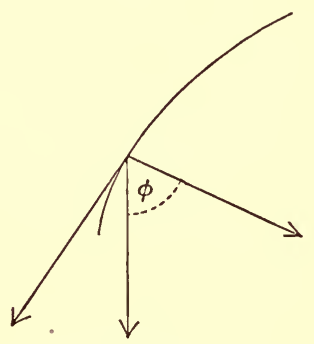

Fig. 51 . is descending.

Let the arc $s$ of the curve be measured from some point of the curve so that it increases in the sense of the velocity, and let $\phi$ be the angle contained between the inwards normal and the downwards vertical. Then $\phi$ increases with $s$, and $d s / d \phi(=\rho)$ is the length of the radius of curvature.

Let $v$ be the velocity of the particle, $m$ its mass, $R$ the pressure of the curve on the particle. The equations of motion are

$$
\left.\begin{array}{c}
m v \frac{d v}{d s}=m g \sin \phi-\mu R, \\
m \frac{v^{2}}{\rho}=m g \cos \phi-R
\end{array}\right\} .
$$

Eliminating $R$ we obtain the equation

$$
\begin{aligned}
& v \frac{d v}{d s}-\mu \frac{v^{2}}{\rho}=g(\sin \phi-\mu \cos \phi), \\
& v \frac{d v}{d \phi}-\mu v^{2}=g \rho(\sin \phi-\mu \cos \phi) .
\end{aligned}
$$


This equation can be integrated after multiplication by the factor $e^{-2 \mu \phi}$, in fact it becomes

$$
\frac{d}{d \phi}\left(\frac{1}{2} v^{2} e^{-2 \mu \phi}\right)=g \rho e^{-2 \mu \phi}(\sin \phi-\mu \cos \phi),
$$

so that $\quad v^{2} e^{-2 \mu \phi}=2 g \int \rho e^{-2 \mu \phi}(\sin \phi-\mu \cos \phi) d \phi+$ const.,

an equation which determines $v$ as a function of $\phi$, and therefore gives the velocity at any point of the curve. The velocity being determined, the second of the equations of motion gives the pressure, and, as in the case of a smooth curve, if $R$ vanishes the particle leaves the curve.

The equations of motion take different forms according as the particle is inside or outside the curve, and according as it is ascending or descending. But in each case the equations can be integrated by the above method. There is accordingly no definite expression for the velocity at any point of the curve in terms of the position, but the expressions obtained are different in the different cases.

\section{Examples.}

1. Write down the equations of motion in the three cases not investigated in Article 200 and the integrating factor in each case.

2. A particle is projected horizontally from the lowest point of a rough sphere of radius $a$, and returns to this point after describing an arc aa, $\left(a<\frac{1}{2} \pi\right)$, coming to rest at the lowest point. Prove that the initial velocity is $\sin a \sqrt{ }\left\{2 g a\left(1+\mu^{2}\right) /\left(1-2 \mu^{2}\right)\right\}$, where $\mu$ is the coefficient of friction.

3. A particle slides down a rough cycloid, whose base is horizontal and vertex downwards, starting from rest at a cusp and coming to rest at the vertex. Prove that, if $\mu$ is the coefficient of friction, $\mu^{2} e^{\mu \pi}=1$.

4. A ring moves on a rough cycloidal wire whose base is horizontal and vertex downwards ; prove that during the ascent the direction of motion at time $t$ makes with the horizontal an angle $\phi$, given by the equation

$$
\frac{d^{2}}{d t^{2}}\left\{e^{\phi \tan \epsilon} \sin (\phi+\epsilon)\right\}=-\frac{g}{4 a} \sec ^{2} \epsilon e^{\phi \tan \epsilon} \sin (\phi+\epsilon),
$$

where $\epsilon$ is the angle of friction.

*202. Motion on a curve in general. When a particle moves on a given curve under any forces, we take $m$ for the mass of the particle, $S$ for the tangential component of the resultant force of the field, $N$ for the component along the principal normal, 
and $B$ for the component along the binormal. Also we take $R_{1}$ for the component of the pressure along the principal normal towards the centre of curvature, and $R_{2}$ for the component of the pressure along the binormal in the same sense as $B$. Further if the curve is rough we take $F$ for the friction.

We take $s$ to be the arc of the curve from some point to the position of the particle at time $t, \rho$ to be the radius of curvature, and $v$ to be the velocity, and we suppose the sense in which $s$ increases to be that of $v$. Then the equations of motion are

$$
\left.\begin{array}{rl}
m v \frac{d v}{d s} & =S-F, \\
m \frac{v^{2}}{\rho} & =N+R_{1}, \\
0 & =B+R_{2}
\end{array}\right\}
$$

When the curve is smooth $F$ is zero, and we can integrate the first equation, in the same way as in Article 195, in the form

$$
\frac{1}{2} m v^{2}=\int S d s+\text { const. }
$$

and this result can be expressed in the form

$$
\text { change of kinetic energy = work done, }
$$

so that the velocity is determined in terms of the position. The other two equations then determine the pressure. As in Article 195 the integral equation is expressed more simply when the system of forces whose components are $S, N, B$ is conservative.

When the curve is rough we have to eliminate $F, R_{1}, R_{2}$ by means of the equation

$$
F^{2}=\mu^{2}\left(R_{1}^{2}+R_{2}^{2}\right)
$$

which expresses that the friction is proportional to the resultant pressure. There results a differential equation for $v^{2}$, and, if we can integrate this equation, we shall obtain an equation giving the velocity in terms of the position. As in Article 200 the velocity in any position depends partly on the way in which that position has been reached.

203. Motion on a smooth surface of revolution under gravity. When a particle moves under gravity on a smooth surface of revolution whose axis is vertical we can always obtain 
from general principles sufficient integrals of the equations of motion to determine the velocity completely.

Let the axis of revolution be the axis $x$ ( $x$ being measured upwards), and let the particle at time $t$ be at distance $y$ from the axis, and be on a meridian curve of the surface in an axial plane making an angle $\phi$ with a given axial plane, and let $\sigma$ be the arc of the meridian from some particular circular section to the position of the particle.

Then it is clear that the velocity along the tangent to the meridian is $\dot{\sigma}$, and the velocity along the tangent to the circular section is $y \dot{\phi}$. Thus the energy equation is

$$
\frac{1}{2}\left(\dot{\sigma}^{2}+y^{2} \dot{\phi}^{2}\right)+g x=\text { const. }
$$

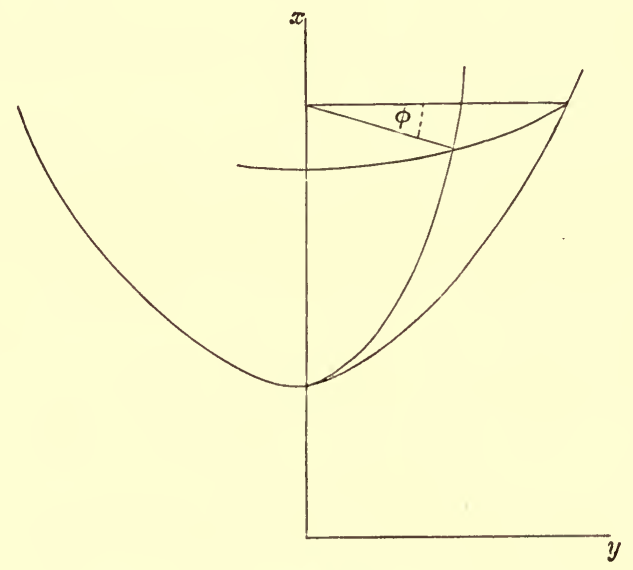

Fig. 52.

Again, since the reaction of the surface on the particle is along the normal to the surface, and the normal meets the axis of revolution, while the weight of the particle acts in a line parallel to this axis, the forces acting on the particle have no moment about this axis.

Hence by the Principle of the Conservation of Moment of Momentum the moment of the velocity about the axis is constant, or we have

$$
y^{2} \dot{\phi}=\text { const. }
$$

The equations written down determine $\dot{\sigma}$ and $\dot{\phi}$, that is they 
determine the two components of the velocity ( $\dot{\sigma}$ and $y \dot{\phi})$ in two directions at right angles which lie in the tangent plane to the surface.

\section{Examples.}

1. Show that a particle projected along a circular section of a smooth surface of revolution with its axis vertical can describe the circle under the action of gravity and the reaction of the surface, provided the velocity $V$ of projection and the radius $y$ of the circle are connected by the equation

$$
V^{2}=g y \tan \beta,
$$

where $\beta$ is the angle which the normal to the surface at any point on the circle makes with the vertical.

Prove also that the reaction of the surface is $m g \sec \beta$, where $m$ is the mass of the particle.

[In the particular case where the surface is spherical this motion is frequently referred to as motion of a "spherical pendulum," and the constraint can be provided by an inextensible string with one end fixed at the centre of the sphere. Since the string describes a right circular cone the name "conical pendulum" is sometimes used.]

2. A train rounds a curve whose radius of curvature is $\rho$ with velocity $v$. Prove that, to prevent the train from leaving the metals the outer rail ought to be raised a height $b v^{2} / \rho g$ above the inner, $b$ being the distance between the rails.

3. A railway carriage is travelling on a curve of radius $r$ with velocity $v, 2 a$ is the distance between the rails and $h$ is the height of the centre of inertia of the carriage above the rails. Show that the weight of the carriage is divided between the rails in the ratio gra $-v^{2} h: g r a+v^{2} h$, and hence that the carriage will upset if $\quad v>\sqrt{ }($ gra $/ h)$.

4. The point of suspension of a simple pendulum of length $l$ is carried round in a horizontal circle of radius $c$ with angular velocity $\omega$; show that when the motion is steady the inclination $a$ of its suspending thread to the vertical is given by the equation

$$
\omega^{2}(c+l \sin a)=g \tan a .
$$

Show that, if $\left(g / \omega^{2}\right)^{\frac{2}{3}}<l^{\frac{2}{3}}-c^{\frac{2}{3}}$, the inclination can be inwards towards the axis.

5. A particle moves on a smooth surface of revolution whose axis is vertical. Prove that the polar equation of the projection of the path on a horizontal plane is given by the equation

$$
\left(\frac{d u}{d \theta}\right)^{2}\left[1+u^{2}\left\{f^{\prime}(u)\right\}\right]^{2}+u^{2}+\frac{2 g}{h^{2}} f(u)=\text { const. }
$$

where $z=f(u)$ is the equation of the meridian curve, $u^{-1}$ being the distance from the axis, and $h$ is a constant. 
*205. Motion on a surface in general. Suppose that a particle moves on a fixed surface under the action of given forces and the reaction of the surface.

We may imagine the surface to be covered with a network of curves belonging to distinct families, in such a way that at each point of the surface one curve of one family meets one curve of the other family, and we may suppose the curves that meet in any point to cut at right angles. At any point we may resolve the forces of the field into components along the tangents to the curves that meet in that point, and along the normal to the surface. We may resolve the acceleration along the same lines.

When the surface is smooth the reaction is simply a pressure along the normal. For a particle moving on a smooth surface in a conservative field there will be an energy equation expressing the velocity in terms of the position. We shall see presently that the pressure is determinate as soon as the velocity is known.

When the surface is rough there will be two components of friction in the directions of the tangents to the two curves that meet at any point, and the resultant friction has the same direction as the velocity but the opposite sense. Also the resultant friction is equal in magnitude to the product of the coefficient of friction and the pressure.

We have thus the means of writing down equations of motion of the particle, but the process can in general be simplified by using methods of Kinematics and Analytical Dynamics which are beyond the scope of the present work. We shall therefore confine ourselves to the simplest cases.

We proceed to investigate a general expression for the resolved part of the acceleration along the normal to the surface.

Let $v$ be the velocity of the particle, $\rho$ the radius of curvature of its path. The tangent to the path touches the surface, and we suppose a normal section of the surface drawn through it. This section is not, in general, the osculating plane of the path; we suppose that it makes an angle $\phi$ with this osculating plane. We take $\rho^{\prime}$ to be the radius of curvature of the normal section of the surface through the tangent to the path.

Since the normal to the surface is at right angles to the 
tangent to the path the resolved part of the acceleration along the normal to the surface is the resolved part in that direction of the acceleration along the principal normal to the path, it is therefore

$$
\frac{v^{2}}{\rho} \cos \phi
$$

Also by a well-known theorem we have $\rho=\rho^{\prime} \cos \phi$.

Hence the acceleration along the normal to the surface is $v^{2} / \rho^{\prime}$, and the pressure is determined by resolving along the normal.

*206. Osculating plane of path. In Example 1 of Article 204 it is stated that a particle may be projected along a horizontal tangent of a smooth surface of revolution whose axis is vertical with such velocity that it describes the circular section under the action of gravity and the reaction of the surface. It is almost obvious that if the velocity exceeds that requisite for description of the circle the path of the particle rises above the circle, otherwise it falls below the circle. We may use the result of Article 205 to find the position of the osculating plane of the path for any velocity of projection.

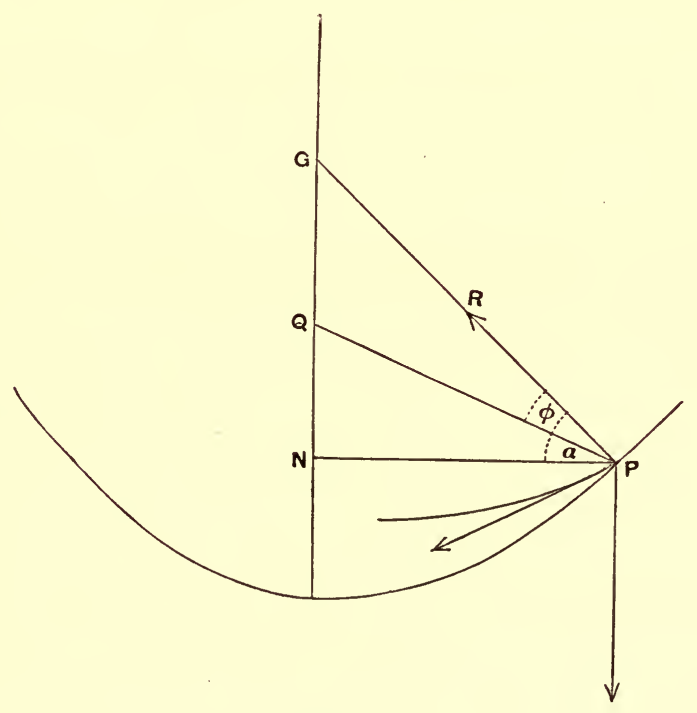

Fig. 53. 
Let $P$ be the point of projection, $P G$ the normal to the surface at $P, P N=y$ the ordinate of $P$ at right angles to the axis of revolution, $Q$ the point where the osculating plane of the path meets the axis. Let $\angle G P N=\alpha$, and $\angle G P Q=\phi$.

When the particle is projected along the tangent to the circular section with velocity $V$ there is initially no acceleration along a line in the meridian plane at right angles to $P Q$.

Hence resolving along this line we have

$$
R \sin \phi-m g \cos (\alpha-\phi)=0,
$$

where $m$ is the mass of the particle, and $R$ is the pressure.

Again, resolving along $P N$, we have

$$
m \frac{V^{2}}{\rho} \cos (\alpha-\phi)=R \cos \alpha
$$

where $\rho$ is the radius of curvature of the path.

Now, with the notation of Article 205,

$$
\rho^{\prime}=P G, \quad \rho=P G \cos \phi \text {. }
$$

Also $\quad y=P N=P G \cos \alpha$.

Hence $\quad \tan \phi=g y / V^{2}$.

This equation determines the position of the osculating plane of the path.

Now if $\tan \phi>\tan \alpha$, or $V^{2}<g y \cot \alpha$, the osculating plane of the path initially lies below the horizontal plane through the point of projection, and if $\tan \phi<\tan \alpha$, or $V^{2}>g y \cot \alpha$, it lies above that plane.

\section{*207. Examples.}

1. A particle moving on a surface (smooth or rough) under no forces but the reaction of the surface describes a geodesic.

2. A particle moves on a rough cylinder of radius $a$ under no forces but the reaction of the surface, starting with velucity $V$ in a direction making an angle $a$ with the generators; prove that in time $t$ it moves over an arc

$$
a \mu^{-1} \operatorname{cosec}^{2} a \log \left(1+\mu V t \alpha^{-1} \sin ^{2} a\right),
$$

$\mu$ being the coefficient of friction.

3. A hollow circular cylinder of radius $a$ is rough on the inside, and is made to rotate uniformly with angular velocity $\omega$ about its axis which makes 
an angle $a$ with the vertical. Show that a particle can slide down a line parallel to the axis with uniform velocity

$$
a \omega \sqrt{ }\left\{\left(\mu^{2}+1\right) /\left(\mu^{2} \tan ^{2} a-1\right)\right\},
$$

where $\mu$ is the coefficient of friction, and $\mu>\cot a$.

4. An ellipsoidal shell whose principal semiaxes are $a, b, c(a>b>c)$ is placed with the greatest axis vertical and a particle is projected from one of the lower umbilics with velocity $v$ along the tangent to the horizontal section within the ellipsoid. Show that the osculating plane of the path is initially above or below this section according as

$$
v^{2}>\text { or }<g a b^{2}\left(b^{2} / c^{2}-1\right) / \sqrt{ }\left\{\left(a^{2}-c^{2}\right)\left(a^{2}-b^{2}\right)\right\} \text {. }
$$

208. Motion in Resisting Medium. In illustration of the class of forces admitted in Rational Mechanics under the name resistances, we consider cases of the motion of a particle in a known field of force when, in addition to the forces of the field, there is exerted on the particle a force proportional to a power of its velocity having the same direction as the velocity and the opposite sense.

Problems of this kind are related to facts of observation in regard to the motions of bodies in the air and in other fluid media. In many cases it is found that the observed facts can be approximately represented by the supposition that the resistance is proportional to the velocity, this is true for instance for the motion of a pendulum swinging in air. There are other cases for which it is found that the facts are better represented by supposing the resistance to be proportional to the cube of the velocity, this appears in fact to be the simplest function of the velocity which gives rise to an approximate representation of the motion of a shot.

In such cases there is not necessarily any dissipation of energy in the system. The motion of the body through the fluid generates motion in the fluid, so that kinetic energy is gained by the fluid, and thus the body must part with kinetic energy at a greater rate than it would do in the absence of the fluid. Work is done by the body against the resistance, and an equal amount of work is done upon the elements of fluid, and its equivalent may be produced in kinetic energy of the fluid, potential energy of strain, \&c. We can in fact conceive cases in which this would happen. On the other hand in actual cases some of the energy is invariably dissipated, i.e. converted into heat, or generally into other forms of 
energy than kinetic energy of visible motion, potential energy of strain, and potential energy of the parts of the system in the field of force.

209. Resistance proportional to the Velocity. Since the velocity of a particle is a vector whose direction and sense are determined by the resolved parts $\dot{x}, \dot{y}, \dot{z}$, the resistance has resolved parts $-\kappa \dot{x},-\kappa \dot{y},-\kappa \dot{z}$, where $\kappa$ is a constant.

Suppose the motion takes place under gravity parallel to the negative direction of the axis $y$, and first suppose the particle to move vertically. The equation of motion is

or

$$
\begin{gathered}
m \ddot{y}=-m g-\kappa \dot{y}, \\
\ddot{y}+\lambda \dot{y}+g=0,
\end{gathered}
$$

where $\lambda$ is written for $\kappa / m$. Multiplying by $e^{\lambda t}$ and integrating, we have

$$
\dot{y} e^{\lambda t}+\frac{g}{\lambda} e^{\lambda t}=C,
$$

where $C$ is a constant of integration. Hence

$$
\dot{y}=C e^{-\kappa t / m}-m g / \kappa \text {. }
$$

If the particle continues to fall for a sufficiently long time the value of $\dot{y}$ will ultimately differ very little from $-g m / \kappa$, or the particle falls with a practically constant velocity when it has been falling for some seconds.

The equation last written can easily be integrated again so as to express $y$ as a function of $t$.

Again suppose that the particle is projected in any other than a vertical direction, then the vertical motion is the same as before, but for the horizontal motion we have an equation

giving

$$
\begin{aligned}
& m \ddot{x}=-\kappa \dot{x}, \\
& \dot{x}=A e^{-\kappa t / m},
\end{aligned}
$$

where $A$ is a constant of integration. This equation can easily be integrated again so as to express $x$ as a function of $t$.

210. Resisted Simple Harmonic Motion. Consider the case where, apart from the resistance, the motion would be simple harmonic in period $2 \pi / n$, and the resistance is proportional to the velocity. 
We have the equation

or

$$
\begin{gathered}
m \ddot{x}=-m n^{2} x-\kappa \dot{x}, \\
\ddot{x}+\lambda \dot{x}+n^{2} x=0,
\end{gathered}
$$

where $\lambda$ is written for $\kappa / m$. The complete primitive of this equation takes different forms according as $n^{2}>$ or $<\frac{1}{4} \lambda^{2}$. In the former case, which is practically the more important, it is

$$
x=e^{-\frac{1}{2} \lambda t}\left[A \cos \left\{t \sqrt{ }\left(n^{2}-\frac{1}{4} \lambda^{2}\right)\right\}+B \sin \left\{t \sqrt{ }\left(n^{2}-\frac{1}{4} \lambda^{2}\right)\right\}\right] .
$$

The motion represented may be roughly described as simple harmonic motion with period $2 \pi / \sqrt{ }\left(n^{2}-\frac{1}{4} \lambda^{2}\right)$, and with amplitude diminishing according to the exponential function $e^{-\frac{1}{2} \lambda t}$. It will be observed that the period is lengthened by the resistance, and that the amplitude falls off in geometric progression as the time increases in arithmetic progression. Thus the motion rapidly dies away.

\section{Examples.}

1. A particle is projected vertically upwards with velocity $v$ in a medium in which the resistance is proportional to the velocity. It rises to a height $h$ and returns to the point of projection with velocity $w$. Prove that

$$
\begin{aligned}
& g h / v^{2}=\frac{1}{2}-\frac{1}{3}(v / V)+\frac{1}{4}(v / V)^{2}-\frac{1}{5}(v / V)^{3}+\ldots \\
& g h / w^{2}=\frac{1}{2}+\frac{1}{3}(w / V)+\frac{1}{4}(w / V)^{2}+\frac{1}{5}(w / V)^{3}+\ldots
\end{aligned}
$$

where $V$ is the terminal velocity in the medium.

2. A particle moves under gravity in a medium whose resistance varies as the velocity, starting with horizontal and vertical component velocities $u_{0}$, $v_{0}$, and returning to the horizontal plane through the point of projection with component velocities $u_{1}, v_{1}$; show that the range $R$ and time of flight $t$ are given by the equations

$$
v_{0}-v_{1}=g t, \quad R=t\left(u_{0}-u_{1}\right) /\left(\log u_{0}-\log u_{1}\right) .
$$

Prove also that $R=u_{0} V t /\left(V+v_{0}\right)$, where $V$ is the terminal velocity in the medium.

3. A body performs rectilinear vibrations under an attractive force to a fixed centre proportional to the distance in a medium whose resistance is proportional to the velocity. Prove that, if $T$ is the period, and $a, b, c$ are the coordinates of the extremities of three consecutive semi-vibrations, then the coordinate of the position of equilibrium and the time of vibration if there were no resistance are respectively

$$
\frac{a c-b^{2}}{a+c-2 b} \text { and } T\left[1+\frac{1}{\pi^{2}}\left(\log \frac{a-b}{c-b}\right)^{2}\right]^{-\frac{1}{2}} \text {. }
$$


4. A particle of unit mass is fastened to one end of an elastic thread of natural length $a$ and modulus $a n^{2}$, in a medium the resistance of which to the motion of the particle is $2 \kappa$ (velocity). The other end of the thread is fixed and the particle is held at a distance $b(>a)$ below the fixed point. Prove that, when set free, (i) it will begin to rise or fall according as $n^{2}(b-a)>$ or $<g$, (ii) in its subsequent motion it will oscillate about a point $O$ which is at a distance $a+g / n^{2}$ below the fixed point, (iii) the distances from $O$ of successive positions of rest form a geometric series of ratio $e^{-\pi \kappa / m}$, (iv) the interval between any two positions of rest is $\pi / m$, where $m^{2}=n^{2}-\kappa^{2}$.

5. A particle rnoves on a smooth cycloid whose axis is vertical and vertex downwards under gravity and a resistance varying as the velocity. Prove that the time of falling from any point to the vertex is independent of the starting point.

6. A particle moves under a central force $\phi(r)$ in a medium of which the resistance varies as the velocity. Investigate the equations

where $h$ and $\mu$ are constants.

$$
\ddot{r}+\mu \dot{r}-\frac{h^{2}}{r^{3}} e^{-2 \mu t}+\phi(r)=0, \quad \frac{h^{2}}{p^{3}} \frac{d p}{d r}=\phi(r) e^{2 \mu t},
$$

*212. Motion in a vertical plane under gravity. For any law of resistance we can make some progress with the equations of motion of a particle moving in a vertical plane under gravity.

Let $m f(v)$ be the magnitude of the resistance when the velocity is $v, m$ being the mass of

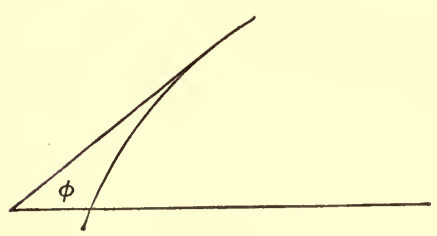

Fig. 54 . the particle, then resolving horizontally we have

$$
\dot{u}=-f(v) \cos \phi
$$

where $\phi$ is the angle the direction of motion at time $t$ makes with the horizontal and $u$ is the horizontal velocity, so that $u=v \cos \phi$.

Again resolving along the normal to path, since the resistance is directed along the tangent, we have

$$
\frac{v^{2}}{\rho}=g \cos \phi,
$$

where $\rho$ is the radius of curvature. This equation may be written and thus, eliminating $t$,

$$
v \dot{\phi}=-g \cos \phi
$$

$$
\frac{d u}{d \phi}=\frac{v f(v)}{g}, \text { where } v=u \sec \phi
$$


This equation can be integrated when $f(v)=\kappa v^{n}$, and we have

$$
\frac{1}{u^{n}}+\frac{n \kappa}{g} \int \frac{d \phi}{\cos ^{n+1} \phi}=\text { const. }
$$

an equation giving $u$, and therefore also $v$, in terms of $\phi$.

Now the equation

$$
v \frac{d \phi}{d t}=-g \cos \phi
$$

gives

$$
t=-\int \frac{v}{g} \sec \phi d \phi+\text { const. }
$$

so that $t$ is found in terms of $\phi$. Also the equations

$$
\frac{d x}{d s}=\cos \phi, \quad \frac{d y}{d s}=\sin \phi, \quad \frac{d s}{d t}=v,
$$

give us $x=-\int \frac{v^{2}}{g} d \phi+$ const., $y=-\int \frac{v^{2}}{g} \tan \phi d \phi+$ const.

and thus the time and the position of the particle are determined in terms of a single parameter $\phi$.

It is not generally possible to integrate the equation for vertical rectilinear motion even for the case here described where $f(v)=v^{n}$. In the special case, however, where the resistance is proportional to the square of the velocity the velocity can be found in any position. We have, when the particle is ascending,

$$
\ddot{y}=-g-\kappa \dot{y}^{2},
$$

$y$ being measured upwards. Now

$$
\ddot{y}=\dot{y} \frac{d \dot{y}}{d y}=\frac{d}{d y}\left(\frac{1}{2} \dot{y}^{2}\right) ;
$$

hence

$$
\frac{d}{d y}\left(\frac{1}{2} \dot{y}^{2}\right)+\kappa \dot{y}^{2}=-g \text {. }
$$

Multiplying by $e^{2 \kappa y}$ and integrating, we have

$$
\frac{1}{2} \dot{y}^{2} e^{2 \kappa y}=-\frac{g}{2 \kappa} e^{2 \kappa y}+\text { const., }
$$

giving

$$
\dot{y}^{2}=C e^{-2 \kappa y}-g / \kappa .
$$

Again when the particle is descending we have, measuring $y$ downwards,

$$
\ddot{y}=g-\kappa \dot{y}^{2},
$$


or

$$
\frac{d}{d y}\left(\frac{1}{2} \dot{y}^{2}\right)+\kappa \dot{y}^{2}=g
$$

giving

$$
\dot{y}^{2}=\frac{g}{\kappa}-C e^{-2 \kappa y} .
$$

As in the case of resistance proportional to the velocity, there is a limiting velocity, $\sqrt{ }(g / \kappa)$, which is practically attained when the particle has fallen through a considerable height.

\section{* 213. Examples.}

1. A particle is projected vertically upwards in a medium whose resistance varies as the square of the velocity. Prove that the interval that elapses before it returns to the point of projection is less than it would be if there were no resistance.

Prove also that, if the particle is let fall from rest, then in time $t$ it acquires a velocity $U \tanh (g t / U)$ and falls a distance $U^{2} g^{-1} \log \cosh (g t / U)$, where $U$ is the terminal velocity in the medium.

2. A particle of weight $W$ moves in a medium whose resistance varies as the $n$th power of the velocity. Prove that, if $F$ is the resistance when the direction of motion makes an angle $\phi$ with the horizon, then

$$
\frac{W}{F}=n \cos ^{n} \phi \int \sec ^{n+1} \phi d \phi \text {. }
$$

3. A particle of unit mass moves in a straight line under an attraction $\mu$ (distance) to a point in the line, and a resistance $\kappa$ (velocity) ${ }^{2}$. Prove that if it starts from rest at a distance $a$ from the centre of force it will first come to rest at a distance $b$, where

$$
(1+2 a \kappa) e^{-2 a \kappa}=(1-2 \kappa b) e^{2 \kappa b} .
$$

4. The bob of a simple pendulum moves under gravity in a medium of which the resistance per unit of mass is $\kappa$ (velocity) ${ }^{2}$, and starts from the lowest point with such velocity that if it were unresisted the angle of oscillation would be $a$. Prove that it comes to rest after describing an angle $\theta$ which satisfies the equation

$$
\left(1+4 \kappa^{2} l^{2}\right) \cos a=4 \kappa^{2} l^{2}-2 \kappa l \sin \theta e^{2 \kappa l \theta}+\cos \theta e^{2 \kappa l \theta},
$$

where $l$ is the length of the pendulum. 


\section{EXAMPLES.}

1. Prove that the time of quickest descent along a straight line from a point on one vertical circle to another in the same plane is

$$
\sqrt{ }\left\{2\left(c^{2}-a^{2}\right) / g(a+h)\right\},
$$

where $c$ is the distance between their centres, $a$ is the sum of the radii, and $h$ the vertical height of the centre of the former circle above that of the latter.

2. Any curve is drawn in a vertical plane, and a second curve is drawn cutting off equal distances along the normals to the first curve. Prove that, if the second curve now receives a certain vertical displacement, the time of quickest descent from one curve to the other is independent of the starting point.

3. Show that when two curves lie in the same vertical plane and do not intersect, the straight line of quickest descent from one to the other is such that the normals and the vertical lines through its extremities form a rhombus; and further that the centres of curvature at the extremities cannot lie on the segments of the normals included between the verticals.

4. A parabola is placed in a vertical plane with its axis inclined to the vertical at an angle $\cos ^{-1} \frac{3}{5}$, the vertex being the highest point of the axis. Prove that the time of sliding down the latus rectum is the same as that of sliding down the chord drawn from the upper end of the latus rectum to the vertex, and that the time down any intermediate chord is less.

5. A parabola is placed in a vertical plane with its vertex downwards and its axis inclined to the vertical at an angle $\beta$. Prove that the time down the chord of quickest descent from the focus to the curve is $\sqrt{ }\left(2 \alpha g^{-1} \sec ^{3} \frac{1}{3} \beta\right)$.

6. A spherical shell has a small hole at its lowest point, and any number of particles start down chords from the interior surface at the same instant, pass through the hole, and then move freely. Prove that at any instant before or after passing through the hole they lie on the surface of a sphere, and find the radius and position of such a sphere.

7. An ellipse is placed with its minor axis vertical. Prove that the normal chord of quickest descent from the curve to the major axis is that drawn from a point subtending a right angle at the foci when there is such a point. Determine the normal chord of quickest descent when there is no such point. 
8. A cycloid is placed with its axis vertical and base upwards, and particles starting from various points of the base run down chords of quickest descent to the curve. Prove that if $x$ is the length of such a chord, and $2 h$ the vertical height through which a particle would fall freely in the time of describing it, then $x^{2}-x \sqrt{ }\left(h^{3} / a\right)-2 h^{2}=0$.

9. Two equal parabolas of latus rectum $2 l$ are placed with their axes vertical at a distance $2 l$ from each other and with the vertex of the lower at a depth $l$ below the vertex of the higher, the convexities being opposed. The line of quickest descent from the higher to the lower is of length $h$ and makes an angle $\phi$ with the vertical. Prove that

$$
h / l=\sec \phi \sec ^{2} 2 \phi=2 \sqrt{2} \operatorname{cosec} \phi \sec 2 \phi \cos \left(\frac{1}{4} \pi+2 \phi\right) .
$$

10. A window is supported by two cords passing over pulleys in the framework of the window (which it loosely fits) and is connected with counterpoises each equal to half the weight of the window. One cord breaks, and the window descends with acceleration $f$. Show that the coefficient of friction between the window and the framework is

$$
a(g-3 f) /\{b(g+f)\}
$$

where $a$ is the height and $b$ the breadth of the window.

11. A train of mass $m$ runs from rest at one station to stop at the next at a distance $l$. The full speed is $V$, and the average speed is $v$. The resistance of the rails when the brake is not applied is $u \mathrm{~V} / \mathrm{lg}$ of the weight of the train, and when the brake is applied it is $u^{\prime} V / l g$ of the weight of the train. The pull of the engine has one constant value while the train is getting up speed, and another constant value while it is running at full speed, prove that the average rate at which the engine works in starting the train is

$$
\frac{1}{2} m \frac{V^{2}}{l}\left\{u-\frac{1}{2 / v-2 / V-1 / u^{\prime}}\right\} \text {. }
$$

12. A train starts from rest at one station and stops at the next, the pull of the engine having one constant value while the train is getting up speed, and another constant value while it is running at full speed." Prove that the work done by the engine in getting up speed exceeds that done by the brake in stopping the train by $(V / v-1)$ times the work done by the resistance during the whole journey, $V$ and $v$ being respectively the full speed and the average speed of the train.

13. It is required to find in horse-power the average rate of working of, and in pounds weight the pull exerted by, each horse of a two-horse omnibus which maintains an average speed of 6 miles an hour without exceeding $7 \frac{1}{2}$ miles an hour and slows down to 1 foot per second every hundred yards to pick up or set down - given the following data-weight of 'bus $=25$ cwt., weight of two horses $=30$ cwt., weight of driver, conductor and passengers $=35 \mathrm{cwt}$., and brake power produces a friction equal to one-fifth of the pressure. The brake is supposed to be applied to one wheel only, and no work is done by the horses when going at full speed. 
14. A bucket of mass $M$ lbs. is raised from the bottom of a shaft of depth $h$ feet by means of a cord which is wound on a wheel of mass $m$ lbs. The wheel is driven by a constant force, which is applied tangentially to its rim for a certain time and then ceases. Prove that if the bucket just comes to rest at the top of the shaft, $t$ seconds after the beginning of the motion, the greatest rate of working in foot-pounds per second is

$$
2 h M^{2} g t /\left\{M g t^{2}-2 h(M+m)\right\},
$$

the mass of the wheel being regarded as condensed uniformly on its rim.

15. An engine is pulling a train and works at a constant horse power doing $H$ units of work per second. If $M$ is the mass of the whole train and $F$ the resistance (supposed constant), prove that the time of generating velocity $v$ from rest is

$$
\left(\frac{M H}{F^{2}} \log \frac{H}{H-F v}-\frac{M v}{F}\right) \text { seconds. }
$$

16. A two-wheeled vehicle is being drawn along a level road with velocity $v$; the wheels (radius $c$ ) are connected by an axle (radius $r$ ) fixed to them, the weight of the vehicle exclusive of the wheels and axle is $W$, and its centre of inertia is vertically over the middle point of the axle. Show that, if the shafts are in a horizontal plane with the tops of the wheels, the horse is working at a rate $W v r \sin \lambda / \sqrt{ }\left(c^{2}-r^{2} \sin ^{2} \lambda\right)$, where $\lambda$ is the angle of friction between the axle and its bearings.

17. Two bodies hang by a cord over a fixed pulley : show that, neglecting the inertia of the pulley, the spaces described by the bodies in successive equal intervals of time are in arithmetic progression.

If instead of one of the bodies a pulley of negligible mass is substituted, and bodies of masses $m$ and $m^{\prime}$ slung over it, find the mass of the single body in order that $m^{\prime}$ may remain at rest if initially so, and prove that the acceleration of the pulley is $\frac{1}{2} \frac{m^{\prime}-m}{m} g$.

18. For one of the moving kodies in an Atwood's machine a pulley is substituted, round which passes a cord connecting two masses $P$, $Q$, which hang freely. Show that, if the ratio $P: Q$ lies between 3 and $\frac{1}{3}$, certain values of the other moving body may be found which will keep either $P$ or $Q$ stationary, and that these values are in the ratio $3 P-Q: 3 Q-P$.

19. If in an Atwood's machine the chain can only support a tension equal to one quarter of the sum of the weights at its two ends, show that the greater weight cannot be much less than six times the smaller, and that the least acceleration possible is $\frac{1}{2} g \sqrt{ } 2$.

20. In an Atwood's machine the groove in the pulley in which the chain runs is cut to that depth at which it is found that the inertia of the pulley may be divided equally between the moving bodies, and $Q$ is the weight 
required to be added to overcome the friction of the axle when equal weights $P$ are hung at the ends of the chain. Prove that an additional weight $R$ will produce acceleration $R g /(2 P+2 Q+R+W)$, where $W$ is the weight of the pulley.

21. Two equal masses $P, P^{\prime}$ are connected by a cord passing over a smooth pulley, and to them are attached equal masses $Q, Q^{\prime}$ by cords. Initially $Q$ lies on a horizontal plane, and $P, P^{\prime}, Q^{\prime}$ are in motion ; $Q$ is raised from the plane and $Q^{\prime}$ caught by it almost simultaneously. If $V$ is the initial subsequent velocity of $P, Q, P^{\prime}$ when $Q$ is raised just first, and $V^{\prime}$ the initial subsequent velocity when $Q^{\prime}$ is dropped just first, prove that

$$
V: V^{\prime}=(2 P+Q)^{2}: 4 P(P+Q) \text {. }
$$

22. Two pulleys each of mass $8 m$ hang at the ends of a chain of negligible mass which passes over a fixed pulley; a similar chain passes over each pulley and carries at its ends bodies of mass $2 m$. A mass $m$ is now removed from one of the bodies and attached to one of those which hang over the other pulley; prove that the acceleration of each pulley is $\frac{1}{11} g$. Prove also that the two descending bodies move with the same velocity, and that the velocity of one of the ascending bodies is five times that of the other.

23. A chain of negligible mass passes over two fixed pulleys and under a moveable pulley and bodies are attached to its ends. Prove that, if all the parts of the chain are vertical, the moveable pulley will remain at rest if its mass is twice the harmonic mean of the other two masses.

24. A chain of negligible mass passes over a fixed pulley $B$ and supports a body of mass $m$ at one end and a pulley $C$ of mass $p$ at the other. A similar chain is fastened to a point $A$ below $B$, passes over $C$, and supports a body of mass $m^{\prime}$. Prove that the acceleration of the pulley is

$$
g\left(2 m^{\prime}-m+p\right) /\left(4 m^{\prime}+m+p\right) .
$$

25. Two pulleys of masses $M$ and $M^{\prime}$ are connected by a cord passing over a fixed pulley. Bodies of masses $m_{1}$ and $m_{2}$ are hung over $M$ by a cord, and bodies of masses $m_{1}^{\prime}, m_{2}^{\prime}$ are hung over $M^{\prime}$. Prove that either pulley moves with acceleration

$$
g\left(M+2 \mu-M^{\prime}-2 \mu^{\prime}\right) /\left(M+M^{\prime}+2 \mu+2 \mu^{\prime}\right),
$$

where $\mu$ is the harmonic mean of $m_{1}$ and $m_{2}$, and $\mu^{\prime}$ is the harmonic mean of $m_{1}^{\prime}$ and $m_{2}^{\prime}$.

26. Two bodies are supported in equilibrium on a wheel and axle, and a body whose mass is equal to that of the greater body is suddenly attached to that body. Prove that the acceleration with which it moves is $a g /(2 a+b)$, $a$ and $b$ being the radii of the wheel and the axle respectively, and the inertia of the machine being neglected.

27. A body of weight $P$ balances a body of weight $W$ in that system of pulleys in which each pulley hangs by a separate cord. Prove that if bodies 
of weights $P^{\prime}$ and $W^{\prime}$ are substituted, $P^{\prime}$ will descend with acceleration $f$, such that

$$
f\left\{2^{2 n} P^{\prime}+W^{\prime}+\frac{1}{3}\left(2^{n}+1\right)\left(2^{n} P-W\right)\right\}=2^{n} g\left\{2^{n}\left(P^{\prime}-P\right)+W^{\prime}-W\right\},
$$

all the pulleys being of equal weight.

28. In any machine without friction and inertia a body of weight $P$ supports a body of weight $W$, both hanging by vertical cords. These bodies are replaced by bodies of weights $P^{\prime}$ and $W^{\prime}$, which, in the subsequent motion move vertically. Prove that the centre of inertia of $P^{\prime}$ and $W^{\prime}$ will descend with acceleration

$$
g\left(W P^{\prime}-W^{\prime} P\right)^{2} /\left(W^{2} P^{\prime}+W^{\prime} P^{2}\right)\left(W^{\prime}+P^{\prime}\right) .
$$

29. Two particles of masses $P$ and $Q$ lie near to each other on a smooth horizontal table, being connected by a thread on which is a ring of mass $R$ hanging just over the edge of the table. Prove that it falls with acceleration

$$
g(1 / P+1 / Q) \div(1 / P+1 / Q+4 / R)
$$

30. Two particles of masses $m, m^{\prime}$ are attached to the ends of a thread passing over a pulley and are held on two inclined planes each of angle $a$ placed back to back with their highest points beneath the centre of the pulley. Prove that if each portion of the thread makes an angle $\beta$ with the corresponding plane the particle of greater mass $m$ will at once pull the other off the plane if

$$
m^{\prime} / m<2 \tan a \tan \beta-1 .
$$

31. Two equal bodies, each of mass $M$, are attached to the chain of an Atwood's machine, and oscillate up and down through two fixed horizontal rings so that each time one of them passes up through a ring it lifts a bar of mass $m$, while at the same instant the other passes down through its ring and deposits on it a bar of equal mass. Prove that, neglecting friction, the period of an excursion of amplitude $\alpha$ is

$$
2 \sqrt{ }\left\{\frac{2 a}{g}\left(1+\frac{2 M+\mu}{m}\right)\right\}
$$

and that the successive amplitudes form a diminishing geometric progression of which the ratio is

$$
\left(1+\frac{m}{2 M+\mu}\right)^{-2}
$$

where $\mu$ is a mass which distributed over the circumference of the pulley will produce the same effect on the motion as the inertia of the actual mechanism.

32. A series of vertical circles touch at their highest points, and smooth particles slide down the ares starting from rest at the highest point; prove that the foci of the free paths lie on a straight line whose inclination to the vertical is $\tan ^{-1}\left(\frac{5}{8} \sqrt{ } 5\right)$.

33. A particle is projected along the circumference of a smooth vertical circle of radius $a$. It starts from the lowest point and leaves the circle before 
reaching the highest point. Prove that, if the coefficient of restitution between the circle and the particle is unity, and if the initial velocity is

$$
\sqrt{ }\left[\operatorname{ag}\left\{2+\frac{3}{2} \sqrt{ }(3-\sqrt{ } 3)\right\}\right],
$$

the particle after striking the circle will retrace its former path.

34. A smooth parabolic cylinder is fixed with its generators horizontal and the axis of each of its normal sections is horizontal. A particle is placed upon it at a height above the axial plane equal to the latus rectum; prove that it will run off at the extremity of the latus rectum, and will then describe a parabola of equal latus rectum.

35. A particle slides under gravity on a smooth parabola whose axis is not necessarily vertical, and is free to leave it and describe a different parabola under gravity alone. Prove that, if the particle leaves the first parabola at all, it will do so at the point where the normal passes through the intersection of the directrices of the two parabolas.

36. A particle moves on the outside of a smooth elliptic cylinder whose generators are horizontal, starting from rest on the highest generator, which passes through extremities of major axes of the normal sections. Prove that it will leave the cylinder at a point whose eccentric angle $\phi$ is given by the equation

$$
e^{2} \cos ^{3} \phi=3 \cos \phi-2,
$$

where $e$ is the eccentricity of the normal sections.

37. A particle moves in an elliptic tube under a force to a focus equal to $\mu r^{-2}+\nu r^{-3}$. Prove that, if it is projected from the nearer vertex with velocity $\sqrt{ }\{\mu(1+e) / a(1-e)\}$, the pressure is given by

$$
\frac{\nu}{\rho}\left\{\frac{1}{r^{2}}+\frac{1}{a^{2}(1-e)^{2}}-\frac{1}{a r}\right\} .
$$

38. A particle is constrained to move in an ellipse about a centre of force in one focus varying inversely as the square of the distance, and its initial velocity is such that if it were free its orbit would pass through the other focus. Prove that if the constraint were removed at any point of its path it would describe an orbit passing through the other focus.

39. A particle is projected horizontally from the lowest point of a smooth elliptic arc, whose major axis $2 \alpha$ is vertical, and moves under gravity along the concave side. Prove that it will leave the curve if the velocity of projection lies between $\sqrt{ }(2 g a)$ and $\sqrt{ }\left\{g a\left(5-e^{2}\right)\right\}$.

40. A ring is free to move on a smooth elliptic wire whose minor axis is vertical. An elastic thread of natural length $l$ and of modulus equal to $n$ times the weight of the ring passes through the ring and has its extremities fixed to the foci of the wire. Prove that if the ring falls from an extremity of the major axis the pressure at the lowest point will vanish if

$$
l=4 n a^{2} b /\left(a^{2}+2 n a b+2 b^{2}\right),
$$

where $2 a, 2 b$ are the major and minor axes of the ellipse, and $l<2 a$. 
41. A smooth cycloid has its axis $A B$ inclined to the vertical and its convexity upwards; a particle begins to slide down the arc from $A$, and leaves the curve at $P$; the perpendicular from $P$ on $A B$ cuts at $Q$ the circle on $A B$ as diameter, and $Q R$ is a diameter of this circle. Prove that $P R$ is horizontal.

42. A particle moves on a smooth curve in a vertical plane, the form of the curve being such that the pressure on the curve is always $m$ times the weight of the particle. Prove that the time of a complete revolution is $2 \pi \frac{m}{\left(m^{2}-1\right)^{\frac{3}{2}}} \sqrt{\frac{a}{g}}$, and that the length of the vertical axis of the curve is $\frac{2 m a}{\left(m^{2}-1\right)^{2}}$, the whole length of the curve being $\pi a \frac{2 m^{2}+1}{\left(m^{2}-1\right)^{\frac{5}{2}}}$.

43. Prove that, if a particle moves in a smooth tube under the action of forces tending to centres, the pressure on the tube at any point will be proportional to

$$
\frac{1}{\rho}\left\{C-\Sigma \frac{1}{p} \frac{d}{d p}\left(p^{2} F\right)\right\}
$$

where $\frac{d F}{d r}$ is the acceleration towards any one of the centres, and $\rho$ is the radius of curvature.

44. A smooth circular tube of radius $a$ is fixed in a vertical plane, and contains a particle, which is attached to the highest point of the tube by an elastic thread inside the tube ; the modulus of elasticity is $\frac{1}{2} \sqrt{ } 3$ of the weight of the particle, and the natural length of the thread subtends an angle $\frac{1}{6} \pi$ at the centre. Prove that, if when the particle is in equilibrium it receives by an impulse a downwards velocity $\sqrt{ }\{(2 \pi \sqrt{ } 3-3) a g\}$, it will just reach the lowest point.

45. Two equal smooth circular tubes are fixed so as to touch at their lowest points the same horizontal plane, their planes being at different inclinations; two small heavy beads are projected at the same instant along these circles from their lowest points, the velocity of each bead being due to falling from the highest point of the other circle. Show that throughout the motion the two beads will always be at the same height.

46. Assuming that the mass of the Moon is $\frac{1}{80}$ of that of the Earth, and that the Moon's distance is 60 times the Earth's radius, prove that owing to the Moon's attraction a seconds pendulum will be losing at a rate $\frac{1}{40} \sigma\left(3 \sin ^{2} a-1\right)$ seconds per day, where $a$ is the altitude of the Moon at the place of observation.

47. A bead moves on a smooth circular wire in a vertical plane its velocity being that due to falling from a horizontal line $H K$ above the circle. Prove that, if $I$ is the internal limiting point of the co-axal system of which the circle and the line $H K$ are members, then any chord through $I$ divides the wire into two parts which are described in equal times. 
48. The bob of a pendulum (weight $W$ ) is suspended by a cord from one end of an inextensible rod of negligible mass, which is constrained to move vertically, and the other end of the rod is attached to a cord passing over a smooth pulley and supporting a body of weight $W$. Prove that the period of small oscillations of the pendulum is the same as when the point of support is at rest, and that when the suspending cord makes an angle $\theta$ with the vertical the tension is

$$
2 W\left\{\frac{\cos \theta}{1+\cos ^{2} \theta}+\frac{2(\cos \theta-\cos a)}{\left(1+\cos ^{2} \theta\right)^{2}}\right\},
$$

where $a$ is the amplitude of the oscillations.

49. A simple pendulum is suspended from the roof of a railway carriage and remains vertical while the train is running uniformly at 30 miles an hour. When the brakes are put on, the pendulum oscillates through an angle of $3^{\circ}$. Prove that the train will come to rest after running about 385 yards, the resistance being assumed constant.

50. Prove that the time of a beat of a circular pendulum of length $a$ oscillating through an angle $2 a$ is equal to the time of complete revolution of a pendulum of length $a \operatorname{cosec}^{2} \frac{1}{2} a$, the height of the line of zero velocity above the lowest point being $2 a \operatorname{cosec}^{4} \frac{1}{2} a$.

51. The bob of a simple pendulum of length $l$ and mass $m$ is acted on by a horizontal force $m p g \cos n t$, where $p$ is a large number, and $l n^{2}$ is large compared with $g$. Show that the pendulum may oscillate about either of two points distant $a$ from the lowest point with an amplitude $\beta$, where

$$
\cos a=2 \ln ^{2} /\left(g p^{2}\right), \quad \beta=2 / p .
$$

52. The point of support of a simple pendulum of length $l$ and weight $w$ is attached to a massless spring so that it can move to and fro in a horizontal line; prove that the time of vibration is

$$
2 \pi \sqrt{ }\left\{\frac{l}{g}\left(1+\frac{w}{W}\right)\right\},
$$

where $W$ is the weight required to stretch the spring a length $l$.

53. A platform is sliding down a smooth spherical hill from rest at the summit. From a point fixed on it a plumb-line is suspended in a tube which is always held perpendicular to the surface of the hill at the point occupied by the platform. Prove that the tension of the cord, when the platform has descended a distance $x$ measured vertically, is $w(a-3 x) / a$, where $a$ is the radius of the sphere, and $w$ is the weight of the lead.

54. A ring slides on a smooth wire bent into the form of a curve in a vertical plane, being attached by an elastic thread to a fixed point in the plane; it starts from a position in which the thread has its natural length, and the modulus of elasticity is twice the weight of the ring. Prove that it 
will descend through a vertical height which is a third proportional to the natural length of the thread, and the increase of its length when in the lowest position, the thread being stretched throughout the motion.

55. A particle hangs in equilibrium under gravity being suspended by an elastic thread whose modulus of elasticity is 3 times the weight of the particle. The particle is slightly displaced in a direction making an angle $\cot ^{-1} 4$ with the horizontal, and is then released. Show that it will oscillate in an arc of a small parabola terminated by the ends of the latus rectum.

56. A particle placed at an end of the major axis of a normal section of a uniform gravitating elliptic cylinder is slightly disturbed in the plane of the section. Prove that it can move round in contact with the cylinder, and that its velocity $v$ when at a distance $y$ from the major axis of the section is given by the equation

$$
v^{2}=4 \pi \gamma \rho y^{2} a(a-b) /\{b(a+b)\},
$$

where $\rho$ is the density of the cylinder, and $2 a, 2 b$ are the principal axes of a normal section.

57. A particle moves in a smooth tube in the form of a catenary being attracted to the directrix with a force proportional to the distance from the directrix. Prove that the period of oscillation is independent of the amplitude.

58. Prove that a hypocycloid, generated by the rolling of a circle of radius $b$ on a circle of radius $a$, is isochronous for a force varying as the distance from the centre of the fixed circle, and that the time of an oscillation is

$$
4 \pi \alpha^{-1} \sqrt{ }\{b(\alpha-b) / \mu\}
$$

where the force per unit of mass at unit distance is $\mu$ (distance).

59. A particle, of unit mass, is at rest in a smooth tube in the form of an equiangular spiral of angle $a$ at a distance $2 d$ from the pole. Prove that, under the action of a force $\mu /(\text { distance })^{2}$ towards the pole, it will reach the pole in time $\pi \sec a d^{\frac{3}{2}} / \sqrt{ } \mu$.

60. A cycloidal wire in a vertical plane, with its axis vertical and vertex upwards is completely occupied by equal small smooth rings. Prove that, if the constraint at the cusps is removed, then in time $t$ the length of the arc cleared of rings will be

$$
2 l \sinh ^{2} \sqrt{ }\left(g t^{2} / 4 l\right)
$$

where $l$ is the length of the cycloid.

61. A particle slides down a smooth cycloidal tube with its axis vertical and vertex downwards, starting from rest at an arc-distance $s_{1}$ from the vertex. After a time $t$, and before the first particle has reached the vertex, a second particle slides down the tube starting from rest at an arc-distance $s_{2}$ from the vertex. Prove that the arc-distance from the vertex of the point where the particles meet is

$$
\sin \frac{2 \pi t}{T} / \sqrt{ }\left(\frac{1}{s_{1}{ }^{2}}+\frac{1}{s_{2}{ }^{2}}-\frac{2}{s_{1} s_{2}} \cos \frac{2 \pi t}{T}\right),
$$

where $T$ is the time of a complete oscillation in the tube.

L. 
62. A cycloidal tube, of which the radius of the generating circle is $a$, is placed with axis vertical and vertex downwards, and contains two elastic threads of natural length $l$ fastened at one end to the extremities of the base and at their other ends to a particle. If the particle is moved a distance $x$ from the vertex, where $x<4 a-l$, it will reach the vertex in time

$$
\pi \sqrt{\left(\frac{a}{g} \frac{l}{8 n a+l}\right),}
$$

where $n$ is the ratio of the modulus of the string to the weight of the particle. Find the time taken if $x>4 a-l$.

63. Two cycloids are placed in the same vertical plane, with their axes vertical, and their vertices downwards and at the same level. Two particles start to describe the cycloids from points at the same level. Show that they will next be at the same level after a time $2 \pi \sqrt{ }\left(a a^{\prime}\right) /\left\{\left(\sqrt{ } a+\sqrt{ } a^{\prime}\right) \sqrt{ } g\right\}$, and next after that at time $4 \pi \sqrt{ }\left(a \alpha^{\prime}\right) /\left\{\left(\sqrt{ } a+\sqrt{ } \alpha^{\prime}\right) \sqrt{ } g\right\}$ or $2 \pi \sqrt{ }\left(a \alpha^{\prime}\right) /\left\{\left(\sqrt{ } a \sim \sqrt{ } \alpha^{\prime}\right) \sqrt{ } g\right\}$, whichever is less, $\alpha$ and $\alpha^{\prime}$ being the radii of the generating circles.

64. An endless thread of length $2 l$, on which are threaded beads of masses $M$ and $m$, passes over two small smooth pegs $A$ and $B$, which are at a distance $a$ apart and in a horizontal line. The lighter bead $m$ is raised to the middle point of $A B$ and is then let go. Show that the beads will just meet if

$$
(M+m) / M=2 \sqrt{ }\{l /(l+a)\} \text {. }
$$

65. Two particles $A, B$ are connected by a thread of length $l$ which passes through a small hole $C$ in a smooth horizontal table on which $A$ moves and supports $B . A$ is projected along the table at right angles to $A C$. Show that, if $A C=\kappa l$, and if $n$ is the ratio of the masses of $B$ and $A, B$ cannot reach the table if the velocity of projection is less than that due to falling through a height $n l /(1+\kappa)$.

66. Two particles of masses $m$ and $\kappa m$ are connected by a thread which passes over the top of a smooth circle, the particles lying on the circle. Show that the motion of $m$ from its position of equilibrium will be the same as that of a free particle starting from the top of the circle, under gravity diminished in the ratio $\sqrt{ }\left(1+\kappa^{2}+2 \kappa \cos a\right): 1+\kappa, a$ being the angle the connecting thread subtends at the centre.

67. A straight smooth groove is cut in a horizontal table, and a straight slit is cut in the bottom of the groove. A thread of length $l$ attached at one end to a particle of mass $m$ resting in the groove passes through the slit and supports a particle of mass $\kappa m$. The second particle is held displaced in the vertical plane containing the slit with the string straight, and is let go. Prove that its path is part of an ellipse of semi-axes $l$, and $l /(1+\kappa)$, the major axis being vertical.

68. Two particles $A, B$ each of mass $m$ slide on a circular wire of radius $a$ fixed in a vertical plane, and are connected with a third particle $C$ of mass $m^{\prime}$ by two threads each equal to the radius. The system starts from rest in a 
position in which the threads and the radii through $A$ and $B$ form a square with $C$ vertically below the centre. Prove that when $A$ and $B$ meet the velocity of either of them is

$$
\sqrt{ }\left\{(2-\sqrt{ } 2) a g\left(1+m^{\prime} / m\right)\right\} \text {. }
$$

69. Two particles of masses $M, m$ are connected by a cord passing over a smooth pulley, the smaller $(m)$ hangs vertically and the other $(M)$ moves in a smooth circular groove on a fixed plane of inclination $a$ to the vertical, the highest point of the groove being vertically under the pulley. $M$ starts from the highest point of the groove without initial velocity. Prove that, if it makes complete revolutions, the radius of the groove must not exceed

$$
h m M \cos a /\left(m^{2}-M^{2} \cos ^{2} a\right),
$$

where $h$ is the height of the pulley above the highest point of the groove.

70. A particle moves from rest at an extremity of the major axis of a smooth elliptic groove of axes $2 a, 2 b$ cut in a horizontal table, being attached to a thread which passes through a small hole at the centre of the ellipse and supports a particle of equal mass. Prove that the horizontal pressure on the groove when the first particle is at an extremity of the minor axis vanishes if

$$
2 \alpha^{3}-a^{2} b-4 a b^{2}+4 b^{3}=0 .
$$

71. A particle of weight $W$ moves in a smooth elliptic groove on a horizontal table, and is attached to two threads which pass through holes at the foci, and each thread supports a body of weight $W$. One of the bodies is pulled downwards with velocity $V e$ when the particle is at an end of the minor axis. Prove that, if $V^{2}<a b^{2} g /\left\{e\left(3 \alpha^{2}-2 b^{2}\right)\right\}$, the threads do not become slack, and that in this case the horizontal pressures, $R$ and $R^{\prime}$, on the groove when the particle is at the ends of the axes are connected by the equation

$$
R b^{3} \sim R^{\prime} a\left(3 a^{2}-2 b^{2}\right)=6 \mathrm{Wa}^{2} b e^{2},
$$

where $2 a$ and $2 b$ are the principal axes, and $e$ is the eccentricity of the ellipse.

72. A smooth parabolic wire, on which is a smooth bead of weight $w$, is fixed in a horizontal plane. To the bead is attached a thread, which passes through a smooth ring fixed at the focus of the parabola and carries, at its other end, a weight $w /(e-1)$. Prove that the tension $T$ of the thread at any stage of the motion is given by an equation of the form

$$
(e T-w)(e r-a)^{2}=\text { const., }
$$

where $r$ is the focal distance of the bead and $4 a$ the latus rectum of the parabola.

73. Two smooth straight horizontal non-intersecting wires are fixed at right angles to each other at a distance $d$ apart. Two small rings of equal mass, connected by an inextensible thread of length $l$, slide on the wires, and they are projected with velocities $u$ and $v$ from points at distances $a$ and $b$ from the shortest distance between the wires. Prove that after the thread becomes tight the motion is oscillatory and of period $2 \pi\left(l^{2}-d^{2}\right) /(a v \sim b u)$. 
74. Two equal particles connected by a massless rigid rod are placed in a vertical circular tube, one being at the highest point. Show that, when the other reaches the lowest point, the velocity of each is the same as if they had been unconnected throughout the motion.

75. A thread of length $a a$ has a particle attached to one extremity, while the other is fastened to the highest point of a horizontal cylinder of radius $a$. The particle is initially supported with the thread in a horizontal line at right angles to the axis of the cylinder and is allowed to fall under gravity. Find the tension when the thread has described a given angle, and show that, if the maximum tension occurs when the thread is vertical, then $a=\frac{1}{2} \pi$.

76. A particle is suspended by an inextensible thread of length $l$ from a point on the circumference of a cylinder of radius $\alpha$ whose axis is horizontal, the thread being tangential to the cylinder. Prove that, if the particle is projected horizontally in a plane perpendicular to the axis of the cylinder so as to pass under the cylinder, the least velocity it can have in order that the thread may wind itself wholly up is $\sqrt{ }[\{g\{a \beta(1-\sin \beta)\}]$, where $a \beta$ is the length of the part to be wound up.

77. One end of a thread of length $l$ is attached to the highest point of a fixed horizontal circular cylinder of radius $\alpha$. A particle attached to the other end is dropped from a position in which the thread is straight and horizontal and at right angles to the axis of the cylinder. Prove that, if $l \nless 2 \pi a$, the thread will become slack before the particle comes to rest, and that it will then have turned through an angle whose circular measure is

$$
\pi+\frac{4}{3} a / l+\frac{4}{3} \pi(a / l)^{2}+\frac{4}{3}\left(\frac{32}{2} 7+\pi^{2}\right)(\alpha / l)^{3}+\ldots
$$

78. Two particles $P, Q$, of equal mass, slide on a smooth endless cord $O P Q$, which passes through a small smooth ring at $O$, and lies on a smooth horizontal plane. Initially $O P=O Q$, and the particles are projected with equal velocities along the external bisectors of the angles $O P Q, O Q P$ respectively. Prove that, throughout the motion, the tension of the cord varies inversely as $O P$.

79. Particles of masses $M$ and $m$ are attached to the ends of a thread, the former being within a smooth fixed horizontal tube and the datter on a smooth table in the horizontal plane of the tube. The thread is initially straight and the particle of mass $m$ is projected at right angles to the thread. Prove that the polar equation of its path is of the form $r \cos \{\theta \sqrt{M+m}\}=c$.

80. Two particles, masses $m, m^{\prime}$, on a smooth horizontal table are connected by a thread passing through a small smooth ring fixed in the table. Initially the thread is just extended and in two straight pieces meeting at the ring, the lengths of the pieces being $a$ and $\alpha^{\prime}$. The particles are projected at right angles to the string with velocities $v$ and $v^{\prime}$. Prove that, if $T$ is the tension at any time and $r, r^{\prime}$ the distances from the ring, then

$$
T\left(\frac{1}{m}+\frac{1}{m^{\prime}}\right)=\frac{a^{2} v^{2}}{r^{3}}+\frac{a^{\prime 2} v^{\prime 2}}{r^{\prime 3}} .
$$

Prove also that the other apsidal distances will be equal if

$$
m v^{2}: m^{\prime} v^{\prime 2}=3 \alpha^{\prime}+a: 3 \alpha+a^{\prime} \text {. }
$$


81. Two particles of equal mass, connected by a thread of length $a$, lie on a smooth table with the thread just straight. One of the particles is set in motion at right angles to the thread with velocity $v$; prove that each of them describes a series of cycloids, the time of describing any one of which is $\pi \alpha / v$.

82. Two particles $P, Q$, are connected by a fine string which passes through a small hole in a smooth inclined plane (inclination a). $Q$ hangs vertically, and $P$ moves on the inclined plane. Show that the differential equation of $P$ 's path is

$$
2 \frac{\sin \theta \sin a}{u^{3}}+\frac{d}{d \theta}\left\{\frac{\kappa-\sin a \cos \theta+\sin a \sin \theta(1+\kappa) \frac{d u}{u d \theta}}{u^{2}(1+\kappa) \frac{d^{2} u}{d \theta^{2}}+u^{3}}\right\}=0,
$$

where $k$ is the ratio (mass of $Q:$ mass of $P$ ).

83. Two particles, of masses $m$ and $m^{\prime}$, are connected by a thread which passes through a hole at the vertex of a smooth right circular cone having its axis vertical and vertex uppermost. The particle of mass $m^{\prime}$ hangs vertically, and $m$ describes a circle of radius $c$ on the cone. Prove that, if slightly disturbed, it will perform small oscillations in time

$$
2 \pi \sqrt{\left\{\frac{c\left(m^{\prime}+m\right)}{3 g\left(m^{\prime}-m \cos a\right) \sin a}\right\}},
$$

$2 a$ being the vertical angle of the cone.

84. A particle of mass $M$ is attached to a cord and is on a smooth table. The cord passes over the edge of the table and supports a pulley, of mass $m$, carrying another cord to the ends of which bodies of masses $m_{1}, m_{2}$ are attached. Prove that the acceleration of $M$ is

$$
\frac{m\left(m_{1}+m_{2}\right)+4 m_{1} m_{2}}{(M+m)\left(m_{1}+m_{2}\right)+4 m_{1} m_{2}} g .
$$

85. A bead of mass $M$ slides on a smooth fixed straight rod, and a thread attached to it passes round a pulley, which is fixed at a point on the rod, and is attached to a particle of mass $m$. Initially $M$ is at rest, the two parts of the thread contain a right angle, and $m$ is projected with velocity $V$ parallel to the rod. Prove that, when $m$ is at a distance $r$ from the pulley, the velocity of $M$ is

$$
r^{-1} V \sqrt{ }\left\{\left(r^{2}-a^{2}\right) m /(m+M)\right\}
$$

where $a$ is the initial value of $r$.

86. A ring can move on a long straight rough rod, the coefficient of friction being $\mu$, under an attraction to a fixed point (not on the rod) varying as the distance. Prove that the time of oscillation is the same as if the rod were smooth, and that the ring will ultimately come to rest at a point within a length $2 \mu d$ of the rod, where $d$ is the distance of the rod from the centre of force. 
87. A particle slides down the arc of a rough circle $\left(\mu=\frac{1}{2}\right)$ fixed in a vertical plane, and the particle starts from rest at an end of the horizontal diameter. Prove that, if $\theta$ is the angle the radius vector through the particle makes with the horizontal when the velocity is a maximum, then

$$
\sin \theta=\frac{1}{3} \cos \theta+e^{-\theta} \text {. }
$$

88. A particle of unit mass moves in a rough straight tube $A B$ under the action of a central repulsive force from a point $C$ of magnitude $\lambda / r$ at a distance $r$ from $C$. The point $A$ is the foot of the perpendicular from $C$ on the tube, and the particle is projected from $A$ along the tube with velocity $v$. Prove that it comes to rest when the radius vector from $C$ makes with $C A$ an angle $\theta$ satisfying the equation

$$
\mu \theta-\log \sec \theta=\frac{1}{2} v^{2} / \lambda,
$$

where $\mu$ is the coefficient of friction.

89. A particle is started with indefinitely small velocity from that point of a rough cycloidal arc (vertex uppermost) at which it could rest in limiting equilibrium. Show that the velocity at a point at which the tangent makes an angle $\phi$ with the horizon is $2 \sqrt{ }(a g) \sin (\phi-\epsilon)$, and that the particle leaves the cycloid when the velocity is $\sqrt{ }(2 a g)\left(\sin \frac{1}{2} \epsilon+\cos \frac{1}{2} \epsilon\right)$, where $\epsilon$ is the angle of friction.

90. A particle slides down a rough cycloid whose axis is vertical and vertex downwards. Prove that the time of reaching a certain point on the cycloid is independent of the starting point.

Prove also that, if $\lambda$ is the angle of friction, and if the tangent at the starting point makes with the horizontal an angle greater than $a$, where $a$ is the least positive angle which satisfies the equation

$$
\sin (a-\lambda)=e^{(a+\lambda) \tan \lambda} \sin 2 \lambda,
$$

the particle will oscillate.

91. A ring moves on a rough cycloidal wire with its axis vertical and vertex downwards. Prove that, if it starts from the lowest point with velocity $u_{0}$, its velocity $u$ when its direction of motion is inclined at an angle $\phi$ to the horizontal is given by

$$
u^{2}=\left(u_{0}^{2}+4 a g \sin ^{2} \epsilon\right) e^{-2 \phi \tan \epsilon}-4 a g \sin ^{2}(\phi+\epsilon),
$$

where $a$ is the radius of the generating circle and $\epsilon$ is the angle of friction.

Prove also that if it starts from a cusp with velocity $v_{0}$, its velocity $v$ during its descent is given by

$$
v^{2}=\left(v_{0}^{2}+4 a g \cos ^{2} \epsilon\right) e^{(\phi-2 \pi) \tan \epsilon}-4 a g \sin ^{2}(\phi-\epsilon) .
$$

92. A particle is projected from a point on the lowest generator of a rough horizontal cylinder of radius $a$ with velocity $V$ at right angles to the generator. Prove that it returns to the point of projection after a time $a\left(e^{2 \mu \pi}-1\right) /(\mu V)$, where $\mu$ is the coefficient of friction.

93. A rough wire in the form of an equiangular spiral whose angle is $\cot ^{-1} 2 \mu$ is placed in a vertical plane, and a heavy particle slides down it, 
coming to rest at its lowest point. Prove that at the starting point the tangent makes with the horizon an angle $2 \tan ^{-1} \mu$, and that the velocity is greatest when the angle $\phi$ which the direction of motion makes with the horizon is given by the equation

$$
\left(2 \mu^{2}-1\right) \sin \phi+3 \mu \cos \phi=2 \mu .
$$

94. Two particles, each of unit mass, attracting each other with a force $\mu$ (distance), are placed in two rough straight intersecting tubes at right angles to each other and the friction is equal to the pressure on each tube: prove that, if they are initially at unequal distances from the point of intersection, one moves for a time $\frac{1}{2} \pi / \sqrt{ } \mu$ before the other starts, and that, while they are approaching the point of intersection of the tubes, they move in the same manner as the projections of the two extremities of the diameter of a circle upon a straight line on which the circle rolls uniformly.

95. A ring moves on a rough elliptic wire, of semi-axes $a, b$, under the attraction of a thin uniform gravitating rod of mass $M$ in the line of foci. Prove that, if it is projected from an end of the minor axis and comes to rest at the end of the major axis through which it first passes, the velocity $v$ of projection is given by the equation

$$
v^{2}=\frac{4 \gamma M \mu \alpha}{(a+b)^{2}} \int_{0}^{\pi} \frac{e^{-\mu \theta} d \theta}{1-2 a \cos \theta+a^{2}},
$$

where $\mu$ is the coefficient of friction, and $a=(a-b) /(a+b)$.

96. A particle on a plane is moving with constant velocity $V$ relative to it, the plane at the same time turning round a fixed axis perpendicular to it with angular velocity $\omega$. Prove that the path of the particle is given by the equation

$$
\frac{V \theta}{\omega}=\sqrt{ }\left(r^{2}-a^{2}\right)+\frac{V}{\omega} \cos ^{-1} \frac{a}{r},
$$

$r$ and $\theta$ being referred to fixed axes, and $a$ being the least distance of the particle from the axis of rotation.

97. A point $P$ moves along a plane curve which rotates in its plane about a point $O$ with uniform angular velocity $\omega$. Prove that the curvature of its path is

$$
\frac{V(\sigma V+2 \omega)(V+r \omega \sin \psi)+r \omega\left(V \omega \sin \psi-f \cos \psi+r \omega^{2}\right)}{\left(V^{2}+r^{2} \omega^{2}+2 V r \omega \sin \psi\right)^{\frac{3}{2}}},
$$

where $r$ is the length $O P, \sigma$ is the curvature of the curve at $P, \psi$ the angle between $O P$ and the tangent, $V$ the velocity of $P$ relative to the curve, and $f$ the rate of increase of $V$.

98. A particle $P$ is free to move on a smooth circular wire whose centre $C$ rotates with constant angular velocity in the plane of the wire about a fixed point $O$. Show that, if $C P=3 O C$ and the particle just makes complete revolutions, the pressure between the particle and the wire vanishes when $C P$ makes with $O C$ an angle $\sec ^{-1} 3$. 
99. A smooth horizontal circular wire rotates uniformly about a point in its plane. Prove that the motion of a bead in the wire will be the same as that of the bob of a simple pendulum.

100. A particle is at rest in a smooth horizontal circular tube, and the tube is made to rotate with uniform angular velocity about a vertical axis through a point on the diameter passing through the particle. Prove that the time of describing any arc bounded by a chord through the centre of rotation is constant.

101. A bead is initially at rest on a smooth circular wire of radius $\alpha$ in a horizontal plane; the wire is made to rotate with uniform angular velocity $\omega$ about an axis perpendicular to its plane and passing through a point on the diameter through the bead at a distance $c$ from the centre. When the bead has moved a distance $a \theta$ on the wire, the wire is suddenly stopped. Prove that the bead will subsequently move with velocity

$$
\omega\left\{\sqrt{ }\left(\alpha^{2}+c^{2}+2 \alpha c \cos \theta\right)-(\alpha+c \cos \theta)\right\} \text {. }
$$

102. Two small beads of masses $m_{1}, m_{2}$ slide along two smooth straight rods which intersect at an angle $a$, and the beads are connected by an elastic thread of natural length $c$ and modulus $\lambda$. The rods are made to revolve uniformly in their plane, about their point of intersection, with angular velocity $\omega$. Prove that throughout the motion

$$
m_{1}\left(\dot{r}_{1}^{2}-r_{1}^{2} \omega^{2}\right)+m_{2}\left(\dot{r}_{2}^{2}-r_{2}^{2} \omega^{2}\right)+\lambda \epsilon^{2} / c=\text { const. }
$$

where $\epsilon$ is the extension of the thread, and $r_{1}, r_{2}$ are the distances of the beads from the intersection of the wires at any time.

103. A smooth elliptic tube rotates about a vertical axis through its centre perpendicular to its plane with uniform angular velocity $\omega$. Prove that a particle can remain at an extremity of the axis major, and if slightly disturbed will oscillate in a period $2 \pi \sqrt{ }\left(1-e^{2}\right) / e \omega$, where $e$ is the eccentricity.

104. A particle can move in a smooth elliptic tube which can turn about its centre in a vertical plane, and, the major axis being vertical and the particle being at rest at the highest point, the tube is sudderily set in rotation with uniform angular velocity $\sqrt{ }\left\{\frac{1}{2} g /(\alpha+b)\right\}$, where $2 a$ and $2 b$ are the axes of the ellipse. Prove that the particle will move on the ellipse as if under a force to the centre varying as the distance.

105. A body is describing an ellipse of semi-axes $a, b$ about a centre of gravitation, and when it is at a distance $r$ from this centre it comes under the influence of a small disturbing force directed to the same point and varying inversely as the cube of the distance. Prove that the effect is the same as if the body described under the original force an orbit which at the same time rotated (with the body) round the centre of force with angular velocity $n$ times the angular velocity of the body, where $n$ is a small constant such that the semi-axes of this new free orbit are equal to those of the original one reduced by fractions $2 n b^{2} / r^{2}$ and $n\left(1+b^{2} / r^{2}\right)$ of themselves. 
106. If while one particle oscillates in a smooth circular tube of radius $a$ in a vertical plane through an arc of height $h$, another particle circulates in a smooth helical tube described on the cylinder of diameter $h$ whose axis is horizontal, touching the circular tube at the lowest point, with velocity due to a height $2 a$ above the lowest point, the two particles can move so as always to be at the same level provided the length of one turn of the helix is equal to the circumference of the circular tube.

107. A particle slides on a smooth helix of angle $a$ and radius $a$ under a force to a fixed point on the axis equal to $\mu$ (distance). Show that the pressure cannot vanish unless the greatest velocity of the particle is $\alpha \sqrt{ } \mu \sec a$.

108. A particle moves on a helical wire whose axis is vertical. Prove that the velocity $v$ after describing an arc $s$ is given by the equations

$$
v^{2}=a g \sec a \sinh \phi, \quad \frac{d s}{d \phi}=\frac{1}{2} a \frac{\sec ^{2} a \cosh \phi}{\tan a-\mu \cosh \phi},
$$

where $a$ is the radius of the cylinder in which the helix lies, $a$ the inclination of the helix to the horizon, and $\mu$ the coefficient of friction.

109. A small smooth groove is cut on the surface of a right circular cone whose axis is vertical and vertex upwards in such a manner as that the tangent is always inclined to the vertical at the same angle $\beta$. A particle slides down the groove from rest at the vertex; show that the time of descending through a vertical height $h$ is equal to the time of falling freely through a height $h \sec ^{2} \beta$. Show also that the pressure is constant and makes with the principal normal to the path a constant angle

$$
\tan ^{-1}\left\{\frac{1}{2} \sin a / \sqrt{ }\left(\cos ^{2} a-\cos ^{2} \beta\right)\right\},
$$

where $2 a$ is the angle of the cone.

110. A smooth helical tube of pitch $a$ has its axis inclined at an angle $\beta(>a)$ to the vertical, and a particle rests in the tube. The tube is made to turn about its axis with uniform angular velocity $\omega$. Prove that the particle makes complete revolutions round the axis if

$$
\frac{1}{2} a \omega^{2} / g>[(\pi-2 \gamma) \sin \gamma-2 \cos \gamma] \sin \beta \cot ^{2} a \operatorname{cosec}^{2} a,
$$

where $\sin \gamma=\tan a \cot \beta$, and $\alpha$ is the radius of the helix.

111. A smooth tube is bent so as to lie on a cone of vertical angle $2 a$ and to cut the generators at a constant angle $\beta$, the axis of the cone being vertical and the vertex uppermost. The tube is made to rotate uniformly about the axis of the cone with angular velocity $\Omega$. Prove that if a particle starts from rest at the vertex it will in time $t$ describe along the tube a distance

$$
\frac{g}{\Omega^{2}} \frac{\cos a}{\sin ^{2} a \cos \beta}[\cosh (\Omega t \sin a \cos \beta)-1] .
$$

112. A particle moves in a smooth tube in the form of a loxodrome on a sphere of radius $a$, while the tube turns uniformly about the polar axis with angular velocity $\omega$. The particle is projected from a point in the equatoreal 
plane with velocity $a \omega$ relative to the tube. Prove that the particle will be at an angular distance $\theta$ from the equatoreal plane after a time

$$
\{\sec a-\log (\sec \theta+\tan \theta)\} / \omega,
$$

and that the pressure on the tube in this position is

$$
2 m a \omega^{2}(1+\sin a) \cos \theta
$$

$m$ being the mass of the particle and $a$ the angle of the loxodrome.

113. A train starts from rest on a level uniform curve, and moves round the curve so that its speed increases at a constant rate $f$. The outer rail is raised so that the floor of a carriage is inclined at an angle $a$ to the horizon. Show that a body cannot rest on the floor of the carriage unless the coefficient of friction between the body and the floor exceeds

$$
f \cos a / \sqrt{ }\left(g^{2}+f^{2} \sin ^{2} a\right) .
$$

114. A locomotive, starting with a constant acceleration $f$ from a point $A$ of a railway, comes to a curve $P Q$ in the line. Prove that, if, in passing along $P Q$, the pressure of the flanges of the wheels on the rails is constant, $P Q$ must be a portion of an equiangular spiral whose pole may be any point on a circle touching $A P$ at $P$ and having its diameter equal to $A P$. Prove also that if the track is tilted up at an angle $\theta$ so that the constant pressure vanishes the angle of the spiral must be $\tan ^{-1}\left(\frac{1}{2} g f^{-1} \tan \theta\right)$.

115. Two particles of masses $m$ and $m^{\prime}$ are attached to the ends of a rigid rod of negligible mass and of length $2 l$, which is freely moveable about its middle point. Show that the inclination $a$ of the rod to the vertical when the particles are moving with uniform angular velocity $\omega$ is given by the equation

$$
\cos \boldsymbol{a}=\left(m-m^{\prime}\right) g /\left\{\left(m+m^{\prime}\right) \omega^{2} l\right\} \text {. }
$$

116. A particle is fastened to one end of a thread of length $l$, the other end being fixed to the top of a smooth sphere of radius $a$; the particle describes a horizontal circle with angular velocity $\omega$, and the length of the thread in contact with the sphere is $a a$. Prove that

$$
\omega^{2}=g \cot a /\{a \sin a+(l-\alpha a) \cos a\} \text {. }
$$

117. A bead can slide on a rough straight wire which is rotating with uniform angular velocity $\omega$ about a fixed vertical axis intersecting it, and $a$ is the inclination of the wire to the horizontal. Prove that for the ring to be in relative equilibrium it must lie between two points in the wire whose distance apart is

$$
g \omega^{-2} \sec a\{\tan (a+\lambda)-\tan (a-\lambda)\},
$$

where $\lambda$ is the angle of friction.

118. A small ring can slide on a smooth plane curved wire which rotates with angular velocity $\omega$ about a vertical axis in its plane. Find the form of the curve in order that the ring may be in relative equilibrium at any point.

Prove that if the angular velocity is increased to $\omega^{\prime}$ the ring will still be in relative equilibrium if the wire is rough and the coefficient of friction between it and the ring not less than $\frac{1}{2}\left(\omega^{\prime} / \omega-\omega / \omega^{\prime}\right)$. 
119. A rod of length $2 a$ rotates in a horizontal plane about one of its ends with uniform angular velocity $\omega$. The ends of a thread of length $2 l$ are attached to the ends of the rod, and a bead can slide on the thread. Prove that when the motion is steady and the bead is at a distance $a+x$ from the axis, the acceleration towards the axis is

$$
g\left\{x \sqrt{ }\left(l^{2}-a^{2}\right) / l \sqrt{ }\left(l^{2}-x^{2}\right)\right\} .
$$

120. A smooth cycloidal tube rotates with uniform angular velocity about its base which is vertical. Prove that a particle cannot rest in the tube anywhere except at the lowest point unless the angular velocity $\omega$ of the tube exceeds $\sqrt{ }(g / \alpha)$, where $a$ is the radius of the generating circle, and that, when $\omega$ exceeds this value, there are two positions of relative equilibrium, the arc-distances of which from the vertex of the cycloid are

$$
2 \omega^{-1} \sqrt{ }\left[2 \alpha^{2} \omega^{2} \pm 2 \alpha \sqrt{ }\left(\alpha^{2} \omega^{4}-g^{2}\right)\right] .
$$

121. A particle moves in a smooth circular tube of radius $a$ which rotates about a fixed vertical diameter with angular velocity $\omega$. Prove that, if $\theta$ is the angular distance of the particle from the lowest point, and if initially it is at rest relative to the tube with the value $a$ for $\theta$ where $\omega \cos \frac{1}{2} a=\sqrt{ }(g / a)$, then at any subsequent time $t$

$$
\cot \frac{1}{2} \theta=\cot \frac{1}{2} a \cosh \left(\omega t \sin \frac{1}{2} a\right) \text {. }
$$

122. A particle of mass $m$ is constrained to remain on the surface of a sphere of radius $a$, and is attached to a fixed point of the sphere by a slightly extensible thread of natural length $a a$ and modulus $\lambda$. Prove that, if the particle is projected at right angles to the unstretched thread with velocity $v$ the greatest subsequent elongation is $2 a \lambda^{-1} m v^{2} \cot a$.

123. A particle is projected horizontally on the interior surface of a smooth cone whose axis is vertical and vertex downwards. Prove that its path when the cone is developed into a plane is the same as the path of a particle under the action of a constant force to a fixed point.

124. A particle moves on a smooth cone under a force to the vertex varying inversely as the square of the distance. Prove that, if the cone is developed into a plane, the path will be a conic having one focus at the vertex of the cone.

125. A particle moves under gravity on a right circular cone with a vertical axis. Show that, if the equations of motion can be integrated without elliptic functions, the particle must be below the vertex, and that its distance $r$ from the vertex at time $t$ is given by an equation of the form

$$
(r \dot{r})^{2}=2 g \cos a\left(r-r_{0}\right)\left(r+2 r_{0}\right)^{2},
$$

where $2 a$ is the vertical angle of the cone.

126. A particle moves on the inside of a smooth circular cone of vertical angle $2 a$ under a force to the vertex varying inversely as the square of the distance. It is projected from an apse at a distance $c$ from the axis with 
velocity $\frac{1}{2} \sqrt{6}$ of that requisite for circular motion. Prove that the polar equation of the projection of the path on a plane perpendicular to the axis is

$$
3 c / r=2+\cos (\theta \sin a),
$$

that the time from one apse to the next is $\pi(2 c \operatorname{cosec} a)^{\frac{3}{2}} / J \mu$, and that the pressure is inversely proportional to the cube of the distance from the vertex.

127. A particle is projected horizontally along the smooth inner surface of a right circular cone, whose axis is vertical and vertex downwards, the initial velocity being $\sqrt{ }\left\{2 g h /\left(n^{2}+n\right)\right\}$ where $h$ is the initial height above the vertex. Prove that the lowest point of its path is at a height $h / n$ above the vertex.

128. A right circular cone of vertical angle $2 a$ is placed with one generator vertical and vertex upwards. From a point on the generator of least slope a particle is projected horizontally and at right angles to the generator with velocity $v$. Prove that it will just skim the surface of the cone without pressure if the distance of the point of projection from the vertex is

$$
\frac{1}{2} v^{2} \operatorname{cosec}^{2} a / g \text {. }
$$

129. A particle is projected horizontally from a fixed point on the interior surface of a smooth paraboloid of revolution whose axis is vertical and vertex downwards. Prove that when it is again moving horizontally its velocity is independent of the velocity of projection.

130. A particle is projected along a small circle on the surface of a smooth uniform oblate spheroid of semi-axes $a$ and $c$. Prove that, if it describes the circle with angular velocity $\omega$, then

$$
\omega^{2}=\left(A a^{2}-C c^{2}\right) / a^{2},
$$

where $A$ and $C$ have their usual meanings in the theory of the attraction of the spheroid.

131. Prove that, when a body of mass $m$ moves under gravity on a smooth sphere of unit radius, the osculating plane of the path makes an angle $\tan ^{-1}\left(g h / m v^{3}\right)$ with the normal, $h$ being the moment of momentum about the vertical diameter and $v$ the velocity, the osculating plane always cutting the vertical diameter below the centre.

132. A particle moves on the inner surface of a smooth bowl in the form of a paraboloid of latus rectum $4 a$ with axis vertical and vertex downwards, being projected along the surface in the horizontal plane through the focus with velocity $\sqrt{ }(2 \mathrm{nag})$. Prove that the initial radius of curvature of the path is $2 \sqrt{ } 2 n a / \sqrt{ }\left(1+n^{2}\right)$.

133. A particle moves inside a smooth paraboloid of revolution whose axis is vertical and vertex downwards, being projected from the level of the focus with velocity due to a height $h$ in a direction making an angle $\frac{l}{6} \pi$ with the meridian. Prove that, if $l$ is the latus rectum, the initial radius of curvature of the path is

$$
\frac{4 l \sqrt{ } 2}{5} \cos \tan ^{-1} \frac{l}{5 h} \text {. }
$$


134. Prove that if the path of a particle moving on a right circular cone cuts the generators at an angle $\chi$ the acceleration in the tangent plane to the surface and normal to the path is

$$
v^{2}\left(d \chi / d s+r^{-1} \sin \chi\right),
$$

where $v$ is the velocity, and $r$ the distance from the vertex.

If the axis of the cone is vertical, and the vertex upwards, and if the velocity is that due to falling from the vertex, prove that, when the particle leaves the cone,

$$
2 \sin ^{2} \chi=\tan ^{2} a,
$$

$2 a$ being the vertical angle of the cone. What happens when $\tan ^{2} a>2$ ?

135. A particle moves on a smooth surface of revolution. The velocity is $v$ at a point where the normal terminated by the axis of revolution is of length $\nu$, and this normal makes an angle $\theta$ with the axis; prove that, if $d s$ is the element of arc of the path, and $\chi$ the angle at which it cuts the meridian, the acceleration in the tangent plane to the surface and normal to the path is

$$
v^{2}\left(\frac{d \chi}{d s}+\frac{\sin \chi \cot \theta}{\nu}\right) \text {. }
$$

136. A particle describes a rhumb line on a sphere in such a way that the longitude increases uniformly; prove that the resultant acceleration varies as the cosine of the latitude, and that its direction makes with the normal an angle equal to the latitude.

137. A particle describes a rhumb line on a smooth sphere under a force parallel to its axis. Show that the force varies inversely as the fourth power of the distance from the axis and directly as the distance from the diametral plane perpendicular to the axis.

138. A particle of unit mass moves on a smooth sphere under two central attractive forces $\mu / r_{1}{ }^{3} r_{2}^{2}$, and $\mu / r_{2}{ }^{3} r_{1}{ }^{2}$ in the distances $r_{1}, r_{2}$ of the point from the two poles. Prove that, if the velocity at starting is that due to falling from an infinite distance, the path on the sphere is a rhumb line.

139. A particle is placed at rest on the smooth inner surface of a vertical circular cylinder, which rotates with uniform angular velocity $\omega$ about the generator furthest from the particle. Prove that the particle leaves the surface when it has descended a distance

$$
\frac{1}{2} \frac{g}{\omega^{2}}\left(\log \frac{2}{3-\sqrt{ } 5}\right)^{2} \text {. }
$$

140. A particle is attached by a thread of length $\alpha$ to a point of a rough fixed plane inclined to the horizon at an angle equal to the angle of friction between the particle and the plane. The particle is projected down the plane at right angles to the thread, which is initially straight and horizontal. Prove that it comes to rest at the lowest point of its path if the square of the initial velocity is $(\pi-2) \mu g a / \sqrt{ }\left(1+\mu^{2}\right)$, where $\mu$ is the coefficient of friction. 
141. A rough hollow circular cylinder is made to rotate uniformly about its axis which is horizontal, and a particle within it is projected from the lowest point in a direction contrary to that of the motion of the neighbouring parts of the cylinder with such velocity that it comes to rest at an end of the horizontal diameter. Prove that, provided the angular velocity is great enough, the next position of instantaneous rest is given by the least positive root of the equation

$$
3 \mu\left(e^{2 \mu \theta}-\cos \theta\right)=\left(2 \mu^{2}-1\right) \sin \theta,
$$

$\theta$ being the angle between the axial planes through the two positions of instantaneous rest, and $\mu$ the coefficient of friction.

142. A particle is projected horizontally with velocity $V$ along the interior surface of a rough vertical circular cylinder. Prove that, at a point where the path cuts the generator at an angle $\phi$, the velocity $v$ is given by the equation

$$
a g / v^{2}=\sin ^{2} \phi\left\{a g / V^{2}+2 \mu \log (\cot \phi+\operatorname{cosec} \phi)\right\},
$$

and the azimuthal angle and the vertical descent are respectively

$$
\int_{\phi}^{\frac{1}{2} \pi} \frac{v^{2}}{a g} d \phi \text { and } \int_{\phi}^{\frac{1}{2} \pi} \frac{v^{2}}{g} \cot \phi d \phi .
$$

143. A particle moves on the surface of a rough right circular cone of vertical angle $2 a$ under no forces but the reaction of the surface. It is projected at a distance $r$ from the vertex with velocity $V$ perpendicular to the generator. Show that, when its path crosses a generator at angle $\chi$, the velocity is $V e^{-\mu \cot \alpha \cos \chi}$, and the time to that point is $\frac{r}{V} \int_{\chi}^{\frac{1}{2} \pi} e^{\mu \cot \alpha \cos \chi} \operatorname{cosec}^{2} \chi d \chi, \mu$ being the coefficient of friction.

144. A particle is projected vertically upwards in a medium in which the resistance is $k$ (velocity) ${ }^{2}$. If $u$ is the initial velocity and $T$ the whole time of motion prove that $\sqrt{ } k(2 u / g-T)$ is positive and increases as $k$ increases.

145. A particle is projected vertically upwards in a medium in which the resistance is $\frac{g}{K^{2}}$ (velocity) ${ }^{2}$. Prove that, if $U, V$ are the velocities with which the particle leaves and returns to the point of projection,

$$
\frac{1}{V^{2}}-\frac{1}{U^{2}}=\frac{1}{K^{2}} \text {. }
$$

146. A particle falls from rest under gravity through a distance $x$ in a medium whose resistance varies as the square of the velocity; $v$ is the velocity acquired by the particle, $V$ the terminal velocity, and $v_{0}$ the velocity that would be acquired by falling through a distance $x$ in vacuo ; prove that

$$
v^{2} / v_{0}^{2}=1-\frac{1}{2} v_{0}^{2} / V^{2}+\frac{1}{2.3} v_{0}^{4} / V^{4}-\frac{1}{2.3 .4} v_{0}^{6} / V^{6}+\ldots
$$


147. A particle is projected vertically upwards from the surface of the Earth with velocity $u$, and when its velocity is $v$ and its height above the surface is $z$ the resistance is $\kappa v^{2} /(\alpha+z)$, where $\alpha$ is the Earth's radius. Prove that, if $z$ is always small compared with $a$, the velocity $V$ with which it returns to the point of projection is approximately given by the equation

$$
V^{2} / u^{2}=1-\kappa v^{2} / g a+\left(4 \kappa^{2}-\frac{2}{3} \kappa\right)\left(v^{2} / 2 g a\right)^{2},
$$

variations of gravity with height being taken into account.

148. A particle is projected vertically upwards in a medium in which the resistance is $\mathrm{kg}$ (velocity) ${ }^{2}$. Prove that it returns to the point of projection with kinetic energy diminished in the ratio $1: 1+k V^{2}$, where $V$ is the velocity of projection.

Prove that in the same medium the angle $\theta$ between the asymptotes of the complete trajectory of a projectile is given by the equation

$$
U^{2} / w^{2}=\cot \theta \operatorname{cosec} \theta+\sinh ^{-1} \cot \theta,
$$

where $U$ is the terminal velocity and $w$ the velocity when the projectile moves horizontally.

149. A particle moves under gravity in a medium whose resistance is proportional to the velocity. Prove that the range on a horizontal plane is a maximum, for given velocity of projection, when the angle of elevation at first and the angle of descent at last are complementary.

150. A particle is projected up a plane of inclination $a$ under gravity and a resistance proportional to the velocity. The direction of projection makes an angle $\beta$ with the vertical, the range $R$ is a maximum and $t$ is the time of flight. Prove that, if $U$ is the terminal velocity and $V$ the velocity of projection, then

(i) $1+(V / U) \sec \beta=\exp .(g t / U)$,

(ii) $U V(U+V \cos \beta) /(V+U \cos \beta)=g(R \sin a+U t)$,

(iii) $U V^{2} \sin \beta /(V+U \cos \beta)=g R \cos a$.

151. A particle of unit mass describes a plane curve under a central attraction equal to $\left(\mu^{2}+\kappa^{2}\right) r$ when it is at a distance $r$ from the origin, in a medium whose resistance is $2 \kappa$ (velocity). Prove that its coordinates at time $t$ are

$$
\begin{aligned}
& e^{-\kappa t}\left\{x_{0} \cos \mu t+\mu^{-1}\left(u_{0}+\kappa x_{0}\right) \sin \mu t\right\}, \\
& e^{-\kappa t}\left\{y_{0} \cos \mu t+\mu^{-1}\left(v_{0}+\kappa y_{0}\right) \sin \mu t\right\},
\end{aligned}
$$

$x_{0}, y_{0}$ being its initial coordinates and $u_{0}, v_{0}$ its initial velocities.

152. A particle moves under gravity in a medium whose resistance varies as the square of the velocity, and $u$ and $v$ are the magnitudes of its velocity at the two instants when its direction of motion makes an angle $\frac{1}{4} \pi$ with the horizontal. Prove that, when it is moving horizontally its velocity is

$$
u v / \sqrt{ }\left(u^{2}+v^{2}\right) \text {. }
$$


153. Defining the instantaneous parabola of a projectile in a medium whose resistance is proportional to the square of the velocity as that which would be described if the resistance ceased to act, prove that its latus rectum diminishes at a rate which varies as $v^{3} \cos ^{2} \theta$, where $\theta$ is the inclination to the horizon of the direction of motion at the point where the velocity is $v$. Prove also that the axis of the parabola moves towards or from the point of projection according as the projectile is ascending or descending.

154. Show that the horizontal and vertical coordinates $x, y$ of a particle moving under gravity in a medium whose resistance is $R$ satisfy the equation

$$
\frac{d^{3} y}{d x^{3}}+\frac{2 g R}{v^{4} \cos ^{3} \phi}=0,
$$

$v$ being the velocity and $\phi$ the inclination of the tangent to the horizontal.

155. Prove that the time $t$, the horizontal abscissa $x$, and the vertical ordinate $y$, at a point where the tangent of the inclination of the velocity to the horizon is $p$, of a trajectory in a medium whose resistance varies as the $n$th power of the velocity, are given by

$$
\begin{gathered}
\frac{g t}{w}=\int_{p}^{a} P^{-\frac{1}{n}} d p, \frac{g x}{w^{2}}=\int_{p}^{a} P^{-\frac{2}{p}} d p, \frac{g y}{w^{2}}=\int_{p}^{a} p P^{-\frac{2}{n}} d p, \\
P=\int_{p}^{a}\left(1+p^{2}\right)^{\frac{n-1}{2}} d p
\end{gathered}
$$

where

$w$ denoting the terminal velocity in the medium, and $a$ the tangent of the inclination to the horizon at the origin, the point of infinite velocity.

156. A particle in a medium whose resistance is small, and equal to $\kappa$ (velocity) ${ }^{2}$, is executing small vibrations. Prove that the period is unaltered, but that in each semi-vibration the amplitude is diminished by $\frac{4}{3} \kappa a^{2}$, where $a$ is the original amplitude.

157. A pendulum oscillates in a medium of which the resistance per unit of mass is $k$ (velocity) ${ }^{2}$. Prove that, when powers of the arc above the first are neglected, the period is the same as in the absence of resistance, but the time of descent exceeds that of ascent by ${ }_{3}^{2} \kappa a \sqrt{ }\left(l^{3} / g\right)$, where $a$ is the angular amplitude of the descent, and $l$ is the length of the pendulum.

158. Prove that in a resisting medium a particle can describe a circle of diameter $a$ under the action of a force to a point on the circumference varying inversely as the fourth power of the distance, the resistance being proportional to $r^{-4} \sqrt{ }\left(a^{2}-r^{2}\right)$ when the distance is $r$.

159. A particle describes an equiangular spiral in a resisting medium under a force $F$ to the pole, and the rate of description of areas is uniformly retarded; prove that

$$
F=\mu r^{-3}-\lambda r^{-1},
$$

where $\lambda$ and $\mu$ are constants, and find the law of resistance. 
160. The resistance of a medium is $\kappa v^{4}$; prove that the orbit described in it by a particle under a central attraction $\mu / r^{2}$ will be an equiangular spiral if the velocity of projection is that in a circle at the same distance, and the angle of projection is $\cos ^{-1}(2 \mu k)$.

161. Two particles move in a medium the resistance of which is proportional to the velocity, under the action of their mutual attraction, which is any function of their distance. Prove that their centre of inertia either remains at rest or moves in a straight line with a velocity which diminishes in geometric progression as the time increases in arithmetic progression.

162. A particle acted on by a central force and moving in a resisting medium in which the resistance is $k$ (velocity) ${ }^{2}$ describes an equiangular spiral whose pole is the centre of force; prove that the force is proportional to $r^{-3} e^{-2 \kappa r \sec \alpha}$, where $a$ is the angle of the spiral.

163. A particle of unit mass moves in a resisting medium, of which the resistance at any point is $R$, under the action of a radial force $F$ and a transversal force $G$. Prove with the usual notation of central orbits that

$$
\begin{gathered}
\frac{d}{d \theta}\left(\frac{1}{2} h^{2}\right)=\frac{G}{u^{3}}-\frac{R}{u^{4}} \frac{d \theta}{d s}, \\
h^{2}\left(\frac{d^{2} u}{d \theta^{2}}+u\right)=-\frac{1}{u^{2}}\left(F+\frac{G}{u} \frac{d u}{d \theta}\right) .
\end{gathered}
$$

164. A particle of mass $m$ moves in a field of force having a potential $V$ in a medium in which the resistance is $k$ times the velocity. Prove that, if $D$ is the quantity of energy dissipated in time $t$,

$$
\frac{d D}{d t}+\frac{2 k}{m}(D-V)=\text { const. }
$$

If the resistance is $k$ (velocity) ${ }^{2}$, and if $d s$ is the element of arc of the path of the particle, then

$$
\frac{d D}{d s}+\frac{2 k}{m}(D-V)=\text { const. }
$$

165. A smooth straight tube rotates in one plane with uniform angular velocity $\omega$ about a fixed end, and a particle moves within it under a resistance equal to $\kappa$ times the square of the relative velocity. Prove that if the particle is projected so as to come to rest at the fixed end the relative velocity at a distance $r$ from that end is

$$
\frac{1}{2} \sqrt{ } 2 \omega \kappa^{-1} \sqrt{ }\left(e^{2 \kappa r}-2 \kappa r-1\right) \text {. }
$$

166. A particle is suspended so as to oscillate in a cycloid whose vertex is at the lowest point, and starts at a distance $a$ from that point measured along the curve. Prove that, if the medium in which it moves gives a small resistance $k$ (velocity) ${ }^{2}$ per unit of mass, then before it next comes to rest energy equal to $\frac{8}{3} k a$ of the original energy will have been dissipated.

L. 
167. A particle moves on a smooth cycloid whose axis is vertical and vertex upwards in a medium whose resistance is $(2 c)^{-1}$ (velocity) $)^{2}$ per unit of mass, and the distance of the starting point from the vertex is $c$; prove that the time to the cusp is $\sqrt{ }\{8 a(4 a-c) / g c\}, 2 a$ being the length of the axis.

168. A particle of mass $m$ moves under equal constant forces $m f$ along the tangent and normal to its path, and the resistance is $m f v^{2} / k^{2}$ when the velocity is $v$. Prove that the intrinsic equation of the path is

$$
k^{2}\left(e^{2 f s / k^{2}}-1\right)=u^{2}\left(e^{2 \phi}-1\right),
$$

where $u$ is the velocity of projection.

169. A particle moves in a medium in which the resistance at any point varies as the density of the medium at the point and as the square of the velocity of the particle, and the particle describes an ellipse under the action of two forces to the foci varying inversely as the $n$th power of the distance; find the density of the medium at any point of the path; and show that if $n=1$ and the forces are equal at equal distances the density varies as the acceleration with which the particle would move if constrained to describe the same ellipse under the same forces but without resistance. 


\section{CHAPTER *XI.}

\section{MOTION OF A RIGID BODY IN TWO DIMENSIONS.}

214. In this Chapter we propose to discuss the motion of a rigid body in cases where every particle of the body moves parallel to a fixed plane, for example the plane $(x, y)$ of a frame of reference. In such a case the $x$ and $y$ of a particle of the body vary with the time, but the $z$ of each particle remains constant throughout the motion. The motion is said to be "in two dimensions," or "in one plane." Now we saw in Article 114 that to determine the position of a rigid body it is requisite and sufficient to determine the positions of a particle of the body, of a line of particles passing through that particle, and of a plane of particles passing through that line. In the case now under discussion we may take the line and plane in question to be parallel to the plane $(x, y)$, then the position of the plane is invariable, and the position of the line is determined by the angle it makes with a fixed line in the plane, for instance the axis of $x$, further the position of the chosen particle is determined by its coordinates $x$ and $y$. Thus the determination of the position of the rigid body (moving in two dimensions) requires the determination of three numbers, representing the coordinates of the position of one of the particles, and the angle which a line of the body through that particle and in the plane of its motion makes with a fixed line.

We can now see what is meant by the angular velocity of a rigid body moving in one plane. Suppose, in fact, that one line of particles in the body and parallel to the plane makes an angle $\theta$ at time $t$ with a line fixed in the plane. Then this angle is increasing at a rate $\dot{\theta}$. Let any other line of particles be drawn also parallel to the plane, and let $\alpha$ be the angle it makes with 
the first line. Then $\alpha$ is invariable, for if it were to change the body would be deformed. Now the second line of particles makes an angle $\theta+\alpha$ with the fixed line, and this angle also increases at a rate $\dot{\theta}$. We thus see that every line of particles parallel to the plane turns with the same angular velocity, and this is the angular velocity of the rigid body.

215. Moment of Inertia. Consider a rigid body turning about an axis with angular velocity $\omega$. Let $m$ be the mass of a particle of a body at a distance $r$ from the axis. Then this particle is describing a circle of radius $r$ with velocity $r \omega$. Hence its moment of momentum about the axis is $m r^{2} \omega$, and its kinetic energy is $\frac{1}{2} m r^{2} \omega^{2}$.

It follows that the moment of momentum of the rigid body about the axis is

$$
\omega \Sigma m r^{2} \text {, }
$$

the summation referring to all the particles, and the kinetic energy is

$$
\frac{1}{2} \omega^{2} \Sigma m r^{2}
$$

These expressions become

$$
\omega \iiint \rho\left(x^{2}+y^{2}\right) d x d y d z
$$

and

$$
\frac{1}{2} \omega^{2} \iiint \rho\left(x^{2}+y^{2}\right) d x d y d z
$$

for a body of density $\rho$ at a point $(x, y, z)$ the axis of rotation being the axis of $z$.

The multiplier of $\omega$ and $\frac{1}{2} \omega^{2}$ in these expressions is called the moment of inertia of the body about the axis. We shall see presently that it enters into the expressions for the kinetic energy and moment of momentum of a rotating body, whether the axis of rotation is fixed or not.

The moment of inertia of a body about an axis depends only on the shape of the body, its situation with reference to the axis, and the distribution of density within it.

216. Theorems concerning Moments of Inertia. I. The moment of inertia of a system about any axis is equal to the moment of inertia about a parallel axis through the centre of inertia together with the moment of 
inertia about the original axis of the whole mass placed at the centre of inertia.

Let $x, y, z$ be the coordinates of any particle of the system, $m$ its mass, $\bar{x}, \bar{y}, \bar{z}$ the coordinates of the centre of inertia, $x^{\prime}, y^{\prime}, z^{\prime}$ those of the particle $m$ relative to the centre of inertia.

Then

$$
\left.\begin{array}{r}
x=\bar{x}+x^{\prime}, y=\bar{y}+y^{\prime}, \quad z=\bar{z}+z^{\prime}, \\
\Sigma m x^{\prime}=0, \Sigma m y^{\prime}=0, \Sigma m z^{\prime}=0 .
\end{array}\right\}
$$

Now

$$
\Sigma m x^{2}=\Sigma m\left(\bar{x}+x^{\prime}\right)^{2}=\bar{x}^{2} \Sigma m+\Sigma m x^{\prime 2} \text {. }
$$

So

$$
\Sigma m y^{2}=\bar{y}^{2} \Sigma m+\Sigma m y^{\prime 2} \text {. }
$$

Hence

$$
\Sigma m\left(x^{2}+y^{2}\right)=\Sigma m\left(x^{\prime 2}+y^{\prime 2}\right)+\left(\bar{x}^{2}+\bar{y}^{2}\right) \Sigma m,
$$

which is the theorem stated.

II. The moment of inertia of a plane disc of any form about any axis perpendicular to its plane is the sum of those about any two rectangular axes in the plane which meet on the first axis.

For, taking the axes to be those of $z, x, y$, and taking the point where they meet for origin, the moments of inertia about the axes of $x$ and $y$ are respectively $\Sigma m y^{2}$ and $\Sigma m x^{2}$, and the moment of inertia about the axis of $z$ is $\Sigma m\left(x^{2}+y^{2}\right)$.

III. To compare the moments of inertia of a disc about different axes in its plane.

For parallel axes we can use Theorem I. and it will therefore be sufficient to consider axes in different directions through the origin. Let $\theta$ be the angle which any line makes with the axis $x$. The distance of any point $(x, y)$ from this line is $-x \sin \theta+y \cos \theta$, and thus the moment of inertia about the line is $\Sigma m(y \cos \theta-x \sin \theta)^{2}=\sin ^{2} \theta \Sigma\left(m x^{2}\right)+\cos ^{2} \theta \Sigma\left(m y^{2}\right)-2 \sin \theta \cos \theta \Sigma m x y$.

The expression for the moment of inertia about a perpendicular line would be

$$
\cos ^{2} \theta \Sigma\left(m x^{2}\right)+\sin ^{2} \theta \Sigma m y^{2}+2 \sin \theta \cos \theta \Sigma(m x y) .
$$

The quantity $\Sigma(m x y)$ is known as the product of inertia with respect to the axes of $x$ and $y$ (in two dimensions). For new axes obtained by turning through an angle $\theta$ it has the value

$$
\left(\cos ^{2} \theta-\sin ^{2} \theta\right) \Sigma(m x y)-2 \sin \theta \cos \theta\left\{\Sigma\left(m y^{2}\right)-\Sigma\left(m x^{2}\right)\right\} .
$$

We can always choose the axes of $(x, y)$ so that this quantity $\Sigma(m x y)$ vanishes. When this is done the axes of $x$ and $y$ are called Principal axes of the disc. The directions of the principal axes vary with the point chosen as origin.

Now suppose the axes of $x$ and $y$ are principal axes of the disc at the origin. Let $A,=\Sigma\left(m y^{2}\right)$, be the moment of inertia with respect to the axis of $x$, and $B,=\Sigma\left(m x^{2}\right)$, be the moment of inertia with respect to the axis $y$. Then the moment of inertia about a line through the origin making an angle $\theta$ with the axis $x$ is $A \cos ^{2} \theta+B \sin ^{2} \theta$.

If an ellipse whose equation is $A x^{2}+B y^{2}=$ const. be supposed drawn on the disc, then the moment of inertia about any diameter of it is inversely 
proportional to the square of the length of that diameter. This ellipse is known as the ellipse of inertia.

IV. If two plane systems in the same plane have the same mass, the same centre of inertia, the same principal axes at the centre of inertia, and the same moments of inertia about these principal axes, they have the same moments of inertia about any axis in or perpendicular to the plane.

For, in the first place, the two systems have by Theorem III. the same moments of inertia about any axis lying in the plane and passing through the common centre of inertia, by Theorem I. they have the same moments of inertia about any other axis in the plane, and by Theorem II. they have the same moment of inertia about any axis perpendicular to the plane.

Such systems are described as momental equivalents.

\section{Calculations of moments of inertia.}

I. Uniform ring. Radius of gyration of a body. For a circular ring of mass $m$, and of very small section, the mass between two sections made by planes through the axis containing an angle $d \theta$ is $\frac{m}{2 \pi} d \theta$. If $a$ is the radius of the ring the moment of inertia about the axis is

$$
\int_{0}^{2 \pi} \frac{m}{2 \pi} a^{2} d \theta=m a^{2}
$$

This might have been seen at once since every element is at distance $a$ from the axis.

In the case of a body of any shape and of mass $m$ we can always express the moment of inertia about any axis in the form $m k^{2}$, where $k$ represents the length of a line, and thus we see that $k$ is the radius of a ring such that, if the mass of the body were condensed uniformly upon the ring, the moment of inertia of the ring about its axis would be the same as the moment of inertia of the body about the axis in question. The quantity $k$ for any body and any axis is known as the radius of gyration of that body about that axis.

II. Uniform rod. Let $m$ be the mass of the rod, and $2 a$ its length, $r$ the distance of any section from the middle point. The mass of the element between the sections $r$ and $r+d r$ is $\frac{m}{2 a} d r$. Therefore, if the thickness of the rod is disregarded, the moment of inertia about an axis through the middle point at right angles to the rod is

$$
\int_{-a}^{a} \frac{m}{2 a} r^{2} d r=\frac{1}{3} m a^{2}
$$

The radius of gyration of the rod is $a / \sqrt{ } 3$.

III. Circular disc. For a uniform circular disc, of radius $a$ and mass $m$, the mass per unit area is $m / \pi a^{2}$, and thus the moment of inertia about an axis through the centre perpendicular to the plane is

$$
\int_{0}^{2 \pi} \int_{0}^{a} \frac{m}{\pi a^{2}} r^{2} \cdot r d r d \theta
$$

or $\frac{1}{2} m a^{2}$. Hence the radius of gyration about the axis of the disc is $a / \sqrt{ } 2$. 
IV. Sphere. Let $a$ be the radius of the sphere, $\rho$ its density (supposed uniform), and let it be referred to a system of rectangular axes with origin at the centre. Then we require the values of such integrals as $\iiint x^{2} \rho d x d y d z$ taken through the volume. From symmetry it is clear that the integrals obtained from this by putting $y$ or $z$ for $x$ will have the same value as the one written.

Now using polar coordinates $r, \theta, \phi$, we know that the polar element of volume is $r^{2} \sin \theta d r d \theta d \phi$, and the $z$ of any point is $r \cos \theta$. Hence

$$
\iiint z^{2} \rho d x d y d z=\iiint \rho r^{2} \cos ^{2} \theta r^{2} \sin \theta d r d \theta d \phi,
$$

where the limits for $r$ are 0 and $\alpha$, the limits for $\theta$ are 0 and $\pi$, and the limits for $\phi$ are 0 and $2 \pi$.

The value of the integral is $\rho \frac{a^{5}}{5} \frac{2}{3} 2 \pi=M_{5}^{a^{2}}$, where $M$ is the mass of the sphere, $=\frac{4}{3} \pi \rho \alpha^{3}$.

Thus, if $k$ is the radius of gyration of the sphere about a diameter, $k^{2}=\frac{2}{5} \alpha^{2}$.

V. Ellipsoid. To find the value of $\iiint x^{2} \rho d x d y d z$ through the volume of an ellipsoid $x^{2} / a^{2}+y^{2} / b^{2}+z^{2} / c^{2}=1, \rho$ being constant, we change the variables by putting $x=a \xi, y=b \eta, z=c \zeta$, and then we require the value of

$$
\rho a^{3} b c \iint \xi^{2} d \xi d \eta d \zeta
$$

through a range of values given by the inequality $\xi^{2}+\eta^{2}+\zeta^{2} \ngtr 1$. The integration may therefore be regarded as taken through the volume of a sphere of radius unity, and thus by No. IV. the result is $\frac{4}{15} \pi \rho \alpha^{3} b c$. Hence, if $M$ is the mass of the ellipsoid, the moments of inertia about the axes are

respectively.

$$
\frac{1}{5} M\left(b^{2}+c^{2}\right), \frac{1}{5} M\left(c^{2}+a^{2}\right), \frac{1}{5} M\left(a^{2}+b^{2}\right),
$$

\section{Examples.}

1. Prove that a uniform rod, of mass $m$ and length $2 a$, has as momental equivalent three particles, one of mass $\frac{2}{3} m$ at its middle point, and one of mass $\frac{1}{6} m$ at each of its ends.

2. Prove that the moments of inertia of a uniform rectangular disc, of mass $m$ and of sides $2 a, 2 b$, about lines through its centre parallel to its edges are $\frac{1}{3} m b^{2}$ and $\frac{1}{3} m a^{2}$.

3. Prove that the radius of gyration of a uniform circular disc about a diameter is half the radius.

4. Prove that the moments of inertia of a uniform elliptic disc, of mass $m$ and semiaxes $a$ and $b$, about these axes are $\frac{1}{4} m b^{2}$ and $\frac{1}{4} m a^{2}$.

5. Prove that a uniform triangular disc has as momental equivalent three particles, each one-third of its mass, placed at the middle points of its sides.

6. Prove that the moment of inertia of a uniform cube about any axis through its centre is $\frac{2}{3} m a^{2}$, where $m$ is the mass and $2 a$ the length of a side. 


\section{Velocity and Momentum of rigid body.}

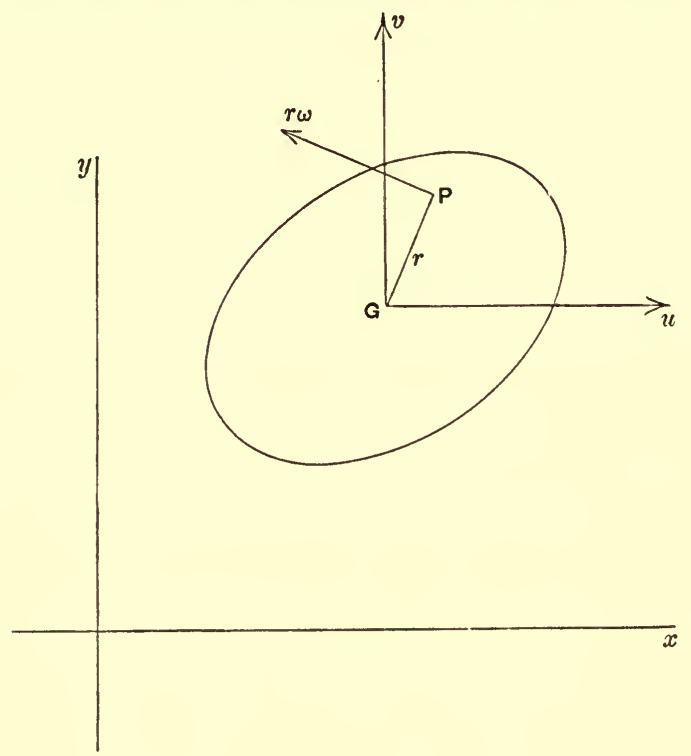

Fig. 55 .

Let $G$ be the centre of inertia of a rigid body moving in two dimensions, and let $u$ and $v$ be resolved parts of the velocity of $G$ parallel to the axes of $x$ and $y$. Let $P$ be any other particle of the body, $r$ its distance from $G$, and $x^{\prime}, y^{\prime}$ its coordinates relative to $G$ at time $t$. Then the line GP is turning with the angular velocity $\omega$ of the rigid body, and the velocity of $P$ relative to $G$ is $r \omega$ at right angles to GP; the resolved parts of this relative velocity parallel to the axes are $-\omega y^{\prime}$ and $\omega x^{\prime}$, since the line GP makes with the axis of $x$ an angle whose cosine is $x^{\prime} / r$ and whose sine is $y^{\prime} / r$.

Hence the resolved velocities of $P$ parallel to the axes are

$$
u-\omega y^{\prime} \text { and } v+\omega x^{\prime} \text {. }
$$

Let $m$ be the mass of the particle at $P$. Then the linear momentum of the body parallel to the axis of $x$ is

$$
\Sigma m\left(u-\omega y^{\prime}\right)=M u
$$

where $M,=\Sigma m$, is the mass of the body. Similarly the linear momentum of the body parallel to the axis of $y$ is $M v$. Thus the linear momentum of the body in any direction is the same as that 
of a particle of mass equal to the mass of the body placed at the centre of inertia and moving with it. (Article 100.)

The moment of momentum of the body about an axis through the centre of inertia perpendicular to the plane of motion is

$$
\begin{gathered}
\Sigma m\left\{x^{\prime}\left(v+\omega x^{\prime}\right)-y^{\prime}\left(u-\omega y^{\prime}\right)\right\} \\
=\omega \Sigma m\left(x^{\prime 2}+y^{\prime 2}\right)=M k^{2} \omega,
\end{gathered}
$$

where $k$ is the radius of gyration about the axis.

The moment of momentum about any parallel axis is the moment about that axis of the momentum of the whole mass placed at the centre of inertia and moving with it together with the moment $M k^{2} \omega$ (Article 102). Thus the momentum of the rigid body is specified by the resultant and couple of a system of vectors localised in lines. The resultant has resolved parts $M u$, $M v$ in the two chosen directions, and the moment of the couple is $M k^{2} \omega$.

Again, the kinetic energy of the body is

$$
\begin{gathered}
\frac{1}{2} \sum m\left\{\left(u-\omega y^{\prime}\right)^{2}+\left(v+\omega x^{\prime}\right)^{2}\right\} \\
=\frac{1}{2} M\left(u^{2}+v^{2}+k^{2} \omega^{2}\right),
\end{gathered}
$$

which is the kinetic energy of the whole mass moving with the centre of inertia together with the kinetic energy of the rotation about the centre of inertia (Article 104).

The formulæ for the velocity of a point show that at each instant the point whose coordinates relative to $G$ are $-v / \omega$ and $u / \omega$ has zero velocity, so that the motion of the body at the instant is a motion of rotation about an axis through this point perpendicular to the plane of motion. The point is called the instantaneous centre of no velocity, or frequently "the instantaneous centre." The fact that the motion of the body at each instant is equivalent to rotation about a point is of importance in many geometrical investigations.

219. Kinetic Reaction of rigid body. With the notation of the last Article, the point $P$ moves relatively to $G$ in a circle of radius $r$ with angular velocity equal to $\omega$ at time $t$; its acceleration relative to $G$ may therefore be resolved into $r \dot{\omega}$ at right angles to $G P$, and $r \omega^{2}$ along $P G$. Hence the resolved parts 
234 MOTION OF A RIGID BODY IN TWO DIMENSIONS. [CHAP. XI. of the acceleration of $P$ parallel to the axes are

$$
\dot{u}-\dot{\omega} y^{\prime}-\omega^{2} x^{\prime} \text {, and } \dot{v}+\dot{\omega} x^{\prime}-\omega^{2} y^{\prime} \text {. }
$$

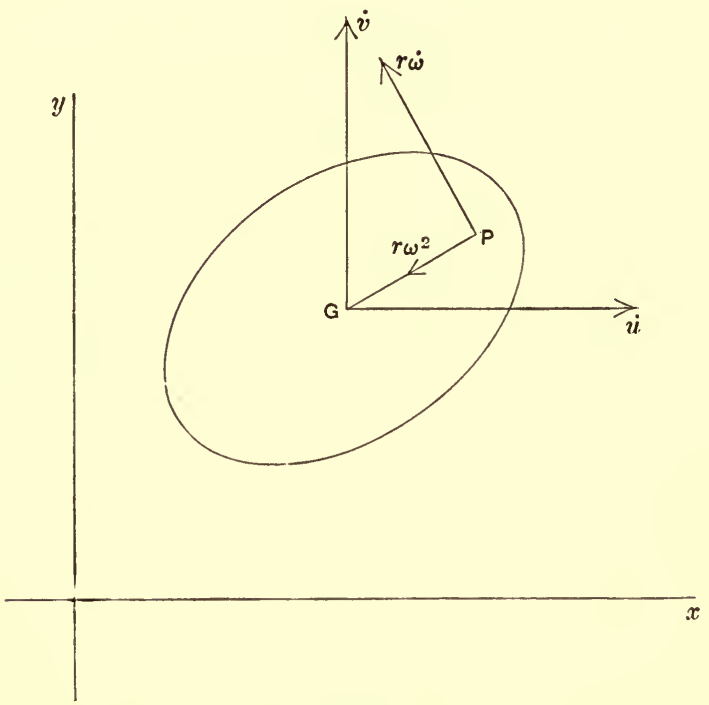

Fig. 56.

The kinetic reactions may be reduced to a vector localised in a line through the centre of inertia and a couple. The vector in question has resolved parts parallel to the axes

$$
\Sigma m\left(\dot{u}-\dot{\omega} y^{\prime}-\omega^{2} x^{\prime}\right)=M \dot{u},
$$

and

$$
\Sigma m\left(\dot{v}+\dot{\omega} x^{\prime}-\omega^{2} y^{\prime}\right)=M \dot{v} ;
$$

and the couple is the moment of the kinetic reactions about a line through the centre of inertia perpendicular to the plane of motion; this moment is

$$
\begin{aligned}
& \sum m\left\{x^{\prime}\left(\dot{v}+\dot{\omega} x^{\prime}-\omega^{2} y^{\prime}\right)-y^{\prime}\left(\dot{u}-\dot{\omega} y^{\prime}-\omega^{2} x^{\prime}\right)\right\} \\
& =M k^{2} \dot{\omega} .
\end{aligned}
$$

The moment of the kinetic reactions about any axis perpendicular to the plane of motion is the moment about that axis of the kinetic reaction of a particle of mass equal to the mass of the body and moving with the centre of inertia, together with the moment of the couple $M k^{2} \dot{\omega}$. (Article 103.)

The formulæ for the acceleration of a point show that at each instant there is a point which has zero acceleration. This point 
is called the instantaneous centre of no acceleration. It is of much less importance than the instantaneous centre of no velocity.

\section{Examples.}

1. Prove that at any instant the normal to the path of every particle passes through the instantaneous centre (of no velocity).

[It follows that this centre can be constructed if we know the directions of motion of two particles.]

2. To calculate the moment of the kinetic reactions about the instantaneous centre (of no velocity).

The coordinates of the instantaneous centre $I$ being $-v / \omega$ and $u / \omega$ referred to axes through the centre of inertia $G$ parallel to the axes of reference, the moment in question is

$$
\frac{v}{\omega} m \dot{v}+\frac{u}{\omega} m \dot{u}+m k^{2} \dot{\omega} .
$$

The velocity of $G$,is $r \omega$ at right angles to the line joining it to $I$, where $r=I G$, or we have $u^{2}+v^{2}=r^{2} \omega^{2}$.

Hence the above is

or

$$
\begin{aligned}
& \frac{1}{\omega} \frac{d}{d t}\left(\frac{1}{2} m r^{2} \omega^{2}\right)+m k^{2} \dot{\omega}, \\
& \frac{1}{\omega} \frac{d}{d t}\left\{\frac{1}{2} m\left(k^{2}+r^{2}\right) \omega^{2}\right\} .
\end{aligned}
$$

If we take an angle $\theta$ such that $\dot{\theta}=\omega$, and write $K$ for the moment of inertia about the instantaneous centre $I$, then $K=m\left(k^{2}+r^{2}\right)$ by I. of Art. 216, and the result obtained may be written $\frac{d}{d \theta}\left(\frac{1}{2} K \omega^{2}\right)$.

When the motion is a small oscillation, or an initial motion from rest, or when the point $I$ is fixed in the body this can be replaced by $K \dot{\omega}$.

3. Prove that those particles which at any instant are at inflexions on their paths lie on a circle.

[This circle is called the "circle of inflexions."]

4. Prove that the curvature of the path of any particle which is not on thel circle of inflexions is $\omega^{3} p^{2} / V^{3}$ where $p^{2}$ is the power with respect to the circle of the position of the particle, $\omega$ is the angular velocity of the body, and $V$ is the resultant velocity of the particle.

5. Prove that, in general, that particle which is at the instantaneous centre (of no velocity) is at a cusp on its path.

221. Equations of motion of rigid body. The equations of motion express that the kinetic reactions and the external forces are equivalent systems of vectors.

Let $M$ be the mass of the body $f_{1}, f_{2}$ the resolved accelerations of the centre of inertia in any two rectangular directions in the plane of motion, $\omega$ the angular velocity of the body. 
Let the forces acting on the body be reduced to a resultant force at its centre of inertia and a couple. Let $P, Q$ be the resolved parts of the force in the directions in which the acceleration of the centroid was resolved, and let $N$ be the couple.

Then the system of vectors expressed by $M f_{1}, M f_{2}, M k^{2} \dot{\omega}$ has the same resolved part in any direction, and the same moment about any axis, as the system $P, Q, N$.

In particular we have

$$
M f_{1}=P, M f_{2}=Q, M k^{2} \dot{\omega}=N,
$$

and the equations of motion of the body can always be written in this form.

In the formation of equations of motion diversity can arise from the choice of directions in which to resolve, and of axes about which to take moments. As in the case of Dynamics of a Particle, the equations arrived at are differential equations, and no rules can be given for solving them in general. If however the circumstances are such that there is an equation of energy, or an equation of conservation of momentum, such equations are first integrals of the equations of motion.

222. Continuance of motion in two dimensions. The question arises whether a body, which at some instant is moving in two dimensions parallel to a certain plane, continues to move parallel to that plane or will presently be found to be moving in a different manner. A general answer to this question cannot be given here, but it is clear that there is a class of cases in which the motion in two dimensions persists. Thus this class includes all the cases in which the body is symmetrical with respect to a plane and the forces applied to it are directed along lines lying in that plane, or, more generally, when the forces can be reduced to a single resultant in the plane of symmetry and a couple about an axis perpendicular to that plane.

223. Rigid Pendulum*. A heavy body free to rotate about a fixed horizontal axis is known as a "compound pendulum"

* Huygens was the first to solve the problem of the motion of the pendulum, and the principles which he invoked were among the considerations which ultimately led to the establishment of the Theory of Energy. 
to distinguish it from the "simple pendulum" whose motion was discussed in Articles 188-193.

Let $G$ be the centre of inertia of the body, $G S$ the perpendicular from $G$ to the axis, $\theta$ the angle GS makes with the vertical at time $t$. Then the whole motion takes place in the vertical plane through $G$ at right angles to the axis, and depends only on the quantity $\theta$.

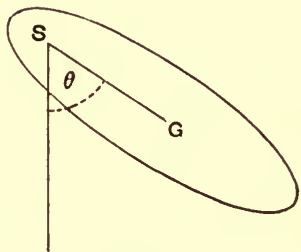

Fig. 57.

Let $G S=h$. Let $M$ be the mass of the body, $k$ its radius of gyration about an axis through $G$ perpendicular to the plane of motion.

The velocity of the centre of inertia is $h \dot{\theta}$, and the kinetic energy is

$$
\frac{1}{2} M\left(h^{2}+k^{2}\right) \dot{\theta}^{2}
$$

The potential energy of the body in the field of the earth's gravitation is

$$
\operatorname{Mgh}(1-\cos \theta) \text {, }
$$

the standard position being the equilibrium position.

Hence the equation of energy can be written

$$
\frac{1}{2} M\left(h^{2}+k^{2}\right) \dot{\theta}^{2}=M g h \cos \theta+\text { const. }
$$

Comparing this equation with that obtained in Article 188, we see that the motion is the same as that of a simple pendulum of length $\left(k^{2}+h^{2}\right) / h$.

A point in the line $S G$ at this distance from $S$ is known as the "centre of oscillation," $S$ is called the "centre of suspension." The distance between these centres is the "length of the simple equivalent pendulum."

\section{Examples.}

1. Prove that if a rigid pendulum, for which $S$ and $O$ are respectively a centre of suspension and the corresponding centre of oscillation, is hung up so that it can oscillate in the same vertical plane as before but with $O$ as centre of suspension, then $S$ will be the centre of oscillation.

2. A uniform rod moves with its ends on a smooth circular wire fixed in a vertical plane. Prove that if it subtends an angle of $120^{\circ}$ at the centre the length of the simple equivalent pendulum is equal to the radius of the circle. 
3. A compound pendulum consists of a rod which can turn about a fixed horizontal axis and a spherical bob which can slide on the rod. Prove that the period of oscillation will be prolonged by sliding the bob up or down according as the length of the equivalent simple pendulum is $>$ or $<$ twice the distance of the centre of gravity of the bob from the axis of rotation.

4. Two rigid pendulums of masses $m$ and $m^{\prime}$ turn about the same horizontal axis. The distances of the centres of gravity and of oscillation from the axis are $h, h^{\prime}$ and $l, l^{\prime}$ respectively. Prove that, if the pendulums are fastened together in the position of equilibrium, the length of the simple equivalent pendulum for the compound body will be $\left(m h l+m^{\prime} h^{\prime} l^{\prime}\right) /\left(m h+m^{\prime} h^{\prime}\right)$.

225. Illustrative Problems. It will be most convenient to exemplify the application of the principles that have been laid down in this chapter and in Chapters VII. and VIII. by partially working out some problems. The most important matters to be illustrated are actions between two rigid bodies whether smooth or rough, and the expression of the effects of the inertia of a rigid body by means of the moment of inertia. Other matters of subsidiary interest are the kinematical expression of velocities and accelerations in terms of a small number of independent geometrical quantities, the expression of kinematical conditions, and the calculation of resultant stresses. Some of the problems selected for discussion are of a complicated character, and have been chosen in order to illustrate a number of points.

I. Inertia of machines. We shall consider Atwood's machine. To avoid having to take account of the motion of the pulley in our preliminary notice of Atwood's machine (Article 186) we assumed the pulley to be perfectly smooth, or that the rope slides over it without frictional resistance and without setting it in motion. It will now be most convenient in order to get some idea of the way the motion of the pulley affects the result to suppose the pulley to be so rough that the particles of the rope and the pulley in contact move with the same velocity along the tangents to the pulley.

Now let $M$ be the mass of the pulley, $a$ its radius, $k$ its radius of gyration about its axis, $\theta$ the angle through which it has turned up to time $t$.

Let $m$ and $m^{\prime}$ be the masses of the bodies attached to the rope, and $x$ the distance through which $m$ has fallen up to time $t$. Then $x=a \theta$.

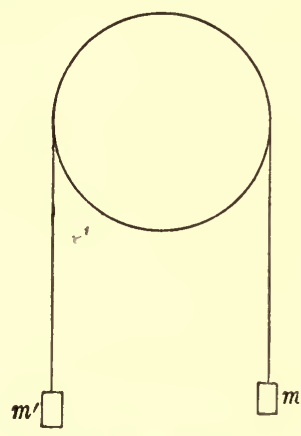

Fig. 48 (bis).

Neglecting the mass of the rope, the kinetic energy is

$$
\frac{1}{2} M k^{2} \dot{\theta}^{2}+\frac{1}{2}\left(m+m^{\prime}\right) \dot{x}^{2},
$$

and the work done is

$$
\left(m-m^{\prime}\right) g x,
$$

so that the energy equation is

$$
\frac{1}{2} M \frac{k^{2}}{a^{2}} \dot{x}^{2}+\frac{1}{2}\left(m+m^{\prime}\right) \dot{x}^{2}=\left(m-m^{\prime}\right) g x+\text { const. }
$$


Thus the acceleration with which $m$ descends is

$$
\frac{m-m^{\prime}}{m+m^{\prime}+M k^{2} / a^{2}} g \text {. }
$$

It appears that the effect of the inertia of the pulley is equivalent to an increase of each of the masses in the simple problem (where the pulley is regarded as smooth) by $\frac{1}{2} M k^{2} / a^{2}$.

II. Rolling and sliding. We take the problem presented by a uniform cylinder of mass $M$ and radius $a$ which is set rolling and sliding on a rough horizontal plane, the angular velocity being initially such that the points on the lowest generator have the greatest velocity.

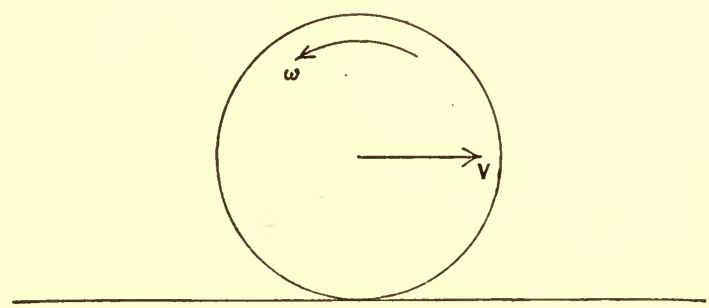

Fig. 58.

Let $V$ be the velocity of the axis, and $\omega$ the angular velocity at time $t$, the senses being those shown in the figure.

The system of kinetic reactions reduces to $M \dot{V}$ horizontally through the centre of inertia, in the sense of $V$, and a couple $M k^{2} \dot{\omega}$ in the sense of $\omega$, where $k$ is the radius of gyration about the axis of the cylinder.

Taking moments about the point of contact we have

$$
M a \dot{V}-M k^{2} \dot{\omega}=0 \text {. }
$$

Now let $F^{\prime}$ be the friction between the cylinder and the plane. The particles on the lowest generator have velocity $V+\alpha \omega$ in the sense of $V$, and therefore $F^{\prime}$ has the opposite sense.

Resolving horizontally we have

$$
M \dot{V}=-F,
$$

where $F$ is positive. Hence $\dot{V}$ is negative and $\dot{\omega}$ is also negative.

The velocity $V$ diminishes and the angular velocity $\omega$ also diminishes according to the equation

$$
k^{2} \omega-\alpha V=k^{2} \omega_{0}-a V_{0},
$$

where $V_{0}$ and $\omega_{0}$ are the values of $V$ and $\omega$ in the beginning of the motion. We shall proceed with the case where $V_{0}<\omega_{0} k^{2} / a$. Then there must come an instant at which $V$ vanishes, and at this instant $\omega$ has the value $\omega_{0}-\alpha V_{0} / k^{2}$. At this instant the lowest point has velocity $\alpha \omega_{0}-V_{0} \alpha^{2} / k^{2}$ in the same sense as before, the friction is still finite and in the same sense as before, and a velocity in the opposite sense begins to be generated. 
At any later stage of the motion let $U$ be the velocity. Then so long as $a \omega>U$ the friction $F$ acts in the same sense, and we have

$$
\left.\begin{array}{l}
M U=F, \\
M a \dot{U}+M k^{2} \dot{\omega}=0,
\end{array}\right\}
$$

whence $U$ increases and $\omega$ diminishes according to the equation

$$
a U+k^{2} \omega=k^{2} \omega_{0}-a V_{0} .
$$

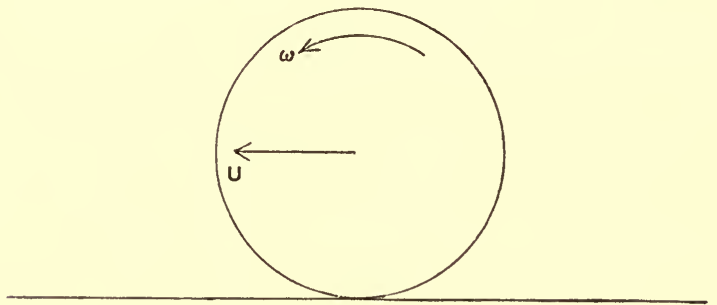

Fig. 59.

When $U$ becomes equal to $a \omega$ the value of either is

$$
a\left(k^{2} \omega_{0}-a V_{0}\right) /\left(a^{2}+k^{2}\right),
$$

and at this instant the cylinder is rolling on the plane. Thereafter the cylinder rolls on the plane uniformly.

It is to be noticed that, in this problem, so long as the cylinder slips, the friction is constantly equal to $\mu M g$, where $\mu$ is the coefficient of friction between the cylinder and the plane.

\section{Examples.}

1. In the problem just considered prove that the time from the beginning of the motion until the motion becomes uniform is $\frac{k^{2}}{a^{2}+k^{2}} \frac{V_{0}+a \omega_{0}}{\mu g}$.

2. A homogeneous cylinder of mass $M$ and radius $a$ is free to turn about its axis which is horizontal, and a particle of mass $m$ is placed upon it close to the highest generator. Prove that when the particle begins to slip, the angle $\theta$ which the radius through it makes with the vertical is given by the equation

$$
\mu\{(M+6 m) \cos \theta-4 m\}=M \sin \theta,
$$

where $\mu$ is the coefficient of friction between the particle and the cylinder.

3. A uniform thin circular hoop of radius $a$ spinning in a vertical plane about its centre with angular velocity $\omega$ is gently placed on a rough plane of inclination $a$ equal to the angle of friction between the hoop and the plane so that the sense of rotation is that for which the slipping at the point of contact is down a line of greatest slope. Prove that the hoop will remain stationary for a time $a \omega / g \sin a$ before descending with acceleration $\frac{1}{2} g \sin a$. 
III. Kinematic condition of rolling. Formation of equations of motion.

A cylinder of radius $b$ rolls on a cylinder of radius $a$, which rolls on a horizontal plane.

Let $m$ and $m^{\prime}$ be the masses, $A$ and $B$ the centres, $V$ the horizontal velocity of $m, \Omega$ the angular velocity of $m, \theta$ the angle $A B$ makes with the vertical, $\omega$ the angular velocity of $m^{\prime}$.

The condition that $m$ rolls on the plane is $V=a \Omega$

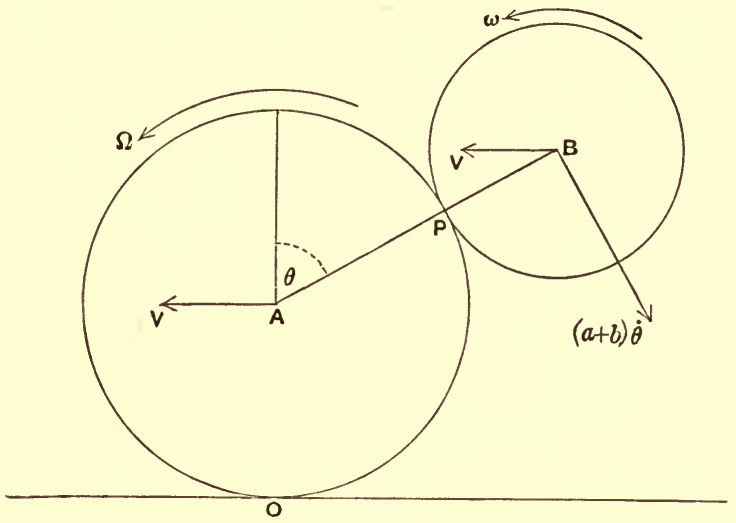

Fig. 60 .

The velocity of $B$ relative to $A$ is $(a+b) \dot{\theta}$ at right angles to $A B$, and its velocity is therefore compounded of this velocity and $V$ horizontally.

The velocity of $P$ (considered as a point of $m^{\prime}$ ) relative to $B$ is $b \omega$ at right angles to $A B$, in the sense of $(a+b) \dot{\theta}$.

The velocity of $P$ (considered as a point of $m$ ) relative to $A$ is $a \Omega$ at right angles to $A B$, but in the opposite sense.

The condition of rolling is that the particles of $m$ and $m^{\prime}$ that are at $P$ have the same velocity along the common tangent to the two circles.

We therefore have $\quad(a+b) \dot{\theta}+b \omega=-a \Omega$

In the diagram of accelerations (Fig. 61) we have introduced the value of $V$ from equation (1).

Since $B$ describes a circle relative to $A$ with angular velocity $\dot{\theta}$, the acceleration of $B$ relative to $A$ is compounded of $(a+b) \ddot{\theta}$ at right angles to $A B$, and $(a+b) \dot{\theta}^{2}$ in $B A$. This gives us the diagram.

Now, to form the equations of motion, take moments about $P$ for $m^{\prime}$, and about $O$ for the system. We have

and

$$
-m^{\prime} b(a+b) \ddot{\theta}+m^{\prime} a \dot{\Omega} b \cos \theta+m^{\prime} k^{\prime 2} \dot{\omega}=-m^{\prime} g b \sin \theta
$$

$m k^{2} \dot{\boldsymbol{\Omega}}+m a^{2} \dot{\boldsymbol{\Omega}}+m^{\prime} a \dot{\Omega}\{a+(a+b) \cos \theta\}+m^{\prime} k^{2} \dot{\boldsymbol{\omega}}$

$-m^{\prime}(a+b) \ddot{\theta}(a+b+a \cos \theta)+m^{\prime}(a+b) \dot{\theta}^{2} a \sin \theta=-m^{\prime} g(a+b) \sin \theta$ 
One of the quantities $\omega$ and $\Omega$ can be eliminated by means of equation (2), and there then remain two unknown quantities in terms of which the motion can be completely expressed by solving the equations (3) and (4).

Two first integrals of these equations can be obtained, one of them being the equation of energy.

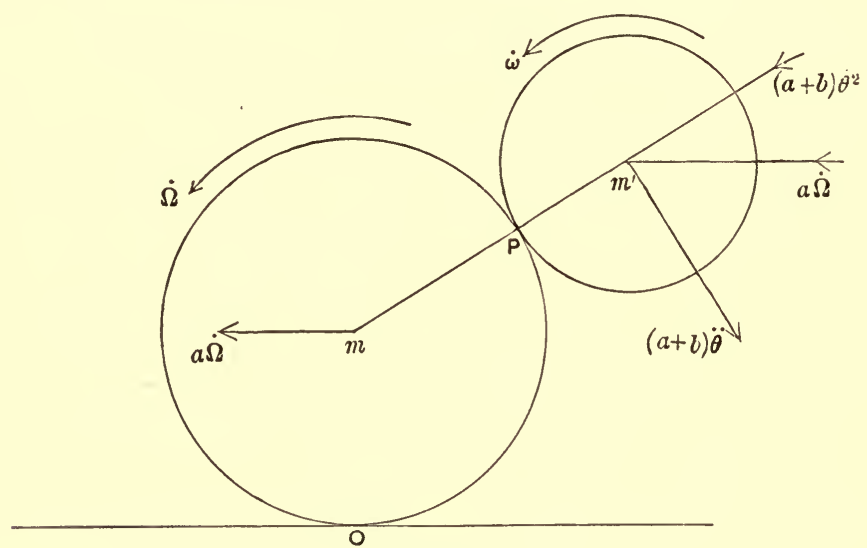

Fig. 61 .

\section{Examples.}

1. Prove that, in the problem just considered, there is an integral equation of the form

$$
m a \Omega\left(1+k^{2} / a^{2}\right)+m^{\prime}\left\{a \Omega-(a+b) \dot{\theta} \cos \theta-\omega k^{\prime 2} / b\right\}=\text { const. }
$$

and that $\dot{\theta}$ and $\theta$ are connected by an equation of the form

$\frac{1}{2}(a+b) \dot{\theta}^{2}\left[\left(1+k^{2} / b^{2}\right)-m\left(\cos \theta-k^{2} / b^{2}\right)^{2} /\left\{M\left(1+k^{2} / a^{2}\right)+m\left(1+k^{2} / b^{2}\right)\right\}\right]+g \cos \theta$ = const.

2. A uniform rod of length $l$ rests on a fixed horizontal cylinder of radius $a$ with its middle point at the top; prove that, if it is displaced in a vertical plane, so as to remain in contact with the cylinder, and if it rocks without slipping, the angle $\theta$ it makes with the horizontal at time $t$ is given by the equation $\quad \frac{1}{2}\left(\frac{1}{12} l^{2}+\alpha^{2} \theta^{2}\right) \dot{\theta}^{2}+g a(\cos \theta+\theta \sin \theta)=$ const., and the length of the simple equivalent pendulum for small oscillations is

$$
\frac{1}{12} l^{2} / \alpha \text {. }
$$

3. A homogeneous sphere rolls down a rough plane of inclination $a$ Prove that the acceleration of its centre is $\frac{5}{7} g \sin a$, and that the ratio of the friction to the pressure is $\frac{2}{7} \tan a$.

4. A thread unwinds from a reel of radius $\alpha$, the uppermost point of the thread being held fixed, the unwound part of the thread being vertical, and the axis of the reel being horizontal. Prove that the acceleration of the 
centre of the reel is $g \alpha^{2} /\left(\alpha^{2}+k^{2}\right)$, where $k$ is the radius of gyration of the reel about its axis, and that the tension of the thread is $\left\{1+k^{2} /\left(k^{2}+a^{2}\right)\right\}$ of the weight of the reel.

5. A thread passes over a smooth peg and unwinds itself from two cylindrical reels freely suspended from it and having their axes horizontal. Prove that each reel descends with uniform acceleration.

6. A ball is at rest in a cylindrical garden roller, when the roller is seized and made to roll uniformly on a level walk; to find the motion of the ball, supposing it does not slip on the roller.

Let $\alpha$ be the radius of the ball, $b$ of the roller, $\theta$ the angle the line of centres makes with the vertical, $V$ the velocity of the roller.

Prove (i) that the angular velocity of the roller is $V / b$,

(ii) that the angular

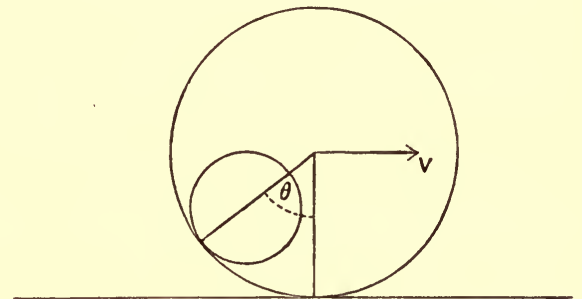

Fig. 62 . velocity $\omega$ of the ball is $V / a-(b-a) \dot{\theta} / a$.

Let $k$ be the radius of gyration of the ball, supposed uniform, about an axis through its centre of inertia, $m$ the mass of the ball. Initially all the impulsive forces acting on the ball pass through the point of contact, and therefore the moment of momentum of the ball about any axis through this point is zero initially. Hence obtain the equation

$$
m k^{2} \omega_{0}-m a\left\{(b-a) \dot{\theta}_{0}-V\right\}=0
$$

for the initial values $\omega_{0}$ of $\omega$ and $\dot{\theta}_{0}$ of $\dot{\theta}$; prove that $\omega_{0}$ vanishes, and find the value of $\dot{\theta}_{0}$.

Obtain the equations of motion

$$
\begin{aligned}
m k^{2} \dot{\omega}-m a(b-a) \ddot{\theta} & =m g \alpha \sin \theta, \\
m(b-a) \dot{\theta}^{2} & =R-m g \cos \theta,
\end{aligned}
$$

where $R$ is the pressure of the roller on the ball. Prove that the motion in $\theta$ is the same as that of a simple pendulum of length $\frac{7}{5}(b-a)$, and show that the value of $R$ in any position is

$$
m g\left(\frac{17}{7} \cos \theta-\frac{10}{7}\right)+m V^{2} /(b-a) .
$$

Deduce the condition that the ball may roll quite round the interior of the roller.

7. A cube containing a spherical cavity slides without friction down a plane of inclination $a$, and a homogeneous sphere rolls in the cavity. Prove that the angle $\theta$ between the normal to the plane and the radius through the point of contact of the sphere with the cavity is connected with the angular velocity $\omega$ of the sphere by the equation $(a-b) \dot{\theta}=b \omega$, where $a$ is the radius of the cavity, and $b$ is the radius of the sphere. 
Further taking $M$ and $m$ for the masses of the cube and sphere, and $x$ for the distance described by the cube in time $t$, obtain the equations of motion by resolving for the system down the plane and at right angles to it and taking moments for the sphere about its point of contact with the cavity.

Finally obtain the equation

$$
\frac{1}{2}\left\{\frac{7}{5}(M+m)-m \cos ^{2} \theta\right\} \dot{\theta}^{2}+(M+m) \cos a \sin \theta g /(a-b)=\text { const. }
$$

8. Prove that, when the plane of Example 7 is rough and $\epsilon$ is the angle of friction between it and the cube, the value of $\theta$ at time $t$ is given by the equation

$$
\begin{aligned}
& \frac{1}{2} \frac{d}{d \theta}\left[\left\{\frac{7}{5}(M+m) \cos \epsilon-m \cos \theta \cos (\theta-\epsilon)\right\} \dot{\theta}^{2}\right]-\frac{1}{2} m \dot{\theta}^{2} \sin \epsilon \\
& \quad+(M+m) \cos a \cos (\theta-\epsilon) g /(a-b)=0 .
\end{aligned}
$$

9. Motion of a circular disc rolling on a given curve under gravity.

Let $c$ be the radius of the disc, $\phi$ the angle the normal at the point of contact makes with the vertical, $\rho$ the radius of curvature of the curve at this point. The centre of the disc describes a curve

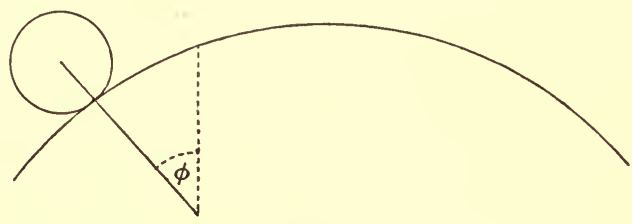

Fig. 63. parallel to the given curve and at a distance $c$ from it, and the instantaneous centre of rotation of the disc is at the point of contact, so that, if $\omega$ is the angular velocity of the disc, we have velocity of centre $=c \omega=(\rho+c) \dot{\phi}$.

Hence obtain the equation of energy

$$
\frac{1}{2}(\rho+c)^{2}\left(1+k^{2} / c^{2}\right) \dot{\phi}^{2}=g \int(\rho+c) \sin \phi d \phi,
$$

where $k$ is the radius of gyration of the disc about its centre, and the centre of inertia is the centre of the disc. Investigate the corresponding equation when the curve is concave to the disc.

Prove that the disc can roll inside a cycloid the radius of whose generating circle is $a$ and whose vertex is lowest with uniform angular velocity

$$
\frac{1}{2} \sqrt{ }\left\{g / a\left(1+k^{2} / c^{2}\right)\right\} \text {. }
$$

Prove that when the disc is uniform and rolls outside a cycloid, the radius of whose generating circle is $\frac{1}{4} c$ and whose vertex is highest, the motion is determined by the equation

$$
3 c \dot{\phi}^{2} \cos ^{4} \frac{1}{2} \phi=g(3+\cos \phi) \sin ^{2} \frac{1}{2} \phi,
$$

and that the disc leaves the cycloid when $\cos \phi=\frac{3}{5}$.

10. A uniform rod slides between a smooth vertical wall and a smooth horizontal plane. Assuming the rod to move in a vertical plane it is required to determine the motion. 
Let $A B$ be the rod, $2 \alpha$ its length, $m$ its mass, and suppose the end $A$ moves vertically in contact with the wall and the end $B$ horizontally in contact with the plane. The instantaneous centre $I$ is the intersection of the horizontal through $A$ and the vertical through $B$, and the figure OBIA is a rectangle, so that the centre of inertia $G$, which is the middle point of $A B$, is always at a distance $a$ from $O$.

The system of kinetic reactions is therefore equivalent to a resultant kinetic

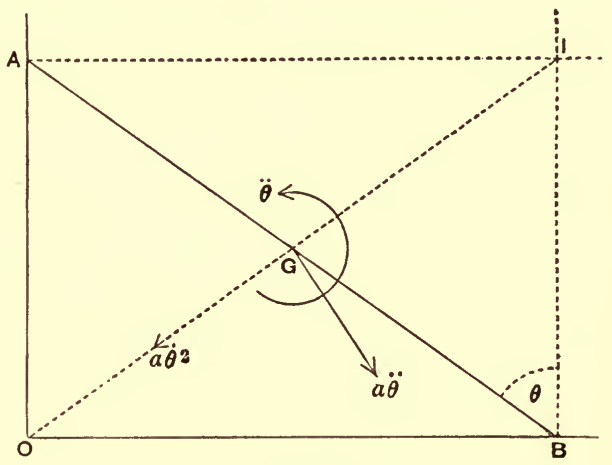

Fig. 64 . reaction at $G$ having components $m a \ddot{\theta}$ and $m a \dot{\theta}^{2}$ perpendicular to $O G$ and along $G O$, and a couple $m k^{2} \ddot{\theta}$ in the sense of increase of the angle $\theta$ which the $\operatorname{rod} B A$ makes with the vertical $B I$.

The forces acting on the rod are its weight at $G$, the horizontal pressure at $A$, and the vertical pressure at $B$. The lines of action of the two latter forces meet in $I$. If then we take moments about $I$ the unknown reactions do not enter.

Hence prove that the motion in $\theta$ is the same as that of a simple pendulum of length $\frac{4}{3} \alpha$.

By resolving horizontally and vertically find the pressures at $A$ and $B$, and show that the rod leaves the wall when $\cos \theta=\frac{2}{3} \cos a, a$ being the initial value of $\theta$.

11. When the plane and the wall of Example 10 are both rough, with the same angle of friction $\epsilon$, prove that the value of $\theta$ at time $t$ is given by the equation

$$
a\left(\frac{1}{3}+\cos 2 \epsilon\right) \ddot{\theta}-a \sin 2 \epsilon \dot{\theta}^{2}=g \sin (\theta-2 \epsilon) .
$$

12. A wheel whose centre of gravity is at its centre rolls down a rough plane of inclination $a$ dragging a particle of mass $m$ which slides on the plane and is connected with the centre of the wheel by a thread, so that the whole motion takes place in a vertical plane, and the thread makes an angle $\beta$ with the line of greatest slope down which the particle slides. Prove that the system descends with uniform acceleration

$$
\frac{M \sin a \cos (\beta-\epsilon)+m \cos \beta \sin (a-\epsilon)}{M\left(k^{2}+\alpha^{2}\right) \cos (\beta-\epsilon)+m a^{2} \cos \beta \cos \epsilon} g \alpha^{2},
$$

where $\alpha$ is the radius of the wheel, $M$ its mass, $k$ its radius of gyration about its axis, $m$ the mass of the particle and $\epsilon$ the angle of friction between it and the plane. 
13. Two smooth spheres are in contact and the lower slides on a horizontal plane. To find the motion.

Let $M, m$ be the masses, $a$ and $b$ the radii, $\theta$ the angle the line of centres

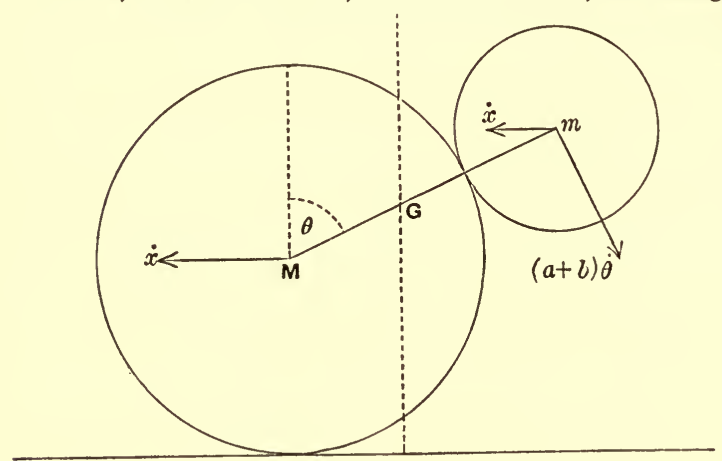

Fig. 65 . makes with the vertical at time $t$. Supposing the whole system to start from rest, the centre of inertia $G$ descends vertically, for there is no resultant horizontal force on the system. Further, since all the forces acting on either sphere pass through its centre, neither acquires any angular velocity. Let $x$ be the distance of the centre of the lower sphere $(M)$ from the vertical through the centre of inertia at time $t$, then the distance of $G$ from the centre of $M$ is $m(\alpha+b) /(M+m)$, and thus the horizontal velocity of $G$ is

and this vanishes.

$$
\dot{x}-\frac{m}{M+m}(a+b) \dot{\theta} \cos \theta
$$

Hence prove that the equation of energy can be put in the form

$$
\frac{1}{2}\left(1-\frac{m}{M+m} \cos ^{2} \theta\right) \dot{\theta}^{2}+\frac{g}{a+b} \cos \theta=\text { const. }
$$

Find the pressure between the spheres in any position, and prove that, if $\theta=a$ initially, the spheres separate when

$$
\cos \theta\left(3-\frac{m}{M+m} \cos ^{2} \theta\right)=2 \cos a .
$$

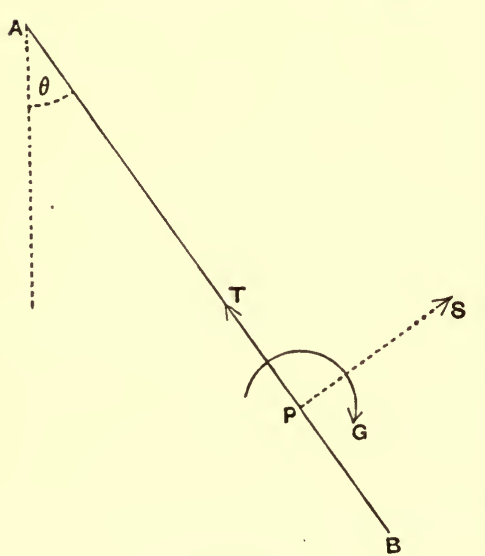

Fig. 66 .

IV. Stress in a rod. As an example of resultant stress across a section of a body wé consider the case of a rigid uniform rod swinging as a pendulum about one end.

If $m$ is the mass of the rod, $2 \alpha$ its length, $\theta$ the angle it makes with the vertical at time $t$, we have, since the radius of gyration about the centre of inertia is $a / \sqrt{ } 3$,

$$
\begin{array}{ll}
{ }_{3}^{4} a^{2} \ddot{\theta} & =-a g \sin \theta, \\
\text { and } \quad & { }_{3}^{2} a \dot{\theta}^{2}=g(\cos \theta-\cos a),
\end{array}
$$
where $a$ is the amplitude of the oscillations.

Now consider the action between the two parts of the rod exerted across 
a section distant $2 x$ from the free end. Let $P$ be the centroid of this section. We may suppose the action of $A P$ on $B P$ reduced to a force at $P$ and a couple, and we may resolve the force into a tension $T$ in the rod, and a shearing force $S$ at right angles to it. We call the couple $G$, and suppose the senses of $T, S$, and $G$ to be those shown in the figure. The action of $B P$ on $A P$ is then reducible to a force at $P$ having components $T, S$, and a couple $G$, in the opposite senses to those shown.

Now $B P$ is a rigid uniform rod of mass $m x / a$, turning with angular velocity $\dot{\theta}$, while its centre describes a circle of radius $2 \alpha-x$ with the same angular velocity. It moves in this way under the action of the forces $T$ and $S$, of its weight $m g x / a$ vertically downwards through its middle point, and of the couple $G$. By resolving along $A B$ and at right angles to it, and by taking moments about $P$, we obtain the equations of motion of $B P$ in the form

$$
\left.\begin{array}{l}
m \frac{x}{a}(2 a-x) \dot{\theta}^{2}=T-m g \frac{x}{a} \cos \theta, \\
m \frac{x}{a}(2 a-x) \ddot{\theta}=S-m g \frac{x}{a} \sin \theta, \\
m \frac{x}{a}\left\{x(2 a-x) \ddot{\theta}+\frac{x^{2}}{3} \ddot{\theta}\right\}=-G-m g \frac{x}{a} x \sin \theta,
\end{array}\right\}
$$

and by these equations $T, S$, and $G$ are completely determined. In particular the couple $G$ resisting bending is

$$
\frac{1}{2} m g \sin \theta \frac{x^{2}}{a^{2}}(a-x) \text {, or } \frac{1}{4} m g \sin \theta \frac{A P . B P^{2}}{A B^{2}} .
$$

\section{EXAMPLES.}

1. If any circle is drawn through the instantaneous centre of no acceleration, prove that the accelerations of all other points on this circle are directed to a common point.

2. A straight rod moves in any manner in its plane. Prove that at any instant the directions of motion of all its particles are tangents to a parabola.

3. A rope passes round a rough pulley which moves in any manner in its plane so that the rope remains tight. Prove that the directions of motion of all the points of the rope in contact with the pulley at any instant are tangents to a conic.

4. A uniform triangular disc $A B C$ is so supported that it can oscillate in its own plane (which is vertical) about the angle $A$. Prove that the length of the simple equivalent pendulum is

$$
\frac{1}{4}\left\{3\left(b^{2}+c^{2}\right)-a^{2}\right\} / \sqrt{ }\left\{2\left(b^{2}+c^{2}\right)-a^{2}\right\} .
$$

5. A uniform triangular disc $A B C$ is constrained to move in a vertical plane with its corners on a fixed circle. Prove that the motion is the same as that of a simple pendulum of length

$$
R(1-2 \cos A \cos B \cos C) / \sqrt{ }(1-8 \cos A \cos B \cos C),
$$

where $R$ is the radius of the circle circumscribing the triangle. 
6. Two circular rings, each of radius $\alpha$, are firmly joined together so that their planes contain an angle $2 \alpha$ and are placed on a rough horizontal plane. Prove that the length of the simple equivalent pendulum is

$$
\frac{1}{2} a \cos a \operatorname{cosec}^{2} a\left(1+3 \cos ^{2} a\right) \text {. }
$$

7. A thin uniform rod one end of which can turn about a smooth hinge is allowed to fall from a horizontal position. Prove that, when the horizontal component of the pressure on the hinge is a maximum, the vertical component is $\frac{11}{8}$ of the weight of the rod.

8. A uniform sphere of mass $M$ and radius $a$ oscillates under gravity about a fixed horizontal tangent as axis. Given the angular velocity $\omega$ of the sphere in the lowest position, find the pressure on the axis in any position, and prove that in a position of rest the resultant pressure will be perpendicular to the radius drawn to the axis if $\omega^{2}=\frac{10}{7} \mathrm{~g} / \alpha$.

9. A uniform rectangular block of mass $M$ stands on a railway truck with two faces perpendicular to the direction of motion, the lower edge of the front face being hinged to the floor of the truck. If the truck is suddenly stopped, find its previous velocity so that the block may just turn over. Prove that in this case the horizontal and vertical pressures on the hinge vanish when the angle the plane through the hinge and the centre of inertia of the block makes with the horizontal has the values $\sin ^{-1} \frac{2}{3}$ and $\sin ^{-1 \frac{1}{3}}$ respectively, and that the total pressure is a minimum, and equal to $\frac{1}{4} M g \sqrt{\frac{7}{11}}$, when the angle is $\sin ^{-1} \frac{20}{33}$.

10. The door of a railway carriage which has its hinges (supposed smooth) towards the engine stands open at right angles to the length of the train when the train starts with an acceleration $f$. Prove that the door closes in time $\sqrt{ }\left(\frac{\alpha^{2}+k^{2}}{2 a f}\right) \int_{0}^{\frac{1}{2} \pi} \frac{d \theta}{\sqrt{(\sin \theta)}}$, with an angular velocity $\sqrt{ }\left\{2 \alpha f /\left(\alpha^{2}+k^{2}\right)\right\}$, where $2 a$ is the breadth of the door, and $k$ the radius of gyration about a vertical axis through the centre of inertia.

11. Two bodies of masses $P$ and $Q$ are attached by ropes to a wheel and axle of mass $M$ and radius of gyration $k$ about its axis, the axle being supported in two rough sockets symmetrically placed which it just fits and touches along a horizontal line. Prove that when $Q$ has ascended a height $x$ its velocity $V$ is given by the equation

$$
\begin{array}{r}
V^{2}\left[P a^{2}(M+Q)+Q b^{2}\left(M+P^{\prime}\right)+Q a b\left(P+P^{\prime}\right)+M k^{2}\left(P^{\prime}+Q+M\right)\right] \\
=2 g b x\left(P-P^{\prime}\right)[M a+Q(a+b)],
\end{array}
$$

where $P^{\prime}$ is the mass of a body which substituted for $P$ would keep equilibrium.

12. A particle is placed on a rough plane lamina which is initially horizontal, and which is free to turn about a horizontal axis through its centre of inertia. Show that the particle will begin to slip when the plane has turned through an angle

$$
\tan ^{-1}\left\{\mu M a^{2} /\left(M a^{2}+9 m c^{2}\right)\right\}
$$

$\mu$ being the coefficient of friction, $2 a$ the length of the plane perpendicular to the axis, $c$ the distance of the particle from that axis, and $M, m$ the masses of the lamina and the particle. 
13. A uniform sphere is placed on the highest generator of a rough cylinder which is fixed with its axis horizontal. Prove that, if slightly displaced, the sphere will roll on the cylinder until the plane through the centre of the sphere and the axis of the cylinder makes with the vertical an angle $a$ satisfying the equation

$$
17 \mu \cos a-2 \sin a=10 \mu
$$

where $\mu$ is the coefficient of friction.

14. A system consisting of a rough uniform circular wire of mass $M$, and a straight uniform rod of mass $m$, whose ends can slide on the wire, moves in one plane under no forces, the rod subtending an angle $2 a$ at the centre of the wire. Prove that if neither of the expressions

$$
(M+m) \sin ^{2} a+3 M \cos ^{2} a \pm \mu \sin a \cos a(m-3 M)
$$

is negative ( $\mu$ being the coefficient of friction), and, if initially the rod has an angular velocity $\Omega$ about the centre while the wire is at rest, the rod will come to rest relatively to the wire after a time

$$
\frac{(M+m)\left[(M+m) \sin ^{2} a+3 M \cos ^{2} \alpha+\mu^{2} m \sin ^{2} a \sim 2 \mu^{2} M \sin ^{2} a\right]}{\mu m \Omega\left[(M+m) \sin ^{2} a+3 M \cos ^{2} \alpha\right]} .
$$

15. A flat circular disc of radius $\alpha$ is projected on a rough horizontal table which is such that the friction on an element $a$ is $c V^{3} m a$, where $V$ is the velocity of the element and $m$ the mass of a unit of area. Prove that, if $u_{0}$ and $\omega_{0}$ are the initial velocity of the centre of inertia and angular velocity of the disc, the corresponding velocities $u, \omega$ at any subsequent time satisfy the equation

$$
\left(3 u^{2}-\alpha^{2} \omega^{2}\right)^{2} /\left(3 u_{0}^{2}-\alpha^{2} \omega_{0}^{2}\right)^{2}=\left(u^{2} \omega\right) /\left(u_{0}^{2} \omega_{0}\right) .
$$

16. A uniform circular ring moves on a rough curve under no forces, the curvature of the curve being everywhere less than that of the ring. The ring is projected from a point $A$ of the curve, and begins to roll at a point $B$. Prove that the angle between the normals at $A$ and $B$ is $\mu^{-1} \log 2$, where $\mu$ is the coefficient of friction.

17. A locomotive engine of mass $M$ has two pairs of wheels of radius $a$ such that the moment of inertia of either pair with its axle about its axis of rotation is $A$. The engine exerts a couple $G$ on the forward axle. Prove that, if both pairs of wheels bite at once when the engine starts, the friction between one of the forward wheels and the line capable of being called into play must not be less than $\frac{1}{2} G\left(A+M \alpha^{2}\right) / \alpha\left(2 A+M \alpha^{2}\right)$. Prove also that, if the only action between an axle and its bearings is a couple varying as the angular velocity of the axle, the final friction called into play between either forward wheel and the line is $G / 4 \alpha$.

18. A homogeneous solid hemisphere of mass $M$ and radius $a$ with a smooth base, is placed with its vertex lowest on a rough horizontal plane, and a particle of mass $m$ is placed on the base at a distance $c$ from the centre. Prove that the hemisphere begins to roll or slide on the plane according as

$$
\mu>\text { or }<25 \mathrm{mac} /\left\{26(M+m) a^{2}+40 m c^{2}\right\},
$$

where $\mu$ is the coefficient of friction between the hemisphere and the plane. 
19. A homogeneous sphere of radius $\alpha$ is initially at rest on a horizontal plane and the plane is made to move backwards and forwards horizontally so that its displacement at time $t$ is $b \cos n t$. Prove that, if $\mu$ the coefficient of friction $<\frac{2}{7} b n^{2} / g$, the changes from rolling to sliding take place at times $(r \pi-a) / n$, where $r$ is a positive integer and $a$ is the least positive root of the equation $\cos a=7 \mu g / 2 b n^{2}$; prove also that the changes from sliding to rolling (except the first) take place at times $(r \pi+\gamma) / n$, where $\gamma$ is the least positive root of the equation

$$
\sin \gamma+\sin a=7 \mu g(\gamma+a) / 2 b n^{2} .
$$

20. A uniform sphere of mass $M$ rests on a rough plank of mass $M^{\prime}$ fyhich is on a rough horizontal plane ; the plank is suddenly set in motion along its length with velocity $V$. Prove that the sphere will first slide and then roll on the plank and that the whole system will come to rest after a time $M^{\prime} V / \mu g\left(M+M^{\prime}\right)$ from the beginning of the motion, where $\mu$ is the coefficient of friction at each of the places of contact.

21. On a smooth table rests a board of mass $M$, having its upper surface rough and the lower smooth. A sphere of mass $m$ is projected on the upper surface of the board so that the direction of projection passes through the centre of inertia of the board; the velocity of projection is $V$ and the sphere has an angular velocity $\Omega$ about a horizontal axis perpendicular to the plane of projection. Prove that after a time

$$
(V-a \Omega) / \mu g\left(1+\frac{a^{2}}{k^{2}}+\frac{m}{M}\right)
$$

the motion will become uniform, and that the velocity of the board will then be

$$
\frac{m}{M}(V-a \Omega) /\left(1+\frac{a^{2}}{k^{2}}+\frac{m}{M}\right) .
$$

22. A reel of mass $M$ and radius $\alpha$ rests on a rough floor, $\mu$ being the coefficient of friction. Fine thread is coiled on the reel so as to lie on a cylinder of radius $b(<a)$ and coaxal with the reel. The free end of the thread is carried in a vertical line over a smooth peg at a height $h$ above the centre of the reel and supports a body of mass $m$. Prove that if either

$$
\mu<m b /(M-m) a \text { or if } M<m\left[1-b^{2}\left(1+a / h-a^{2} / b h\right) /\left(a^{2}+k^{2}\right)\right],
$$

the thread will be unwound from the reel.

23. A garden roller, in which the mass of the handle may be neglected, is pulled with a force $P$ in a direction making an angle $a$ with the horizontal plane on which it rests. Show that it will not roll unless

$$
P\left\{\sin a \sin \phi+\cos a \cos \phi k^{2} /\left(a^{2}+k^{2}\right)\right\} \leqslant W \sin \phi,
$$

where $a, k, W$ are the radius, the radius of gyration about the axis, and the weight of the roller, and $\phi$ is the angle of friction between it and the ground.

24. Two rough cylinders of radii $r_{1}, r_{2}$ are put on a rough table and on them is placed a rough plank. Prove that, under certain conditions, the 
system can start from rest and move so that each cylinder rolls on the table with the constant acceleration

$$
M g \sin 2 a(1+\cos 2 a) /\left\{m_{1}\left(1+k_{1}^{2} / r_{1}^{2}\right)+m_{2}\left(1+k_{2}{ }^{2} / r_{2}{ }^{2}\right)+4 M \sin ^{2} 2 a\right\},
$$

where $\sin a=\left(r_{1} \sim r_{2}\right) / d$, and $d$ is the initial distance between the axes of the cylinders.

25. A circular cylinder of radius $a$ whose centre of inertia is at a distance $b$ from its axis rolls on a horizontal plane. Prove that if it oscillates its angular motion is given by an equation of the form

$$
\frac{1}{2} \dot{\theta}^{2}\left(k^{2}+a^{2}+b^{2}-2 a b \cos \theta\right)=g b(\cos \theta-\cos a) .
$$

26. A curve is drawn on the convex side of a hypocycloid parallel to it, the distance between the curves being $a$, and a circular disc of radius $a$ rolls on the concave side under the action of a force to the centre varying as the distance. Prove that the motion is isochronous for oscillations of all amplitudes.

27. On the top of a fixed smooth sphere rests a fine uniform ring with its centre in the vertical diameter, and its diameter subtends an angle $2 a$ at the centre of the sphere. Prove that, if the ring is slightly displaced, it will first begin to leave the sphere when its plane has turned through an angle $\theta$ given by the equation

$$
\sin (\theta+a) \sin a=2 \cos ^{2} a(2-3 \cos \theta) .
$$

28. A uniform rod lying at rest in a smooth sphere is of such length that it subtends a right angle at the centre. The rod is projected so that its ends remain on the sphere and make complete revolutions in a vertical plane. Prove that, if $V$ is the initial velocity of the centre, and $a$ the radius of the sphere,

$$
V^{2}>g a\left(\frac{3}{4} \sqrt{ } 2+\frac{1}{8} \sqrt{202}\right) .
$$

29. Two uniform rods of equal length $(a \sqrt{ } 2)$ and of equal mass are firmly fixed at one extremity of each and are at right angles. The rods are placed over two smooth pegs, distant, $c$ apart, in a horizontal line and move in the vertical plane through the pegs. Prove that the angular motion of the right angle on the semicircle it describes is given by one of the equations

$$
\dot{\phi}^{2}\left(\frac{2}{3} \alpha^{2}-a c \cos \frac{1}{2} \phi+c^{2}\right) \pm 4 g\left(a \cos \frac{1}{2} \phi-c \cos \phi\right)=\text { const. }
$$

and if the motion is a small oscillation the length of the simple equivalent pendulum is

$$
\frac{2}{3}\left(2 a^{2}+3 c^{2} \sim 3 a c\right)(4 c \sim a) .
$$

30. Two equal uniform rods of mass $m$ and length $2 a$ are free to turn about their middle points which are fixed at a distance $2 a$ apart in a horizontal line. The rods being horizontal a uniform sphere of mass $M$ and radius $c$ is gently placed upon them at the point where their ends meet. Prove that, if $9 M\left\{a^{2}+c^{2}\right\}^{2}=2 m\left\{a^{2}-c^{2}\right\}^{2}$, the sphere will, as it leaves the rods, have half the velocity which it would have had after falling freely through the same height. 
31. An elastic thread of modulus $\lambda$ is wound round the smooth rim of a homogeneous circular disc of mass $m$, one end being fastened to the rim, and the other to the top of a smooth fixed plane of inclination $a$ to the horizontal, down which the disc moves in a vertical plane through a line of greatest slope, which is the line of contact of the straight portion of the thread with the plane. Initially the thread has its natural length $l$ and is entirely wound on the rim of the disc which is at rest at the top. Prove that at any time $t$ before the thread is entirely unwound the tension is

$$
\frac{2}{3} m g \sin a \sin ^{2}\left\{\frac{1}{2} t \sqrt{ }(3 \lambda / l m)\right\} \text {. }
$$

32. Two equal cylinders of mass $m$, bound together by a light elastic band of tension $T$, roll with their axes horizontal down a rough plane of inclination $a$. Show that their acceleration down the plane is

$$
\frac{2}{3} g \sin a\left(1-\frac{2 \mu T}{m g \sin a}\right),
$$

$\mu$ being the coefficient of friction between the cylinders.

33. A waggon runs down a road inclined at an angle $a$ to the horizon, and the road is crushed uniformly by the wheels, prove that the acceleration is

$$
\frac{(M+2 m) \sin (a-\beta)}{(M+2 m) \cos \beta+2 m k^{2} / \alpha^{2}} g,
$$

the centre of inertia being midway between the wheels, $M$ denoting the mass of the body of the waggon, $m$ the mass, $m k^{2}$ the moment of inertia, and $\alpha$ the radius of each pair of wheels, and $\beta$ being an angle depending on the nature of the road.

34. A $\operatorname{rod} A B$, whose density varies in any manner, is swung as a pendulum about a horizontal axis through $A$. Prove that the couple resisting bending is greatest at a point $P$ determined by the condition that the centre of inertia of the part $P B$ is the centre of oscillation of the pendulum.

35. A semicircular wire $A C B$ whose line density varies as the distance from the diameter $A B$ rotates in its plane, which is vertical, with uniform angular velocity $\omega$ about the fixed point $A$. Prove that the stress couple at the middle point $C$ of the $\operatorname{arc} A B$ vanishes when $A B$ is vertical if

$$
\omega=\sqrt{ }\{(4-\pi) g /(6-\pi) a\} \text {. }
$$

36. A uniform rod of mass $m$ has one extremity fastened by a pivot to the centre of a uniform circular disc of mass $M$ which rolls on a horizontal plane, the other extremity being in contact with a smooth vertical wall at right angles to the plane containing the disc and the rod. Prove that the inclination $\theta$ of the rod to the vertical when it leaves the wall is given by the equation

$$
9 M \cos ^{3} \theta+6 m \cos \theta-4 m \cos a=0,
$$

the system starting from rest in a position in which $\theta=a$. 
37. A homogeneous sphere of mass $M$ and radius $a$ rests on a horizontal plane in contact with a vertical wall, and a second homogeneous sphere of mass $m$ and radius $b(<\alpha)$ is placed in contact with it and the wall, the centres being in a vertical plane at right angles to the wall. Prove that, if all the surfaces are smooth, the spheres will separate when the line joining their centres makes with the horizontal an angle $\theta$ given by the equation

$$
(a+b)\left\{(M-m) \sin ^{3} \theta+3 m \sin \theta\right\}=4 m \sqrt{ }(a b) .
$$

38. A smooth circular cylinder of mass $M$ and radius $c$ is at rest on a smooth horizontal plane, and a heavy straight rail of mass $m$ and length $2 a$ is placed so as to rest with its length in contact with the cylinder and to have one extremity on the ground. Prove that the inclination of the rail to the vertical in the ensuing motion (supposed to be in a vertical plane) is given by the equation

$$
\frac{1}{2} \dot{\theta}^{2}\left[\left(\frac{1}{3}+\sin ^{2} \theta\right) a^{2}+\frac{M}{M+m}\left(\frac{c}{1-\sin \theta}-a \cos \theta\right)^{2}\right]=g a(\cos a-\cos \theta),
$$

where $a$ is the initial value of $\theta$.

39. The outer surface of a uniform spherical shell of mass $M$ is rough and of radius $a$, and the inner (concentric) surface is smooth and of radius $b$. A particle of mass $m$ moves inside the shell while the shell rolls on a horizontal plane. Show that the angular distance $\theta$ of the particle from the vertical diameter at time $t$ is given by the equation

$$
\frac{1}{2}\left(\frac{7}{5} M+m \sin ^{2} \theta\right) \dot{\theta}^{2}=\left(\frac{7}{5} M+m\right)(\cos \theta-\cos a)(g \mid b),
$$

where $a$ is the greatest value of $\theta$.

40. A circular cylinder of radius $a$ and radius of gyration $k$ rolls inside a fixed horizontal cylinder of radius $b$. Prove that the plane through the axes moves like a simple pendulum of length

$$
(b-a)\left(1+k^{2} / a^{2}\right) \text {. }
$$

When the second cylinder can turn about its axis, and when the first cylinder is of mass $m$ and the moment of inertia of the second about its axis is $M K^{2}$, prove that the length of the equivalent simple pendulum is $(b-a)(1+n) / n$, where $n=a^{2} / k^{2}+m b^{2} / M K^{2}$; prove also that the pressure between the cylinders is proportional to the depth of the point of contact below a plane which is at a depth $2 n b \cos a /(1+3 n)$ below the fixed axis, where $2 a$ is the angle of oscillation.

41. A perfectly rough inelastic sphere is dropped on the lowest generating line of a horizontal circular cylinder which is revolving freely about its axis, which is fixed, with angular velocity $\Omega$. Prove that the plane through the axis of the cylinder and the point of contact will move like a simple pendulum of length

$$
(a-c)\left\{\frac{M K^{2}\left(k^{2}+c^{2}\right)+m k^{2} a^{2}}{M K^{2} c^{2}+m k^{2} a^{2}}\right\}
$$

where $a$ and $c$ are the radii, $K$ and $k$ the radii of gyration, $M$ and $m$ the 
masses of the cylinder and sphere respectively. Prove also that the angular velocity of the pendulum at the lowest point is

$$
\frac{a}{a-c} \frac{\Omega}{1+\frac{c^{2}}{k^{2}}+\frac{m a^{2}}{M K^{2}}} .
$$

42. A garden roller stands at rest on a level path with the handle vertical; the handle is pulled down into a horizontal position held at rest and then released. Prove that the angular motion of the handle about the axis of the roller is given by the equation

$$
\frac{1}{2} \dot{\phi}^{2}\left[l-\frac{h \cos ^{2} \phi}{1+(M / m)\left(1+K^{2} / R^{2}\right)}\right]=g \cos \phi,
$$

where $R$ is the radius of the roller, $K$ its radius of gyration about its axis, $M$ its mass, and $m$ is the mass of the handle, $h$ the distance of its centre of gravity from the axis, and $l$ the length of the simple equivalent pendulum of the handle when the roller is held fixed.

43. A uniform circular hoop of radius $a$ is so constrained that it can only move by rolling in a horizontal plane on a fixed horizontal line, and a particle whose mass is $1 / \lambda$ of that of the hoop can slide on the hoop without friction. Prove that, if initially the hoop is at rest, and the particle is projected along it from the point furthest from the fixed line with velocity $v$, then the angle turned through by the hoop in time $t$ will be

$$
(v t / a-\sin \psi) /(2 \lambda+1)
$$

where $\psi$ is the angle the diameter through the particle has turned through in the same interval. Prove also that

$$
v t \sqrt{ }(2 \lambda)=a \int_{0}^{\psi} \sqrt{ }\left(2 \lambda+\sin ^{2} \theta\right) d \theta .
$$

44. A uniform rod swings in a vertical plane hanging by two cords attached to its ends and to points $A, B$ in a horizontal line, $A B$ being equal to the length of the rod, and the cords not being crossed. Prove that, if the cords attached to $A$ and $B$ are of lengths $a$ and $a+\lambda$ respectively, where $\lambda$ is small, the angular velocity of the cord attached to $A$ when inclined to the vertical at an angle $\theta$ is greater than it would be if $\lambda$ were zero by

$$
\lambda \sqrt{ }\left(g / 2 a^{3}\right)(\cos \theta-\cos a)^{\frac{1}{2}}\left(\tan ^{2} \theta-\frac{1}{2} \sec \theta \sec a\right)
$$

approximately, $a$ being the value of $\theta$ in a position of rest and not being nearly equal to a right angle.

45. A uniform rod which is free to turn about a point fixed in it touches, at a distance $c$ from the fixed point, the rough edge of a disc of mass $m$, radius $a$, and radius of gyration $k$ about its centre. The system being at rest on a smooth horizontal plane, an angular velocity $\Omega$ is suddenly communicated to the rod so that the disc also is set in motion. Prove that in the subsequent motion the distance $r$ of the point of contact from the fixed point satisfies the equation

$$
\left(M K^{2}+m r^{2}\right)\left(1+k^{2} / a^{2}\right) \dot{r}^{2}=\left(M K^{2}+m c^{2}\right)\left(k^{2}+a^{2}+r^{2}-c^{2}\right) \Omega^{2},
$$

where $M K^{2}$ is the moment of inertia of the rod about the fixed point and the edge is rough enough to prevent slipping. 
46. A uniform rod has its lower end on a smooth table and is released from rest in any position. Show that the velocity of its centre on arriving at the table is $\sqrt{ }\left(\frac{3}{2} g h\right)$, where $h$ is the height through which the centre has fallen, and that at the instant when the centre reaches the table the pressure on the table is one quarter of the weight of the rod.

47. If a particle is moving in a circular tube held at rest on a smooth horizontal plane, and the tube is let go, the centre of the tube will describe a trochoid.

48. A uniform sphere of mass $m$ is rolling on the horizontal upper surface of a wedge of mass $M$ whose under surface slides without friction on a fixed plane inclined at an angle $a$ to the horizontal. Assuming the system to move from rest, and the whole motion to be in a vertical plane, prove that, if at time $t$ the wedge has slipped a length $x$ along the plane, and the sphere has rolled a length $s$ along the surface of the wedge, then

$$
x=\frac{7}{5} s \sec a=\frac{7}{2} \frac{(M+m) \sin a}{7 M+\left(7-5 \cos ^{2} a\right) m} g t^{2} .
$$

49. A wheel can turn freely about a horizontal axis, and a fly of mass $m$ is at rest at the lowest point; if the fly suddenly starts off to walk along the rim of the wheel with constant velocity $V$ relative to the rim, show that he cannot ever get to the highest point of the rim unless $V$ is at least as great as

$$
2 \sqrt{ }\left\{g a\left(m \alpha^{2} / M K^{2}\right)\left(1+m \alpha^{2} / M K^{2}\right)\right\},
$$

where $a$ is the radius of the wheel, and $M K^{2}$ its noment of inertia about its axis.

50. A hollow thin cylinder of radius $\alpha$ and mass $M$ is maintained at rest in a horizontal position on a rough plane of inclination $a$, and an insect of mass $m$ is at rest in the cylinder on the line of contact with the plane. The insect starts to crawl up the cylinder with velocity $V$, and the cylinder is released at the same instant. Prove that if the relative velocity is maintained and the cylinder rolls uphill, then it will come to instantaneous rest when the angle the radius through the insect makes with the vertical is given by the equation

$$
V^{2}\{1-\cos (\theta-a)\}+\alpha g(\cos a-\cos \theta)=(1+M / m) \alpha g(\theta-a) \sin a .
$$

51. A rigid square $A B C D$ of four uniform rods each of length $2 a$ lies on a smooth horizontal table and can turn freely about one angular point $A$ which is fixed. An insect whose mass is equal to that of either rod starts from the corner $B$ to crawl along the $\operatorname{rod} B C$ with uniform velocity $V$ relative to the rod. Prove that in any time $t$ before the insect reaches $C$ the angle turned through by the square is

$$
\sqrt{\frac{3}{13}} \tan ^{-1}\left(\sqrt{\frac{3}{52}} \frac{I^{\prime} t}{a}\right)
$$




\section{CHAPTER XII.}

\section{MISCELLANEOUS METHODS AND APPLICATIONS.}

226. We propose in this Chapter to bring together a number of methods and theories relating to general classes of problems which can be solved by the principles laid down in previous Chapters. One of the great difficulties of our subject is the integration of the differential equations of motion of a system of bodies, but there are a number of cases in which all the information desired can be obtained without any integration. Such cases include impulsive motions, and initial motions. There are other cases in which the method of integration is known. Such cases include small oscillations, and problems in which the principles of energy and momentum supply all the first integrals of the equations of motion. We shall consider such cases here. Another general problem on which much light can be thrown by the theory of momentum is presented in the motion of a string or chain, and this problem at the same time forms an introduction to the dynamics of systems capable of continuous deformation. We shall devote some space to it at the end of the Chapter.

\section{IMPULSES.}

\section{Nature of impulsive action between bodies in} contact. When two bodies collide, at first their surfaces come into contact at a point of each, but a little observation shows that, before separation, they must be in contact over a finite area; for example, if one body is smeared over with soot, the other, after separation, will show a sooty patch. It is clear therefore that during the impact the bodies undergo deformation. There are numberless cases in which the deformation is permanent, there 
are others in which the recovery of form is practically complete. Now it is clear that if the bodies are rigid no deformation can take place, and accordingly we shall be unable to give an account of the circumstances if we treat the bodies as rigid. On the other hand, the problem of calculating the deformation from the elastic properties of the bodies is generally beyond our power. Further, we shall find that one inevitable result of every impulsive action between parts of a system is a loss of kinetic energy in the system, and this apparent loss of energy can frequently be calculated. Considering the bodies as elastic we might expect to find the energy transformed partly into potential energy of strain, and partly into kinetic energy of relative motions of the parts of the bodies. Now there is one particular case in which the calculation can be made by means of the Theory of Elasticity, the case of the longitudinal impact of thin elastic bars, and in that case it is found that the apparent loss of energy as calculated depends on the masses of the bars in a way quite different to that which is experimentally found to hold good. It appears to follow that, even if we could in every case calculate from the Theory of Elasticity the deformations of impinging bodies, we should nevertheless not be in a position to give a complete account of the nature of the action that takes place in impact, and we are led to expect, in impulsive changes of motion, a transformation of energy into some other form than kinetic and potential energies of the bodies and their parts, regarded as continuous. Nor have we far to seek for the form of energy that is developed in compensation for the apparent loss. It is a fact of observation that when one body strikes against another the temperature of both is raised, and it has been abundantly proved that the production of thermal effects of this kind is of the nature of just such a transformation of energy as we have described. We must therefore expect that in impulsive changes of motion some mechanical energy will be transformed into heat. In order to formulate in a simple and general manner the mechanical effects produced in two bodies by collision it is necessary to have recourse to special experiments and subsidiary hypotheses.

228. Newton's experimental Investigation. Newton made an elaborate series of experiments* on the impact of

* Principia, Lib. 1., Axiomata sive Leges Motus.

L. 
spheres. The experiments were conducted with the aid of the ballistic balance (Article 93), and the spheres came into contact when moving in the line joining their centres. He found that the relative velocity of the spheres after impact was oppositely directed to that before impact, and that its amount was diminished in a ratio depending only on the materials.

To express this result, let $u$ and $u^{\prime}$ be the velocities of the two bodies in the line of centres and in the same sense before impact, $v$ and $v^{\prime}$ their velocities in the same line and in the same sense after impact, then

$$
v-v^{\prime}=-e\left(u-u^{\prime}\right)
$$

where $e$ is a positive fractional coefficient depending on the materials of the two bodies, and independent of their masses and velocities.

If $m$ and $m^{\prime}$ are the masses of the bodies, we have by the Principle of the Conservation of Linear Momentum (Article 111)

$$
m v+m^{\prime} v^{\prime}=m u+m^{\prime} u^{\prime} .
$$

Equations (1) and (2) determine the velocities after impact and we find

$$
\begin{aligned}
v & =\frac{\left(m-e m^{\prime}\right) u+m^{\prime}(1+e) u^{\prime}}{m+m^{\prime}}, \\
v^{\prime} & =\frac{\left(m^{\prime}-e m\right) u^{\prime}+m(1+e) u}{m+m^{\prime}} .
\end{aligned}
$$

The impulsive pressure exerted by $m^{\prime}$ on $m$ is $m(u-v)$, in the sense opposite to that of $u$, and this is

$$
(1+e) m m^{\prime}\left(u-u^{\prime}\right) /\left(m+m^{\prime}\right) \text {. }
$$

The hypothesis adopted by Poisson and others as to the action between two bodies in collision is founded on the result last obtained. Poisson imagined that when the bodies come into contact they begin to be compressed, that the compression continues to increase until the end of a certain interval, called the "period of compression," and that at the end of this interval the two bodies have the same velocity along the common normal to the surfaces in contact. Further, he imagined that after the period of compression the bodies begin to recover their form and continue to do so until they cease to be in contact. The interval in which the recovery takes place is called the "period of restitution." Poisson supposed that the pressure 
between the bodies at any instant during restitution is less than that at the corresponding instant during compression in such a way that the impulse of the pressure exerted during restitution is the product of $e$ and the impulse of the pressure exerted during compression. On this hypothesis the impulse of the pressure exerted between the two bodies while in contact is greater than it would be if $e$ were zero in the ratio $1+e: 1$. Poisson* supposed this hypothetical account of the motion to apply equally whether the impact is direct or not, and whether the bodies are smooth or rough provided they are not sufficiently rough to prevent sliding.

229. Coefficient of restitution. The number $e$ is called the "coefficient of restitution." For very hard elastic solids, such as glass and ivory, $e$ is little different from unity; for very soft materials, such as wool or putty, it approaches zero. The connexion between $e$ and the elasticity of the impinging bodies has led to its being sometimes called the "coefficient of elasticity," but we avoid this phrase because it has a different and very definite meaning in the Theory of Elasticity. For a like reason we avoid the phrase "coefficient of resilience" which has also been sometimes used. Materials for which $e$ is zero or unity may be regarded as ideal limits to which some bodies approach. We shall speak of such materials as being "without restitution" and "of perfect restitution" respectively, ordinary materials we shall speak of as having "imperfect restitution." It is, of course, to be understood that any such phrase refers to an action between two bodies of the same or different materials. The coefficient $e$ depends on both the materials just as the coefficient of friction between two bodies depends on the materials and degree of polish of both.

\section{Oblique impact} of smooth spheres. Let two smooth uniform spheres, of masses $m, m^{\prime}$, impinge.

Let $U, V$ be the resolved velocities of $m$ in the line of centres and at right angles thereto before impact, $U^{\prime}, V^{\prime}$ corresponding velocities of $m^{\prime}$, and let $u, v$ and $u^{\prime}, v^{\prime}$ be

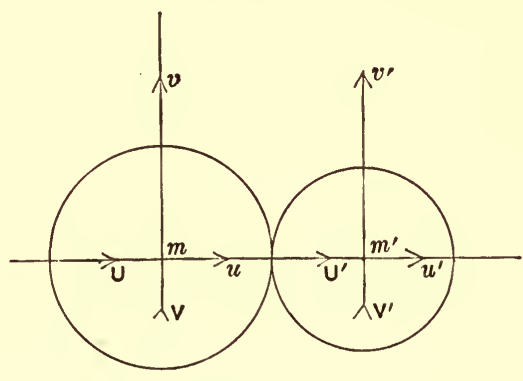

Fig. 67.

* Traité de Mécanique, t. II., pp. 273 et seq. Second Edition, Paris 1833. 
corresponding velocities for $m$ and $m^{\prime}$ after impact. Let $u_{0}, v_{0}$ and $u_{0}^{\prime}, v_{0}^{\prime}$ be the velocities that would replace $u, v$ and $u^{\prime}, v^{\prime}$ if $e$ were zero.

Now if $e$ were zero there would be no restitution or the bodies would have the same velocities in the line of centres immediately after the impulse, we should therefore have

$$
u_{0}^{\prime}=u_{0} \text {. }
$$

The equation of conservation of linear momentum of the system in the line of centres is in this case

$$
\left(m+m^{\prime}\right) u_{0}=m U+m^{\prime} U^{\prime},
$$

and the impulsive pressure, $R_{0}$, between the bodies is given by the equation

$$
\begin{aligned}
R_{0} & =m\left(U-u_{0}\right) \\
& =\frac{m m^{\prime}}{m+m^{\prime}}\left(U-U^{\prime}\right),
\end{aligned}
$$

the sense of $R_{0}$ being opposite to that of $U$.

Now suppose that $e$ is not zero, but the impulsive pressure between the bodies is $R_{0}(1+e)$. We have the equations

$$
\left.\begin{array}{l}
m u+m^{\prime} u^{\prime}=m U+m^{\prime} U^{\prime}, \\
m(U-u)=\frac{m m^{\prime}}{m+m^{\prime}}(1+e)\left(U-U^{\prime}\right),
\end{array}\right\}
$$

which may be written

$$
\frac{U-u}{m^{\prime}}=\frac{U^{\prime}-u^{\prime}}{-m}=\frac{U-U^{\prime}}{m+m^{\prime}}(1+e)
$$

whence

$$
u-u^{\prime}=-e\left(U-U^{\prime}\right)
$$

exactly as in direct impact.

Thus, at any rate for the case of smooth spheres, Poisson's hypothesis is equivalent to the following generalisation of Newton's experimental result :-

The relative velocities, after and before impact, of the points of two impinging bodies that come into contact, resolved along the common normal to their surfaces at these points, are in the ratio $-e: 1$, where $e$ is the coefficient of restitution.

In what follows we shall refer to the statement just made as 
the "generalised Newton's rule," and we shall apply it instead of Poisson's hypothesis. We shall show later on that its equivalence with this hypothesis is not limited to the cases of smooth bodies or of spheres.

231. Elastic systems. The method followed in applying this rule is to treat the impact as instantaneous, and the impinging bodies as rigid both before and after it. This method is adequate for the discussion of many questions. It cannot however be applied to the transmission of stress in elastic systems capable of large deformations. In such systems no internal stress is developed except after a finite deformation has taken place, so that at the beginning of a motion impulsively produced some part of the system yields at once, and starts to move with a finite velocity; after a finite time a finite strain is produced, and is opposed by a finite elastic stress, this stress continuing as long as there is any strain. This statement may conveniently be summed up in the proposition:-An elastic system cannot support an impulse. It is now clear that the method founded on Newton's result is of the nature of a compromise, the whole time of the action in which the elasticity of the bodies is concerned being treated as infinitesimal. An example of the statement that an elastic system cannot support an impulse will be found in the action of elastic strings attached to rigid bodies whose motion is altered suddenly. There is no impulsive tension in such a string, and the motion of the body immediately after the impulse is exactly the same as if the string were not attached to it (cf. Article 258). On the other hand, an inelastic string is conceived as capable of supporting an impulsive tension.

232. Impact of smooth spheres. We return to the problem presented by the collision of two smooth uniform spheres, and we shall show how to apply the generalised Newton's rule and the equations of momentum to determine the whole motion, and shall estimate the loss of kinetic energy. The notation is the same as that in Article 230.

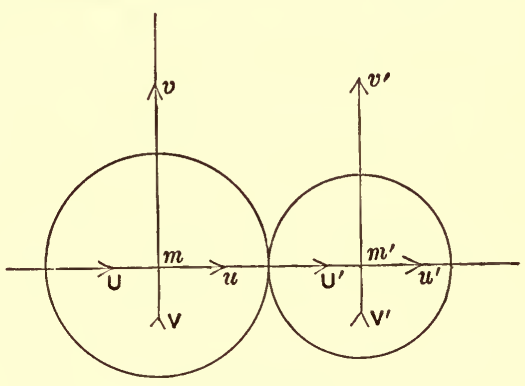

Fig. 67 (bis).

The generalised Newton's Rule gives the equation

$$
u-\iota^{\prime}=-e\left(U-U^{\prime}\right) \text {. }
$$

The equation of conservation of momentum parallel to the line of centres is

$$
m u+m^{\prime} u^{\prime}=m U+m^{\prime} U^{\prime} .
$$


The reaction between the spheres, being localised in the common normal, has no resolved part at right angles thereto, and thus the momentum of each sphere at right angles to the line of centres is unaltered by the impact. This gives us

$$
v=V \text {, and } v^{\prime}=V^{\prime} \text {. }
$$

Now we may solve the first two equations for $u$ and $u^{\prime}$, and we shall obtain

$$
\left.\begin{array}{c}
u=\frac{\left(m-m^{\prime} e\right) U+m^{\prime}(1+e) U^{\prime}}{m+m^{\prime}}, \\
u^{\prime}=\frac{\left(m^{\prime}-m e\right) U^{\prime}+m(1+e) U}{m+m^{\prime}} .
\end{array}\right\}
$$

Thus the motion after impact is determined.

The impulsive pressure on $m$ is

$$
m(U-u)=\frac{m m^{\prime}}{m+m^{\prime}}(1+e)\left(U-U^{\prime}\right)
$$

in the sense opposite to that of $U$, and there is an equal impulsive pressure on $m^{\prime}$ in the opposite sense. Let the magnitude of this pressure be denoted by $R$.

To find the kinetic energy lost, we have

kinetic energy before impact - kinetic energy after impact

$=\frac{1}{2} m\left(U^{2}+V^{2}\right)+\frac{1}{2} m^{\prime}\left(U^{\prime 2}+V^{\prime 2}\right)-\left\{\frac{1}{2} m\left(u^{2}+v^{2}\right)+\frac{1}{2} m^{\prime}\left(u^{\prime 2}+v^{\prime 2}\right)\right\}$

$=\frac{1}{2}\left\{m\left(U^{2}-u^{2}\right)+m^{\prime}\left(U^{\prime 2}-u^{\prime 2}\right)\right\}$

$=\frac{1}{2} R\left(U+u-U^{\prime}-u^{\prime}\right)$, since

$$
m(U-u)=m^{\prime}\left(u^{\prime}-U^{\prime}\right)=R .
$$

Hence the kinetic energy lost is $\frac{1}{2} R\left(U-U^{\prime}\right)(1-e)$

$$
=\frac{1}{2} \frac{m m^{\prime}}{m+m^{\prime}}\left(1-e^{2}\right)\left(U-U^{\prime}\right)^{2} .
$$

It is to be noted that the expression

$$
\frac{1}{2} R(U+u)-\frac{1}{2} R\left(U^{\prime}+u^{\prime}\right)
$$

for the change of kinetic energy produced by the impulse is in accordance with that obtained in the general case of a system of bodies in Article 155. The impulse in the present case is an internal one but it contributes something to the change of kinetic energy, on the other hand the internal impulsive actions between parts of the spheres contribute nothing to the change of kinetic energy, and we shall be able to prove later on that this is always the case for a rigid body. 


\section{Deduction of Newton's Rule from a particular} assumption. Without assuming any law of restitution we may throw the expression for the kinetic energy lost in impact into a form depending only on the relative velocities along the line of centres before and after impact. With the notation of the last Article, we have

$$
\frac{R}{m m^{\prime}}=\frac{U-u}{m^{\prime}}=\frac{u^{\prime}-U^{\prime}}{m}=\frac{\left(U-U^{\prime}\right)-\left(u-u^{\prime}\right)}{m+m^{\prime}},
$$

and the kinetic energy lost is

$$
\frac{1}{2} \frac{m m^{\prime}}{m+m^{\prime}}\left\{(U-u)^{2}-\left(U^{\prime}-u^{\prime}\right)^{2}\right\}
$$

Hence, if $W$ and $W^{\prime}$ denote the relative velocities resolved parallel to the line of centres before and after impact, the kinetic energy lost is

$$
\frac{1}{2} \frac{m m^{\prime}}{m+m^{\prime}}\left(W^{2}-W^{\prime 2}\right)
$$

or in words it is the product of one quarter of the harmonic mean of the masses and the difference of the squares of these relative velocities.

The same result may be obtained from general principles.

Since the motion of the centre of inertia of the spheres is unaltered by the impact, and since the motions of the spheres at right angles to the line of centres are also unaltered, we only require the velocities of the spheres relative to the centre of inertia resolved along the line of centres, the kinetic energy being that of the whole mass moving with the centre of inertia together with that of the relative motion (Article 104).

Now the velocity of $m$ relative to the centre of inertia, resolved along the line of centres, is $m^{\prime} W /\left(m+m^{\prime}\right)$ and the velocity of $m^{\prime}$ relative to the centre of inertia, resolved along the same line, is

$$
-m W /\left(m+m^{\prime}\right) \text {. }
$$

Just after the impact the velocities of $m$ and $m^{\prime}$ relative to the centre of inertia, resolved in the same direction, are respectively

$$
m^{\prime} W^{\prime} /\left(m+m^{\prime}\right) \text { and }-m W^{\prime} /\left(m+m^{\prime}\right) \text {. }
$$

Hence the kinetic energy lost is

$$
\begin{aligned}
& \frac{1}{2} m\left(\frac{m^{\prime}}{m+m^{\prime}} W\right)^{2}+\frac{1}{2} m^{\prime}\left(\frac{m}{m+m^{\prime}} W\right)^{2} \\
- & \frac{1}{2} m\left(\frac{m^{\prime}}{m+m^{\prime}} W^{\prime}\right)^{2}-\frac{1}{2} m^{\prime}\left(\frac{m}{m+m^{\prime}} W^{\prime}\right)^{2},
\end{aligned}
$$

leading to the same result as before. 
It follows that the statement $W^{\prime}=-e W$, which is the generalised Newton's rule, is equivalent to the statement that the kinetic energy lost is proportional to the square of the relative velocity of approach, and, if this could be assumed, the generalised Newton's rule could be deduced. This rule would thus be obtained from an hypothesis as to the amount of energy dissipated instead of an hypothesis as to the impulsive pressure.

\section{General theory of impulsive changes of motion.}

So far we have been confining our attention to the impulsive action between impinging bodies, but there are many other changes of motion which take place so rapidly that it is convenient to regard them as impulsively produced. The general method of treating such changes of motion has been indicated in Articles 82 and 113; it depends simply on repeated applications of the statement that for every particle in a connected system, and for each rigid body in such a system, the changes of momentum are a system of vectors equivalent to the impulses that produce them. We shall illustrate the application of this statement in a number of problems.

\section{Illustrative problems.}

I. Two equal smooth balls, whose centres are $A$ and $B$, lie nearly in contact on a smooth table, and a third ball of equal size and mass impinges directly on $A$, so that the line joining its centre $C$ to $A$ makes with the line $A B$ an angle $C A B,=\pi-\theta$. Prove that, if $\sin \theta>(1-e) /(1+e)$, the ball $A$ will start off in a direction making with $A B$ an angle $\tan ^{-1}\{2 \tan \theta /(1-e)\}, e$ being the coefficient of restitution for either pair of balls.

Let $V$ be the velocity of $C$ before striking $A$; since the impact is direct, $V$ is localised in $C A$. Let $w$ be its velocity after striking $A$; the direction of $w$ is that of $V$. Let $u^{\prime}$ be the velocity of $A$ immediately after $C$ strikes it, $u$ its velocity just after $A$ strikes $B, v$ the velocity of $B$ after $A$ strikes it, then the direction of $u^{\prime}$ makes an angle $\theta$ with $A B$. Suppose that the direction of $u$ makes an angle $\phi$ with $A B$. The direction of $v$ is $A B$.

We have the equations of momentum

$$
V=u^{\prime}+v, \quad u^{\prime} \cos \theta=u \cos \phi+v, \quad u^{\prime} \sin \theta=u \sin \phi,
$$

and the equations given by Newton's Rule

$$
u^{\prime}-w=e V, \quad u \cos \phi-v=-e u^{\prime} \cos \theta ;
$$

whence $2 w=V(1-e), \quad 2 u^{\prime}=V(1+e), \quad 2 u \cos \phi=(1-e) u^{\prime} \cos \theta$, and

$$
\tan \phi=\frac{2 \tan \theta}{1-e} \text {. }
$$




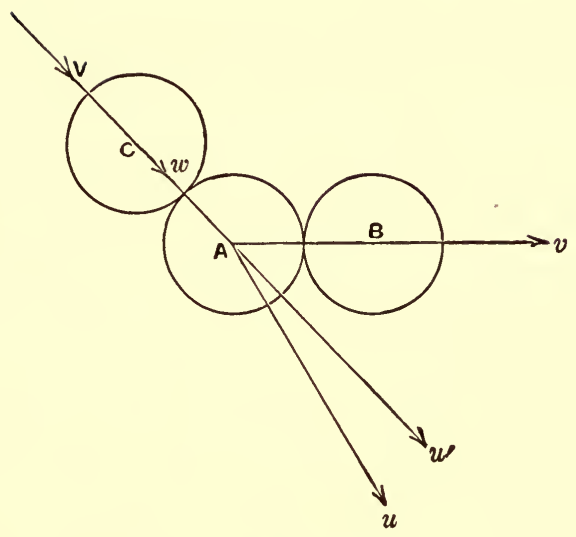

Fig. 68 .

Thus $A$ moves off as stated provided there is no second impact between $A$ and $C$. The condition for this is $u \cos (\phi-\theta)>w$,

or

$$
\frac{1}{2}(1-e) u^{\prime} \cos ^{2} \theta+u^{\prime} \sin ^{2} \theta>\frac{1-e}{1+e} u^{\prime},
$$

which leads to

$$
\sin \theta>(1-e) /(1+e) \text {. }
$$

II. A particle is projected with velocity $V$ from the foot of a smooth fixed plane of inclination $\theta$ in a direction making an angle a with the horizon $(\boldsymbol{a}>\theta)$. Find the condition that it may strike the plane $n$ times striking it at right angles at the nth impact, $e$ being the coefficient of restitution between the plane and the particle.

Since the velocity parallel to the plane is unaltered by impact, the motion of the particle parallel to the plane is determined by the same equation as if there were no impacts, thus at the end of any interval $t$ from the beginning of the motion the velocity parallel to the plane is $V \cos (a-\theta)-g t \sin \theta$.

Let $t_{1}, t_{2}, \ldots t_{n}$ be the times of flight before the first impact, between the first and second, and so on. Then $t_{1}$ is given by

$$
V t_{1} \sin (a-\theta)-\frac{1}{2} g t_{1}{ }^{2} \cos \theta=0,
$$

and thus $t_{1}=2 V \sin (a-\theta) / g \cos \theta$. The velocity perpendicular to the plane at time $t_{1}$ is $V \sin (a-\theta)-g t_{1} \cos \theta$ or $-V \sin (a-\theta)$. Immediately after the impact the velocity at right angles to the plane becomes $e V \sin (a-\theta)$ away from the plane. We thus find that $t_{2}=e t_{1}, t_{3}=e t_{2}, \ldots$.

Hence $t_{1}+t_{2}+\ldots+t_{n}=\frac{1-e^{n}}{1-e} \frac{2 V \sin (a-\theta)}{g \cos \theta}$ is the interval from the beginning of the motion till the $n$th impact. By supposition, at the end of 
this interval the velocity parallel to the plane vanishes, or this interval is $V \cos (a-\theta) / g \sin \theta$. The required condition is therefore

$$
\tan \theta=2 \tan (\alpha-\theta)\left(1-e^{n}\right) /(1-e) \text {. }
$$

III. A smooth sphere of mass $m$, is tied to a fixed point by an inextensible thread, and another sphere of mass $m^{\prime}$ impinges directly on it with velocity $v$ in a direction making an acute angle a with the thread. Find the velocity with which $m$ begins to move.

The impulse between the spheres acts in the line of centres so that the

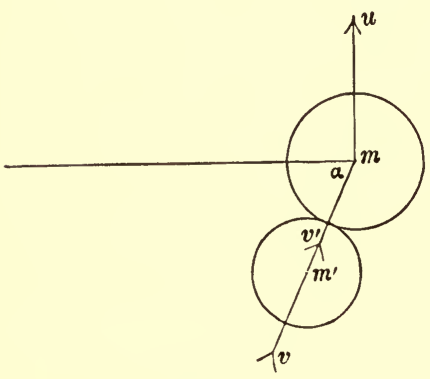

Fig. 69 . direction of motion of $m^{\prime}$ is unaltered. Let its velocity after impact be $v^{\prime}$.

There is an impulsive tension in the thread and the sphere $m$ is constrained to describe a circle about the fixed end. It therefore starts to move at right angles to the thread. Let $u$ be its velocity.

Resolving for the system at right angles to the thread we have the equation of momentum

$$
m u+m^{\prime} v^{\prime} \sin \alpha=m^{\prime} v \sin \alpha .
$$

By the generalised Newton's Rule we have

Whence

$$
\begin{gathered}
v^{\prime}-u \sin a=-e v . \\
u=\frac{m^{\prime} \sin a(1+e)}{m+m^{\prime} \sin ^{2} \alpha} v .
\end{gathered}
$$

IV. Two particles $A, B$ of equal mass are connected by a rigid rod of negligible mass, and a third equal particle $C$ is tied to a point $P$ of the rod at distances $a, b$ from the two ends. $C$ is projected with velocity u perpendicular to $A B$. Find the velocity of $C$ immediately after the string becomes tight.

Let $v$ be the velocity of $C$ immediately after the string becomes tight.

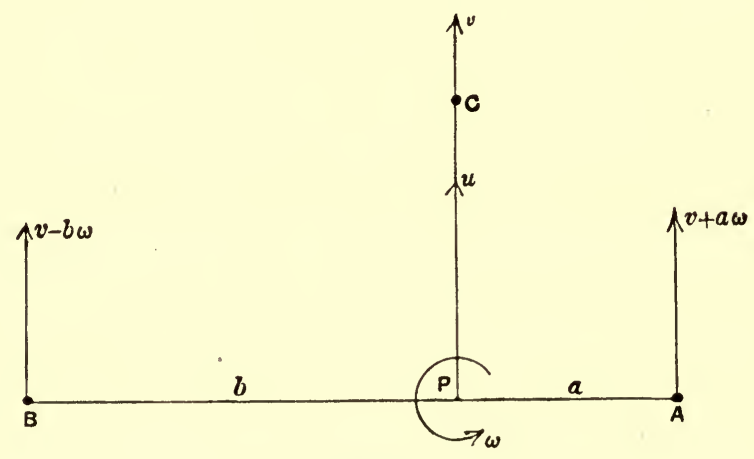

Fig. 70. 
Since the impulse on $C$ is along the string its direction of motion is unaltered. The velocity with which $P$ starts to move is $v$ along the string.

Let $\omega$ be the angular velocity with which the rod begins to turn. The velocity of $A$ is compounded of the velocity of $P$ and the velocity of $A$ relative to $P$. Thus $A$ starts with velocity $v+a \omega$. So $B$ starts with velocity $v-b \omega$.

The equation of linear momenturn parallel to the string is

$$
m v+m(v+a \omega)+m(v-b \omega)=m u,
$$

$m$ being the mass of either particle.

The equation of moment of momentum about $P$ is

giving

$$
\begin{gathered}
m a(v+a \omega)-m b(v-b \omega)=0, \\
\omega=(b-a) v /\left(a^{2}+b^{2}\right) .
\end{gathered}
$$

Eliminating $\omega$ we find

or

$$
\begin{aligned}
& 3 v-\frac{(a-b)^{2}}{a^{2}+b^{2}} v=u, \\
& v=\frac{1}{2} \frac{a^{2}+b^{2}}{a^{2}+b^{2}+a b} u .
\end{aligned}
$$

\section{Examples.}

[In these examples $e$ is the coefficient of restitution between two bodies.]

1. The sides of a rectangular billiard table are of lengths $a$ and $b$. If a ball is projected from a point on one of the sides of length $b$ to strike all four sides in succession and continually retrace its path, show that the angle of projection $\theta$ with the side is given by $a e \cot \theta=c+e c^{\prime}$, where $c$ and $c^{\prime}$ are the parts into which the side is divided at the point of projection.

2. Prove that in order to produce the greatest deviation in the direction of a smooth billiard ball of diameter $\alpha$ by impact on another equal ball at rest, the former must be projected in a direction making an angle

$$
\sin ^{-1}\left(\frac{a}{c} \sqrt{\frac{1-e}{3-e}}\right)
$$

with the line (of length $c$ ) joining the two centres.

3. A particle is projected from a point at the foot of one of two smooth parallel vertical walls so that after three reflexions it may return to the point of projection, and the last impact is direct. Prove that $e^{3}+e^{2}+e=1$, and that the vertical heights of the three points of impact are in the ratios

$$
e^{2}: 1-e^{2}: 1 \text {. }
$$

4. A particle is projected from the foot of an inclined plane and returns to the point of projection after several rebounds, one of which is at right angles to the plane; prove that, if it takes $r$ more leaps in coming down than in going up, the inclination $\theta$ of the plane and the angle of projection $a$ are connected by the equation

$$
\cot \theta \cot (a-\theta)=2\left\{\sqrt{ }\left(1-e^{r}\right)-\left(1-e^{r}\right)\right\} /\left\{e^{r}(1-e)\right\} .
$$


5. A particle is projected from the foot of a plane of inclination $\gamma$ in a direction making an angle $\beta$ with the normal to the plane, in a plane through this normal making an angle $a$ with the line of greatest slope on the inclined plane. Prove that, for the particle to be on the horizontal through the point of projection when it meets the plane for the $n$th time, the angles $a, \beta, \gamma$ must satisfy the equation

$$
\left(1-e^{n}\right) \tan \gamma=(1-e) \cos a \tan \beta .
$$

6. Three equal spheres are projected simultaneously from the corners of an equilateral triangle with equal velocities towards the centre of the triangle, and meet near the centre. Prove that they return to the corners with velocities diminished in the ratio $e: 1$.

7. A smooth uniform hemisphere of mass $M$ is sliding with velocity $V$ on a plane with which its base is in contact; a sphere of smaller mass $m$ is dropped vertically and strikes the hemisphere on the side towards which it is moving so that the line joining their centres makes an angle $\pi / 4$ with the vertical. Show that, if the coefficient of restitution between the plane and the hemisphere is zero, and that between the sphere and the hemisphere is $e$, the height through which the sphere must have fallen if the hemisphere is stopped dead is

$$
\frac{V^{2}}{2 g} \frac{(2 M-e m)^{2}}{(1+e)^{2} m^{2}} .
$$

8. A particle of mass $M$ is moving on a smooth horizontal table with uniform speed in a circle, being attached to the centre by an inextensible thread, and strikes another particle of mass $m$ at rest. Show that, if the two particles adhere, the tension of the thread is diminished in the ratio

$$
M /(M+m) \text {. }
$$

If there is restitution between the particles and the second one is describing the same circle as the first, prove that the tensions $T$ and $t$ in the two threads after impact are connected with their values before impact by the equation

$$
T+t=T_{0}+t_{0}-\left(1-e^{2}\right)\left\{\sqrt{ }\left(m T_{0}\right)-\sqrt{ }\left(M\left(t_{0}\right)\right\}^{2} /(M+m) .\right.
$$

9. A bucket and a counterpoise, of equal mass $M$, connected by a chain of negligible mass passing over a smooth pulley, just balance each other, and a ball, of mass $m$, is dropped into the centre of the bucket from a height $h$ above it; find the time that elapses before the ball ceases to rebound, and show that the whole distance descended by the bucket during this interval is

$$
4 m e h /\left\{(2 M+m)(1-e)^{2}\right\} \text {. }
$$

10. Three equal particles are attached to the ends and middle point of a rod of negligible mass, and one of the end ones is struck by a blow so that it starts to move at right angles to the rod. Prove that the velocities of the particles at starting are in the ratios, $5: 2: 1$.

11. An impulsive attraction acts between the centres of two spheres which are approaching each other so as to generate kinetic energy $E$. If $v$ is 
their relative velocity before the impulse, and $\theta, \theta^{\prime}$ the angles which the directions of the relative velocity, before and after, make with the line of centres, prove that

$$
\sin \theta=\sin \theta^{\prime} \sqrt{ }\left(1+\frac{4 E}{M v^{2}}\right)
$$

where $M$ is the harmonic mean of the masses.

12. Two small bodies of equal mass are attached to the ends of a rod of negligible mass; the rod is turning uniformly about its centre, which is supported, so that each of the bodies is describing a horizontal circle, when one of the bodies is struck by a vertical blow equal in magnitude to twice its momentum. Prove that the direction of motion of each of the bodies is instantaneously deflected through half a right angle.

*237. Impulsive motion of rigid bodies. The theory already explained in this Chapter and the theory of the momentum of a rigid body considered in Article 218 are sufficient for the discussion of the impulsive motion of rigid bodies in two dimensions.

For each body we have three equations of impulsive motion expressing that the change of momentum of the body is equivalent to the impulses exerted upon it.

The momentum of the body was shown to be equivalent to a single vector localised in a line through the centre of inertia, and equal to the momentum of the mass of the body moving with the centre of inertia, and a couple of amount equal to the product of the angular velocity of the body and the moment of inertia about an axis through the centre of inertia perpendicular to the plane of motion.

Let $m$ be the mass of the body $U, V$ the resolved velocities of the centre of inertia in two rectangular directions in the plane of motion, and $\Omega$ the angular velocity before impact; let $u, v$ be the resolved velocities of the centre of inertia in the same two directions after impact, and $\omega$ the angular velocity; also let $k$ be the radius of gyration of the body about an axis through the centre of inertia perpendicular to the plane.

Then the change of momentum of the system can be expressed as a vector localised in a line through the centre of inertia, whose resolved parts in the two specified directions are $m(u-U)$ and $m(v-V)$; and a couple, in the plane of motion, of moment

$$
m k^{2}(\omega-\Omega)
$$


The impulses exerted on the body can be expressed as a single impulse at any origin and an impulsive couple.

The equations of impulsive motion express the equivalence of the two systems of vectors.

Thus if the impulses are reduced to an impulse at the centre of inertia, whose resolved parts in the specified directions are $X$ and $Y$, and a couple $\underline{N}$, we can take the equations of motion to be

$$
m(u-U)=X, \quad m(v-V)=\underline{Y}, \quad m k^{2}(\omega-\Omega)=\underline{N} .
$$

More generally, the resolved part, in any direction of the vector whose resolved parts, in the specified directions, are $m(u-U)$ and $m(v-V)$ is equal to the resolved part, in the same direction, of the vector whose resolved parts, in the specified directions, are $X$ and $Y$; and the moment about any axis of the vector system determined by $m(u-U), m(v-V), m k^{2}(\omega-\Omega)$, is equal to the moment about the same axis of the vector system determined by $X, Y, \underline{Y}, \underline{.}$.

\section{*238. Kinetic energy produced by impulses applied to} rigid body. Suppose the body to move in one plane. Let $m$ be the mass of the body, $U, V$ resolved velocities of its centre of inertia parallel to the axes of reference, and $\Omega$ its angular velocity just before the impulses act, $u, v, \omega$ corresponding quantities just after.

Let $X_{1}, \underline{Y}_{1}$ be the resolved parts parallel to the axes of the impulse applied to the body at any point whose coordinates relative to the centre of inertia are $x_{1}, y_{1}$.

The equations of impulsive motion are

$$
\left.\begin{array}{rl}
m(u-U) & =\Sigma \dot{X}, \\
m(v-V) & =\Sigma \underline{Y}, \\
m k^{2}(\omega-\Omega) & =\Sigma(x \underline{Y}-y \underset{X}{X}) .
\end{array}\right\}
$$

Multiply these equations in order by

$$
\frac{1}{2}(u+U), \frac{1}{2}(v+V), \frac{1}{2}(\omega+\Omega),
$$

and let $T$ be the kinetic energy of the body after the impulses, $T_{0}$ that before. Then we have

$$
T-T_{0}=\Sigma \frac{1}{2}\{X(u-\omega y+U-\Omega y)+\underline{Y}(v+\omega x+V+\Omega x)\} .
$$

The right-hand member of this equation is the sum of the 
products of the external impulses and the arithmetic means of the velocities of their points of application resolved in their directions before and after.

Now the theorem of Article 155 asserts that the change of kinetic energy is equal to the value of the like sum for all the impulses internal and external. It follows that the internal impulses between the parts of a rigid body, which undergoes a sudden change of motion, contribute nothing to this sum.

\section{*239. Examples.}

1. A uniform rod at rest is struck at one end by an impulse at right angles to its length. Prove that, if the rod is free, it begins to turn about the point of it which is distant one-third of its length from the other end, and that the kinetic energy generated is greater than it would be if the other end were fixed in the ratio $4: 3$.

2. A free rigid body is rotating about an axis through its centre of inertia for which the radius of gyration is $k$ when a parallel axis at a distance $c$ becomes fixed. Prove that the angular velocity of the body is suddenly diminished in the ratio $k^{2}: c^{2}+k^{2}$.

3. An elliptic disc is rotating in its plane about one end $P$ of a diameter $P P^{\prime}$, when $P^{\prime}$ is suddenly fixed. Find the impulse at $P$ and the angular velocity about it, and prove that, if the eccentricity exceeds $\sqrt{ } \frac{2}{3}$, the diameter $P P^{\prime}$ may be so chosen that the disc is reduced to rest.

4. A uniform rod of length $2 a$ and mass $m$ is constrained to move with its ends on two smooth fixed straight wires at right angles to each other, and is set in motion by an impulse of magnitude $m V$. Prove that the kinetic energy generated is $\frac{3}{8} m V^{2} p^{2} / a^{2}$, where $p$ is the perpendicular from the intersection of the fixed wires on a line parallel to the line of the impulse and such that the centre of inertia is midway between the two parallels.

*240. Rigid bodies with restitution. Let two rigid bodies moving in the same plane come into contact at a point $P$ and suppose the bodies to be smooth at $P$. Let $R$ be the impulsive pressure between the bodies at $P$. The direction of $R$ is the common normal at $P$ to the two surfaces. Let the axis of $x$ be taken in this direction, the axis of $y$ being any fixed line in a perpendicular direction.

Let $m$ and $m^{\prime}$ be the masses of the bodies, $U, V, \Omega$ the velocity system of $m$ before impact, $u, v, \omega$ corresponding quantities after impact, and let accented letters denote similar quantities for $m^{\prime}$. Also let $x, y$ be the coordinates of the centre of inertia of $m$ and $x^{\prime}, y^{\prime}$ those of $m^{\prime}$ at the instant of impact, and let $\xi, \eta$ be coordinates of $P$ at the same instant. Also suppose that, as acting on $m$, the sense of $R$ is the negative sense of the axis of $x$. 
The velocity of $P$, considered as a point of $m$, has components

$$
\begin{array}{ll}
U-\Omega(\eta-y), & V+\Omega(\xi-x) \text { before impact, and } \\
u-\omega(\eta-y), & v+\omega(\xi-x) \text { after impact. }
\end{array}
$$

The velocity of $P$, considered as a point of $m^{\prime}$, has components

$$
\begin{array}{cc}
U^{\prime}-\Omega^{\prime}\left(\eta-y^{\prime}\right), & V^{\prime}+\Omega^{\prime}\left(\xi-x^{\prime}\right) \text { before impact, and } \\
u^{\prime}-\omega^{\prime}\left(\eta-y^{\prime}\right), & v^{\prime}+\omega^{\prime}\left(\xi-x^{\prime}\right) \text { after impact. }
\end{array}
$$

The equation provided by the generalised Newton's Rule is accordingly

$$
u-\omega(\eta-y)-u^{\prime}+\omega^{\prime}\left(\eta^{\prime}-y^{\prime}\right)=-e\left\{U-\Omega(\eta-y)-U^{\prime}+\Omega^{\prime}\left(\eta-y^{\prime}\right)\right\} .
$$

The equations of motion of the two bodies by resolving parallel to the axis of $x$ are

$$
m(u-U)=-R, \quad m^{\prime}\left(u^{\prime}-U^{\prime}\right)=R .
$$

The equations of motion by resolving parallel to the axis of $y$ are

$$
m(v-V)=0, \quad m^{\prime}\left(v^{\prime}-V^{\prime}\right)=0 .
$$

The equations of moments about axes through the centres of inertia perpendicular to the plane of motion are

$$
m k^{2}(\omega-\Omega)=R(\eta-y), \quad m^{\prime} k^{\prime 2}\left(\omega^{\prime}-\Omega^{\prime}\right)=-R\left(\eta-y^{\prime}\right),
$$

where $k$ and $k^{\prime}$ are the radii of gyration of the bodies about the axes in question.

On substituting for $u, u^{\prime}, \omega, \omega^{\prime}$ in the equation containing $e$, we find

$$
R\left[\frac{1}{m}\left\{1+\frac{(\eta-y)^{2}}{k^{2}}\right\}+\frac{1}{m^{\prime}}\left\{1+\frac{\left(\eta-y^{\prime}\right)^{2}}{k^{\prime 2}}\right\}\right]=(1+e)\left[U-U^{\prime}-\Omega(\eta-y)+\Omega^{\prime}\left(\eta-y^{\prime}\right)\right],
$$

and this equation shows that the impulsive pressure with any value of $e$ is $(1+e)$ times what it would be if $e$ were zero.

The result of this Article can be expressed in the statement that the generalised Newton's rule and the rule derived from Poisson's hypothesis are equivalent for any two smooth bodies moving in one plane.

*241. Impulsive action between rough bodies. The impulsive action between two rough bodies which come into contact, when there is sliding at the point of contact, is assumed to be expressible by means of an impulsive pressure of the kind we have met with in the case of smooth bodies, and an impulsive friction tending to resist sliding, the friction and the pressure having a constant ratio, the coefficient of friction. We shall suppose the geometrical condition as regards the relative velocity to be the same as in the case of smooth bodies, viz. the generalised Newton's rule.

In the case of bodies rough enough to prevent sliding, the elastic action cannot be so simple as in the case considered by Poisson, and accidental circumstances probably play an important part.

We shall now show that when there is sliding at the points that come into contact the rule deduced from Poisson's hypothesis is equivalent to the generalised Newton's rule, for the impulsive action between rough bodies. 
Writing $F$ for the impulsive friction at the point of contact, and taking the same notation as in the last Article, we have the equations of impulsive motion

and

$$
\left.\begin{array}{rl}
m(u-U) & =-R, m(v-V)=-F, \\
m k^{2}(\omega-\Omega) & =-(\xi-x) F+(\eta-y) R
\end{array}\right\}
$$

$$
\left.\begin{array}{rl}
m^{\prime}\left(u^{\prime}-U^{\prime}\right) & =R, \quad m^{\prime}\left(v^{\prime}-V^{\prime}\right)=F, \\
m^{\prime} k^{\prime 2}\left(\omega^{\prime}-\Omega^{\prime}\right) & =\left(\xi-x^{\prime}\right) F-\left(\eta-y^{\prime}\right) R
\end{array}\right\}
$$

Also we have the equation of sliding friction

$$
F=\mu R
$$

and the equation provided by the generalised Newton's Rule

$$
u-\omega(\eta-y)-u^{\prime}+\omega^{\prime}\left(\eta-y^{\prime}\right)=-e\left\{U-\Omega(\eta-y)-U^{\prime}+\Omega^{\prime}\left(\eta-y^{\prime}\right)\right\} \ldots
$$

From these equations we obtain, by elimination of $u, u^{\prime}, v, v^{\prime}, \omega, \omega^{\prime}, F$, an equation for $R$, viz.

$$
\begin{aligned}
R\left[\left(\frac{1}{m}+\frac{1}{m^{\prime}}\right)+\frac{\eta-y}{m k^{2}}\{(\eta-y)-\mu(\xi-x)\}\right. & \left.+\frac{\eta-y^{\prime}}{m^{\prime} k^{\prime 2}}\left\{\left(\eta-y^{\prime}\right)-\mu\left(\xi-x^{\prime}\right)\right\}\right] \\
& =(1+e)\left[U-\Omega(\eta-y)-U^{\prime}+\Omega^{\prime}\left(\eta-y^{\prime}\right)\right],
\end{aligned}
$$

showing that $R$ contains $(1+e)$ as a factor and is otherwise independent of $e$, and thus proving the equivalence of the two rules.

Further we can show that, when there is sufficient friction to prevent sliding, the rules are not in general equivalent.

We shall assume the generalised Newton's rule as a basis of discussion. We have not in this case any relation between $F$ and $R$, but equations (1), (2), (4) still hold, with the additional equation of no sliding

$$
v+\omega(\xi-x)=v^{\prime}+\omega^{\prime}\left(\xi-x^{\prime}\right)
$$

From equations (1), (2), (4), (5) we can form two equations for $R$ and $F$, viz.

$$
\begin{aligned}
R\left[\left(\frac{1}{m}+\frac{1}{m^{\prime}}\right)+\frac{(\eta-y)^{2}}{m k^{2}}+\frac{\left(\eta-y^{\prime}\right)^{2}}{m^{\prime} k^{\prime 2}}\right]- & F\left[\frac{(\xi-x)(\eta-y)}{m k^{2}}+\frac{\left(\xi-x^{\prime}\right)\left(\eta-y^{\prime}\right)}{m^{\prime} k^{\prime 2}}\right] \\
& =(1+e)\left[U-\Omega(\eta-y)-U^{\prime}+\Omega^{\prime}\left(\eta-y^{\prime}\right)\right], \\
F\left[\left(\frac{1}{m}+\frac{1}{m^{\prime}}\right)+\frac{(\xi-x)^{2}}{m k^{2}}+\frac{\left(\xi-x^{\prime}\right)^{2}}{m^{\prime} k^{\prime 2}}\right] & -R\left[\frac{(\xi-x)(\eta-y)}{m k^{2}}+\frac{\left(\xi-x^{\prime}\right)\left(\eta-y^{\prime}\right)}{m^{\prime} k^{\prime 2}}\right] \\
& =V+\Omega(\xi-x)-V^{\prime}-\Omega^{\prime}\left(\xi-x^{\prime}\right) .
\end{aligned}
$$

It is clear that the solution of these equations will give an expression for $R$ consisting of two terms, one of them having $(1+e)$ as a factor and the other not containing that factor.

\section{*242. Examples.}

[In these examples $e$ is the coefficient of restitution between two bodies.]

1. From the rule deduced from Poisson's hypothesis obtain the generalised Newton's rule for smooth bodies, and for bodies with sliding friction. 
2. Prove that when the common normal at the points that come into contact passes through the centres of inertia of the impinging bodies the rules are equivalent even if the bodies are rough enough to prevent sliding.

[This includes the case of the impact of rough spheres.]

3. A uniform sphere of radius $\alpha$ and mass $m$ moving without rotation impinges directly on a smooth uniform cube of side $2 \alpha$ and mass $m^{\prime}$, the line of motion of the sphere being at a distance $b$ from the centre of inertia of the cube. Prove that, if $e=0$, the kinetic energy lost in the impact is to that of the sphere before impact in the ratio

$$
1: 1+\left(m / m^{\prime}\right)\left(1+\frac{3}{2} b^{2} / a^{2}\right) \text {. }
$$

4. A uniform rod, falling without rotation, strikes a smooth horizontal plane. Prove that, for all values of $e$, the angular velocity of the rod immediately after impact is a maximum if the rod before impact makes with the horizontal an angle $\cos ^{-1} 1 / \sqrt{ } 3$.

5. A sphere whose centre of inertia coincides with its centre of figure is moving in a vertical plane and rotating about an axis perpendicular to that plane when it strikes against a horizontal plane which is sufficiently rough to prevent sliding. Prove that, for all values of $e$, the sphere will rebound at an angle greater or less than if there were no friction according as the lowest point of it at the instant of impact is moving forward or backward.

6. A disc of any form of mass $m$, moving in its plane without rotation and with velocity $V$ at right angles to a fixed plane, strikes the plane so that the distances of the centre of inertia from the point of impact and from the plane are $r$ and $p$. Prove that, if the plane is sufficiently rough to prevent sliding, the impulsive pressure is

$$
m V(1+e)\left(k^{2}+p^{2}\right) /\left(k^{2}+r^{2}\right)
$$

where $k$ is the radius of gyration of the disc about its centre of inertia.

7. A ball spinning about a vertical axis moves on a smooth table, and impinges directly on a vertical cushion. Prove that, if $\theta$ is the angle of reflexion, the kinetic energy is diminished in the ratio

$$
10+14 \tan ^{2} \theta: 10 e^{-2}+49 \tan ^{2} \theta,
$$

the cushion being sufficiently rough to prevent sliding.

8. A circular disc of mass $M$ and radius $c$ impinges on a rod of mass $m$ and length $2 a$ which is free to turn about a pivot at its centre, and the point of impact is distant $b$ from the pivot. Prove that, if the direction of motion of the centre of the sphere makes angles $a$ and $\beta$ with the rod before and after collision, then

$$
2\left(3 M b^{2}+m a^{2}\right) \tan \beta=3\left(3 M b^{2}-e m a^{2}\right) \tan a,
$$

the edges in contact being sufficiently rough to prevent sliding.

9. A uniform sphere is let drop upon a uniform smooth hoop which is free to turn in its own vertical plane about its highest point, the centre of the sphere moving in the plane of the hoop. Prove that, for the sphere to rebound horizontally, it must strike the hoop at an angular distance $\tan ^{-1} \sqrt{ }(2 e / 3)$ from the highest point. 
*243. Impulsive motion of connected systems. In illustration of the application of the equations of impulsive motion to systems of rigid bodies with invariable connexions we take the following problems. In the first it will be observed that we do not need to introduce explicitly the reactions between the connected bodies. The second illustrates the choice of equations; for, although some of the unknown reactions must be introduced, it is unnecessary to form equations for each body separately.

I. Three uniform rods of masses proportional to their lengths are freely jointed together and laid out straight, and one of the end rods is struck at the free end at right angles to its length. It is required to find how they begin to move.

Let $2 a, 2 b, 2 c$ be the lengths of the rods, the last being struck, and let

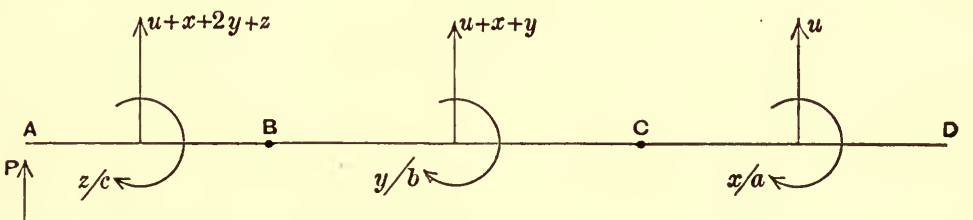

Fig. 71.

$x / a, y / b, z / c$ be the angular velocities with which they begin to move, $u$ the velocity of the centre of inertia of the first. Then the system of velocities is as shown in the figure. Let $P$ be the impulse applied at the end $A$, and $\kappa a, \kappa b, \kappa c$ the masses of the rods.

We take moments about $C$ for the rod $C D$, about $B$ for the rods $B C, C D$, and about $A$ for the three rods, and we resolve for the whole system at right angles to the rods. We thus obtain the equations

$u-\frac{1}{3} x=0$,

$b\left[b(u+x+y)-\frac{1}{3} b y\right]+a\left[(2 b+a) u-\frac{1}{3} a x\right]=0$,

$c\left[c(u+x+2 y+z)-\frac{1}{3} c z\right]+b\left[(2 c+b)(u+x+y)-\frac{1}{3} b y\right]$

$\kappa c(u+x+2 y+z)+\kappa b(u+x+y)+\kappa \alpha u=P$.

$$
\begin{aligned}
& -a\left[(2 c+2 b+a) u-\frac{1}{3} a x\right]=0, \\
& \text {, }
\end{aligned}
$$

Subtracting the second and third we get, on dividing by $c$,

$$
c\left(u+x+2 y+\frac{2}{3} z\right)+2 b(u+x+y)+2 a u=0,
$$

and, on simplifying this and the second by using the first, we get

$$
x(a+4 b+2 c)+y(3 c+3 b)+z c=0,
$$

and

$$
(2 b+a) x+b y=0 \text {. }
$$


Hence we have

$$
\begin{aligned}
\frac{u}{\frac{1}{3} b}=\frac{x}{b} & =\frac{y}{-(2 b+a)}=\frac{c z}{2 a b+3 a c+2 b^{2}+4 b c} \\
& =\frac{P}{\kappa\left(\frac{4}{3} a b+a c+\frac{4}{3} b c+\frac{4}{3} b^{2}\right)^{2}} .
\end{aligned}
$$

II. A rhombus formed of four equal uniform rods freely jointed at the corners is set in motion by an impulse applied to one rod at right angles to it. To find how the rhombus begins to move.

Let $2 a$ be the length of each side of the rhombus $A B C D, a$ the angle

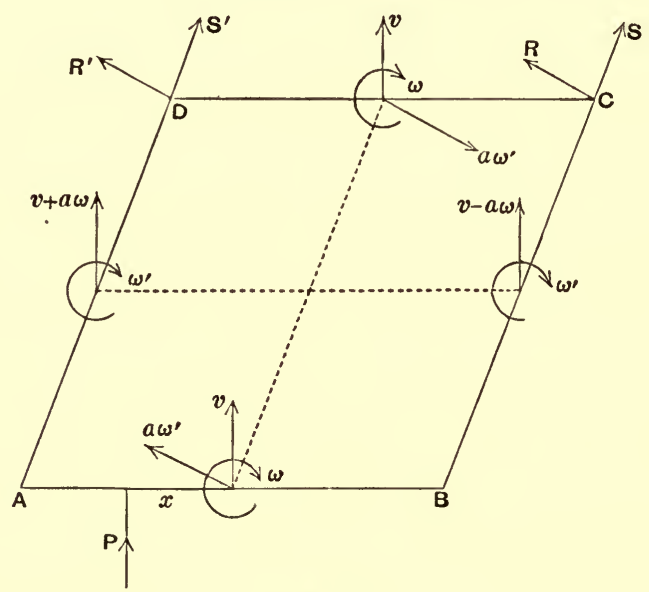

Fig. 72.

$D A B, x$ the distance of the point struck from the middle point of the side $A B$ containing it, $P$ the impulse, $m$ the mass of each rod.

The centre of inertia is the point of intersection of the lines joining the middle points of opposite sides. Since the figure is alway's a parallelogram, opposite sides have the same angular velocities, and the lines joining the middle points of opposite sides are of constant length $2 a$ and turn with the angular velocities of the sides to which they are parallel. Let these angular velocities be $\omega$ and $\omega^{\prime}$, and let $v$ be the velocity of the centre of inertia. Then the velocities of the centres of inertia of the rods and their angular velocities are as shown.

Now let the impulsive reaction of the hinge at $C$ be resolved into $S$ in $B C$ and $R$ at right angles to $B C$, and the impulsive reaction of the hinge at $D$ into $S^{\prime}, R^{\prime}$ in the same directions. These impulses act in opposite senses on the two rods which meet at a hinge. The figure shows the senses in which we take them to act on the rod $C D$.

We form two equations of motion by resolving for the system in the direction of the impulse and by taking moments about the centre of inertia. 
We thus obtain

$$
\left.\begin{array}{rl}
4 m v & =P, \\
\frac{8}{3} m \alpha^{2}\left(\omega+\omega^{\prime}\right) & =P(x+a \cos a) .
\end{array}\right\}
$$

Again, we can form three equations containing $R$ and $R^{\prime}$ by resolving for $C D$ at right angles to $B C$, and taking moments for $B C$ and $A D$ about $B$ and $A$ respectively. We thus obtain

$$
\left.\begin{array}{rl}
m\left(v \cos a-a \omega^{\prime}\right) & =R+R^{\prime}, \\
m\left[(v-a \omega) \alpha \cos a-\frac{1}{3} a^{2} \omega^{\prime}\right] & =-2 \alpha R, \\
m\left[(v+a \omega) \alpha \cos a-\frac{1}{3} a^{2} \omega^{\prime}\right] & =-2 \alpha R^{\prime}
\end{array}\right\}
$$

from which, on elimination of $R$ and $R^{\prime}$, we get

$$
v \cos a=\frac{2}{3} a \omega^{\prime} \text {. }
$$

Hence

$$
v=\frac{1}{4} P / m, \omega=\frac{3}{8} P x / m \alpha^{2}, \omega^{\prime}=\frac{3}{8} P \cos a / m \alpha \text {. }
$$

\section{*244. Examples.}

1. Two equal rods $A B, A C$ freely jointed at $A$ are at rest with the angle $B A C$ a right angle, and $A C$ is struck at $C$ by an impulse in a direction parallel to $A B$. Prove that the velocities of the centres of inertia of $A B$ and $A C$ in the direction of $A B$ are in the ratio $2: 7$.

2. Two equal uniform rods freely hinged at a common end are laid out straight, and one end of one of them is struck by an impulse at right angles to their length. Prove that the kinetic energy generated is greater than it would be if the rods were firmly fastened together so as to form a single rigid body in the ratio $7: 4$.

3. Four equal uniform rods are freely hinged together so as to form a rhombus of side $2 \alpha$ with one diagonal vertical, and the system falling in a vertical plane with velocity $V$ strikes against a fixed horizontal plane. Taking $a$ to be the angle which each rod makes with the vertical and assuming no restitution, prove that (i) the impulsive action between the two upper rods is directed horizontally, (ii) the angular velocity of each rod after the impulse is $\frac{3}{2}(V / a) \sin a /\left(1+3 \sin ^{2} a\right)$, (iii) the impulsive action between the two upper rods is to the momentum of the system before impact in the ratio

$$
\sin a\left(3 \cos ^{2} a \sim 1\right): 8 \cos a\left(1+3 \sin ^{2} a\right),
$$

(iv) the impulsive action at either of the hinges in the horizontal diagonal makes with the horizontal an angle $\tan ^{-1}\left\{\left(3 \cos ^{2} a \sim 1\right) \cot a\right\}$.

4. In Example 3, prove that, if the coefficient of restitution between the rhombus and the ground is $e$, the angular velocity of each rod after the impulse is

$$
3(1+e)(V / a) \sin a /\left(1+3 \sin ^{2} a\right) \text {. }
$$

5. A square framework $A B C D$ is formed of uniform rods freely jointed at $B, C$, and $D$, the ends at $A$ being in contact but free. Prove that, if $A B$ is struck by a blow at $A$ in direction $D A$, the initial velocity of $A$ is 79 times that of $D$. 
6. A rectangle formed of four uniform rods, of lengths $2 a$ and $2 b$ and masses $m$ and $m^{\prime}$, freely hinged together, is rotating in its plane about its centre with angular velocity $n$ when a point in one of the sides of length $2 a$ becomes suddenly fixed. Prove that the angular velocity of the sides of length $2 b$ instantly becomes $\frac{1}{2} n\left(3 m+m^{\prime}\right) /\left(3 m+2 m^{\prime}\right)$, and find the angular velocity of the sides of length $2 a$.

\section{INITIAL Motions.}

245. Nature of the problems. We suppose that a system is held in some definite position in a field of force, and that at a particular instant some one of the constraints ceases to be applied, then the system begins to move, each particle of it with a certain acceleration. Our first object in such a case is to determine the accelerations with which the parts of the system begin to move. When the accelerations have been found there is generally no difficulty in determining the initial values of the reactions of supports, or internal actions between different bodies of the system; and the determination of the unknown reactions is our second object.

Again, we may enquire as to approximate expressions for the coordinates of a particle in terms of the time elapsed since the instant when the constraint was removed. For motions starting from rest, a knowledge of the accelerations alone determines the initial tangents to the paths of the particles, and gives values for the displacements of the particles after a short time which are correct to the second order of small quantities, the time being of the first order. If the approximation could be continued beyond the second order we could state the curvature of the path of each of the particles. Thus a convenient way of referring to problems of this character is to regard as their object the determination of initial curvatures.

Finally we may remark that the problem of determining the curvature of the path of a particle whose velocity is not zero offers no difficulty when the velocity and acceleration are known, since the resolved acceleration along the normal to the path is the product of the square of the resultant velocity and the curvature. This remark enables us easily to determine the initial curvature of the path of a particle when its motion is changed impulsively. 
246. Method for initial accelerations. It is always possible to determine expressions for the accelerations of all the points of a connected system in terms of a small number of independent accelerations, and there is always the same number of equations of motion free from unknown reactions, so that all the accelerations can be found. The expression of the initial accelerations in the manner proposed is facilitated by observing (1) that the velocity of every particle initially vanishes, (2) that every composition and resolution may be effected by taking the position of the system to be that from which it starts. The method laid down will be better understood after the study of an example. We purposely choose one of a somewhat complicated character in order to illustrate the points mentioned.

247. Mlustrative Problem. Four equal rings $A, B, C, D$ are at equal distances on a smooth fixed horizontal rod, and three other equal and similar rings $P, Q, R$ are attached by pairs of equal inestensible threads to the pairs of rings $(A, B),(B, C),(C, D)$. The system is held so that all the threads initially make the same angle a with the horizontal, and is let go. It is required to find the acceleration of each ring.

From the symmetry of the system the accelerations of $A, D$ are equal and

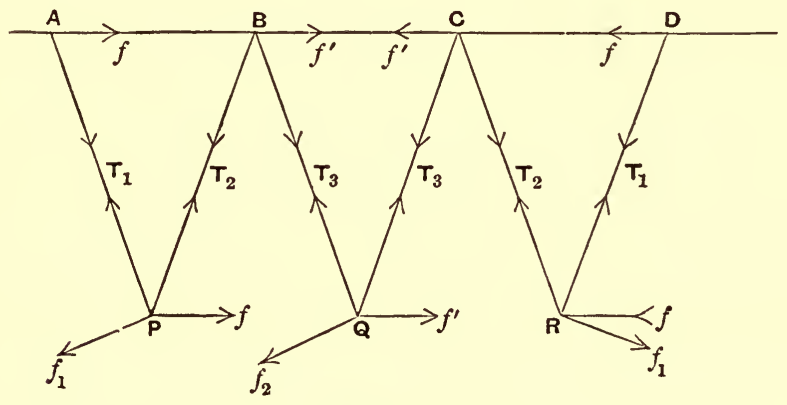

Fig. 73.

opposite, so are those of $B, C$, and those of $P, R$. Also the acceleration of $Q$ is vertical.

Let $f, f^{\prime}$ be the accelerations of $A, B$ along the smooth horizontal rod.

Now relative to $A, P$ describes a circle, and thus the acceleration of $P$ relative to $A$ is made up of a tangential acceleration $f_{1}$ at right angles to $A P$, and a normal acceleration proportional to the square of the angular velocity of $A P$. Since the initial angular velocity vanishes, we have, as the relative acceleration, $f_{1}$ at right angles to $A P$. Again, since the threads $A P, B P$ are 
equal, the particle $P$ is always vertically under the middle point of $A B$ and thus its horizontal acceleration is $\frac{1}{2}\left(f+f^{\prime}\right)$.

Hence giving

$$
\begin{aligned}
\frac{1}{2}\left(f+f^{\prime}\right) & =f-f_{1} \sin a, \\
f_{1} \sin a & =\frac{1}{2}\left(f-f^{\prime}\right) .
\end{aligned}
$$

Again, the horizontal acceleration of $Q$ vanishes, and we have therefore the acceleration $f_{2}$ of $Q$ relative to $B$ given by the equation

$$
f_{2} \sin a=f^{\prime} \text {. }
$$

Thus the accelerations of the particles are expressed in terms of $f$ and $f^{\prime}$; in particular the vertical accelerations of $P$ and $Q$ are $\frac{1}{2}\left(f-f^{\prime}\right) \cot a$ and $f^{\prime} \cot a$ downwards.

Now let $m$ be the mass of each particle and $T_{1}, T_{2}, T_{3}$ the tensions in the threads as shown in the figure. Then resolving horizontally for $A, P$, and $B$ we have

$$
m f=T_{1} \cos a, \frac{1}{2} m\left(f+f^{\prime}\right)=\left(T_{2}-T_{1}\right) \cos \alpha, m f^{\prime}=\left(T_{3}-T_{2}^{\prime}\right) \cos a \ldots(1) ;
$$

and resolving vertically for $P$ and $Q$ we have

$$
\frac{1}{2} m\left(f-f^{\prime}\right) \cot a=-\left(T_{1}+T_{2}\right) \sin a+m g, m f^{\prime} \cot a=-2 T_{3} \sin a+m g \ldots \text { (2). }
$$

From the set of equations (1) we have

$$
T_{1} \cos a=m f, \quad T_{2} \cos a=m\left(\frac{3}{2} f+\frac{1}{2} f^{\prime}\right), \quad T_{3} \cos a=m \frac{3}{2}\left(f+f^{\prime}\right) ;
$$

and from (2), on substituting for $T_{1}, T_{2}, T_{3}$, we have

$$
\left(f-f^{\prime}\right) \cot a+\left(5 f+f^{\prime}\right) \tan a=2 g, f^{\prime} \cot a+3\left(f+f^{\prime}\right) \tan a=g ;
$$

whence

$$
\frac{f}{4-\cos 2 a}=\frac{f^{\prime}}{\cos 2 a}=\frac{g \sin 2 a}{12-11 \cos 2 a+\cos ^{2} 2 a} \text {. }
$$

248. Initial curvature. As an example of initial curvatures when the motion does not start from rest we take the following problem:

Two particles of masses $m, m^{\prime}$ connected by an inextensible thread of length $l$ are placed on a smooth table with the thread straight, and are projected at right angles to the thread in opposite senses. To find the initial curvatures of their paths.

Let $u, v$ be the initial velocities of the particles, and $\omega$ the initial angular velocity of the thread, then

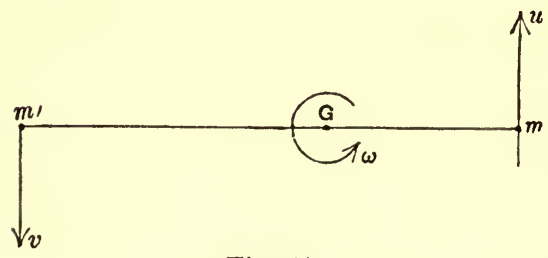

Fig. 74.

$$
u+v=l \omega \text {. }
$$

Let $G$ be the centre of inertia of the two particles. Then $G$ moves uniformly on the table with velocity

$$
\left(m u-m^{\prime} v\right) /\left(m+m^{\prime}\right)
$$

The acceleration of $G$ vanishes, and the acceleration of $m$ relative to $G$ is that of a particle describing a circle of radius $m^{\prime} l /\left(m+m^{\prime}\right)$ with angular velocity $\omega$; thus the acceleration of $m$ along the thread is $m^{\prime} l \omega^{2} /\left(m+m^{\prime}\right)$, and 
this is the acceleration of $m$ along the normal to its path. Hence, if $\rho$ is the initial radius of curvature of the path of $m$,

$$
\frac{u^{2}}{\rho}=\frac{m^{\prime} l}{m+m^{\prime}}\left(\frac{u+v}{l}\right)^{2},
$$

giving

$$
1 / \rho=m^{\prime}(u+v)^{2} /\left\{\left(m+m^{\prime}\right) l u^{2}\right\} .
$$

In like manner the initial curvature of the path of $m^{\prime}$ is

$$
m(u+v)^{2} /\left\{\left(m+m^{\prime}\right) l v^{2}\right\} .
$$

\section{Examples.}

1. Two bodies $A$ and $B$ of equal weight are suspended from the chains of an Atwood's machine; $A$ is rigid, while $B$ consists of a vessel full of water in which is a cork attached to the bottom by a string. Supposing the string to be destroyed in any manner, determine the sense in which $A$ begins to move.

2. A particle is supported by equal threads inclined at the same angle $a$ to the horizontal. One thread being cut, prove that the tension in the remaining thread is suddenly changed in the ratio $2 \sin ^{2} a: 1$.

3. Particles of equal mass are attached to the points of trisection $C, D$ of a thread $A C D B$ of length $3 l$, and the system is suspended by its ends from points $A, B$ distant $l(1+2 \sin a)$ apart in a horizontal line. Prove that, if the portion $D B$ of the thread is cut, the tension of $A C$ is instantly changed in the ratio $2 \cos ^{2} a: 1+\cos ^{2} a$, and that the initial direction of motion of $D$ is inclined to the vertical at an angle $\phi$ such that

$$
\cot \phi=\tan a+2 \cot a \text {. }
$$

4. Three small equal rings rest on a smooth vertical circular wire at the corners of an equilateral triangle with one side vertical, the uppermost being connected with the other two by inextensible threads. Prove that, if the vertical thread is cut through, the tension in the other thread is instantly diminished in the ratio $3: 4$.

5. A set of $2 n$ equal particles are attached at equal intervals to a thread, and the ends of the thread are attached to equal small smooth rings which can slide on a horizontal rod. The rings are initially held in such a position that the lowest part of the thread is horizontal, and the highest parts make equal angles $\gamma$ with the horizontal, and the rings are let go. Prove that in the initial motion (i) the acceleration of each particle is vertical, (ii) the tension in the lowest part of the thread is to what it was in equilibrium in the ratio $m^{\prime}: m n \cot ^{2} \gamma+m^{\prime}$, where $m$ is the mass of a particle and $m^{\prime}$ the mass of a ring.

6. Three particles $A, B, C$ of equal masses are attached at the ends and middle point of a thread so that $A B=B C=a$, and the particles are moving at right angles to the thread, which is straight, with the same velocities, when $B$ impinges directly on an obstacle. Prove that, if there is perfect restitution, the radii of curvature of the paths which $A$ and $C$ begin to describe are equal to $\frac{1}{4} a$. 
7. Two particles, of masses $M$ and $n M$, are attached respectively to a point of a thread distant $a$ from one end and to that end, and the other end is fixed to a point on a smooth table on which the particles rest, the thread being in two straight pieces containing an obtuse angle $\pi-a$. Prove that, if the particle $n M$ is projected on the table at right angles to the thread, the initial radius of curvature of its path is $a(1+n \sin a \cos a)$.

8. Two particles $P, Q$ of equal mass, are connected by a thread of length $l$ which passes through a small hole in a smooth table; $P$ being at a distance $c$ from the hole and $Q$ hanging vertically, $P$ is projected on the table at right angles to the thread with velocity $v$; prove that the initial radius of curvature of $P$ 's path is $2 c v^{2} /\left(v^{2}+c g\right)$. Prove also that, if $Q$ is projected horizontally with velocity $v$, the initial radius of curvature of $Q$ 's path is

$$
2 v^{2}(l-c) /\left\{g(l-c) \sim v^{2}\right\} \text {. }
$$

\section{*250. Initial motions of Rigid bodies and connected}

systems. No new method is required for the solution of problems concerning rigid bodies of the same kind as those considered in Articles 247 and 248; the only point to be attended to that did not occur in those Articles is that the system of kinetic reactions for a rigid body reduce to a couple together with the kinetic reaction of a particle of mass equal to that of the body and moving with the centre of inertia. It is however worth while to remark that the expression for the moment of the kinetic reaction of a rigid body about the instantaneous centre at an instant when the velocity vanishes is the product of the angular acceleration and the moment of inertia of the body about an axis through the instantaneous centre perpendicular to the plane of motion [Example 2 of Article 220], and thus the equation of moments about the instantaneous centre takes, in initial motions from rest, a very simple form. Further it is worth while to notice that the kinetic reactions can always be expressed in terms of a finite number of geometrical quantities which are unconnected by any geometrical equations. This can usually be effected in a simple manner by help of the principles of relative motion laid down in Article 40, and the methods are of the same character as that adopted in Article 247. It may however happen that such methods are difficult of application, and when this is so we may begin by writing down the geometrical relations that hold between the coordinates of the points in any position. If we differentiate these relations twice with respect to the time, and, in the results obtained, substitute for every first differential coefficient of a 
geometrical quantity the value 0 , and for every geometrical quantity the value that it has in the initial position, we shall obtain the relations between the initial accelerations of the various geometrical quantities involved. Thus if $x, y$ are the coordinates of any particle whose acceleration is required, and $\theta, \phi, \ldots$ are a series of geometrical quantities which define the position of the system, there will be certain values $\theta_{0}, \phi_{0}, \ldots$ for these quantities in the initial position. Now the geometrical equations provide the means of expressing the $x$ and $y$ of the particle in any position in terms of the values of $\theta, \phi, \ldots$ for that position. Let $x=f(\theta, \phi, \ldots)$ be the form of one of the equations we can obtain. On differentiating we have

$$
\begin{aligned}
\dot{x}= & \frac{\partial f}{\partial \theta} \dot{\theta}+\frac{\partial f}{\partial \phi} \dot{\phi}+\ldots \\
\ddot{x}=\left(\frac{\partial^{2} f}{\partial \theta^{2}} \dot{\theta}+\frac{\partial^{2} f}{\partial \theta \partial \phi} \dot{\phi}\right. & +\ldots) \dot{\theta}+\left(\frac{\partial^{2} f}{\partial \theta \partial \phi} \dot{\theta}+\frac{\partial^{2} f}{\partial \phi^{2}} \dot{\phi}+\ldots\right) \dot{\phi}+\ldots \\
& +\frac{\partial f}{\partial \theta} \ddot{\theta}+\frac{\partial f}{\partial \phi} \ddot{\phi} \ldots
\end{aligned}
$$

Reducing, as explained, we obtain

$$
\ddot{x}_{0}=\left(\frac{\partial f}{\partial \theta}\right)_{0} \ddot{\theta}_{0}+\left(\frac{\partial f}{\partial \phi}\right)_{0} \ddot{\phi}_{0}+\ldots
$$

where $\ddot{x}_{0}, \ddot{\theta}_{0} \ldots$ denote the initial values of $\ddot{x}, \ddot{\theta}, \ldots$, and $\left(\frac{\partial f}{\partial \theta}\right)_{0}$, $\left(\frac{\partial f}{\partial \phi}\right)_{0}, \ldots$ denote the values of $\frac{\partial f}{\partial \theta}, \frac{\partial f}{\partial \phi}, \ldots$ when $\theta=\theta_{0}, \phi=\phi_{0}, \ldots$

Now this process can be carried further, and arranged as a process of approximation for expressing the values of $x, y, \ldots$ as series in ascending powers of the time. We have in fact as a first approximation $x=\frac{1}{2} \ddot{x}_{0} t^{2}, y=\frac{1}{2} \ddot{y}_{0} t^{2}$.

It will be easier to understand how this process is carried out after studying its application to a particular problem, and it will at the same time be seen how simplifications may at times suggest themselves. The problem chosen is intentionally complicated.

The method here sketched is of course applicable to systems of particles with invariable connexions as well as to rigid bodies with such connexions. 
*251. Illustrative problem. Two uniform rods $A B, B C$ of masses $m_{1}$, $m_{2}$ and lengths $a, b$ are freely hinged at $B$, and $A B$ can turn about $A$ in $a$ vertical plane. The system starts from rest in a position in which $A B$ is horizontal and $B C$ vertical. To discuss the initial curvature of the path of any point of $B C$.

Let $A B$ make an angle $\theta$ with the horizontal, and $B C$ an angle $\phi$ with the

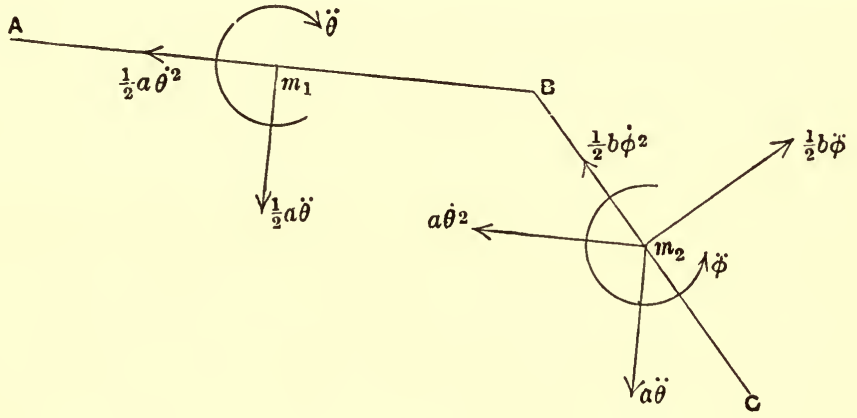

Fig. 75.

vertical at time $t$. Since $B$ describes a circle of radius $a$ about $A$, and since the centre of inertia of $B C$ describes a circle of radius $\frac{1}{2} b$ relative to $B$, the system of kinetic reactions will be as shown in the diagram.

By taking moments about $B$ for $B C$, and about $A$ for the system, we obtain the two equations

$$
\begin{array}{r}
m_{2}\left(\frac{1}{4} b^{2}+\frac{1}{12} b^{2}\right) \ddot{\phi}-m_{2} a \ddot{\theta} \frac{1}{2} b \sin (\theta+\phi)-m_{2} a \dot{\theta}^{2} \frac{1}{2} b \cos (\theta+\phi)=-\frac{1}{2} m_{2} g b \sin \phi, \\
m_{1}\left(\frac{1}{4} a^{2}+\frac{1}{12} a^{2}\right) \ddot{\theta}+m_{2} a \ddot{\theta}\left\{a+\frac{1}{2} b \sin (\theta+\phi)\right\}+m_{2} a \dot{\theta}^{2} \frac{1}{2} b \cos (\theta+\phi) \\
-m_{2} \frac{1}{12} b^{2} \ddot{\phi}-m_{2}\left\{\frac{1}{2} b+\alpha \sin (\theta+\phi)\right\} \frac{1}{2} b \ddot{\phi}-m_{2} a \cos (\theta+\phi) \frac{1}{2} b \dot{\phi}^{2} \\
\quad=\frac{1}{2} m_{1} g a \cos \theta+m_{2} g\left(a \cos \theta+\frac{1}{2} b \sin \phi\right) .
\end{array}
$$

Adding the equations, and dividing out common factors; we have

$$
\left(\frac{1}{3} m_{1}+m_{2}\right) a \ddot{\theta}-\frac{1}{2} m_{2} b \ddot{\phi} \sin (\theta+\phi)-\frac{1}{2} m_{2} b \dot{\phi}^{2} \cos (\theta+\phi)
$$

and the first equation is

$$
=g \cos \theta\left(\frac{1}{2} m_{1}+m_{2}\right) \ldots \ldots(1),
$$

$$
\frac{1}{3} b \ddot{\phi}-\frac{1}{2} a \ddot{\theta} \sin (\theta+\phi)-\frac{1}{2} a \dot{\theta}^{2} \cos (\theta+\phi)=-\frac{1}{2} g \sin \phi \text {. }
$$

In the initial position $\theta=0, \phi=0, \dot{\theta}=0, \dot{\phi}=0$, and we have

$$
\phi_{0}=0, \ddot{\theta}_{0}=\frac{3 m_{1}+6 m_{2}}{2 m_{1}+6 m_{2}} \frac{g}{a} \text {. }
$$

In any position we have, by Maclaurin's theorem,

also

$$
\begin{array}{ll}
\theta=\frac{1}{2} \ddot{\theta}_{0} t^{2}+\frac{1}{6} \dddot{\theta}_{0} t^{3}+\ldots, & \phi=\frac{1}{2} \ddot{\phi}_{0} t^{2}+\frac{1}{8} \dddot{\phi}_{0} t^{3}+\ldots ; \\
\dot{\theta}=\ddot{\theta}_{0} t+\frac{1}{2} \ddot{\theta}_{0} t^{2}+\ldots, & \dot{\phi}=\dddot{\phi}_{0} t+\frac{1}{2} \dddot{\phi}_{0} t^{2}+\ldots .
\end{array}
$$

Now, taking equation (2), we see that if $\ddot{\phi}_{0}$ were finite, $\phi$ would be of order $t^{3}$, and $\theta$ of order $t^{2}$, so that the terms would be respectively of orders $1,2,2$, 
3. This shows that $\dddot{\phi}_{0}$ must be zero. Again, if $\phi_{0}{ }^{\text {iv }}$ is finite the equation can be reduced, by picking out the terms of order 2 , to

$$
\frac{1}{6} b \phi_{0}^{\text {iv }}-\frac{1}{2} a \ddot{\theta}_{0}\left(\frac{1}{2} \ddot{\theta}_{0}\right)-\frac{1}{2} a \ddot{\theta}_{0}^{2}=0,
$$

giving

$$
\phi_{0}^{\mathrm{iv}}=\frac{9 a}{2 b} \ddot{\theta}_{0}^{2}=\frac{9 a}{2 b}\left(\frac{3 m_{1}+6 m_{2}}{2 m_{1}+6 m_{2}} \frac{g}{a}\right)^{2} \text {. }
$$

Again, taking equation (1) and observing that $\cos \theta=1-\frac{\theta^{2}}{2 !}+\frac{\theta^{4}}{4 !}-\ldots$ we see that the lowest power of $t$ in this series is the fourth, and then it appears from equation (1) that the lowest power of $t$ in $\ddot{\theta}$ is the fourth, so that the series for $\theta$ begins

$$
\theta=\frac{1}{2} \ddot{\theta}_{0} t^{2}+\frac{1}{6 !} \theta_{0}^{\mathrm{vi}} t^{6}+\ldots
$$

Going back now to equation (2) it is clear that $\ddot{\phi}$ contains no term in $t^{3}$ but there is a term in $t^{4}$. In fact, picking out the terms in $t^{4}$ in equation (2) we have

$$
\frac{1}{3} b \phi_{0}{ }^{\mathrm{v} 1} \frac{t^{4}}{4 !}-\frac{1}{2} a \ddot{\theta}_{0} \phi_{0}^{\mathrm{iv}} \frac{t^{4}}{4 !}=-\frac{1}{2} g \phi_{0}^{\mathrm{iv}} \frac{t^{4}}{4 !},
$$

giving

$$
\phi_{0}^{\mathrm{vi}}=\frac{3}{2}\left(\frac{a}{b} \ddot{\theta}_{0}-\frac{g}{b}\right) \phi_{0}^{\mathrm{iv}}=\frac{9}{4} \frac{m_{1}}{m_{1}+2 m_{2}} \ddot{\theta}_{0} \frac{a^{2}}{b^{2}} .
$$

Now, in the figure, taking as origin the initial position of $B$, and taking the axes of $x$ and $y$ horizontal and vertical, we can write for the coordinates of a point of $B C$ distant $r$ from $B$,

$$
x=-a(1-\cos \theta)+r \sin \phi, \quad y=a \sin \theta+r \cos \phi ;
$$

expanding these we have approximately

$$
x=-a\left(\frac{\theta^{2}}{2}-\frac{\theta^{4}}{24}\right)+r\left(\phi-\frac{1}{6} \phi^{3}\right), \quad y=a\left(\theta-\frac{\theta^{3}}{6}\right)+r\left(1-\frac{\phi^{2}}{2}+\frac{\phi^{4}}{24}\right),
$$

giving

$$
\begin{aligned}
& \left.x=-\frac{a}{8} t^{4} \ddot{\theta}_{0}{ }^{2}+\frac{r}{24} t^{4} \phi_{0}{ }^{\mathrm{iv}}=\frac{a}{16} \ddot{\theta}_{0}{ }^{2} t^{4}\left(\frac{3 r}{b}-2\right),\right\} \\
& y-r=\frac{1}{2} a t^{2} \ddot{\theta}_{0},
\end{aligned}
$$

correct as far as $t^{4}$. Hence the initial path of the point is approximately a parabola

$$
(y-r)^{2}=4 a x \frac{b}{3 r-2 b},
$$

and the radius of curvature of the path is $2 a b /(3 r-2 b)$ unless $r=\frac{2}{3} b$.

If however $r=\frac{2}{3} b$, in order to get an approximate equation to the path we must expand to a higher order. We find

$$
x=\frac{2}{3} b \phi_{0}{ }^{\mathrm{vi}} \frac{t^{6}}{6 !}=\frac{m_{1}}{m_{1}+2 m_{2}} \frac{a^{2}}{b} \frac{\ddot{\theta}_{0}^{3}}{480} t^{6},
$$

correct as far as $t^{6}$, and thus the initial path is given by the approximate equation.

$$
\left(y-\frac{2}{3} b\right)^{3}=60 a b x\left(1+2 m_{2} / m_{1}\right) \text {. }
$$


*252. Examples.

1. A uniform rod of length $2 \alpha$ and mass $m$ is symmetrically supported in a horizontal position by two inextensible cords attached to its ends each of length $l$ and making an angle $a$ with the vertical. Prove that if one cord is cut the tension in the other immediately becomes $m g \cos a /\left(1+3 \cos ^{2} a\right)$ and that the ratio of the initial angular accelerations of the cord and rod is $\frac{1}{3}(a / l) \tan a \sec a$.

2. Two equal uniform rods are freely jointed at common ends, the other end of the first is fixed so that the rods can turn about it, and the other end of the second is held at the same level as the fixed end of the first, so that the rods make equal angles $a$ with the horizontal, and this end is let go. Prove that the initial angular accelerations of the rods are in the ratio

$$
6-3 \cos 2 a: 9 \cos 2 a-8 \text {. }
$$

3. A uniform triangular disc is supported horizontally by three equal vertical cords attached to its corners. Prove that, if one cord is cut, the tension of each of the others is instantly halved.

4. Three equal uniform rods are freely jointed at $B$ and $C$ so as to form a quadrilateral $A B C D$, and the ends $A$ and $D$ can slide on a smooth horizontal rod. The system is initially held (by means of horizontal forces applied at $A$ and $D$ ) in a symmetrical position with $B C$ lowest and horizontal, and with $A B$ and $C D$ equally inclined at an angle $a$ to the horizontal. Prove that, when the ends $A$ and $D$ are released, the pressures at $A$ and $D$ are changed in the ratio $1+\sin ^{2} a: 5-3 \sin ^{2} a$.

5. A uniform rod of length $2 a$ is placed at an inclination $a$ to the horizontal in contact with a smooth peg at its middle point. Prove that the initial radius of curvature of the path of a particle distant $r$ from the middle point is $\left(\alpha^{2} / r\right) \tan a$.

6. Two equal uniform rods $A B, B C$ each of length $a$ are freely jointed at $B$ and can turn freely about $A$. Prove that, if the system is released from a horizontal position the initial radius of curvature of the path of $C$ is $\frac{2}{5} \alpha$.

\section{Small Oscillations.}

253. We have to consider the small motion of a system which is slightly displaced from a position of equilibrium. We confine our attention to cases where any position of the system is determined by assigning the value of a single geometrical quantity $\theta$, as in the case of the simple circular pendulum (Article 189). We can always choose $\theta$ to vanish in the position of equilibrium; for, if it has been chosen in any other way so that its value in the position of equilibrium is $\theta_{0}$, then $\theta-\theta_{0}$ can be used instead of $\theta$. 
Now the velocity of each particle of the system can be expressed in terms of $\theta$ and $\dot{\theta}$, and the kinetic energy $T$ is thus of the form $\frac{1}{2} A \dot{\theta}^{2}$ where $A$ may depend upon $\theta$, but does not vanish with $\theta$.

Also the potential energy $V$ vanishes with $\theta$, if the standard position is the position of equilibrium. Thus $V$ is a function of $\theta$ which may be expanded* in powers of $\theta$ and the series contains no term independent of $\theta$. Again, the principle of virtual work shows that $\frac{\partial V}{\partial \theta}$ vanishes with $\theta$, or that the term of the first order is missing from the series for $V$. Thus $V$ can be expressed as a series beginning with the term in $\theta^{2}$, and more generally we may say that, when $\theta$ is sufficiently small,

$$
V=\frac{1}{2} C \theta^{2} \text {, }
$$

where $C$ is a function of $\theta$ which is finite when $\theta=0$.

The equation of energy accordingly is

$$
\frac{1}{2} A \dot{\theta}^{2}+\frac{1}{2} C \theta^{2}=\text { const., }
$$

and on differentiating we have

$$
A \ddot{\theta}+\frac{1}{2}\left(\frac{\partial A}{\partial \theta}\right) \dot{\theta}^{2}+C \theta+\frac{1}{2}\left(\frac{\partial C}{\partial \theta}\right) \theta^{2}=0 .
$$

Omitting small quantities of an order higher than the first we have

$$
A \ddot{\theta}+C \theta=0 \text {, }
$$

where $A$ and $C$ have their values for $\theta=0$. Thus, if these two quantities have the same sign, the motion in $\theta$ is simple harmonic with period $2 \pi \sqrt{ }(A / C)$.

Now $A$ must be positive since otherwise the expression $\frac{1}{2} A \dot{\theta}^{2}$ could not represent an amount of kinetic energy. Hence there are oscillations in a real period if $C$ is positive.

The value $C$ (for $\theta=0$ ) is the value of $\frac{\partial^{2} V}{\partial \theta^{2}}$ for $\theta=0$, and thus the conditions for a real period of oscillation are the same as the conditions that $V$ may have a minimum value in the position of equilibrium.

* The possibility of the expansion is assumed. The function' $V$ is in fact supposed known for any value of $\theta$, and is assumed to be a function capable of expansion in Maclaurin's series in powers of $\theta$. We do not require to consider any other case. 
Exactly as in Article 65 we can see that, if the period is real, the motion can be indefinitely small; otherwise it soon becomes so large that we cannot simplify our equations by neglecting $\theta^{2}$. In the former case the equilibrium is stable and in the latter unstable.

The process adopted shows that we might have reduced the expression for $T$ by substituting zero for $\theta$ in $A$, and the expression for $V$ might have been taken to be simply the term of the series which contains $\theta^{2}$. These simplifications might have been made before differentiating the energy equation. Thus if we express the kinetic energy correctly to the second order of small quantities in the form $\frac{1}{2} A \dot{\theta}^{2}$, and the potential energy also correctly to the second order of small quantities in the form $\frac{1}{2} C \theta^{2}$, the period of the small oscillations is $2 \pi \sqrt{ }(A / C)$. In the case of a simple pendulum of mass $m$ and length $l, A$ is $m l^{2}$ and $C$ is $m g l$, so that

$$
A / C=l / g \text {. }
$$

In any other case we may compare the motion with that of a simple pendulum and then the quantity $g A / C$ is the length of a simple pendulum which oscillates in the same time as the system. It is called the length of the simple equivalent pendulum for the small oscillations of the system.

\section{Examples.}

1. Two rings of masses $m, m^{\prime}$ connected by a rigid rod of negligible mass are free to slide on a smooth vertical circular wire of radius $a$, the rod sub. tending an angle $a$ at the centre. Prove that the length of the simple equivalent pendulum for the small oscillations of the system is

$$
\left(m+m^{\prime}\right) a / \sqrt{ }\left(m^{2}+m^{\prime 2}+2 m m^{\prime} \cos a\right) \text {. }
$$

2. One end of an inextensible thread is attached to a fixed point $A$, and the thread passes over a small pulley $B$ fixed at the same height as $A$ and at a distance $2 a$ from it and supports a body of mass $P$. A ring of mass $M$ can slide on the thread and the system is in equilibrium with $M$ between $A$ and $B$. Prove that the time of a small oscillation is

$$
4 \pi \sqrt{ }\left\{a M P(M+P) / g\left(4 P^{2}-M^{2}\right)^{\frac{3}{2}}\right\} .
$$

3. A particle is suspended from two fixed points at the same level by equal elastic threads of natural length $a$, and hangs in equilibrium at a depth $h$ with each thread of length $l$. Prove that, if it is slightly displaced, parallel to the line joining the fixed ends of the threads, the length of the simple equivalent pendulum for the small oscillation is

$$
h l^{2}(l-a) /\left(l^{3}-h^{2} a\right) \text {. }
$$


4. Prove that, if the fixed points in Example 3 are at a distance $2 c$ apart and the particle is displaced vertically, the length of the simple equivalent pendulum is

$$
h l^{2}(l-a) /\left(l^{3}-c^{2} a\right) .
$$

5. A pulley of negligible mass is hung from a fixed point by an elastic cord of modulus $\lambda$ and natural length $a$, and an inextensible cord passing over the pulley carries at its ends bodies of masses $M$ and $m$. Prove that the time of a small oscillation in which the pulley moves vertically is $4 \pi \sqrt{ }\{M m a /(M+m) \lambda\}$.

*255. Rigid bodies and connected systems. In the application of the method of Article 253 to problems of oscillations of rigid bodies and connected systems usually the most important matter to attend to is that the potential energy $V$ must be expressed correctly to the second order of the small quantity $\theta$.

If we formed the equation of motion by a direct process it would be necessary in it to retain only the first power of $\theta$.

Now there are cases in which the equations of motion of a single rigid body can be readily obtained in a form free from unknown reactions by taking moments about the instantaneous centre, but in case this method is adopted there is again a matter to attend to, in that the equation obtained becomes nugatory if moments are taken about the instantaneous centre in the position of equilibrium. This position is, of course, occupied by the instantaneous centre at a single instant during the period, viz. : at the instant when $\theta=0$, and at any other instant during the period the instantaneous centre is in a slightly different position. The method which is now effective is to form the equation of moments about the instantaneous centre in a displaced position. In the application of this method it is worth while to remark that the moment of the kinetic reactions about the instantaneous centre is expressed, correctly to the first order of small quantities, by the product of the angular acceleration and the moment of inertia of the body about an axis through the instantaneous centre perpendicular to the plane of motion. [Example 2 of Article 220.]

We shall illustrate the application of the method just described and of that described in Article 253 by solving some problems.

\section{*256. Illustrative problems.}

I. A uniform rod can slide with its ends on two smooth straight wires equally inclined to the horizontal and fixed in a vertical plane. To find the oscillation about the horizontal position.

L. 
Let $O A, O B$ be the two wires, $a$ the angle which each of them makes with the horizontal, $A B$ the horizontal position of equilibrium of the rod. $A^{\prime} B^{\prime}$ a

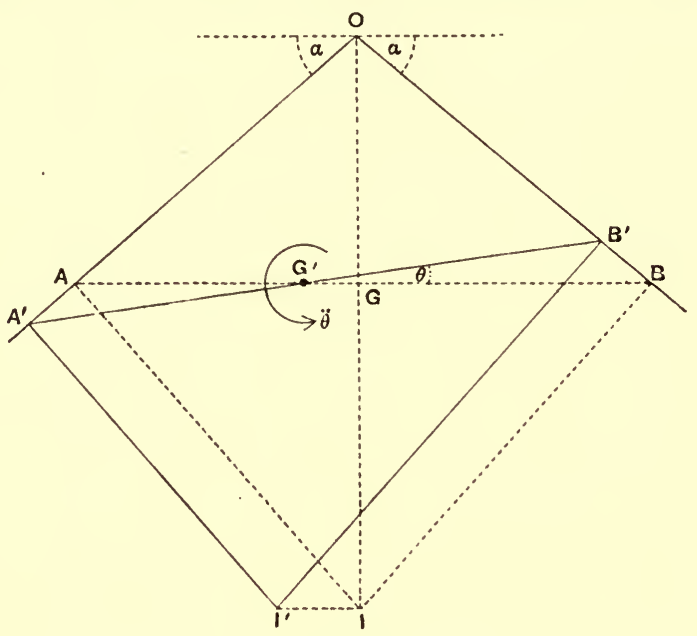

Fig. 76.

displaced position, $\theta$ the angle between $A B$ and $A^{\prime} B^{\prime}$. Then $\dot{\theta}$ is the angular velocity, and $\ddot{\theta}$ the angular acceleration of the rod. The instantaneous centre in any position is the point of intersection of perpendiculars to $O A, O B$ drawn from the ends of the rod. We denote by $I, I^{\prime}$ the positions of the instantaneous centre corresponding to $A B$ and $A^{\prime} B^{\prime}$, and by $G, G^{\prime}$ the corresponding positions of the centre of inertia.

The moment of the kinetic reaction about $I^{\prime}$ is $m\left(k^{2}+I^{\prime} G^{\prime 2}\right) \ddot{\theta}$, where $m$ is the mass of the rod and $k$ its radius of gyration about its centre of inertia. With sufficient approximation we may put $I G$ for $I^{\prime} G^{\prime}$.

The forces acting on the rod are pressures at its ends, whose lines of action pass through $I^{\prime}$, and its weight. Now $O I^{\prime}$ is a diameter of a circle of which $A^{\prime} B^{\prime}$ is a chord subtending an angle $\pi-2 a$ at the circumference, and thus $O I^{\prime}$ is of constant length and $I I^{\prime}$ is therefore ultimately at right angles to $O I$ and horizontal. Also $G G^{\prime}$ being ultimately at right angles to $I G$ is horizontal, and thus the moment of the weight about $I^{\prime}$ is $-m g\left(I I^{\prime}-G G^{\prime}\right)$. Hence we have the equation of moments

$$
m\left(k^{2}+I G^{2}\right) \ddot{\theta}=-m g\left(I I^{\prime}-G G^{\prime}\right) .
$$

Now let $2 a$ be the length of the rod. We find

$$
\begin{aligned}
I I^{\prime}=B B^{\prime} \sec a & =I B \theta \sec a=a \theta \operatorname{cosec} a \sec a, \\
G G^{\prime}=I G \theta & =a \theta \cot a,
\end{aligned}
$$

and the equation becomes

$$
m a^{2}\left(\frac{1}{3}+\cot ^{2} a\right) \ddot{\theta}=-m g a \theta(\sec a \operatorname{cosec} a-\cot a),
$$


where the right-hand member is $-m g a \theta \tan a$, and this equation shows that the motion in $\theta$ is the same as for small oscillations of a simple pendulum of length

$$
a \cot a\left(\frac{1}{3}+\cot ^{2} a\right) .
$$

II. A uniform rod is supported at its ends by two equal vertical cords suspended from fixed points. To find the small oscillations when the middle point moves vertically and the rod, remaining horizontal, turns round its middle point.

Let $2 a$ be the length of the rod, $l$ the length of either cord, $z$ the distance through which the middle point has risen at time $t, \theta$ the angle through which the rod has turned in the same time. The depth of either end $A$ or $B$ below the corresponding point of support is $l-z$, and the distance $A M$ or $B N$ of an end from the vertical plane through the points of support is $2 a \sin \frac{1}{2} \theta$. Hence we have

$$
(l-z)^{2}+4 a^{2} \sin ^{2} \frac{1}{2} \theta=l^{2} ;
$$

this equation shows that when $z$ and $\theta$ are small $z=\frac{1}{2}\left(a^{2} / l\right) \theta^{2}$ to the second order, and $\dot{z}=0$ to the first order.

Now, if $m$ is the mass of the rod, the kinetic energy in any position is

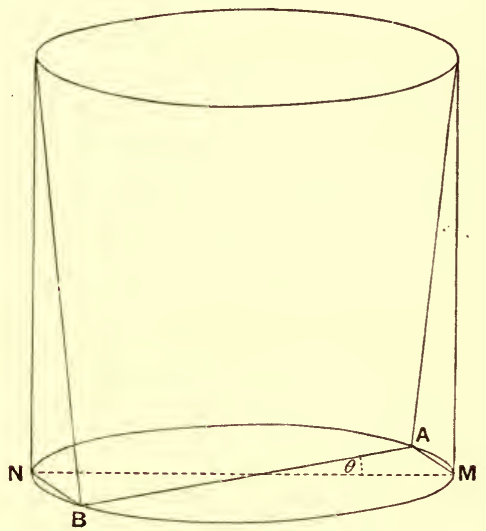

Fig. 77.

$$
\frac{1}{2} m\left(\dot{z}^{2}+\frac{1}{3} \alpha^{2} \dot{\theta}\right),
$$

and the potential energy is

$$
m g z
$$

the lowest position being the standard position.

Hence in the small oscillations the kinetic energy is, with sufficient approximation,

$$
\frac{1}{6} m a^{2} \dot{\theta^{2}}
$$

and the potential energy is, with sufficient approximation,

$$
\frac{1}{2} m g\left(\alpha^{2} / l\right) \theta^{2} \text {. }
$$

The motion in $\theta$ is therefore the same as for small oscillations of a simple pendulum of length $\frac{1}{3} l$.

\section{*257. Examples.}

1. A uniform rod of length $2 \alpha$ rests in a smooth bowl in the form of a surface of revolution whose axis is vertical, so that the ends are at points where the radius of curvature of the meridian curve is $\rho$ and the normal makes an angle $a$ with the vertical. Prove that the length of the simple 
equivalent pendulum for oscillations in the vertical plane through the equilibrium position of the rod is

$$
\frac{1}{3} \rho \alpha \cos a\left(1+2 \cos ^{2} a\right) /\left(\alpha-\rho \sin ^{3} a\right),
$$

provided this expression is positive.

2. A uniform rod of length $2 \alpha$ passes through a smooth ring fixed at a height $b$ above the vertex of a smooth bowl in the form of a surface of revolution whose axis is vertical, and rests in a vertical position. Prove that, if $c$ is the radius of curvature of the meridian curve at the vertex, the length of the simple equivalent pendulum for small oscillations is

$$
\frac{1}{3} c\left\{a^{2}+3(a-b)^{2}\right\} /\left(b^{2}-a c\right),
$$

provided this expression is positive.

3. A uniform rod of length $2 a$ is supported symmetrically by two cords each of length $l$ attached to its ends and to two fixed points at the same level distant $2(a+l \cos a)$ apart. Prove that the length of the simple equivalent pendulum for small oscillations in the vertical plane through the cords is

$$
\frac{1}{3} a l \sin a\left(1+3 \sin ^{2} a\right) /\left(\alpha+l \cos ^{3} a\right) .
$$

4. A number of equal uniform rods each of length $2 a$ are freely jointed at a common end and arranged at equal intervals like the ribs of an umbrella, and this cone of rods is placed in equilibrium over a smooth sphere so that the angle of the cone is $2 a$. Prove that for small vertical oscillations of the joint the length of the simple equivalent pendulum is

$$
\frac{1}{3} \alpha \cos a\left(1+3 \cos ^{2} a\right) /\left(1+2 \cos ^{2} a\right) \text {. }
$$

5. Prove that the length of the simple equivalent pendulum for small oscillations of the handle of a garden roller rolling on a horizontal walk is

$$
l-\frac{h}{1+M\left(k^{2}+a^{2}\right) / m a^{2}},
$$

where $a$ is the radius of the roller, $M$ the mass of the roller alone, $k$ its radius of gyration about its axis, $h$ the distance of the centre of inertia of the handle from the axis of the roller, and $l$ the length of the simple equivalent pendulum for the oscillations of the handle when the roller is held fixed.

6. Four equal uniform rods are freely jointed so as to have a common extremity, and four other like rods are similarly jointed; the free ends of the rods are then jointed in pairs so as to form eight edges of an octahedron: One of the joints where four rods meet is fixed and the other is attached to it by an elastic thread, so that in equilibrium the octahedron is regular and the thread vertical. Prove that the length of the simple equivalent pendulum for small vertical oscillations of the lowest point is $\frac{5}{6}\left(l-l_{0}\right)$, where $l$ and $l_{0}$ are the equilibrium length and the natural length of the thread. 


\section{Principles of Energy and Momentum.}

258. We have remarked that there are numerous cases in which the principles of energy and momentum supply all the first integrals of the equations of motion of a system, and thus suffice to determine the velocities of the parts of the system in any position. Applications of this method have already been made in parabolic motion, elliptic motion, the problem of two bodies, and other cases.

To illustrate further take the following problem:

Two particles $A$ and $B$ placed on a smooth horizontal table are connected by an elastic string of negligible mass. When the string is straight, and of its natural length, one of the particles is struck by a blow in the line of the string and away from the other particle; determine the subsequent motion.

Let $m$ be the mass of the particle struck, $m^{\prime}$ that of the other, $V$ the velocity with which $m$ begins to move. There is no tension in the string until it is extended, and thus at first $m^{\prime}$ has no velocity.

The centre of inertia moves on the table with uniform velocity $u$, $=m V /\left(m+m^{\prime}\right)$, in the line of the string. Let $x$ be the increase in the length of the string at time $t$, then the velocities of the particles are

$$
u+\frac{m^{\prime} \dot{x}}{m+m^{\prime}}, u-\frac{m \dot{x}}{m+m^{\prime}} .
$$

Hence the kinetic energy is $\frac{1}{2}\left(m+m^{\prime}\right) u^{2}+\frac{1}{2} \frac{m m^{\prime}}{m+m^{\prime}} \dot{x}^{2}$;

the potential energy is $\frac{1}{2} \frac{\lambda}{a} x^{2}$ so long as $x$ is positive, $a$ being the natural length of the string, and $\lambda$ the modulus of elasticity.

Thus the energy equation is

$$
\frac{1}{2} \frac{m^{2}}{m+m^{\prime}} V^{2}+\frac{1}{2} \frac{m m^{\prime}}{m+m^{\prime}} \dot{x}^{2}+\frac{1}{2} \frac{\lambda}{\alpha} x^{2}=\frac{1}{2} m V^{2},
$$

showing that the motion in $x$ is simple harmonic motion of period

$$
2 \pi \sqrt{ }\left\{m m^{\prime} \alpha /\left(m+m^{\prime}\right) \lambda\right\},
$$

so long as $x$ remains positive. Whenever the string is unstretched we have $\dot{x}= \pm V$. When $\dot{x}$ vanishes the string has its greatest length

$$
a+V \sqrt{ }\left\{m m^{\prime} a /\left(m+m^{\prime}\right) \lambda\right\} \text {. }
$$

We can thus describe the whole motion:- $m$ moves off with velocity $V$ which gradually diminishes, and $m^{\prime}$ moves in the same direction from rest with gradually increasing velocity; the string begins to extend, and continues to do so until it attains its greatest length; this happens at the end of a quarter of the period of the simple harmonic motion, and at this instant the particles have equal velocities $u$. The velocity of $m$ continues to diminish 
until it is reduced to $V\left(m-m^{\prime}\right) /\left(m+m^{\prime}\right)$, and the velocity of $m^{\prime}$ continues to increase until it has the value $2 m V /\left(m+m^{\prime}\right)$, these values are attained at the same instant; in the meantime the string contracts to its natural length $\alpha$, which it attains at the instant in question, and this happens at the end of half a period from the beginning of the motion. The particles then move with the velocities they have attained until $m^{\prime}$ overtakes $m$, when a collision takes place. The subsequent motion depends on the coefficient of restitution between the two particles; if this is unity, the relative motion is reversed. In any case the description of the subsequent motion involves nothing new.

\section{Examples.}

1. A shot of mass $m$ is fired from a gun of mass $M$ placed on a smooth horizontal plane and elevated at an angle $a$. Prove that, if the muzzle velocity of the shot is $V$, the range is

$$
\frac{2 V^{2}}{g} \frac{(1+m / M) \tan a}{1+(1+m / M)^{2} \tan ^{2} a} .
$$

2. A smooth wedge of mass $M$ whose base angles are $a$ and $\beta$ is placed on a smooth table, and two particles of masses $m$ and $m^{\prime}$ move on the faces being connected by an inextensible thread which passes over a smooth pulley at the summit. Prove that the wedge moves with acceleration

$$
g \frac{\left(m \sin \alpha \sim m^{\prime} \sin \beta\right)\left(m \cos \boldsymbol{a}+m^{\prime} \cos \beta\right)}{\left(m+m^{\prime}\right)\left(M+m+m^{\prime}\right)-\left(m \cos \boldsymbol{\alpha}+m^{\prime} \cos \beta\right)^{2}} .
$$

3. Two bodies of masses $m_{1}, m_{2}$ are connected by a spring of such strength that when $m_{1}$ is held fixed $m_{2}$ makes $n$ complete vibrations per second. Prove that, if $m_{2}$ is held, $m_{1}$ will make $n \sqrt{ }\left(m_{2} / m_{1}\right)$, and that, if both are free, they will make $n \sqrt{ }\left\{\left(m_{1}+m_{2}\right) / m_{1}\right\}$ vibrations per second, the vibrations in all cases being in the line of the spring.

4. Three equal particles are attached at equal intervals to an inextensible thread, and when the thread is straight, the two end ones are projected with equal velocities in the same sense at right angles to the thread. Prove that, if there are no external forces, the velocity of each of the end particles at the instant when they impinge is $\frac{1}{3} \sqrt{ } 3$ of their initial velocity.

5. A particle is attached by an elastic thread of natural length $a$ to a point of a smooth plank which is free to slide on a horizontal table, and the thread is stretched to a length $a+c$ in a horizontal line passing over the centre of inertia of the plank and the system is let go from rest. Prove that, if the plank and particle have equal masses, and the modulus of elasticity of the thread is equal to the weight of the particle, the velocity of the particle relative to the plank when the thread has its natural length is that due to falling through a height $c^{2} / \alpha$.

6. A spherical shell of radius $\alpha$ and mass $m$ contains a particle of the same mass which is attached to the highest point by an elastic thread of natural length $a$ stretched to length $a+c$ and also attached to the lowest 
point by an inextensible thread, and the shell rests on a horizontal plane. Suddenly the lower thread breaks, the particle jumps up to the highest point of the shell and adheres there, and it is observed that the shell jumps up through a height $h$. Prove that the modulus of elasticity of the upper thread is

$$
2 m g a(a+c+4 h) / c^{2} \text {. }
$$

Explain what external forces produce momentum in the system as a whole.

7. Three equal particles are connected by an inextensible thread of length $a+b$ so that the middle one is at distances $a$ and $b$ from the other two. The middle one is held fixed and the other two describe circles about it with the same uniform angular velocity so that the two portions of the thread are always in a straight line. Prove that, if the middle particle is set free, the tensions in the two parts of the thread are altered in the ratios $2 a+b: 3 a$ and $2 b+a: 3 b$, there being no external forces.

8. Two equal particles are connected by an inextensible thread of length $l$; one of them $A$ is on a smooth table and the other is just over the edge, the thread being straight and at right angles to the edge. Find the velocities of the particles inmediately after they have become free of the table, and prove (i) that in the subsequent motion the tension of the thread is always half the weight of either particle, and (ii) that the initial radius of curvature of the path of $A$ immediately after it leaves the table is $\frac{5}{12} \sqrt{ } 5 l$.

*260. Stability of steady motions. The principles we are now illustrating may frequently be applied to problems concerning the stability of steady motions of which we had an example in Article 65. We shall illustrate the method by considering the steady motion of a spherical pendulum.

Let $\theta$ be the angle the radius vector from the centre of the sphere to the particle makes with the downwards vertical at time $t, a$ the radius of the sphere, $\phi$ the angle contained between the plane through the particle and the vertical diameter and a fixed plane through the same diameter.

The energy equation is

$$
\begin{aligned}
\frac{1}{2} m a^{2}\left(\dot{H}^{2}+\sin ^{2} \theta \dot{\phi}^{2}\right)+m g a(1 & -\cos \theta) \\
& =\text { const. }
\end{aligned}
$$

and the equation of momentum about the vertical diameter is

$$
m a^{2} \sin ^{2} \theta \dot{\phi}=\text { const. }
$$

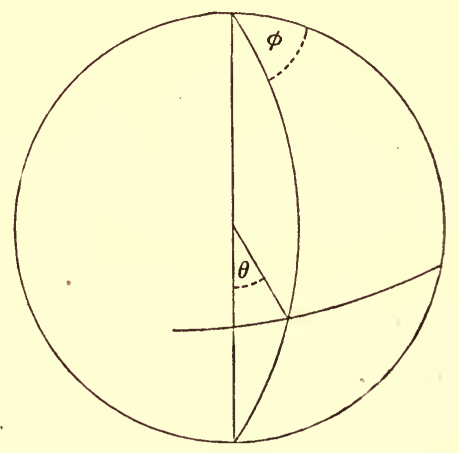

Fig. 78. 
We wish to discover the condition that motion in a horizontal circle, $\theta=\alpha$, with angular velocity $\omega$ may be possible. We have

$$
\sin ^{2} \theta \dot{\phi}=\sin ^{2} \alpha \omega,
$$

so that the energy equation may be written

$$
\frac{1}{2} a\left(\dot{\theta}^{2}+\frac{\sin ^{4} \alpha \omega^{2}}{\sin ^{2} \theta}\right)-g \cos \theta=\text { const. }
$$

Differentiating with respect to the time we obtain the equation

$$
\ddot{\theta}-\omega^{2} \frac{\sin ^{4} \alpha \cos \theta}{\sin ^{3} \theta}+\frac{g}{a} \sin \theta=0
$$

Now the steady motion is possible if $\omega$ is so adjusted that $\ddot{\theta}=0$ when $\theta=\alpha$. This gives us the condition

$$
a \omega^{2}=g \sec \alpha \text {. }
$$

(Cf. Example 1, Article 204.)

If the particle is projected from a point for which $\theta$ is nearly equal to $\alpha$, in a nearly horizontal direction, with an angular momentum $m a^{2} \omega \sin ^{2} \alpha$ about the vertical diameter, then either it tends to remain always very near the circle $\theta=\alpha$, or to depart widely from it. Supposing it to remain near the circle, we may put $\theta=\alpha+\chi$, expand the terms of equation (1), and reject powers of $\chi$ above the first. We thus find

$$
\ddot{\chi}+\chi \frac{g}{a} \frac{1+3 \cos ^{2} \alpha}{\cos \alpha}=0,
$$

showing that the particle oscillates about the state of steady motion in a period equal to that of a simple pendulum of length

$$
a \cos \alpha /\left(1+3 \cos ^{2} \alpha\right) \text {. }
$$

\section{*261. Examples.}

1. Prove that the steady motion with angular velocity $\omega$ of a conical pendulum of length $l$ is stable, and that, if a small disturbance is made, oscillations take place in time

$$
2 \pi l \omega / \sqrt{ }\left(3 g^{2}+l^{2} \omega^{4}\right)
$$

2. A particle describes a horizontal circle of radius $r$ in a smooth paraboloid of revolution whose axis is vertical and vertex downwards. Prove that, if it is slightly disturbed, its period of oscillation is

$$
\pi \sqrt{ }\left\{\left(r^{2}+4 a^{2}\right) / 2 g a\right\},
$$

where $4 a$ is the latus rectum. 
3. A small ring can slide on a smooth circular wire of radius $\alpha$ which rotates uniformly about a vertical diameter. Prove that, if in the position of relative equilibrium the radius through the ring makes an angle $a$ with the vertical, the period of small oscillations about the state of steady motion is the same as for a simple pendulum of length

$$
a \sec a(c+a \sin a) /\left(c+a \sin ^{3} a\right) .
$$

4. An elastic circular ring of mass $m$ and modulus of elasticity $\lambda$ rotates uniformly in its own plane about its centre under no external forces. Prove that, if $a$ is the radius in steady motion, and $l$ is the radius when the ring is unstrained, the period of the small oscillations about the state of steady motion is

$$
\sqrt{ }\{2 \pi \operatorname{lam} / \lambda(4 a-3 l)\} .
$$

*262. Illustrative problem. In further illustration of the principles of Energy and Momentum consider the following problem:

A uniform rod and a particle are connected by an inextensible thread attached to one end of the rod, the system is laid out straight, and the particle is projected at right angles to the thread. To find the motion when there are no forces.

Let $2 a$ be the length of the rod, $l$ the length of the thread, $\chi$ the angle the thread makes with the line of the rod produced at time $t$. Consider first

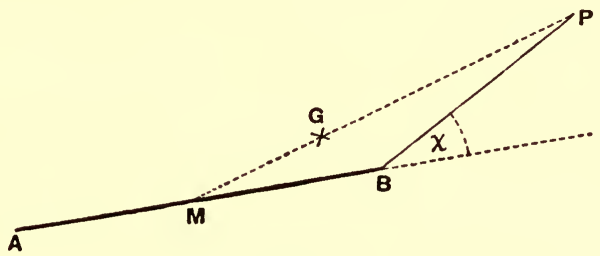

Fig. 79 .

the motion of the particle $P$ relative to the centre of inertia $M$ of the rod $A B$.

Let $\theta$ be the angle which $A B$ makes at time $t$ with its initial direction. Then the velocity of $B$ relative to $M$ is $a \dot{\theta}$ at right angles to $A B$, and, since $B P$ makes an angle $\theta+\chi$ with a line fixed in the plane of motion, the velocity of $P$ relative to $B$ is $l(\dot{\theta}+\dot{\chi})$ perpendicular to $B P$. The velocity of $P$ relative to $M$ is the resultant of these two velocities. Its resolved parts along and perpendicular to $A B$ are accordingly

$$
-l(\dot{\theta}+\dot{\chi}) \sin \chi \text { and } a \dot{\theta}+l(\dot{\theta}+\dot{\chi}) \cos \chi \text {. }
$$

Now the centre of inertia $G$ is always at the point dividing $M P$ in the ratio of the masses of the particle and the rod, and, if these masses are $p$ and $m$ respectively, the velocity of $M$ relative to $G$ has components

$$
\frac{p}{m+p} l(\dot{\theta}+\dot{\chi}) \sin \chi \text { and }-\frac{p}{m+p}\{a \dot{\theta}+l(\dot{\theta}+\dot{\chi}) \cos \chi\}
$$


along and perpendicular to $A B$, and the velocity of $P$ relative to $G$ has components

$$
-\frac{m}{m+p} l(\dot{\theta}+\dot{\chi}) \sin \chi \text { and } \frac{m}{m+p}\{a \dot{\theta}+l(\dot{\theta}+\dot{\chi}) \cos \chi\}
$$

in the same directions.

Hence the moment of momentum in the motion relative to $G$ is

$$
{ }_{3}^{1} m a^{2} \dot{\theta}+\frac{m p}{m+p}[(a+l \cos \chi)\{a \dot{\theta}+l(\dot{\theta}+\dot{\chi}) \cos \chi\}+l \sin \chi\{l(\dot{\theta}+\dot{\chi}) \sin \chi\}],
$$

or

$$
\frac{1}{3} m a^{2} \dot{\theta}+\frac{m p}{m+p}[(a+l \cos \chi) a \dot{\theta}+(l+a \cos \chi) l(\dot{\theta}+\dot{\chi})] ;
$$

also twice the kinetic energy in the motion relative to $G$ is

$$
\frac{1}{3} m a^{2} \dot{\theta}^{2}+\frac{m p}{m+p}\left[\alpha^{2} \dot{\theta}^{2}+l^{2}(\dot{\theta}+\dot{\chi})^{2}+2 \alpha l \dot{\theta}(\dot{\theta}+\dot{\chi}) \cos \chi\right] .
$$

Now the centre of inertia moves with uniform velocity in a straight line; and thus the kinetic energy of the whole mass placed at the centre of inertia and moving with it is constant, and the moment about any fixed axis of the momentum of the whole mass placed at the centre of inertia and moving with it is also constant. Also the kinetic energy of the system and its moment of momentum about any fixed axis are constants. Hence the moment of momentum in the motion relative to $G$ and the kinetic energy in the same relative motion are constants.

Let $V$ be the velocity with which the particle was initially projected at right angles to the thread; then the initial values of the moment of momentum and kinetic energy in the motion relative to $G$ are

$$
(a+l) V m p /(m+p) \text {, and } \frac{1}{2} V^{2} m p /(m+p) \text {. }
$$

Hence throughout the motion we have the equations

$$
\left.\begin{array}{l}
\frac{1}{3}(1+m / p) a^{2} \dot{\theta}+a \dot{\theta}(a+l \cos \chi)+l(\dot{\theta}+\dot{\chi})(l+a \cos \chi)=(a+l) V, \\
\frac{1}{3}(1+m / p) a^{2} \dot{\theta}^{2}+a^{2} \dot{\theta}^{2}+l^{2}(\dot{\theta}+\dot{\chi})^{2}+2 a l \dot{\theta}(\dot{\theta}+\dot{\chi}) \cos \chi=V^{2} .
\end{array}\right\}
$$

*263. Kinematical Note. It is sometimes convenient in calculating the velocities of points in a connected system to use the coordinates of a point referred to axes which do not retain the same direction. Thus in the problem of Article 262 we might have obtained the velocity of $P$ relative to $M$ by taking as axes lines through $M$ along and perpendicular to $A B$. When we wish to calculate the velocity of a point in this way we have to attend to the fact that the component velocities parallel to the moving axes are not the differential coefficients (with respect to the time) of the coordinates referred to the same axes.

Consider the motion of a particle $l^{\prime}$ whose coordinates at time $t$ are $x^{\prime}, y^{\prime}$ referred to rectangular axes rotating in their own plane about the origin; let $\phi$ be the angle which the axis of $x^{\prime}$ makes with a fixed axis of $x$ in the plane at time $t$, and $x, y$ the coordinates of the particle referred to fixed 
rectangular axes of $x$ and $y$. Also let $u, v$ be component velocities of the particle parallel to the axes of $x^{\prime}$ and $y^{\prime}$.

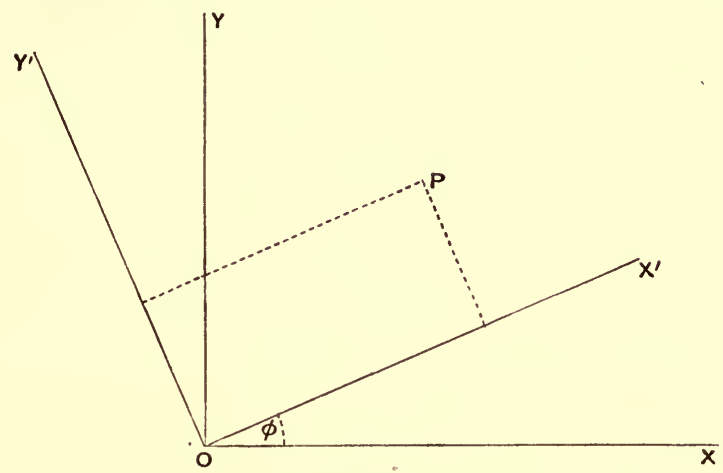

Fig. 80 .

We have

whence

$$
x=x^{\prime} \cos \phi-y^{\prime} \sin \phi, \quad y=y^{\prime} \cos \phi+x^{\prime} \sin \phi,
$$

$$
\left.\begin{array}{l}
\dot{x}=\left(\dot{x}^{\prime}-y^{\prime} \dot{\phi}\right) \cos \phi-\left(\dot{y}^{\prime}+x^{\prime} \dot{\phi}\right) \sin \phi, \\
\dot{y}=\left(\dot{y}^{\prime}+x^{\prime} \dot{\phi}\right) \cos \phi+\left(\dot{x}^{\prime}-y^{\prime} \dot{\phi}\right) \sin \phi .
\end{array}\right\}
$$

Also

$$
\dot{x}=u \cos \phi-v \sin \phi, \quad \dot{y}=v \cos \phi+u \sin \phi \text {. }
$$

Hence we find

$$
u=\dot{x}^{\prime}-y^{\prime} \dot{\phi}, \quad v=\dot{y}^{\prime}+x^{\prime} \dot{\phi} .
$$

Now, if we write $\omega$ for $\dot{\phi}, \omega$ is the angular velocity of the moving axes, and the resolved parts parallel to the moving axes of the velocity of the particle whose coordinates are $x^{\prime}, y^{\prime}$ are

$$
\dot{x}^{\prime}-\omega y^{\prime} \text { and } \dot{y}^{\prime}+\omega x^{\prime} \text {. }
$$

We may prove in precisely the same way that, if $a, \beta$ are the resolved parts of the acceleration of $P$ parallel to the axes of $x^{\prime}, y^{\prime}$, then

$$
a=\dot{u}-\omega v \text { and } \beta=\dot{v}+\omega u \text {. }
$$

In the problem of Article 262, taking axes through $M$ along and perpendicular to $A B$, the angular velocity of the moving axes is $\dot{\theta}$, and the coordinates of $P$ are $a+l \cos \chi$ and $l \sin \chi$. From these the component velocities of $P$ relative to $M$ otherwise obtained in that Article might be deduced.

\section{*264. Examples.}

1. Two equal circular rings each of radius $a$ and radius of gyration $k$ about its centre are freely pivoted together at a point of their circumferences so that their planes are parallel, and the rings are so thin they may be regarded as in the same plane. The system being at rest on a smooth table with the pivot in the line of centres, the pivot is struck by a blow perpendicular to the line of centres so that it moves off with velocity $V$. Prove that the angle $\theta$ which either radius through the pivot makes with its initial direction at any subsequent time is given by the equation

$$
k^{2}\left(k^{2}+\alpha^{2} \sin ^{2} \theta\right) \dot{\theta}^{2}=V^{2} \alpha^{2} .
$$


2. A uniform straight tube of length $2 \alpha$ contains a particle of equal mass, and, the particle being at the middle point, the tube is started to rotate about that point with angular velocity $\omega$. Prove that, if there are no external forces, the velocity of the particle relative to the tube when it leaves it is $a \omega \sqrt{ } \frac{2}{5}$.

3. Two horizontal threads are attached to a circular cylinder of negligible mass whose axis is vertical, are coiled in opposite directions round it, and carry equal particles which are initially at rest on two smooth horizontal planes. One of the particles is struck at right angles to its thread so that it starts off with velocity $V$ and its thread begins to unwind from the cylinder. Prove that, if the initial length of the straight portion of the thread attached to the particle struck is $c$, its length $r$ at time $t$ is given by the equation

$$
r^{2}=c^{2}+2 a V t+\frac{1}{2} V^{2} t^{2},
$$

the cylinder being free to turn about its axis.

4. A thread is attached to a rigid cylinder of radius $a$ and moment of inertia $I$ about its axis, and carries a particle of mass $m$ which is free to move on a smooth plane perpendicular to the axis, while the cylinder is free to rotate about the axis. The particle is projected on the plane at right angles to the thread with velocity $V$ so that the thread tends to wind up round the cylinder. Prove that the length $r$ of the straight portion at any subsequent time is given by the equation

$$
\left(I+m a^{2}\right) r^{2} \dot{r}^{2}=\left\{I+m\left(r^{2}+a^{2}-c^{2}\right)\right\} a^{2} V^{2},
$$

where $c$ is the initial value of $r$, and deduce that

where $M=I / a^{2}$.

$$
r^{2}-c^{2}=2 a V t+V^{2} t^{2} m /(M+m),
$$

5. A cone of vertical angle $2 a$ is free to turn about its axis, and a smooth groove is cut in its surface so as to make with the generators an angle $\beta$. A particle of mass $m$ moves in the groove, and starts at a distance $c$ from the vertex. Prove that, if at any subsequent time the particle is at a distance $r$ from the vertex and the cone has turned through an angle $\theta$, $r$ and $\theta$ are connected by the equation

$$
\left(I+m c^{2} \sin ^{2} a\right) e^{2 \theta \sin \alpha \cot \beta}=\left(I+m r^{2} \sin ^{2} a\right),
$$

where $I$ is the moment of inertia of the cone about its axis.

6. An elliptic tube of latus rectum $2 l$, eccentricity $e$, and moment of inertia $I$ about its major axis, is rotating freely about its major axis, which is fixed, with angular velocity $\Omega$, and contains a particle of mass $m$ which is

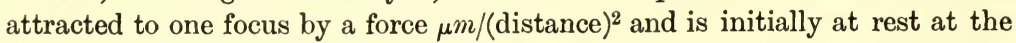
end of the major axis nearest the centre of force. Prove that, if the particle is slightly displaced, and if $\mu e(1+e)^{2}<l^{3} \Omega^{2}$, it will come to rest relatively to the tube at an end of the nearer latus rectum, provided

$$
\Omega^{2} l=2 \mu m e\left(1 / m l^{2}+1 / I\right) \text {. }
$$


7. Four equal uniform rods freely hinged together so as to form a rhombus of side $2 \alpha$ rotate about one diagonal which is fixed in a vertical position, the highest point being fixed and the lowest being free to slide on the diagonal. Find the angular velocity in the steady motion in which each rod makes an angle $a$ with the vertical, and prove that the period of the small oscillations about this state of steady motion is the same as for a simple pendulum of length $\frac{2}{3} a \cos a\left(1+3 \sin ^{2} a\right) /\left(1+3 \cos ^{2} a\right)$.

\section{Motion of a String or Chain.}

265. Suppose in the first place that a uniform inextensible chain is in rectilinear motion in such a way that every particle moves along the line of the chain. Then, if $V$ is the velocity of any particle, the condition of inextensibility requires that every particle moves with the same velocity $V$; and since this condition holds at all times every particle moves with the same acceleration. In such cases any part of the string which is in continuous rectilinear motion moves like a particle at its centre of inertia under the action of the resultant of the forces acting on the part, every particle having the velocity and acceleration of the centre of inertia. The special interest presented by problems of this kind attaches to the magnitude of the tension at a place where the character of the motion changes discontinuously.

The principle to be adopted in such cases is that the increase of momentum of any system in any time is equal to the impulse of the resultant force acting on the system during that time (cf. Article 113). Taking, as the system, a small element of the chain whose motion is changing discontinuously, this element passes in a very short interval from one state of motion to another, and the product of the interval and the resultant of the tensions on the ends is equal to the momentum generated in the element. The method of application of this principle will be made clearer by means of an example.

\section{Illustrative Problems.}

I. A chain is coiled at the edge of a table with one end just hanging over. To find the motion.

At any time $t$ let $x$ be the length which has fallen over the edge, $T$ the tension at the edge in the falling portion. There is no tension in the part coiled up. Let $m$ be the mass per unit length of the chain. 
In time $d t$ a portion $m \dot{x} d t$ is set in motion with velocity $\dot{x}$. We therefore have

$$
T d t=m \dot{x} d t \cdot \dot{x},
$$

or

$$
T=m \dot{x}^{2} \text {. }
$$

The equation of motion of the falling portion is therefore

Writing $v$ for $\dot{x}$, this is

$$
m x \ddot{x}=m x g-m \dot{x}^{2} .
$$

or

$$
\begin{aligned}
& x v \frac{d v}{d x}+v^{2}=g x, \\
& \frac{d}{d x}\left(x^{2} v^{2}\right)=2 g x^{2} .
\end{aligned}
$$

Integrating, and observing that $v$ and $x$ vanish together, we have

$$
v^{2}=\frac{2}{3} g x,
$$

giving the velocity of the falling portion when its length is $x$.

The time until the length is $x$ is

$$
\int_{0}^{x} \frac{d x}{\sqrt{ }\left(\frac{2}{3} g x\right)}=\sqrt{\frac{6 x}{g}}
$$

We note that in this and similar problems energy is dissipated in the impulsive action at the place where the discontinuous change of motion occurs.

II. A chain, one end of which is held fixed, is initially held with the other end close to the fixed end, and the other end is then let go. To find the motion.

Let $2 l$ be the length of the chain, $m$ the mass per unit length, $l+x$ the length of the part that has come to rest at time $t, T$ the tension

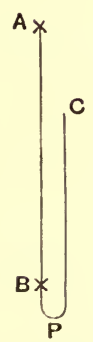
at its lower end.

The free end has fallen throngh $2 x$ under gravity, so that

$$
2 x=\frac{1}{2} g t^{2},
$$

and the falling portion is free from tension.

An element, of mass mgtdt, passes from motion with velocity $g t$ to rest in an interval $d t$, so that the momentum destroyed by the impulse $T d t$ is $m g^{2} t^{2} d t$.

$$
\text { Hence } \quad T=m g^{2} t^{2} \text {. }
$$

Fig. 81. Thus the motion and the tension at any time are determined.

267. Constrained motion of a chain under gravity. For a chain moving in a tube or in a groove on a surface, the condition of inextensibility has the same form as before, i.e. every particle of the chain has the same velocity along the tangent to the chain at the point occupied by the particle.

Let $m$ be the mass per unit length of the chain, $T$ the tension at any particle distant $s$ from a chosen particle, $v$ 
the velocity, $\phi$ the angle between the vertical and the normal at any point, $R$ the pressure on the curve per unit length, $\mu R$ the

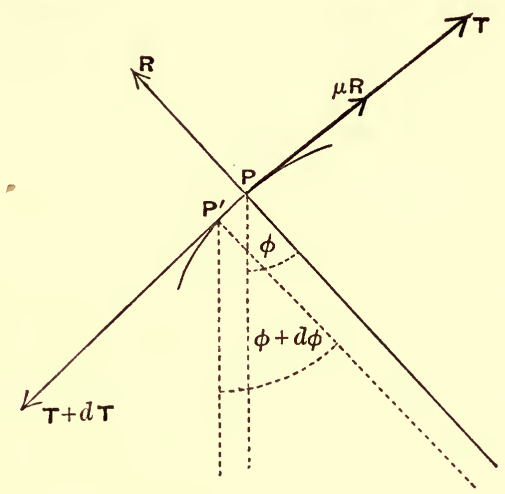

Fig. 82 .

friction also per unit length, and suppose that the sense of $v$ is that in which $s$ increases. The equations of motion of an element are found by resolving along the tangent and normal, in the forms

$$
\begin{aligned}
m d s \dot{v} & =m g d s \sin \phi+(T+d T) \cos (d \phi)-T-\mu R d s, \\
m d s \frac{v^{2}}{\rho} & =m g d s \cos \phi+(T+d T) \sin d \phi-R d s,
\end{aligned}
$$

and these are

$$
\begin{gathered}
m \dot{v}=m g \sin \phi+\frac{d T}{d s}-\mu R \\
m \frac{v^{2}}{\rho}=m g \cos \phi+\frac{T}{\rho}-R \ldots .
\end{gathered}
$$

where $\rho$ is the radius of curvature of the curve of the chain at the point distant $s$ from the chosen particle.

In the case of a chain with free ends, and on a smooth curve, the first equation (with $\mu R$ omitted) can be integrated, and the integral is the equation of energy. When $v$ is found from this equation we can find the tension at any point by substituting in equation (1), and then equation (2) determines the pressure.

\section{Examples.}

1. A uniform chain of length $a$ is laid out straight on a smooth table at right angles to the edge, and one end is put just over the edge. Prove that, if the edge of the table is rounded off so that the part of the chain which 
has run off at any time is vertical, the velocity of the chain as the last element leaves the table is $\sqrt{ }(a g)$.

2. A uniform chain of length $l$ and weight $W$ is suspended by one end and the other end is at a height $h$ above a smooth table. Prove that, if the upper end is let go, the pressure on the table as the coil is formed increases from $2 h W / l$ to $(2 h+3 l) W / l$.

3. A uniform chain $A B$ is held with its lower end fixed at $B$ and its upper end $A$ at a vertical distance above $B$ equal to the length of the chain. The end $A$ is released, and at the instant when it passes $B$ the end $B$ is also released. Prove that the chain becomes straight after an interval equal to three-quarters of that in which $A$ fell to $B$.

4. Two uniform chains whose masses per unit of length are $m_{1}$ and $m_{2}$ are joined by a thread passing over a fixed smooth pulley. Initially the chains are held up in coils and they are released simultaneously without causing any finite impulse in the thread. Prove that, until one of the chains has become entirely uncoiled, the thread slips over the pulley with uniform acceleration

$$
g\left(\sqrt{ } m_{1} \sim \sqrt{ } m_{2}\right) /\left(\sqrt{ } m_{1}+\sqrt{ } m_{2}\right),
$$

and that the portions of the chains which have become straight increase during the interval with uniform accelerations

$$
2 g \sqrt{ } m_{2} /\left(\sqrt{ } m_{1}+\sqrt{ } m_{2}\right) \text { and } 2 g \sqrt{ } m_{1} /\left(\sqrt{ } m_{1}+\sqrt{ } m_{2}\right) .
$$

5. A uniform chain of length $l$ and weight $W$ is placed on a line of greatest slope of a smooth plane of inclination $a$ to the horizontal so that it just reaches to the bottom of the plane where there is a small smooth pulley over which it can run off. Prove that when a length $x$ has run off the tension at the bottom of the plane is

$$
W(1-\sin a) x(l-x) / l^{2} .
$$

6. A uniform chain is held with its highest point on the highest generator of a smooth horizontal circular cylinder, and lies on the cylinder in a vertical plane, subtending an angle $\beta$ at the centre of the circular section on which it lies. Prove that, when the chain is let go, the lower end is the first part of it to leave the cylinder, and that this happens when the radius drawn through the upper end makes with the vertical an angle $\phi$ given by the equation

$$
\frac{1}{2} \beta \cos (\phi+\beta)=\sin \beta+\sin \phi-\sin (\phi+\beta) .
$$

*269. Chain moving freely in one plane. Let $s$ be the length of the chain measured from a chosen particle to any particle $P, s+d s$ the length up to a neighbouring particle $P^{\prime}, u$ the component velocity of the particle $P$ along the tangent to the chain in the sense in which $s$ increases, $v$ the component velocity of the same particle along the normal inwards, $\phi$ the angle the tangent at $P$ makes with a line fixed in the plane, $\rho$ the radius of curvature of the curve of the chain at $P$. Then there are two conditions 
of inextensibility. In the first place the velocity of $P^{\prime}$ has components

$$
u+\frac{\partial u}{\partial s} d s, v+\frac{\partial v}{\partial s} d s
$$

along the tangent and normal at $P^{\prime}$, and these lines make angles $\frac{\partial \phi}{\partial s} d s$ with the tangent and normal at $P$. Now the velocity of $P^{\prime}$

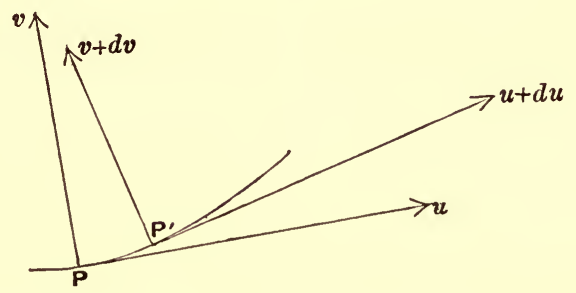

Fig. 83 .

relative to $P$ must ultimately be at right angles to $P P^{\prime}$, and therefore we have

$$
\left(u+\frac{\partial u}{\partial s} d s\right) \cos \left(\frac{\partial \phi}{\partial s} d s\right)-u-\left(v+\frac{\partial v}{\partial s} d s\right) \sin \left(\frac{\partial \phi}{\partial s} d s\right)=0,
$$

or

$$
\frac{\partial u}{\partial s}-\frac{v}{\rho}=0 \ldots \ldots \ldots \ldots \ldots \ldots \ldots \ldots \ldots(1) .
$$

Again, since $P P^{\prime}$ is a constant length and turns in the plane with angular velocity $\frac{\partial \phi}{\partial t}$, the velocity of $P^{\prime}$ relative to $P$ resolved at right angles to $P P^{\prime}$ is ultimately $d s \frac{\partial \phi}{\partial t}$, and thus we have

$$
\left(v+\frac{\partial v}{\partial s} d s\right) \cos \left(\frac{\partial \phi}{\partial s} d s\right)+\left(u+\frac{\partial u}{\partial s} d s\right) \sin \left(\frac{\partial \phi}{\partial s} d s\right)-v=d s \frac{\partial \phi}{\partial t},
$$

or

$$
\frac{\partial v}{\partial s}+\frac{u}{\rho}=\frac{\partial \phi}{\partial t}
$$

The equations (1) and (2) are the kinematic conditions of inextensibility of the chain.

To form the equations of motion of the chain under any forces we have to observe that $u, v, \phi$ are functions of two independent variables $s$ and $t$, and that the centre of inertia of every element of the chain moves under the action of the resultant of the tensions at its ends and of the bodily forces exerted upon it. 
Let $m$ be the mass per unit of length, and $T$ the tension at any point, $S$ and $N$ the tangential and normal components of the bodily force per unit mass in the senses of $u$ and $v$.

For the motion of the element between $s$ and $s+d s$ resolve parallel to the line from which $\phi$ is measured. We have $m d s \frac{\partial}{\partial t}(u \cos \phi-v \sin \phi)=(S \cos \phi-N \sin \phi) m d s$

$$
-T \cos \phi+(T+d T) \cos \left(\phi+\frac{\partial \phi}{\partial s} d s\right) \text {. }
$$

Performing the differentiations, and replacing $\partial \phi / \partial s$ by $1 / \rho$, we may equate coefficients of $\sin \phi$ and $\cos \phi$ on the two sides of the equation, since the line from which $\phi$ is measured is any line in the plane of motion. We thus have

$$
\left.\begin{array}{l}
m\left(\frac{\partial u}{\partial t}-v \frac{\partial \phi}{\partial t}\right)=m S+\frac{\partial T}{\partial s} \\
m\left(\frac{\partial v}{\partial t}+u \frac{\partial \phi}{\partial t}\right)=m N+\frac{T}{\rho},
\end{array}\right\}
$$

These are the equations of motion of the element by resolution parallel to the lines which are the tangent and normal at one end of it at time $t$.

It is to be noticed that the left-hand members of these equations might have been written down in accordance with the result of Article 263.

*270. Invariable form. Interesting cases of the motion of a chain arise in which the shape of the curve formed by the chain is invariable, but the chain moves along the curve. In discussing such cases it conduces to clearness to imagine the chain to be enclosed in a rigid tube, of the shape in question, and to move along the tube while the tube moves in its plane. The velocity of any point of the tube is then determined as the velocity of a point of a rigid body moving in two dimensions, and the velocity of any element of the chain will be found by compounding a certain velocity $w$ relative to the tube with the velocity of any point of the tube. The direction of $w$ is that of the tangent to the tube at the point, and its magnitude is variable from point to point in accordance with the kinematic conditions of inextensibility.

Taking now the special case of a uniform chain moving under gravity, we show that the chain can move steadily in the form of a common catenary, the curve retaining its position as well as its form. The velocity $w$ is in this case the velocity of an element of the chain, and, with the notation of Article 269, we have

$$
u=w, \quad v=0 .
$$


The equations (1) and (2) of that Article then give us

$$
\frac{\partial w}{\partial s}=0, \quad \frac{w}{\rho}=\frac{\partial \phi}{\partial t},
$$

so that the chain moves uniformly along itself.

The equations of motion (3) of the same Article are satisfied by

$$
T=m g c \sec \phi+m w^{2}
$$

provided the form of the curve is the catenary $s=c \tan \phi$.

\section{*271. Examples.}

1. Prove that any curve which is a form of equilibrium for a uniform chain under conservative forces is a form which the chain can retain when moving uniformly along itself under the same forces and that the tension is greater in the steady motion than in equilibrium by $m w^{2}$, where $m$ is the mass per unit length of the chain, and $w$ is the velocity with which the chain moves along itself.

2. A uniform chain moves over two smooth parallel rails distant $2 \alpha$ apart at the same level and is transferred from a coil at a distance $h$ vertically below one rail to a coil at a distance $h+b$ vertically below the other. Prove that the portion between the rails can be a common catenary provided the velocity of the chain along itself is $\sqrt{ }(g b)$.

3. A uniform chain moves in a plane under no forces in such a way that the curve of the chain retains an invariable form which rotates about a fixed point in the plane with uniform angular velocity $\omega$, while the chain advances relatively to the curve with uniform velocity $V$. Prove that the general $(p, r)$ equation of the curve must be of the form

where $a$ and $b$ are constants.

$$
(p+2 V / \omega) r^{2}=a p+b
$$

4. A uniform chain falls in a vertical plane under gravity. Prove that the square of the angular velocity of the tangent at any element is

$$
\frac{1}{m}\left(\frac{T}{\rho^{2}}-\frac{\partial^{2} T}{\partial s^{2}}\right)
$$

the notation being that of Article 269 .

5. A uniform chain hangs in equilibrium over a smooth pulley with one end fixed to the extremity of the vertical diameter and portions hanging vertically on both sides. Prove that if the end is set free the distance $y$ of the lowest point from the horizontal diameter during the first part of the motion satisfies the equation

$$
\left(l-y+\frac{1}{2} g t^{2}\right) \ddot{y}-(\dot{y}-g t)^{2}=g\left(y+\frac{1}{2} c\right),
$$

where $l$ is the length of the chain and $2 c$ is the circumference of the circle.

6. A uniform chain of length $2 L$ and mass $2 L \mu$ has its ends attached to two points $A, C$ and passes over a smooth peg $B$ between $A$ and $C$ and in the same horizontal line with them, the points $A, B, C$ being so close together 
that the parts of the chain between them may be considered vertical. Elastic threads of natural lengths $l$ and $l^{\prime}$ and moduluses $\lambda$ and $\lambda^{\prime}$ are fastened to points $P$ and $P^{\prime}$ of the chain on opposite sides of $B$ and their other ends are fixed to points $O$ and $O^{\prime}$ vertically below $P$ and $P^{\prime}$. The system oscillates so that the threads are always stretched and the points $P$ and $P^{\prime}$ are never for any finite time at rest. Prove that the time of a complete oscillation is

$$
2 \pi \sqrt{ }\left\{L l l^{\prime} \mu /\left(\lambda l^{\prime}+\lambda^{\prime} l-\mu g l l^{\prime}\right)\right\} \text {. }
$$

7. A fine elliptic tube is constrained to rotate with uniform angular velocity $\omega$ about its major axis which is vertical, and contains a uniform chain whose length is equal to a quadrant of the ellipse. Prove that, if $\omega^{2}=4 g / l$, where $l$ is the latus rectum of the ellipse, the chain will be in stable relative equilibrium with one end at the lowest point.

8. A rough helical tube of pitch $a$ and radius $a$ is placed with its axis vertical, and a uniform chain is placed within it, the coefficient of friction between the tube and the chain being $\tan a \cos \epsilon$. Prove that when the chain has fallen a vertical distance $m a$ its velocity is $\sqrt{ }(a g \sec a \sinh 2 \mu)$, where $\mu$ is determined by the equation

$$
\cot \frac{1}{2} \epsilon \tanh \mu=\tanh \left(\mu \sin \epsilon+\frac{1}{2} m \cos a \sin 2 \epsilon\right) .
$$

*272. Initial Motion. When the chain starts from rest in a position which is not one of equilibrium the initial velocities are zero, and the equations of motion are simplified by the omission of $\partial \phi / \partial t$. At the same time the kinematic conditions are altered in form. Since

$$
\frac{\partial}{\partial t}\left(\frac{1}{\rho}\right)=\frac{\partial^{2} \phi}{\partial s \partial t},
$$

this quantity is initially zero, and we may therefore differentiate equation (1) of Article 269, and write our result

$$
\frac{\partial^{2} u}{\partial s \partial t}=\frac{1}{\rho} \frac{\partial v}{\partial t} .
$$

Writing equations (3) of the same Article in the form

$$
\begin{aligned}
& \left.\frac{\partial u}{\partial t}=S+\frac{1}{m} \frac{\partial T}{\partial s},\right) \\
& \left.\frac{\partial v}{\partial \bar{t}}=N+\frac{1}{m} \frac{T}{\rho},\right\}
\end{aligned}
$$

differentiating the first with respect to $s$, multiplying the second by $1 / \rho$, and subtracting, we obtain an equation

$$
\frac{\partial}{\partial s}\left(\frac{1}{m} \frac{\partial T}{\partial s}\right)-\frac{1}{m} \frac{T}{\rho^{2}}=-\frac{\partial S}{\partial s}+\frac{N}{\rho} .
$$


This equation serves to determine the initial tension at any point of the chain. To determine the arbitrary constants which enter into the solution of the equation we have to use the conditions which hold at the ends, or at other special points, of the chain. Thus if one end of the chain is guided to move on a given curve the acceleration of the extreme particle must be directed along the tangent to the curve. Cases arise in which this method cannot be applied; thus, in the case of a heavy chain with an end moveable on a smooth straight wire not perpendicular to the tangent at the end, the equation of motion of an element at the end, found by resolving along the wire, cannot be satisfied if the acceleration of the element is finite (not infinite) and the tension is finite (not zero). The conclusion in such cases must be that the chain becomes slack at the end, and it may become slack throughout. In such cases it is usually convenient to suppose the end of the chain attached to a ring which can slide on the wire, and to take the mass of the ring, at first, to be finite: when the problem has been solved with this condition we can pass to the case above described by supposing the mass of the ring to be diminished without limit.

*273. Impulsive Motion. The equations of impulsive motion when the chain is suddenly set in motion follow at once by the method of Article 269. We have only to regard $S$ and $N$ as the resolved parts of an impulse reckoned per unit of mass applied to an element, and $T$ as impulsive tension. The equations are

$$
\left.\begin{array}{l}
m u=\frac{\partial T}{\partial s}+m S, \\
m v=\frac{T}{\rho}+m N .
\end{array}\right\}
$$

The kinematic conditions are the same as for a chain in continuous motion, viz.: equations (1) and (2) of Article 269.

In case no impulses are applied to the chain except at its ends, $S$ and $N$ vanish, and we can eliminate $u$ and $v$, obtaining an equation for $T$ in the form

$$
\frac{\partial}{\partial s}\left(\frac{1}{m} \frac{\partial T}{\partial s}\right)-\frac{T}{m \rho^{2}}=0 .
$$

The solution of this equation subject to the given terminal conditions gives the impulsive tension at any point of the chain. 


\section{*274. Examples.}

1. In the initial motion of a chain under gravity prove that the tension satisfies the equation

$$
\frac{\partial}{\partial s}\left(\frac{1}{m} \frac{\partial T}{\partial s}\right)-\frac{T}{m \rho^{2}}=0 .
$$

2. A uniform chain hangs under gravity with its ends attached to two rings which are free to slide on a smooth horizontal bar. Prove that, if the rings are initially held so that the tangents to the chain just below them make equal angles $\gamma$ with the horizontal, and are let go, the tension at the lowest point is changed in the ratio $2 M^{\prime}: 2 M^{\prime}+M \cot ^{2} \gamma$, where $M$ is the mass of the chain, and $M^{\prime}$ that of either ring. [Cf. Example 5, p. 281.]

3. If the ends of the chain of Example 2 are held fixed, and the chain is severed at its vertex, prove that the tension at a point where the tangent makes an angle $\phi$ with the horizontal immediately becomes

$$
\frac{1}{2} M g \phi \sec \phi \cos \gamma /(\cos \gamma+\gamma \sin \gamma) \text {. }
$$

4. Impulsive tensions $T_{\alpha}, T_{\beta}$ are applied at the ends of a piece of chain of mass $M$ hanging in the form of a common catenary with terminal tangents inclined to the horizontal at angles $a$ and $\beta$. Prove that the kinetic energy generated is

$$
\frac{1}{2} \frac{\tan \alpha-\tan \beta}{M}\left\{\frac{\left(T_{\alpha} \cos \alpha-T_{\beta} \cos \beta\right)^{2}}{a-\beta}+\left(T_{\alpha}^{2} \sin \alpha \cos \alpha-T_{\beta}^{2} \sin \beta \cos \beta\right)\right\} .
$$

\section{EXAMPLES.}

1. A ball is projected vertically with velocity $v_{1}$ from a point in a rigid horizontal plane, and when its velocity is $v_{2}$ a second ball is projected from the same point with velocity $v_{1}$; assuming the restitution in each impact to be perfect, prove (i) that the time between successive impacts of the two balls is $v_{1} / g$, (ii) that the heights at which they take place are alternately $\left(3 v_{1}-v_{2}\right)\left(v_{1}+v_{2}\right) / 8 g$ and $\left(3 v_{1}+v_{2}\right)\left(v_{1}-v_{2}\right) / 8 g$, (iii) that the velocities of the balls at the impacts are equal and opposite and alternately $\frac{1}{2}\left(v_{1}-v_{2}\right)$ and $\frac{1}{2}\left(v_{1}+v_{2}\right)$.

2. Two equal balls of radius $a$ are in contact and are struck simultaneously by a ball of radius $c$ moving in the direction of their common tangent; prove that, if all the balls are of the same material, the impinging ball will be reduced to rest if the coefficient of restitution is

$$
\frac{1}{2} c^{2}(a+c)^{2} / a^{3}(2 a+c) \text {. }
$$

3. Two equal balls lie in contact on a table. A third equal ball impinges on them, its centre moving along a line nearly coinciding with a horizontal common tangent. Assuming that the periods of the impacts do not overlap, prove that the ratio of the velocities which either ball will receive according as it is struck first or second is $4: 3-e$, where $e$ is the coefficient of restitution. 
4. Two spheres of equal radius and of masses $\lambda_{1} m$ and $\lambda_{2} m$ are lying in contact on a smooth horizontal plane. A third sphere of the same radius and of mass $m$ falls freely with its centre in the vertical plane containing the centres of the other two so as to strike them simultaneously. Assuming that there is no restitution in any of the impacts prove that the velocity produced in the sphere of mass $\lambda_{1} m$ is

$$
v \sqrt{ } 3\left(1+2 \lambda_{2}\right) /\left(1+4 \lambda_{1}+4 \lambda_{2}+12 \lambda_{1} \lambda_{2}\right),
$$

where $v$ is the velocity of the falling sphere just before impact.

5. From one corner $A$ of a rectangular billiard table a ball is projected in a direction making an angle $a$ with the side $A B$; it strikes first the side $B C$, then $A D$, then $D C$, then $B C$ again, and then returns to $A$. Prove that, if $e$ is the coefficient of restitution, $A B: A D=e^{2} \cot a: 1+e^{2}$.

6. Three smooth billiard balls of perfect restitution, each of radius $d$, rest on a smooth table, their centres forming a triangle $A B C$; prove that, if the ball $A$ is to cannon off $B$ on to $C$, the angle of impact at $B$ must lie between

$$
B-\frac{1}{2} \pi-\tan ^{-1} \frac{2 d \cos B}{c-2 d \sin B} \text { and } B+\delta-\frac{1}{2} \pi-\tan ^{-1} \frac{2 d \cos (B+\delta)}{c-2 d \sin (B+\delta)},
$$

where $\delta=\sin ^{-1} 4 d / a$.

7. Two bodies of masses $m, m^{\prime},\left(m>m^{\prime}\right)$, are connected by a string passing over a smooth pulley, the coefficient of restitution between the greater and the plane being unity and that between the smaller and the obstacle zero. They start from rest at the same distance $a$ above a fixed horizontal plane, and, when $m$ impinges on the plane, $m^{\prime}$ strikes against a fixed obstacle. Show that the two bodies are again in a position of instantaneous rest when $m$ is at a height $m^{2} a /\left(m+m^{\prime}\right)^{2}$ above the plane.

8. Show that it is possible to project a small elastic ball inside a regular polygon of $n$ sides so as to describe a regular polygon of the same number of sides, and prove that the ratio of the sides of the two polygons is

$$
2 \cos \frac{\pi}{n}:\left\{\sqrt{ }\left(1+\frac{1-e}{1+e} \sin \frac{2 \pi}{n}\right)+\sqrt{ }\left(1-\frac{1-e}{1+e} \sin \frac{2 \pi}{n}\right)\right\},
$$

where $e$ is the coefficient of restitution.

9. A ball is projected with velocity $V$ from a point of a plane inclined at an angle $a$ to the horizontal, the direction of projection is at right angles to the plane, and the coefficient of restitution between the ball and the plane is $e$. Prove that, before ceasing to bound, it will have described a length $2 V^{2} \sin a / g \cos ^{2} a(1-e)^{2}$ along the plane.

10. A hollow elliptic cylinder stands on a horizontal plane with its axis vertical. From the focus of a horizontal section a particle is projected in a horizontal direction with velocity $v$. Prove that if it returns to the point of projection the height of the section above the table is $2 m^{2} g a^{2} / n^{2} v^{2}$, where $m, n$ are any integers and $2 \alpha$ is the major axis, the coefficient of restitution in each impact being unity. 
11. A particle is projected inside a smooth tube of equal mass which is closed at both ends and lies on a smooth table. Prove that the distance travelled through by the tube when the particle has made $(n+1)$ impacts is $\alpha\left(1-e^{n}\right) /\left(e^{n}-e^{n+1}\right)$ or $a\left(1-e^{n+1}\right) /\left(e^{n}-e^{n+1}\right)$ according as $n$ is odd or even, $2 \alpha$ being the length of the tube, and $e$ the coefficient of restitution for each impact.

12. In a row of $n$ equal spheres, the coefficient of restitution between any two of which is $e$, one of the terminal spheres impinges directly on its neighbour. Prove that their final velocities are in the ratios

$$
a: a \beta: a \beta^{2}: a \beta^{3}: \ldots a \beta^{n-2}: \beta^{n-1},
$$

where $a=\frac{1}{2}(1-e)$ and $\beta=\frac{1}{2}(1+e)$.

13. Two unequal particles are attached to a thread which passes over a smooth pulley. Initially the smaller is in contact with a fixed horizontal plane, and the other at a height $h$ above the plane. Prove that, if the coefficient of restitution for each impact is $e$, and if $e$ is a root of any equation of the form $e^{n}-2 e+1=0$ with $n$ integral, the system will come to rest after a time $2 h(1+e) / v(1-e)$, where $v$ is the velocity of the particle of greater mass immediately before its first impact on the plane.

14. Two equal spheres are in contact, and are attached by equal threads to two other equal spheres at rest. The lines of the threads pass through the centres of the spheres to which they are attached and make angles of $30^{\circ}$ with that common tangent to the first two at their point of contact which lies in the plane of the four centres. A fifth equal sphere running along this common tangent strikes the first two symmetrically so that the threads become tight. Prove that the velocity of the impinging sphere is diminished in the ratio $7-12 e: 19$, where $e$ is the coefficient of restitution.

15. Two balls of masses $M, m$ and of equal radii, connected by an inextensible thread, lie on a smooth table with the thread straight, and a ball of the same radius and of mass $m^{\prime}$ moving parallel to the thread with velocity $v$ strikes the ball $m$ so that the line of centres $\left(m^{\prime}, m\right)$ makes an acute angle $a$ with the line of centres $(M, m)$. Prove that, if $e$ is the coefficient of restitution between $m$ and $m^{\prime}, M$ starts with velocity

$$
v m m^{\prime}(1+e) \cos ^{2} a /\left\{M m^{\prime} \sin ^{2} a+m\left(M+m+m^{\prime}\right)\right\} .
$$

16. Two balls are attached by inextensible threads to fixed points, and one of them, of mass $m$ describing a circle with velocity $u$ impinges on the other of mass $m^{\prime}$ at rest, so that the line of centres makes an angle $a$ with the thread attached to $m$ and the threads cross each other at right angles. Prove that $m^{\prime}$ will start to describe a circle with velocity

$$
m u \sin a \cos a(1+e) /\left(m \cos ^{2} a+m^{\prime} \sin ^{2} a\right),
$$

where $e$ is the coefficient of restitution between the balls. 
17. A particle, describing an ellipse about a focus, strikes a fixed plane through the focus at right angles to the major axis. Prove that, if the coefficient of restitution is equal to the eccentricity, the major axis of the new orbit is half that of the old.

18. Prove that the impulse necessary to make a particle of unit mass, moving in an equiangular spiral of angle $a$ under the action of a force to the pole, describe a circle under the action of the same force, is

$$
2 \sqrt{ }(F r) \sin \left(\frac{\pi}{4} \pm \frac{a}{2}\right),
$$

$r$ being the distance from the pole, and $F$ the force at the moment of impact.

19. A particle is describing an ellipse of eccentricity $e$ about a focus and when its radius vector is half the latus rectum it receives a blow which makes it move towards the other focus with a momentum equal to that of the blow. Find the position of the axis of the new orbit and show that its eccentricity is $\frac{1}{2}\left(e^{-1}-e\right)$.

20. A particle of mass $m$ is projected from a point $P$ with velocity $V$ and moves under a force to a fixed point $S$ varying inversely as the square of the distance. $P P^{\prime}$ is the chord through the other focus of the path. When the particle reaches $P^{\prime}$ the kinetic energy is increased by $\frac{1}{2} m V^{2} R /(4 a-R)$ by a tangential impulse, $R$ being the distance $S P$ and $2 a$ the major axis of the orbit. Prove that the new path is independent of the direction of projection.

21. A comet describing a parabola of latus rectum $2 l$ before it has reached the apse collides at a point of its orbit distant $R$ from the Sun with another comet of equal mass falling from rest at an infinite distance directly towards the Sun, and the two comets coalesce. Prove that the subsequent orbit is an ellipse of major axis $2 a$, given by $(1-l / R)^{2}=2 R / a$.

22. A particle is describing an ellipse about a centre of force in one fous $S$, and when it is at the end $E$ of the further latus rectum it receives a blow in direction $S E$ which makes it move at right angles to $S E$. Find the momentum generated by the blow, and prove that the particle will proceed to describe an ellipse of eccentricity $\sqrt{ }\left\{2 e^{2} /\left(1+e^{2}\right)\right\}$.

23. A particle is describing an ellipse about a focus $S$, and when it is at one end of the latus rectum it receives a blow which makes it describe a confocal hyperbola. Prove that the direction of the blow makes with the tangent to the ellipse an angle $\cot ^{-1} e$, where $e$ is the eccentricity of the ellipse.

24. A shell of mass $M$ is moving with velocity $V$. An internal explosion generates an amount $E$ of energy and thereby breaks the shell into two fragments whose masses are in the ratio $m_{1}: m_{2}$. The fragments continue to move in the original line of motion of the shell. Prove that their velocities are

$$
V+\sqrt{ }\left(2 m_{2} E / m_{1} M\right), \quad V-\sqrt{ }\left(2 m_{1} E / m_{2} M\right) .
$$


25. Weights $P$ and $W$ equilibrate on a wheel and axle of negligible mass. A weight $W$ is attached to $P$ and after the lapse of one second another weight $W$ is attached to the ascending weight $W$. Prove that, after the lapse of another second, the velocity of the ascending weight $2 \mathrm{~W}$ is

$$
g b(2 a-b) /\left(a^{2}+a b+2 b^{2}\right)
$$

$a$ being the radius of the wheel, and $b$ the radius of the axle.

26. A particle of mass $m$ is attached by inextensible threads to particles of masses $m^{\prime}$ and $m^{\prime \prime}$. The particles are placed on a smooth table with the threads in two perpendicular straight lines, and the particle $m$ is struck by a blow in the direction of the bisector of the angle between the threads so that both threads are jerked. Prove that the initial velocities of $m^{\prime}$ and $m^{\prime \prime}$ are in the ratio $m+m^{\prime \prime}: m+m^{\prime}$.

27. A particle of mass $M$ is projected with velocity $V$ in a direction making an angle $\theta$ with the horizontal, being attached to the point of projection by an inextensible thread of length $V^{2} \operatorname{cosec}^{2} \theta / 2 g$. Prove that the impulsive tension when the thread becomes tight is $M V \cos ^{2} \theta \operatorname{cosec} \theta$, and that immediately after the change of motion the tension is $M g\left(1-2 \sin ^{4} \theta\right)$.

28. Three particles $A, B, C$ of equal mass are placed on a smooth plane inclined at an angle $a$ to the horizontal, and $B, C$ are connected with $A$ by threads of length $h \sec a$ which make equal angles $a$ with the line of greatest slope through $A$ on opposite sides of it. If $A$ is struck by a blow along the line of greatest slope so as to start to move downwards with velocity $V$, find when the threads become tight, and prove that the velocity of $A$ immediately afterwards is

$$
V /\left(3-2 \sin ^{2} a\right)+2 g h \sin a / T^{r}
$$

29. Four equal particles are attached at the corners of a rhombus formed of four threads each of length $a$, and the system is moving on a horizontal plane with uniform velocity $u$ in the direction of the longer diagonal $A C$ when the end $A$ of that diagonal is suddenly fixed. Prove that the sides of the rhombus begin to turn with angular velocity $2 u \sin a / a\left(1+2 \sin ^{2} a\right)$, where $2 a$ is the acute angle of the rhombus.

30. A set of $2 n-1$ particles connected by inextensible threads are suspended from two fixed points in a horizontal line so as to hang symmetrically, their weights being such that each of the two lowest threads makes an angle $a$ with the horizontal and each of the threads makes an angle $a$ with the one below it. Prove that, if the lowest particle (mass $m$ ) is struck by a vertical impulse $P$, the horizontal component of the initial velocity of any particle will vary inversely as its mass, and the vertical component of the velocity of the $r$ th from the lowest will be

$$
\frac{P \sin a}{2 m \cos ^{2} a}\{(2 n-2 r-1) \sin a+2 \cos a \cot n a-\sin (2 r+1) a\} .
$$


31. Three particles of equal mass are attached at equal intervals to a rigid rod of negligible mass, and the system being at rest one of the extreme particles is struck by a blow at right angles to the rod. Prove that the kinetic energy imparted to the system when the other extreme particle is fixed and the rod turns about it is less than that which arises when the system is free in the ratio $24: 25$.

32. Two equal rigid rods $A B, B C$ of negligible masses carry equal particles attached at $A, C$ and the middle points of the rods, and, the rods being freely hinged at $B$ and laid out straight, the end $A$ is struck with an impulse at right angles to the rods. Prove that the velocities of the particles are in the ratios $9: 2: 2: 1$.

33. Four particles of equal masses are tied at equal intervals to a thread, and the system is placed on a smooth table so as to form part of a regular polygon whose angles are each $\pi-a$. Prove that if an impulse is applied to one of the end particles in the direction of the thread attached to it the kinetic energy generated is greater than it would be if the particles were constrained to move in a circular groove and the impulse were applied tangentially in the ratio $\cos ^{2} a+4 \sin ^{2} a: \cos ^{2} a+2 \sin ^{2} a$.

34. A rod of length $2 a$ is held in a position inclined at an angle $a$ to the vertical, and is then let fall on a smooth horizontal plane (no restitution). Prove that the end of the rod which strikes the plane will leave it immediately after impact if the height through which the rod falls is greater than

$$
\frac{1}{18} a \sec a \operatorname{cosec}^{2} \alpha\left(1+3 \sin ^{2} a\right)^{2} .
$$

35. A particle of mass $m$ impinges directly on a smooth uniform spheroid of mass $M$ and semiaxes $a, b$ at rest, no energy being lost in the impact. Show that, if

$$
1<M / m<6-10 a b /\left(a^{2}+b^{2}\right),
$$

the point of impact may be so chosen that the particle is reduced to rest.

36. A circular cylinder rocks between two parallel rails whose distance apart is less than the diameter of the cylinder. Prove that the greatest heights of the axis above its equilibrium position diminish in geometrical progression.

37. Any number of equal uniform rod $2 a$

37. Any number of equal uniform rods are jointed together so as to have a common extremity and placed symmetrically so as to be generators of a cone of vertical angle $2 a$, and the system falling with velocity $V$ strikes symmetrically a smooth fixed sphere of radius $c$ (no restitution). Prove that the angular velocity with which each rod begins to turn is

$$
V\left(c \cos a \sim a \sin ^{3} a\right) /\left(\frac{4}{3} a^{2} \sin ^{2} a+c^{2} \cot ^{2} a-a c \sin 2 a\right) .
$$


38. The corners $A, B$ of a uniform rectangular disc $A B C D$ are free to slide on two smooth fixed rigid wires $O A, O B$ at right angles to each other in a vertical plane and equally inclined to the vertical. The disc being in a position of equilibrium with $A B$ horizontal, find the velocity produced by an impulse applied along the lowest edge $C D$.

Prove that, if $A B=2 a, B C=4 a$, then $A B$ will just rise to coincidence with a wire if the impulse is such as would impart to a mass equal to that of the disc a velocity $\frac{2}{3} \sqrt{ }\{a g(4-2 \sqrt{ } 2)\}$.

39. A uniform rigid semicircular wire is rotating in its own plane about a hinge at one end, and is suddenly brought to rest by an impulse applied at the other end along the tangent at that end. Prove that the impulsive stress couple is greatest at a point whose angular distance from the hinge is $\phi$, where $\phi \tan \frac{1}{2} \phi=1$.

40. A heavy ring of rudius $a$ rolls with its plane vertical down a plane of inclination $a$ on which lie a series of pointed obstacles which are equal and at equal distances from each other, and which are sufficiently high to prevent the ring from ever touching the plane. Prove that, if the ring starts from rest in a position in which it is in contact with two obstacles, and if there is no slipping, its angular velocity $\omega$ as it leaves the $(n+1)$ th obstacle is given by

$$
\alpha \omega^{2}=2 g \sin a \sin \gamma \cos ^{4} \gamma\left(1-\cos ^{4 n} \gamma\right) /\left(1-\cos ^{4} \gamma\right),
$$

where $2 \gamma$ is the angle subtended at the centre by two adjacent obstacles when the ring touches both.

41. A circular disc, with $n$ spikes projecting from it in its plane at equal angular intervals, is projected with its plane vertical so as to strike a rough horizontal plane (zero restitution) so that the line joining the point of contact to the centre makes an angle $\pi / n$ with the vertical. Show that, if at the instant the velocity of the centre is $V$ and the angular velocity is $\omega$, the number of its spikes which strike the plane is the greatest integer in the value of $m$ given by the equation

$$
\left(1-2 a^{2} \kappa^{-2} \sin ^{2} \pi / n\right)^{m}\left(\kappa^{2} \omega+a V\right)=2 \kappa \sqrt{ }(a g) \sin _{r^{+}} \pi / 2 n,
$$

where $a$ is the radius of the circle on which the ends of the spikes lie, $k$ is the radius of gyration about the end of a spike and the radius of the disc is less than $a \cos \pi / n$.

42. A uniform ball moving without rotation with velocity $V$ strikes the ground at an angle $a$ with the vertical, and subsequently meets a bat whose plane is vertical and perpendicular to the plane of the ball's motion, and which is kept moving in the vertical plane of the ball's motion with a uniform velocity in a direction making a given angle with the horizontal. Prove that after striking the bat the ball will descend if the vertical velocity of the bat is greater than

$$
\frac{5}{2} V \cos a\left(e+\frac{2}{7} \tan a\right)
$$

gravity being neglected, and $e$ being the coefficient of restitution between the ball and the ground; the bat and the ground are supposed to be sufficiently rough to prevent sliding. 
43. A sphere of radius $a$ rolling on a rough table with velocity $V$ comes to a slit of breadth $b$ perpendicular to its path. Prove that, if there is no restitution, the condition that it should cross the slit without jumping is

$$
V^{2}>1 \frac{0}{7} g a(1-\cos a) \sin ^{2} a\left(14-10 \sin ^{2} a\right) /\left(7-10 \sin ^{2} a\right)^{2},
$$

where $b=2 a \sin a$ and $17 g a \cos a>V^{2}+10 g a$.

44. A sphere centre $O$ with its centre of gravity at a point $G$ distant $c$ from $O$ is dropped vertically upon a plane of inclination $a$ to the horizontal so that $G$ is above $O$ and $G O$ is normal to the plane. Prove that, if the plane is rough enough to prevent sliding, the kinetic energy lost in the impact is to that of the sphere before impact in the ratio

$$
\left(1-e^{2}\right) \cos ^{2} a+\left(\kappa^{2} \sin ^{2} a\right) /\left\{\kappa^{2}+(a+c)^{2}\right\}: 1,
$$

where $\kappa$ is the radius of gyration of the sphere about an axis through $G$ at right angles to $G O$.

45. A circular disc of mass $M$, radius $a$, and moment of inertia $M K^{2}$ about its centre, spinning with angular velocity $\Omega$ impinges normally on a rough rod of mass $m$. Prove that the angular velocity immediately after impact is $(M+m) K^{2} \Omega /\left\{(M+m) K^{2}+m a^{2}\right\}$, there being no restitution.

46. Two rough circular discs of masses $M_{1}, M_{2}$, radii $\alpha_{1}, a_{2}$, and radii of gyration $k_{1}, k_{2}$ about their centres, spinning about their centres with angular velocities $\Omega_{1}, \Omega_{2}$ impinge directly, the relative velocity of the centres before impact being $V$. Prove that, if there is no restitution, the kinetic energy lost in impact is

$$
\frac{1}{2} \frac{V^{2}}{1 / M_{1}+1 / M_{2}}+\frac{1}{2} \frac{\left(a_{1} \Omega_{1}+a_{2} \Omega_{2}\right)^{2}}{1 / M_{1}\left(1+a_{1}^{2} / k_{1}^{2}\right)+1 / M_{2}\left(1+a_{2}^{2} / k_{2}^{2}\right)} .
$$

47. A truck of mass $M_{1}$ which has $n_{1}$ pairs of wheels, each pair having an axle, the mass of the axle and pair of wheels being $m_{1}$, the radius of gyration of the axle and pair of wheels about the axis of the axle being $k_{1}$, and the radius of either wheel being $a_{1}$, impinges directly on another truck running on the same metals and for which the corresponding quantities are $M_{2}, n_{2}$, $m_{2}, k_{2}, a_{2}$. Prove that, if

$$
N_{1}=M_{1}+n_{1} m_{1} k_{1}^{2} / a_{1}^{2} \text { and } N_{2}=M_{2}+n_{2} m_{2} k_{2}{ }^{2} / a_{2}^{2} \text {, }
$$

the impulse between thern is $N_{1} N_{2} V(1+e) /\left(N_{1}+N_{2}\right)$, where $V$ is the relative velocity before impact and $e$ is the coefficient of restitution.

48. Assuming that in the impact of the two trucks the force of restitution in any state of strain of a buffer is $\beta$ times that during compression, prove that the relative velocity after impact is reversed in sense and is in magnitude $\sqrt{ } \boldsymbol{\beta}$ times that before. Further, if the forces of compression for the two buffers are the products of $E_{1}, E_{2}$ and the contractions of the buffers and the forces of restitution are the products of $E_{1} \beta, E_{2} \beta$ and the contractions, the periods of compression and restitution are $T$ and $T / \sqrt{ } \beta$, where

$$
T=\pi \sqrt{ }\left\{\left(E_{1}^{-1}+E_{2}^{-1}\right) /\left(N_{1}^{-1}+N_{2}^{-1}\right)\right\},
$$

the notation $N_{1}, N_{2}$ being the same as in Example 47. 
49. A sphere of mass $m$ falls vertically and impinges with velocity $V$ against a board of mass $M$ which is moving with velocity $U$ on a horizontal plane. The upper face of the board is rough and the lower face smooth, and the coefficient of restitution is $e$. Prove that, if the coefficient of friction exceeds $2 M U /(7 M+2 m)(1+e) V$, the kinetic energy lost in the impact is

$$
\frac{1}{2} m\left(1-e^{2}\right) V^{2}+m M U^{2} /(7 M+2 m) \text {. }
$$

50. A ball is let fall upon a hoop, of which the mass is $1 / n$ of that of the ball, and which is suspended from a point in its circumference, about which it can turn freely in a vertical plane; prove that, if $e$ is the coefficient of restitution, and $a$ the inclination to the vertical of the radius passing through the point at which the ball strikes the hoop, the ball rebounds in a direction making with the horizontal an angle $\tan ^{-1}\left\{\left(1+\frac{1}{2} n\right) \tan a-e \cot a\right\}$.

51. A homogeneous sphere is allowed to fall on one end of a uniform horizontal beam balanced on a horizontal axis through its centre of inertia. Prove that the sphere will not rebound unless the mass of the beam is at least three times as great as that of the ball, the coefficient of restitution being unity.

52. A plank of length $2 \alpha$ is turning about a horizontal axis through its centre of gravity and a particle strikes the rising half, rebounds, and strikes the other half, the coefficient of restitution being unity. Prove that, if the motion indefinitely repeats itself, the inclination of the plank to the horizontal must never exceed $a$ where $I(\pi+2 a) \tan a=m \alpha^{2}, I$ being the moment of inertia of the plank about its axis, and $m$ the mass of the particle.

53. A wedge of mass $M$ and angle $a$ with a smooth face and a rough face is placed with the smooth face on a table and a uniform sphere of mass $m$ is dropped upon it symmetrically. Prove that, if there is no restitution, the kinetic energy is diminished by the impact in the ratio

$$
(M+m) \sin ^{2} a: M+m \sin ^{2} a+\frac{2}{5}(M+m) .
$$

54. Two equal rigid uniform discs, each in the shape of an equilateral triangle, rest with two edges in contact. They are struck at the same instant with equal blows $P$ in opposite directions bisecting the common edge and one other edge of each, so that they are pressed together and begin to slide one over the other. Find the velocity $v$ of the point of application of either blow resolved in its direction, and prove that, if $\mu$ is the coefficient of friction, the kinetic energy generated in the system is $(1-\mu \sqrt{ } 3) P v$, assuming no restitution.

55. A smooth oval disc is rotating with angular velocity $\omega$ on a smooth horizontal plane about its centre of inertia which is fixed, when it strikes a smooth rod of mass $m$ at the middle point of the rod. Prove that the new angular velocity is $\left(I-m e p^{2}\right) \omega /\left(I+m p^{2}\right)$, where $I$ is the moment of inertia of the disc about an axis through its centre perpendicular to its plane, $p$ the perpendicular from the centre of inertia to the normal at the point of contact, and $e$ the coefficient of restitution. 
56. A small smooth ring of mass $m$ slides on the side $A B$ of a square $A B C D$ formed of four rigidly connected rods. An impulse $R$ is applied to $C$ in direction $D C$. Prove that the initial velocity of the ring is

$$
R a c /\left\{m c^{2}+(M+m) k^{2}\right\}
$$

where $2 \alpha$ is the length of a side, $c$ is the distance of the ring from the middle point of $A B, M$ is the mass of the square and $k$ its radius of gyration about its centre.

57. A uniform rod of length $2 a$ moving in a vertical plane falls on a horizontal smooth plane so as to make with it an angle $\theta$ at the instant of impact, and there is perfect restitution. Prove that, if at the instant of impact, the rod is turning about any point in the vertical line through that point of the rod which is distant $a\left(1+\frac{1}{3} \sec ^{2} \theta\right)$ from the lower end, the angular velocity $\omega$ and the vertical component of the velocity of the centre of inertia will be immediately reversed, and further that if $3 \theta \cos \theta=\alpha \omega^{2} / g$ the subsequent impacts on the plane will take place at equal intervals of time $2 \theta / \omega$.

58. A smooth uniform cube of side $2 a$ and radius of gyration $k$ about an axis through its centre is free to turn about an axis which is horizontal and passes through the centres of two opposite faces, and the cube is at rest with two faces horizontal. An equal cube falls without rotation and with velocity $V$, and strikes the upper face of the first cube along a line parallel to the fixed axis and at a distance $c$ from the vertical plane through it. Prove that, if $e$ is the coefficient of restitution and $a$ the angle which the lower face of the falling cube makes with the horizontal, the angular velocity imparted to the first cube is $c V(1+e) /\left(c^{2}+k^{2}+a^{2}-a^{2} \sin 2 a\right)$.

59. Two uniform rods $A B, B C$ of masses $m, m^{\prime}$ lie on a smooth table inclined to each other at an angle $a$; they are jointed at $B$, and the end $A$ turns on a pivot fixed to the table. If $A B$ is struck at the middle by a blow $P$ perpendicular to $A B$ the kinetic energy of the resulting motion is

$$
\frac{1}{2} P^{2} /\left(\frac{4}{3} m+4 m^{\prime}-3 m^{\prime} \cos ^{2} a\right) \text {. }
$$

If there is a smooth peg touching $B C$ at its middle point on the proper side to give constraint the kinetic energy is

$$
\frac{1}{2} P^{2} /\left(\frac{4}{3} m+4 m^{\prime}-\frac{8}{3} m^{\prime} \cos ^{2} a\right) \text {. }
$$

60. Two uniform rods $A B, B C$ hinged together at $B$ are moving about the middle point of $A C$ as instantaneous centre of rotation, with no motion relative to each other, when a point in one of the rods is suddenly fixed, $A B C$ being at the moment a right angle. Prove that, if after impact the relative motion of the rods is initially zero, the point must be the hinge.

61. Two lengths $2 \alpha$ and $2 b$ are cut from the same uniform rod of mass $M$ and freely jointed at one end of each. The rods being at rest in a straight line, an impulse $M V$ is applied at the free end of $a$. Prove that the kinetic energy when $b$ is free is to that when the further end of $b$ is fixed in the ratio $(4 a+3 b)(3 a+4 b) / 12(a+b)^{2}$. 
62. An equilateral triangle formed of three equal uniform rods hinged at their ends is held in a vertical plane with one side horizontal and the opposite corner downwards. Prove that, if after falling through any height the middle point of the highest rod is suddenly stopped, the impulsive stresses at the upper and lower hinges will be in the ratio $\sqrt{ } 13: 1$.

63. A rectangle, sides $2 a$ and $2 b$, formed of four uniform rods of the same material and section, smoothly hinged at the ends, is moving without rotation on a smooth horizontal plane, when a side- of length $2 a$ impinges on a small rough peg (zero restitution). Prove that for that side to acquire the greatest possible angular velocity, the point of impact must be at a distance $a\{(3 b+a) /(3 b+3 a)\}^{\frac{1}{2}}$ from its centre. Prove also that the rectangle cannot begin to rotate as a rigid body unless the direction of motion before impact makes with the impinging side an angle greater than

$$
\tan ^{-1} \frac{a(3 b+a)^{\frac{1}{2}}(3 b+3 a)^{\frac{1}{2}}}{b(2 b+3 a)} .
$$

64. A rhombus formed of four similar uniform rods freely hinged at the angular points is rotating on a smooth horizontal plane about its centre when one corner is suddenly fixed. Prove that the relative angular velocities of, the rods just before and just after the impulse are in the ratio $5-3 \cos a: 2$, where $a$ is the angle of the rhombus at the corner fixed.

65. A rhombus formed of four equal uniform rods each of length $2 a$ freely jointed at common extremities is moving with velocity $v$ in the direction of one of its diagonals of length $4 a \cos a$, when the middle point of one of the front sides is suddenly fixed. Prove that the initial angular velocity of that side is zero, and that of the adjacent sides is $\frac{3}{5}(v / a) \sin a$.

66. Eight equal uniform rods $A B, B C, \ldots H K$ freely jointed at their extremities are placed on a table in the form of a square with two rods in each side, the ends $A, K$ being in contact but free. Prove that if the end $A$ is set in motion with a given velocity in a direction making an angle $\theta$ with $A B$, then $K$ will start in a direction making an angle $\phi$ with $A B$, where

$$
3409 \tan \phi=433 \tan \theta \text {. }
$$

67. Twelve equal rods each of length $2 a$ are so jointed together that they can be the edges of a cube, and the framework moves symmetrically through a configuration in which each rod makes an angle $\theta$ with the vertical; prove that, if $u$ is velocity of the centre of inertia, the kinetic energy is $\frac{1}{2} M\left(\frac{7}{3} a^{2} \dot{\theta}^{2}+u^{2}\right)$, where $M$ is the mass of the framework, and that, if the frame strikes the ground when $u=V$ and $\dot{\theta}=0$, then $u$ is reduced to

$$
V /\left(1+\frac{7}{27} \operatorname{cosec}^{2} \theta\right) \text {. }
$$

68. An indefinite number of equal uniform rods are loosely jointed together and are in a straight line and at rest when a blow $P$ is struck at the free end of the extreme rod in a direction perpendicular to its length. Prove 
that the impulsive force exerted at the hinge at the further end of the $n^{\text {th }}$ rod is

$$
(-)^{n} P 2^{2 n} \sin ^{2 n} \frac{\pi}{12} \text {. }
$$

69. A set of $(2 n+1)$ equal rods $O A, O B, O C, \ldots$ each of mass $m$ and length $2 a$ are freely jointed at $O$, and lie in one plane so that any two neighbouring rods are inclined at an angle $a,=2 \pi /(2 n+1)$; an impulse $P$ acts along $O A$ and $\omega_{1}, \omega_{2}, \ldots$ are the initial angular velocities communicated to the rods on each side of $O A$ in order. Prove that

$$
\omega_{1} \operatorname{cosec} a=\omega_{2} \operatorname{cosec} 2 a=\ldots=\frac{3}{4} u / a,
$$

where $u,=\frac{8}{5} P i\{(2 n-1) m\}$, is the initial velocity of $O A$.

70. Four small smooth rings of equal mass are attached at equal intervals to a thread and rest on a circular wire in a vertical plane. The radius of the wire is one-third of the length of the thread, and the rings are at the four upper corners of a regular hexagon inscribed in the circle, the two lower rings being at the ends of the horizontal diameter. Prove that, if the thread is cut between one of the extreme particles and one of the middle ones the tension in the horizontal part is suddenly diminished in the ratio $5: 9$.

71. Particles of masses $m$ and $m^{\prime}$ are fastened to the ends of a thread which rests in a vertical plane on the surface of a smooth horizontal circular cylinder of mass $M$ which can slide on a horizontal plane. The system is initially held at rest so that the radii of the circular section through the particles make with the vertical angles $a$ and $\beta$. Prove that when the system is released the tension of the thread immediately becomes

$$
m m^{\prime} g \frac{M(\sin a+\sin \beta)+\left(m \sin a+m^{\prime} \sin \beta\right)\{1-\cos (a+\beta)\}}{\left(m+m^{\prime}\right)\left(M+m \sin ^{2} a+m^{\prime} \sin ^{2} \beta\right)-m m^{\prime}(\cos a-\cos \beta)^{2}} .
$$

72. A particle $P$, of mass $M$, rests in equilibrium on a smooth horizontal table being attached to three particles of masses $m, m^{\prime}, m^{\prime \prime}$ by cords which pass over smooth pulleys at points $A, B, C$ at the edge of the table. Prove that, if the cord supporting $m^{\prime \prime}$ is cut, $M$ will begin to move in a direction making with $C P$ an angle

where

$$
\tan -1 \frac{\mu\left(m \sim m^{\prime}\right)\left\{\left(m+m^{\prime}\right)^{2}-m^{\prime \prime 2}\right\}}{4 M m m^{\prime} m^{\prime 2}+\left(m+m^{\prime}\right) \mu^{2}},
$$

73. Two particles $A, B$ of masses $m, m^{\prime}$ are connected by a thread which passes through a smooth ring $C$ at the top of a smooth plane of inclination $a$ to the horizontal. Initially $A C(=\alpha)$ is along a line of greatest slope, and $B C$ is vertical. Prove that, if $A$ is projected at right angles to $A C$ with velocity $v, B$ will begin to ascend or descend according as

$$
m^{\prime} / m<\text { or }>\sin a+v^{2} / g a \text {. }
$$

L. 
74. A sphere of mass $m$ hangs by a chain of length $b$ and negligible mass to one end of a rigid horizontal arm of length $c$, which is free to rotate about a fixed vertical axis passing through its other end. The arm is seized and made to rotate with angular velocity $\Omega$. Prove that the tension of the chain immediately becomes $m\left(g+\Omega^{2} c^{2} / b\right)$, and that the plane through the chain and the radius from the centre of the sphere to the point of attachment starts to rotate with angular velocity $\frac{1}{2} \Omega$ about the radius.

75. A thread $A B C$ is fixed at $A$ and has particles of masses $m, m^{\prime}$ attached to it at $B$ and $C$, and the system is held in a vertical plane so that $A B$ and $B C$ make acute angles $a$ and $a+\beta$ with the vertical. Prove that, when $B$ and $C$ are let go, the initial tension of $A B$ is

$$
m\left(m+m^{\prime}\right) g \cos \boldsymbol{a} /\left(m+m^{\prime} \sin ^{2} \beta\right) .
$$

76. A circular wire of mass $M$ is held at rest in a vertical plane, on a smooth table, and a particle of mass $m$ rests against it being supported by an inextensible thread which passes over the wire and is secured to a fixed point in the plane of the wire at the same level as the highest point of the wire. Prove that if the wire is set free the pressure of the particle upon it is immediately diminished by an amount $m^{2} g \sin ^{2} a /\left(M+4 m \sin ^{2} \frac{1}{2} a\right)$, where $a$ is the angular distance of the ring from the highest point of the wire.

77. Four particles $A, B, C, D$ of equal mass connected by equal threads are placed on a smooth plane of inclination $a\left(<\frac{1}{4} \pi\right)$ to the horizontal, so that $A C$ is a line of greatest slope and $A B, A D$ make angles $a$ with $A C$ on opposite sides of it. If the uppermost particle $A$ is held, and the particles $B$ and $D$ are released, prove that the tension in each of the lower threads is instantly diminished in the ratio

$$
\left(1-2 \sin ^{2} a\right) /\left(1+2 \sin ^{2} a\right) \text {. }
$$

78. A bead of mass $m^{\prime}$ can slide on a thread one end of which is fixed while the other end carries a particle of mass $m$. Initially $m$ is held at the level of the fixed end, and the two parts of the thread make equal angles $a$ with the vertical. Prove that, if the particle $m$ is released, the initial tension in the thread is $m m^{\prime} g \cos a /\left(m^{\prime}+4 m \cos ^{2} a\right)$, and the initial acceleration of the bead $m^{\prime}$ is

$$
g\left(m^{\prime}+2 m \cos ^{2} a\right) /\left(m^{\prime}+4 m \cos ^{2} a\right) .
$$

79. One end of a thread $P Q$ is fixed to a point $P$ on a smooth horizontal plane, and the other end $Q$ is attached to a small smooth ring of mass $m$ which rests on the plane; another thread passes through the ring and is fixed at one end to a point $R$ of the plane while its other end $S$ carries a particle of mass $M$. Initially the angle $P Q R$ is obtuse and equal to $\beta$, and the angle $R Q S$ is right; the particle $M$ is projected parallel to $Q R$ with velocity $V$. Prove that the initial tension in $P Q$ is

$$
M m V^{2}(\sin \beta-\cos \beta) / \alpha(m+M+M \sin 2 \beta),
$$

where $a$ is the length of $Q S$. 
80. There is a system of $n$ moveable pulleys $m_{1}, m_{2}, \ldots m_{n}$ and $n$ corresponding counterpoises of masses $\mu_{1}, \mu_{2}, \ldots \mu_{n}$. Each pulley and its counterpoise are suspended by a cord passing over the preceding pulley. The highest cord (connecting $m_{1}$ and $\mu_{1}$ ) passes over a fixed pulley, and no cord passes over the lowest pulley $m_{n}$. The suffixes indicate the order in which the pulleys are slung. The pulleys are simultaneously set free. Prove that, if $T_{1}, T_{2}, \ldots T_{n}$ are the tensions in the cords,

$$
2\left(T_{p+1} / m_{p}+T_{p-1} / m_{p-1}\right)=T_{p}\left(1 / m_{p}+1 / \mu_{p}+4 / m_{p-1}\right) ;
$$

further, if the mass of each pulley $(m)$ is to the mass of each counterpoise $(\mu)$ as $5: 3$, prove that the downward acceleration of the $p^{\text {th }}$ moveable pulley is

$$
\frac{3^{2 n-p+1}-5}{3^{2 n+1}+5}\left(3^{p}-1\right) g \text {. }
$$

81. Two equal particles connected by an inextensible thread lie on a smooth table with the thread straight; prove that, if one of them is projected on the table at right angles to the thread, the initial radius of curvature of its path is twice the length of the thread.

82. A small ring of mass $m$ rests on a smooth straight wire, and another particle of mass $m^{\prime}$ is connected with it by a thread of length $a$. Prove that, if $m^{\prime}$ is projected in a direction at right angles to the wire from a point on it at a distance $a$ from $m$, the initial radius of curvature of the path is

$$
a\left(m+m^{\prime}\right) / m \text {. }
$$

83. An inextensible thread passes through two smooth rings $A, B$ on a smooth table; particles of masses $p$ and $q$ are attached to the ends, and a particle of mass $m$ is attached to a point $O$ between $A$ and $B$. Prove that, if $m$ is projected horizontally at right angles to the thread, the initial curvature of its path is $(p / O A \sim q / O B) /(p+q+m)$.

84. A particle of mass $m$ on a smooth table is joined to a particle of mass $m^{\prime}$ hanging just over the edge by a thread of length $a$ at right angles to the edge. Prove that, if the system starts from rest, the radius of curvature of the path of $m$ immediately after it leaves the table is

$$
\frac{2 m^{\prime} a}{\left(m+m^{\prime}\right)^{2}} \frac{\left\{\left(m+m^{\prime}\right)^{2}+m^{\prime 2}\right\}^{\frac{3}{2}}}{\left(m+m^{\prime}\right)^{2}+2 m^{\prime 2}} .
$$

85. Two particles $A, B$ are connected by a fine string; $A$ rests on a rough horizontal table (coefficient of friction $=\mu$ ) and $B$ hangs vertically at a distance $l$ below the edge of the table. If $A$ is on the point of motion, and $B$ is projected horizontally with velocity $u$, show that $A$ will begin to move with an acceleration $\mu u^{2} /\{(\mu+1) l\}$, and that the initial radius of curvature of $B$ 's path will be $(\mu+1) l$. 
86. Three particles $A, B, C$ are connected by two threads $A B, A C$ and placed in a line on a smooth table. The extreme particles are projected at right angles to the thread with velocities $u, v$. Prove that, if $m, p, q$ are the masses, and $a, b$ the lengths of the threads, the initial curvatures of the paths of $B$ and $C$ are

$$
\frac{(q+m) u^{2} / a+q v^{2} / b}{(p+q+m) u^{2}} \text { and } \frac{(p+m) v^{2} / b+p u^{2} / a}{(p+q+m) v^{2}} .
$$

87. A particle of mass $m$ is attached to one end of a thread which passes through a bead of mass $M$ and the other end is secured to a point on a smooth horizontal table on which whole rests. Initially the two portions of the thread are straight and contain an obtuse angle $a$, the portion between $m$ and $M$ being of length $a$, and $m$ is projected at right angles to this portion. Prove that the initial radius of curvature of the path of $m$ is

$$
a\left(1+4 m M^{-1} \cos ^{2} \frac{1}{2} a\right) \text {. }
$$

88. Into the top of a smooth fixed sphere of radius $a$ is fitted a smooth vertical rod. A uniform rod of length $2 b$ rests on the sphere with its upper end constrained to remain on the vertical rod, the centre of gravity being at a distance $c$ from the point of contact. Prove that if the constraint is removed the pressure on the sphere is instantaneously diminished in the ratio

$$
b(b-c):\left(b^{2}+3 c^{2}\right) .
$$

89. Two equal uniform rods each of length $2 a$ are freely hinged at one extremity and their other extremities are connected by an inextensible thread of length $2 l$. The system rests on two smooth pegs distant $c$ apart in a horizontal line. Prove that, if the thread is severed, the initial angular acceleration of either rod is

$$
\left(8 a^{2} c-l^{3}\right) g /\left(\frac{8}{3} a^{2} l^{2}+32 a^{4} c^{2} / l^{2}-8 a^{2} c l\right) .
$$

90. Six equal uniform rods each of mass $m$ are freely jointed and are kept in the form of a regular hexagon by a thread joining two opposite corners. The hexagon is in a vertical plane with one rod fixed and horizontal and a particle of mass $p$ is attached to the middle point of the lowest rod. The thread is then destroyed. Prove that $p$ descends with initial acceleration

$$
(9 m+3 p) g /(10 m+3 p) \text {. }
$$

91. A uniform circular disc is supported in a vertical plane by two cords attached to the ends of a horizontal diameter, each making an angle $a$ with the horizontal. One of the cords being cut, prove that the tension of the other is suddenly diminished in the ratio $2 \sin ^{2} a: 1+2 \sin ^{2} a$.

92. A uniform circular disc is symmetrically suspended by two elastic cords of natural length $c$ inclined at an angle $a$ to the vertical and attached to the highest point of the disc. Prove that, if one of the cords is cut, the initial radius of curvature of the path of the centre of the disc is

$$
3 \cos a b(b-c) /(c \sin 4 a-b \sin 2 a),
$$

where $b$ is the equilibrium length of each cord. 
93. A uniform equilateral triangular board is suspended by three equal cords from the corners of a similar triangle in a horizontal plane; prove that, if one of the cords is cut, the tensions in the remaining two are instantly diminished in the ratio $3 \sin ^{2} a: 2+4 \sin ^{2} a$, where $a$ is the angle which the plane through the two cords makes with the horizontal.

94. A thin uniform rectangular board hinged along a line in itself parallel to one side is opened out to any angle and placed on a smooth horizontal plane so that the cross section of the board made by a vertical plane perpendicular to the hinge is a triangle $A B C$, of which the side $A B$ is in the horizontal plane, and the angle $C$ in the hinge. Prove that $C$ starts to move in a direction making with the vertical an angle whose tangent is $\frac{1}{2} \tan A \tan B \tan \frac{1}{2}(A-B)$, and find its initial vertical and horizontal accelerations.

95. A circular ring hangs in a vertical plane on two pegs in a horizontal line subtending an angle $2 a$ at the centre. One peg is suddenly removed. Find the pressure on the remaining peg when it is (1) smooth, (2) rough, and prove that these pressures are in the ratio $1: \sqrt{ }\left(1+\frac{1}{4} \tan ^{2} a\right)$.

96. Four uniform rods two of length $2 a$ and two of length $2 b$ whose masses are proportional to their lengths are freely jointed so as to form a parallelogram. One of the rods of length $2 \alpha$ is free to turn about a pivot at a distance $c$ from its middle point, and is initially held in a horizontal position so that the figure is a rectangle, and is let go. Prove that the initial angular acceleration of each of the horizontal rods is

$$
g c(a+b) /\left\{c^{2}(a+b)+a^{2}\left(\frac{1}{3} a+b\right)\right\} \text {. }
$$

97. A sphere resting on a rough horizontal plane is divided into an indefinitely great number of solid segments by planes through a common diameter and is kept in shape with this diameter vertical by means of a band round the horizontal great circle. Prove that, if the band is cut the pressure on the plane is instantly diminished by the fraction $45 \pi^{2} / 2048$ of itself.

98. Two uniform rods $A B, B C$ of masses $m, m^{\prime}$ and lengths $2 a, 2 b$ are freely jointed at $B$. The end $A$ is fixed and the system is supported by a string attached to $C$ in a position in which $A B, B C$ make angles $a$ and $\beta$ with the vertical. Prove that, if the string is cut, the initial angular accelerations of $A B$ and $B C$ are

and

$$
\begin{gathered}
\frac{\frac{4}{3}\left(m+2 m^{\prime}\right) \sin a-2 m^{\prime} \sin \beta \cos (a-\beta)}{\frac{16}{9}\left(m+3 m^{\prime}\right)-4 m^{\prime} \cos ^{2}(a-\beta)} \frac{g}{a}, \\
\frac{\frac{4}{3}\left(m+3 m^{\prime}\right) \sin \beta-2\left(m+2 m^{\prime}\right) \sin a \cos (a-\beta)}{\frac{16}{9}\left(m+3 m^{\prime}\right)-4 m^{\prime} \cos ^{2}(a-\beta)} \frac{g}{b} .
\end{gathered}
$$

99. A uniform rod of length $2 \alpha$ and weight $W$ rests on a rough horizontal plane with its pressure on the plane uniformly distributed. A horizontal force $P$, large enough to produce motion, is suddenly applied at one end perpendicularly to the length of the rod. Prove that the rod begins to turn 
about a point distant $x$ from its middle point, where $x$ is the positive root of the equation

$$
x^{3}-\left(\frac{1}{3}-2 P / \mu W\right) a^{2} x-\frac{2}{3} P a^{3} / \mu W=0,
$$

where $\mu$ is the coefficient of friction.

100. A thin uniform rectangular plank of mass $M$ is suspended from four points in the same horizontal plane by four parallel chains of equal length and negligible mass attached to the corners, and a uniform cylinder of mass $m$ is on the plank with its axis parallel to an edge and its centre of inertia vertically over that of the plank. The whole system is drawn aside in the vertical plane at right angles to the axis of the cylinder till the chains make an angle $a$ with the vertical, and is then let go. Prove that the initial tension of each chain is

$$
\frac{1}{4}(M+m)(3 M+m) g \cos a /\left\{3(M+m)-2 m \cos ^{2} a\right\},
$$

or

$$
\frac{1}{4} M(M+m) g \cos a /\left\{M+m \sin ^{2} a-m \mu \sin a \cos a\right\},
$$

according as $\mu$ the coefficient of friction is greater or less than

$$
(M+m) \tan \boldsymbol{a} /(3 M+m) \text {. }
$$

101. A uniform circular disc (mass $M$ ) rotates in a horizontal plane with angular velocity $\omega$. Close round it moves a ring of mass $m$ and radius $c$ rotating about its centre with angular velocity $\nu(<\omega)$. The ring carries a massless smooth spoke along a radius, and a bead of mass $p$ can move on the spoke under the action of a force to the centre of the ring equal to $\mu /(\text { distance })^{2}$, and the bead is in relative equilibrium at a distance $a$ from the centre. Prove that, if a slight continuous action now begins between the disc and the ring, of the nature of friction and proportional to the relative angular velocity, the distance of the bead from the centre, and the angular velocity of the ring, will at first increase, and their values after a short time $t$ will be

and

$$
a+\frac{1}{3} t^{3} a \nu \lambda(\omega-\nu) /\left(m c^{2}+p a^{2}\right),
$$

$$
\nu+t \lambda(\omega-\nu) /\left(m c^{2}+p a^{2}\right)-\frac{1}{2} \lambda t^{2}\left[\lambda(\omega-\nu) /\left(m c^{2}+p a^{2}\right)\right]\left[2 / \Delta \dot{L}^{2}+1 /\left(m c^{2}+p a^{2}\right)\right],
$$

where $\lambda \theta$ is the frictional couple when the relative angular velocity is $\theta$.

102. A series of $2 n$ equal uniform rods each of masis $m$ are hinged together and held so that they are alternately horizontal and vertical, each vertical rod being lower than the preceding; the highest rod is horizontal and can turn freely round its end which is fixed. Prove that, when the rods are let go, the horizontal component $X_{2 r}$ and the vertical component $Y_{2} r$ of the initial action between the $2 r$ th and the $(2 r+1)$ th rods are given by

$$
\begin{aligned}
& X_{2 r}=B(-5+2 \sqrt{ } 6)^{r}+C(-5-2 \sqrt{ } 6)^{r}, \\
& Y_{2 r}=B^{\prime}(-5+2 \sqrt{ } 6)^{r}+C^{\prime}(-5-2 \sqrt{ } 6)^{r},
\end{aligned}
$$

the constants $B, C, B^{\prime}, C^{\prime}$ being determined by the equations

$$
X_{2 n}=0, Y_{2 n}=0, X_{2}+2 X_{0}=0,2 Y_{2}+16 Y_{0}-5 m g=0 .
$$


103. A chain is formed of $n$ equal symmetrical rods, each of length $2 a$ and radius of gyration $k$ about its centre of mass. One end is fixed and the whole is supported in a horizontal line. Prove that, if the supports are simultaneously removed, the free end begins to move with acceleration

$$
g\left[1+(-)^{n+1} \operatorname{sech} \log \left(\tanh ^{n} \frac{1}{2} \theta\right)\right] \text {, where } \theta=\log (\alpha / k) .
$$

104. A mass $M$ rests on a smooth table and is connected with a particle of mass $m$ by an inextensible thread passing through a hole in the table. Prove that, if $m$ is released from rest in a position in which its polar coordinates are $a, a$ referred to the hole as origin and the vertical as initial line, then in the initial motion

$$
\begin{gathered}
(M+m) \ddot{r}_{0}=m g \cos a, a \ddot{\theta}_{0}=-g \sin a, \\
a(M+m) r_{0}^{\text {iv }}=3 m g^{2} \sin ^{2} a, a^{2} \theta_{0}^{\text {iv }}=g^{2} \sin a \cos a(M+3 m) /(M+m) .
\end{gathered}
$$

Also prove that the initial radius of curvature of the path of $m$ is

$$
36\left(\ddot{x}_{0}^{2}+\ddot{y}_{0}^{2}\right)^{\frac{3}{2}} /\left(x_{0}^{\mathrm{i}} \ddot{y}_{0}-y_{0}^{\mathrm{iv}} \ddot{\ddot{x}}_{0}\right) \text {, }
$$

where

$$
\ddot{x}_{0}=\ddot{r}_{0}, \ddot{y}_{0}=a \ddot{\theta}_{0}, x_{0}^{\mathrm{iv}}=r_{0}^{\mathrm{iv}}-3 \alpha \ddot{\theta}_{0}^{2}, y_{0}^{\mathrm{iv}}=\alpha \theta_{0}^{\mathrm{iv}}+6 \ddot{r}_{0} \ddot{\theta}_{0} \text {. }
$$

105. One end of a uniform rod of length $2 \alpha$ and mass $m$ is freely jointed to a board of mass $M$ at its centre of inertia and the board is placed on a smooth table. The rod is held so as to make an angle $a$ with the vertical, and is let go. Prove that the initial radius of curvature of the path of its middle point is

$$
a\left\{M^{2} \cos ^{2} a+(M+m)^{2} \sin ^{2} a\right\}^{\frac{3}{2}} / M(M+m)^{2} .
$$

106. A garden roller is at rest on a horizontal plane rough enough to prevent slipping, the handle being so held that the plane through the axis of the cylinder and the centre of inertia of the handle makes an angle $a$ with the horizon. Show that, if the handle is let go, the initial radius of curvature of the path described by its centre of inertia is $c n^{-2}\left(\sin ^{2} a+n^{2} \cos ^{2} a\right)^{\frac{3}{2}}$, where $(n-1) M\left(K^{2}+a^{2}\right)=m a^{2}$, and $c$ is the distance of the centre of inertia of the handle from the axis of the cylinder, $m$ its mass, and $M K^{2}$ the moment of inertia of the cylinder about its axis, the cylinder being homogeneous and of radius $a$.

107. Two uniform rods of lengths $2 a, 2 b$ and masses $A, B$ are freely hinged at a common extremity and the other extremity of $A$ is fixed. The rods fall from a horizontal position of rest. Prove that the initial radius of curvature of the further extremity of $B$ is

$$
2 a b(A+B)^{2} /\left\{a A^{2}+b(2 A+B)^{2}\right\} \text {. }
$$

108. A rough plank of mass $M$ is free to turn in a vertical plane about a horizontal axis distant $c$ from its centre of inertia, and a uniform sphere of mass $m$ is placed on the plank at a distance $b$ from the axis on the side remote from the centre of inertia, the plank being held horizontal. Prove that when the plank is let go the initial radius of curvature of the path of the centre of the sphere is $21 b \theta /(5-11 \theta)$, where $\theta=(m b-M c) /(m b+M a)$, and $M a b$ is the moment of inertia of the plank about the axis. 
109. Two rods $A C, C B$ of equal length $2 a$ are freely jointed at $C$, the rod $A C$ being free to turn in a vertical plane about the point $A$, and the end $B$ of the rod $C B$ being attached to $A$ by an inextensible string of length $4 a / \sqrt{ } 3$. The system being in equilibrium the string is cut. Show that the initial radius of curvature of the path of $B$ is $a \frac{4}{181} \sqrt{\frac{41^{3}}{3}}$.

110. A set of $n$ equal rods are jointed together in one straight line and have initial angular accelerations $\omega_{1}, \omega_{2}, \ldots \omega_{n}$ in one plane. Prove that, if one end is fixed, the initial radius of curvature of the path of the free end is

$$
\left(a_{1} \omega_{1}+a_{2} \omega_{2}+\ldots+a_{n} \omega_{n}\right)^{2} /\left(a_{1} \omega_{1}^{2}+a_{2} \omega_{2}^{2}+\ldots+a_{n} \omega_{n}^{2}\right) .
$$

111. A system of two equal uniform rods $A B, C D$ and a sphere of diameter $B C$ equal to the length of either $\operatorname{rod}$ is free to turn about $A$, the bodies being freely jointed at $B$ and $C$, and $A B C D$ being initially a horizontal straight line. Prove that, if the mass of the sphere is equal to that of either rod, the initial radius of curvature of the path of $D$ is $\frac{256}{81} A B$.

112. Three particles, of masses $m_{1}, m_{2}, m_{3}$, are symmetrically attached to a circular wire of negligible mass and of radius $a$ which can move in a smooth circular tube of the same radius fixed in a vertical plane. Prove that the length of the simple equivalent pendulum of the small oscillations of the system is

$$
\left(m_{1}+m_{2}+m_{3}\right) a / \sqrt{ }\left\{m_{1}^{2}+m_{2}^{2}+m_{3}^{2}-m_{2} m_{3}-m_{3} m_{1}-m_{1} m_{2}\right\} .
$$

113. Two equal particles of mass $P \sin a$ are attached, at distance $2 a \sin a$ apart, to a thread, to the ends of which particles of mass $P$ are attached. The thread is hung over two pegs distant $2 a$ apart in a horizontal line. Prove that the period of the small oscillations about the position of equilibrium is the same as that for a simple pendulum of length $a \tan a$.

114. Three particles of masses $m, N, m$ are attached to the points $B, C$, $D$ of a thread $A E$ of length $4 a$, and rest suspended by the ends $A, E$ from two points at the same level. The portions $A B, B C, C D, D E$ are each of length $\alpha$ and make with the horizontal angles $a, \beta, \beta, a$ respectively. Prove that $M \tan a=(M+2 m) \tan \beta$, and that, if $M$ receives a small vertical displacement, the period of the small oscillations is the same as for a simple pendulum of length

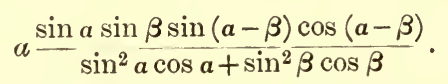

115. A particle of mass $M$ is placed near the centre of a smooth circular horizontal table of radius $a$; cords are attached to the particle and pass over $n$ smooth pulleys placed symmetrically round the circumference, and each cord supports a mass $M$. Show that the time of a small oscillation of the system is

$$
\pi \sqrt{\frac{a}{g}\left(1+\frac{2}{n}\right)}
$$


116. Two equal particles are connected by a string of length $2 l$, which passes over a fixed pulley, and they rest on a smooth inclined plane; $\beta$ is the inclination of the two portions of the string to the plane when the particles are together, and $\boldsymbol{a}$ that of the plane to the horizon. Prove that when they are slightly displaced the length of the simple equivalent pendulum is

$l \cot \beta \operatorname{cosec} \beta \operatorname{cosec} a$.

117. A triangle $A B C$ is formed of equal smooth rods each of length $2 \alpha$, and small equal rings rest on the rods at the middle points of $A B, A C$, being attached to $A$ by equal elastic threads of natural length $l$, and connected together by an inextensible thread passing through a fixed smooth ring at the middle point of $B C$. Prove that, if there are no external forces, and if one of the rings is slightly displaced, the period of the small oscillations is

$$
2 \pi \sqrt{ }\{2 \alpha l m / E(5 \alpha-3 l)\},
$$

where $m$ is the mass of each ring and $E$ is the modulus of elasticity.

118. A particle is attached to the middle point of an elastic thread whose ends are attached to two points in the same horizontal plane. The distance between the points and the unstretched length of the thread are each equal to $2 \alpha$, and in the position of equilibrium the two parts of the thread contain a right angle. Prove that the time of a small oscillation is the same as for a simple pendulum of length

$$
a(2 \sqrt{ } 2-2) /(2 \sqrt{ } 2-1)
$$

119. A uniform elastic ring of mass $m$ modulus $\lambda$ and natural length $2 \pi c$ in the form of a circle is under the action of a force $\mu$ (distance) per unit mass directed from its centre. Prove that its radius will vary harmonically about a mean length $2 \pi \lambda c /(2 \pi \lambda-m \mu c)$, provided $2 \pi \lambda>m \mu c$. What happens if this condition is not satisfied?

120. Three small equal rings are fitted on three smooth rods, which are parallel and in the same plane, one being midway between the other two, and the distance between neighbouring rods being $\alpha$. Prove that, if the rings attract each other according to the law of gravitation and are placed so that the line joining any two of them is nearly perpendicular to the rods, the middle ring and the centre of inertia of the other two will oscillate in a period $2 \pi / \sqrt{ }(3 \mu)$, and the other two relatively to each other in period $4 \pi / \sqrt{ }(5 \mu), \mu \alpha$ being the attraction at distance $a$.

121. A circular hoop of negligible mass and of radius $b$ carries a particle rigidly attached to it at a point distant $c$ from its centre, and its inner surface is constrained to roll on the outer surface of a fixed circle of radius $a,(b>a)$, under the action of a repulsive force from the centre of the fixed circle equal to $\mu$ times the distance. Prove that the period of small oscillations of the hoop will be

$$
2 \pi \frac{b+c}{a} \sqrt{\frac{b-a}{c \mu}} .
$$


122. Two particles of masses $M, m$ are attached to a thread which hangs vertically from a fixed point, $m$ being above $M$; (1) $m$ is held slightly pulled aside a distance $h$ from the position of equilibrium, and, being let go, the system performs small oscillations; (2) $M$ is held slightly pulled aside a distance $k$, without disturbing $m$, and, being let go, the system performs small oscillations. Prove that the angular motion of the lower thread in the first case will be the same as that of the upper thread in the second case if

$$
M k=(M+m) h \text {. }
$$

123. A particle is placed on one of the plane faces of a uniform gravitating circular cylinder at a very small distance from the centre of the face; prove that it will make small oscillations in a period

$$
2\left(\alpha^{2}+h^{2}\right)^{\frac{1}{4}}(\pi / \gamma \rho h)^{\frac{1}{2}},
$$

where $a, h, \rho$ are the radius of the cylinder, its height, and the density of its material.

124. The lower end of a uniform rod of length $a$ slides on an inextensible thread of length $2 a$ whose ends are fixed to two points distant $2 \sqrt{ }\left(a^{2}-b^{2}\right)$ apart in a horizontal line, and the upper end of the rod slides on a fixed vertical rod which bisects the line joining the two fixed points. Prove that the time of a small oscillation about the vertical position of equilibrium is

$$
2 \sqrt{ } 2 \pi a / \sqrt{ }\{3 g(2 b-a)\} \text {. }
$$

125. The extremities of a uniform rod of length $4 a$ slide without friction on the circumference of a three-cusped hypocycloid whose plane is vertical, one of the cusps being at the highest point of the circumscribing circle (radius $3 a$ ). Prove that the length of the simple equivalent pendulum is $\frac{4}{3} a$.

126. A number $n$ of uniform isosceles triangular discs are smoothly jointed at a common vertex so as to form a pyramid whose base is a regular polygon inscribed in a circle of radius $a$, and whose edges lie on a cone of vertical angle $2 \cot ^{-1} \sqrt{ }\left(3+\sin ^{2} \pi / n\right)$. Prove that, if the system is placed so as to rest on a smooth sphere with each of its planes inclined to the vertical at an angle $a\left[>\sin ^{-1}\left(\frac{1}{2} \cos \pi / n\right)\right]$, the length of the simple equivalent pendulum for its small oscillations is

$$
\frac{1}{6} a \cos a\left(1+8 \cos ^{2} a\right) /\left(1+2 \cos ^{2} a\right) .
$$

127. In a heavy plane lamina, whose centre of gravity is $G$, are two narrow straight slits $B A, A C$, such that $A G$ bisects the angle $B A C$. Through each slit passes a fixed peg, the pegs, $P, Q$, being in the same horizontal line. Prove that the time of a small oscillation of the lamina in its own plane, about a position of equilibrium in which the vertex $A$ of the triangle $A P Q$ is upwards, is

$$
2 \pi \sqrt{\frac{2 P Q\left(P Q^{2}+k^{2} \sin ^{2} A\right)}{g \sin A\left(4 P Q^{2}-A G^{2} \sin ^{2} A\right)},}
$$

where $k$ is the radius of gyration of the lamina about a line through $G$ perpendicular to its plane. 
128. A uniform solid right circular cone of height $h$, vertical angle $2 a$, and radius of gyration $k$ about an axis through its centre of inertia at right angles to its axis of figure, rests with its vertex downwards between two rough parallel rails at a distance $2 c$ apart in a horizontal plane. Prove that, if the equilibrium is stable, the period of the small oscillations about it is

$$
\pi \sqrt{ }\left[\left\{16 k^{2} \sin ^{2} a+(3 h \sin a-4 c \cos a)^{2}\right\} / g \sin a \cos a(4 c-3 h \tan a)\right] .
$$

129. A uniform sphere of radius $c$ is placed on a horizontal wire in the form of an ellipse of axes $2 a, 2 b$. Prove that, if the wire is rough enough to prevent slipping, the length of the simple equivalent pendulum of the small oscillation about the position of equilibrium is

$$
\left(a^{2}-b^{2}\right)\left(d^{2}+k^{2}\right) / b^{2} d,
$$

where $k^{2}=\frac{2}{5} c^{2}$, and $d^{2}=c^{2}-b^{2}$.

130. Two equal wheels each of mass $M$, radius $a$, and radius of gyration $k$ about its axis, are rigidly connected by an axle of length $c$ and run on a horizontal plane. Two particles, each of mass $m$, are connected, one to each of the centres of the wheels by cords which pass over smooth pegs in the line of centres. Prove that if the wheels are symmetrically placed between the pegs, and slightly displaced by rolling on the plane, the time of a small oscillation is

$$
2 \pi \sqrt{ }\left\{M b\left(\alpha^{2}+k^{2}\right) / m g a^{2}\right\},
$$

where $2 b+c$ is the distance between the pegs.

131. A solid circular cylinder, bounded by two planes making given angles with the axis, is laid on its curved surface on a rough horizontal plane. Find the position of stable equilibrium, and prove that, if $l$ is the length of the simple equivalent pendulum for a small oscillation, and $d$ the diameter of the cylinder, then the ratio of the longest and shortest generators is

$$
l+4 d: l-2 d \text {. }
$$

132. Four equal rods each of length $2 \alpha$ are jointed so as to form a rhombus which is set up in a vertical plane with its lowest corner fixed and one diagonal vertical, being kept in shape by an elastic thread in the other diagonal, and in the position of equilibrium the thread is stretched to twice its natural length and the rods make equal angles $a$ with the vertical. Prove that the period of a small symmetrical oscillation is the same as that of a pendulum of length

$$
\frac{2}{3} a \sec 2 a \cos a\left(1+3 \sin ^{2} a\right) .
$$

133. Four equal uniform rods, each of length $2 \alpha$ and weight $W$, are freely jointed so as to form a rhombus, and the opposite corners are joined by two similar elastic threads of equal unstretched lengths and of modulus $\lambda$. Prove that, if the system is laid on a smooth horizontal plane and the threads never become slack, each rod swings about its position of equilibrium like a simple pendulum of length $\frac{2}{3} \sqrt{ } 2 \mathrm{~W} a / \lambda$. 
134. A cubical framework of twelve rods freely jointed at the corners is suspended from a corner and held in shape by an elastic string occupying the vertical diagonal. Prove that, if small oscillations take place with the string remaining vertical, their period is the same as that of a simple pendulum of length $\frac{25}{36}\left(l-l_{0}\right)$, where $l$ and $l_{0}$ are the equilibrium length and natural length of the string.

135. A uniform rod rests in equilibrium on a rough gravitating uniform sphere under no forces but the attraction of the sphere. Prove that, if slightly displaced, it will oscillate in time

$$
2 \pi l\left(a^{2}+l^{2}\right)^{\frac{3}{4}} / a \sqrt{ }(3 \gamma m)
$$

where $m$ is the mass of the sphere, $a$ its radius, and $2 l$ the length of the rod.

136. A uniform rod of length $2 a$ moves in a smooth fixed tube under the action of a fixed gravitating particle of mass $m$ at a point distant $c$ from the tube. Prove that the period of small oscillations is

$$
2 \pi\left(a^{2}+c^{2}\right)^{\frac{3}{4}} / /(\gamma m) .
$$

137. A series of $n$ infinitely long uniform circular cylinders, each of radius $c$ and mass $M$ per unit of length, is ranged symmetrically round a rigid framework freely moveable about a fixed axis $A$, the axis of each cylinder being parallel to $A$ and at distance $a$ from it. They are attracted by a similar gravitating fixed cylinder with a parallel axis at a distance $b(>a)$ from $A$. Find the positions of stable equilibrium, and prove that the period of small oscillation about such a position is

$$
\left.2 \pi \sqrt{ } \frac{M}{X} \frac{2 a^{2}+c^{2}}{2 b}\left(\frac{b^{n}}{a^{n}}-1\right)\right\},
$$

where $X$ is the pressure on the axis per unit of length, and the mass of the framework is neglected.

138. Two equal spheres, each of radius $a$ and moment of inertia $I$ about an axis through its centre, have their centres connected by an elastic thread passing through holes in their surfaces, and are set to vibrate symmetrically. Prove (i) that, if in equilibrium the tension of the thread is $T$, then the time of an oscillation of small amplitude $a$ is $2 \pi a^{-1} \sqrt{ }(I / T a)$, and (ii) that, if the natural length of the thread is $2 a$ and $\lambda$ is its modulus of elasticity, then the period is

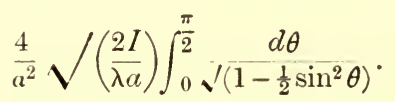

139. Two equal uniform balls are fixed to the ends of a rod $A B$ of negligible mass which is suspended by its middle point $O$ by means of a wire of such torsional elasticity that the system makes a complete oscillation about $O$ in a horizontal plane in time $T$. Two equal fixed uniform spheres of radius $a$ are fixed with their centres at $C, D$ so that $A C$ and $B D$ are each of length $b$ and are in the same horizontal plane with $A B$ and perpendicular to it on opposite sides. The attraction of the spheres alters the position of 
equilibrium of the balls by a small distance $x$. Prove that the density of the spheres is $3 \pi b^{2} x / a^{3} \gamma T^{2}$,

where $\gamma$ is the constant of gravitation.

140. A number of uniformly distributed particles move with the same velocity $v$ in the same direction; in this medium is placed a body of any form and such that all the particles impinging on it adhere. Show that, if $M$ is the mass of the body at any time, and $u$ its velocity, then $M(v-u)$ will remain constant.

141. An umbrella whose surface is smooth and spherical is held in rain which falls vertically with velocity $v$ and the umbrella itself is drawn vertically downwards with velocity $V(<v)$. Prove that the average pressure per unit area of the rain falling on the umbrella at a point whose distance from the highest point is $\theta$ is $p \cos ^{2} \theta(v-V)^{2} / v^{2}$, where $p$ is the average pressure per unit area of the rain falling on a fixed horizontal plane.

142. Three equal particles are attached at equal intervals to a thread, and, when the thread is straight, the two extreme ones are projected in parallel directions with the same velocity $v$ at right angles to the thread. Prove that, if there are no external forces, the angular velocity of the portions of the thread when they have turned through an angle $\theta$ is $1 / \sqrt{ }\left(1+2 \sin ^{2} \theta\right)$ of its initial value.

143. Two particles on a smooth table are connected by an elastic thread of natural length $a$ and are initially at rest at a distance $a$ apart. One particle is projected at right angles to the thread. Prove that, if the greatest length of the thread during the subsequent motion is $2 a$, the velocity of projection is $\sqrt{ }(8 \alpha \lambda / 3 m)$, where $\lambda$ is the modulus of elasticity of the thread, and $m$ is the harmonic mean between the masses of the particles.

144. An equilateral wedge of mass $M$ is placed on a smooth table with one of its lower edges in contact with a smooth vertical wall, and a smooth ball of mass $M^{\prime}$ is placed in contact with the wall and with one face of the wedge so that motion ensues without rotation of the wedge. Prove that the ball will descend with acceleration

$$
3 M^{\prime} g /\left(M+3 M^{\prime}\right) \text {. }
$$

145. Two particles $A, B$ of masses $2 m$ and $m$ are attached at equal intervals to an inextensible thread $O A B$, and lie on a smooth table with the thread straight and the end $O$ fixed. The particle $B$ is projected on the table at right angles to $A B$. Prove that in the subsequent motion the angle $O A B$ is never less than a right angle, and that when $O A B$ is again a straight line the velocity of $B$ is half that of $A$.

146. Two particles of masses $m, m^{\prime}$ are placed close together on a smooth horizontal plane and are connected by an elastic thread of modulus $\lambda$ which passes round a smooth peg in the plane, and is of its natural length $\alpha$. The two particles are projected away from the peg with equal momenta. Prove 
that they will come to rest at the same time and that their distance apart will then be $\left(m \sim m^{\prime}\right) \sqrt{ }\left\{a u v / \lambda\left(m+m^{\prime}\right)\right\}$, where $u$ and $v$ are their initial velocities.

147. Find the charge of powder required with an elevation of $15^{\circ}$ to send a $32 \mathrm{lb}$. shot over a range of 1600 yards, being given that the initial velocity is 1600 feet per second when the charge is half the weight of the shot.

Prove that, if the gun is moveable on a smooth horizontal plane, and if the weight of the gun is $n$ times that of the shot, while the charge is that just found, then the range is

$$
6400 n /(4 n+2-\sqrt{ } 3) \text { yards. }
$$

148. A gun is suspended freely at an inclination $a$ to the horizontal by two equal parallel cords in a vertical plane containing the gun, and a shot whose mass is $1 / n$ of that of the gun is fired from it. Prove that the range on a horizontal plane through the muzzle is $4 n(1+n) h \tan a$, where $h$ is the height through which the gun rises in the recoil.

149. A regular polygon of $n$ sides is placed in a vertical plane with its two highest sides equally inclined to the vertical. A particle slides down from the highest point and $V_{\kappa}$ is its velocity on arriving at the $\kappa$ th angular point from the top, there being no restitution in the impacts. Prove that the particle will or will not pass through the $(\kappa+1)$ th angular point according as

$$
V_{\kappa}^{2}<\text { or }>\frac{1}{2} \operatorname{ag} \operatorname{cosec} \frac{2 \pi}{n} \cos ^{2} \frac{(2 \kappa+1) \pi}{n} \sec \frac{(2 \kappa-1) \pi}{n} .
$$

150. A wedge of mass $M$ and angle $a$ rests on a smooth horizontal table, and a particle of mass $m$, moving on the table in a vertical plane through the centre of inertia of the wedge and a line of greatest slope on its inclined face, comes to the edge of the wedge. Prove that, if there is no restitution between the wedge and the particle, and if the wedge is high enough, the particle will ascend through a vertical height

$$
h M^{2} \cos ^{2} a /\left\{(M+m)\left(M+m \sin ^{2} a\right)\right\},
$$

where $h$ is the height to which the velocity of the particle before reaching the wedge is due.

151. A wedge of angle $a$ and mass $M$ is free to move on a fixed horizontal plane. Another wedge of angle $a$ and of mass $M^{\prime}$ is laid upon it so that its upper surface, on which there is a particle of mass $m$, is horizontal. The surfaces are all smooth and the motion takes place in a vertical plane. Prove that the pressure of the particle $m$ on the plane with which it is in contact is

$$
M M^{\prime} m g /\left\{M M^{\prime}+\left(M+M^{\prime}\right)\left(m+M^{\prime}\right) \tan ^{2} a\right\} \text {. }
$$

152. Prove that, in the system of Example 151, the total weight exceeds the pressure on the fixed horizontal plane by

$$
\left\{\left(M+M^{\prime}\right)\left(M^{\prime}+m\right)^{2} g \sin ^{2} a\right\} /\left\{\left(M+M^{\prime}\right)\left(M^{\prime}+m\right) \sin ^{2} a+M M^{\prime} \cos ^{2} a\right\} .
$$


153. A smooth bore gun and carriage, together of mass $M$ tons, are placed on a railway truck of mass $M^{\prime}$ tons which runs on a smooth level railway. A projectile of mass $m$ tons is fired from the gun parallel to the rails. Show that, if the gun is fixed to the carriage, if the powder gas exerts a uniform thrust equal to the weight of $Q$ tons on the shot and gun, lasting till the shot has traversed the bore, a length $l$ feet, and if the resistance to sliding between the gun carriage and the truck is constant and equal to the weight of $R$ tons, then the velocity imparted to the shot is

$$
Q \sqrt{ }\left[2 M g l /\left\{m(m+M) Q-m^{2} R\right\}\right] \text { feet per second, }
$$

and the total length of recoil of the gun carriage on the truck is

$$
l Q m\left(M^{\prime}(Q-R)-M R\right) /\left[R\left(M+M^{\prime}\right)\{(M+m) Q-m R\}\right] \text { feet. }
$$

154. In a truck of mass $M$ is fixed a fine vertical tube inside which is fastened a particle of mass $m$. The truck is made to slide on a smooth horizontal plane by a massless horizontal chain which passes over a fixed smooth pulley and supports a body of mass $M^{\prime}$. Motion ensues for time $t$ after which the particle is allowed to fall down the tube. Prove that the path of the particle is a parabola of latus rectum

$$
2 M^{\prime 2}\left(M+M I^{\prime}+m\right) g t^{2} /\left\{\left(M+M^{\prime}+n\right)^{2}+M^{\prime 2}\right\}^{\frac{3}{2}}
$$

155. A railway carriage of mass $M$ moving with velocity $v$ impinges on a carriage of mass $M^{\prime}$ at rest. The force necessary to compress a buffer through the full extent $l$ is equal to the weight of a mass $m$. Assuming that the compression is proportional to the force, prove that the buffers will not be completely compressed if

$$
v^{2}<2 m g l\left(1 / M+1 / M^{\prime}\right) .
$$

Prove also that if $v$ exceeds this limit, and if the backing against which the buffers are driven is inelastic, the ratio of the final velocities of the carriages is

$$
M v-\sqrt{ }\left\{2 m M^{\prime} g l\left(1+M^{\prime} \mid M\right)\right\}: M v+\sqrt{ }\left\{2 m M g l\left(1+M / M^{\prime}\right)\right\} .
$$

156. Two particles of masses $m$ and $m^{\prime}$, joined by an elastic thread of natural length $l$ and modulus $\lambda$, are placed on a smooth table with $m$ at the edge and $m^{\prime}$ at a distance $l$ in a line perpendicular to the edge. The particle $m$ is then just pushed over the edge. Prove that, if the length of the thread at any time is $l+s$, then

$$
\dot{s}^{2}=2 g s-\lambda s^{2}\left(m+m^{\prime}\right) / m m^{\prime} l .
$$

Also, if at time $t, m$ has fallen through $z$ and $m^{\prime}$ is at a distance $x$ from the edge, prove that

$$
m^{\prime}(l-x)+m z=\frac{1}{2} m g t^{2} .
$$

157. An elastic circular ring of radius $c \sin a$ is placed unstretched in a horizontal plane over a smooth sphere of radius $c$. Prove that if it just slips over the sphere the position of equilibrium is defined by the equation

$$
4(\sin \theta-\sin a)^{2}(1+\sin a)=\tan ^{2} \theta(1-\sin a)^{3} .
$$


158. Two equal particles are connected by a thread one point of which is fixed, and the particles are describing circles of radii $a$ and $b$ about this point with the same angular velocity so that the thread is always straight. Prove that, if the thread is suddenly released, the tensions in the two portions are altered in the ratios $(a+b): 2 a$ and $(a+b): 2 b$.

159. Three equal particles are attached at equal intervals to a thread. One extreme particle $A$ is held fixed, and the other two are describing circles about it with the same angular velocity so that the thread is straight. Prove that, if the particle $A$ is let go, the tensions in the two portions of the thread are diminished in the ratios $1: 3$ and $1: 2$.

160. Two particles each of mass $m$ are connected by a rod of negligible mass and of length $l$, and lie on a rough horizontal plane (coefficient of friction $\mu$ ). One of the particles is projected vertically upwards with velocity $V$, prove that the other particle will begin to move when the rod makes with the plane an angle $a$, where $a$ is the least angle which satisfies the equation

$$
\left(V^{2}-3 g l \sin a\right)(\cos a+\mu \sin a)=\mu g l,
$$

provided ${V^{2}}^{2} / g l$ is less than $3 \sin a+\operatorname{cosec} a$, and find the radius of curvature of the path immediately after.

161. Two particles, each of mass $m$, are connected by an inextensible thread of length $l$ passing over a smooth pulley at the top of a smooth plane of inclination $a$ on which one of the particles rests at a distance $a$ from the top $(a<l)$. Prove that in the motion which ensues after the system is free of the plane the tension of the thread is constant and equal to

$$
\frac{1}{2} m g a l^{-1} \cos ^{2} a(1-\sin a),
$$

and that the radius of curvature of the path of the upper particle immediately after it leaves the plane is

$$
a \frac{1-\sin a}{\cos a} \frac{\left[\cos ^{2} a+\frac{1}{4}(1-\sin a)^{2}\right]^{\frac{3}{2}}}{1+\frac{1}{2} a l^{-1} \cos ^{2} a(1-\sin a)} .
$$

162. A spherical shell contains a particle of equal mass supported by springs of equal length and strength, attached at opposite ends of a diameter, and the system, all parts of which are moving in the line of the springs with the same velocity, strikes directly a fixed plane. Show that, if the coefficient of restitution between the shell and the plane is unity, the shell will strike the plane again after an interval of time equal to half the period of free oscillation.

163. In Example 162 the spherical shell is of mass $\mathrm{km}$ and the particle of mass $m$. Prove that the shell will or will not strike the plane again according as $k<$ or $>1+2 \cos a$, where $a$ is the least positive root of the equation $\tan a=a+\pi$.

164. In Example 162 the particle and the shell have equal masses but there is imperfect restitution (coefficient $e$ ) between the shell and the plane. 
Prove that, if $2 \pi / n$ is the period of the free oscillations of the system, the time $t$ until the shell again strikes the plane is the smallest positive root of the equation

$$
(1+e) \sin n t=(1-e) n t \text {. }
$$

165. In Example 163 prove that the period of oscillation when the shell is free to move is less than it would be if the shell were held fixed in the ratio

$$
1: \sqrt{ }(1+1 / k) \text {. }
$$

166. In a smooth table are two small holes $A, B$ at a distance $2 \alpha$ apart; a particle of mass $M$ rests on the table at the middle point of $A B$, being connected with a particle of mass $m$ hanging beneath the table by two inextensible threads, each of length $a(1+\sec a)$, passing through the holes. A blow $J$ is applied to $M$ at right angles to $A B$. Prove that, if

$$
J^{2}>2 M m a g \tan a,
$$

$M$ will oscillate to and fro through a distance $2 \alpha \tan a$, but if

$$
J^{2}=2 M m a g(\tan \alpha-\tan \beta)
$$

where $\tan \beta$ is positive, the distance through which $M$ oscillates will be

$$
2 \alpha \sqrt{ }\{(\sec a-\sec \beta)(\sec a-\sec \beta+2)\} \text {. }
$$

167. A particle can move in a smooth plane tube which rotates uniformly with angular velocity $\omega$ about a vertical axis. Prove that the time of a small oscillation about a position of relative equilibrium is

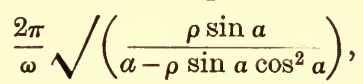

where $\rho$ is the radius of curvature at the point of equilibrium, $a$ the angle the normal at this point makes with the vertical, and $a$ the distance of the point from the axis.

168. A smooth circular wire is made to rotate uniformly about a vertical diameter. A bead of mass $m$ can move on the wire, and is attached to a thread, which passes through a fixed smooth ring at the lowest point of the circle and supports a body of mass $m$. Prove that, if $a$ is the angle which the radius through the bead in steady motion makes with the vertical, the steady motion is stable or unstable according as

is negative or positive.

$$
1-6 \sin ^{2} \frac{1}{2} a-8 \sin ^{3} \frac{1}{2} a
$$

169. A particle describes a horizontal circle in steady motion at a depth $d$ below the centre of a smooth oblate spheroid of axes $2 a, 2 b$, the axis of revolution being vertical. Prove that, if the tangent plane at any point of the circle makes an angle $\psi$ with the vertical, the velocity is $a \cot \psi \sqrt{ }(g d) / b$, and the period of the small oscillations when the steady motion is slightly disturbed is the same as that of a simple pendulum of length

$$
a^{2} d /\left(\alpha^{2} \cos ^{2} \psi+4 b^{2} \sin ^{2} \psi\right)
$$

170. A particle is describing a circle of radius $r$ in a smooth bowl in the form of a surface of revolution whose axis is vertical. Prove that, if the 
particle is slightly disturbed, the length of the simple equivalent pendulum for the small oscillations is

$$
r \rho \cos a /\left(r+3 \rho \cos ^{2} a \sin a\right),
$$

where $\rho$ is the radius of curvature of the meridian curve and $a$ the inclination of the normal to the vertical at any point on the horizontal circle.

171. A thread of length $l$ has its ends attached to two points distant $c$ apart on a vertical axis, and a bead can slide on the thread; the system rotates about the vertical axis with angular velocity $\omega$. Prove that, if

$$
\omega^{2}>2 g l /\left(l^{2}-c^{2}\right)
$$

the time of a small oscillation about a position of relative equilibrium is

where

$$
2 \pi \sqrt{ }\left\{A\left(l^{4}-A^{2} c^{2}\right) / 2 l^{2} g\left(l^{2}+3 A^{2}\right)\right\},
$$

$$
A=2 g l^{2} / \omega^{2}\left(l^{2}-c^{2}\right) \text {. }
$$

172. A particle describes a circle uniformly under the influence of two centres of force which attract inversely as the square of the distance. Prove that the motion is stable if $3 \cos \theta \cos \phi<1$, where $\theta, \phi$ are the angles which a radius of the circle subtends at the centres of force.

173. A straight uniform rod passes through a ring on a smooth horizontal plane, and an elastic thread whose natural length is equal to that of the rod has its ends fastened to the ends of the rod and its middle point fixed to the ring. Prove that, if the rod is rotating about its centre with an angular velocity such that the steady motion is unstable, then if it is slightly disturbed its centre will describe the curve whose polar equation is

$$
\left(1+\kappa^{2} / r^{2}\right) \sin ^{2} a=\cosh ^{2}(\theta \sin a),
$$

where $\theta$ is measured from the apse line, $\kappa$ is the radius of gyration of the rod about its centre, and $\kappa \tan a$ is the value of $r$ at the apse.

174. A uniform rod of length $2 b$ can slide with its ends on a smooth vertical circular wire of radius $a$ and the wire is made to rotate about a vertical diameter with uniform angular velocity $\omega$. Prove that the lowest horizontal position is stable if

$$
9 \omega^{2}\left(a^{2}-\frac{4}{3} b^{2}\right)<g \sqrt{ }\left(a^{2}-b^{2}\right) .
$$

175. Four equal uniform rods are freely jointed so as to form a rhombus $A B D C ; A B, A C$ are connected with a vertical spindle ly means of a hinge at $A$, permitting free motion in the vertical plane $B A C$. An elastic thread, of natural length ${ }_{3}^{2} A D$ when $A B$ is inclined to the vertical at an angle $a$, and of modulus of elasticity equal to twice the weight of a rod, joins $A$ to $D$. Prove that if the system is started to rotate with angular velocity $\omega$,

$$
=\sqrt{ }(3 g \mid A D),
$$

when each rod makes an angle $a$ with the. vertical the system will move steadily, and that the time of a small oscillation about the steady motion is

$$
(\pi / \omega) \sqrt{ }\left(1+3 \sin ^{2} \alpha\right) .
$$


176. A plane disc of any form has in its surface a flat circular cavity of radius $a$, and it is free to turn about a vertical axis through a point in the circumference of the cavity. A circular disc of mass $m$ and radius $c(<a)$ with a smooth face and a rough edge is in the cavity, and the whole system is rotating steadily about the vertical axis with angular velocity $\omega$. Prove that the length of the simple equivalent pendulum for small oscillations about the state of steady motion is

$$
\frac{g(a-c)}{a c^{2} \omega^{2}} \cdot I+m\left\{k^{2}+(2 a-c)^{2}\right\},
$$

where $I$ is the moment of inertia of the first disc about the axis, and $k$ is the radius of gyration of the circular disc about a vertical axis through its centre.

177. A plane tube the equation of which is $y^{2}=f(x)$ is turning freely about the axis of symmetry which is vertical with angular velocity $\sqrt{ }(g / c)$, and a particle of mass $m$ is in the tube close to the lowest point. Prove that, if the radius of curvature of the tube at the lowest point is greater than $c$, the particle will rise in the tube to a vertical height $h$ which is the least positive root of the equation

$$
2 I c h=(I-2 m c h) f(h),
$$

where $I$ is the moment of inertia of the tube about the axis of symmetry.

178. One end of a rigid uniform rod of length $2 \alpha$ formed of gravitating matter is constrained to move uniformly in a circle of radius $c$ with angular velocity $\omega$, and the rod is attracted to a fixed particle of mass $m$ at the centre of the circle. Prove that the rod can move steadily projecting inwards towards the centre, and that this steady motion is stable if

$$
\gamma m>\omega^{2} c(c-2 a)^{2} \text {. }
$$

179. If an elastic thread whose length is the same as that of a uniform rod is attached to the rod at both ends, and suspended by the middle point, prove that the rod will sink until the parts of the thread are inclined to the horizon at an angle $\theta$ which satisfies the equation

$$
\cot ^{3} \frac{1}{2} \theta-\cot \frac{1}{2} \theta=2 n
$$

where $n$ is the ratio of the modulus of elasticity of the thread to the weight of the rod.

180. A bead is free to slide on a rod whose ends slide without friction on a fixed circle. Prove that it moves relatively to the rod as if repelled from the middle point with a force varying inversely as the cube of the distance.

181. A smooth rigid uniform circular tube of mass $M$ contains two particles of masses $m_{1}, m_{2}$ and being placed on a table is set in motion by a blow in a line passing through the centre of inertia of the system. Prove that, if $\theta_{1}, \theta_{2}$ are the angles which the radii to the particles make at time $t$ with a fixed line on the table, then throughout the motion

$$
M\left(m_{1} \dot{\theta}_{1}+m_{2} \dot{\theta}_{2}\right)+2 m_{1} m_{2}\left(\dot{\theta}_{1}+\dot{\theta}_{2}\right) \sin ^{2} \frac{1}{2}\left(\theta_{1}-\theta_{2}\right)=0 \text {. }
$$


182. Two equal uniform rods $A B, B C$ each of mass $m$ and length $2 \alpha$ are freely jointed at $B$ and have their middle points joined by an elastic string, and the system moves in one plane under no forces. Prove that; if $\theta$ is the angle between the string and either rod at any time, $\phi$ the angle the string makes with a fixed line, and $V$ the potential energy of the stretched string, then throughout the motion

$$
\begin{gathered}
\left(\frac{1}{3}+\cos ^{2} \theta\right) \dot{\phi}=\text { const. } \\
m a^{2}\left\{\left(\frac{1}{3}+\cos ^{2} \theta\right) \dot{\phi}^{2}+\left(\frac{1}{3}+\sin ^{2} \theta\right) \dot{\theta}^{2}\right\}+V=\text { const. }
\end{gathered}
$$

183. Two equal uniform rods $A C, C B$, hinged at $C$, and having their extremities $A, B$ connected by a thread so that $A C B$ is a right angle, are revolving in their own plane with uniform angular velocity about the angle $A$ which is fixed. Prove that if the thread is severed the reaction at the hinge is instantaneously changed in the ratio $\sqrt{ } 5: 4$.

184. A smooth uniform tube contains a smooth uniform rod and the system moves under no external forces being set in motion by an impulse at right angles to the tube when the distance between the middle points of the rod and tube is $a$. Prove that the distance $r$ between the middle points when the system has turned through an angle $\theta$ is given by the equation

$$
\left(a^{2}+b^{2}\right)\left\{r^{2}+b^{2}+\left(\frac{d r}{d \theta}\right)^{2}\right\}=\left(r^{2}+b^{2}\right)^{2},
$$

where $b$ is a certain constant depending on the masses and moments of inertia of the rod and tube.

185. A smooth circular tube lying in a horizontal plane contains a particle at a point $C$ and can turn about a point $A$ of its circumference. Prove that, if the tube is struck by a horizontal blow, the particle can oscillate about the point $B$ furthest from $A$, and that, if $C B$ subtends at the centre an angle $a$ and the line joining the particle to the centre at time $t$ makes with the radius to $B$ an angle $\beta$, the limits of oscillation are given by

$$
\cos \frac{1}{2} \beta= \pm \frac{1}{2} \sin a \text {. }
$$

186. One end of an inextensible thread of length $\alpha$ is attached to a smooth circular wire of radius $a$ whose plane is vertical at one end of a horizontal diameter, and the other end is attached to one end of a rigid uniform rod of length $a$ whose other end can slide on the wire. The system starts from rest with the thread and rod in a horizontal position; find the velocity of the rod when its middle point has fallen through any distance.

187. A uniform rod of mass $m$ and length $2 \alpha$ moves at right angles to itself on a smooth table, and impinges symmetrically on a uniform circular disc of mass $m^{\prime}$ and radius $a$ spinning freely about its centre. Prove that, if there is no restitution, and the edge of the disc is rough enough to prevent slipping; the bodies will separate after an interval in which the unmolested disc would have turned through an angle whose circular measure is

$$
\left(m^{\prime}+3 m\right) /\left(m^{\prime}+m\right) \text {. }
$$


188. A solid paraboloid of revolution is free to turn round its axis which is vertical, and has a groove cut in its surface which makes a constant angle $a$ with the axis. A particle of mass $m$ is placed in the groove at a depth $h_{0}$ below the vertex. Prove that when the particle has descended a depth $h$ the angular velocity of the paraboloid is

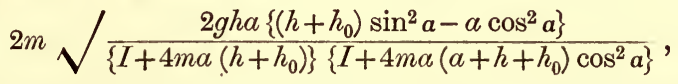

where $4 a$ is the latus rectum of the paraboloid and $I$ is its moment of inertia about its axis.

189. A uniform cube, of mass $M$, and radius of gyration $\kappa$ about an axis through its centre, rests on a smooth horizontal plane, and a smooth circular groove of radius $a$ is cut on the upper face and passes through the centre $O$ of that face. A particle of mass $m$ is projected along the groove from $O$ with velocity $V$. Prove that, if $a \psi$ is the arc traversed by the particle, and $\phi$ the angle turned through by the block in any time,

where

$$
\cot \frac{\sqrt{ }\left(1+\beta^{2}\right)}{\beta}\left(\frac{1}{2} \phi-\theta\right)=\frac{\beta}{\sqrt{\left(1+\beta^{2}\right)}} \cot \frac{1}{2} \phi,
$$

190. Two uniform rods each of length $2 a$ are freely jointed and placed on a smooth table in a straight line parallel to an edge. A cord is attached to the joint and passing over the edge of the table at right angles supports a body of mass $1 / n$ of that of either rod. Prove that the angle $\theta$ through which either rod has turned at time $t$ is given by the equation

$$
\left\{2+n\left(1+3 \sin ^{2} \theta\right)\right\} a \dot{\theta}^{2}=3 g \sin \theta .
$$

191. Six equal uniform rods are freely jointed at a point $O$ and have their other ends at the corners of a regular hexagon on a smooth horizontal plane, these ends being connected by six similar elastic threads in the sides of the hexagon. Initially all the threads have their natural lengths, and the rods are inclined at an angle $a$ to the vertical. Prove that the joint will or will not reach the plane according as the ratio of the modulus of elasticity of each thread to the weight of each $\operatorname{rod}<$ or $>\sin a \cos a /(1-\sin a)^{2}$.

192. A rifled gun is mounted on a carriage without wheels. Prove that, if $a$ is the elevation of the gun, $p$ the pitch of the barrel, $k$ the radius of gyration of the shot, and $U, V$ the muzzle velocities of the shot when the carriage is (1) fixed and (2) allowed a free recoil, then

$V^{2}\left\{k^{2} / p^{2}+\sin ^{2} a+M \cos ^{2} a /(M+m)\right\}=U^{2}\left(1+k^{2} / p^{2}\right)\left\{\sin ^{2} a+M^{2} \cos ^{2} a /(M+m)^{2}\right\}$, where $m$ is the mass of the shot, and $M$ is the mass of the gun and carriage.

193. A particle is placed in a smooth elliptic tube of $n$ times its mass at an end of the major axis, and the tube is struck by a blow parallel to the minor axis so that it starts to move parallel to this axis with velocity $V$. Prove that the eccentric angle $\phi$ of the position of the particle at any time is given by the equation

$$
\left[a^{2} \sin ^{2} \phi+b^{2} \cos ^{2} \phi+\frac{a^{2}\left(a^{2}-b^{2}\right) \sin ^{2} \phi}{(1+n) k^{2}-\left(a^{2}-b^{2}\right) \sin ^{2} \phi}\right] \dot{\phi}^{2}=V^{2},
$$


where $2 a$ and $2 b$ are the principal axes of the tube, and $k$ is its radius of gyration about its centre.

194. Two rough horizontal cylinders each of radius $c$ are fixed with their axes inclined to each other at an angle $2 a$ and a uniform sphere of radius $a$ rolls between them, starting with its centre very nearly above the point of intersection of the highest generators. Prove that the vertical velocity of its centre in a position in which the radii to the two points of contact make angles $\phi$ with the horizontal is

$$
\sqrt{ }\left\{10 g(a+c)(1-\sin \phi) /\left(7-5 \cos ^{2} a \cos ^{2} \phi\right)\right\} .
$$

195. Two equal right circular cones of semivertical angle $a$ are fixed with their axes horizontal so as to touch along a horizontal generator and to have their vertices coincident. A sphere of radius $\alpha$ rolls between them. Prove that the height $z$ of its centre above the plane of the axes satisfies the equation

$$
\frac{1}{2} \dot{z}^{2}\left\{1+\frac{z^{2}}{a^{2}} \cot ^{2} a+\frac{k^{2}}{4 a^{2}} \cot ^{2} a\left(\sec ^{2} a+\frac{z^{2}}{a^{2}}\right)^{2}\right\}=g\left(z_{0}-z\right) .
$$

196. A circular tube of mass $m$ and radius $a$ contains a particle of mass $n m$, and the tube rotates freely about a vertical chord $A B(A$ above $B)$ which subtends an angle $2 a$ at the centre. Initially the particle is at the highest point $C$ of the tube and the tube is set rotating with angular velocity $\Omega$. Prove that, if the particle oscillates between $C$ and $B$, then

$$
a \Omega^{2}\left\{(n+1) \cos ^{2} a+\frac{1}{2}\right\} \cos ^{2} a=g(1+\sin a)\left(1+2 \cos ^{2} a\right) .
$$

197. A rhombus of four equal uniform rods each of length $a$ freely jointed together is laid on a smooth horizontal table with one angle equal to $2 a$, and the opposite corners are joined by similar elastic threads of natural lengths $2 \alpha \cos a$ and $2 \alpha \sin a$. Prove that, if one thread is slightly extended and the rhombus left free, the periods during which the threads are extended in the subsequent mation are in the ratio $(\cos a)^{\frac{3}{2}}:(\sin a)^{\frac{8}{2}}$.

198. A particle of mass $m$ is placed in a smooth straight tube which can rotate in a vertical plane about its middle point, and the system starts from rest with the tube horizontal. Prove that the angle $\theta$ which the tube makes with the vertical when its angular velocity is a maximum and equal to $\omega$ is given by the equation $4\left(m r^{2}+I\right) \omega^{4}-8 m g r \omega^{2} \cos \theta+m g^{2} \sin ^{2} \theta=0$, where $I$ is the moment of inertia of the tube about its middle point, and $r$ is the distance of the particle from that point.

199. Four equil rods of length $a$ and mass $m$ are freely jointed so as to form a rhombus one of whose diagonals is vertical; the ends of the horizontal diagonal are joined by an elastic thread at its natural length, and the system falls through a height $h$ to a horizontal plane. Prove that, if any rod makes an angle $\theta$ with the vertical at time $t$ after the impact, then

$$
\left(1+3 \sin ^{2} \theta\right) \dot{\theta}^{2}=\frac{18 g h}{a^{2}} \frac{\sin ^{2} a}{1+3 \sin ^{2} a}+\frac{6 g}{a}(\cos a-\cos \theta)-\frac{3 \lambda}{2 m a} \frac{(\sin \theta-\sin a)^{2}}{\sin a},
$$

where $a$ is the initial value of $\theta$, and $\lambda$ is the modulus of the thread. 
200. A square formed of four similar uniform rods freely jointed at their extremities, is laid on a smooth horizontal table, one of its corners being fixed; show that, if angular velocities $\omega, \omega^{\prime}$ in the plane of the table are communicated to the rods that meet at this corner, the greatest value of the angle between them is

$$
\frac{1}{2} \cos ^{-1}\left\{-\frac{5}{6}\left(\omega-\omega^{\prime}\right)^{2} /\left(\omega^{2}+\omega^{\prime 2}\right)\right\} .
$$

201. A homogeneous hemisphere of radius $a$ and mass $M$ falls from rest with its base vertical on to a smooth horizontal plane (no restitution). Prove that its pressure on the plane when its base is horizontal is equal to

$$
\frac{173}{83} M g+\frac{675}{132} M V^{2} / \alpha
$$

where $V$ is the velocity with which it strikes the plane.

Prove that the hemisphere will leave the plane immediately upon its base becoming vertical if $15 \mathrm{~V}>16 \sqrt{ }(a g)$, and that, if $675 \mathrm{~V}^{2} /(1024 \pi a g)$ is an integer, the hemisphere will again strike the plane with its base vertical.

202. Two equal homogeneous cubes are moving on a smooth table with equal and opposite velocities $V$ in parallel lines, and impinge so that finite portions of opposing faces come into contact; show that so long as they remain in contact the line joining their centres meets the opposing faces at a distance $x$ from the centres of the faces where

$$
\dot{x}^{2}\left(x^{2}+\frac{2}{3} a^{2}\right)\left(x_{0}^{2}+\frac{2}{3} a^{2}\right)=V^{2} x_{0}^{2}\left(a^{2}+x^{2}-x_{0}^{2}\right),
$$

where $2 a$ is a side of either cube, and $x_{0}$ is the initial value of $x$.

Prove further that, if the line joining the centres at the instant of impact cuts the opposing faces at an angle $\frac{1}{3} \pi$, then while the faces are in contact they slip with uniform relative velocity, and separate after an interval $(1+\sqrt{ } 3) a / V$ after turning through an angle

$$
2 \sqrt{ } \frac{3}{5}\left\{\tan ^{-1} \sqrt{ } \frac{3}{5}+\tan ^{-1} \sqrt{\frac{1}{5}}\right\} \text {. }
$$

203. Two equal rigid inelastic uniform hooks $A B C D, A^{\prime} B^{\prime} C^{\prime} D^{\prime}$ each in the form of three sides of a square of side $2 a$ moving with equal velocities $V$ in opposite directions parallel to $A B$ or $A^{\prime} B^{\prime}$ impinge, so that the points $D$ and $D^{\prime}$ strike the middle points of $B^{\prime} C^{\prime}$ and $B C$. Show that they separate immediately after impact with the velocities of their centres of inertia reduced in the ratio $9: 53$.

Prove that if the ends $D$ and $D^{\prime}$ are provided with apparatus for clipping the sides $B^{\prime} C^{\prime}$ and $B C$ so that they can slide on these sides without friction, then in the subsequent motion $D$ and $D^{\prime}$ will come to relative rest after moving over distances $\frac{1}{3}(3-\sqrt{ } 5) a$ on $B^{\prime} C^{\prime}$ and $B C$, and that the sides $C D$ and $C^{\prime} D^{\prime}$ will impinge upon $A^{\prime} B^{\prime}$ and $A B$ after an interval

$$
\frac{\sqrt{ } 53}{18} \frac{a}{V} \int_{\sqrt{ } 5}^{6} \frac{\sqrt{ }\left(44+x^{2}\right)}{\sqrt{ }\left(x^{2}-5\right)} d x
$$

from the instant when $D$ and $D^{\prime}$ were at rest relative to the hooks.

204. Two smooth rigid uniform hemispheres, each of radius $a$ and of equal masses, moving at right angles to their bases with the same velocity $V$, impinge so that lengths $\frac{5}{4} a$ of the diameters of their bases in the plane 
through their centres perpendicular to their bases come into contact. Prove that the distance $x$ between their centres at time $t$ is given by the equations

$$
\begin{gathered}
80 x^{2}=19 a^{2}\left(z^{2}-1\right), \\
\frac{15}{\sqrt{ } 19} \frac{V t}{a}=\frac{8}{\sqrt{ } 19}-z-\frac{1}{2} \log \left(\frac{8+\sqrt{ } 19}{8-\sqrt{ } 19} \frac{z-1}{z+1}\right) .
\end{gathered}
$$

205. A uniform chain of length $l$ is held by its upper end so that its lower end is at a height $l$ above a fixed horizontal plane, and is let drop on the plane. Prove that when half the chain is on the plane the pressure on the plane is $\frac{7}{2}$ of the weight of the chain.

206. A uniform chain of length $l$ is coiled at the edge of a table; one end is attached to a particle of mass equal to that of the chain, and the other end is put over the edge of the table. Prove that immediately after leaving the table the particle is moving with velocity $\frac{1}{2} \sqrt{ }\left(\frac{5}{6} g l\right)$.

207. Two uniform chains, lengths $l, l^{\prime}$, masses $m l, m^{\prime} l^{\prime}$, are coiled in loose heaps and lie close together. They are projected with velocities $v, v^{\prime}$ in directions containing an angle $a$ and move under no forces. Prove that, if $m l^{2}>m^{\prime} l^{2}$, the former will be uncoiled before the latter, that the tension so long as neither is completely uncoiled is $m m^{\prime}\left(v^{2}+v^{\prime 2}-2 v v^{\prime} \cos a\right) /\left(\sqrt{ } m+\sqrt{ } m^{\prime}\right)^{2}$, and that the whole time which elapses before both are uncoiled is

$$
\frac{1}{2}\left(m l^{2}+m^{\prime} l^{\prime 2}+2 m^{\prime} l^{\prime}\right) / m l \sqrt{ }\left(v^{2}+v^{\prime 2}-2 v v^{\prime} \cos a\right) \text {. }
$$

208. A coil of uniform chain of mass $m_{1}$ per unit of length is placed on a smooth table and one end of it is joined by a thread passing over the edge of the table to one end of another coil of mass $m_{2}$ per unit of length, which is held just at the edge. Prove that if the second coil is let go the straight parts of the chains increase with uniform accelerations

$$
g \sqrt{ } m_{2} /\left(\sqrt{ } m_{1}+\sqrt{ } m_{2}\right) \text { and } g \sqrt{ } m_{1} /\left(\sqrt{ } m_{1}+\sqrt{ } m_{2}\right),
$$

so long as neither is completely uncoiled.

209. A chain of length $l$ slides from rest down a line of greatest slope on a smooth plane of inclination $a$ to the horizontal, the end of the chain hanging initially just over the edge. Prove that the time of leaving the plane is $\sqrt{ }\{l / g(1-\sin a)\} \log \left(\cot \frac{1}{2} a\right)$.

210. A smooth circular cylinder is fixed with its axis horizontal and vertically over the edge of a table, on which a length $a$ of a uniform chain of mass $m l$ and length $l$ is coiled, the chain passing over the cylinder and having its free end on a level with the table. Prove that, if this end is slightly displaced downwards, the amount of energy that will have been dissipated by the time the chain leaves the table is $\frac{1}{6} m g a^{3} / l$.

211. A smooth circular cylinder is fixed with its axis horizontal and vertically above the edge of a table on which lies a chain of length $l$ and mass $\mu$, one end of which is attached to a thread passing over the cylinder and supporting a body of mass $M$. If all the chain is off the 
table before any of it reaches the cylinder, prove that the amount of energy dissipated by the time the chain leaves the table is

$$
\frac{1}{6} m g l(3 M-\mu) /(M+\mu) \text {. }
$$

212. A quantity of uniform chain is coiled on a horizontal plane, and a body of mass equal to that of a length $l$ of the chain is fastened to one end and projected vertically upwards with the velocity due to falling through a height $h$; prove that it will rise to a height

$$
\left\{l^{2}(l+3 h)\right\}^{\frac{1}{3}}-l .
$$

213. A length $k$ of a uniform chain of length $l+k$ and mass $\mu(l+k)$ is coiled at the edge of a table and the length $l$ hangs over the edge. Prove that the amount of energy dissipated by the time the chain leaves the table is $\frac{1}{6} \mu g k^{2}(3 l+k) /(l+k)$.

214. A great length of uniform chain is coiled at the edge of a horizontal platform, and one end is allowed to hang over until it just reaches another platform distant $h$ below the first. The chain then runs down under gravity. Prove that it ultimately acquires a finite terminal velocity $V$, that its velocity at time $t$ is $V \tanh (V t / h)$, and that the length of chain which has then run down is $h \log \cosh (V t / h)$.

215. A thread of length $2 h-l$ passing over a smooth peg at a height $h$ above a table has attached to its ends two uniform chains, and the system is released from rest when a length $l$ of one chain is vertical and the rest of that chain and the other chain are coiled on the table. Prove that the chains will be momentarily at rest when the length of the vertical portion $l$ is reduced to $l-x$, where

$$
\log \{l /(l-x)\}=2 x / l,
$$

and that the maximum velocity is acquired when $2 x / l=\log 2$.

216. A chain whose density varies uniformly from $\rho$ at one end to $2 \rho$ at the other end is placed symmetrically on a small smooth pulley and is then let go. Prove that it leaves the pulley with velocity $\frac{1}{3} \sqrt{ }(11 l g)$, where $2 l$ is its length.

217. Two buckets each of mass $M$ are connected by a chain of negligible mass which passes over a fixed smooth pulley. On the bottom of one of them lies a length $l$ of uniform chain, whose mass is $\mu l$, one end of which is attached to a fixed point just above the bottom of the bucket. Prove that, if the system starts to move from rest, the velocity of the bucket when there remains upon it a length $y$ of chain is $V$, where

$$
V^{2}=2 g(l-y)-\frac{4 M g}{\mu} \log \frac{2 M+\mu l}{2 M+\mu y} .
$$

218. Two scale-pans each of mass $M$ are supported by a cord of negligible mass passing over a smooth pulley, and a uniform chain of mass $m$ and length $l$ is held by its upper end above one of the scale-pans so that it just reaches the pan. Find the acceleration of the pan when a length $x$ of chain has fallen upon it, and prove that the whole chain will have fallen upon it after an interval $\sqrt{ }\left\{\frac{1}{2} l(4 M+m) / M g\right\}$. 
219. A chain of length $a$ is coiled up on a ledge at the top of a rough plane of inclination $a$ to the horizontal, and one end is allowed to slide down. Prove that, if the inclination of the plane is double the angle of friction $(\lambda)$, the chain will be moving freely at the end of a time $\sqrt{ }\{6 a /(g \tan \lambda)\}$.

220. One end of a uniform chain of length $l$ and mass $m$ is fixed to a horizontal platform of mass $(2 k-1) m$, the chain passes over a smooth fixed pulley, and is coiled on the platform. As the platform descends vertically, the chain uncoils, rises vertically and passes over the pulley. Prove that at any time $t$ before the chain is completely uncoiled the depth $x$ of the platform satisfies an equation of the form $\dot{x}^{2}=a+\beta x+\gamma e^{-k x / l}$, where $a, \beta$, and $\gamma$ are constants.

221. A uniform chain is placed on the arc of a smooth cycloid whose axis is vertical and vertex upwards. Show that so long as the chain is wholly in contact with the cycloid the tension at any point of the chain is constant throughout the motion, and is a maximum at the middle point.

222. A string without weight is coiled round a rough horizontal uniform solid cylinder of mass $M$ and radius $\alpha$ which is free to turn about its axis. To the free extremity of the string is attached a uniform chain of mass $m$ and length $l$; if the chain is gathered close up and then let go, prove that the angle, $\theta$, through which the cylinder has turned after a time $t$, before the chain is fully stretched, satisfies the equation $M l a \theta=m\left(\frac{1}{2} g t^{2}-a \theta\right)^{2}$.

223. A piece of uniform chain is placed on a four-cusped hypocycloid occupying the are between two cusps the tangents at which are horizontal and vertical, and the chain runs off the curve at the lower cusp. Prove that the velocity of the chain when it has just all run off is that due to falling through $\frac{9}{10}$ of its length.

224. An elastic circular ring of which the radius when unstrained is $a$ rests on a smooth surface of revolution whose axis is vertical in the form of a circle of radius $r$. Prove that the period of the small oscillations in which each element moves in a vertical plane is the same as for a simple pendulum of length $l$, where $1 / l=\sin a \cos a /(r-a)-\sec a / \rho, \rho$ being the radius of curvature of the meridian curve at a point on the ring, and $a$ the inclination of the normal to the vertical.

225. An endless flexible and inextensible chain, of which the mass per unit length is $\mu$ through one continuous half and $\mu^{\prime}$ through the other, is stretched over two equal rough discs each of mass $M$ and radius $a$, which can turn freely about their centres at a distance $b$ apart in a vertical line. Prove that the time of a small oscillation of the chain under gravity is

$$
2 \pi \sqrt{\frac{M+(\pi a+b)\left(\mu+\mu^{\prime}\right)}{2\left(\mu-\mu^{\prime}\right) g},}
$$

the discs being rough enough to prevent slipping. 
226. An elastic string (modulus $\lambda$, mass $m a$, unstretched length $\alpha$,) is confined within a straight tube to one end of which it is fastened, and the tube rotates about that end with uniform angular velocity $\omega$ in a horizontal plane. Show that the length of the string in equilibrium is

$$
\alpha \frac{\tan \theta}{\theta}, \text { where } \theta=a \omega \sqrt{\lambda} .
$$

227. A smooth circular tube of radius $\alpha$ having a narrow slit on its inner side rotates about a fixed vertical diameter $A B$ with angular velocity $\omega$, $A$ being the highest point, and a uniform chain which subtends an angle $2 a$ at the centre can move in the tube. To its upper end is attached an elastic thread which passes through the slit and through a ring at $A$ and is fastened at $B$, its natural length is $2 \alpha$ and its modulus of elasticity equal to a weight $4 a \tan a$ of the chain. Prove that the chain is at rest in the tube in the same position for all values of $\omega$, and that, if slightly disturbed, it oscillates in a period

$$
2 \pi \sqrt{ }\left\{\alpha a \operatorname{cosec} a /\left(g \tan a+\alpha \omega^{2} \cos a\right)\right\} .
$$

228. A cone of vertical angle $2 a$, whose moment of inertia about its axis is $I$, is free to turn about its axis which is vertical, and a fine smooth groove is cut on its surface so as to make a constant angle $\beta$ with the generators. A uniform chain of mass $\mu$ and length $l$ moves in the groove under gravity, one end being initially at the vertex. Prove that, if $\theta$ is the angle through which the cone has turned when the upper end is at a distance $r$ from the vertex,

$$
\left\{I \operatorname{cosec}^{2} a / \mu+\frac{1}{3} l^{2} \cos ^{2} \beta\right\} e^{2 \theta \sin \alpha \cot \beta}=r^{2}+r l \cos \beta+\frac{1}{3} l^{2} \cos ^{2} \beta+I \operatorname{cosec}^{2} \alpha / \mu .
$$

229. A uniform chain of mass $m$ and length $2 l$ is in a tube of uniform bore in the form of an equiangular spiral which revolves in its plane about its pole with uniform angular velocity $\omega$. Prove that the tension at any point of the chain is $\frac{1}{4} m \cos ^{2} a\left(l^{2}-x^{2}\right) \omega^{2} / l$, where $a$ is the angle of the spiral and $x$ the arcual distance of the point from the middle point of the chain.

230. A smooth tube in the form of a cycloid generated by a circle of radius $\alpha$ rotates uniformly about the base of the cycloid with angular velocity $\boldsymbol{\Omega}$, and a piece of uniform chain of length $2 l$ is in the tube. Prove that, if the chain is under no forces but the pressure of the tube, the time of a small oscillation about the position of relative equilibrium is

$$
(8 \pi / \Omega) \sqrt{ }\left\{2 \alpha^{2} /\left(16 \alpha^{2}-l^{2}\right)\right\} \text {. }
$$

231. A rough circular cylinder of radius $c$ is fixed with its axis vertical, and a uniform chain lying on a smooth horizontal plane has a length $c \beta$ in contact with the cylinder, its end portions of lengths $a$ and $b$ being straight. The free end of the length $\alpha$ is pulled by a constant force $F$ in the direction of its length. Prove that when the free end of the length $b$ reaches the cylinder it will be moving with a velocity

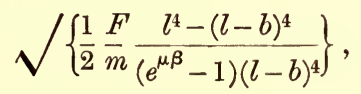


where

$$
l=c / \mu+\left(a+b e^{\mu \beta}\right) /\left(e^{\mu \beta}-1\right),
$$

$\mu$ being the coefficient of friction, and $m$ the mass of a unit of length of the chain.

232. A uniform chain falls in a vertical plane with uniform acceleration $f$ retaining an invariable form, while the chain advances along itself with a velocity which at any instant is the same for all points of the chain. Prove that the angle $\phi$ which the tangent at any point of the chain makes with the horizontal, considered as a function of the time $t$ and of the arc $s$ measured up to this point from some definite point of the chain, satisfies the two partial differential equations

$$
\begin{gathered}
(f-g)\left\{\cos \phi \frac{\partial^{2} \phi}{\partial s^{2}}+2 \sin \phi\left(\frac{\partial \phi}{\partial s}\right)^{2}\right\}+\frac{\partial^{2} \phi}{\partial t^{2}} \frac{\partial \phi}{\partial s}-\frac{\partial \phi}{\partial t} \frac{\partial^{2} \phi}{\partial s \partial t}=0, \\
\frac{\partial \phi}{\partial s} \frac{\partial^{2} \phi}{\partial s \partial t}-\frac{\partial \phi}{\partial t} \frac{\partial^{2} \phi}{\partial s^{2}}=0 .
\end{gathered}
$$

233. A uniform flexible chain passes over and under two rough equal pulleys of radius $a$ whose centres are at a distance $d$ apart in the same horizontal line; part of the chain is coiled up on a horizontal platform at a depth $h$ below this line, the part between one pulley and the platform is vertical, the part below the pulleys is a catenary of parameter $c$, and the chain hangs from the second pulley to a platform at a lower level $h^{\prime}$, the vertical parts being between the pulleys. Show that steady motion with this configuration is possible the pulleys rotating with angular velocity $\sqrt{ }\left\{g\left(h-h^{\prime}\right)\right\} / a$, and that the relation between $c, d$, and $h$ can be found by eliminating $a$ between the equations

$$
h=c \sec a+a \cos a, \quad d=2 c \sinh ^{-1}(\tan a)-2 a \sin a .
$$

234. A uniform chain hanging under gravity receives a tangential impulse at one end. Prove that the initial velocity at any point in the direction at right angles to the directrix is proportional to the curvature at the point.

235. A chain of variable density is in the form of an arc of a circle less than a semicircle and subtending an angle $2 a$ at the centre, and the line density varies inversely as the square of the distance from the diameter parallel to the chord joining the ends. The chain is set in motion by equal tangential impulses $T$ applied at its ends; prove that the kinetic energy generated is $2 T^{2} \sin ^{2} a / M$, where $M$ is the mass of the chain.

236. The ends of a chain of variable density are held at the same level, and the chain hangs in the form of an arc of a circle subtending an angle $2 \theta(<\pi)$ at the centre. If equal tangential impulses are applied at the ends the initial normal velocities at the lowest point and at either end are in the ratio $1: \cos \theta$.

237. A uniform chain lying in a curve on a smooth horizontal plane is set in motion by impulsive tension applied at one end in the direction of the tangent. If the initial direction of motion of every element makes the same angle with the tangent prove that the curve is an equiangular spiral. 
238. A string is laid on a smooth table in the form of a catenary, and an impulse is communicated to one extremity in the direction of the tangent; prove (1) that the initial velocity of any point, resolved parallel to the directrix, is proportional to the inverse square of the distance of that point from the directrix, (2) that the velocity of the centre of gravity of any arc, resolved in the same direction, is proportional to the angle between the tangents at the ends of the are directly and to the length of the arc inversely.

239. A uniform chain lies in a part of the curve $r=\alpha e^{\frac{3}{2} \theta}$ from $\theta=0$ to $\theta=\beta$ and receives a tangential impulse $T_{0}$ at $\theta=0$, the other end being free. Prove that the impulsive tension at any point is

$$
T_{0}\left(e^{\frac{5}{2} \beta-\frac{1}{2} \theta}-e^{2 \theta}\right) /\left(e^{\frac{5}{2} \beta}-1\right) .
$$

240. An endless uniform chain lying in the form of a circle receives a tangential pluck at one point $A$ which gives it an impulsive tension $T_{0}$ at that point; prove that the impulsive tension at any point $P$ is

$$
T_{0} \frac{\sinh (2 \pi-\theta)}{\sinh 2 \pi}
$$

$\theta$ being the angle which $A P$ subtends at the centre, and that $P$ starts in a direction making an angle $\phi$ with the tangent, where

$$
\tan \phi=\left(e^{4 \pi}-e^{2 \theta}\right) /\left(e^{4 \pi}+e^{2 \theta}\right) \text {. }
$$

241. A thin chain of variable density is placed on a smooth table in the form of the curve in which it would hang under gravity, and two impulsive tensions are applied at its extremities, which are to each other in the ratio of the tensions at the same points in the hanging chain. Prove that the whole will move without change of form parallel to the line which was vertical in the hanging chain.

242. A uniform flexible inextensible chain of density $\rho$ rests on a smooth plane; a part of its length is in contact with a smooth circular disc of radius $a$ which lies on the plane, the length of this part being $\alpha(a+\beta)$; the remainder is in two straight portions which touch the disc at the ends of the arc of contact; and particles of masses $m$ and $m^{\prime}$ are attached at the ends. Prove that, when the disc is suddenly moved with velocity $V$ in a direction making an angle $a$ with the radius to the point at which the portion carrying $m$ leaves the disc, $m$ begins to move with velocity

$$
M^{-1} V\left[\left(m^{\prime}+\rho l^{\prime}\right)(\sin a+\sin \beta)+\rho \alpha\{(a+\beta) \sin a+(\cos a-\cos \beta)\}\right],
$$

where $M$ is the mass of the whole system, and $l^{\prime}$ is the length of the straight portion of chain to which $m^{\prime}$ is attached.

243. A uniform inextensible chain is stretched nearly straight with two ends at the same level; suddenly one end is released. Prove that, to a first approximation, half the product of the tensions at the other end before and after release is equal to the square of the weight of the chain. 
244. A uniform chain hangs in equilibrium with its ends fastened to two points nearly in the same vertical line. One end is released, and when the velocity of this end is $u$ the lengths of the two portions of the chain are $l$ and $l^{\prime}$. Prove that, if the other end is released at this instant, the chain becomes straight after a time $l\left(l+2 l^{\prime}\right) / l^{\prime} u$.

245. A uniform chain is suspended from two points in the same horizontal line so that the tangents at the ends make angles $a$ with the horizontal. Prove that, if the support at one end is removed, that end starts to move in a direction making with the horizontal an angle $\theta$, where

$$
\tan \theta=\left(1+\sin ^{2} a+2 a \tan a\right) / \sin a \cos a,
$$

and that the tension at the other end is diminished in the ratio

$$
1: 1+\frac{1}{2} a^{-1} \cot a \text {. }
$$

246. A chain is attached to two fixed points and rests in an arc of a circle of angle $2 a$ under a repulsive force varying inversely as the cube of the distance from the extremity of the diameter bisecting the arc. Prove that, if the chain is cut through at its middle point, the initial tension at any point is proportional to

$$
\sec ^{2} \frac{1}{2} \phi-\operatorname{sech} a \cosh (a-\phi),
$$

where $\phi$ is the angular distance of the point from the point of section.

247. A heterogeneous chain hangs under gravity in the form of a circle, its ends being free to slide on two smooth straight wires which make equal angles $\gamma$ with the vertical. Prove that, if the chain is severed at its vertex, the tension at a point where the tangent makes an angle $\phi$ with the horizontal is diminished in the ratio

$$
\phi: \gamma+\cot \gamma
$$

248. A chain of variable density has its ends fixed at points $A, B$ and hangs freely, the tangents at $A$ and $B$ making angles $a$ and $\beta$ with the horizontal. Prove that, if the end $A$ is released, the tension at a point $P$, where the tangent makes an angle $\phi$ with the horizontal, is instantaneously changed in the ratio

$$
(\phi+a) \sin \beta: \cos \beta+(a+\beta) \sin \beta \text {. }
$$




\section{CHAPTER XIII.}

\section{RELATIVE MOTION AND UNIVERSAL GRAVITATION.}

275. In the course of this book we have, in order to avoid interruptions of the argument, made a number of statements of a provisional character. Such, for example, were the statement in Article 3 concerning the method of measuring time, the statements in Articles 90, 91, and 94 concerning the relations between weight, mass, acceleration due to gravity, and gravitational force exerted by the Earth upon a body, and the statement in Article 88 that frames of reference can be chosen for which the Postulates of Mechanics apply to natural bodies. We shall now reconsider all these matters, and we shall find it convenient to begin with an account of the motion known as the rotation of the Earth, and its effects as observed in the cases of falling bodies and pendulums.

276. Rotation of the Earth. It is a fact of observation that there is a relative motion of the Earth and stars by which every star moves relatively to the Earth continually from East to West, or, what is the same thing, by which any part of the Earth's surface moves relatively to the stars continually from West to East. This motion can be precisely described by saying that, relatively to the stars, the Earth rotates about its polar axis; the magnitude of the angular velocity is such that the Earth turns through four right angles in a sidereal day, and the sense is such that the positive sense of the axis of rotation is from South Pole to North Pole. We shall use the letter $\Omega$ to denote the angular velocity of the Earth's Rotation.

In the statement of the Law of Gravitation in Article 97 we said that the forces between bodies in the solar system are 
expressed by that law if the frame of reference is fixed relatively to the stars. If then we wish to describe the motion of a body by applying the Law of Gravitation we must refer to axes which are not fixed in the Earth, but which, relatively to the Earth, rotate with the stars.

277. Gravity. We have given a provisional account of the acceleration due to gravity, and of the weight of a body. We said that relatively to axes fixed in the Earth a falling body near the Earth's surface moves very nearly in a vertical direction, and with an acceleration which is very nearly equal to $\gamma E / R^{2}$, where $\gamma$ is the constant of gravitation, $E$ the Earth's mass, and $R$ the Earth's radius. In this statement the vertical at a place was understood to mean the line of a flexible cord freely supporting a body so that it remains at rest relative to the Earth, and this line makes with a line drawn to the Earth's centre of inertia an angle which is very nearly zero. Treating these approximate statements as exact, we said that the weight of a body is the force with which the Earth attracts it, that its direction at any place is the vertical at the place, and that its magnitude is the product of the mass of the body and the acceleration due to gravity; further with the same degree of inexactness the weight of a body is equal to the force exerted by a cord or by a spring supporting the body so that it remains at rest relatively to the Earth. Also we said that the most exact process for experimentally determining the acceleration due to gravity is provided by pendulum experiments.

We wish as far as possible to replace these provisional statements by exact ones, and to exhibit the relations between the quantities involved. We accordingly give the following definitions :-

(i) The vertical at a place is the direction of the plumb-line there, i.e. the direction of a flexible cord which supports a body so that it remains at rest relatively to the Earth.

(ii) The weight of a body at a place is a force equal and opposite to the force which must be applied to support the body in equilibrium relatively to the Earth. Its direction is that of the vertical at the place.

(iii) The acceleration due to gravity at a place is the initial value of the acceleration (relative to axes fixed in the Earth) of a 
free body falling from relative rest in the neighbourhood of the place.

We shall write $W$ for the weight of the body, $m$ for its mass, and $g$ for the acceleration due to gravity, and we shall regard the Earth as a sphere of radius $R$ and mass $E$ attracting like a particle of the same mass placed at its centre. We shall find that $W$ is not equal to the force $\gamma m E / R^{2}$ with which the Earth attracts a body, and we shall find that $W$ is equal to $m g$.

278. Weight of a body. The difference between $W$ and $\gamma m E / R^{2}$ arises from the Earth's rotation. We may say roughly that some part of $\gamma m E / R^{2}$ is required to maintain the circular motion of the body about the polar axis, and that the remainder is the force $W$.

Let $\lambda$ be the angle which the radius of the Earth drawn to the place makes with the plane of the equator. The body describes a

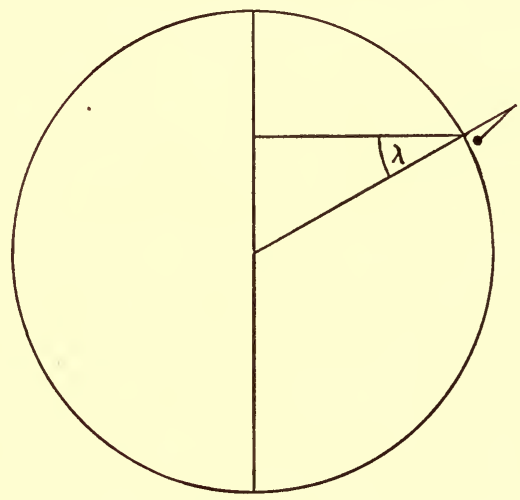

Fig. 84.

circle of radius $R \cos \lambda$ with angular velocity $\Omega$, and thus the acceleration $R \Omega^{2} \cos \lambda$ towards the axis must be produced by the force of magnitude $W$ supporting the body, and the force $\gamma m E / R^{2}$ towards the Earth's centre.

Let $l$ be the angle which the direction of $W$ makes with the plane of the equator. Then $l$ is the (astronomical) latitude of the place. 
For the motion of the body, we have, by resolving parallel to the direction of $W$,

$$
-W+\left(\gamma m E / R^{2}\right) \cos (l-\lambda)=m R \Omega^{2} \cos \lambda \cos l \ldots \ldots(1),
$$

and, by resolving at right angles to the radius,

$$
W \sin (l-\lambda)=m R \Omega^{2} \cos \lambda \sin \lambda .
$$

Now $m R \Omega^{2} / W$ is, in all latitudes, a small fraction, approximately equal to $1 / 290$, and thus the angle $l-\lambda$ is very small. From equation ( 1 ) we have, when $\Omega$ is neglected altogether,

$$
W=\gamma m E / R^{2},
$$

and for a second approximation

$$
W=\gamma m E / R^{2}-m R \Omega^{2} \cos ^{2} l
$$

To this order of approximation, the weight of a body differs from the force with which the Earth attracts it by the vertical component of the force required to maintain the circular motion about the polar axis.

279. Deviation of the plumb-line. The latitude $l$ and the angle $\lambda$, which the Earth's radius makes with the plane of the equator, are connected by an equation, which is most readily obtained by resolving for the motion of the body at right angles to the direction of $W$. We find

$$
\left(\gamma m E / R^{2}\right) \sin (l-\lambda)=m R \Omega^{2} \cos \lambda \sin l,
$$

so that

$$
\tan \lambda=\tan l\left(1-\frac{R \Omega^{2}}{\gamma E / R^{2}}\right)
$$

The angle $l-\lambda$ is the deviation of the plumb-line, i.e. the angle between the vertical and a line drawn to the Earth's centre.

The components of the force supporting the body parallel and perpendicular to the polar axis are determined by resolving in those directions. We find

$$
\left.\begin{array}{l}
-W \sin l+\left(\gamma m E / R^{2}\right) \sin \lambda=0, \\
-W \cos l+\left(\gamma m E / R^{2}\right) \cos \lambda=m R \Omega^{2} \cos \lambda,
\end{array}\right\}
$$

so that we have

$$
\frac{\left(\gamma E / R^{2}\right) \sin \lambda}{\sin l}=\frac{\left\{\left(\gamma E / R^{2}\right)-R \Omega^{2}\right\} \cos \lambda}{\cos l}=W / m \ldots \ldots(2),
$$

and these equations are in accord with equation (1). 
*280. Motion of a free body near the Earth's surface. We form first the equations of motion of the body referred to axes fixed in the Earth. We take the origin to be at the centre of the Earth, the axis of $z$ to be the polar axis (from South Pole to North Pole), the axis of $x$ to be the intersection of the plane of the equator and the meridian plane near which the motion takes place, the positive sense being from the centre to the meridian in question, also we take the axis of $y$ to be at right angles to this meridian plane and directed towards the East. This system is a right-handed system. By the results of Article 263, the component velocities of the body parallel to these axes are not $\dot{x}, \dot{y}, \dot{z}$, but they are

$$
\dot{x}-\Omega y, \quad \dot{y}+\Omega x, \quad \dot{z},
$$

and the component accelerations are

$$
\frac{d}{d t}(\dot{x}-\Omega y)-\Omega(\dot{y}+\Omega x), \quad \frac{d}{d t}(\dot{y}+\Omega x)+\Omega(\dot{x}-\Omega y), \quad \ddot{z} .
$$

Hence the equations of motion of the body are

$$
\left.\begin{array}{rl}
m\left(\ddot{x}-2 \Omega \dot{y}-\Omega^{2} x\right) & =-\left(\gamma m E / R^{2}\right) \cos \lambda, \\
m\left(\ddot{y}+2 \Omega \dot{x}-\Omega^{2} y\right) & =0, \\
m \ddot{z} & =-\left(\gamma m E / R^{2}\right) \sin \lambda,
\end{array}\right\}
$$

where $\lambda$ is the angle which the radius drawn through the body makes with the plane of the equator. Now, as the body remains near a place, we may take $\lambda$ to be constant, and we may in the terms containing $\Omega^{2}$, put $x=R \cos \lambda$ and $y=0$. Then, using equations (2) of Article 279, we shall have

$$
\begin{aligned}
\ddot{x}-2 \Omega \dot{y} & =-(W / m) \cos l, \\
\ddot{y}+2 \Omega \dot{x} & =0, \\
\ddot{z} & =-(W / m) \sin l .
\end{aligned}
$$

Since these equations contain only differential coefficients of $x, y, z$ with respect to the time, we may, without making any alteration, suppose the origin to be on the Earth's surface in the latitude and longitude near which the motion takes place. We shall now, taking the origin as just explained, transform to the horizontal drawn southwards as axis of $x^{\prime}$, the horizontal drawn eastwards as axis of $y^{\prime}$, and the vertical drawn upwards as axis of $z^{\prime}$. We have

$$
x^{\prime}=x \sin l-z \cos l, \quad y^{\prime}=y, \quad z^{\prime}=z \sin l+x \cos l .
$$


We thus obtain the equations

$$
\left.\begin{array}{l}
\ddot{x}^{\prime}-2 \Omega \dot{y}^{\prime} \sin l=0, \\
\ddot{y}^{\prime}+2 \Omega\left(\ddot{x}^{\prime} \sin l+\ddot{z}^{\prime} \cos l\right)=0, \\
\ddot{z}^{\prime}-2 \Omega \dot{y}^{\prime} \cos l=-W / m,
\end{array}\right\}
$$

these equations determine the motion of the body relative to the axes at the place of observation.

*281. Acceleration due to gravity. Suppose the body to fall from rest relative to the Earth. Then the initial velocities relative to the axes at the place of observation are

$$
\dot{x}^{\prime}=0, \quad \dot{y}^{\prime}=0, \quad \dot{z}^{\prime}=0,
$$

and we shall suppose that the initial value of the coordinate $y^{\prime}$ is zero. The motion is determined by equations (1) of Article 280. Integrating the first of these, we have

$$
\dot{x}^{\prime}=2 \Omega y^{\prime} \sin l
$$

and integrating the third equation, we have

$$
-\dot{z}^{\prime}=(W / m) t-2 \Omega y^{\prime} \cos l \text {. }
$$

where $t$ is the time from the beginning of the motion. Substituting in the second equation, and neglecting $\Omega^{2} y^{\prime}$, we have, on integration

$$
\dot{y}^{\prime}=\Omega(W / m) t^{2} \cos l,
$$

so that

$$
y^{\prime}=\frac{1}{3} \Omega(W / m) t^{3} \cos l
$$

Substituting in equations (1) and (2), and neglecting terms of the same order as before, we have, on integration,

$$
\left.\begin{array}{l}
x^{\prime}=x_{0}^{\prime}, \\
z^{\prime}=z_{0}^{\prime}-\frac{1}{2}(W / m) t^{2},
\end{array}\right\}
$$

where $x_{0}^{\prime}$ and $z_{0}^{\prime}$ are the initial values of $x^{\prime}$ and $z^{\prime}$.

In the beginning the motion of the acceleration relative to axes fixed on the Earth is directed vertically downwards and is precisely $(W / m)$, or we have

$$
W=m g,
$$

and, to the order of approximation here adopted, this equation holds throughout the motion. 
It appears that the body falls a little to the East of the starting point, the eastward deviation in a fall through a height $h$ being very approximately

$$
\frac{2}{3} \sqrt{ } 2 \Omega \sqrt{ }\left(h^{3} / g\right) \cos l .
$$

This result accords well with observed facts.

*282. Motion of a Pendulum. Suppose that a simple circular pendulum of length $L$ is free to move about its point of support which is fixed relatively to the Earth, and let $T$ be the tension of the suspending fibre.

Taking $x^{\prime}, y^{\prime}, z^{\prime}$ to be the coordinates of the bob referred to the system of axes described in Article 280, the origin being at the equilibrium position, the line of action of $T$ makes with the axes angles whose cosines are

$$
-x^{\prime} / L, \quad-y^{\prime} / L, \quad\left(L-z^{\prime}\right) / L,
$$

and we have the relation

$$
x^{\prime 2}+y^{\prime 2}+\left(L-z^{\prime}\right)^{2}=L^{2}
$$

Now the equations of motion are, by Article 280,

$$
\left.\begin{array}{l}
m \ddot{x}^{\prime}-2 m \Omega \ddot{y}^{\prime} \sin l=-T\left(x^{\prime} / L\right), \\
m \ddot{y}^{\prime}+2 m \Omega\left(\ddot{x}^{\prime} \sin l+\ddot{z}^{\prime} \cos l\right)=-T\left(y^{\prime} / L\right), \\
m \ddot{z}^{\prime}-2 m \Omega \ddot{y}^{\prime} \cos l=-W+T\left(L-z^{\prime}\right) / L .
\end{array}\right\}
$$

We shall integrate these equations on the assumption that the pendulum makes small oscillations. On this assumption we have approximately

$$
z^{\prime}=\frac{1}{2}\left(x^{\prime 2}+y^{\prime 2}\right) / L
$$

Multiply the equations (2) in order by $\dot{x}^{\prime}, \dot{y}^{\prime}, \ddot{z}^{\prime}$, and add. The terms containing $T$ vanish identically by (1), the terms containing $\Omega$ also vanish identically, and the equation can be integrated. Omitting $\dot{z}^{\prime 2}$ in the integral equation, and substituting for $z^{\prime}$ from (3), we have

$$
\frac{1}{2} m\left(\dot{x}^{\prime 2}+\dot{y}^{\prime 2}\right)=\text { const. }-\frac{1}{2} W\left(x^{\prime 2}+y^{\prime 2}\right) / L
$$

Again, multiplying the first of equations (2) by $-y^{\prime}$, and the second by $x^{\prime}$, adding, and omitting the term in $y^{\prime} z^{\prime}$, we have on integration

$$
x^{\prime} \dot{y}^{\prime}-y^{\prime} \ddot{x}^{\prime}=-\Omega \sin l\left(x^{\prime 2}+y^{\prime 2}\right)+\text { const. }
$$

Introducing polar coordinates in the horizontal plane given by

$$
x^{\prime}=r \cos \theta, \quad y^{\prime}=r \sin \theta,
$$


and putting $g$ for $W / m$, we obtain from equations (4) and (5), equations of the form

and, if we put

$$
\left.\begin{array}{rl}
\dot{r}^{2}+r^{2} \dot{\theta}^{2} & =A-r^{2}(g / L) \\
r^{2} \dot{\theta} & =B-r^{2} \Omega \sin l,
\end{array}\right\}
$$

we shall have

$$
\theta+\Omega t \sin l=\phi
$$

$$
\begin{aligned}
\dot{r}^{2}+r^{2} \dot{\phi}^{2} & \left.=(A+2 \Omega B \sin l)-r^{2}\left\{(g / L)+\Omega^{2} \sin ^{2} l\right\},\right\} \ldots \\
r^{2} \dot{\phi} & =B .
\end{aligned}
$$

These equations completely determine the motion. It is to be noticed that $r$ and $\phi$ are polar coordinates referred to an initial line rotating about the vertical from East to West with an angular velocity $\Omega \sin l$, viz., with the component of the angular velocity of diurnal rotation with which the stars rotate about the vertical relative to the Earth. Thus the initial line from which $\phi$ is measured remains parallel to a plane fixed with reference to the stars.

*283. Foucault's Pendulum. When the pendulum can turn freely about its point of support and is set oscillating so as to pass through its equilibrium position, the system is known as a Foucault's Pendulum.

Since $r$ can vanish, it follows by the second of equations (7) of the last Article that $B$ must vanish, and thus $\dot{\phi}$ vanishes throughout the motion. Hence the pendulum oscillates so that its plane of vibration is fixed in direction relatively to the stars, and this plane turns round the vertical relatively to the Earth with angular velocity $\Omega \sin l$ from East to West.

The first of equations (7) of the last Article then becomes, on neglecting $\Omega^{2} \sin ^{2} l$ in comparison with $g / L$,

$$
\dot{r}^{2}=A-r^{2}(g / L)
$$

showing that the horizontal motion in the plane of vibration is simple harmonic motion of period $2 \pi \sqrt{ }(L / g)$.

If $a$ is the amplitude of the simple harmonic motion, so that the pendulum has no velocity in the plane of vibration when $r=a$, it will not move as here described unless its angular velocity relative to the Earth is $\Omega \sin l$ from East to West. To start the pendulum, therefore, it is not sufficient to hold it aside from its 
equilibrium position; it must be projected at right angles to the vertical plane containing it with velocity $a \Omega \sin l$. When thus set going it moves like a simple pendulum of the same length in a plane which turns about the vertical from East to West with angular velocity $\Omega \sin l$.

This result accords well with observed facts.

\section{*284. Examples.}

[In these examples the Earth is regarded as a homogeneous sphere.]

1. If the Earth were to rotate so fast that bodies at the equator had no weight, prove that in any latitude the plumb-line would be parallel to the polar axis.

2. If the acceleration due to gravity at the poles is $g_{0}$ and at the equator $g_{e}$, prove that in (geocentric) latitude $\lambda$ the value of $g$ is

$$
\sqrt{ }\left(g_{0}^{2} \sin ^{2} \lambda+g_{e}^{2} \cos ^{2} \lambda\right)
$$

and that the deviation of the plumb-line from the (geometrical) vertical is

$$
\tan ^{-1}\left\{\left(g_{0}-g_{e}\right) \sin \lambda \cos \lambda /\left(g_{0} \sin ^{2} \lambda+g_{e} \cos ^{2} \lambda\right)\right\} \text {. }
$$

3. Prove that a pendulum which beats seconds at the poles will lose approximately $30 \mathrm{~m} \cos ^{2} l$ beats per minute in latitude $l$, where $1+m: 1$ is the ratio of the weight of a body at the poles to its weight at the equator.

4. A train of mass $m$ is travelling with uniform speed $v$ along a parallel of latitude in latitude $l$. Prove that the difference between the pressures on the rails when the train travels due East and when it travels due West is $4 m v \Omega \cos l$ approximately.

5. A projectile is projected from a point on the Earth's surface with velocity $V$ at an elevation $a$ in a vertical plane making an angle $\beta$ with the meridian (East of South). Prove that after an interval $t$ it will have moved southwards through $x$, eastwards through $y$, and upwards through $z$, where

$$
\left.\begin{array}{l}
x=V t \cos a\{\cos \beta+\Omega t \sin l \sin \beta\}, \\
y=V t\{\cos a \sin \beta-\Omega t(\sin l \cos \beta \cos a+\cos l \sin a)\}+\frac{1}{3} \Omega g t^{3} \cos l, \\
z=V t\{\sin a+\Omega t \cos l \sin \beta \cos a\}-\frac{1}{2} g t^{2},
\end{array}\right\}
$$

approximately, $\Omega^{2} y$ being neglected.

6. Prove that, if the bob of a pendulum of length $L$ is let go from a position of rest relative to the Earth when its displacement from its equilibrium position is $\alpha$, and the vertical plane through it makes an angle $\beta$ with the meridian (East of South), its path is given approximately by the equation

$$
(\beta-\theta)=\Omega \sqrt{ }(L / g) \sin l\left\{\sqrt{ }\left(x^{2}-r^{2}\right) / r-\cos ^{-1}(r / a)\right\},
$$

higher powers of $L \Omega^{2} / g$ being neglected. 
7. A particle being observed to move, relatively to a certain frame, with a simple harmonic motion of period $2 \pi / n$ in a line, which turns uniformly about the mean position of the particle in a plane fixed relatively to the frame with angular velocity $\omega$, prove that the acceleration of the particle when at distance $r$ from its mean position is compounded of a radial acceleration $\left(n^{2}+\omega^{2}\right) r$, and a transverse acceleration $2 \omega \dot{r}$ in the sense in which the line turns.

*285. Relativity of Force. Passing now from this particular set of facts connected with the rotation of the Earth about its polar axis and their dynamical formulation, we consider next the influence of the doctrine of the relativity of motion in respect of the notion of force, and we say at once that force, like motion and position, is relative, meaning thereby that the force acting on a body is force relative to a frame; in other words, just as we hold that no meaning can be attached to any phrase of the kind-" a body is moving with such a velocity" or "a body is at rest" or "a body is rotating about an axis"- - until the frame of reference is specified, so no meaning can be attached to any phrase of the kind-" a body is acted upon by a force of such and such a magnitude" or "the force acting on a body is directed to a certain other body "- until the frame of reference is specified. This conclusion is at once established when we reflect that (1) the force on a body is the resultant of the forces on its particles, (2) the force on a particle is made up of components each of which is the product of the mass of the particle and the acceleration induced in it by some other particle, and (3) the acceleration of a particle is the rate of change of its velocity relative to a frame; in fact "acceleration," and by consequence "force," have no meaning except as dependent on a frame; "acceleration" means "acceleration relative to a frame," and similarly with force.

But now it is important to observe that, whereas the position of a point, or the velocity or acceleration of a particle, can be described by reference to any frame determined by parts of natural bodies, this is not the case for forces; but the notion of force requires that the frame of reference should be properly chosen. Thus there are frames of reference for which the phrase "force on a particular body" would have no meaning. We shall now illustrate and enforce this theory by considering the choice of the origin and of the lines of reference of a frame of reference. 
*286. Choice of Origin. In choosing the origin of a frame of reference for the motion of any system of bodies, it is in the first place necessary to observe that the Postulates of Mechanics (Article 87) will in general be inapplicable if the origin is taken at the position of any definite particle of the system. For, if this were done, that particle would have no motion, and could not be subject to any resultant force. Now the masses of parts of a system are determined by observing the ratios of their accelerations relative to a properly chosen frame, and to secure that any part shall always have zero acceleration we should have to take the mass of that part to be infinite, and then no definite number however great could represent this mass. It is one of our Postulates that every body and every part of a body has a definite mass, and it thus appears that this Postulate implies a restriction upon the position of the origin of the frame of reference; it may not be taken at the position of one of the particles.

To take an example:-In discussing the motions of bodies relative to the Earth, the Postulates do not apply if the origin is taken at the centre of the Earth, unless the mass of the Earth is taken to be infinite. The mass of the Earth is, however, assumed to be a determinate multiple of the mass of any particular body, and this multiple can be determined in accordance with the Law of Gravitation. In general, it appears that the postulates cannot be applied without error to the motion of a body if the origin of the frame of reference is fixed relatively to the Earth, and the error that would arise in such an application consists in the neglect of the fraction (mass of body : mass of Earth). In Articles 278 to 284 this fraction is neglected.

Again, we have said that the interaction between two particles consists of two equal and opposite forces in the line joining them, the force on one particle being measured by the product of the mass of that particle and a component of its acceleration, and we have seen how the theory of the motion of the centre of inertia enables us to replace a body by a certain particle. Now, if in a system of two bodies with relative accelerations we took the origin of a frame of reference at the centre of inertia of one body, then the magnitude of the force exerted by the second body upon the first would be zero, and accordingly the first 
body would exert no force on the second, and the acceleration of the second body relative to the frame would not be produced by a force arising from interactions between parts of a system. It is one of our Postulates that all accelerations are so produced, and it thus appears that this Postulate implies a restriction upon the position of the origin of the frame of reference.

To take the same example as before:-If the origin of the frame of reference were fixed in the Earth, then any other body would exert zero force on the Earth, and the Earth consequently would not act on the body. The "acceleration due to gravity" could not be regarded as produced by forces exerted by the parts of the Earth on the parts of a falling body.

We have so far explained and illustrated the relativity of force by showing that, unless the frame of reference is properly chosen, bodies will not have definite masses, and action and reaction will not be equal and opposite.

We may evade these difficulties by taking the origin at the centre of inertia of a system so remote from all other bodies that it may be considered to be independent of them. Thus for the motion of a body near the Earth's surface it will be sufficient to consider the body and the Earth as such a system; for the motions of the planets it will be sufficient to consider the solar system as such a system. When however the origin of the frame of reference is taken at the centre of inertia of a system the system must be regarded as independent; in other words, no meaning could be attached to the phrase "action of external bodies on the system." For example, so long as the origin of the frame of reference is the centre of inertia of the solar system no meaning could be attached to the phrase "force exerted on the Sun by a star."

*287. Choice of lines of reference. We have seen that a frame of reference is determined by the origin, a line through the origin, and a plane through the line; and we have recorded, by way of examples and otherwise, a number of theorems relating to changes introduced into the descriptions of particular motions by changing the frame of reference. Thus in Example 4, p. 52, and Example 2, p. 186, we noticed a transformation from uniform rectilinear motion to motion with a central acceleration 
varying inversely as the cube of the distance; in Article 198 we noticed a transformation from one law of central acceleration to another.

Now it is one of our Postulates that accelerations are produced by interactions between bodies, and the choice of the frame of reference is restricted by the condition that the accelerations of the parts of a system must be such as reduce to components in the lines joining the particles. This condition is however in general extremely difficult of application, and we make use of the possibility of changing the acceleration by changing the frame of reference to simplify the description of motions.

We refer the motion to be described to such a frame among possible frames that the description of the observed motion may be more easily brought under such general principles as the Law of Gravitation and the Conservation of Energy than it would be for others.

To see how this might have been done in a particular case, let us imagine what might have been the course of history in respect of the theory of the motion of falling bodies. Let us suppose that after Galilei's experiments on falling bodies the next considerable step was Foucault's pendulum experiment. It would have been known (1) that bodies tend to fall with uniform acceleration in a vertical direction, (2) that this statement is not exact, but, at any rate in one case there is, for a supported body, a westerly component acceleration as well as a vertical component (Example 7, p. 360). Further, if the fact of the eastward deviation of a falling body (Article 281) had been observed, it would have been known that for another case there was, for an unsupported body, an easterly component as well as a vertical component. It is also perfectly possible that it might have been found that, relative to lines of reference pointing to fixed stars, the acceleration in all such cases was simply directed to the Earth's centre. It would then have appeared much simpler to choose such lines of reference for the description of the motion in terms of acceleration than to choose axes fixed in the Earth.

*288. Relativity of Force and Conservation of Momentum. The theory of the relativity of force makes it necessary 
to reconsider the statements of the Principles of Conservation of Momentum and of Moment of Momentum. These principles were stated in Articles 111 and 112 for the motion of a system in a given field of force, and they hold provided the field of force is subject to certain conditions. They consequently imply that the frame of reference has been so chosen that the field of force obeys the conditions, and of course it is understood that the frame of reference has been so chosen that the Postulates of Mechanics apply to the system under discussion. We accordingly arrive at the following statements of these principles:-

(1) When the motion of a system can be referred to a frame for which the Postulates of Mechanics are applicable, and which is such that the field of force relative to the frame gives rise to zero resultant force in a direction fixed relatively to the frame, the linear momentum relative to this frame resolved in this direction is constant.

(2) When the motion of a system can be referred to a frame for which the Postulates of Mechanics are applicable, and which is such that the field of force relative to the frame gives rise to zero moment about an axis fixed relatively to the frame, the moment of momentum relative to this frame about this axis is constant.

It is worth while here to remark that if one frame has been chosen for which the Postulates apply they will still apply to a new frame moving relatively to the first with uniform velocity in a direction fixed relatively to the first, and if either of the above principles has an application to the former frame it has an application also to the latter.

Now when any system can be treated as independent (Article 286) both the principles apply to it, and the moment of momentum about any axis fixed relatively to the frame of reference, and the linear momentum in any direction fixed relatively to the frame of reference are constants. Taking the origin of the frame of reference at the centre of inertia of the system, we see that the linear momentum in any direction is zero. On the other hand the moment of momentum about any axis being the moment of a couple (of vectors localised in lines), the moment about any axis is the resolved part of an unlocalised vector parallel to that axis. 
The direction and sense of this unlocalised vector are fixed relatively to the frame of reference, and might therefore be used to determine one of the lines of reference.

Thus an independent system presents a natural origin for a frame of reference, viz., its centre of inertia, and one natural line of reference, viz., the axis of resultant moment of momentum.

The plane through the centre of inertia of an independent system at right angles to the axis of resultant moment of momentum is known as the invariable plane.

This discussion and that in the last Article show that there are cases of practical importance in which it is possible to determine the frame of reference, at least partly, by dynamical considerations. The rule is that among possible frames that one is to be taken for which the field of force has the simplest expression.

*289. Law of Gravitation. In connexion with the doctrine of the relativity of force, the matter which is of the greatest importance is the statement of the Law of Gravitation in a precise form. It is not sufficient to say, "The force between two particles is the product of a constant, the masses of the particles, and the reciprocal of the square of the distance between them," for this is just the kind of statement which has no meaning until the frame of reference is specified.

We shall make some advance towards a more precise statement if we say, "There is a frame of reference relative to which the force between two particles of masses $m, m^{\prime}$ at distance $r$ is $\gamma m m^{\prime} / r^{2}$." The objection to this statement is that we shall not be able to say where that frame of reference is. Our statement will imply forces between particles in the stars and particles in the solar system, and we shall be unable at present $†$ to fix the position of either a possible origin or the directions of possible axes relative to the sun and stars. Our statement then will either involve a pretence of knowing something which we do not know, or we shall be reduced to saying that there is a frame of

+ The objection needs qualification in view of the fact that it has been suggested that the required frame of reference is presented in the undisturbed position of parts of the luminiferous medium. If an Electrical and Optical Theory on the basis of an ether " at rest" becomes established, it will perhaps be possible to free the statement in the above form from the objection here urged. 
reference which fulfils a certain condition but that we do not know where its origin is or how its axes point. This is very like saying that there is an absolute space in which bodies move, or that bodies have absolute positions, but that we never can know what those absolute positions are. It is equivalent to denying the relativity of motion.

Now if we content ourselves with a statement of the Law of Gravitation as an abstract formula representing observed facts, it will take the form given to it in Article 97, viz.:- "If the frame of reference has its origin at the centre of inertia of the Solar System. and its lines of reference determined by stars so remote as to have no observable annual parallax, the force between two particles within the system is $\gamma m m^{\prime} / r^{2}$, where $m$ and $m^{\prime}$ are the masses of the particles and $r$ is their distance."

The law thus applies to forces within a particular system which can be treated as independent (Article 286). The similarity of chemical constitution of the bodies outside the solar system and of those within it suggests that the law probably has an application to such bodies as well. It is thus suggested that, for any system of bodies which can be treated as isolated, the force between two particles is expressed by the law of gravitation when their motions are referred to a frame whose origin is at the centre of inertia of the system and whose axes point to bodies external to the system and at sufficiently great distances.

With the understanding referred to in Article 97, that the law applies only to particles at measurable distances, the above is probably the most general statement that we can make. The law as extended to bodies outside the solar system is not proved to be an expression of facts of observation. For example, in the motions of double stars, the relative paths are oval curves, but they are not proved to be ellipses, nor is there any evidence to show that one component star occupies a focus of the path of the other relative to it $\dagger$. On the other hand there are no facts of observation in this department of Astronomy which conflict with the proposed extension of the law to bodies outside the solar system.

*290. Measurement of Time. Relatively to the stars the Earth rotates about its polar axis as we have explained in

+ See Tisserand, Mécanique Céleste, Tome I., Chapter 1. Paris, 1889. 
Article 276. This process of relative rotation is that which is taken as time measuring process (Article 3), and accordingly we say of this process that it takes place uniformly. Time so measured is called sidereal time, and the interval in which the Earth turns through four right angles relatively to the stars is a sidereal day.

Now we have said (Article 3) that the process used for measuring time is the average rotation of the Earth relative to the Sun. To explain this statement, consider in the first place the motion of the Sun relative to a frame whose origin is the centre of the Earth and whose lines of reference go out thence to stars so distant as to have no observable annual parallax. The path and motion of the Sun relative to this frame are the same as the path and motion of the Earth relative to a frame whose origin is in the Sun and whose lines of reference go out thence to the same stars (cf. Example 1, p. 61), and this latter motion is a planetary motion of the kind described in Article 177. There is thus for the motion of the Sun, relative to the frame of Earth and stars, at any instant an instantaneous ellipse osculating the Sun's actual path relative to the frame, and the motion is very nearly an elliptic motion about a focus at the centre of the Earth. The sense in which the Sun describes his orbit is the same as the sense in which any particular meridian plane of the Earth turns about the polar axis, i.e. the Sun is always moving from stars which have a more westerly position towards stars which have a more easterly position in the plane of his path. The elements of the elliptic orbit are not quite constant; in particular the apse line has a small progressive motion in the sense in which the orbit is described, and the line of intersection of the plane of the orbit with the plane of the Earth's equator (known as the line of nodes) has a small progressive motion in the opposite sense. The Sun passes the line of nodes at the Equinoxes, and the periodic time in the orbit is a year. Now it is to be observed that, relatively to a frame fixed in the Earth, the Sun makes about $365 \frac{1}{4}$ revolutions round the Earth in a year, and the stars make about $366 \frac{1}{4}$ revolutions, but the time of revolution of the Sun is not a constant multiple of the time of revolution of the stars. The variability arises in the first place from the fact that the motion 
of the Sun in his path, relative to the frame of Earth and stars, is much more nearly elliptic motion about a focus than uniform circular motion, and in the second place from the fact that the plane of the Sun's path is inclined to the equator. To define the measurement of time by the average rotation of the Earth relative to the Sun, we imagine a point to move (relatively to the frame of Earth and stars) in the Sun's path, with a uniform angular motion about the centre of the Earth (i.e. so that the time of describing any angle is a constant multiple of the time in which the Earth turns through the same angle), and at such a rate as always to coincide with the Sun at the nearer apse of his path; then we imagine a second point to move in the plane of the Earth's equator with a uniform angular motion about the centre of the Earth, and at such a rate as always to coincide with the first point at the node corresponding to the Vernal Equinox. This second point is called the Mean Sun. We may determine a frame of reference by taking the centre of the Earth as origin, the line joining the origin to the Mean Sun as a line of reference, and the plane through this line and the polar axis as a plane of reference. Relatively to this frame the Earth rotates about its polar axis in an interval called a mean solar day; this rotation can be used instead of the rotation relative to the stars as time measuring process, and time so measured is mean solar time. The unit of time is the time in which the Earth rotates relatively to this frame through an angle equal to $1 / 86400$ of four right angles, and this unit is the mean solar second.

*291. Change of the time measuring process. In the last Article we have explained how in a particular case it is convenient to take as the measurement of time a multiple of the time measured by some particular process, or, in other words, to change the variable which expresses elapsed time by multiplying it by a constant number. In the same way we might conceivably take as time measurer the value of any definite onevalued function of the number expressing sidereal time, provided the function always increases as the number expressing sidereal time increases. In general such a procedure would introduce a needless complication, but it is on the other hand conceivable that it might produce a simplification. 
So long as the time measuring process is the rotation of the Earth relative to the stars, the question whether the length of the day is liable to change is meaningless. Now it has been stated by astronomers that the day is increasing in length, or that, in long past ages, the angular velocity of the Earth was sensibly greater than it is now. This statement should imply that there is some other process for measuring time than the rotation of the Earth, and that at one time less of this process would have taken place while the Earth turned through four right angles than would be the case now. It might be meant, for example, that such a process is the vibration of the luminiferous medium in transmitting a certain kind of light, and that a particular measurable interval is the period of such a vibration, and then the statement would be that the number of such periods in a day is increasing. In such a form however the statement would be quite unverifiable. Again, it might be meant by the statement (that the length of the day is increasing) that time measured by a function of sidereal time fulfils better than sidereal time the requirements of the Law of Gravitation and the theory of energy, and that this function has a positive second differential coefficient with respect to sidereal time.

Now it is quite conceivable that the change made in the equations of motion of bodies of the solar system by changing the independent variable from the number representing sidereal time to a function of that number which varies very slowly, while producing no change of importance in the mechanical description of most of the motions of the solar system, might help to bring some particular facts under the Law of Gravitation. In such a case it would be simpler to say that time can be measured so that the length of the day varies and the particular fact accords with the law than to keep to the measurement of time by the diurnal rotation and seek a formulation of the fact as an exception to the application of the law. It is known that the existence of one of the inequalities in the motion of the Moon can thus be accounted for.

Again, the Earth and Moon, with the fluid ocean on the Earth, form a system showing various internal relative motions, among which the tides are conspicuous. Such internal relative motions generally involve dissipation of energy in a system, for they do 
not take place without friction; and thus we are led to expect that the kinetic energy of the Earth's rotation is being dissipated at a finite rate. To give a meaning to this statement we must suppose time to be measured by some process other than the diurnal rotation of the Earth. As before we may take the number expressing time to be a slowly varying function of the number expressing sidereal time, and then the sense of the variation is again such that the function has a positive second differential coefficient with respect to sidereal time. As before, it is simpler to say that time can be measured so that the length of the day varies and the tidal motions obey the laws of energy than to keep to the measurement of time by the diurnal rotation and seek a theory of the tides in conflict with the theory of energy. 


\section{APPENDIX.}

\section{MEASUREMENT AND UNITS.}

292. Measurement. The mathematical theory of measurement rests on the assumed possibility of dividing an object into an integral number of parts which are identical in respect of some property. Thus, to measure the length of a segment of a line, we must suppose the segment divided into a number of equal segments, where the test of equality of length is congruence; to measure the mass of a body we must suppose it capable of division into a number of bodies of equal mass, where equality of mass is tested by weighing; to measure an interval of time we measure the angle turned through by the Earth in the interval; this requires the division of an angle into a number of equal angles, and the test of equality of angles is congruence.

The measurement of an object in respect of any property requires (1) a unit or standard of comparison, and (2) a mode of referring to the standard. The standard must be an object which possesses the property in question. The mode of referring to the standard must be such that it determines a positive number (integral, rational but not integral, or irrational) which is the measure of the object in respect of the property. The number is determined by the following rules:-

(a) When the object can be divided into an integral number $n$ of parts, each of which is identical with the standard in respect of the property in question, the measure of the object in respect of that property is $n$.

(b) When the object and the standard can be divided into $p$ and $q$ parts respectively ( $p$ and $q$ being integers), such that all the parts are identical in respect of the property in question, the measure of the object in respect of that property is the rational fraction $p / q$.

Here it is to be noted (1) that the rule $(a)$ is the case of the rule $(b)$ for which $q=1$, and (2) that in practice the integer $q$ may be taken so large that an integer $p$ may be found for which the fraction $p / q$ measures the object within the limits of experimental error.

In the mathematical theory of measurement the case where no rational fraction $p / q$ can measure the object may not be so simply dismissed. It may happen that however great $q$ is taken there is no corresponding number $p$, but that, while the fraction $p / q$ would measure an object somewhat smaller 
than that to be measured, the fraction $(p+1) / q$ would measure an object somewhat greater than that to be measured. When this is the case we say that the measure sought is an irrational number. Now we may take two series of rational numbers $a_{1}, a_{2}, \ldots a_{n}, \ldots$ and $b_{1}, b_{2}, \ldots b_{n}, \ldots$ such that each $a$ is greater than every $b$ and that, by taking $n$ sufficiently great, $a_{n}-b_{n}$ shall become less than any rational number assigned beforehand. All the numbers $a$ may be greater than the measure of the object, and all the numbers $b$ less than that measure. The two series determine an irrational number $a$ which is the common limit of the two series. In such a case we define the measure of the object to be the irrational number $a$.

Thus, suppose we wish to measure the diagonal of a square whose side is the unit of length. The process of extracting the square root of 2 gives $1 \cdot 41421 \ldots$, and thus the series $b$ may be taken to consist of the numbers

$$
1,1 \cdot 4,1.41,1.414,1.4142,1.41421, \ldots \text {, }
$$

and the series $a$ may be taken to consist of the numbers

$$
2,1 \cdot 5,1 \cdot 42,1.415,1.4143,1 \cdot 41422, \ldots \text {, }
$$

and the nature of the process shows that the excess of any $a$ above the corresponding $b$ diminishes without limit. These two series define a limit, which is $\sqrt{ } 2$, and this irrational number is the required measure.

293. Number and Quantity. When the unit is stated the magnitude of an object is precisely determined by its measure in terms of the unit, and this measure is always a number. The "object" may be anything which we can think of as measurable in respect of any property, and the phrase "magnitude of an object" is thus coextensive in meaning with the word "quantity." The quantity does not change when the unit chosen to measure it changes, and thus the quantity is not identical with the number expressing it.

A number can express a quantity only when the unit of measurement is stated or understood. When the unit is stated or implied the number expresses the quantity.

Mathematical equations, and inequalities, are relations between numbers, expressing that a certain number which has been arrived at in one way is equal to, greater than, or less than, a certain number which has been arrived at in another way.

Mathematical equations, and inequalities, between numbers expressing quantities are valid expressions of relations between the quantities only if they hold good for all systems of units.

294. Fundamental and derived Quantities. The fundamental Physical quantities are lengths, times, and masses. In Dynamics, as considered in this book, all the other magnitudes which occur are derived from these. Thus, velocity is measured by a fraction of which the numerator is a number expressing a length and the denominator is a number expressing an interval of time; acceleration is measured by a fraction of which the 
numerator is a number expressing a velocity and the denominator is a number expressing an interval of time; force is measured by the product of a number expressing a mass and a number expressing an acceleration; and all the other magnitudes that occur are in similar ways dependent upon lengths, times, and masses.

295. Dimensions. A number which expresses a quantity is said to be of one dimension in that quantity. If the unit of measurement is altered so that the new unit is a certain multiple $x$ of the old, the number expressing the quantity in terms of the new unit is the quotient by $x$ of the number expressing the quantity in terms of the old unit.

The number expressing a derived quantity is, in every case, the product of three numbers $A, B, C$, of which $A$ is a homogeneous expression of degree $p$ in numbers expressing lengths, $B$ is a homogeneous expression of degree $q$ in numbers expressing intervals of time, and $C$ is a homogeneous expression of degree $r$ in numbers expressing masses. We say that the quantity is of $p$ dimensions in length, $q$ dimensions in time, and $r$ dimensions in mass. We express this shortly by saying that the dimension symbol of the quantity is $[L]^{p}\left[T^{T}\right]^{q}[M]^{r}$. The numbers $p, q, r$ may be positive or negative, integral or fractional, or zero.

If the units of length, time, and mass are changed so that the new units are respectively $x, y, z$ times the old, the measure of any quantity in terms of the new units is obtained from its measure in terms of the old units by dividing by $x^{p} y^{q} z^{r}$, where $[L]^{p}[T]^{q}[M]^{r}$ is the dimension symbol of the quantity.

The condition that a mathematical equation or inequality between numbers expressing quantities may be a valid expression of a relation between the quantities is that every term in it must be of the same dimensions.

296. Physical Quantities. We give here a list showing the principal derived quantities that occur in Dynamics and their dimension symbols.

$\begin{array}{ll}\begin{array}{ll}\text { Velocity } \\ \text { Acceleration }\end{array} & {[L]^{1}[T]^{-1} .} \\ \left.\begin{array}{l}\text { Momentum } \\ \text { Impulse }\end{array}\right\} & {[L]^{1}[T]^{-2} .} \\ \begin{array}{l}\text { Moment of Momentum } \\ \text { Impulsive Couple }\end{array} & {[L]^{1}[T]^{-1}[M]^{1} .} \\ \left.\begin{array}{l}\text { Kinetic Reaction } \\ \text { Force }\end{array}\right\} & {[L]^{2}[T]^{-1}[M]^{1} \text {. }} \\ \begin{array}{l}\text { Kinetic Energy } \\ \text { Work }\end{array} & {[L]^{1}[T]^{-2}[M]^{1}} \\ \begin{array}{l}\text { Power } \\ \text { Density }\end{array} & {[L]^{2}[T]^{-2}[M]^{1} .} \\ \text { Constant of Gravitation } & {[L]^{3}[T]^{-2}[M]^{-1} .}\end{array}$


297. Method of Dimensions. We can frequently determine the forn of a result by consideration of the dimensions of the quantities involved. This will be made clear by the consideration of some examples. Thus, if we assume that the period of oscillation of a pendulum can depend only on its mass, its length, and the acceleration due to gravity, we can prove that it is proportional to the square root of the length. Since the quantity to be expressed is an interval of time its expression cannot involve any power of a mass, and we have assumed that no mass but the mass of the body can enter into the expression; the period is therefore independent of the mass of the body. Now $g$ has dimension symbol $[L]^{1}[T]^{-2}$, and therefore $1 / \sqrt{ } g$ has dimension symbol $\left[T^{1}\right]^{1}[L]^{-\frac{1}{2}}$, hence the only way in which the expression of the period can contain the length $l$ of the pendulum is by being proportional to its square root. This argument would prove that the period is a numerical multiple of $\sqrt{ }(l / g)$. Again, to take another example, consider the ellipticity of the Earth supposed to depend on the angular velocity of rotation $\omega$, the mean density $\rho$, and the constant of gravitation $\gamma$. The product $\gamma \rho$ has dimension symbol $[T]^{-2}$, and thus $\omega^{2} / g \rho$ is a number (angles being measured in circular measure); the ellipticity being a number, must be a function of $\omega^{2} / \gamma \rho$. The method of dimensions supplies also a useful means of verification. In any piece of mathematical reasoning where the numbers represent quantities all the terms in each equation must be of the same dimensions.

298. Units. Throughout this book, except occasionally in examples, it has been assumed that the unit of length is one centimetre, the unit of time one mean solar second, and the unit of mass one gramme. This system of units is known as the c. G.s. (centimetre, gramme, second) system. Some of the derived units have received names which have met with general acceptance, such as the names for the units of force and work.

The c.G.S. unit of force is the dyne, it is the force which acting on a mass of one gramme for one second generates in it a velosity of one centimetre per second. The weight in London of a body whose mass is one gramme is about $981 \cdot 2$ dynes. The gravitational attraction between two spheres each of mass one gramme with their centres at a distance of one centimetre is $(6 \cdot 65) 10^{-8}$ dynes. This is the value of the constant of gravitation $\gamma$ in c. G.s. units*.

The C.G.S. unit of work is the erg, it is the work done by a force of one dyne acting over a displacement of one centimetre.

299. British Gravitation Units. The c. G. s. system, although generally used for scientific work, is frequently not employed in practical applications of science. Engineers in this country use a system of units adapted to their purposes and associated with the commercial measures of quantities here adopted. British tradesmen do not generally estimate lengths in centimetres (or metres) nor do they sell things by the gramme (or kilogramme). The 
commercial units of length and mass in this country are the foot and the pound, and corresponding to these there is a system of dynamical units. There is however another important respect in which this system differs from the C.Q.S. system, and that is in respect of the unit of force. The unit of force employed by British engineers is statical, it is the force that must be applied to support a body whose mass is one pound. This involves a change in the measurement of force. Instead of saying, as we have in Article 75, that the force acting on a body of mass $m$ and producing an acceleration $f$ is measured by the product of the numbers $m, f$, we should have to say that the measure of the force is the product of the numbers $m, f$, and a constant coefficient. The coefficient is then the reciprocal of the numerical measure of the acceleration due to gravity in terms of the unit of length employed (the foot). This constant is accordingly $1 /(32 \cdot 2)$. Thus the equation which we write $P=m f$, where $P$ is the force producing acceleration $f$ in a body of mass $m$, could be written in these units $P=(m / g) f$, where $g$ is the said constant*. In the system of units we are now describing certain measures of quantities of work and power have received special names.

The unit of work is the foot-pound, it is the work done by a force which would support a body of mass one pound acting through a displacement of one foot. The potential energy of a body which weighs $m$ pounds raised to a height $h$ above the Earth's surface is $m h$ foot-pounds. According to Article 149 this is the measure of the "potential energy of the body in the field of the Earth's gravitation."

The unit of power employed in the same system is the horse-power. It

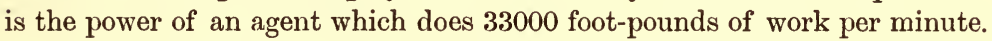

The units of the system here described are called "gravitation units" because the quantities expressed by the units at any place depend on the local numerical value of the acceleration due to gravity. They are unsuited to the general expression of dynamical equations, which are required to hold without modification not merely for relatively small bodies at a place on the Earth's surface but for bodies of any size in any place.

* It does not tend to simplicity that the writers who use these units also use the word "weight" for the quantity we call "mass," and the letter $W$ where we use $m$, and thus they write the above equation $P=(W / g) f$. In the notation of Article 281 this would be a valid equation in c. G. s. units, or in any units in which the unit force produces unit acceleration in unit mass, but all the letters except $f$ would have different meanings. Much confusion has thus been produced. 



\section{INDEX.}

The numbers refer to pages.

Acceleration, Definition of, 33 ; along normal to curve, 36 ; along normal to surface, 193; uniform, 33, 42; central, 51; radial and transversal, 60 ; of rigid body, 233; initial, 279 ; relative to rotating frame, 299,306 , 355

Angular momentum, 104

Angular velocity, 38,227

Apses, 65

Atwood's Machine, 177; Correction for inertia of pulley, 238

Axis, Principal, 229; Polar, 351, 355

Ballistic Balance, 96, 101, 258

Bodies, conception of, 85, 87, 142

Central forces, motion under several, 152

Central Orbits, 51, 62, 148

Centre, Instantaneous, 233, 235, 282, 289 ; of Oscillation, 237; of Inertia, 102 ; Motion of, 89, 107

Centroid, 14

Chain, Tension of, 120; Motion of, 301

Conservative forces, 125, 126; Examples of, 130; Motion of a Particle under, 147,183

Collision, 256

Conic, construction of from certain conditions, 57

Constraint, definition of, 114, 133 ; onesided, 173, 184

L.
Couples, 21; Theorems concerning, 22, 24,26

Curve, motion on a, 172, 182, 187, 188, 302

Curvature, initial, 280, 284

Cycloid, Isochronism of, 176

D'Alembert's Principle, 101, 107

Density, 88

Descent, Line of quickest, 173

Differential Notation, 35

Dimensions, 33, 34, 373

Displacement, 6,30

Elasticity, Theory of, 116, 257

Elements of planetary orbit, 159

Elliptic motion, 54; under gravitation, 97; of two bodies, 156; disturbed, 159

Energy, Kinetic, 105; Potential, 126; Equation of, 133; Conservation of, 136; Forms of, 137; Dissipation of, 136, 257; essential to Mechanics, 143; Problems illustrating, 293

Envelopes, of trajectories, 47, 53, 55

Equations of motion, 90, 106; Variational, 140, 143; of rigid body, 235 ; of chain, 306; impulsive, 91, 269, 309

Field of Force, 93; Potential energy of body in, 132

Force, Definition of, 89; Resultant, 89; Original notion of, 100; Internal, 106, 112, 139; Transmissibility of, 113; 
Bodily, 118; Positional, 135, 152; Motional, 135; Relativity of, 360

Foucault's Pendulum, 358

Friction, 114; Coefficient of, 115; on curve, 174, 187, 189; on surface, 192; in rolling and sliding, 239 ; Impulsive, 272

Frame of Reference, 5, 7, 93, 361

\section{Galilei, 45, 100}

Gravitation, 96; Constant of, 97, 374; Work done by, 128; Motion of two bodies under, 155, 156; Frame of reference for, 97, 366 ; Universal, 365; Units, 374

Gravity, 43, 94, 179, 352, 356; Work done by, 127; Free motion under, 148; Corrections of, 151, 353

Gyration, radius of, 230

Harmonic motion, 47; Composition of, 49; Production of, 149 ; of Pendulum, 178 ; of oscillating system, 287 ; resisted, 196; in line turning uniformly, 360

Heat, generated in collision, 257

Hertz, 141

Huygens, 236

Impulse, 90 ; internal, 110, 271; effect of, on elastic system, 261

Impulsive motion, $91,92,109,264,275$, 309

Inertia, 99; Moment of, 228; Ellipse of, 230

Inflexions, Circle of, 235

Initial Motion, 278, 308

Inverse Square, Law of, $56,58,63$

Kinematic Conditions, 241, 301, 302, 305 Kinetic Energy, 105; Change of, 134, 183, 189; produced by impulse, 138, 270 ; lost in collision, 262

Kinetic Reaction, 90, 103; of rigid body, 233 ; Moment of, 104

\section{Lagrange, 143}

Laws of Motion, 100

Mass, Notion of, 87; Determination of,
95; Measurement of, 98 ; essential to Mechanics, 101, 142

Measurement, Theory of, 371

Mechanics, Postulates of, 93, 361

Molecular hypothesis, 142

Momental equivalents, 230

Moment, of Localised Vector, 18; Theorem of, 21

Momentum, 90, 103; of rigid body, 232; Moment of, 104 ; Conservation of, 108, 147, 364; Change of, 264, 269; Problems illustrating, 293

Newton, 51, 56, 100, 154, 257

Oscillation, 178, 180, 286, 296

Osculating plane, of path of particle, 193

Parabolic motion, 44, 148

Parallelogram, of localised vectors, 17; of forces, 101

Particle, Notion of, 42, 86, 87; Dynamics of, 145, 172; inadequacy of, 142

Path, 32

Pendulum, Simple, 178; Conical, 191 ; Rigid, 236; Simple equivalent, 288; Foucault's, 358

Planetary motion, 97, 157

Plumb-line, 352, 354

Poisson, 259

Position, Determination of, 3

Potential, 129

Potential energy, 126; Localisation of, 132 ; of body in'field, 132; of gravitating system, 129; due to gravity, 130 ; of stretched string, 131

Power, 138

Pressure, 99, 114; on a curve, 172,183 , 187,189 ; on a surface, 193

Problem of Two Bodies, 154

Projectile, 44, 196, 199

Pull, of a locomotive, 174

Quantity, 372 ; of matter, 98

Range, of projectile, 46, 197

Reaction, of bodies in contact, 113, 117; of string in contact with surface, 120 ; initial, 278 
INDEX.

Relative, Coordinates, 39, 102; Motion, $39,40,154,184,263,293,298,305$, 355

Resistance, 115

Resisting medium, 195

Restitution, 259; between rigid bodies, 271

Rigid Body, Force applied to, 89, 112; general motion of, 111 ; in two dimensions, 227, 236; impulses applied to, 269 ; initial motion of, 282 ; oscillation of, 289 ; energy and momentum, 297

Rolling, 239, 241

Rotation, of frame, 184, 186, 298, 363; of rigid body, 227, 233; of the Earth, 351

Screw, right-handed, 19

Second, Mean Solar, 3, 368

Seconds Pendulum, 179

Sliding, 115, 239, 272

Speed, 33

Spheres, impact of, 258, 259, 261, 274

Spring, 121; Potential energy of, 132

Stability, of circular orbit, 68 ; of equi-

librium, 288; of steady motion, 295

Strain, 116, 122

Stress, 116,122 ; in a rod, 246
String, 119, 301; elastic, 120, 293; Potential energy of, 131

Tension, 119; of extensible string, 121; of chain at place of discontinuity, 301

Thread, 120

Time, Measurement of, 3, 366

Traction, Surface, 118

Trajectory, 32

Translation and Rotation, Independence of, 108

\section{Uniformity of Nature, 1}

Unit, of time, 3 ; of velocity, 33 ; of acceleration, 34 ; of mass, 98

Units, Systems of, 374

Vectors, Definition of, 8; Composition and Resolution of, 10, 15; Systems of, 13, 27, 28; Localised, 16; in parallel lines, 23

Velocity, Definition of, 30, 32; terminal, 196, 200; of rigid body, 232; relative to rotating frame, 299

Virtual Work, 139

Weight, 94, 352

Work, Definition of, 123; of internal forces, 128

Work Function, 125 
8 



RETURN Astronomy/Mathematics/Statistics/Computer Science Library

TO $\rightarrow 100$ Evans Hall 642.3381 LOAN PERIOD 1

\begin{tabular}{l|l|l}
\multicolumn{1}{l|}{ 7 DAYS } & & \\
\hline 4 & 5 & 6 \\
\hline
\end{tabular}

ALL BOOKS MAY BE RECALLED AFTER 7 DAYS

\section{DUE AS STAMPED BELOW}

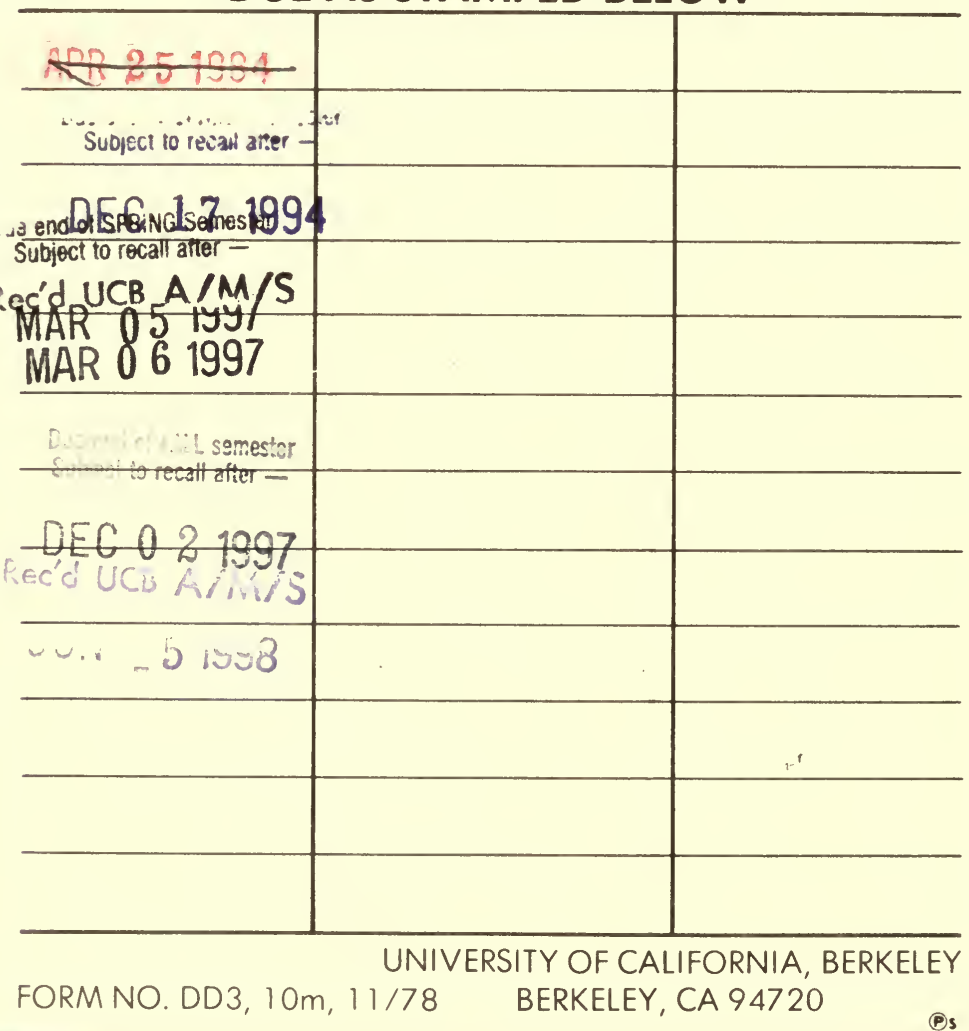


U.C. BERKELEY LIBRARIES

c037251915

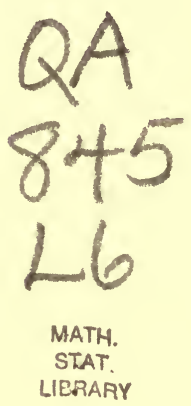

$-877$ 
